\title{
Final Report
}

\section{Integrated Intelligent Industrial Process Sensing and Control: Applied to and Demonstrated on Cupola Furnaces}

US Department of Energy Contract \# DE-FC02-99CH10975

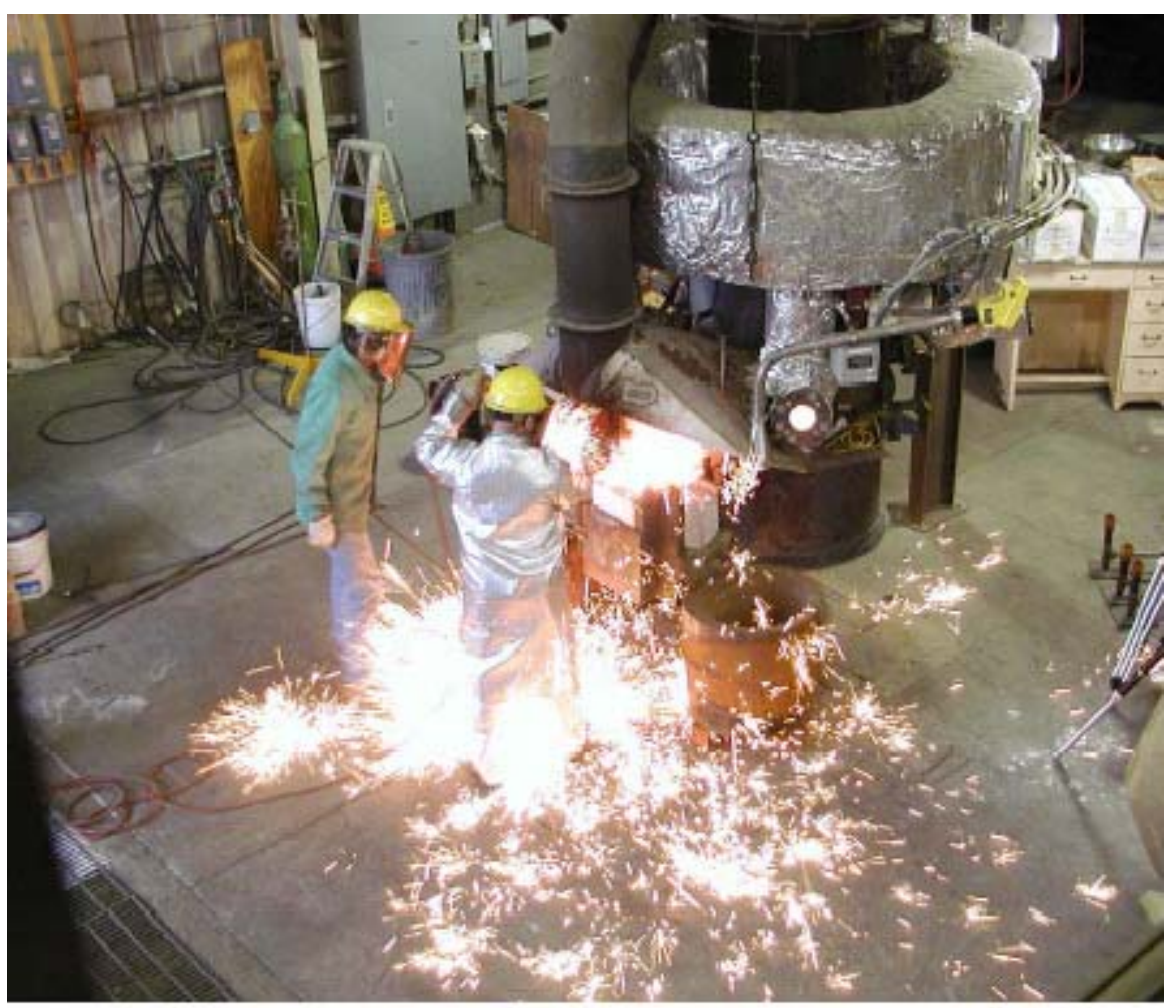

Tennessee Technological University

Utah State University

Idaho National Environmental Engineering Laboratory

Albany Research Center 


\section{Final Report}

\section{Integrated Industrial Process Sensing and Control: Applied to and Demonstrated on Cupola Furnaces}

US Department of Energy Contract \# DE-FC02-99CH10975

Center for Manufacturing Research, Tennessee Technological University Mohamed Abdelrahman, Principal Investigator

Roger Haggard and Wagdy Mahmoud

Utah State University

Kevin Moore

INEEL

Denis Clark and Eric Larsen

Albany Research Center

Paul King 
Section 1

Algorithms and Software Development 


\section{Table of Contents}

ALGORITHMS AND SOFTWARE DEVELOPMENT …...................................

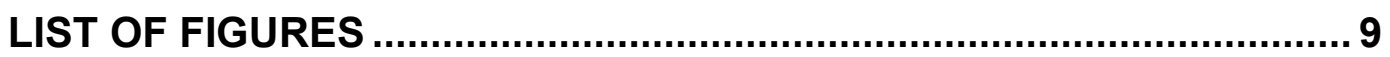

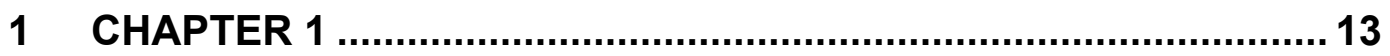

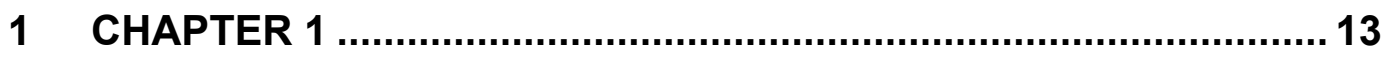

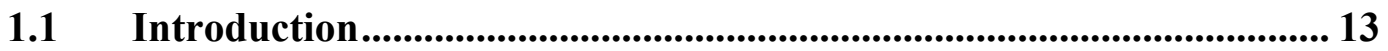

1.2 Objectives and Scope of the Project ............................................................ 15

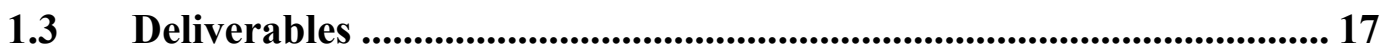

1.4 Project Organization, Administration, and Execution.......................... 18

1.4.1 Management Organization................................................................ 18

1.4.2 External Advisory Board .................................................................. 19

1.4.3 Coordination of Teams Efforts …………………………………...... 20

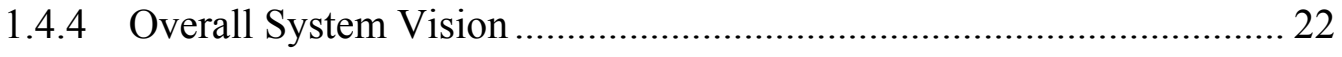

1.5 Evaluation based on Proposed objectives:.............................................. 23

1.6 Summary and Report Organization............................................................. 26

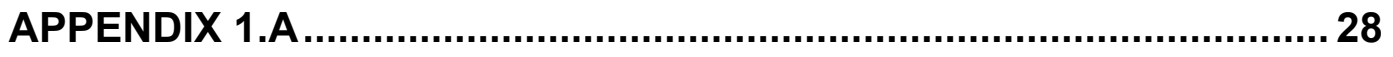

LIST OF PUBLICATIONS SUPPORTED BY THE PROJECT ................. 28 


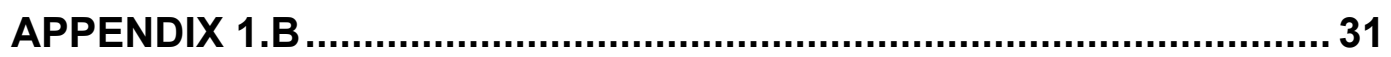

THESES SUPPORTED BY THE PROJECT ...................................... 31

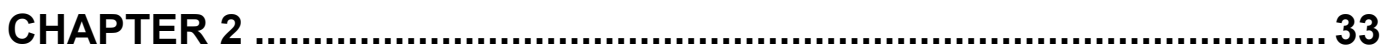

2 MOTIVATION AND OVERVIEW ............................................... 33

2.1 Motivation............................................................................................ 34

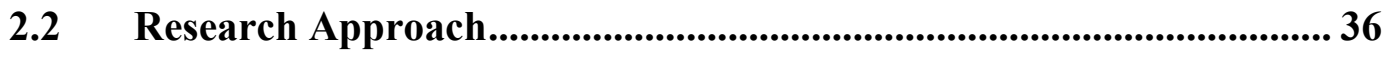

2.3 Multiple Sensor Fusion and Signal Validation...................................... 37

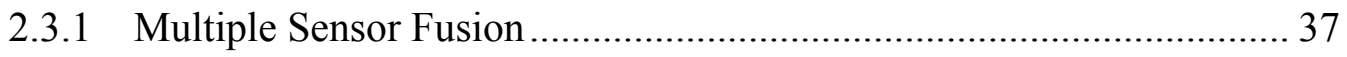

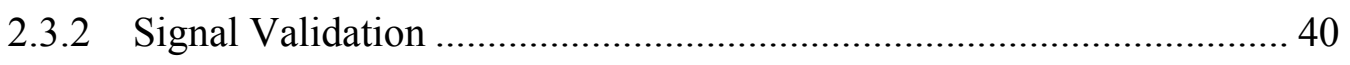

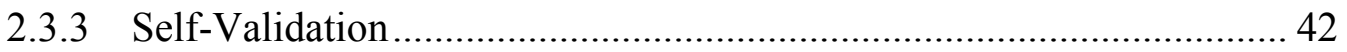

2.4 Adaptive controllers................................................................. 45

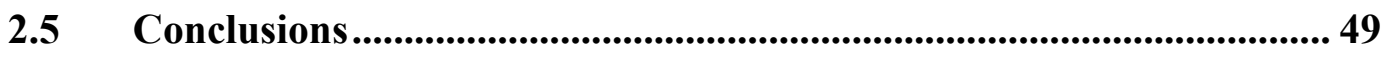

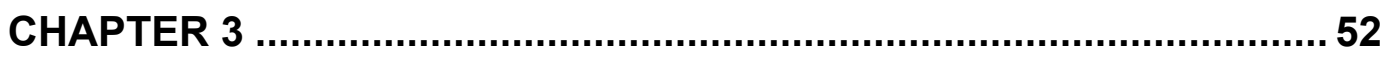

3 MULTIPLE SENSOR FUSION .............................................. 52

3.1 Parzen-like Methodology for Redundant Sensor Fusion ..................... 53

3.1.1 Description of Parzen Estimator ...................................................... 53

3.1.2 Estimation of Measurand Value from PDF ...................................... 54

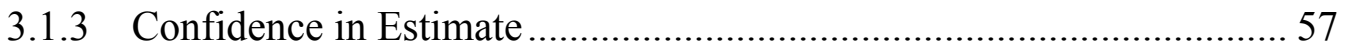

3.2 Considering Self-Confidence in Redundant Sensor Fusion ................ 59 
3.3 Application and Testing ......................................................................62 62

3.3.1 Results of the Sensor Fusion Methodology without Considering SelfConfidence 62

3.3.2 Results of the Sensor Fusion Methodology Considering SelfConfidence 65

3.4 A unified Framework for Multi-Modal Sensor Fusion ........................ 68

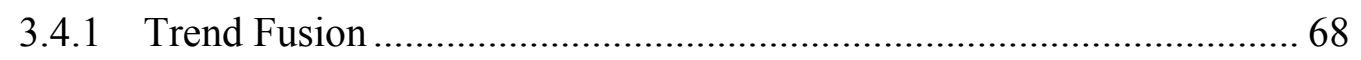

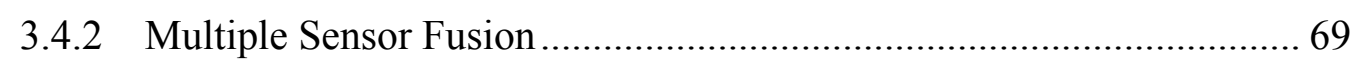

3.4.3 Fusion based on Trend .................................................................. 72

3.4.4 Confidence based on agreement among the Sensors ......................... 77

3.4.5 Measure of Fused Confidence ........................................................ 80

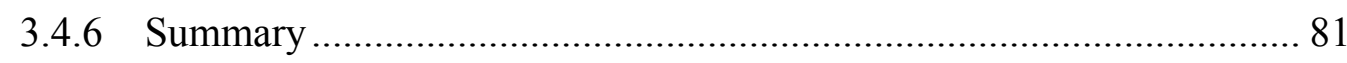

3.5 Fusion of Linguistic Sources ...................................................... 81

3.5.1 Linguistic Information on Trend............................................... 81

3.5.2 Fusion of Linguistic Information on the Measurand Value................. 84

3.6 Wavelet-Based Sensor Fusion for Data having Different Sampling Rates 88

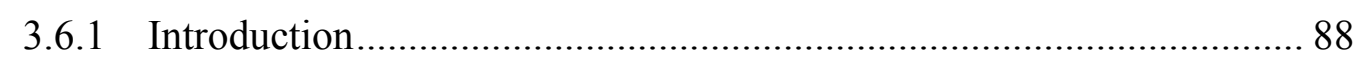

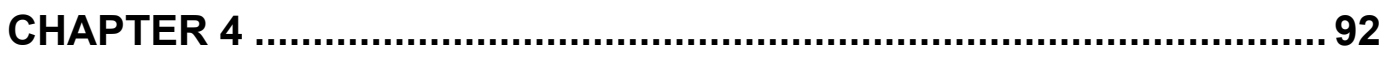

\section{INTEGRATION OF MULTIPLE SENSOR FUSION IN CONTROLLER DESIGN 92}

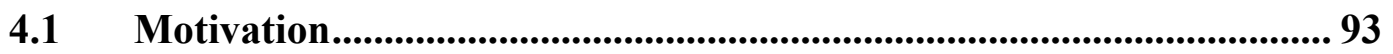

4.2 Controller Design ........................................................................................... 94 


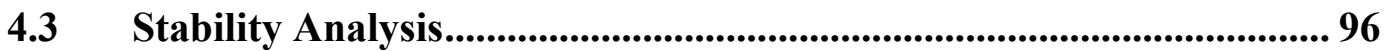

4.4 Fuzzy Controller ............................................................................... 104

4.4.1 Controller design................................................................... 105

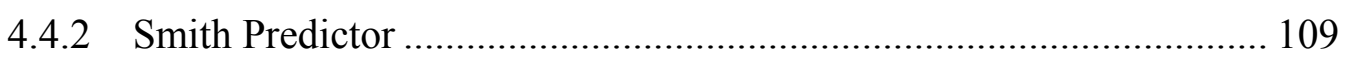

4.4.3 Integration of Sensor Fusion in Controller Design .......................... 110

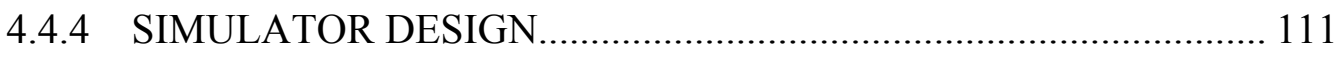

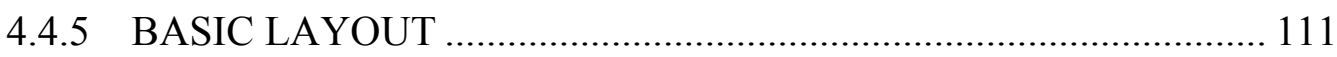

4.4.6 NOISE, DISTURBANCES, AND VARYING PARAMETERS ....... 112

4.4.7 RESULTS ........................................................................ 113

4.4.8 Integration of Sensor Fusion In Controller Design ........................... 118

4.4.9 VARYING MODEL PARAMETERS .......................................... 121

4.4.10 Varying Pure Time Delay of the CMR ........................................ 122

4.4.11 COMBINING ALL NOISES AND DISTURBANCES .................. 124

APPENDIX 4.A

CHAPTER 5 ............................................................................ 129

5 DEMONSTRATION PLANS ................................................... 129

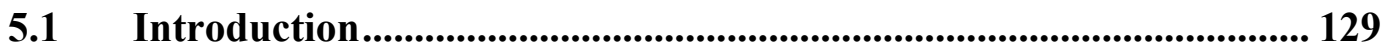

5.2 Setup of $I^{3}$ PSC for Demonstration Runs ...................................... 132

5.3 Results and Analysis of Demo Runs ..................................................... 134

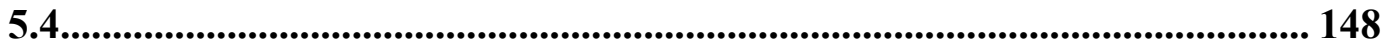

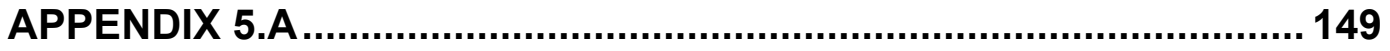


CHAPTER 6

6.1 SUMMARY AND CONCLUSIONS

REFERENCES

161

APPENDIX A: USER MANUAL . 166 


\section{List of Figures}

Figure 1-1: Graphical Representation of Project Organization .................................... 21

Figure 1-2 Detailed representation of Project Organization ......................................... 21

Figure 1-3 Overall System Vision for I3PSC applied to Cupola Furnaces ..................... 23

Figure 2-1 Schematic Diagram of a Feedback Control System.................................... 33

Figure 2-2 A Feedback Control System with Multiple Sensor Fusion ............................ 35

Figure 2-3 Block Diagram of Proposed System ........................................................... 37

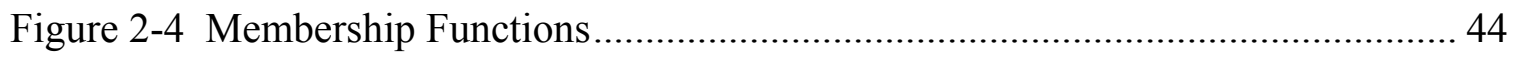

Figure 2-5 Block Diagram of the Self-Validation Technique ..................................... 44

Figure 3-1 Individual Gaussian Functions and the Cumulative PDF ............................ 54

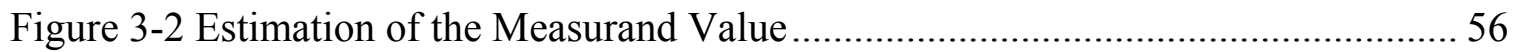

Figure 3-3 Comparison of Estimate with the Centroid ............................................. 57

Figure 3-4 Comparison of Estimate with Peak Values .............................................. 57

Figure 3-5 Measurand Estimate with High Confidence ............................................ 58

Figure 3-6 Measurand Estimate with Low Confidence ........................................... 59

Figure 3-7 Estimation of Measurand without Considering Self-Confidence ................. 60

Figure 3-8 Estimation of Measurand Considering Self-Confidence............................ 61

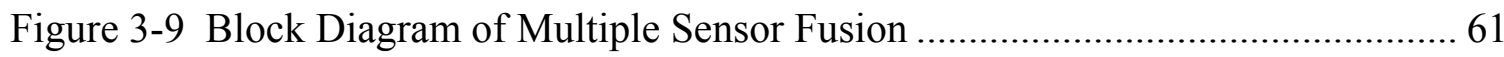

Figure 3-10 Estimated Value from PDF without Considering Self-Confidence ............. 63

Figure 3-11 Estimated Measurand Value Using Average Method................................ 64

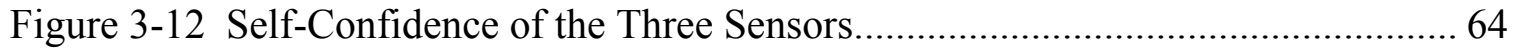

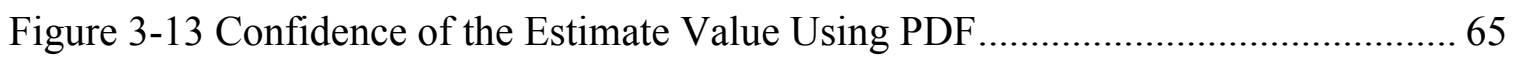

Figure 3-14 A Closeup that Shows Effect of Not Considering Self-Confidence ........... 65

Figure 3-15 Estimated Value using PDF Considering Self-Confidence ....................... 66

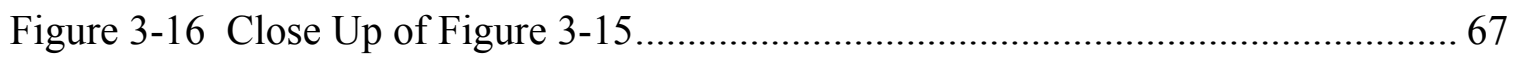

Figure 3-17 Confidence of the Estimate from PDF including the Self-Confidence........ 67

Figure 3-18 Multiple Sensor Fusion without trend information .................................. 70 
Figure 3-19 Confidence Plot..................................................................................... 71

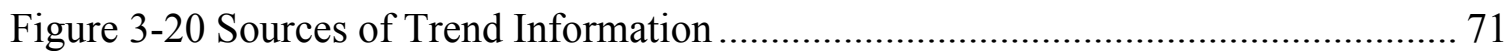

Figure 3-21 General Methodology for Sensor Fusion using Trend................................ 74

Figure 3-22 Distributions of Trends and Fused Trend..................................................... 75

Figure 3-23 Distribution of Temperatures at the previous instant................................... 76

Figure 3-24 Final Distribution of Temperature ............................................................ 76

Figure 3-25 Multiple Sensor Fusion Considering Trend ............................................... 77

Figure 3-26 Distributions of Trend after accounting for agreement between sensor trends 78

Figure 3-27 Multiple Sensor Fusion after filtering ...................................................... 79

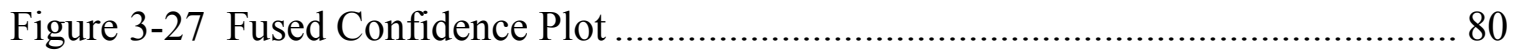

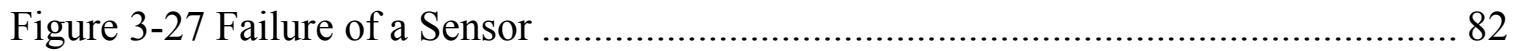

Figure 3-27 Trends after considering Linguistic Source …………………………....... 84

Figure 3-27 Sensor Fusion with Linguistic Trend Information ......................................... 84

Figure 3-27 Another Case of Sensor Failure .............................................................. 86

Figure 3-27 Multi-Modal Sensor Fusion with Linguistic Sources .................................... 86

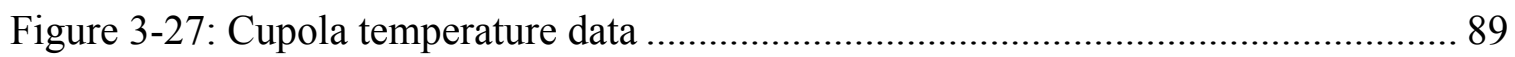

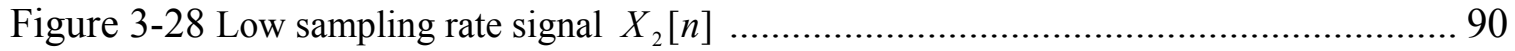

Figure 4-1 Wrong Estimate from the Multiple Sensor Fusion .......................................... 93

Figure 4-2 Schematic Diagram of the System with Sensor Fusion Integrated with the

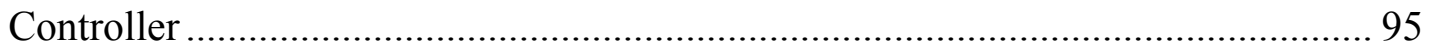

Figure 4-3 Uncertainty in the Estimate from Multiple Sensor Fusion........................... 100

Figure 4-4 Region of Stability .................................................................................... 101

Figure 4-5 Membership functions of the error in melt rate (eMRate) ............................ 108

Figure 4-6 Membership functions of the change in error for the melt rate (deMRate) .. 108

Figure 4-7 Implementing a Smith predictor.................................................................. 109

Figure 4-8 Simulation layout ............................................................................... 112 
Figure 4-9 Step response under ideal conditions ....................................................... 117

Figure 4-10 Step response with noisy outputs ........................................................ 118

Figure 4-11 Step response with input disturbances generated with square waves ......... 118

Figure 4-12 Response for melt rate confidence of 0.9 and -0.1 pulse for 600 seconds 120

Figure 4-13 Response for melt rate confidence of 0.5 and -0.1 pulse for 600 seconds . 120

Figure 4-14 The results of varying the model parameters ....................................... 122

Figure 4-15 Smith predictor with a +600 second time delay plant offset...................... 123

Figure 4-16 Smith predictor with a -600 second plant time delay offset....................... 124

Figure 4-17 System Performance Under Effect of All Disturbances .......................... 125

Figure 5-1 Configuration for Interfacing I3PSC with ALRC DAQ for Demo Runs...... 132

Figure 5-2 Control of Carbon Content, Run \#2 ….................................................. 135

Figure 5-3 Metal Stream Changes suggested by I3PSC for control of \%C for Run \#1 . 135

Figure 5-4 Individual Measurements and Fused Melt Rate for Run \#1 ....................... 136

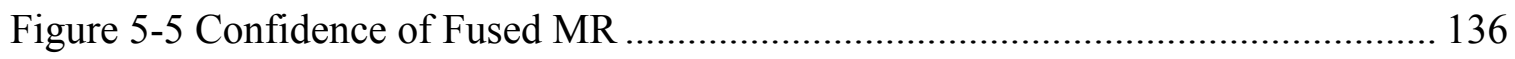

Figure 5-6 Individual Measurements and Fused Temperature ................................... 137

Figure 5-7 Confidence of Fused Temperature ...................................................... 137

Figure 5-8 Oxygen Enrichment for Temperature Control for Run \#1 .......................... 138

Figure 5-9 Blast Rate for Melt Rate Control for Run \#1 .......................................... 138

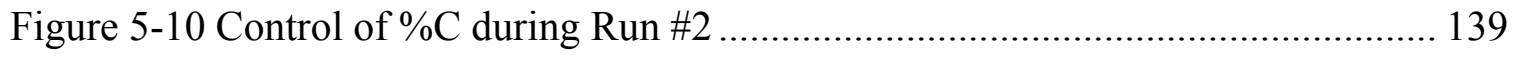

Figure 5-11 Changes in MR during Run \#2 ........................................................... 140

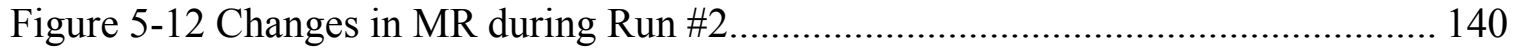

Figure 5-13 Metal Stream Changes control of \%C for Run \#2 ................................. 142

Figure 5-14 Forward Change in CMR to Achieve Large Change in MR (Run \#3) ....... 143

Figure 5-15 Changes in Metal Stream to compensate for Change in CMR .................. 143

Figure 5-16 Changes in Oxygen Enrichment (SCFM) during Run \#3 ......................... 144

Figure 5-17 Changes in Blast Rate (SCFM) during Run \#3 ..................................... 144

Figure 5-18 Control of Melt Rate during Run \#3 .................................................... 145

Figure 5-19 Changes in Iron Temperature deg F during Run \#3 ................................ 145 
Figure 5-20 Changes in \% Carbon during Run \#3 ..................................................... 146

Figure 5-21: Detection of Bridging in the Cupola-Changes in Exit Temperature ............................. 147

Figure 5-22: Detection of Bridging in the Cupola-Changes in Cupola Pressure.............................. 148

Figure 5-23: Opening the Tap-hole at ALRC Cupola .................................................. 149

Figure 5-24: Cupola Always Provides Operational Challenges ................................... 149

Figure 5-25: An Overview of the ALRC Research Cupola ...................................... 150

Figure 5-26: Manual Sampling and Quick Analysis of Molten Iron ........................... 151

Figure 5-27: Manual Measurement of Temperature of Molten Iron ............................ 151

Figure 5-28: Optical Pyrometers for Continuous Measurements of Iron Temperature` 151

Figure 5-29: A Dip Thermocouple for Continuous Temperature Measurement ........... 152

Figure 5-30: Charging Deck of the Cupola at ALRC …............................................. 152

Figure 5-31: Measurement of Melt rate, Chemical Composition, and Temperature .... 153

Figure 5-32: Remote Monitoring and Control of the Cupola during Demo Runs......... 153 


\section{Chapter 1}

\subsection{Introduction}

The cupola furnace is used by the iron foundry industry to melt scrap steel, cast iron and alloying materials into a consistent grade of iron for casting purposes. There are approximately 400 cupolas in the United States, which accounts for $70 \%$ of cast iron production [28]. With an industry estimate of $60 \%$ yield on castings, this equates to the direct production 1.204 million tons of carbon generating 4.412 million tons of carbon dioxide per year. This amounts to $1-2 \%$ of the total annual national production of green house gas [29]. The cupola has maintained its competitiveness for several reasons. Compared with competing technologies such as arc or induction melting, the cupola uses the energy in coal more efficiently because it does not have to go through the intermediate step of producing electricity, and the required coke making consumes little energy. The combustion products in cupola melting are easily contained, another advantage over arc melting. The cupola is a relatively simple device that can be made in many sizes to suit the molten metal needs of foundries of various sizes.

While cupola melting is simple in principle - burning coke with an air blast and melting metal - the actual physical and chemical details of the process are quite complex, and the phenomena occurring in the melt zone are difficult to measure directly 
because of the aggressive chemical environment that exists inside the cupola. Controlling these phenomena is desirable, however, for efficient energy use, for producing iron of acceptable quality, and for reducing the environmental impact of the melting process. The inevitable random variations in charge composition, blast effectiveness, and even local meteorological conditions, however, lead to a degree of variability in the cupola output. This variability can be reduced by expert operation of the cupola by experienced personnel. Reducing this variability is more important for some cast products than for others; where iron temperature and composition are crucial, as in the production of automotive parts, holding furnaces, sometimes hundreds of tons in size, are used to pool the output of one or more cupolas, and temperature and composition can be adjusted before the hot metal goes to the casting line [28].

The economic and environmental costs of this variability can be substantial. Iron that fails to meet specifications can cause substandard castings or even casting failure; the material may be re-melted, but the energy spent melting it the first time is wasted. The costs of installing, maintaining, and operating large holding furnaces to level out the variability is an additional cost of producing iron. Materials such as coke breeze (fines from the handling of coke) that would cause poor operation if charged from above can also be injected through the tuyeres for added energy; the incinerator-like nature of the cupola incorporates these, and even other hazardous wastes unrelated to cupola operation, into the relatively benign cupola outputs: cast iron, $\mathrm{CO}, \mathrm{CO} 2$, and slag. 


\subsection{Objectives and Scope of the Project}

The purpose of the project was to develop and demonstrate an intelligent, integrated industrial process sensing and control system, or $I^{3} P S C$ for short, for the foundry cupola, the primary industrial process used for producing cast iron. However, the $I^{3} P S C$ is a generic, enabling, cross-cutting technology that can be broadly applied to advanced process sensing and control problems in the ferrous metal casting industries as well as in other industrial environments. The project addressed two main objectives:

A. Development of a generic architecture for the integrated, intelligent industrial process sensing and control system. The proposed $I^{3} P S C$ architecture is characterized by

- Intelligent signal processing capabilities and sensor fusion methodologies,

- Intelligent algorithms for hybrid model fusion,

- Methodologies for integrating intelligent signal analysis and sensor and model fusion algorithms with intelligent model-based control methodologies,

- An object oriented generic architecture for integrating all system components.

- Implementation of the intelligent signal processing and sensor fusion algorithms through hardware realization using reconfigurable logic,

B. Demonstration of the application of $I^{3} P S C$ to the specific industrial setting of cupola iron melting furnaces). The demonstration will include: 
- Testing of the developed algorithms using experimental data, and static and dynamic models available from a production cupola and the ALRC research cupola,

- Implementation of the developed algorithms on the 18-inch research cupola at DOE’s Albany Research Center (ALRC),

- Testing the developed $I^{3} P S C$ for regulations of melt rate, temperature, and selected iron composition on the ALRC experimental cupola furnace. 


\subsection{Deliverables}

The final goal of this project was the development of a system that is capable of controlling an industrial process effectively through the integration of information obtained through intelligent sensor fusion and intelligent control technologies. The industry of interest in this project was the metal casting industry as represented by cupola iron-melting furnaces. However, the developed technology is of generic type and hence applicable to several other industries. The system architecture was divided into the following four major interacting components:

1. An object oriented generic architecture to integrate the developed software and hardware components

2. Generic algorithms for intelligent signal analysis and sensor and model fusion

3. Development of supervisory structure for integration of intelligent sensor fusion data into the controller

4. Hardware implementation of intelligent signal analysis and fusion algorithms

Table 1-1 lists the deliverables as they appeared in the proposal. They are listed here for completeness. As will be illustrated in the current report, the objectives stated in the proposal have been achieved. 
Table 1-1 I3PSC Project Tasks

\begin{tabular}{|c|c|}
\hline Task \# & Description \\
\hline 1 & $\begin{array}{l}\text { Generic Structure for Integrating Sensor Fusion and Control } \\
\text { System Components }\end{array}$ \\
\hline 2 & $\begin{array}{l}\text { Algorithms for Intelligent Signal Preprocessing, Multi-Modal } \\
\text { Sensor Fusion, Model Fusion, Sensor and Model Fusion }\end{array}$ \\
\hline 3 & $\begin{array}{l}\text { Re-configurable Logic Implementation for Intelligent Signal } \\
\text { Processing and Sensor Fusion Algorithms }\end{array}$ \\
\hline 4 & $\begin{array}{l}\text { Algorithms for Integration of Intelligent Sensor Fusion Data into } \\
\text { the Controller }\end{array}$ \\
\hline 5 & Prototype Implementation and Testing for ALRC Cupola \\
\hline
\end{tabular}

1.4 Project Organization, Administration, and Execution

\subsubsection{Management Organization}

This project represented a model for collaboration between technical developers, industry oversight, and end users as represented in Figure 1. The technical expertise was provided by:

1- Tennessee Technological University ((TTU) as the main contractor, 
2- Utah State University (USU) as a subcontractor,

3- Idaho National Environmental and Engineering Laboratory (INEEL) as a subcontractor, and

4- Albany Research Center (ALRC) as a subcontractor.

The industry oversight was provided by American Foundry Society (AFS) and the end users represented by General Motors (GM).and US Pipe.

Detailed management organization of the project technical development team is shown in Figure 2. The main tasks of the project are listed in Table 1 along with the groups responsible for the completion of each task.

\subsubsection{External Advisory Board}

In a kickoff meeting in Detroit in January 1999, TTU, USU, INEEL, GM and AFS agreed to create an external advisory board for the project under the direction of the AFS. This board had representatives from foundries and industrial control companies and will serve to assess the progress of the project and the achievement of its goals. Collaborators arranged several meetings with the advisory board over the period of the project. These meeting were coordinated with meetings of the AFS cupola steering committee. The purposes of these meetings were to review the status of the completion of the project, exchange ideas among collaborators and external advisory board and to inform the industry about the benefits of the technology and its potential advantages. 


\subsubsection{Coordination of Teams Efforts}

Coordinating the efforts among the teams working on the project was the responsibility of the principal investigator. This coordination was achieved through continuous communication through:

- Use of Email and Phone, as needed, to address individual teams concerns, problems or achievements. Emails could be addressed to a specific team leader or to the PI.

- Conference calls were scheduled as needed, among TTU investigators and investigators from USU and INEEL to discuss the progress and coordinate the efforts.

- The meetings with the advisory boards were used to have technical meeting among the technical developers at TTU, USU, INEEL, and ALRC.

- A web site and ftp sites were developed where technical materials were exchanged among the collaborators. (www.ece.tntech.edu/I3PSC) 


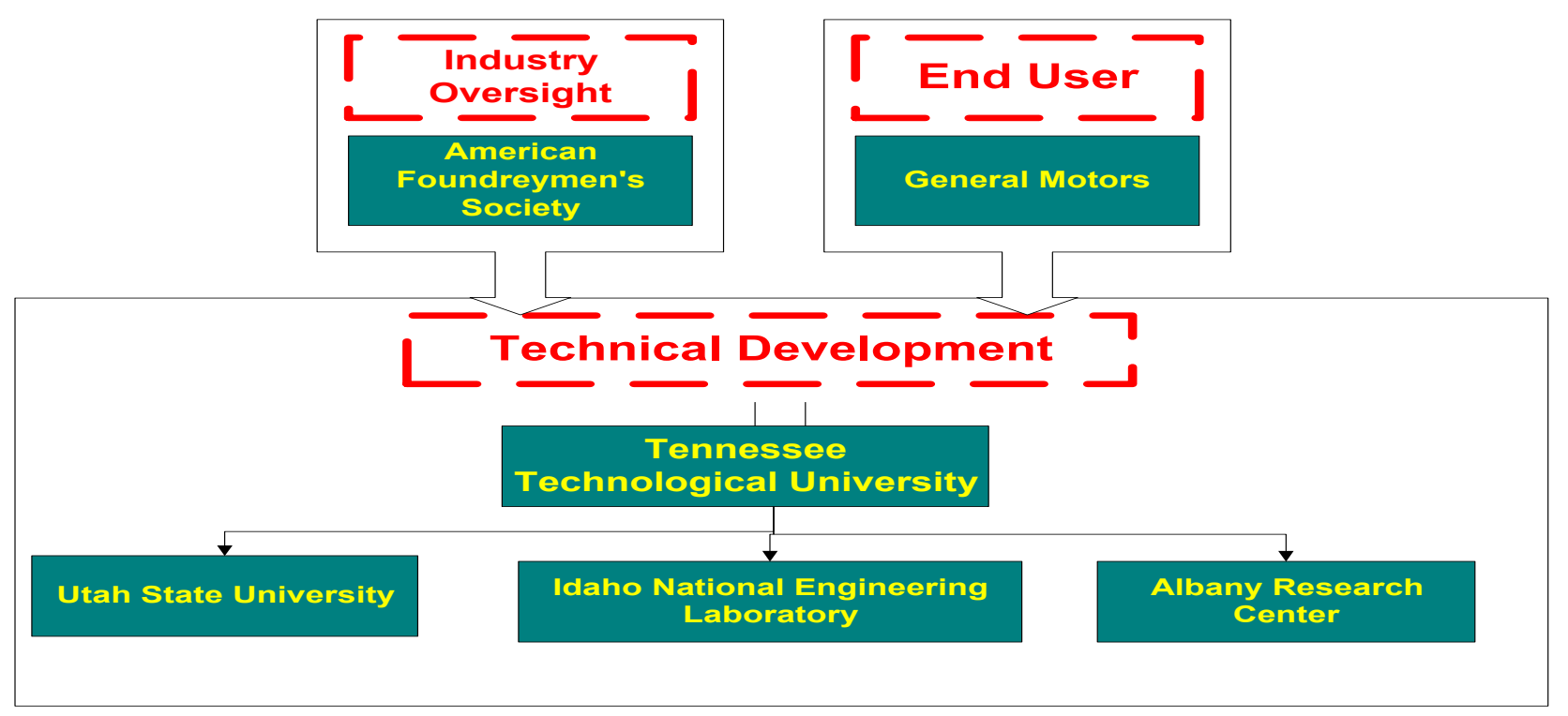

Figure 1-1: Graphical Representation of Project Organization

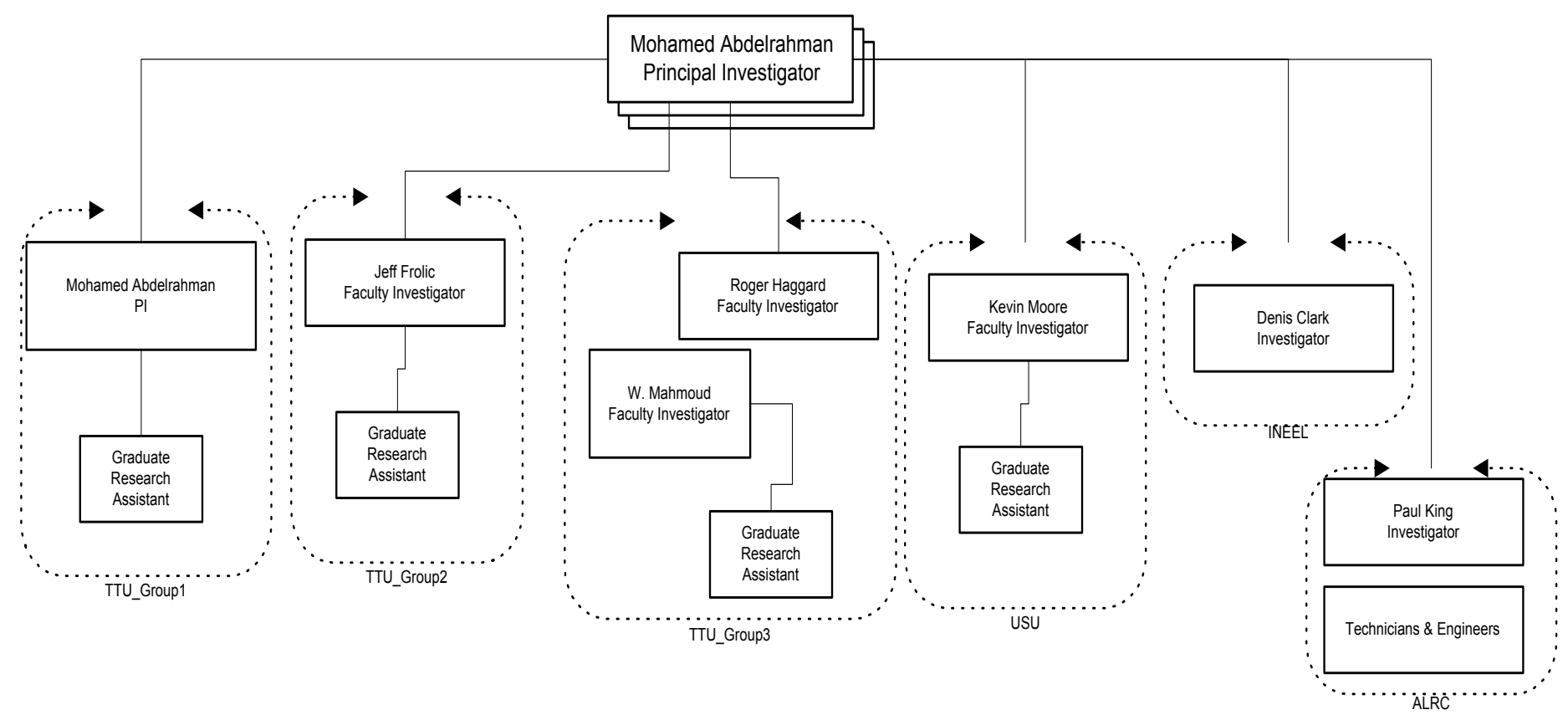

Figure 1-2 Detailed representation of Project Organization 


\subsubsection{Overall System Vision}

Figure 3 shows a schematic of the different components developed in this project and how they are tied together for a cupola furnace application. The system is generally divided into online and offline components. The offline analysis component is aimed at analyzing the data collected during a heat and plan for next heats. This analysis is based mainly on cupola models. The model currently in use is the model developed by the American Foundry Society (AFS). However, the developed tool can be adapted to accept other models as they become available. The online component is aimed at actual analysis and control of the cupola furnace during a heat. It is composed of several modalities that handle the data as it is collected from the sensors, fuse this data along with other data that are pertinent to the cupola operation such as data coming from other sensors, virtual sensors, models, or expert systems (MMSF). The data is then fed to an intelligent controller, which decides based on the required operational parameters, which input variables to manipulate. The required operational parameters are fed to the controller using the planner. The planner can be used, by the user, to plan, offline, how the heat will be conducted. However, it can also be used online to make changes, as appropriate, to the heat plan. 


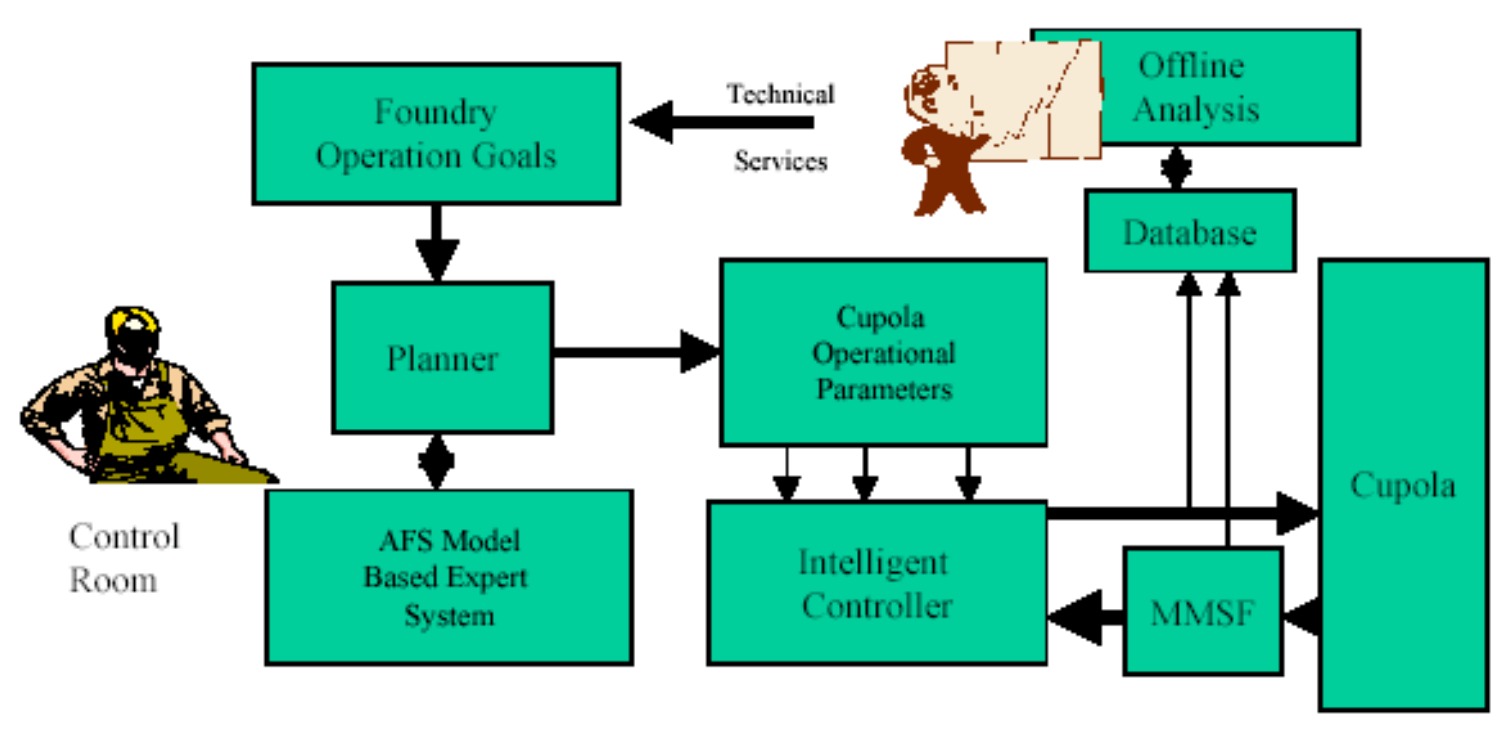

Figure 1-3 Overall System Vision for I3PSC applied to Cupola Furnaces

1.5 Evaluation based on Proposed objectives:

In fulfilling the proposed objectives, the following has been achieved:

- Innovative sensor fusion algorithms based on a new concept has been developed, implemented and tested. These Algorithms allow for the fusion of quasiredundant sensors data and produces a best estimate and a parameter indicating the degree of confidence in the measurement. The algorithms were presented in conference publication namely, the American Control Conference (ACC), and 
published in the prestigious journal of IEEE Transactions on Instrumentation and Measurements.

- The developed algorithms were improved to incorporate trend information as well as linguistic information. This allows for the fusion of information from sources other than physical sensors such as virtual sensors, models and expert system information.

- Generic algorithms for the integration of sensing and control based on the previously developed algorithms for sensor fusion were developed and implemented.

- The developed generic algorithms for sensor fusion and integration of sensing and control represent advances in basic science. The researchers have also presented application specifically for cupola furnaces. These results were presented at professional conferences with audience interested in the advancement of melting methods.

- Algorithms for the implementation of the sensor validation and multiple sensor fusion algorithms on hardware were developed, simulated and tested .

- A generic data structure and an object oriented based software package were developed for the incorporation of the different algorithms. The current package incorporates the following modules:

- A Data Acquisition modality for interfacing with existing data acquisition system in a cupola or other industrial plant, 
- A planner modality where a plan for the cupola heat can be developed,

- A sensor fusion modality,

- A virtual sensors modality for predicting values of some important parameters based on other system measurements,

- A controller modality for producing suggested values for the manipulated variables based on the system requirements,

- A monitoring modality for monitoring trends of specific variables and alerting operator when certain patterns take place.

The software and data structure were designed to allow for easily incorporating other modalities and modifying the existing ones.

- The integrated system was successfully demonstrated on a research cupola facility in Albany Research Center, Albany, Oregon. The demo involved successful interface of the developed system to the existing DAQ system, monitoring and controlling the main parameters of the cupola furnace, namely, molten iron temperature, melt rate and Carbon composition using manipulated variables, namely, oxygen enrichment, blast rate, steel/cast ration and coke/metal ratio. The control system ability to achieve and maintain operational parameters as well as reject disturbances and minimize transition periods was illustrated.

A list of the papers supported by the project and published in refereed journals and conferences is presented in Appendix 1.A. A list of academic theses supported by 
the project is listed in Appendix 1.B. Other information pertaining to the project achievements were presented in previous reports [32] - [34].

\subsection{Summary and Report Organization}

In summary, the project has achieved all the proposed objectives starting from development of algorithms for sensor validation and fusion, integration of sensing and control, development of a package for integrating system components and a proof of concept of using FPGA to implement sensor fusion algorithms. The project has supported the development of basic science in the form of publications in professional refereed journals and conferences as well as practical and applied science with reference to cupola furnace as evidenced by demonstration using cupola furnace data and models and actual demo plans on a research cupola.

This report is divided into two sections. Section 1 describes a subset of the developed algorithms and results of demonstration runs. Chapter 1 summarizes the project organization, objectives and results of the project. Chapters 3 and 4 provide a description of the basic algorithms that were developed for sensor fusion and control. More information can be found in the published work listed in Appendices 1.A and 1.B. A brief description of the developed software package is provided in the form of a user manual in Appendix A of Section One. Section 2 describes the hardware implementation. Chapters 1-3 describe work accomplished during project years. Chapter 4 gives a summary of the 
work accomplished and future recommendations. The section ends with Appendices that describes more details of the algorithms hardware implementation. 


\section{Appendix 1.A}

\section{List of Publications Supported by the Project}

1. Jeff Frolik, Mohamed Abdelrahman, and Param Kandasamy, "A confidence Based Approach to the self-validation, fusion and reconstruction of quasiredundant sensors," IEEE Transaction on Instrumentation and Measurement., Vol. 50, No. 6, December 2001.

2. Mohamed Abdelrahman and Param Kandasamy, "Integration of Multiple Sensor Fusion In Controller Design," Accepted for Publication in the Transactions of Instrumentation Society of America, 2002.

3. Mike Baswell and Mohamed Abdelrahman, "Fuzzy Control Of A Cupola Iron Melting Furnace," To Appear in Transactions of American Foundry Society, $\underline{2003 .}$.

4. Mohamed Abdelrahman and Param Kandasamy, "Integration Of Intelligent Industrial Process Sensing and Control for Cupola Iron Melting Furnace," in proceedings of the $7^{\text {th }}$ Mechatronic Forum International Conference, Atlanta, $6-$ 8 September, Atlanta, GA, 2000. 
5. Mike Baswell and Mohamed Abdelrahman, "Fuzzy Control Of A Cupola Iron Melting Furnace,” AFS Congress, Kansas City, MO, May 2002.

6. Min Luo and Mohamed Abdelrahman, "Wavelet-Based Sensor Fusion for Data with Different Sampling Rates," ," in Proceedings of American Control Conference, Washington D.C., June 2001.

7. Mohamed Abdelrahman et al, "A Methodology For Fusion Of Redundant Sensors," in Proceedings of American Control Conference, Chicago, IL, June 2000.

8. Jeff Frolik and Mohamed Abdelrahman, "Synthesis of quasi-redundant sensor data: a probabilistic approach," , " in Proceedings of American Control Conference, Chicago, IL, June 2000.

9. Steve Orth, Jeff Frolik and Mohamed Abdelrahman, "Fuzzy rules for automated sensor self-validation and confidence measure,, in Proceedings of American Control Conference, Chicago, IL, June 2000.

10. Vipin Vijayakumar and Mohamed Abdelrahman, "A convenient methodology for the hardware implementation of fusion of quasi-redundant sensors," Proceedings of 32nd SSST Conference, Tallahassee, FL, Mar 2000, pp. 349-353.

11. Param Kandasamy and Mohamed Abdelrahman, "A Methodology for Integrating Multiple Sensor Fusion in the Controller Design," in Proceedings Of $32^{\text {nd }}$ SSST conference, Tallahassee, FL, March 2000, pp. 115 -118. 
12. Mike Baswell and Mohamed Abdelrahman, "Intelligent Control of Cupola Furnaces," in Proceedings of the 34th SSST conference, Huntsville, AL, March 2002, pp. 435-440.

13. Wagdy Mahmoud, "Hardware Implementation of Automated Sensor Selfvalidation System For Cupola Furnaces", in Proceedings of 31st conference on Computers and Industrial Engineering, San Francisco, CA, Feb 2-4, 2003.

14. Mohamed Abdelrahman et al, "A Methodology For Multi-Modal Sensor Fusion Incorporating Trend Information", in Proceedings of 31st conference on Computers and Industrial Engineering, San Francisco, CA, Feb 24, 2003. 


\section{Appendix 1.B}

\section{Theses supported by the project}

1. Min Luo, Fusion of Multi-resolution Sensors using Wavelet Transform, Tennessee Technological university, September 2001.

2. Vipin Vijayakumar. A Methodology for Multi-Modal Sensor Fusion. June 2001

3. Parameshwaran Kandasamy. Development of Sensor Fusion Algorithms for Redundant Sensors and Integration in Controller Design, Tennessee Technological university, May 2000.

4. Avinash Seegehalli. Multi Dimensional Data Structure for Cupola Furnace Information Processing, USU, 2000.

5. Jie Chen. Detection and Extraction of Parallel Hardware During C to VHDL Translation, Tennessee Technological University, May 2003.

6. Sobha Sankaran. Hardware/Software Codesign - Efficient Algorithms for Hardware Synthesis from C to VHDL, Tennessee Technological University, 2001.

7. Srikala Vadlamani. Comparison of Cordic Algorithms Implementation on FPGA Families, Tennessee Technological University, 2002 


\section{Chapter 2}

\section{MOTIVATION and OVERVIEW}

Feedback control systems have gained extreme importance in modern engineering world. Feeding back the output has made it possible for systems to perform their assigned tasks with better reliability. A number of control techniques have been developed to achieve the desired response from a feedback control system. These techniques achieve accurate tracking of the system output along a specified reference value [1]. There are also robust techniques that can achieve good performance even if the system is not modeled accurately [2]. Robust feedback control system also reduces the sensitivity of the system with respect to the system parameter variation and external disturbances. A schematic diagram of a general feedback control system is shown in Figure 2-1.

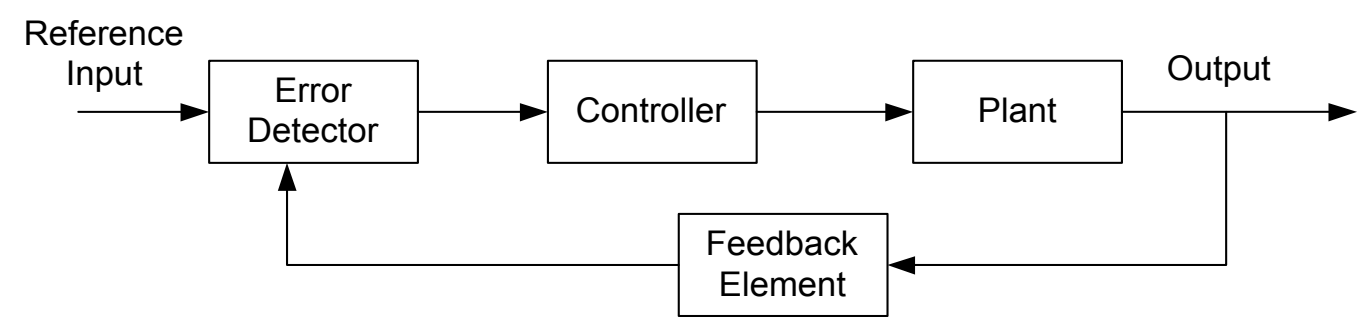

Figure 2-1 Schematic Diagram of a Feedback Control System 


\subsection{Motivation}

Sensors are used to measure and feedback output data in feedback control systems. The feedback data are used to decide the necessary control action. The performance of a feedback control system depends heavily on the reliability of the sensors' readings. There are different reasons why the sensor data may not be reliable. These reasons include:

1) Sensors may be prone to high levels of noise and disturbances during measurement and transmission of the data;

2) Sensors' characteristics may vary with changes in environmental parameters, such as the temperature, humidity, or due to aging;

3) Accurate measurement of some variables may not be possible due to the physical nature of the process; and

4) Failure of electronic circuitry of the sensor.

There are several methods available to increase the reliability of process measurements using redundant sensors. The redundancy may be achieved through physical sensors, analytical sensors, or inferential sensors. Analytical sensors depend on a model of the physical process to estimate the value of the intended system parameter. Inferential sensors utilize other output variables to infer estimates for different variables. Techniques such as signal validation and multiple sensor fusion are usually used to get a better estimate for the desired variable. These techniques will be discussed in detail in 
Chapter 2. A schematic diagram of a closed loop system that utilizes the multiple sensor fusion is presented in Figure 2-2.

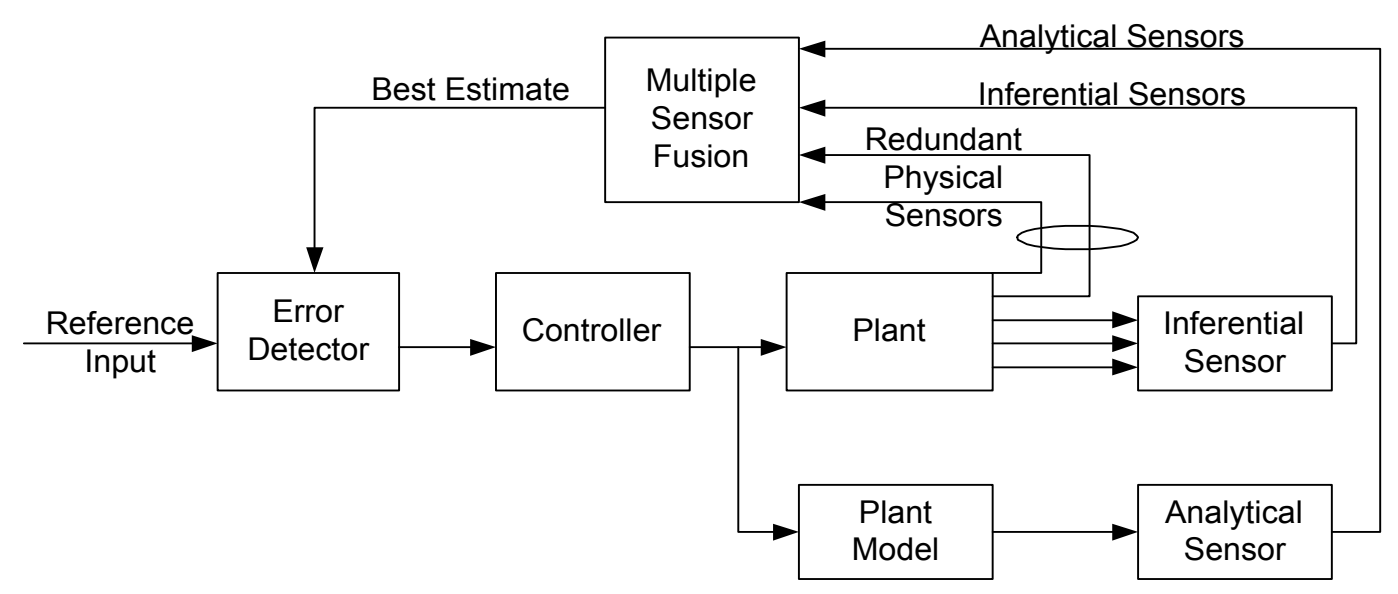

Figure 2-2 A Feedback Control System with Multiple Sensor Fusion

The above techniques are used to reduce the sensitivity of the system performance with respect to sensors' failures. This is accomplished by not relying on a single sensor measurement. For multiple sensor fusion or signal validation techniques to work satisfactorily, certain conditions need to be satisfied. These include, for example, the availability of redundant sensors, an accurate plant model, or known relations between variables. Since most techniques still rely back on other sensors for the feedback value, there will be situations where the feedback value is not reliable. A measure for the performance of the signal validation or multiple sensor fusion technique needs to be developed and utilized in the controller structure. 


\subsection{Research Approach}

The research focus of this report is to develop a methodology to prevent the performance degradation of an automatic control system due to unreliable sensor data. The suggested solution to the problem is twofold:

1) The development of a multiple sensor fusion algorithm that can produce a best estimate and reliability measure for the estimate of the sensor data.

2) The development of a controller structure, which utilizes the estimate and the reliability measure to change its performance, so as to prevent costly mistakes.

The methodology developed should reduce the sensitivity of the system to the sensor data when the reliability of the sensor data is found to be low. This is achieved by changing the controller's dependability on the sensor signal according to the reliability measure from the multiple sensor fusion. A block diagram of the feedback control system to be developed is shown in Figure 2-3. It resembles Figure 2-2, but for the additional flow of information, the confidence, from the multiple sensor fusion block to the controller. 


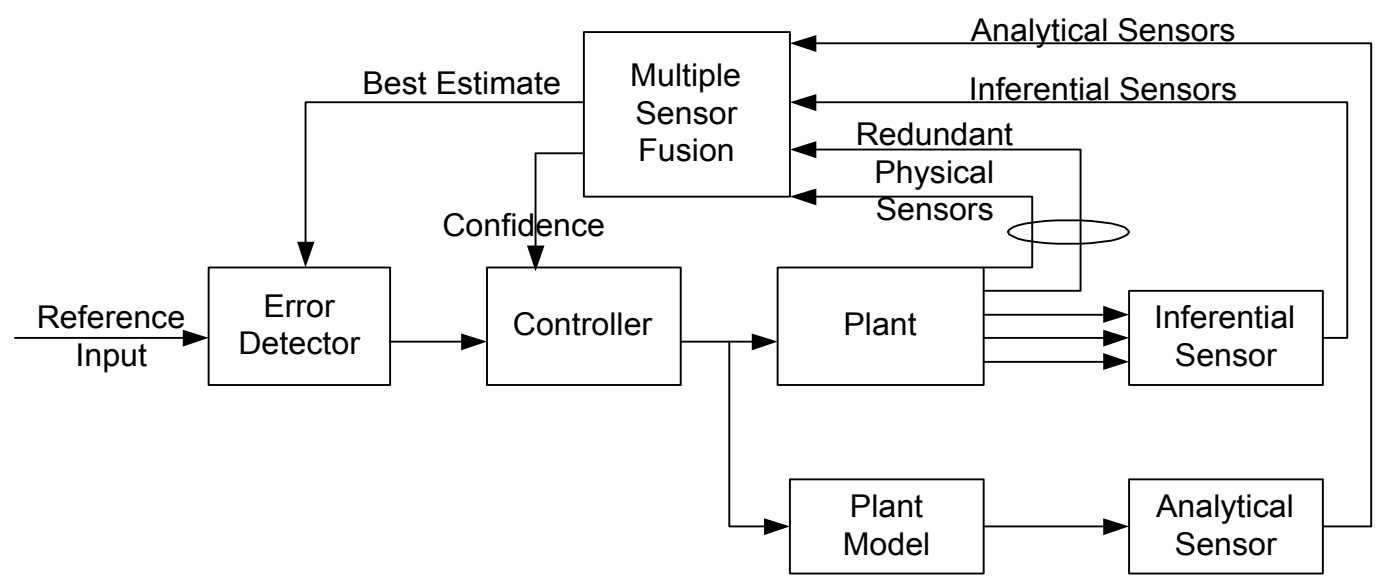

Figure 2-3 Block Diagram of Proposed System

The problem considered in this report is that the performance of the feedback controller degrades when the feedback signal from the sensor data is unreliable. The problem of increasing the reliability of the feedback signal was tackled in many ways. The most common method used to increase the reliability of the feedback signal is multiple sensor fusion. One other approach is to check the reliability of each sensor by using self-validation. In this chapter, a quick review of some of these multiple sensor fusion and self-validation techniques is presented. A basic overview of adaptive controllers and some adaptive methods are also discussed.

\subsection{Multiple Sensor Fusion and Signal Validation}

\subsubsection{Multiple Sensor Fusion}

Sensor fusion is defined as the method to fuse or manipulate information from different sensors and come up with one value of interest. These sensors may measure the 
desired measurand or may measure different values, which should be combined to get the required information. If the different sensors are measuring the same quantity, then these sensors are called redundant sensors. In this report multiple sensor fusion is constrained to mean only the fusion of redundant sensors.

There are many reasons why multiple sensor fusion is used. Combining several sensors' data will give more accurate information of a measurand improving the reliability of measurement data. The measurement data become less sensitive to noise and disturbances that might not affect all the sensors, when many sensors are used. Efficiency and performance of the measured data are enhanced [3].

Several techniques are available to fuse the values from the redundant sensors [4]. The most obvious approach is to find the average of the sensor data. In this case, however, the estimate will be affected by the invalid sensor data. A simple improvement to this was to have a weighted average of the redundant information. A weight is given to each sensor depending upon a threshold. The threshold for the current decision is usually the previous estimate. This helps in eliminating the spurious data. The choice of threshold is important in this method. If the process data has large variations between adjacent values, the threshold technique may result in removing valid sensor data.

Kalman filtering technique is generally used for sensor fusion where Gaussian noise exists. The performance of the Kalman filter technique depends upon the accuracy of the system model [Chapter 12, [3]]. It gives better results if there exists a linear model to the system and if both the system and the sensor noise can be modeled as Gaussian 
noise. Finding an accurate model for systems is not always possible in many cases and most of the real time systems are nonlinear.

A method developed by Luo and Lin [5] finds the estimate from the multiple sensor fusion of only consensus sensors. The method first eliminates those sensors' data that are likely to be erroneous. This is accomplished by using a probability density function(PDF) around each sensor's data. This PDF around each sensor's data is used to find the distance from other sensors' data. This distance measure is stored as a matrix for each sensor, which are combined later to find a combined matrix from which the optimal fusion estimate is found. This method of having an individual matrix and forming a combined large matrix that is reduced to get optimal value is called the Bayesian approach [5].

Many others also approach the multiple sensor fusion problem by finding the best combination of sensors that are to be fused. The search is based on the distance between the sensors, each sensor's failure rate and its previous data. Algorithms like neural based search and genetic algorithms were used [Chapter 10, [3]]. The combination of selected sensors is then usually averaged to find the estimate. The performance of these approaches depends on the search algorithm. These are best suited for decision-making sensor fusion problems.

As an extension to the above search first and then fuse, multiple sensor fusion is implemented using approximate agreement approach in [Chapter 11, [3]]. The approach first establishes an agreement set on each sensor data. This is done by each sensor 
broadcasting its value to other sensors. Each sensor then forms the agreement set based on the distance from other sensor data. This agreement set helps in eliminating invalid sensor data and find the estimate on which most sensors agree. This approach requires $3 t+1$ sensors with $t+1$ giving accurate reading, where $t$ is the number of faulty sensors. The mean of the agreement set after removing $t$ lower and $t$ higher data gives the estimate. This algorithm is again best suited for binary decision-making (Target or no Target).

Multiple sensor fusion techniques use the redundant data and come up with one value. Each sensor data have an effect on the final estimate. A failed sensor will have adverse effect on the estimate if not removed. So it is necessary to validate the sensor data before fusing the redundant data and remove the sensor. The next section discusses some of the signal validation techniques that achieve this.

\subsubsection{Signal Validation}

Signal validation is a technique by which the sensor's signal is validated for its accuracy. Signal validation may involve all or one of the following: detection, isolation, and characterization of faulty sensors [6]. Most of the initial researches depended on finding an additional measure for the sensor, either by having redundant sensors or by producing an analytical redundancy to the sensor data by using a model for the process.

A detailed survey of the signal validation using redundant sensors based on statistical methods is described in Ray and Luck [7]. The statistical approach is based on the difference between the current sensor data and other redundant sensor data. Fuzzy 
logic (FL) is also used for signal validation using redundant sensors. The advantage of using FL is that the strict boundary posed by the numerical sensor data can be replaced with linguistic terms [6].

Analytical redundancy is used in situations where physical redundancy is not possible. Analytical redundancy is created using a model for the process. Neural networks have been used to create the analytical redundancy using historical data of the process [8]. Combinations of fuzzy and neural systems have been used to create analytical redundancy for a specific sensor. Another type of redundancy is created using inferential sensors. The redundancy is obtained from using sensors that measure other variables and the relationship between the variables and the variable of intent. Genetic algorithm is used to find empirically the variables best suited for use in the inferential redundancy while neural based fuzzy system is trained to estimate the monitored sensor signal [9]. These analytical and inferential redundancy are then treated as physical redundancy and used in validating physical sensor data.

There are many difficulties in creating physical or analytical redundancy for sensor signals like increased cost, complexity in hardware implementation for the sensors, and uncertainty in modeling the plant. Moreover, the reliability of the sensors that are used for the redundant measurement cannot be assumed. Hence, few researchers' started to work on the validation of sensors using data from the sensor that is being validated. This is discussed in the next section. 


\subsubsection{Self-Validation}

The technique of validating a sensor using the historical data from that sensor alone is called self-validation. These self-validating sensors are called intelligent sensors and many researches are taking place to create intelligent measurements. Yang and Clarke in their section [10] have defined the self-validating sensors, their rationale, and how they can evolve into intelligent measurements.

Initial research in this area started by considering the invalid data of sensors as noise and hence using filtering techniques for the self-validation. Kalman filter was found to give good results for self-validation. A detailed description of self-validating sensors was given in Henry and Clarke [11]. The research by Tsai and Chou [12] uses the correlation of system dynamics with multistep readings of a sensor.

Using historical data of the sensors for self-validation was used by Mercadal [13]. This reference paper uses the historical data to create an analytical model for the sensor depending upon its previous values. The actual sensor data are then validated by comparing it with the value predicted from the model.

The paper [14] develops a fuzzy based self-validating algorithm based on the validated historical data of the sensor. The algorithm developed in this paper is described in detail as it is implemented and used as a part in this report. In this paper a measure of reliability of the sensor data, called self-confidence, is obtained. Self-confidence is a measure of the agreement between the characteristics of current sensor data and historical 
sensor data that are deemed valid. This self-confidence can be used for the detection and isolation of faulty sensors.

An FL-based system is developed based on same basic rules that characterize the sensor data, namely:

1) The data from the sensor should be within a valid range;

2) The absolute value of the rate at which data varies should not be higher than a given threshold that is determined using historical data;

3) The standard deviation of the sensor data within a certain window should be less than a given threshold; and

4) The standard deviation of the sensor data should not be zero, which would indicate a constant value. This indicates that the sensor is not working properly.

These requirements are coded as rules in the fuzzy system. The input variables used are the data, rate of change in the data, and the standard deviation of a certain window. The membership functions for these input variables are defined by finding the variation and the trend in the historical data. The membership functions are shown in Figure 2-4. The limits in the membership function, namely MT1, MT2, etc., are found from the processing of the historical data. The deviation of the data from the curve is considered as the standard deviation of the sensor. The block diagram of the selfvalidation algorithm from the paper is shown in Figure 2-5. 

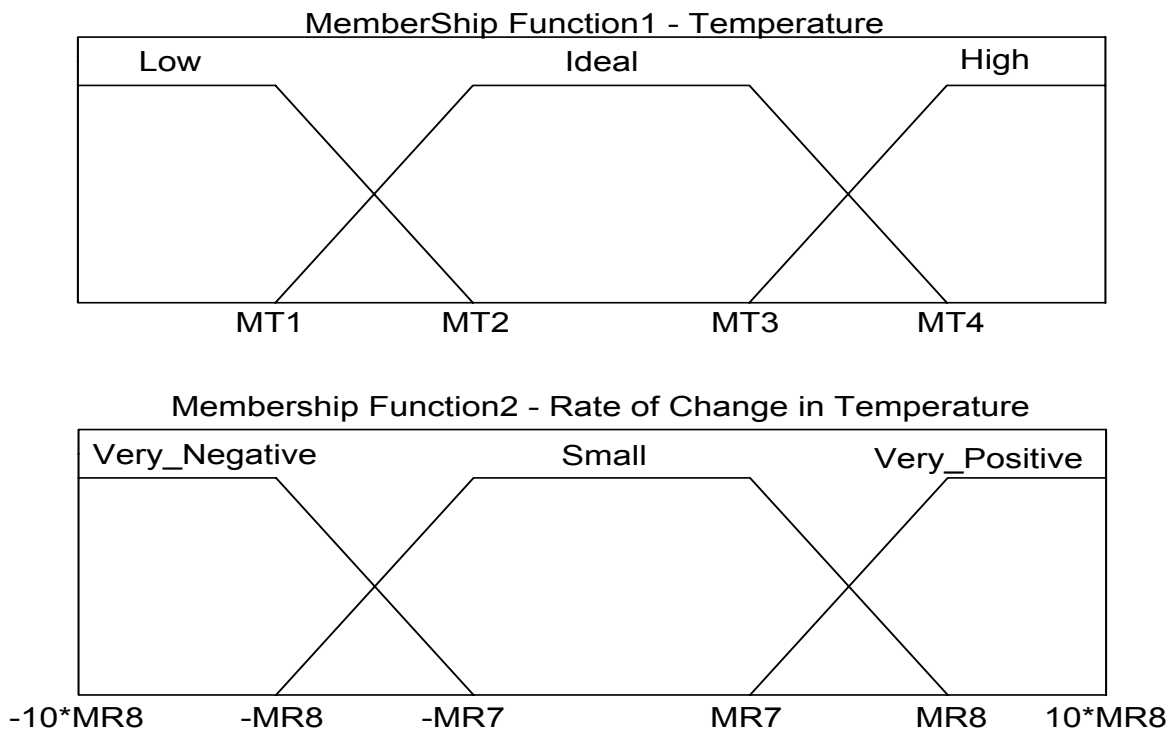

Membership Function 3 - standard Deviation

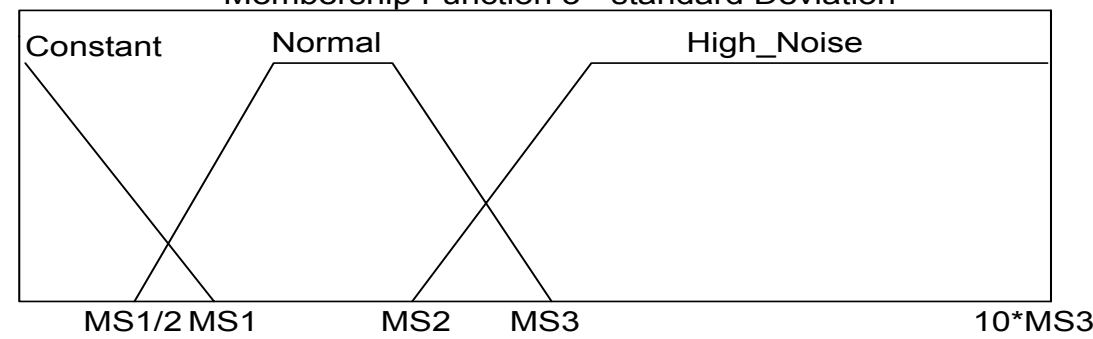

Figure 2-4 Membership Functions

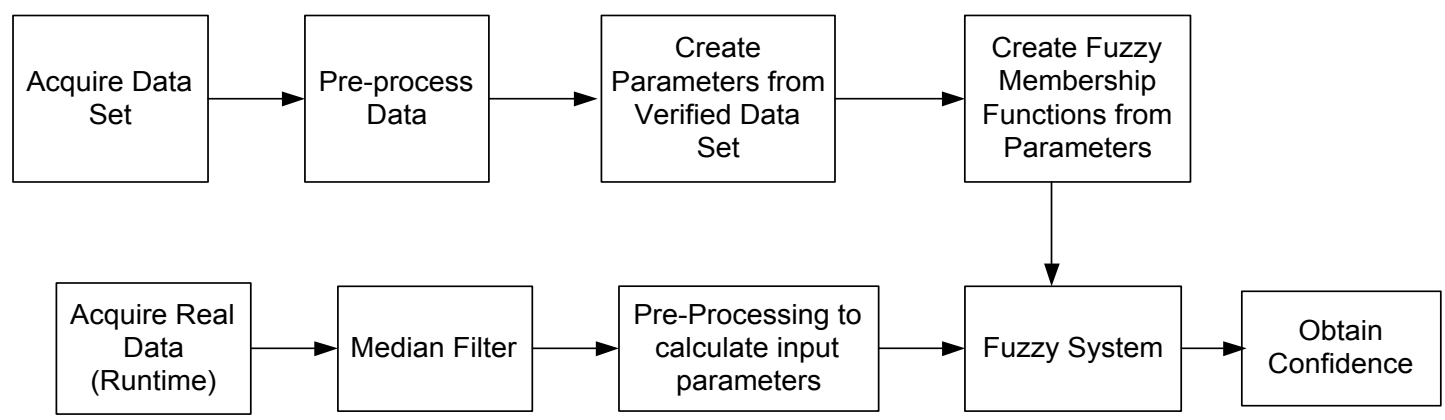

Figure 2-5 Block Diagram of the Self-Validation Technique 
The rules of the fuzzy system remains the same for all sensors while the membership function varies from one sensor to another sensor depending upon the sensor's historical data. The fuzzy output gives the self-confidence of the sensor. The median output of the data is the sensor data output of the self-validation.

\subsection{Adaptive controllers}

Controllers are designed based on the model of the plant. Earlier control designers assumed exact knowledge of the plant and that the plant is modeled accurately. These controllers demanded accurate measurement of the state variables that are used for controlling the performance of the plant. It was soon realized that meeting these requirements is not possible in all situations and people tried to design controllers that are robust. Many robust control design techniques were developed and these controllers were able to tolerate the variation in the model, system parameters, and state variable estimations. But, these controllers achieved them at the cost of performance [15].

Adaptive controllers provide an answer to the problem. Adaptive controllers are basically a controller whose control law adapts its own behavior as it learns about the process it is designed to control or as the process changes with its environment. The field of adaptive control is a very wide and what is presented in this section is a brief introduction to adaptive control. It is not intended to be a thorough literature review.

Adaptive control methods are classified into two broad categories [15]: 
1) Indirect or explicit control. - The basic requirement in this method is the availability of a design model, but the parameters of the model are not known. The plant parameters are estimated explicitly on-line and the control parameters are then adjusted based on these estimations. Indirect control methods utilize separate parameter identification and control schemes.

2) Direct or implicit control. - This method does not assume the availability of the design model. The controller parameters are adjusted directly, only using plant input and output signals. The identification and control functions are merged into one scheme.

Many adaptive control approaches have been developed. Gain scheduling, selftuning of the controller, model reference adaptive control, and variable structure adaptive control are some of the most commonly used approaches. All of these approaches fall in one of the two categories mentioned above [16]. Few approaches that involve both the direct and indirect method have also been developed.

Gain scheduling is the simplest type of the adaptive control. In this approach, the controller gains are made dependent on the parameters that can be measured or inferred from other measurements. This approach is very conservative and poses many problems if the dependent parameter has high rate of variation [15].

Parameter estimation forms the base for self-tuning. The required parameter is modeled and an observer is implemented to estimate the parameter. The controller is designed as a dependent on the estimated parameter. This method requires all the state 
variables for parameter estimation, which is not possible in all systems [16]. Using state estimator may help, but it results in a complex system.

Model reference adaptive control is based on a reference model for the plant. The error between the actual output and the output from the reference model is used to change the controller parameters. This approach introduces a lot of nonlinearity through multipliers and additional error processing. Hence, determining the stability of the system is very difficult [16].

Variable structure adaptive control from input and output variables has been discussed in the paper [17]. Variable structure is similar to model reference adaptive control but instead of using parameter estimation, it uses signal synreport. A discontinuous switching control function is designed to generate the sliding surface for the variable structure adaptive control. The paper derives the stability of the adaptive control. The disadvantage of the variable structure control is that it requires the knowledge of all state variables. State estimator may be used, but it results in a complex system.

In [18] Burdet and Codourey compares most of these adaptive control algorithms and have tested experimentally two of the best algorithms. It was shown that the Adaptive FeedForward Controller (AFFC) is well suited for learning the parameters of the dynamic equation. The resulting control performance is compared with the measured parameters for any trajectory in the workspace and was said to give better results. The 
paper also introduces an adaptive look-up-table memory and was shown to be simpler and better for tasks that requires repeating the same trajectory.

In [19], another type of adaptive control based on switching the controllers is developed. In this paper the output of the plant with unknown parameters were made to track the reference signal through switched nonlinear feedback control strategy. Many controllers were designed and the controllers are selected online through a performance evaluation procedure that uses the output prediction error. The paper also discusses sufficient conditions under which the closed loop control system is exponentially stable. This approach achieved asymptotically stable control and the results of this approach were illustrated with three examples.

Automatic synthesizing of controllers other than gain scheduling was used in [20]. The paper describes a method that automatically derives controllers. The controllers were derived for timed discrete-event systems with non-terminating behavior modeled by timed transition graphs. The specifications of control requirements were expressed by metric temporal logic (MTL) formulas. The syntheses of the controllers were performed by using, a forward-chaining search and a control-directed backtracking. The synreport process does not require explicit storage of an entire transition structure. This feature of automatic synthesizing of the controllers for the above procedure of switching controllers may compliment each other for obtaining superior performance from an adaptive controller. 
Adaptive controllers give better performance even when the system parameters or the environment changes. Adaptive controllers have gained importance with rigorous proofs for stability. Their tolerance to large parameter variations has made them more suitable for many industrial applications.

\subsection{Conclusions}

Most of the literatures in multiple sensor fusion exist for detection purposes and are developed for target or enemy detection in military-based research. Few literatures are available on fusing redundant sensors for non-military applications. These are commonly based on averaging the redundant data. Kalman and Bayesian methods are based on probability density function (PDF). Kalman filtering technique, however, needs a good model of the system, which is not always available. The Bayesian method considers two data points at a time for a confidence measure and also involves lot of matrix manipulations. The approximate agreement approach explains the advantages of finding agreement between the sensors. One other factor that is required is the degree of agreement between the sensors on the estimate value. There was no literature discussing an algorithm to find such a measure.

Adaptive control methods are available to improve performance. The controller parameters are adapted based on the system parameter variation, environment changes and even with performance. However, not much of research exists in the area of adapting the controllers based upon the sensor reliability. 
This chapter discussed some of the self-validation techniques, multiple sensor fusion algorithms and adaptive control approaches. The problem of improving the performance of the system even under the failure of sensors is solved using adaptive control approach. The self-validation technique and multiple sensor fusion algorithm is used to decide upon the adaptation of the controller. The self-validation technique developed in Year 1 of the project was reviewed in section 2.4. In Chapter 3, the developed methodology for redundant as well as multi-modal sensor fusion is presented. 


\section{Chapter 3}

\section{MULTIPLE SENSOR FUSION}

In Chapter 2, several multiple sensor fusion algorithms were discussed. Sensor fusion is used to reduce the effect of a sensor failure over the operation of the system considered. The signals from sensors are fused to get a better estimate of the measurand value. Thus, sensor fusion helps in improving the reliability of the measurements that primarily affects the performance of a system. This is especially true in the case of feedback control systems.

Among the multiple sensor fusion algorithms discussed in Chapter 2, many techniques build on averaging the redundant sensors' readings. However, averaging the sensors' data would still mean that a failed sensor would affect the estimate value. So, the factor that should be considered in the sensor fusion is the confidence in the data obtained from each sensor. This is the concept that was introduced in section 2.2 as the self-confidence. A multiple sensor fusion algorithm incorporating this self-confidence will be less affected by a sensor failure.

Some of the algorithms that were studied in Chapter 2, produces an estimate that represents the value that most sensors agree. But, these techniques do not specify the degree of agreement on the estimate by the sensors. Hence, a multiple sensor fusion algorithm that reflects the degree of agreement among sensors will be more appropriate. 
In this chapter, a multiple sensor fusion algorithm that produces a measure of the confidence in the estimated value of the measurand is developed. The confidence measure reflects the degree of agreement among the sensors.

First, the discussion on redundant sensor fusion algorithm and how a measure of confidence in the estimate is produced, are presented. Next, the integration of the selfconfidence into the multiple sensor fusion algorithms to mitigate the effect of sensor failure are presented. The multiple sensor fusion algorithm is tested using data from an experimental run of a cupola furnace. A comparison of the results with that of the averaging method is also discussed. A unified framework for multi-modal sensor fusion is also presented in this chapter of the report.

\subsection{Parzen-like Methodology for Redundant Sensor Fusion}

\subsubsection{Description of Parzen Estimator}

The Parzen estimator is a nonparametric method for estimating probability density functions without making any assumptions about the nature of the distribution [21]. Given a set of sensor data, the Parzen estimator utilizes parametric functions such as Gaussian functions that are centered at each of the sensors' readings. The functions are then added up and normalized. The resulting Probability Density Function (PDF) reflects the distribution of the sensors' data. The PDF energy is more concentrated where more data points exist. This is illustrated in Figure 3-1. 


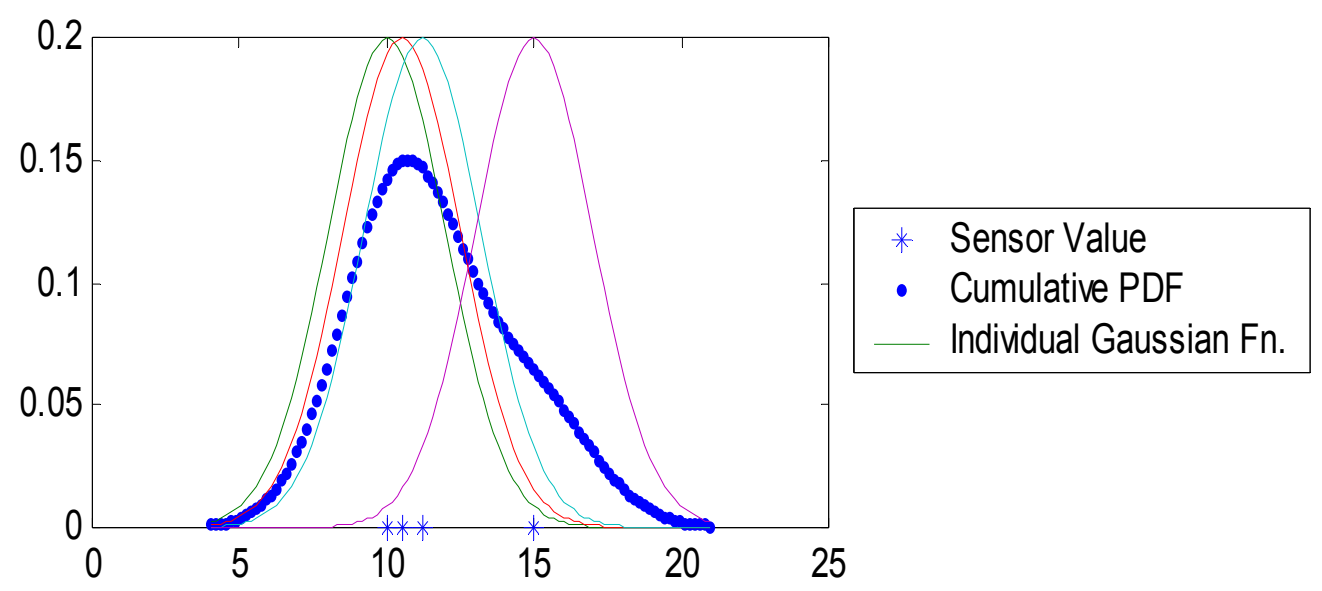

Figure 3-1 Individual Gaussian Functions and the Cumulative PDF

For this research a Gaussian function (GF) is selected as the parametric function. The mean value of the GF is equal to the sensor reading and the standard deviation is estimated from the noise level in the sensor [14]. The PDF is given by

$$
P D F(x)=\frac{1}{N} \sum_{k=1}^{N} \frac{1}{\sqrt{2 \pi} \sigma_{k}} \exp \left(-\frac{\left(x-x_{k}\right)^{2}}{2 \sigma_{k}{ }^{2}}\right)
$$

where $N$ is the number of sensors, $x_{k}$ is the $\mathrm{k}^{\text {th }}$ sensor data, and $\sigma_{\kappa}$ is the standard deviation. The parameter $\sigma_{\kappa}$ is estimated based on the standard deviation of the noise associated with each of the sensors considered.

\subsubsection{Estimation of Measurand Value from PDF}

The estimate value of the measurand is calculated from the PDF obtained as explained in previous section. There are several ways to get an estimate of the 
measurand value similar to defuzzification methods such as average, centroid, maximum, and sum of the maximum [22]. In this research an algorithm was developed for estimating the measurand value. The algorithm is an integration of the peak and centroid methods.

1. Find the range $X$ which contains $95 \%$ of the PDF energy.

2. Find the centroid of the PDF using:

$$
\text { Centroid }=\frac{\int_{X} x \cdot P D F d x}{\int_{X} P D F d x} \text {. }
$$

3. Find the area on each side of the centroid.

4. The estimate is found as the value of measurand that corresponds to the supremum of the PDF on that side of the centroid that has the higher area, thus

5. Measurand Estimate $=\arg (\operatorname{Sup}(\mathrm{PDF})))$.

The function arg corresponds to finding the $\mathrm{x}$ co-ordinate at which the maximum value occurs in the PDF. This procedure is illustrated in Error! Reference source not found. 


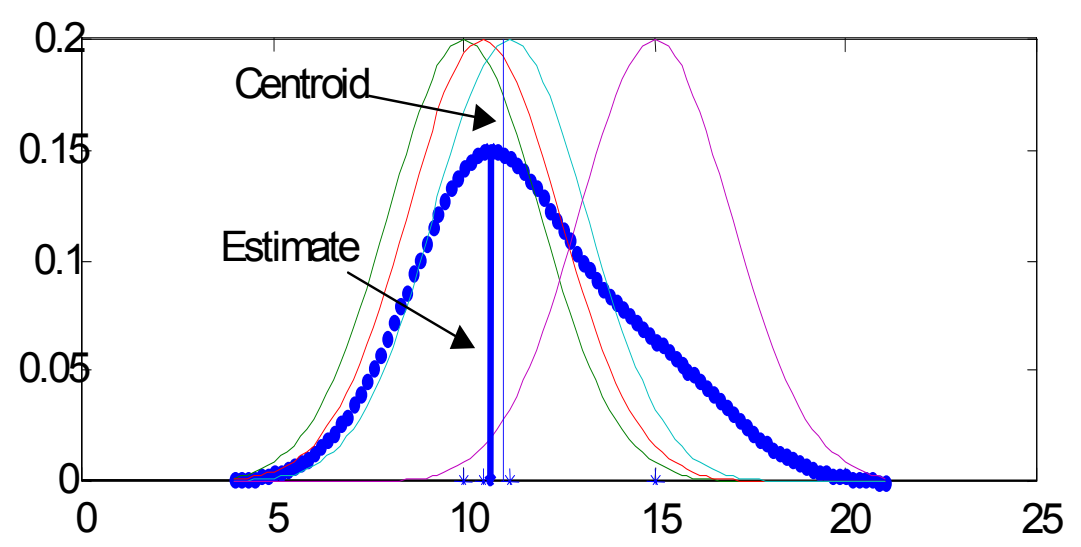

Figure 3-2 Estimation of the Measurand Value

This particular method of finding the estimate is found to be more advantageous than other methods as explained in this section. The estimate of the multiple sensor fusion algorithm should be the value on which most of sensors agree, and at the same time the estimate should not be adversely affected by invalid sensors. Other methods for estimating the measured value from the PDF such as centroid and peak allow faulty sensors to have an effect on the estimate or may not give the most probable value on which the sensors agree. Figure 3-3 shows how the estimate, if chosen, using the centroid would be affected by the faulty sensor. Figure 3-4 shows that the peak value does not always correspond to the value on which most sensors agree, at all cases. 


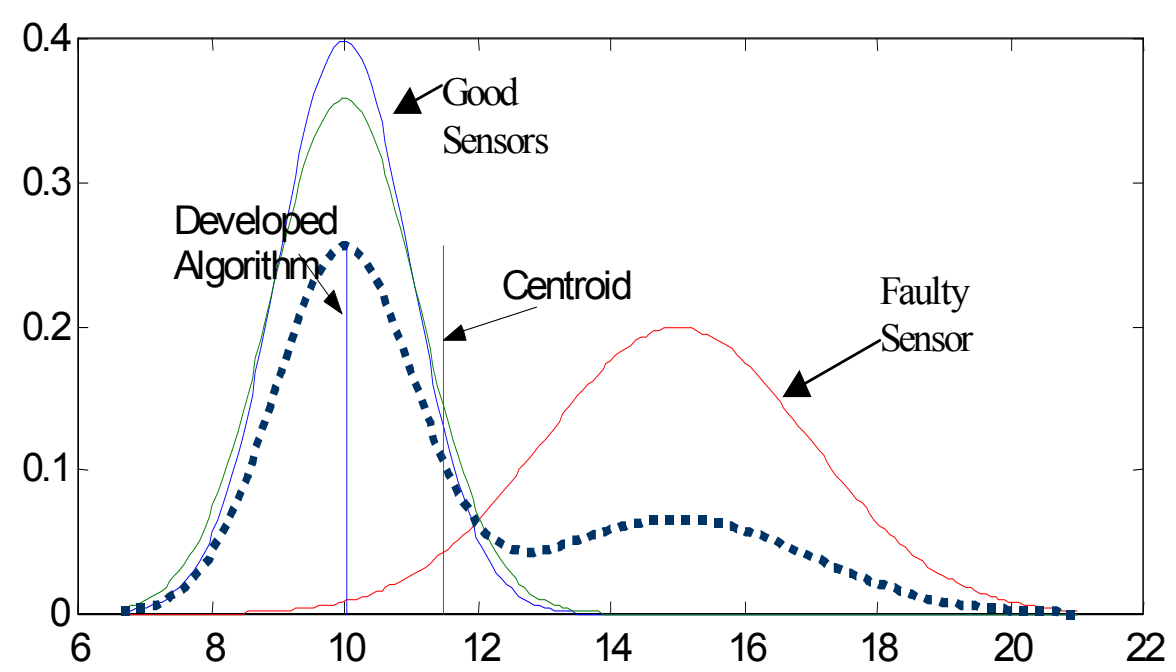

Figure 3-3 Comparison of Estimate with the Centroid

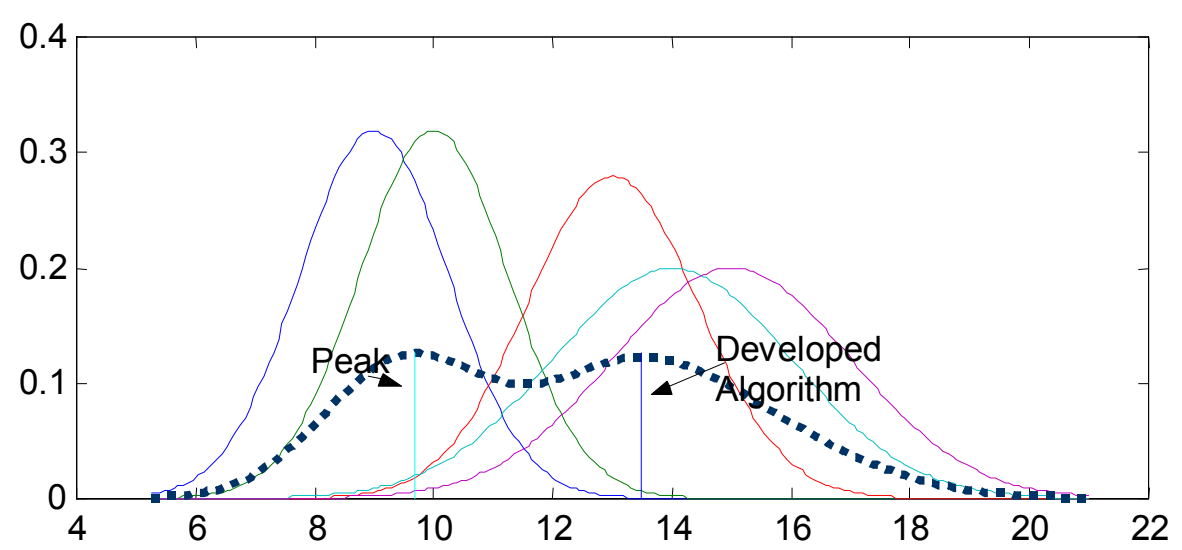

Figure 3-4 Comparison of Estimate with Peak Values

\subsubsection{Confidence in Estimate}

The estimate value from the previous procedure takes into account the agreement between the sensor data. However, the estimated value does not explicitly reflect the 
degree of agreement between the sensors and hence the confidence in the estimated value. The agreement between the sensors is reflected in the width of the PDF function estimated according to the process previously described. Thus, the confidence is calculated using the area of the PDF that is enclosed within three standard deviations on each side of the estimated measurand value. In the ideal case, where all the sensors agree exactly, this will be approximately equal to one. As the agreement between the sensors' decrease, this area will decrease as well. This is illustrated in Figure 3-5 and Figure 3-6.

The confidence is related to the PDF function width according to the relation:

$$
\text { Confidence }=\int_{\text {Estimate }-3 \sigma}^{\text {Estimate }+3 \sigma} P D F(x) d x
$$

where $\sigma$ is the maximum standard deviation of the parametric functions used in forming the PDF.

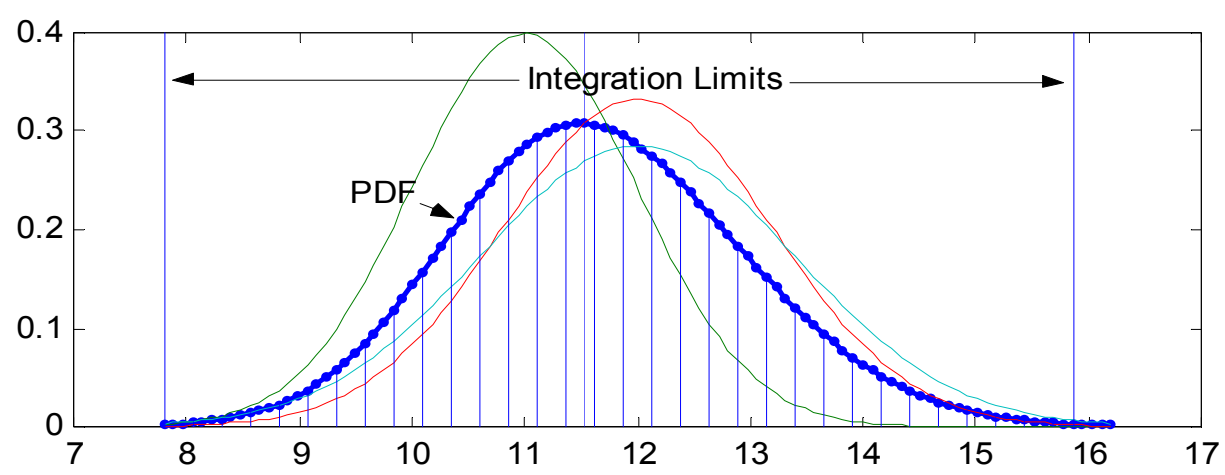

Figure 3-5 Measurand Estimate with High Confidence 


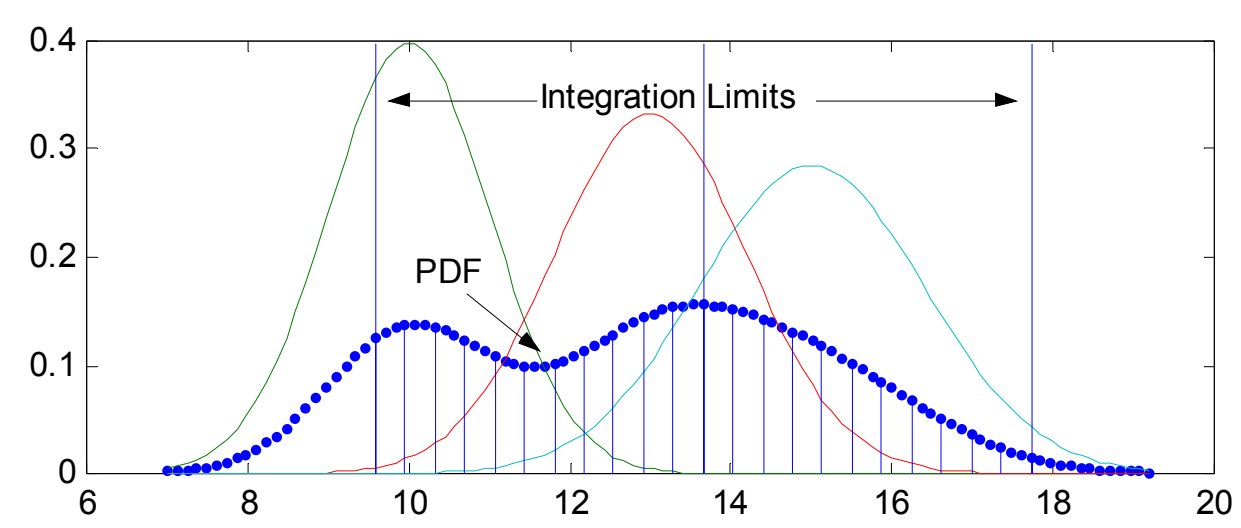

Figure 3-6 Measurand Estimate with Low Confidence

\subsection{Considering Self-Confidence in Redundant Sensor Fusion}

The self-confidence of a sensor data obtained from the self-validation technique explained in section 2.2 is a measure of how much this data agrees with the expected characteristics of the sensor as estimated from historical data. Thus, a change in the sensor noise level or if the sensor data or its rate of change exceeds the expected limits, the self-confidence value decreases. Integration of this self-confidence into the redundant sensor fusion is necessary to decrease the effect of the failed sensors on the estimated value.

In Section 3.1 the Parzen like procedure for estimating the PDF which was then used to get a best estimate for the measurand value was presented. The function used in Parzen estimation was a Gaussian function with a standard deviation that depends upon the sensor noise. This Gaussian function can be thought of as a representation of the 
probability in finding the true value of the measurand data around the sensor reading. As the self-confidence decreases, the probability of finding the true value of the measurand in the neighborhood of the sensor measurement decreases. In other words, the region in which the true value could be with respect to the sensor reading becomes wider. This could be reflected by scaling up the standard deviation of the Gaussian function, used in building the PDF, using the self-confidences of the sensors. Thus, the PDF function becomes

$$
P D F(x)=\frac{1}{N} \sum_{k=1}^{N} \frac{1}{\sqrt{2 \pi}\left(\sigma_{k} / S C\right)} \exp \left(-\frac{\left(x-x_{k}\right)^{2}}{2\left(\sigma_{k} / S C\right)^{2}}\right)
$$

where SC is the self-confidence of the sensor and the rest of the parameters are defined as in (3.1). Figure 3-7 and Figure 3-8 illustrate the effect of the change in selfconfidence over the shape of the Gaussian functions and hence the PDF. It should be noted that this change is not used in the standard deviation used for finding the Confidence.

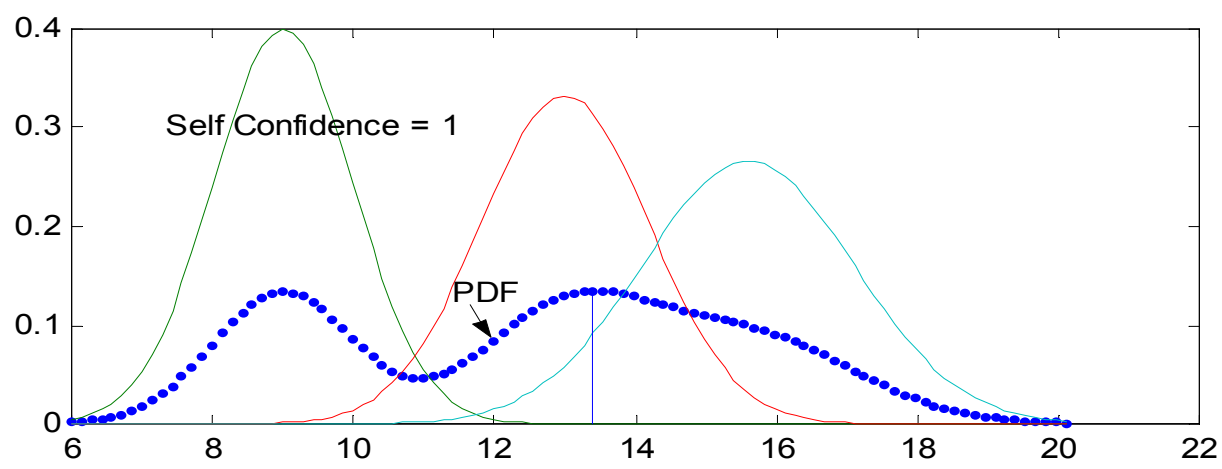

Figure 3-7 Estimation of Measurand without Considering Self-Confidence 


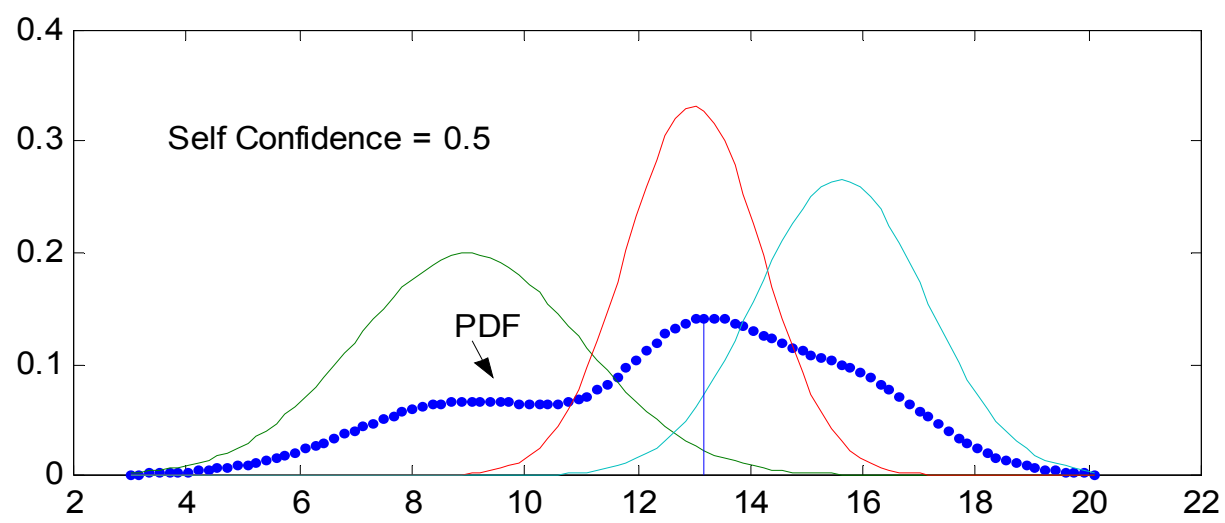

Figure 3-8 Estimation of Measurand Considering Self-Confidence

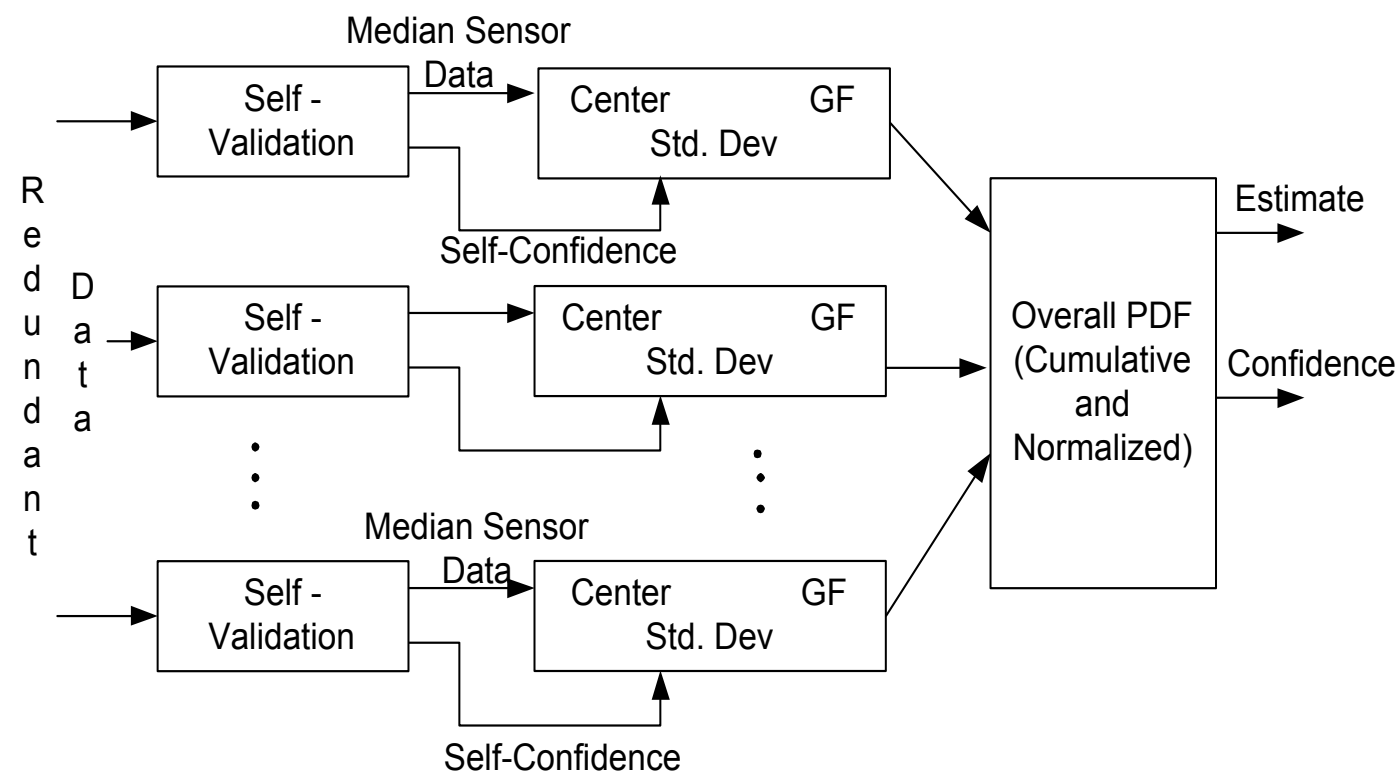

Figure 3-9 Block Diagram of Multiple Sensor Fusion 
A block Diagram of the Multiple Sensor Fusion Algorithm Developed including the integration of self-confidence is shown in Figure 3-9.

\subsection{Application and Testing}

In the following section the testing results are presented for the redundant sensor fusion methodologies presented in sections 3.1 and 3.2. The testing was performed using data from an experimental cupola iron-melting furnace in Albany, Oregon. The system uses three temperature sensors that measure the temperature of the iron melt produced from the furnace. These sensors were quasi-redundant as explained in [23]. The sensors' data of two of the temperature sensors were translated using a linear regression relation to give an estimate of the third sensor. The resulting data were then treated as if the sensors were redundant sensors.

\subsubsection{Results of the Sensor Fusion Methodology without Considering Self- Confidence}

The results of testing the methodology of integrating redundant sensors presented in Section 3.1 are presented first. Figure 3-10 shows the results of the test. The data from one of the sensors (TC5) were artificially perturbed by injecting sudden disturbance at $\mathrm{t}=$ 10 minutes and high noise level into the sensor in the range $t=40$ to 70 minutes. For comparison purposes, an estimate of the measurand value using the average of the sensor data is presented in Figure 3-11. The self-confidences of the three sensors are presented in Figure 3-12. Figure 3-13 shows the total confidence in the estimate. It is clear from 
Figure 3-11 that the average method results in the estimated value to be affected by the sudden disturbances as well as by the noise introduced into one of the sensors. In comparison Figure 3-10 shows that the effect of the disturbances were mitigated to some extent. However, a close up of the data in Figure 3-10 shows that the estimated value of the measurand is still affected by the readings of the sensor, which was artificially injected with the high noise level. This close up is shown in Figure 3-14. It should be noted, however, that the confidence in the estimates are lower in periods where the agreement between the considered sensors decreases as shown in Figure 3-13.

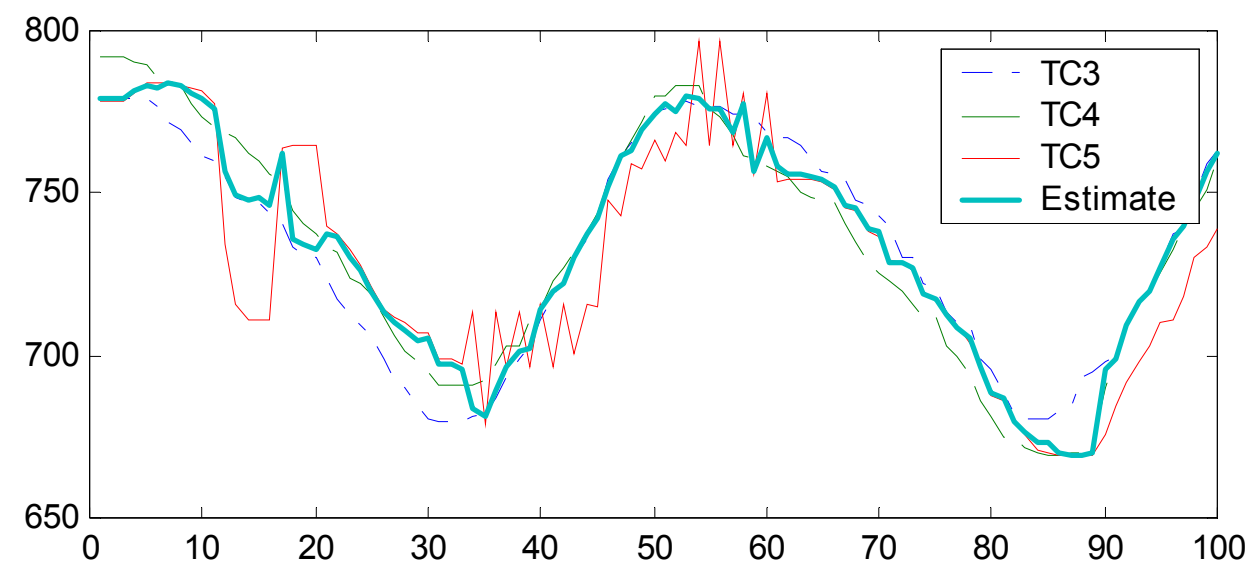

Figure 3-10 Estimated Value from PDF without Considering Self-Confidence 


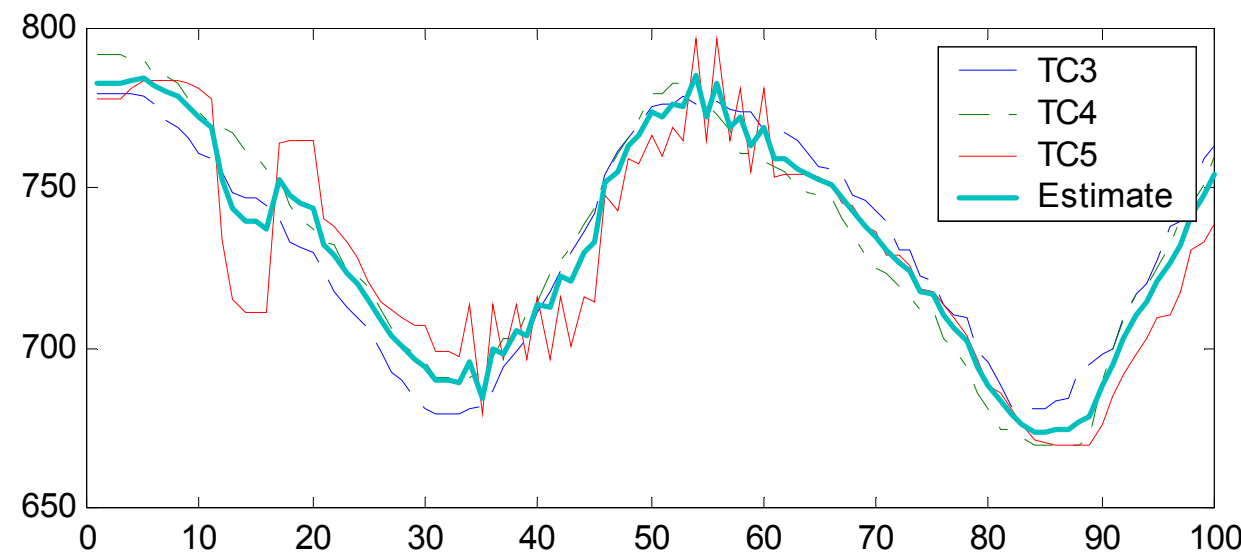

Figure 3-11 Estimated Measurand Value Using Average Method

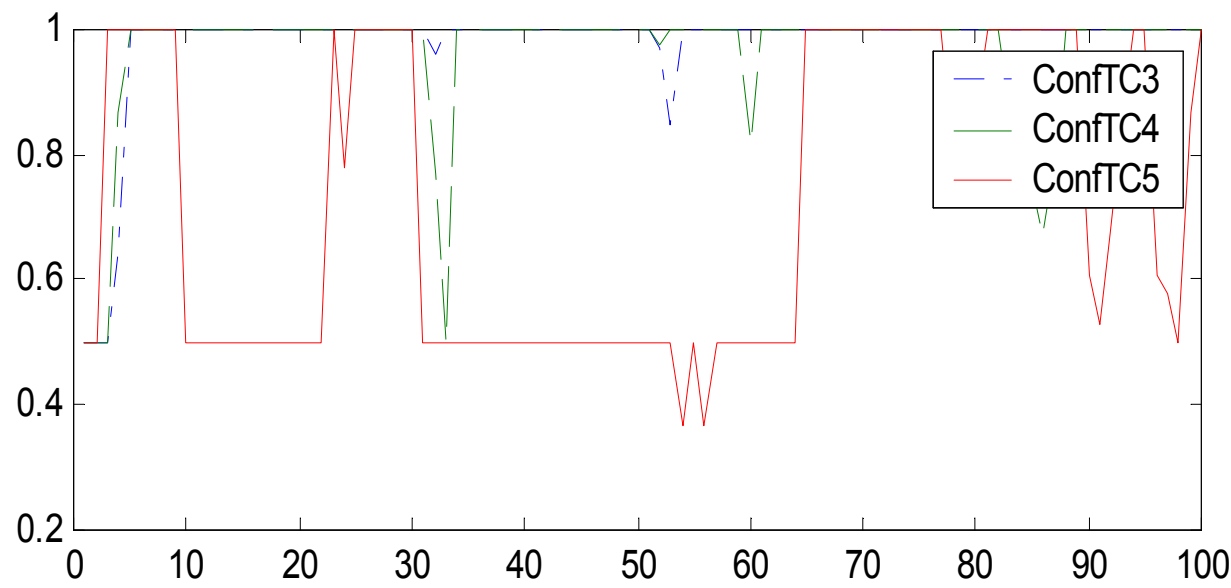

Figure 3-12 Self-Confidence of the Three Sensors 


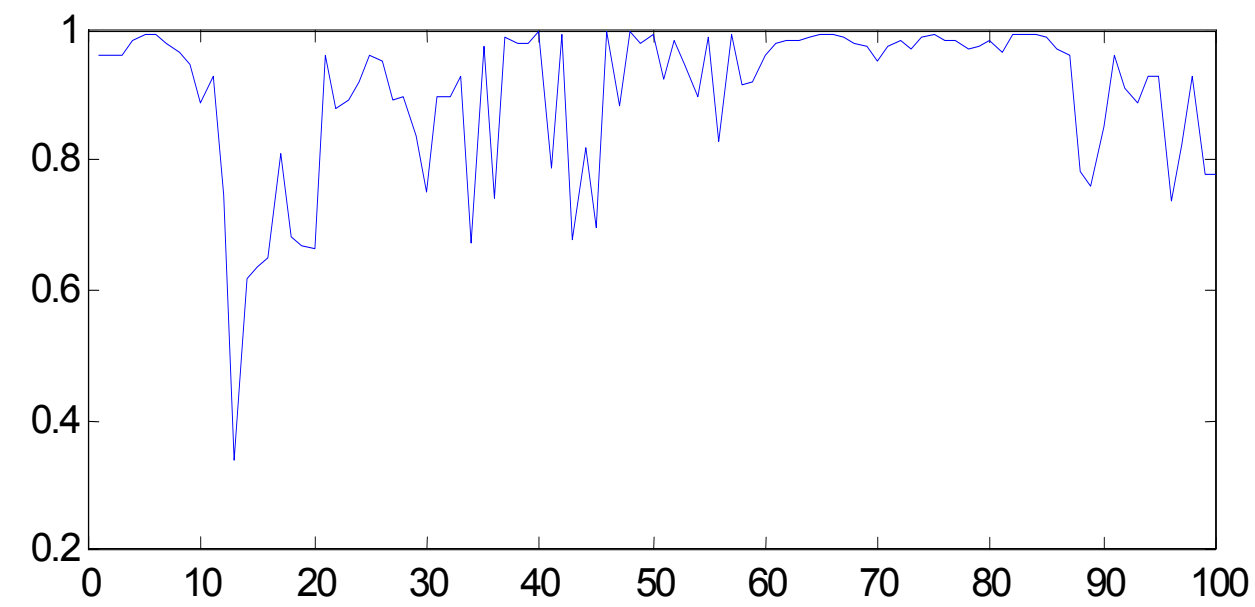

Figure 3-13 Confidence of the Estimate Value Using PDF

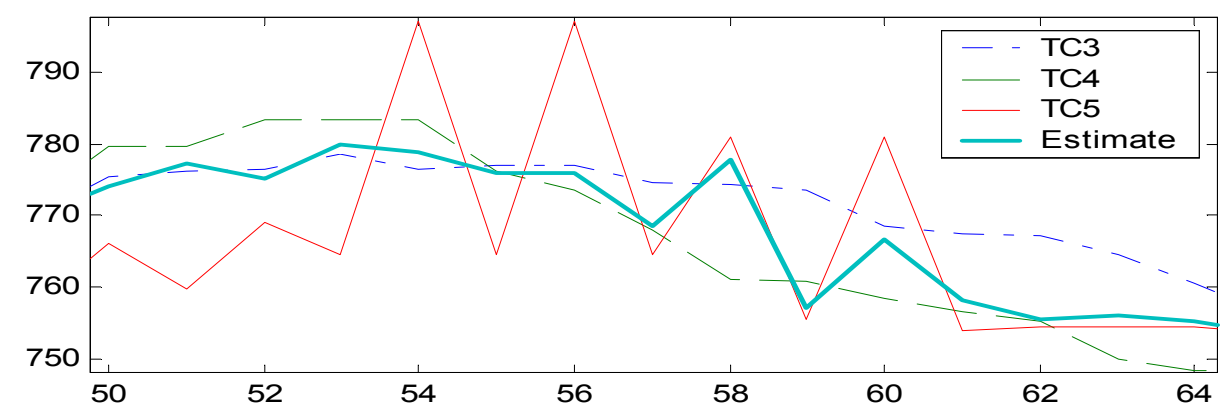

Figure 3-14 A Closeup that Shows Effect of Not Considering Self-Confidence

\subsubsection{Results of the Sensor Fusion Methodology Considering Self-Confidence}

The test is repeated using the same data presented in Section 3.3.1. However, this time the methodology presented in Section 3.2 is used. The self-confidences of the sensors over the considered time period is shown in Figure 3-12. The estimates of the 
measurand value are shown in Figure 3-15. A close up of Figure 3-15 is shown in Figure 3-16. A Comparison between Figure 3-14 and Figure 3-16 shows the advantages of including the self-confidence in the sensor fusion methodology. When the high noise level was injected the self-confidence of the sensor affected by the noise is reduced (See Figure 3-12) and in turn its effect over the PDF function is reduced. This leads the estimate of measurand value to depend more on the other two sensors with higher selfconfidence parameters. Moreover, the overall confidence of the estimate increases (See Figure 3-17). This is because the energy around the third sensor is decreased by the inclusion of the self-confidence.

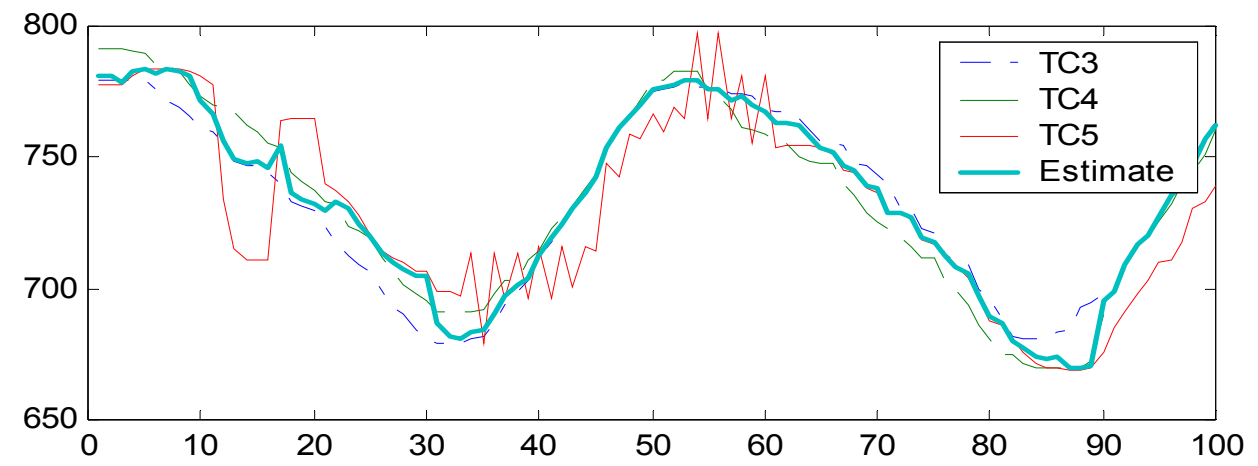

Figure 3-15 Estimated Value using PDF Considering Self-Confidence 


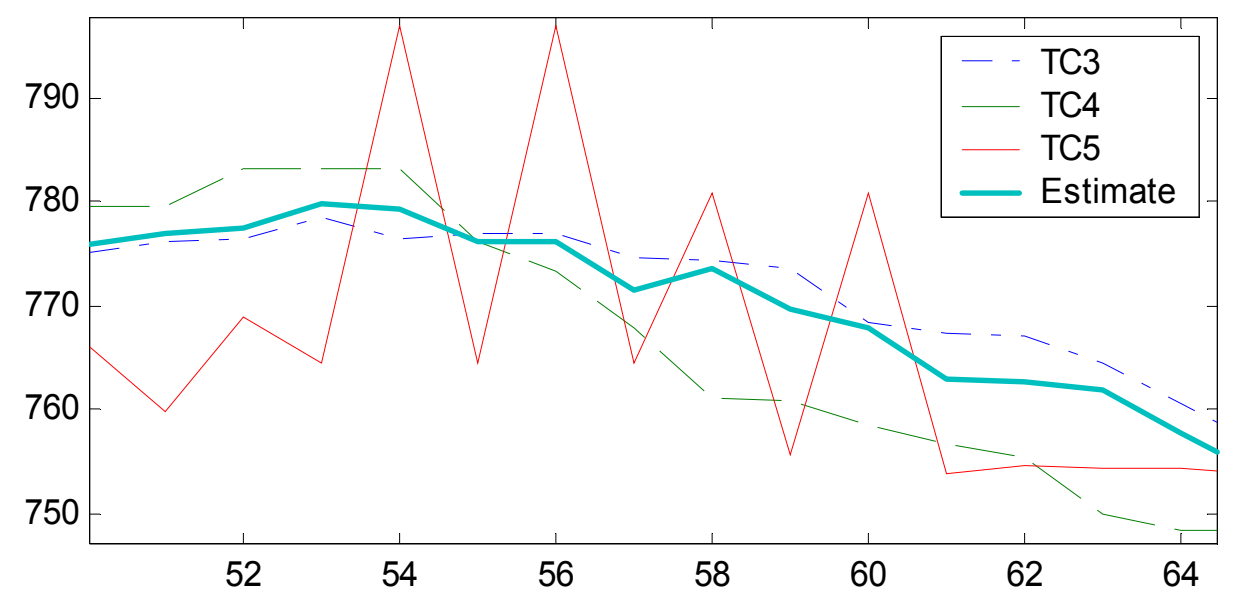

Figure 3-16 Close Up of Figure 3-15

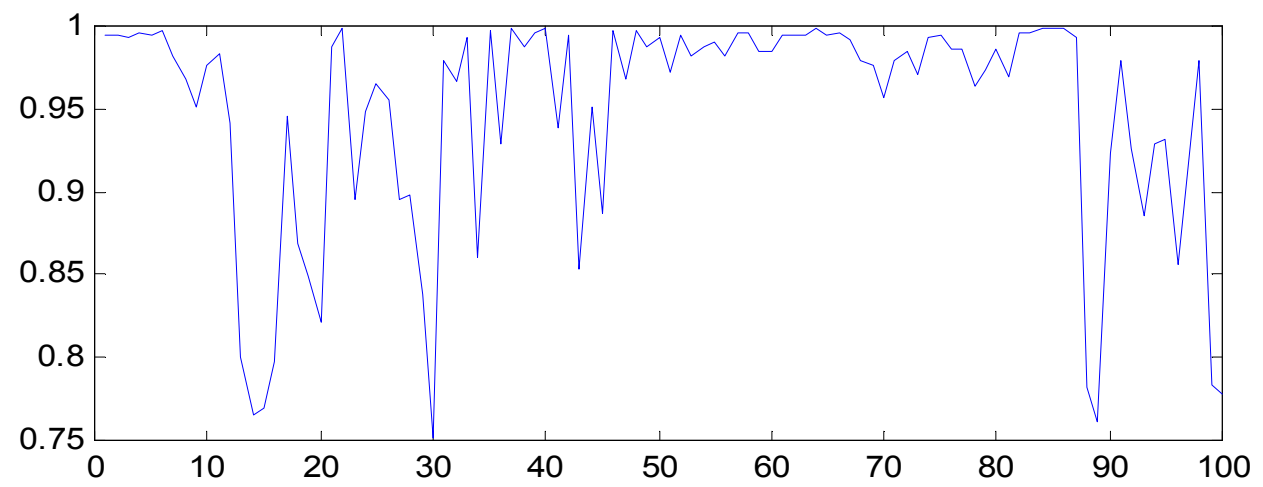

Figure 3-17 Confidence of the Estimate from PDF including the Self-Confidence

Multiple sensor fusion helps the feedback controller by giving a better estimate to the sensor's data, but there might be conditions where even this estimate may be poor. In other words, multiple sensor fusion does not assure reliability at all conditions. At these 
conditions, the feedback controller will fail, degrading the performance of the system. In the algorithm developed, the reliability on the estimate is reflected by the confidence. This measure of confidence can be used in a way to achieve a better performance of the feedback controller even when the estimate from the sensor fusion fails.

\subsection{A unified Framework for Multi-Modal Sensor Fusion}

\subsubsection{Trend Fusion}

The independent sources of information for sensor fusion considered, in this section, include the real sensors themselves and/or information regarding the trend of the measurand as provided by other sources such as models or virtual sensors. Our goal, here, is the development of a methodology wherein information regarding trend as is fused with the absolute measurements from the sensors.

Motivation for developing this trend fusion algorithm stems from the fact that the estimates of virtual sensors and models developed for the cupola furnace were found to provide more accurate information on the measurand trend rather than on its value.

This section is arranged as follows: a brief description of the previous work on multiple sensor fusion is presented. This is followed by description of the algorithm of fusion based on trend. Simulations that illustrate the algorithm and its effectiveness are presented throughout the paper. 


\subsubsection{Multiple Sensor Fusion}

The process of multiple sensor fusion (MSF) based on Parzen estimator, presented in the previous sections [28], provides an algorithm for fusing data from multiple sensors. In this methodology, no emphasis is given on the trend of the system.

Based on the measure provided by the sensor, the reading is fed to a fuzzy engine [27]. The fuzzy engine looks at the median value, the rate-of-change, and the variance of the parameter and assigns it a confidence measure. The fuzzy engine assigns each sensor self-confidence value based on the agreement between current and historical behavior [27]. In the MSF algorithm, a trapezoidal distribution is constructed around each sensor measurement [30]. The spread of the distribution depends on the slef-confidence measure of that sensor. Such a distribution is constructed for each of the sensors and these distributions are added up and normalized. The reading corresponding to the peak on the larger side of the centroid of the joint distribution is the fused measure. The confidence in the fused value was considered as the area enclosed within three standard deviations on either side of the fused value. 


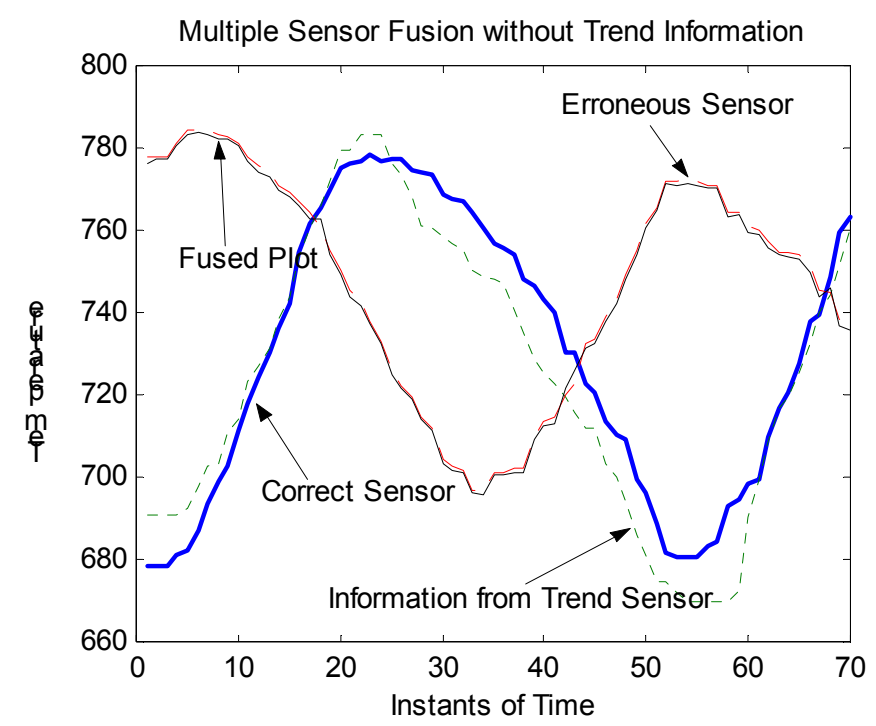

Figure 3-18 Multiple Sensor Fusion without trend information

In the MSF algorithm, the major emphasis was on the absolute measurements of the sensors and their self-confidence. There however, are cases where trend information, if available, can provide useful information. Consider, for example, a sequence of sensor measurements as shown in Figure 3.18. The fused value at points where all sensors meet would be of very high confidence and the confidence values at all other points would be low. This is illustrated in Figure 3.19.

Figure 3.20, shows the plots of Information regarding the trend. In this figure, we assume that we have an additional source of information on the trend, but the algorithm does not use this information for the fusion process. Examining this figure, it is clear that the erroneous sensor is effecting the calculation of the fused value. The methodology 
introduced in this section aims at handling such situations by using the available trend information.

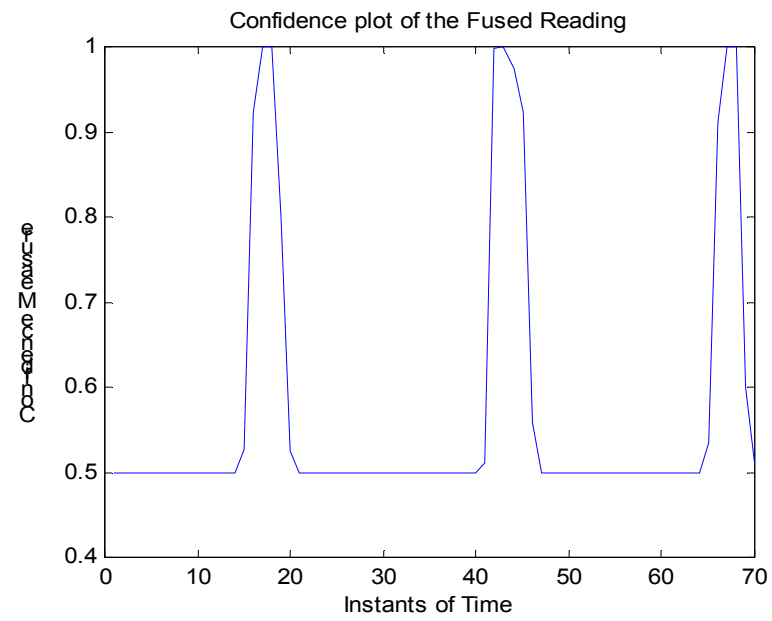

Figure 3-19 Confidence Plot

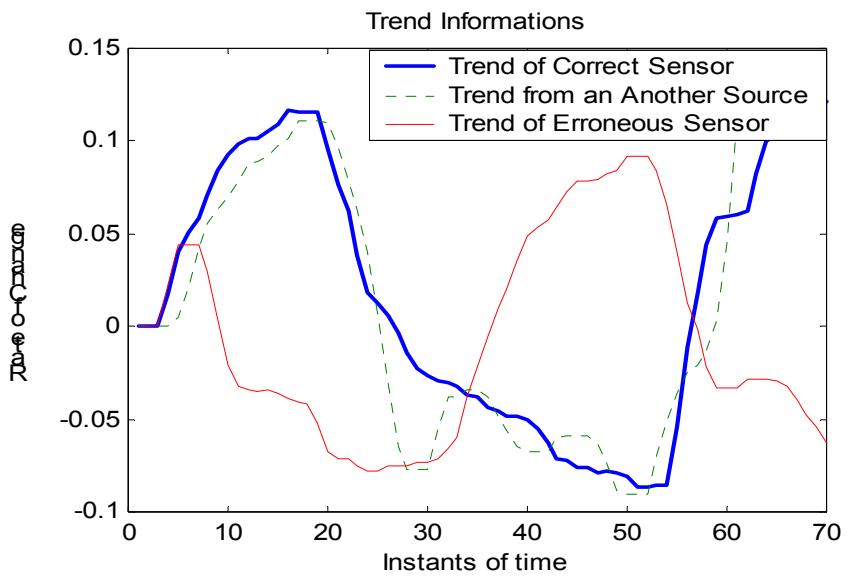

Figure 3-20 Sources of Trend Information 


\subsubsection{Fusion based on Trend}

As explained earlier, the MSF algorithm presented in [27] considered only the agreement between the sensor values rather than their trend. By examining Figure 3.18 it is obvious that one of the used sensors is trending differently. Obviously this sensor is erroneous and its value should not be allowed to affect the fused value. The trend fusion algorithm introduced thereafter minimizes the influence of that erroneous sensor on the fusion process by including the trend of the sensor as a source of information for the fusion process.

The algorithm proposed in this section looks at the trend of the parameter along with the measured value. Based on the trend information provided by each of the real sensors, a fused value for the trend is calculated using the Parzen estimator algorithm presented in [27]. Using this trend, and the previous distribution of the measurand at a previous instant, an estimate of the current value of the measurand is estimated. This estimated distribution is further used for the estimation of the final fused value of the measurand.

The sequence of steps used in this algorithm is as follows:

- Determine the measure of self-confidence from the fuzzy engine [26].

- Estimate the fused trend from the individual sensors using the Parzen Estimator Algorithm [27]. 
- Based on the parameter value at the previous instant and measure of trend, an estimate of the parameter at this instant is calculated. The distribution for the expected temperature is obtained from the distribution of the fused temperature at the previous instant and the distribution of the fused trend at the current instance. The distribution of the expected value is obtained from the formula

$$
\hat{P}^{i}=P^{i-1}+\left(\frac{d P}{d t}\right)^{i} \Delta t
$$

Where,

$\hat{P}^{i}$ - Fuzzy distribution of the expected measurand value the $\mathrm{i}^{\text {th }}$ instant.

$P^{\mathrm{i}-1}-$ Fuzzy distribution of the measurand at the $(\mathrm{i}-1)^{\text {th }}$ instant.

$\mathrm{dP} / \mathrm{dt}$ - rate of change (trend).

$\Delta \mathrm{t}$ - change in time.

The above equation can be considered as a fuzzy arithmetic operation with the result being the distribution for the expected temperature. The distribution obtained for the expected measurand value is normalized.

- Using this measurand estimate and the distribution obtained from actual sensor measurements the sensor fusion algorithm is performed to obtain the fused value and a measure of confidence using the algorithms described in details in [27] and summarized in previous section. 


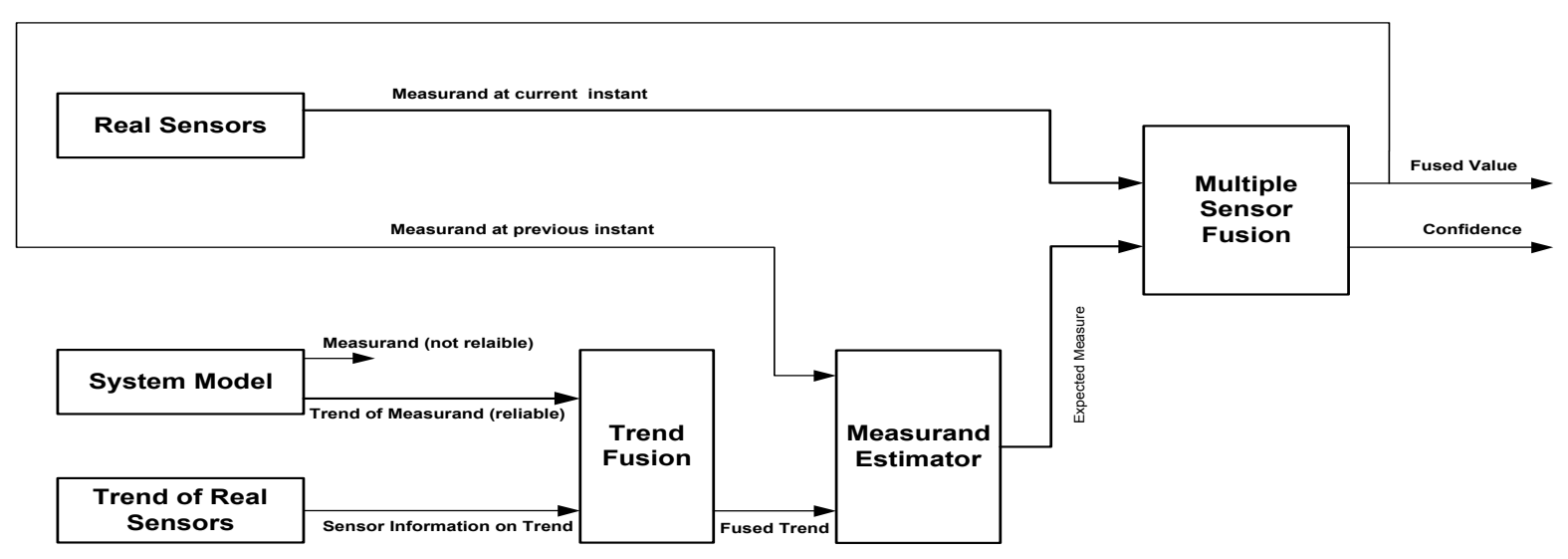

Figure 3-21 General Methodology for Sensor Fusion using Trend

Figure 3-21 summarizes the sequence of steps proposed in the incorporation of trend in sensor fusion.

Figure 3.22 shows the distribution of the trends of three sources of information at one instant, namely 60 . The distribution of the fused trend is shown and this distribution is convoluted with the fused distribution of the temperature at the previous instant, which is shown in Figure 3.23. 


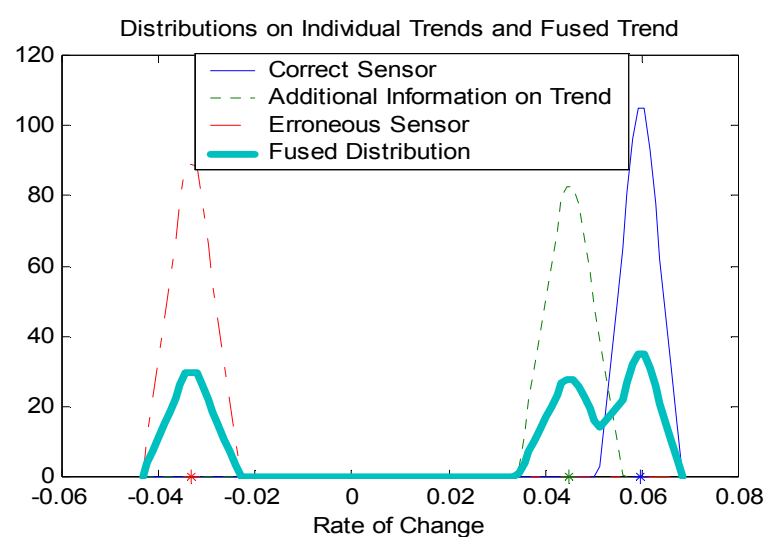

Figure 3-22 Distributions of Trends and Fused Trend

The final temperature distribution is shown in Figure 3.24. This information on the expected temperature helps in deciding the correct value in the fusion process. Using this estimated measure of the parameter along with the actual measure, it can be observed that the effectiveness of the sensor fusion algorithm has improved. This is illustrated in Figure 3.25. It can be seen that the fused value determined by the algorithm coincide to a good extent with the correct sensor for several instances of time. However, the algorithm still fails at multiple points.

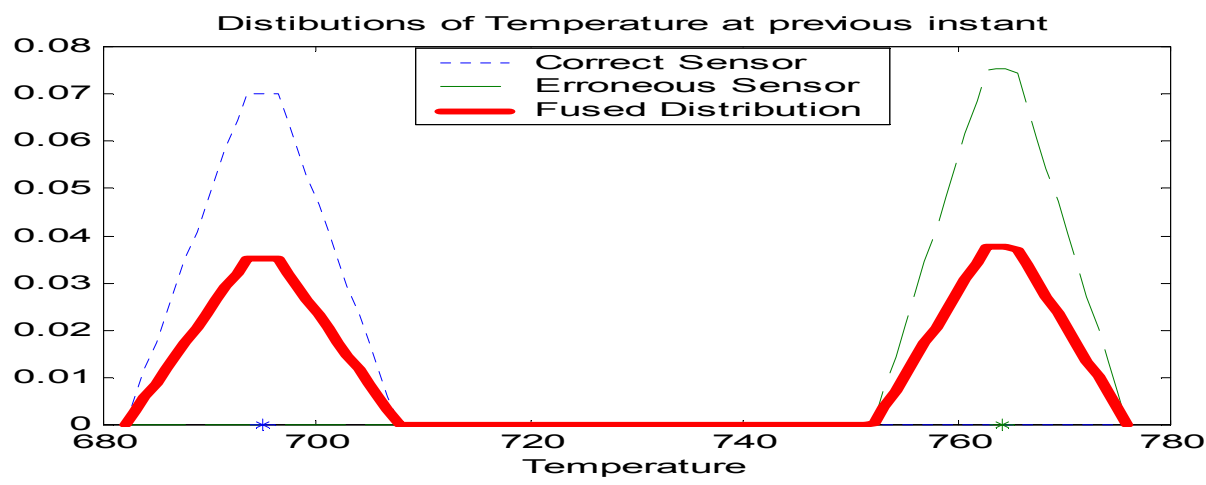


Figure 3-23 Distribution of Temperatures at the previous instant

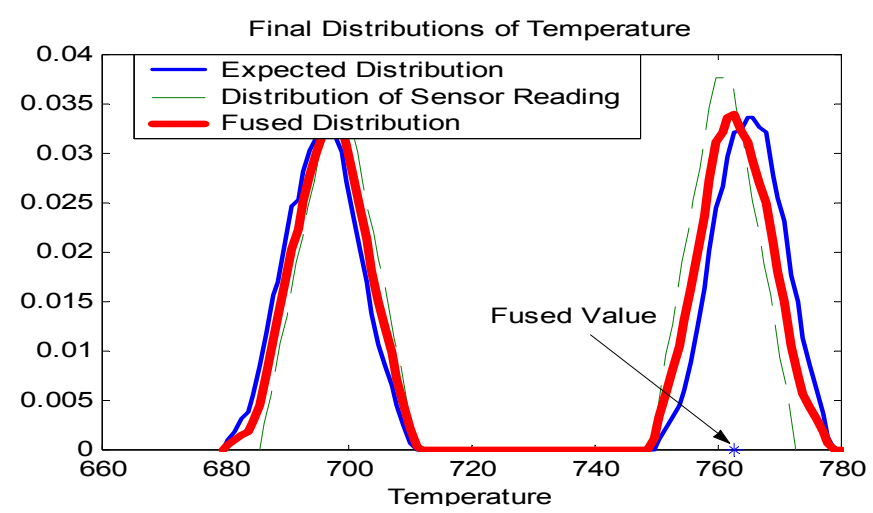

Figure 3-24 Final Distribution of Temperature

It was observed that the failure of the algorithm is accountable mainly to the effect the erroneous sensor has on the fused distribution. Since the erroneous sensor has a very steady performance, the fuzzy engine assigns it a very high confidence. An improvement to the previous algorithm would consider the degree of agreement among the trend sensors. This agreement would be used to modify the self confidence of each sensor. This is presented in the following section. 


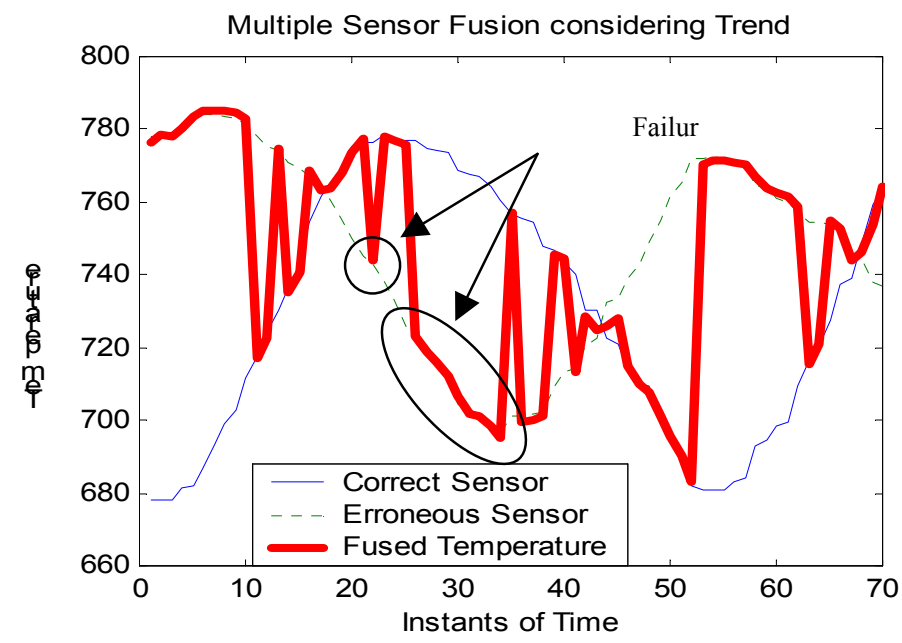

Figure 3-25 Multiple Sensor Fusion Considering Trend

\subsubsection{Confidence based on agreement among the Sensors}

A calculation is proposed wherein the self-confidence measure of each sensor is modified based on the degree of agreement among the trends of the sensors. Using the Parzen estimator, the fused distribution is estimated and the area enclosed by each of the sensor within three standard deviation from the fused value is calculated. The self-confidence measure of each sensor is modified using the formula

confidence $^{i}=\max \left(\min \left(S C^{i}, \frac{\text { Area }^{i}}{\max (\text { Area }) * \text { TotalArea }}\right), 0.5\right)$

Where,

$\mathrm{SC}-$ Self Confidence of the Sensor, Area ${ }^{\mathrm{i}}$ - area enclosed by $\mathrm{i}^{\text {th }}$ sensor in Fused Distribution. 
Total Area - area enclosed in three standard deviation around the fused value. An arbitrary minimum value of 0.5 was assigned to the sensor confidence.

In developing the normalized distribution of the fused data, the contribution of the erroneous sensor is reduced when compared to the contribution made by other sensors. This can be observed from Figure 3.26. It can be observed that the distribution of erroneous sensor spreads further and its contribution to the final fused distribution is reduced.

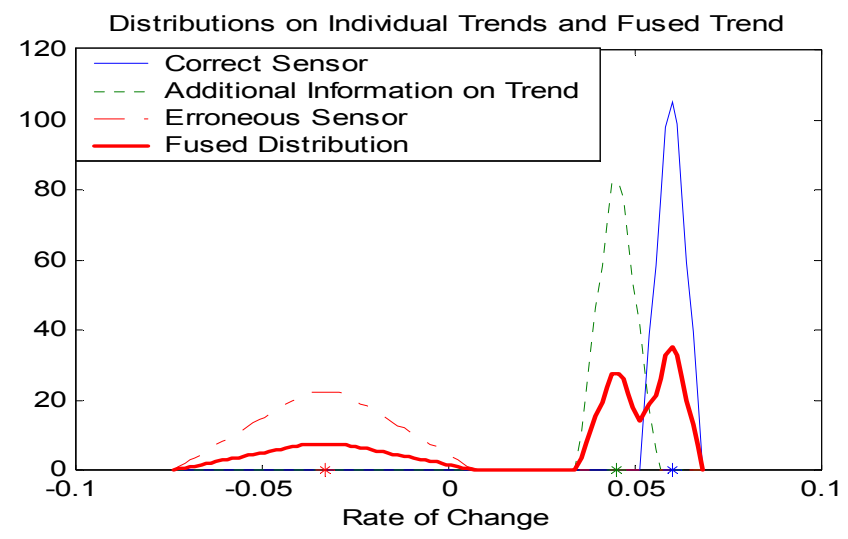

Figure 3-26 Distributions of Trend after accounting for agreement between sensor trends

Using the new confidence measure, the fusion distribution is recomputed. Since the self-confidence of the each sensor is dependent on the degree of agreement between all the sensors, the effect of the erroneous sensor is largely eliminated and there is a great improvement in the performance of the sensor fusion algorithm. From the combined 
distribution, the fused value is the argument of the peak of the distribution on the larger side of the centroid of the distribution. The equations are as shown below:

$$
\begin{gathered}
\text { Centroid }=\frac{\int_{X} x P D F(x) d x}{\int_{X} P D F(x) d x} \\
\text { Measurand Estimate }=\arg (\operatorname{Peak}(\operatorname{PDF}(x)))
\end{gathered}
$$

Where, $\mathrm{x}$ is the parameter whose fused value is to be estimated PDF ( $\mathrm{x}$ ) is the estimated density function of the parameter.

The developed fusion algorithm-incorporating trend was tested for various sets of data and the results were in agreement to those expected. It was also observed that the performance of the sensor fusion algorithm could be improved further by incorporating the fused value at previous instants. This would be akin to low-pass filtering of the data coming out of the sensor fusion module. Figure 3.27 shows the performance of the final algorithm. It is clear that the algorithm has picked the correct sensor for all instances of time.

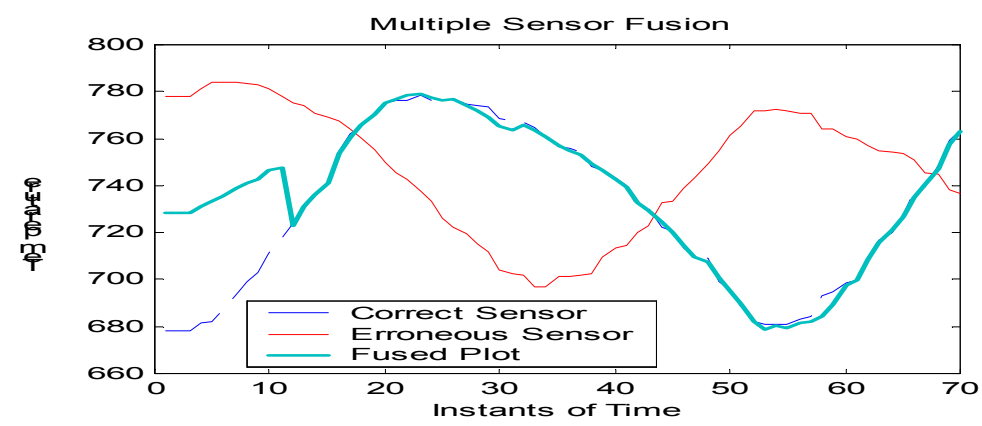

Figure 3-27 Multiple Sensor Fusion after filtering 


\subsubsection{Measure of Fused Confidence}

In this algorithm, it can be observed that we have different types of information sources, namely trend sensors and value. Thus, in evaluating the measure of the fused confidence, it is necessary, to weigh the confidence obtained from trend fusion and that obtained from value fusion. The formula used to evaluate the overall confidence of the measurement is given by:

$$
\text { FusedConfidence }=\frac{N_{T} * C_{T}+N_{V} * C_{V}}{N_{T}+N_{V}}
$$

Where, $\mathrm{N}_{\mathrm{T}}=$ Number of Trend Sources, $\mathrm{C}_{\mathrm{T}}=$ Confidence of Fused Trend, $\mathrm{N}_{\mathrm{V}}=$ Number of Sources of Value, $\mathrm{C}_{\mathrm{V}}=$ Confidence of Fused Value; Figure 3.28 illustrate the calculated overall confidence for the previous example.

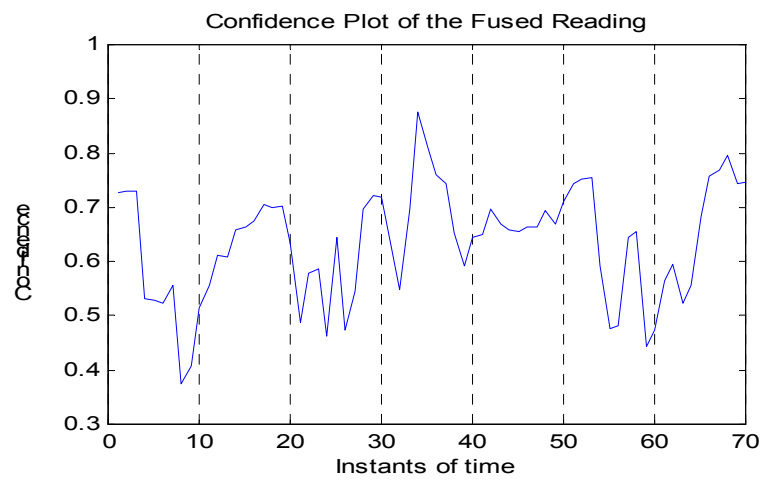

Figure 3-28 Fused Confidence Plot 


\subsubsection{Summary}

The section introduced the concept of incorporating trend in Sensor Fusion to deal with cases where reliable trend information sources such as virtual sensors or model are available. The algorithm was tested using data obtained under various circumstances and the results are shown. The results clearly indicate that the algorithm performed correctly under circumstances of sensors malfunctioning by incorporating trend information.

\subsection{Fusion of Linguistic Sources}

In this section, the algorithm for multi-modal sensor fusion is further strengthened using expert systems as additional sources of information. An expert system provides linguistic information on the parameter, which has to be converted to numerical form so that the sensor fusion algorithm can fuse it along with information from the other sources.

We start by fusing linguistic information on the trend of the measurand and then continue with fusing information on the value of the measurand itself.

\subsubsection{Linguistic Information on Trend}

Consider an expert system, may be an operator who can predict the trend of the parameter. This source of information would be in linguistic form and would be quite reliable. This source of information need not be available at every instant of time. It is 
possible that the operator can intervene at certain instants of time when there is a sudden change in the operating conditions.

This section proposes a methodology that acquires linguistic information from an expert system and converts it to numerical form that can be fused along with the other numerical information sources on trend.

Consider a parameter being monitored by a single sensor that gives information about its value. Considering that the sensor fails at some particular instant as shown in Figure 3.29. We assume an additional source of trend information. The result of the sensor fusion presented earlier is shown in the same figure. It partially corrects the faulty sensor readings, but the performance is still not satisfactory.

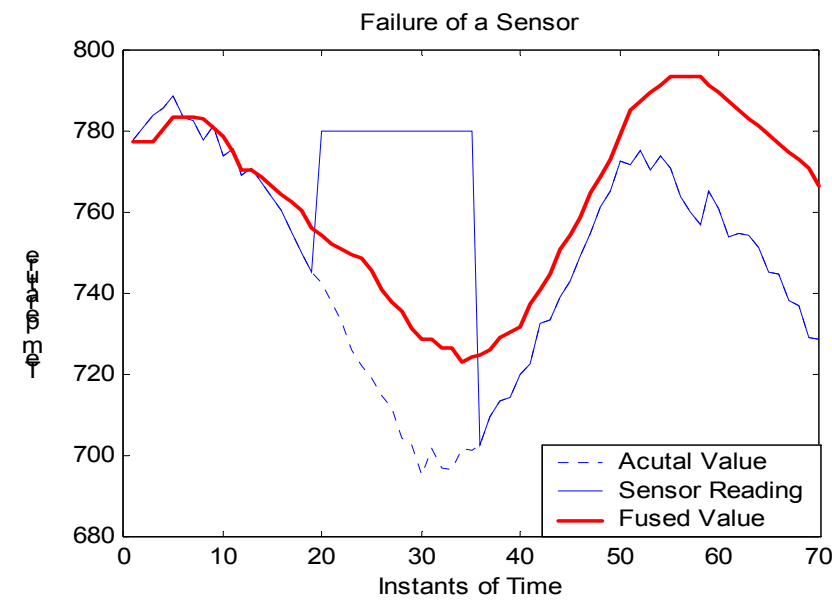

Figure 3-29 Failure of a Sensor 
Considering that we have a linguistic source of information on trend. Each linguistic variable has a pre-defined range of measurand trend values. These ranges are defined based on the behavior of measurand. An example of a set of ranges defined for the trend of a measurand could be:

Sharply decreasing: [-0.1752-0.0584]

Decreasing: [-0.1168 0]

Steady: [-0.0584 0.0584]

Increasing: [0 0.1168]

Sharply increasing: [0.0584 0.1752]

So the linguistic information provided by the operator or expert system is converted to give the operating range of operation of the trend. A Triangular distribution is constructed around this range with the peak at the center of each range. This distribution is then combined with the distributions of the sensor data and normalized to get the fused Distribution from which the centroid and the confidences are calculated as discussed in earlier sections.

Figure 3.30 shows the additional linguistic trend information. The effect of incorporating the linguistic trend information in the sensor fusion algorithm is illustrated in Figure 3.31 it can be observed that the performance of the fusion algorithm has improved when compared to Figure 3.29. 


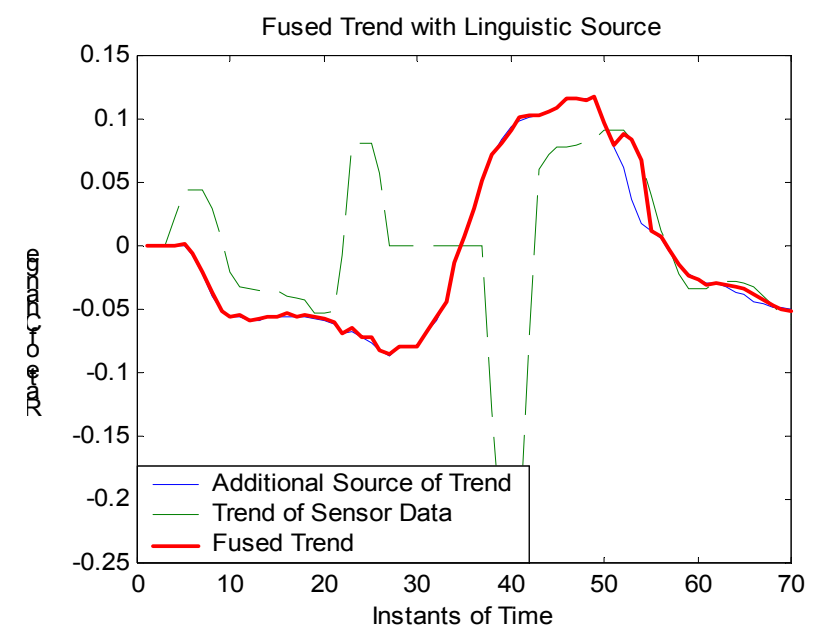

Figure 3-30 Trends after considering Linguistic Source

This increase in reliability of the Fused Trend further improves the calculation of the expected value of the parameter. This causes the Fused value to be more reliable.

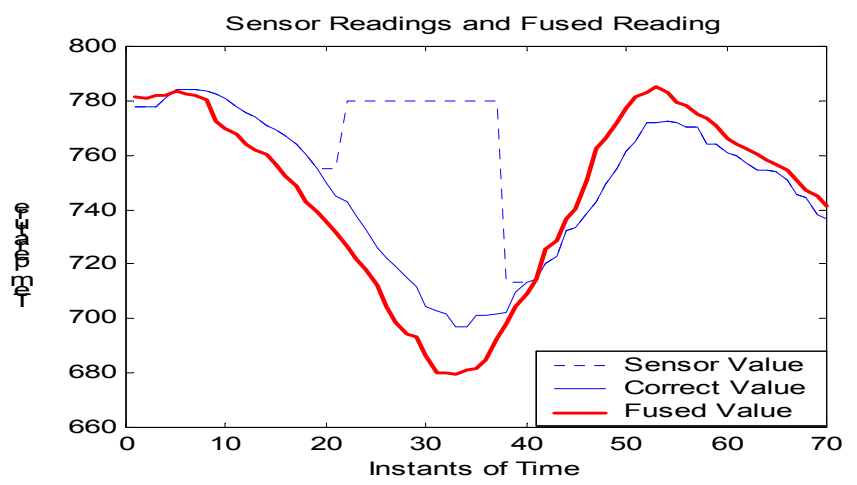

Figure 3-31 Sensor Fusion with Linguistic Trend Information

\subsubsection{Fusion of Linguistic Information on the Measurand Value}


In this section, the effectiveness of having an expert system to enhance the fusion process by considering the measurand value.

Consider a case of sensor failure as shown in Figure 3.32. In this case, the sensor performs satisfactorily till a particular instant and from then on, it has a shift in its readings. But, the sensor still continues to have the similar trend as indicated by the other linguistic sources for trend. As a result of this, the algorithm tends to start following erroneous reading provided by the sensor.

In this section, a similar methodology as that of the linguistic source on trend is considered for the parameter value as well. The operator provides the algorithm with a linguistic value, which as before has a predefined range. The ranges for the measurand values could, for example be:

$$
\begin{gathered}
\text { Very low: }[667.5692 .5] \\
\text { Low: }[680715] \\
\text { Normal: }[692.5737 .5] \\
\text { High: }[715760] \\
\text { Very high: }[750.5775]
\end{gathered}
$$

These ranges are obtained from the historical data considering the behavior of the parameter. Figure 3.33 shows that the performance of algorithm after considering linguistic information on the parameter also. 


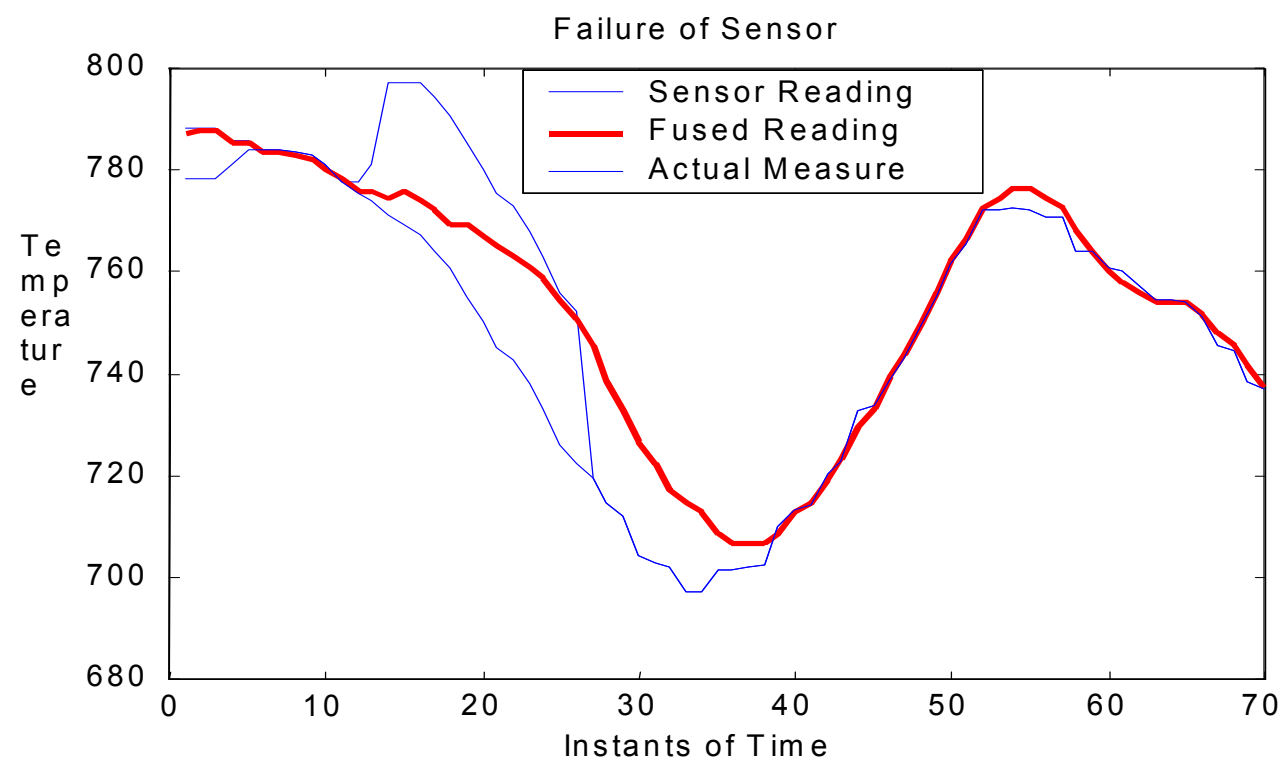

Figure 3-32 Another Case of Sensor Failure

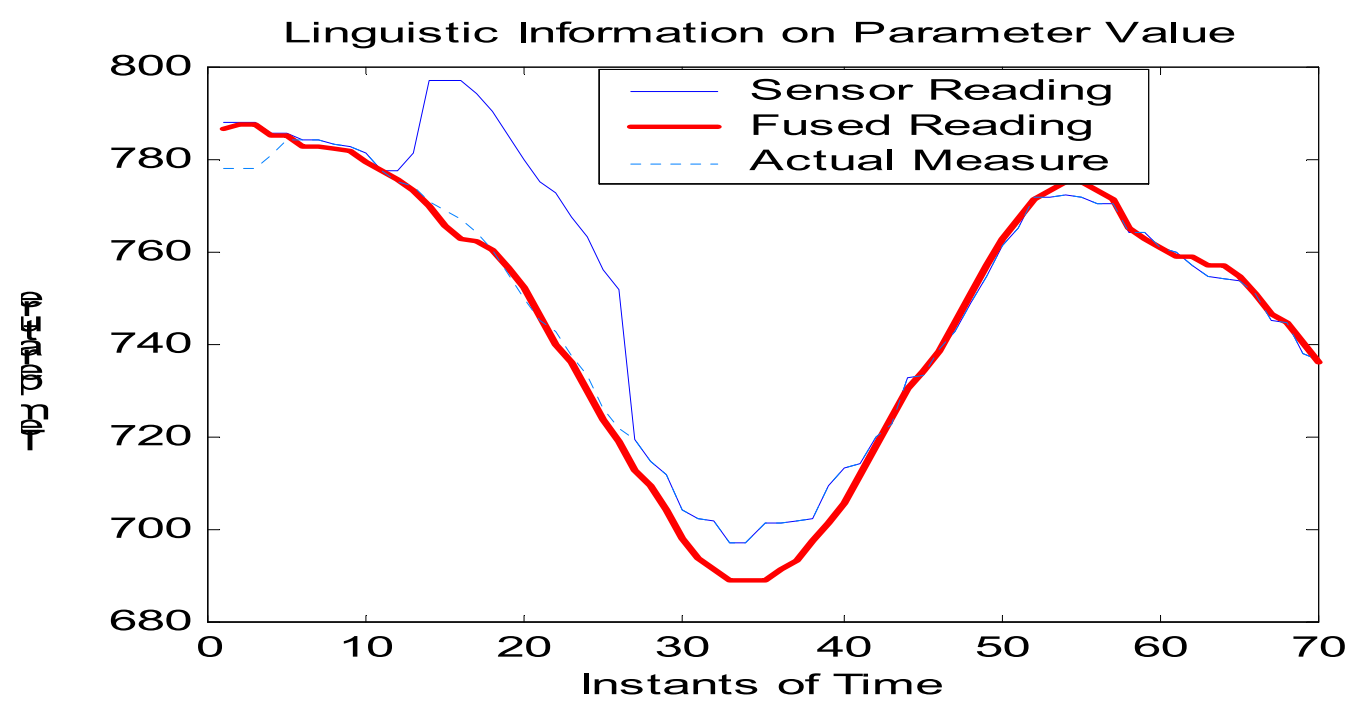

Figure 3-33 Multi-Modal Sensor Fusion with Linguistic Sources 
It can be observed that the algorithm with this linguistic source of information on the parameter value provides a very reliable fused value. 


\subsection{Wavelet-Based Sensor Fusion for Data having Different Sampling Rates}

\subsubsection{Introduction}

Data obtained from numerous sensors can be used to provide more reliable evaluation of physical data than a single sensor. In many industrial settings, several sources of data regarding a certain parameter may be collected. However, information from these sources may not always be available at the same points in time due to physical limitations. In a cupola furnace, for example, the temperature of the molten iron is measured both using a thermocouple and a pyrometer. The thermocouple (TC) measurements are made on a physical sample extracted from the furnace output and thus performed relatively infrequently as compared to the near-continuous collection of pyrometer data. However, the pyrometer data is considered to be a less reliable measure and is susceptible to gross corruption. Figure 1 shows a sample of cupola temperature data obtained using a pyrometer (Pyro_Temp) and a thermocouple (Bath_Temp). For this data, the sampling ratio between pyrometer and TC data is approximately 16:1.

Our objective in this fusion algorithm is to provide a generic methodology to fuse two data sources with different time resolution as motivated by this previous example. In this work, the pyrometer data is said to have high time resolution and the TC is said to have as low time resolution sensor. The assumption made is that the reliability of low-rate 
signal is higher than high-rate signal. Under this scenario, the fused data will be both higher in accuracy and have higher time resolution than can be gleaned from either source on its own.

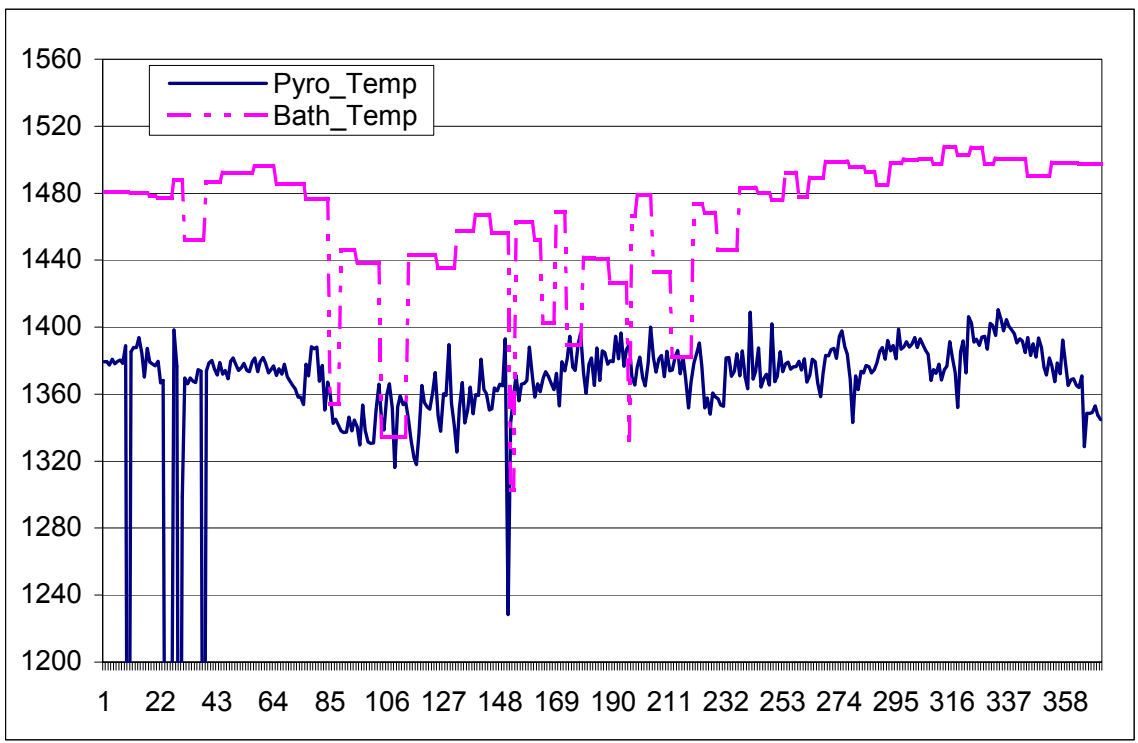

Figure 3-34: Cupola temperature data

Wavelet transforms can be used to project the data features into different levels of time resolution. The fusion process is thus performed at the appropriate time-base of resolution common to data from sensors having different sampling rates. By fusing the data features of different levels, the sampling rate difference between two data sources can be compensated. Figure 3-35, Figure 3-35 show an example of such situation in which a multi-rate fusion algorithm would be needed. The high sampling rate signal is corrupted while the low sampling rate signal is not. The result of a wavelet based fusion algorithm is shown in Figure 3-36. The effectiveness of the developed algorithm is 
further discussed in Table 3.1. The table shows the RMS error between the original signal, the corrupted and fused signal. The details of the algorithm is given in [31].

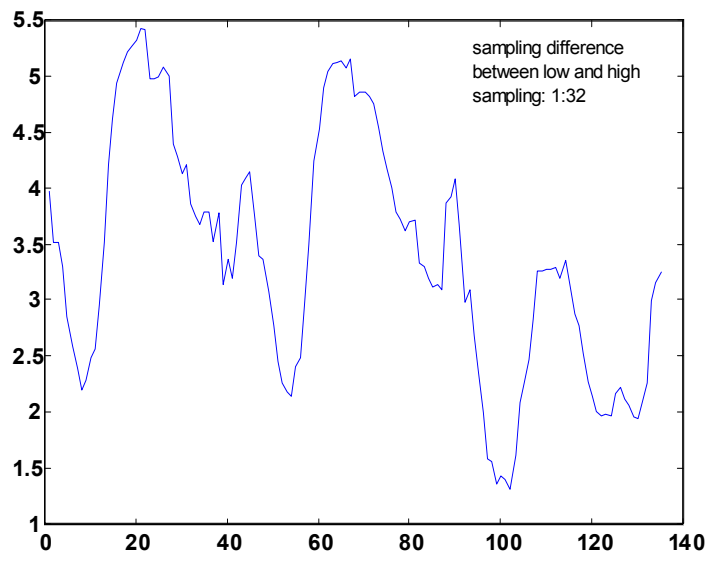

Figure 3-35 Low sampling rate signal $X_{2}[n]$

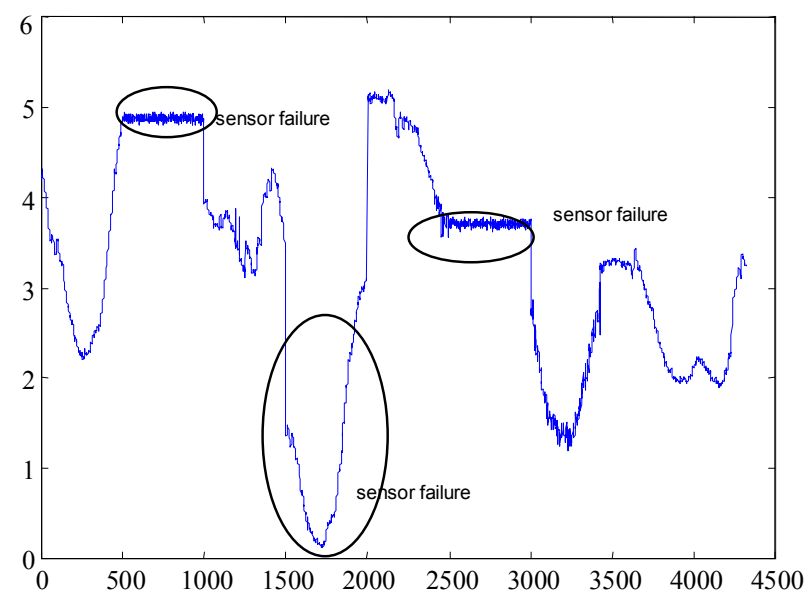

Figure 3-36: Corrupted high sampling rate signal $X_{1}[n]$ 


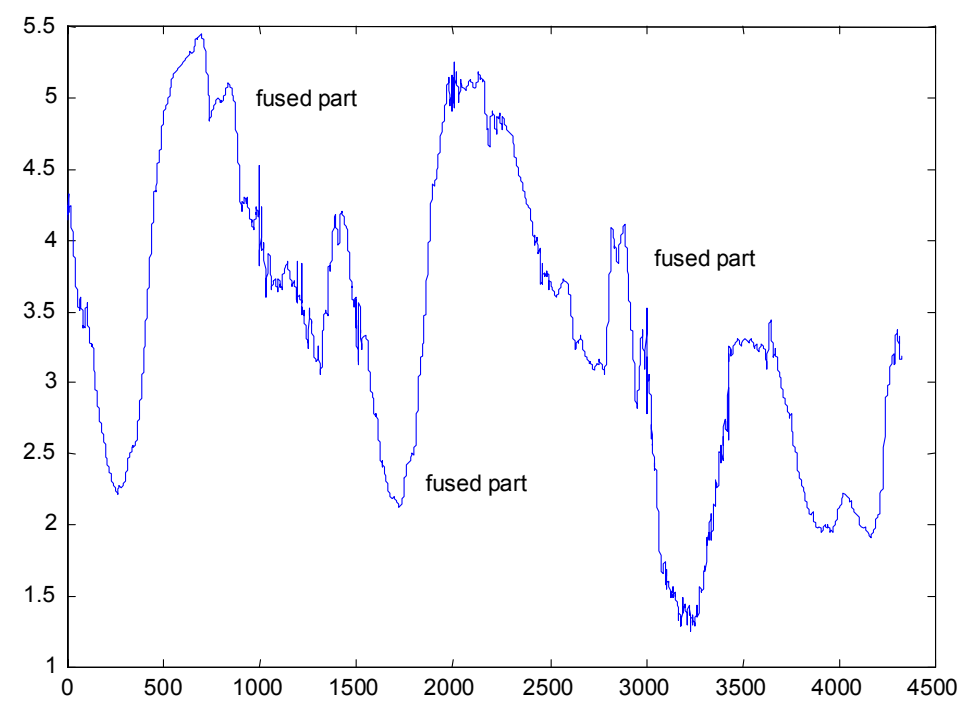

Figure 3-37: Fused signal

Table 3.1: Mean square error of fusion methods

\begin{tabular}{|c|c|}
\hline Method & MSE \\
\hline No Fusion (corrupted signal) & 1.0856 \\
\hline Spline Interpolation & 0.0273 \\
\hline Proposed Wavelet-based Method & 0.0063 \\
\hline
\end{tabular}




\section{Chapter 4}

\section{INTEGRATION OF MULTIPLE SENSOR FUSION IN CONTROLLER DESIGN}

The main focus of this project is to reduce the risk of a catastrophic response of a feedback control system when the feedback data from the sensors is not reliable, while maintaining a reasonable performance of the control system. An algorithm for multiple sensor fusion was presented in Chapter 3. Sensor fusion helps in improving the reliability of the measurement. It does not, however, address the control problem when the data are known to be unreliable. In certain conditions, even multiple sensor fusion could produce an incorrect estimate. So, the problem still exists even if multiple sensor fusion is used.

This chapter starts with the study of a case where the multiple sensor fusion algorithm that was developed in Chapter 3 produces a bad estimate. Then, a methodology for the controller design to improve the system performance in these situations is developed. The stability of the closed loop system with the developed controller is studied using Lyapunov stability theory in the final sections of the chapter. A linear plant model is considered while studying the stability of the system with the controller. 


\subsection{Motivation}

The multiple sensor fusion algorithm is developed, based on the fact that the correct estimate lies at the highest probability value as determined using the sensors' data.

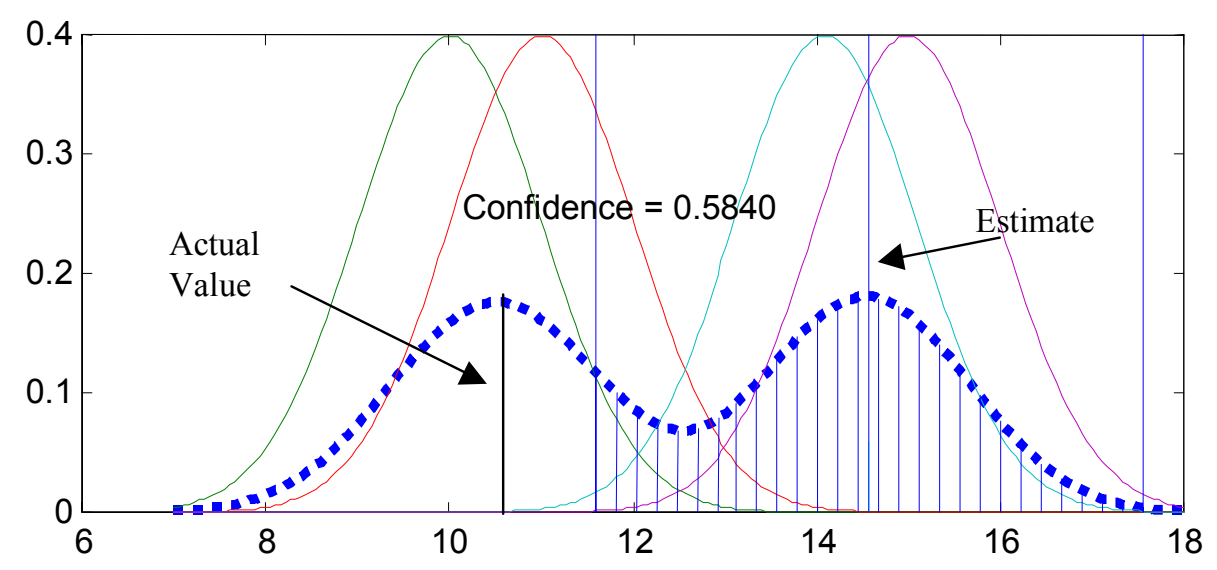

Figure 4-1 Wrong Estimate from the Multiple Sensor Fusion

In the case shown in Figure 4-1, two sensors read the correct value (near 10) while the other two read the wrong value (near 14). The estimate from the sensor fusion can be seen to be closer to the wrong value, just because the readings from the two sensors that have failed are closer to each other than those of the correct sensors.

Although the estimate from the sensor fusion algorithm was wrong, it can be seen from the figure that the corresponding confidence is low. This confidence can thus be used as an important parameter that can be integrated into the controller so as to improve the performance of the system when the multiple sensor fusion fails. 


\subsection{Controller Design}

The controller, designed with the assumption that the estimate from the sensor fusion is reliable, may drive the system fast into the wrong direction if the estimate was wrong. On the other hand, if the controller is designed considering the worst case scenario, it will result in sluggish response. One method that can be used to improve the performance of the system is to design the controller such that it has fast response when the confidence is high and a slow response when the confidence is low. Hence the controller should be able to adapt itself and produce a controller that adapts its response depending upon the confidence in the estimate.

The required performance of the controller after the integration of the confidence can be summarized as

1. When the confidence is high: the feedback signal is reliable. So, the controller should be fast enough to track the reference value.

2. When the confidence is low: the feedback signal is not reliable, which implies that even if the controller has tracked the value fed to it, the state that the system has reached may not be the correct reference value. So, the controller should not try to reach the reference value very fast.

One way to achieve this requirement on the controller is by designing two controllers, a fast controller that will be active when the confidence is high and a slow controller, which will be used when the confidence is low. The controller is then 
implemented by changing its parameters between those of fast controller and slow controller using the confidence as the weighting parameter between the two controllers. A schematic diagram of the system with the controller designed is shown in Figure 4-2. The resulting expression for the controller parameter, with the confidence as the weighing parameter, is given below.

$$
K=\alpha K_{h}+(1-\alpha) K_{l}
$$

and

$$
\alpha=\mathrm{f}(\text { confidence })
$$

where,

$K$ - State feedback matrix of dimension $\mathrm{m} \mathrm{x} \mathrm{n,} \mathrm{m}$ is the number of output variables and $\mathrm{n}$ is the number of state variables.

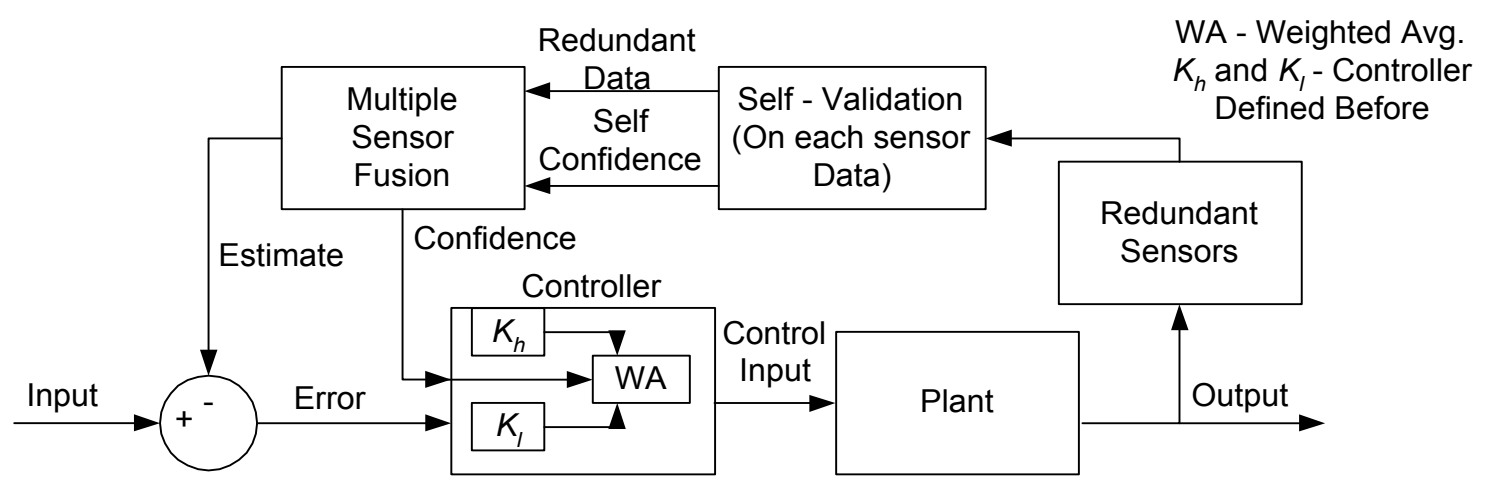

Figure 4-2 Schematic Diagram of the System with Sensor Fusion Integrated with the Controller 
$f$ - Nonlinear piecewise continuous function operating on the minimum of the confidence on each state variable estimate from multiple sensor fusion.

$K_{h}$ - State feedback matrix corresponding to fast controller ( $\mathrm{m} \times \mathrm{n}$ )

$K_{l}$ - State feedback matrix corresponding to slow controller ( $\mathrm{m} \mathrm{x} \mathrm{n)}$

The conditions that the nonlinear function, $f$, should satisfy to meet the controller requirements specified above are

$$
\begin{aligned}
& \alpha=1 \text { when confidence is } 1, \\
& \alpha=0 \text { when confidence is } 0,
\end{aligned}
$$

and $\quad 0<\alpha<1$, for all other confidence between 0 and 1 .

\subsection{Stability Analysis}

The controller designed as discussed above will be helpful only if the closed loop system with the controller is stable. This application is developed for a cupola furnace plant in Albany research center, which has a linear model. Hence a linear model is considered in the stability analysis. The stability conditions for the closed loop system are not trivial since the system is time variant as the controller parameters change with the time. A theorem is stated and proved in this section. The application of the theorem discusses the conditions on the stability of the closed loop system. 


\section{Theorem 4.1}

Consider a linear time varying system

$$
\begin{aligned}
\dot{x}(t) & =A x(t)+B u(t) \\
u(t) & =-K(t) y(t) \\
y(t) & =x(t)
\end{aligned}
$$

where, $x \in R^{n}, y \in R^{m}, u \in R^{k}$ are the state, output, and input variables, respectively. $A, B$, and $K$ are the matrices of appropriate dimensions. The system will be asymptotically stable, if

1. The controller parameter $K$ is given by expression (4.1).

2. There exists a Lyapunov function of the form given in (4.5) where $\mathrm{P}$ is a positive definite matrix that proves the stability of the system for both matrices $K_{h}$ and $K_{l}$.

$$
V=x^{T} P x
$$

Proof: The system equation after combining all the expressions is

$$
\dot{x}=(A-B K(t)) x .
$$

It is given that the closed loop system with the time invariant controller parameters $K_{h}$ and $K_{l}$, are asymptotically stable with the same Lyapunov function in (4.5). Hence the Lyapunov equations for these systems will be satisfied.

Equations (4.6) and (4.7) gives the respective Lyapunov equation.

$$
\left(A-B K_{h}\right)^{T} P+P\left(A-B K_{h}\right)=-Q_{1}
$$




$$
\left(A-B K_{l}\right)^{T} P+P\left(A-B K_{l}\right)=-Q_{2}
$$

where, $Q_{1}$ and $Q_{2}$ are positive definite matrices.

For the controller $K$ given by Equation (4.1), the derivative of the Lyapunov function is

$$
\dot{V}=-x^{T}\left[(A-B K)^{T} P+P(A-B K)\right] x .
$$

Substituting (4.1) in (4.8), gives

$$
\dot{V}=x^{T}\left(A-B\left(\alpha K_{h}+(1-\alpha) K_{l}\right)\right)^{T} P+P\left(A-B\left(\alpha K_{h}+(1-\alpha) K_{l}\right)\right) x .
$$

Writing $\mathrm{A}=\alpha \mathrm{A}+(1-\alpha) \mathrm{A}$ and separating the $K_{h}$ and $K_{l}$ terms, gives

$$
\dot{V}=x^{T}\left\{\alpha\left[\left(A-B K_{h}\right)^{T} P+P\left(A-B K_{h}\right)\right]+(1-\alpha)\left[\left(A-B K_{l}\right)^{T} P+P\left(A-B K_{l}\right)\right]\right\} x
$$

which, by (4.6) and (4.7), gives

$$
\mathrm{Q}_{3}=\alpha \mathrm{Q}_{1}+(1-\alpha) \mathrm{Q}_{2}
$$

The derivative of the Lyapunov function is hence,

$$
\dot{V}=-\alpha x^{T} Q_{1} x-(1-\alpha) x^{T} Q_{2} x=-x^{T} Q_{3} x .
$$

In expression (4.11), both the terms in RHS are negative since $0 \leq \alpha \leq 1$. Hence $\dot{V}$ is always negative. Q.E.D

The closed loop system with the controller designed in section (4.2) can be proved asymptotically stable by the direct application of the above theorem. As the confidence in the estimate changes, the controller $K$ changes with time. But, the controller parameters 
are bounded by $K_{h}$ - for high speed and $K_{l}$ - for low speed and the intermediate value varies between these bounds. Finding a single positive definite matrix $P$ for the Lyapunov function in (4.5) that satisfies the Lyapunov criteria for both $K_{h}$ and $K_{l}$ is sufficient to prove the stability of the closed loop system, where $K$ is given by (4.1).

The above proof for stability is based on the assumptions that the state of the system is known exactly. This is not always true in the closed loop system. Multiple sensor fusion gives a good estimate for the measurand. However, there exists an uncertainty in the state of the system as illustrated in Figure 4-3. The stability conditions should incorporate this uncertainty in the state of the system. Theorem 4.1 is extended to include the uncertainty in the states.

\section{Theorem 4.2}

Let us consider a linear time varying system given by the system equation

$$
\begin{aligned}
& \dot{x}(t)=A x(t)+B u(t) \\
& u(t)=-K(t)(x(t)-g(x))
\end{aligned}
$$

where $g(x)$ is the uncertainty in the output. If the system satisfies

1. The conditions of Theorem 4.1 .

2. The Lyapunov function $V(x)=x^{T} P x$, satisfies

$$
k_{1}\|x\|^{2} \leq V(t, x) \leq k_{2}\|x\|^{2}
$$




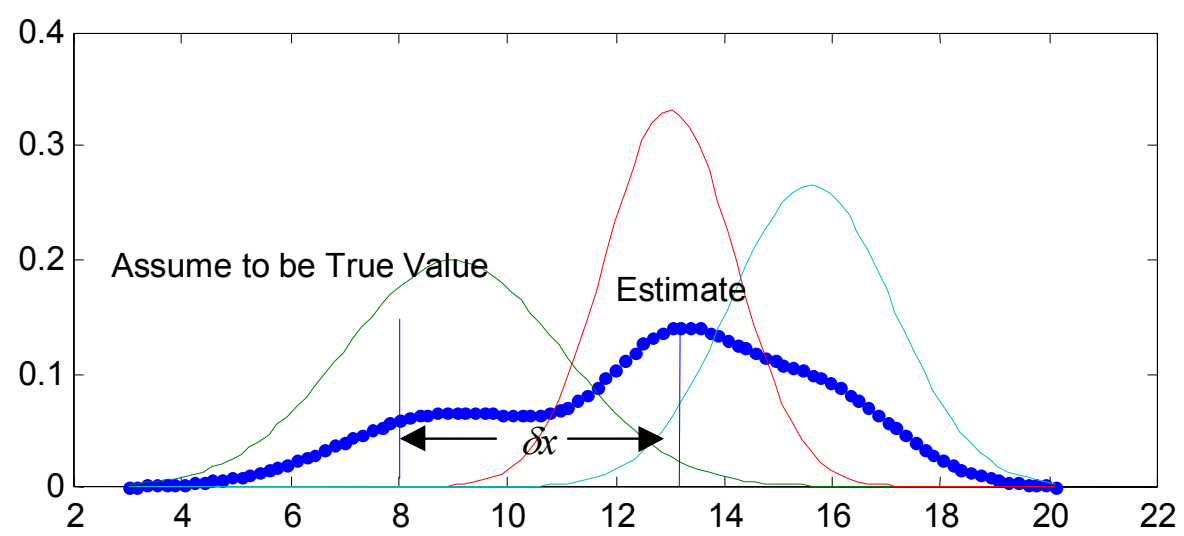

Figure 4-3 Uncertainty in the Estimate from Multiple Sensor Fusion

$$
\frac{\partial V}{\partial t}+\frac{\partial V}{\partial x} f(t, x) \leq-W_{3}(x) \quad \forall\|x\|_{2} \geq \mu>0
$$

$\forall t \geq 0, \forall x \in \mathrm{D}, \mathrm{D}=R^{n}, W_{3}(x)$ is a positive definite function and $\mu$ is a positive constant.

3. The uncertainty is piecewise continous and locally Lipschitz in $x$ and satisfies the following conditions,

$$
\|g(x)\|_{2}<\delta_{0}+\delta_{1}\|x\|_{2}
$$

where the bound on $\delta_{0}$ and $\delta_{l}$ is given by the expressions

$$
\delta_{0}<r \frac{\left(\lambda_{\min }\left(Q_{3}\right)-2\|P B K\|_{2} \delta_{1}\right)}{2\|P B K\|_{2}} \sqrt{\frac{k_{1}}{k_{2}}}
$$




$$
\delta_{1}<\frac{\lambda_{\min }\left(Q_{3}\right)}{2\|P B K\|_{2}}
$$

where $\mathrm{r}$ is the radius of a Ball $B_{r} \subset \mathrm{D}$. Then, the state of the system is given by

$$
\begin{gathered}
\|x(t)\| \leq \beta\left(\left\|x\left(t_{o}\right)\right\|, t-t_{0}\right), \quad \forall \quad t_{0} \leq t<t_{1} \\
\|x(t)\| \leq r, \quad \forall \quad t>t_{1}
\end{gathered}
$$

where $\beta$ is a class KL function.

In other words the steady state of the system lies within the ball, $B_{r}$ shown in Figure 4-4 for all time $t>t_{1}$.

\section{Proof:}

The system equation can be separated into the feedback-stabilized part and the uncertain term as

$$
\dot{x}=(A-B K) x+B K g(x) .
$$

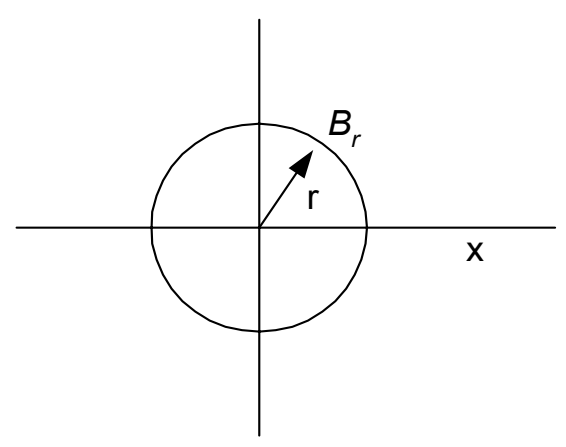

Figure 4-4 Region of Stability 
The derivative of the Lyapunov function is

$$
\dot{V}=x^{T} P(A-B K) x+x^{T}(A-B K)^{T} P x+x^{T} P B K g(x)+(P B K g(x))^{T} x .
$$

Since the last two terms are scalar, they can be combined and using the proof of the Theorem 4.1, the derivative of the Lyapunov function is

$$
\dot{V}=-x^{T} Q_{3} x+2 x^{T} \operatorname{PBKg}(x)
$$

Using Holder's Inequality, gives

$$
\dot{V} \leq-x^{T} Q_{3} x+2\|x\|_{2}\|P B K\|_{2}\|g(x)\|_{2} .
$$

The uncertainty $g(x)$ is given by the expression (4.14). Using this relation in the expression gives

$$
\begin{aligned}
& \dot{V} \leq-x^{T} Q_{3} x+2\|x\|_{2}\|P B K\|_{2} \delta_{0}+2\|x\|_{2}\|P B K\|_{2} \delta_{1}\|x\|_{2} \\
& \dot{V} \leq-\lambda_{\text {min }}\left(Q_{3}\right)\|x\|_{2}^{2}+2\|P B K\|_{2} \delta_{1}\|x\|_{2}^{2}+2\|x\|_{2}\|P B K\|_{2} \delta_{0} \\
& \dot{V} \leq-\|x\|_{2}\left\{\|x\|_{2}\left(\lambda_{\text {min }}\left(Q_{3}\right)-2\|P B K\|_{2} \delta_{1}\right)-2\|P B K\|_{2} \delta_{0}\right\} .
\end{aligned}
$$

The term $\lambda_{\text {min }}\left(Q_{3}\right)-2\|P B K\|_{2} \delta_{1}$ is always positive as $\delta_{1}$ is bounded as given in (4.16). This gives an expression for $\mu$ defined in (4.13)

$$
\mu=\frac{2\|P B K\|_{2} \delta_{0}}{\left(\lambda_{\min }\left(Q_{3}\right)-2\|P B K\|_{2} \delta_{1}\right)} .
$$


Substituting the bound on $\delta_{0}$, and $\delta_{1, \mu} \mu$ satisfies the relation

$$
\mu<r \sqrt{\frac{k_{1}}{k_{2}}}
$$

The constants $k_{i}$ are: $k_{1}=\lambda_{\min }(P), k_{2}=\lambda_{\max }(P)$.

This satisfies the condition in Theorem 5.1 of [24]

$$
\max _{\|x\| \leq \mu} k_{2}\|x\|^{2}<\min _{\|x\|=r} k_{1}\|x\|^{2} .
$$

Thus all the conditions for the Theorem 5.1 in Error! Reference source not found. are satisfied. Applying the theorem and corollary 5.3 in Error! Reference source not found. completes the proof.

This theorem can be applied to the closed loop system developed. As mentioned before the system satisfies all the conditions of the Theorem 4.1. Thus, all the conditions for the Theorem 4.2 are satisfied. The closed loop with the controller designed as specified in Equation (4.1) is stable within the Ball $B_{r}$. The asymptotic stability of the closed loop system with respect to the origin cannot be specified.

Thus, the confidence parameter from the multiple sensor fusion algorithm presented in Chapter 3 is integrated into the controller design and the closed loop system with such a controller is proved to be asymptotically stable though not to the origin, but to a ball of radius $r$. The confidence from the multiple sensor fusion is integrated into the controller to prevent the degradation of the system's performance when the multiple 
sensor fusion fails. The next chapter deals with the implementation of this theory in a multi-variable feedback control system and simulates the performance of the system.

\subsection{Fuzzy Controller}

In the previous sections a traditional controller was designed and a procedure for integrating the sensor fusion in the controller design was presented. The stability of the designed controller was rigorously proven. Traditional controllers, however, are more suitable for linear systems with well-defined models. Although linear models in a specified range of operation can describe cupola furnaces, they are in general nonlinear systems with a lot of uncertainty in the inputs. In this section an alternative to the previously designed traditional controller is given. The alternative design is based on fuzzy control principles. Using the pairing of cupola inputs to outputs as $\mathrm{CMR} / \% \mathrm{C}$, $\mathrm{O}_{2} / \mathrm{T}$, and $\mathrm{BR} / \mathrm{MR}$, three fuzzy controllers were designed. The fuzzy controller is composed of a fuzzy inference system and an integrator. The fuzzy inference system suggests changes to the inputs in order to achieve the desired changes in the outputs. The integrator accumulates these changes and presents it to the cupola. The inputs to each fuzzy inference system are: 1) the error which represents the difference between the desired output and the current output and 2) the change in the error averaged over a period of time. The outputs of the fuzzy inference system of each controller can take on five values: large positive (LgPos), small positive (SmPos), zero, small negative (SmNeg), or large negative (LgNeg). These values represent the required change in the 
input to achieve the desired change in output. Each fuzzy inference system contains five trapezoidal membership functions for each input: a large positive and negative, a small positive and negative, and an zero range. Since there are two inputs (error and change in error) with five membership functions each, there are twenty-five rules relating the inputs and outputs of each fuzzy inference system used in this paper.

\subsubsection{Controller design}

In order to control the system properly, some knowledge of the desired response for each output such as MR, $\mathrm{T}$ and $\% \mathrm{C}$ and limitations on the ranges and possible rates of change of the available inputs such as $\mathrm{O} 2, \mathrm{BR}$ and $\mathrm{CMR}$ is required. This is important since the controller might request changes in those input parameters that might not be achievable or might cause erroneous response of the cupola.

A normal settling time for a moderate change in melt rate was selected to be 5 minutes. This means that small changes in MR could be achieved within 5 minutes. Changes in molten metal temperature were also selected to be achieved in 5 minutes. For changes in the $\% \mathrm{C}$, the long time delay associated with changes in the charge composition forces changes in the $\% \mathrm{C}$ to take a longer time period. The time selected to achieve changes in $\% \mathrm{C}$ is 50 minutes. 
The process of designing the fuzzy controller is an iterative one. An initial guess was made to the membership function parameters, the output values, and the rules so that simulations could be performed. Plots of the fuzzy inputs and system outputs were then used to tune the controller parameters. For example, an initial definition of a small change in the error or a large change in error is readjusted after looking at the response of the system during a simulation. A narrow value for the ideal range would cause the system to be very sensitive to noise while a wide range for the ideal membership function would allow the system to deviate considerably from the desired output. Using the simulations, the fuzzy output parameters were chosen such that: the settling times were close to 600 seconds, the system inputs would not change too quickly, and overshoots were minimized. Figure 4-5 and Figure 4-6 are examples of the membership functions of the two inputs for the melt rate fuzzy inference system. Figure 4-5 represents the error in the MR while Figure 4-6 represents the change in error in the MR.

An iterative method for changing the rules from the initial guess is similarly followed. These rules were updated based on examining plots generated through simulations. The list of rules for the melt rate controller is shown in Appendix 4.A. The rules for the other two outputs of interest in this paper, namely $\mathrm{T}$ and $\% \mathrm{C}$ are very similar. Examining a subset of these rules illustrates the main idea behind the fuzzy controller. The error in an output is positive when the set point is higher than the actual value and the rate of change in the error is negative if the error is decreasing and vice versa. Consider a case when the MR is at steady state and an increase in the MR is 
requested. The error in the MR is positive and change in the error is zero, then, according to rule 23 in Appendix 4.A, the blast rate should be increased at its maximum rate. Another example is if the error in MR is zero, but the output is heading toward an overshoot because the rate of change in the error is a large positive value, then the change in the BR should either be a small negative according to rule 15 in Appendix 4.A.

One of the main objectives of this research was to obtain a controller that could be used for any cupola, not just the Albany Research Center's experimental cupola in Albany, Oregon. Therefore, equations were developed to reconfigure the fuzzy membership function and output parameters. These use the steady state parameters and the limitations on the system inputs to achieve the reconfiguration of the parameters. 


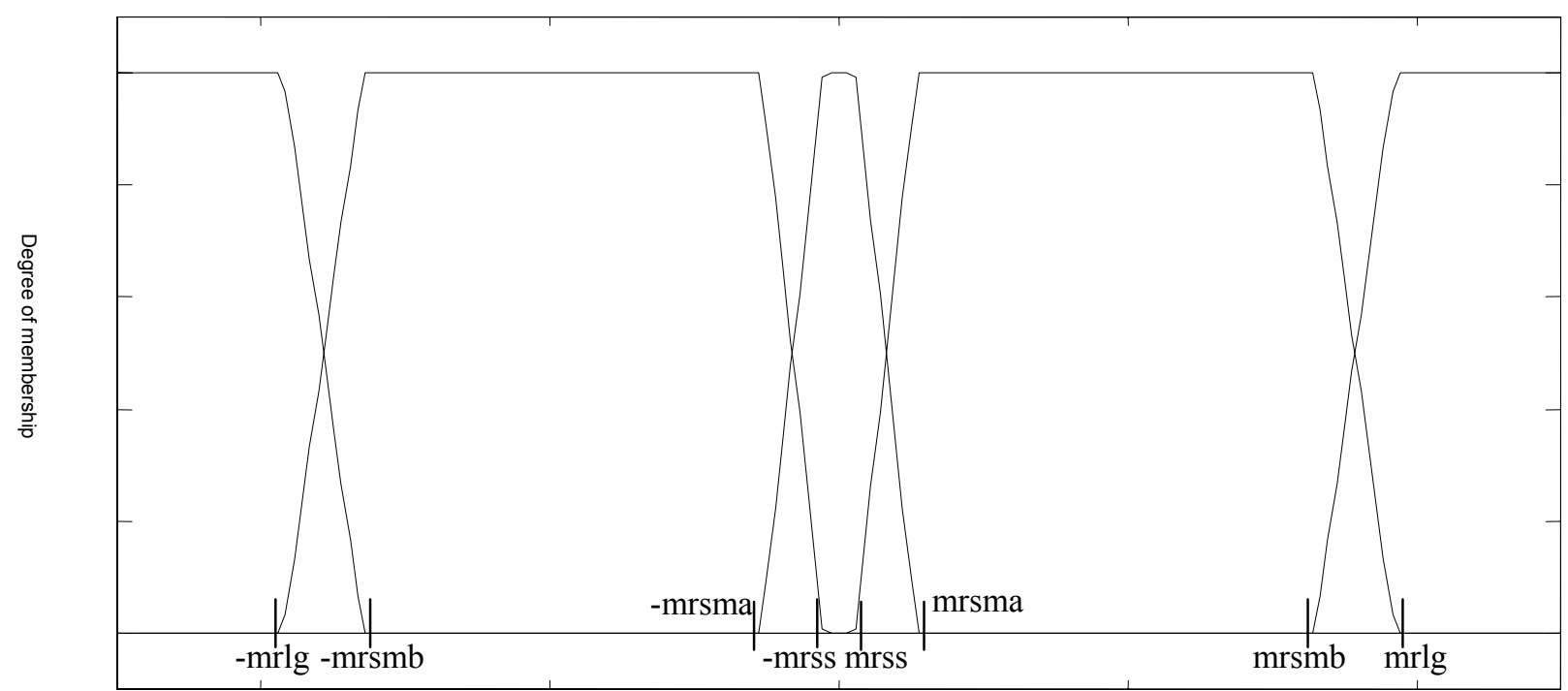

Figure 4-5 Membership functions of the error in melt rate (eMRate)

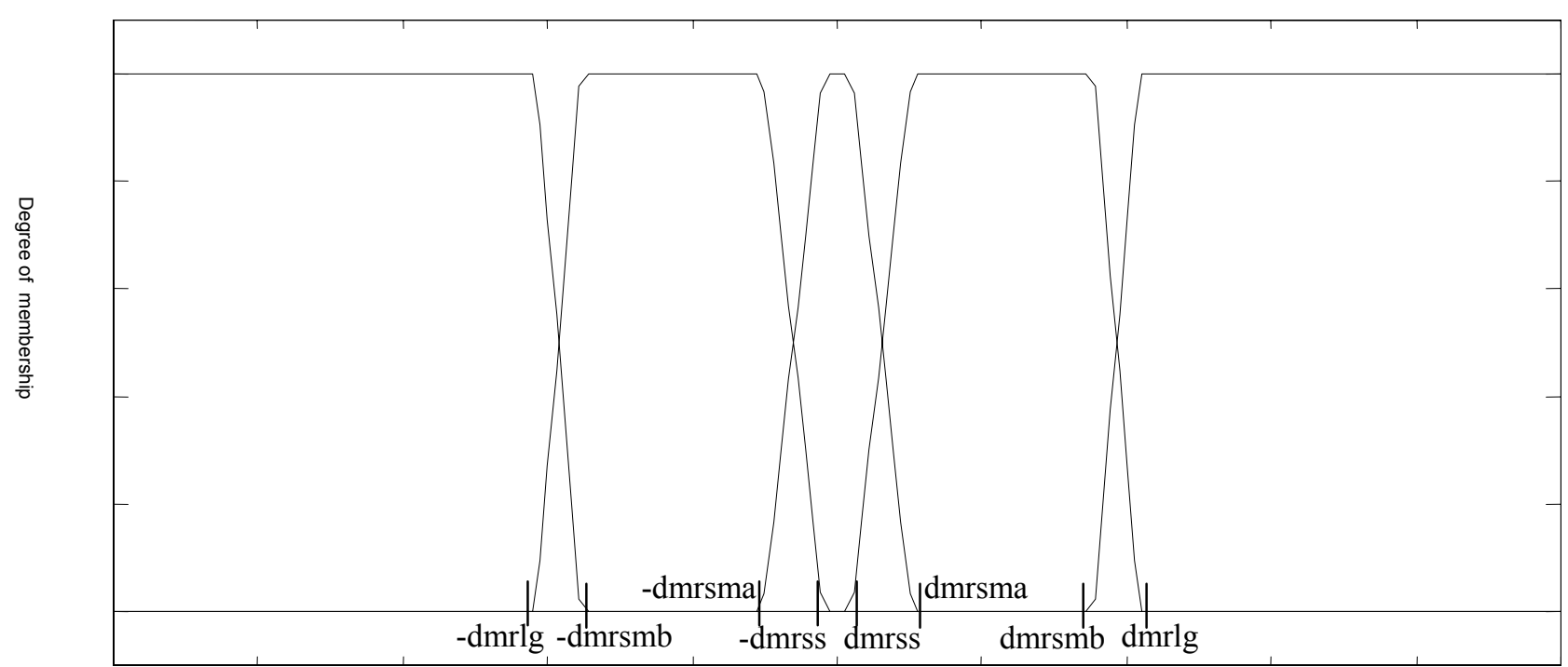

Figure 4-6 Membership functions of the change in error for the melt rate (deMRate) 


\subsubsection{Smith Predictor}

Due to the nature of the cupola in loading the fuel at the top and burning at the bottom, a long time delay exists between changing the CMR and that change affecting the melting zone. In order to accommodate that time delay a predictive model based strategy is utilized. This was suggested first by Smith (Smith, 1957) and thus the method is referred to as a Smith predictor. The Smith predictor is utilized with the $\% \mathrm{C}$ controller. The schematic diagram of the system with the Smith predictor is shown in Figure 4-7. As shown in this figure, an estimate of the input to the cupola is fed to a duplicate transfer function relating the $\mathrm{CMR}$ to the $\% \mathrm{C}$. The signal from the duplicate transfer function has a time delay applied to it and then is subtracted from the $\% \mathrm{C}$ output signal. The resulting signal is then added to the undelayed signal from the duplicate transfer function. The final result is the feedback signal for the system. The time delay in the Smith predictor is representative of the best-known or average delay measured.

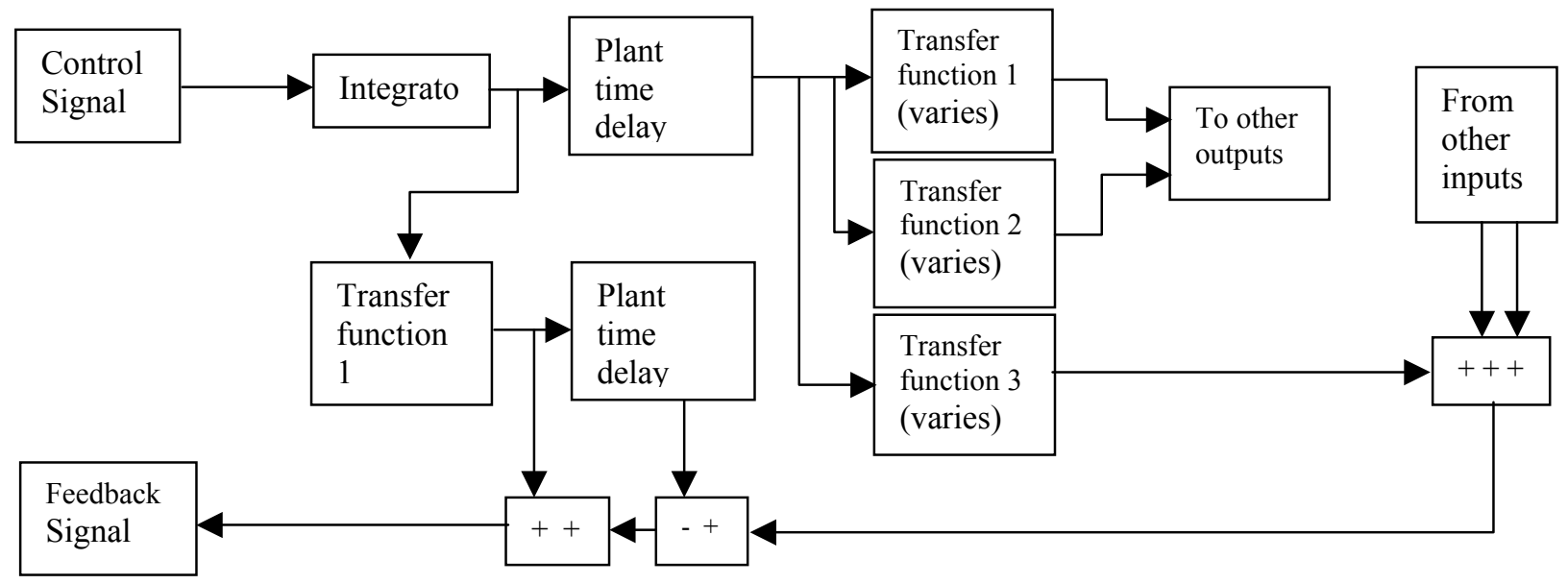

Figure 4-7 Implementing a Smith predictor 


\subsubsection{Integration of Sensor Fusion in Controller Design}

The harsh environment at the output of the cupola results in sensor drift and failure. By using several sensors and the sensor fusion technique developed earlier a confidence between zero and one can be determined that reflects the accuracy of the output value. It is desirable to use the confidence level to adapt the speed of response of the controller. This is achieved by scaling the output of the fuzzy inference in the fuzzy controller by a function related to the confidence. In this paper, the confidence is raised to a power and this is multiplied into the output of the fuzzy inference system. Assume for example that the confidence level in a signal is $90 \%$, it could be raised to the fifth power which results in reducing the change in the input to $60 \%$ of the amount requested by the fuzzy inference system. If the confidence is $50 \%$, the change in input is reduced $3 \%$ of the amount requested by the fuzzy engine. Thus, the effect of using the confidence in the measurement to scale the fuzzy engine output is to adapt the speed of response of the controller based on our confidence in the measurements. This can be effective in mitigating the effects of failed sensor or external disturbances over the performance of the closed loop system. 


\subsubsection{SIMULATOR DESIGN}

The model was implemented in Simulink for the transfer matrix first order responses and the time delay of the CMR. The model requires loading the data of the steady state variables, time constants, CMR time delay, operating point, and input boundaries.

\subsubsection{BASIC LAYOUT}

The simulation is to represent a change in the outputs and inputs from a normal operating point. The output of the controller reflects the desired rate of change of the system inputs. The controller output is integrated to reflect the total change in the system input. The value of the integrator is bounded to prevent integral windup. Each integrator output then passes its value to three transfer functions obtained from the transfer function matrix. Each system output receives three values, one from each integrator after passing through a transfer function. These values are added to give the total change in the output. Error signals, which represent deviation of the actual signal from the desired output, are one of the inputs that get fed to the controllers. The change in the error signal is averaged over a period of time and is sent to the controller as a second input. Figure 4-8 illustrates the layout. 


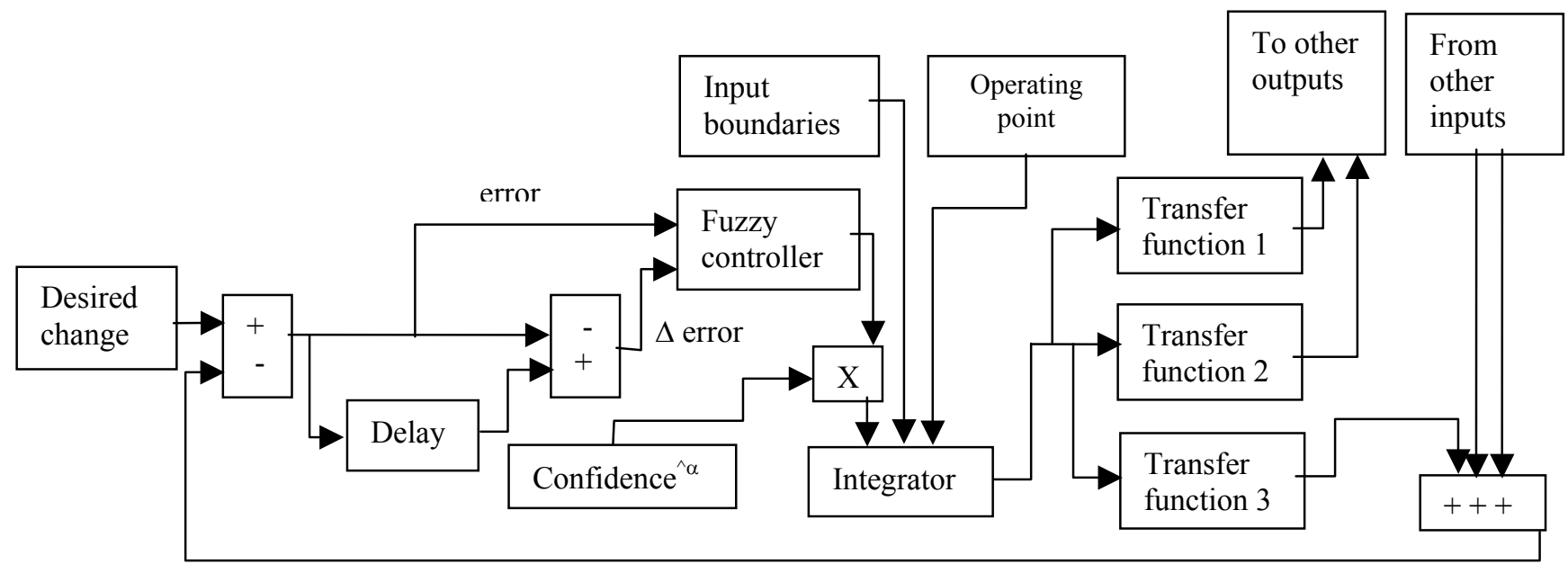

Figure 4-8 Simulation layout

\subsubsection{NOISE, DISTURBANCES, AND VARYING PARAMETERS}

The actual system is non-linear and has been approximated by a linear system with constant parameters in the transfer matrix. Sensors that measure the system outputs are subject to noise and the system inputs may not perform exactly as the controller demands. The simulation must take these factors into consideration.

\section{Noise}

Gaussian noise was added to the system outputs before sampling for feedback. This represents the fact that the sensors do not measure the outputs perfectly. For such a harsh environment as a cupola, this noise could be considerable. 


\section{Disturbances}

Disturbances in the form of a square wave were added to each of the integrator signals. This represents not knowing exactly the system inputs. An example would be setting the blast to increase and not knowing one of the fans were down or the feed of metal into the cupola could have different densities and could be difficult for the human loaders to approximate its weight.

\section{Varying Parameters}

There was no research done on finding how the parameters changed non-linearly. In order to test for all possible cases, a sine wave with an offset close to one was multiplied to the transfer functions, except for the duplicate transfer function of the Smith predictor. For each of the transfer functions, the sine wave was at a different initial phase and all were at different frequencies. With all at different frequencies, the simulation could be run long enough so that all combinations of the parameters within a range could be studied.

\subsubsection{RESULTS}

The controller was tested under ideal conditions, output noise, input disturbance, sensor fusion noise, by varying the model parameters over a wide range, and actual CMR time delay being different than that used in the Smith predictor. The tests of the controller's robustness and dependability are described below with generated plots of the results. 


\section{IDEAL CONDITIONS}

The model was tested without noise and with step inputs that would reflect a change in the operation of the cupola. The temperature was requested to undergo a change of $50^{\circ} \mathrm{C}$ at 3000 seconds, the melt rate to change by 0.1 (tons/hr) at the same time. A change of $0.1 \%$ in $\% \mathrm{C}$ was requested at 100 seconds. The reason the changes in temperature and melt rate were requested at a much later time was that the CMR has a much longer settling time due to the long time delay of the charge. With these change times, the three outputs changed and settled at close to the same time. Figure 4-9 shows the plots of the outputs, inputs, error, and the change in error. Most of the fuzzy parameters were fine-tuned using these plots.

Figure 4-9 shows that the settling time is very close to 600 seconds for the melt rate and temperature. The carbon has a settling time of 2000 seconds, after a pure time delay of 1800 seconds. This long settling time is necessary for the Smith predictor to work properly.

\section{OUTPUT NOISE}

Gaussian noise was added to the output signal. This represents the fact that the sensors are subject to extreme noise because of the nature of the cupola. Even when averaging several sensors measuring the same output, there is noise. Figure 4-10 is the plots generated with noise. Notice the error plot is basically the noise after the outputs reach steady state. The change in error reflects a noisy output, which had to be taken into 
consideration when choosing the membership function parameters. An example is that if the output noise caused the change in error signal to enter the large change in error range in the fuzzy system, then the inputs may change significantly to correct for it. It then leads to an oscillating input and output.

As can be seen in Figure 4-10, the controller rejects output noise efficiently. The inputs move seldom under the noise conditions introduced, increasing the life of the actuators.

\section{INPUT DISTURBANCE}

Input disturbances can be common in the cupola environment. The scrap iron is typically lifted with a human operated front-end loader. Since the scrap iron will have varying densities, there will be a disturbance at the input. The blast rate can be affected by its ability to penetrate the charge in the cupola. The configuration of the charge is constantly changing in the cupola causing a disturbance. Mechanical problems in producing the blast can cause disturbances. The oxygen enrichment will be disturbed if the blast is disturbed because it is a percentage increase of oxygen in the blast air. Mechanical problems in the oxygen delivery can also cause disturbances.

Figure 4-11 shows plots of the inputs and outputs with the inputs disturbed. The disturbances are in the form of a square wave. This gives periodic negative and positive disturbances to the inputs. Figure 4-11 reflects the ability of the controller to adapt to disturbances. The frequencies of the square waves were taken to be 6000 seconds for the 
$\mathrm{O}_{2}, 9000$ seconds for the BR, and 30000 seconds for the CMR. The controller reacts to, and corrects for, these disturbances. The outputs show a $20 \%$ error due to the time it takes to correct for the instantaneous changes in the inputs. The plot shows a long history because the frequencies of the square waves were all different, allowing worst possible cases to arise. 

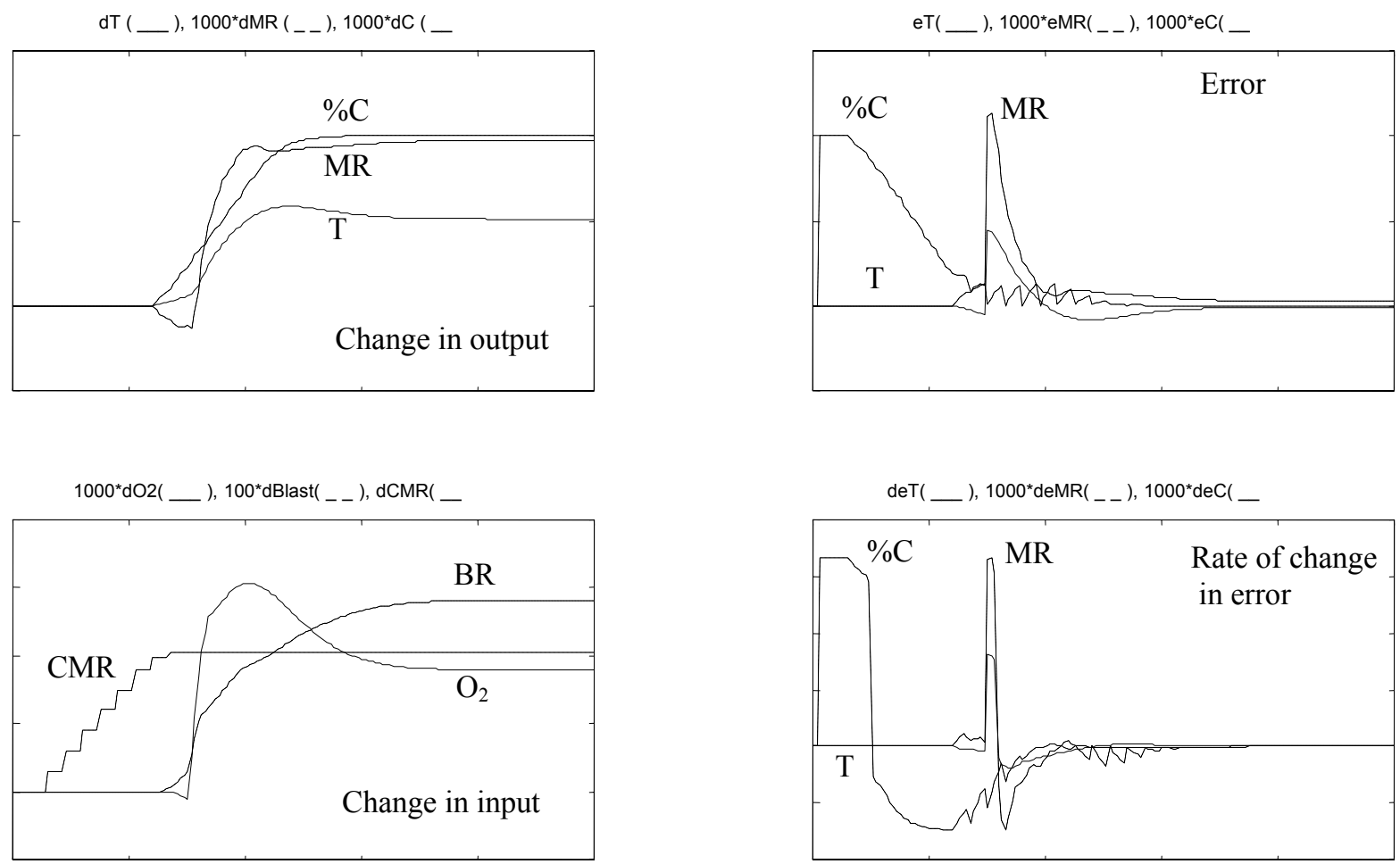

Figure 4-9 Step response under ideal conditions

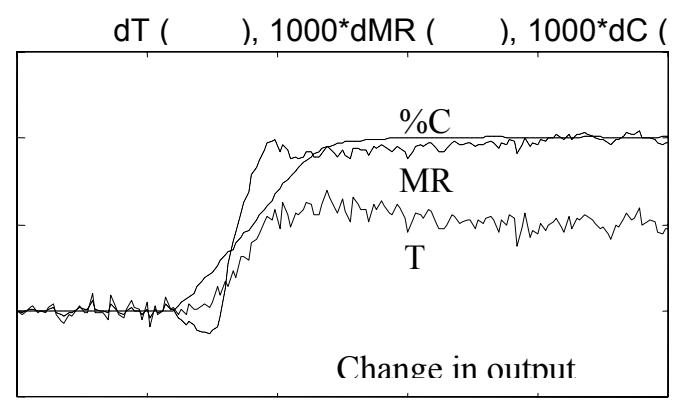

ti

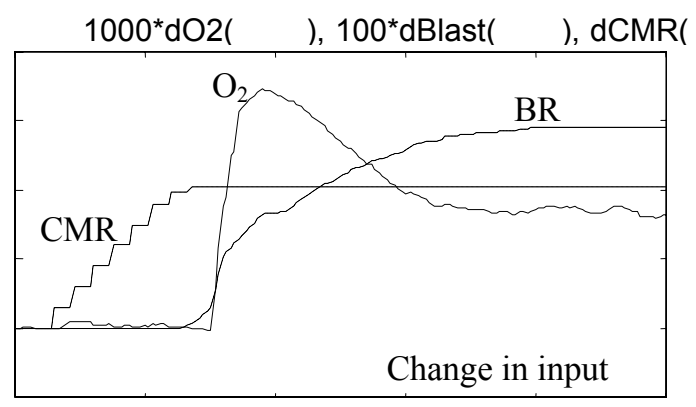

ti

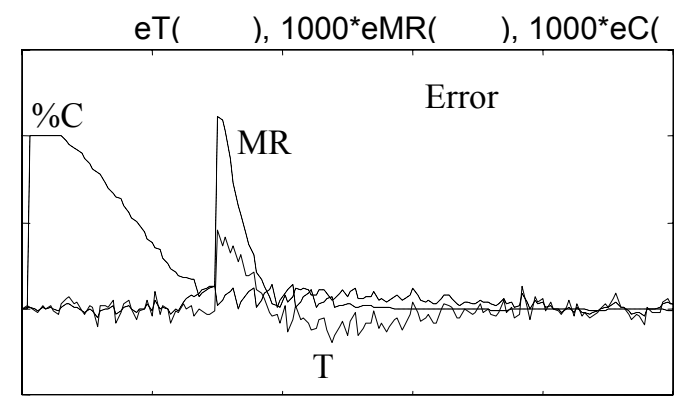

ti

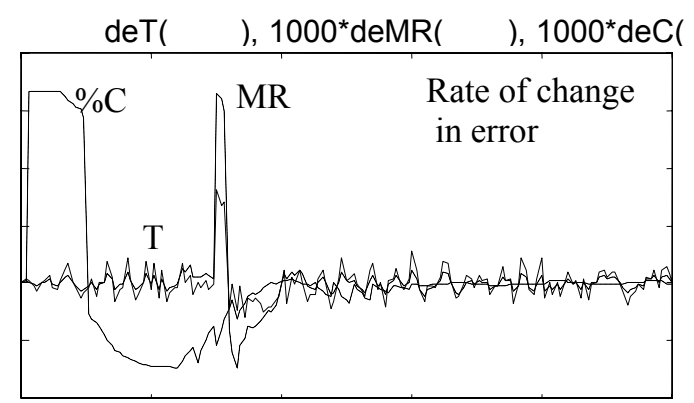

ti 
Figure 4-10 Step response with noisy outputs
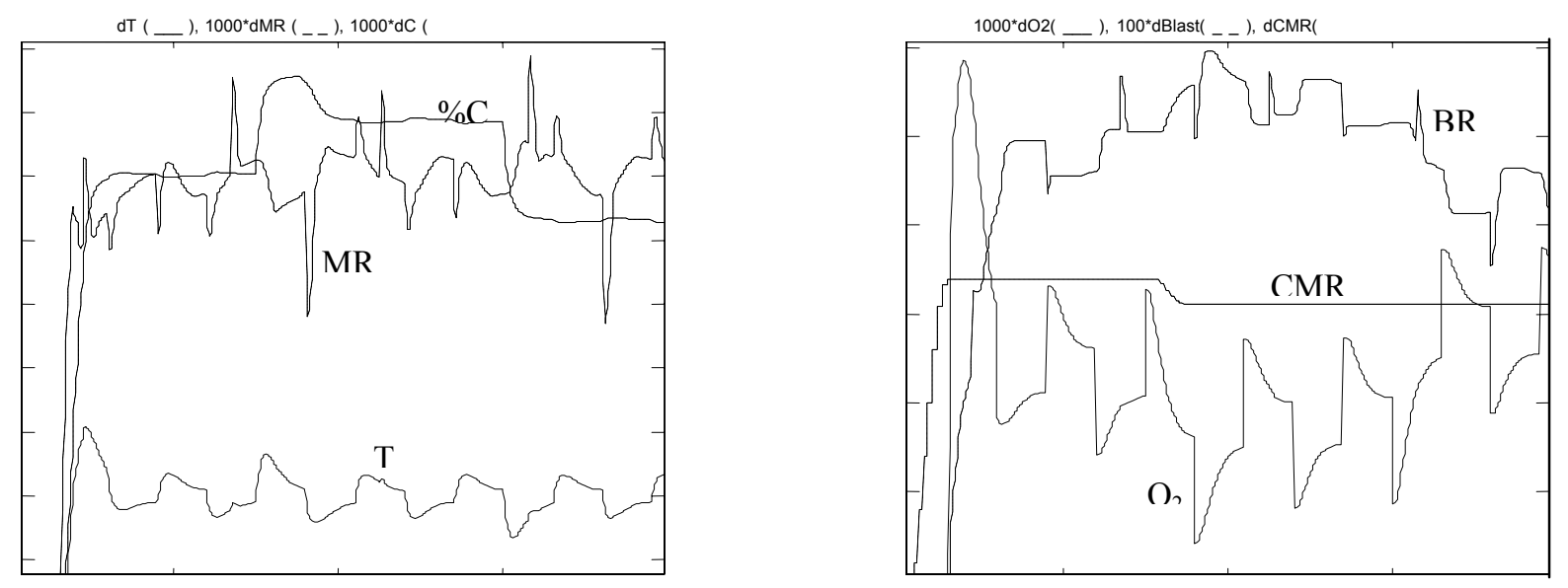

Figure 4-11 Step response with input disturbances generated with square waves

\subsubsection{Integration of Sensor Fusion In Controller Design}

The sensor fusion technique provides the controller with a confidence level in the measurements of the output values. As explained earlier, the confidence level reflects how trustworthy the measurements are. If the confidence is close to zero, then the response should be slow. If the confidence is close to one, the response should be normal. To test the integration of the sensor fusion in the controller design, a relatively large disturbance is applied to the output with the cupola at steady state. This disturbance represents a failure in the sensors rather than an actual disturbance. Figure 4-12 and 
Figure 4-13 are plots of the inputs and outputs of the melt rate for the confidence levels of 0.9 and 0.5 respectively during the disturbance. The confidence is taken to the fifth power and multiplied to the controller's rate of change for the blast rate.

The change in the melt rate due to the disturbance in the measurement at a $90 \%$ confidence is 0.038 (tons/hr) and responds much like it would without sensor fusion. The change in the melt rate for a $50 \%$ confidence was only 0.0038 . The figures show that the reduced confidence slows the controller down so that the output changes are $10 \%$ of those at a confidence of $90 \%$. It does not stop the changes and if the sensors that cause the confidence to go down are not replaced or corrected, the output could eventually reach an erroneous value. With sensor fusion, it is shown that the cupola's operators will have much more time to fix bad sensors before lowering the quality of the product. The controller can be easily adjusted by changing the power that the confidence is taken to. 

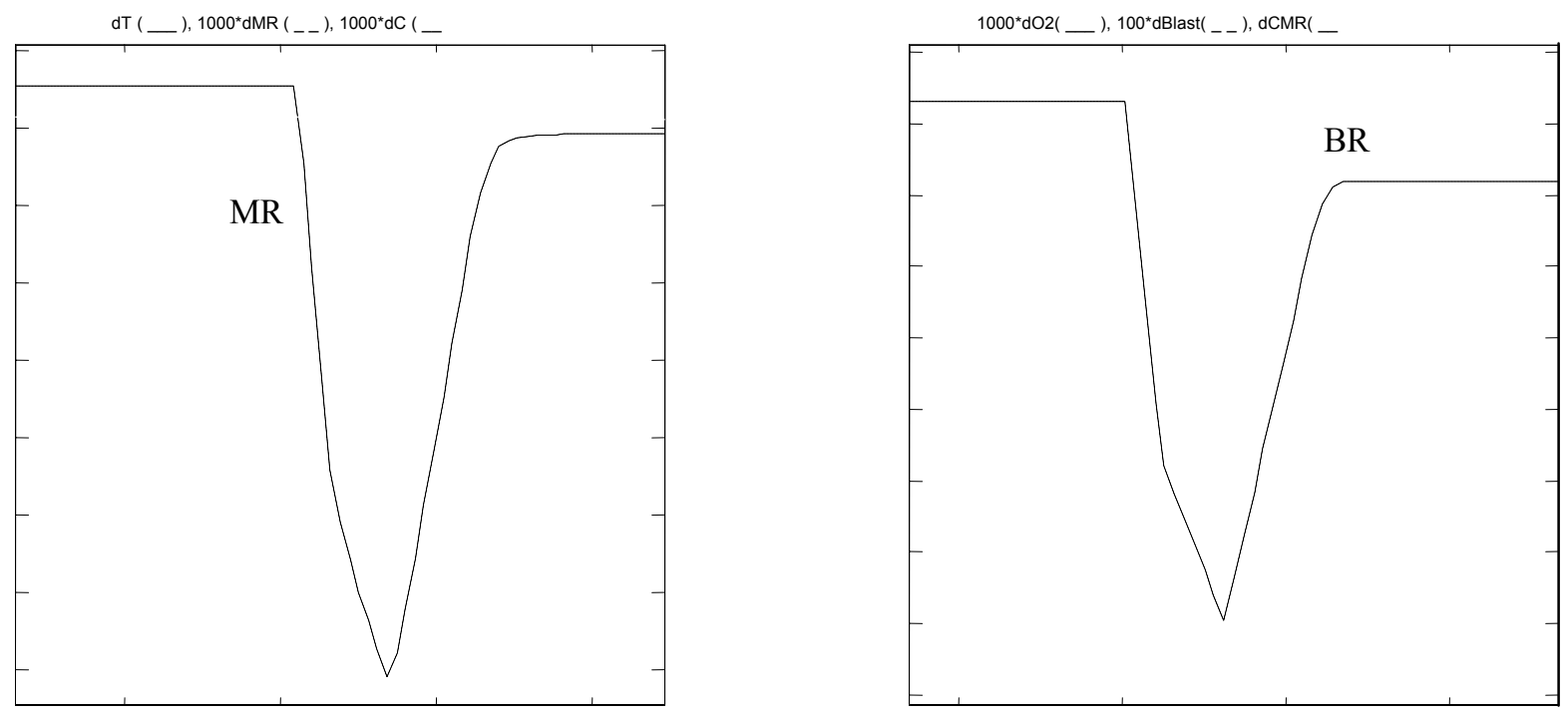

Figure 4-12 Response for melt rate confidence of 0.9 and -0.1 pulse for 600 seconds
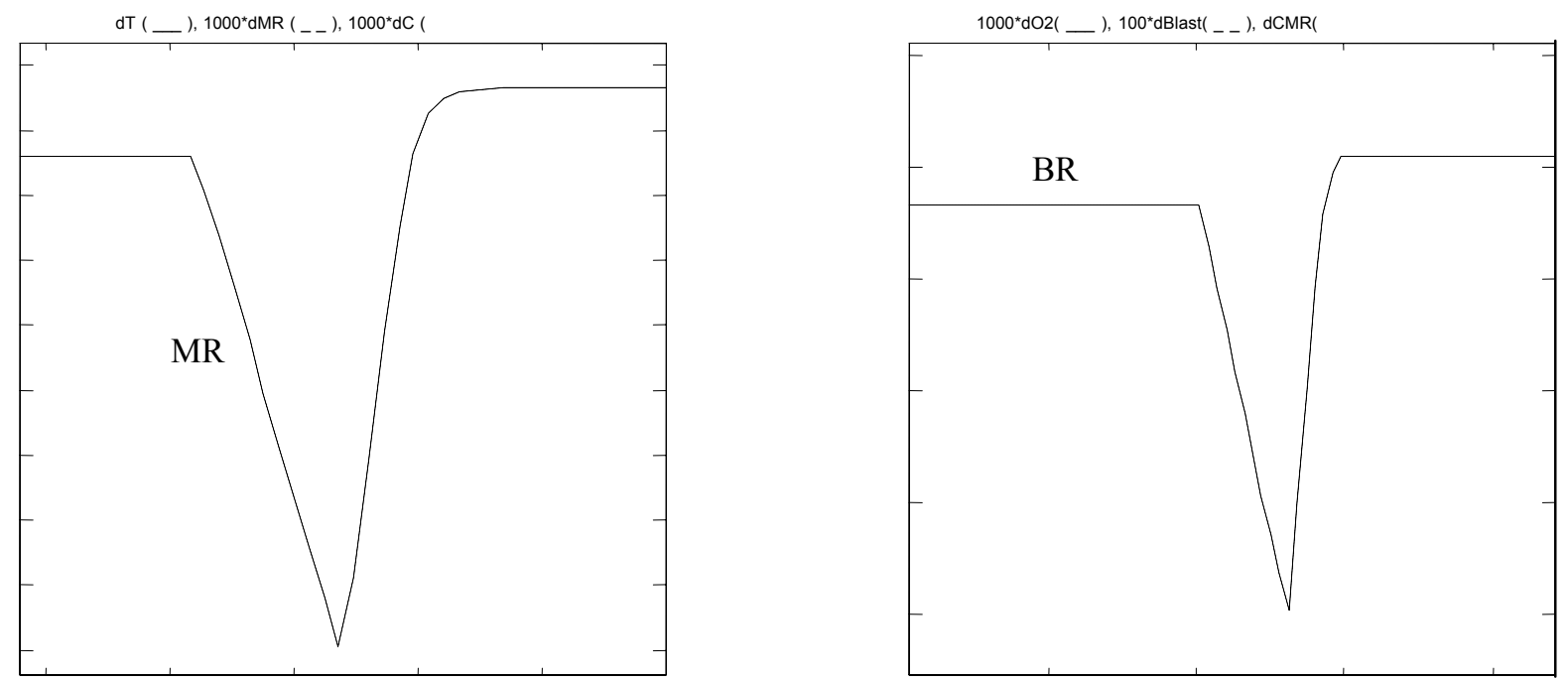

Figure 4-13 Response for melt rate confidence of 0.5 and -0.1 pulse for 600 seconds 


\subsubsection{VARYING MODEL PARAMETERS}

A linear model has approximated the non-linearity of the cupola furnace. The experimental data shows that the model is good only for a narrow operating range. This problem could be solved by designing many controllers and then use a look-up table to choose the best controller for a certain operating point. It would be better if one controller would work under all the normal operating ranges. This is one reason for using fuzzy logic control, that is, it is robust. In Figure 4-14, a sine wave disturbance, with an offset of 1.125 and amplitude of 0.375 is multiplied to each of the nine transfer functions at different frequencies. This varies the steady state transfer function response from $75 \%$ to $150 \%$ of the original value. Since they are varied at different frequencies, a worst-case combination will align if the simulation runs long enough.

The non-linearities in the actual cupola are not a problem for the fuzzy controller. Figure 4.14 shows the results of a widely varying model. Applying a sinusoidal varying gain to the nine individual transfer functions varies the model. The frequencies are all different in order to study the worst case scenario. The controller performs excellent in this test. The one case at 12000 seconds indicates the melt rate cannot be controlled. At this point the input plot shows the blast rate is at its maximum level, therefore the desired operating point cannot be reached. 

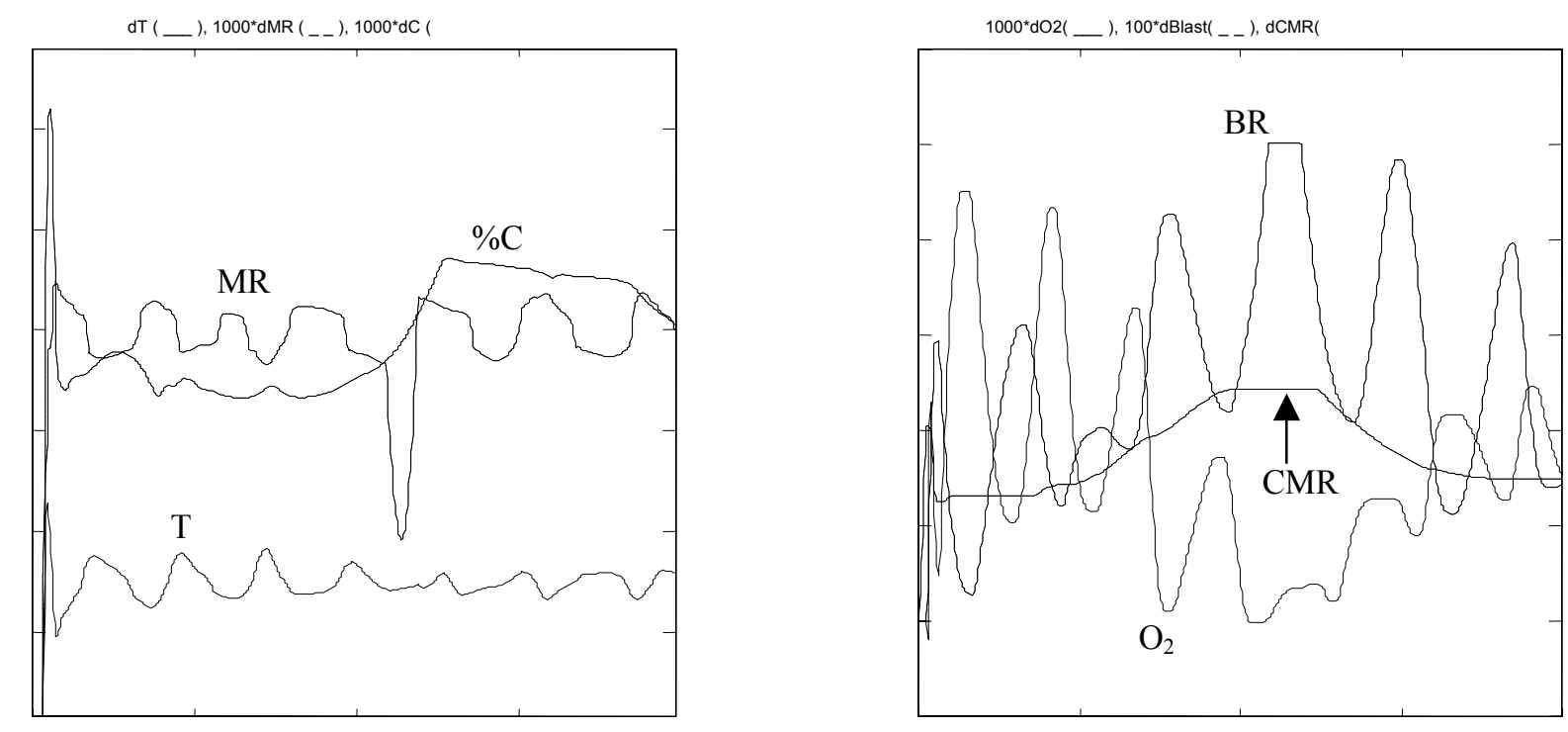

Figure 4-14 The results of varying the model parameters

\subsubsection{Varying Pure Time Delay of the CMR}

The Smith predictor depends on previous knowledge of the time it takes for the charge to burn down to the output level. It can be an average for the range of the cupola's operation or it could be a function of the inputs and outputs. Even if it were given by a function, the penetrability of the charge by the blast cannot be absolutely known, clumping of charge materials in the cupola, and coke consistency can all lead to inaccuracy in the calculation. Therefore results with the cupola given a 2400 second time delay with the Smith predictor given an 1800 second time delay is given in Figure 4-15. 
Figure 4-16 is the results of a pure time delay of 1200 seconds in the cupola and 1800 seconds in the Smith predictor.
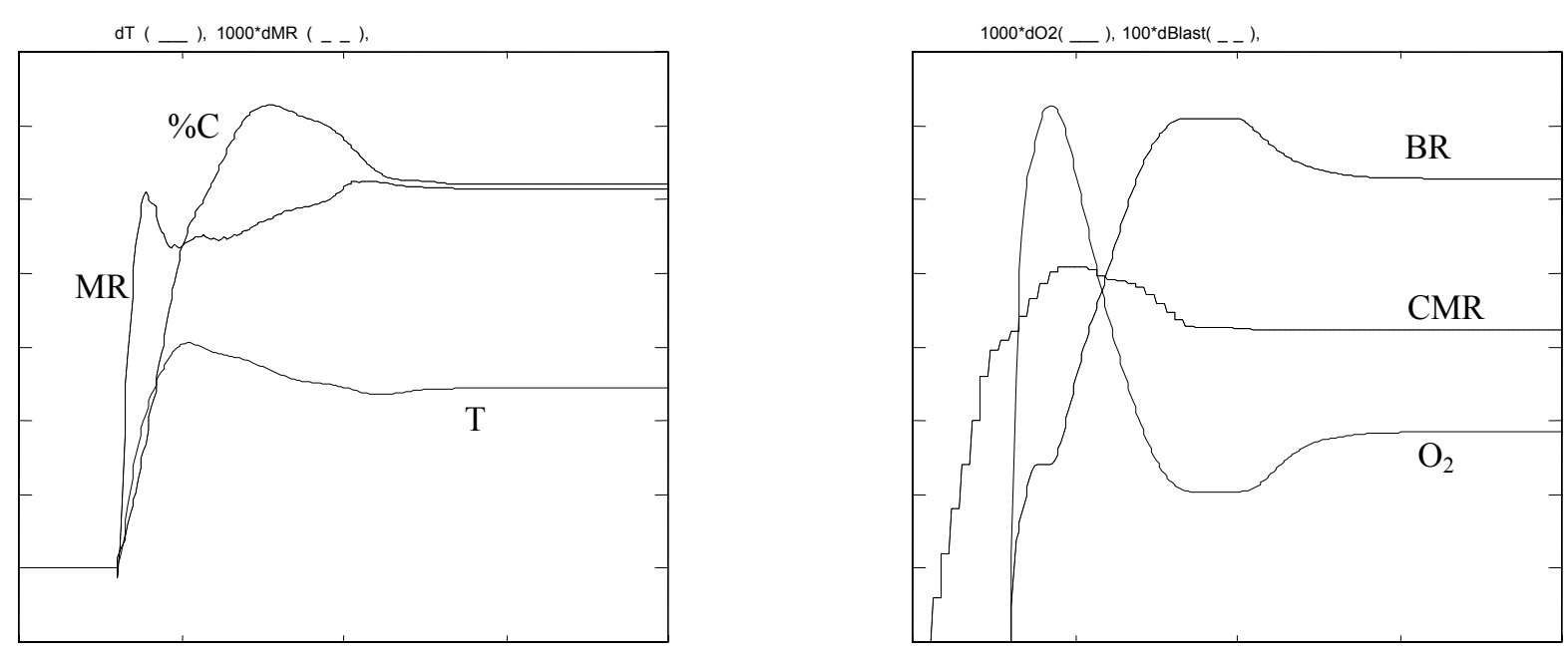

Figure 4-15 Smith predictor with a +600 second time delay plant offset

Observing Figure $4-15$, a $25 \%$ overshoot can be seen due to the 600 -second difference between the cupola's and the Smith predictor's time delay. Figure 4-16 shows the controller's reaction to a -600 second in the same difference, which increases the settling time by $100 \%$. These results are good since the $\% \mathrm{C}$ remained within $25 \%$ of the required change in set point after a 2000 second settling time. A 600 second difference in the time delays would indicate a serious problem and would probably not occur in the actual cupola. Therefore, this controller is acceptable for an average time delay given for the Smith predictor. 

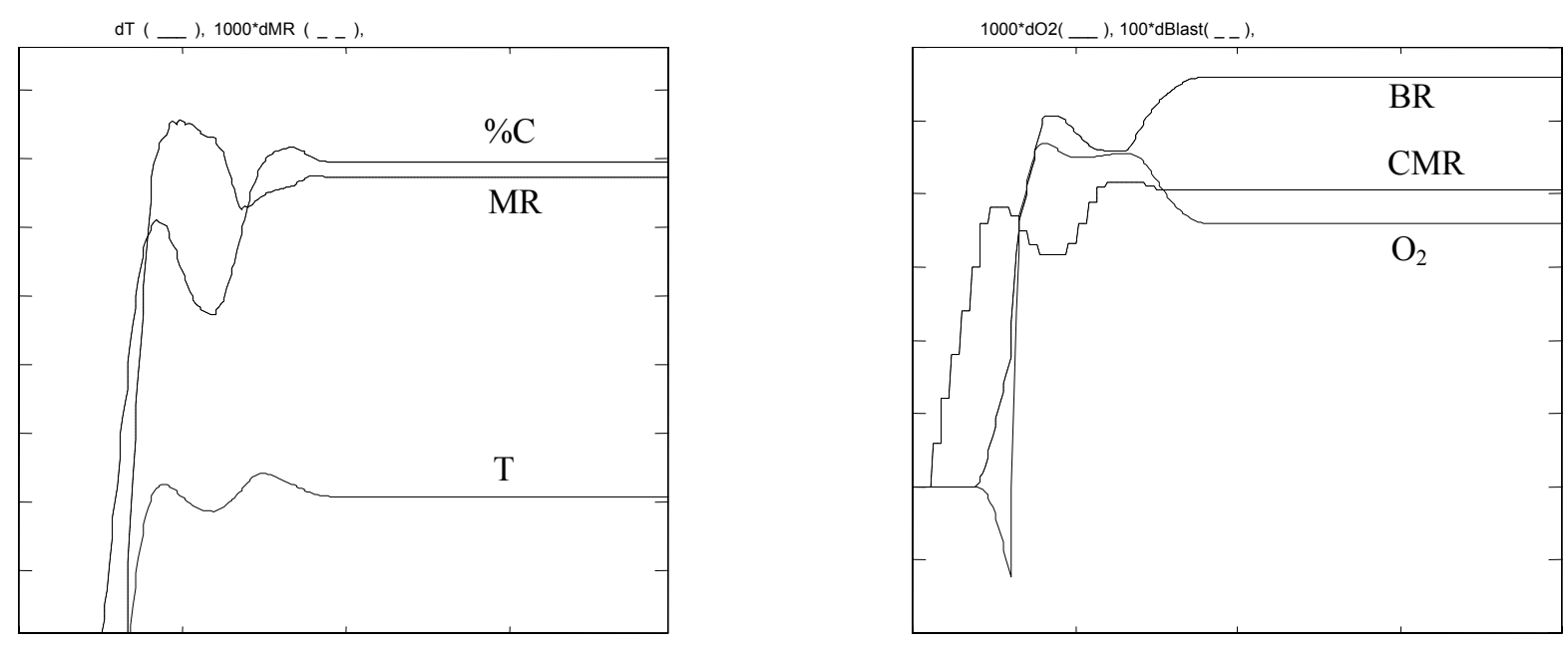

Figure 4-16 Smith predictor with a -600 second plant time delay offset

If a function is available for calculating and updating the Smith predictor's time delay, then this controller is conservative and should be adjusted to lower the settling time.

\subsubsection{COMBINING ALL NOISES AND DISTURBANCES}

Figure 4-17 is a plot of all the above noises and disturbances added in. This represents the cupola in a worst case scenario where many disturbances are simultaneously taking place. The Smith predictor's time delay is 200 seconds more than the cupola's and the sensor fusion confidence is $50 \%$. The figure shows the result of applying all the noises, 
disturbances, and varying parameters to the system. The controller works satisfactorily under these extreme conditions.
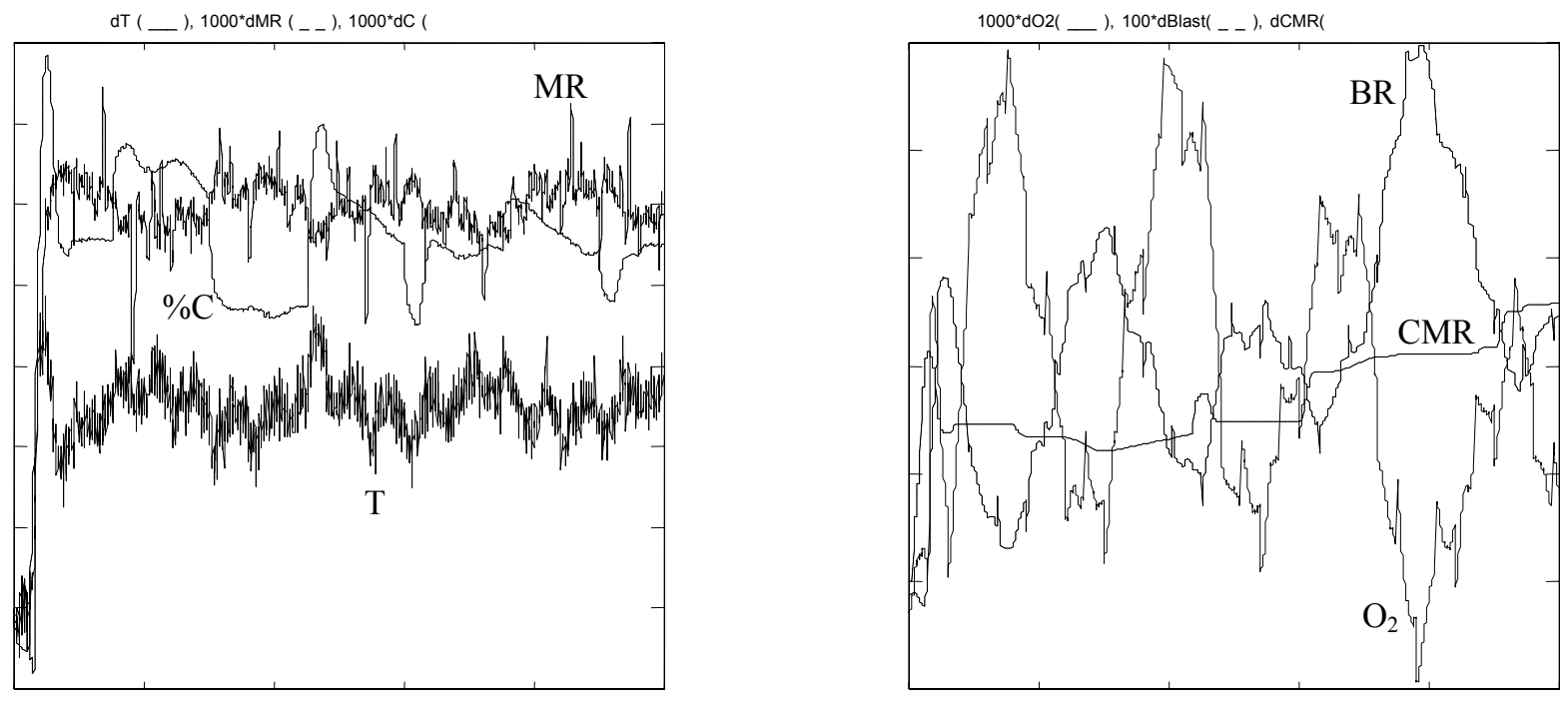

Figure 4-17 System Performance Under Effect of All Disturbances 


\section{Appendix 4.A}

1. If (eMRate is LgNegMR) and (deMRate is LgNegdMR) then (ChangeBlast is LgNegBR)

2. If (eMRate is LgNegMR) and (deMRate is SmNegdMR) then (ChangeBlast is LgNegBR)

3. If (eMRate is LgNegMR) and (deMRate is ZerodMR) then (ChangeBlast is LgNegBR)

4. If (eMRate is LgNegMR) and (deMRate is SmPosdMR) then (ChangeBlast is SmNegBR)

5. If (eMRate is LgNegMR) and (deMRate is LgPosdMR) then (ChangeBlast is SmNegBR)

6. If (eMRate is SmNegMR) and (deMRate is LgNegdMR) then (ChangeBlast is SmNegBR)

7. If (eMRate is SmNegMR) and (deMRate is SmNegdMR) then (ChangeBlast is SmNegBR)

8. If (eMRate is SmNegMR) and (deMRate is ZerodMR) then (ChangeBlast is SmNegBR)

9. If (eMRate is SmNegMR) and (deMRate is SmPosdMR) then (ChangeBlast is SmNegBR)

10. If (eMRate is SmNegMR) and (deMRate is LgPosdMR) then (ChangeBlast is SmNegBR)

11. If (eMRate is IdealMR) and (deMRate is LgNegdMR) then (ChangeBlast is SmPosBR)

12. If (eMRate is IdealMR) and (deMRate is SmNegdMR) then (ChangeBlast is ZeroBR)

13. If (eMRate is IdealMR) and (deMRate is ZerodMR) then (ChangeBlast is ZeroBR)

14. If (eMRate is IdealMR) and (deMRate is SmPosdMR) then (ChangeBlast is ZeroBR)

15. If (eMRate is IdealMR) and (deMRate is LgPosdMR) then (ChangeBlast is SmNegBR)

16. If (eMRate is SmPosMR) and (deMRate is LgNegdMR) then (ChangeBlast is SmPosBR)

17. If (eMRate is SmPosMR) and (deMRate is SmNegdMR) then (ChangeBlast is SmPosBR)

18. If (eMRate is SmPosMR) and (deMRate is ZerodMR) then (ChangeBlast is SmPosBR)

19. If (eMRate is SmPosMR) and (deMRate is SmPosdMR) then (ChangeBlast is SmPosBR)

20. If (eMRate is SmPosMR) and (deMRate is LgPosdMR) then (ChangeBlast is SmPosBR)

21. If (eMRate is LgPosMR) and (deMRate is LgNegdMR) then (ChangeBlast is SmPosBR)

22. If (eMRate is LgPosMR) and (deMRate is SmNegdMR) then (ChangeBlast is SmPosBR)

23. If (eMRate is LgPosMR) and (deMRate is ZerodMR) then (ChangeBlast is LgPosBR)

24. If (eMRate is LgPosMR) and (deMRate is SmPosdMR) then (ChangeBlast is LgPosBR)

25. If (eMRate is LgPosMR) and (deMRate is LgPosdMR) then (ChangeBlast is LgPosBR)

The fuzzy inputs are eMRate for the error in the melt rate and deMRate for the

rate of change in the error. The membership function names are $\operatorname{LgNegMR}$ and

SmNegMR for large and small negative melt rates, $\operatorname{Lg}$ PosMR and SmPosMR for large and

small positive melt rates, $\operatorname{LgNegdMR}$ and $S m N e g d M R$ for large and small negative rate of

change in the melt rates, $L g P o s d M R$ and SmPosdMR for large and small positive rate of

change in the melt rates, IdealMR is about a zero error, ZerodMR is about a zero rate of 
change in the error of the melt rate. The outputs are constants for large or small, negative or positive, and zero changes in the blast rate. 


\section{Chapter 5}

\section{Demonstration Plans}

\subsection{Introduction}

The demonstration plans aimed at illustrating the functionality of $I^{3} \mathrm{PSC}$ technology as it is applied to cupola iron melting furnaces. The plans were carried out as proposed at a research facility operated by the US DOE in Albany Oregon (ALRC). ALRC operates an 18" research Cupola furnace equipped with state of the art instrumentation for measurement of various cupola parameters. Moreover, in order to carry out the $\mathrm{I}^{3} \mathrm{PSC}$ demo plans, several new instrumentations such as a continuous immersion thermocouple and an ultrasonic radar were installed and tested on the furnace as promising technologies that could be recommended for use in the cupola foundries. The parameters of importance to the current demonstration plans were:

1- Iron Temperature,

2- Melt Rate,

3- Carbon content of molten iron,

4- Off gas temperature and composition

5- Cupola back pressure

6- Blast rate 
7- Oxygen enrichment

8- Metal stream composition (SCR)

9- Coke to metal ratio (CMR)

Instrumentation used for measurement of the above parameters included: dip thermocouples, continuous immersion thermocouple and optical Pyrometers for measurement of molten iron temperature, ultrasonic radar for measurement of molten iron level, electronic scale for measurement of molten iron weight, thermal arrest equipment for quick measurement of carbon, silicon and carbon equivalent of the molten iron. Moreover, other parameters such as CMR and SCR were manually monitored and calculated.

The demonstration plans aimed at addressing the following questions:

1- Can I I PSC system be successfully interfaced and integrated into an existing cupola with its own instrumentation and data acquisition system with minimal effort?

2- Can the I I PSC system provide reliable information regarding the cupola parameters and state of operation?

3- Can I PSC system be used to integrate sensing and control algorithms in order to provide an automatic control system that can successfully operate cupola furnaces in order to avoid some of the problems that currently occur in cupola foundries? 
The last question is the most important one as its success automatically indicates a positive answer to the first two questions.

The $I^{3}$ PSC system was interfaced through an Ethernet network connection to the existing Data Acquisition Computer at Albany Research Center (ALRCDAQ). A special software module was written to specifically exchange the important cupola parameters between the I ${ }^{3}$ PSC computer and the ALRCDAQ. This software module is what needs to be customized if the $\mathrm{I}^{3} \mathrm{PSC}$ system is to be used at a different facility. This arrangement ensured that no changes to the ALRCDAQ were required and that any changes to the number of parameters monitored can be done quickly. This arrangement is illustrated in Figure 5-1. 


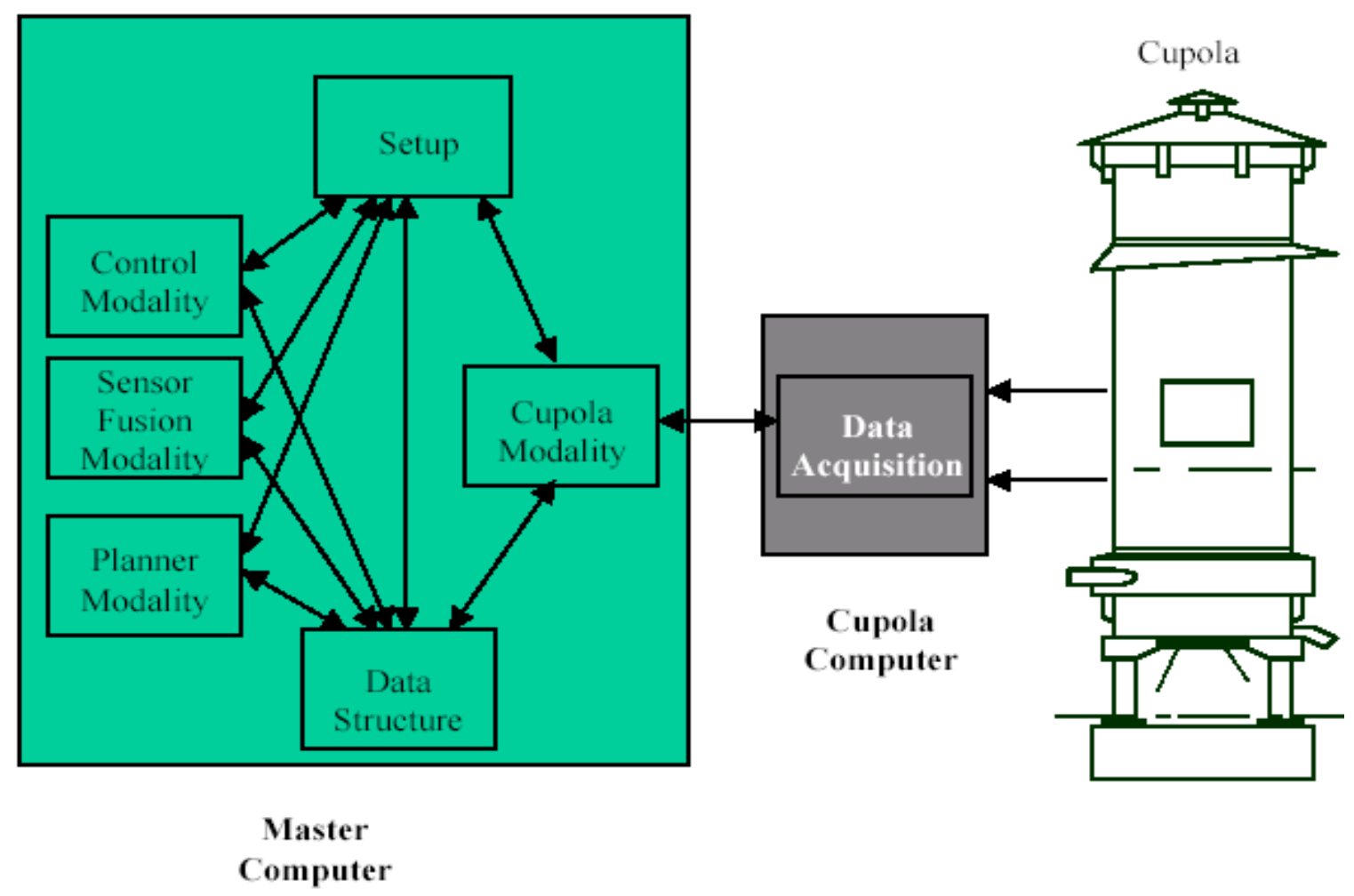

Figure 5-1 Configuration for Interfacing I3PSC with ALRC DAQ for Demo Runs

Three of the demonstration runs were focused on illustrating the integration of sensing and control of cupola parameters.

\subsection{Setup of I ${ }^{3}$ PSC for Demonstration Runs}

$I^{3} \mathrm{PSC}$ system was configured with the following modalities:

a) Data Acquisition Modality: A modality whose function is to collect and send raw data and $\mathrm{I}^{3} \mathrm{PSC}$ control parameters from and to the ALRCDAQ. 
b) Virtual Sensor Modality: A modality using models to predict values of important cupola parameters. Two virtual sensors were configured. One for molten iron temperature and the other for the iron melt rate.

c) Sensor Fusion Modality: For fusion of data collected from the cupola. Three parameters were of interest in the demo runs, namely, iron temperature, melt rate, and Carbon content of the molten metal.

d) Monitoring modality: This modality monitors trend of important variables and displays the current trend of such variables such as increasing, decreasing, constant, etc. This modality can also be setup to monitor for conditions such as bridging that would be reflected in changes in operational parameters such as cupola back pressure and exit temperature. An example of such situation is shown later in this chapter.

e) Planner modality: This is the modality which specifies the run plan in terms of the requirements on the variables of importance. This was limited during the demo runs to the three variables specified earlier, , namely, iron temperature, melt rate, and Carbon content of the molten metal.

f) Controller Modality: This modality uses information from the sensor fusion modality as well as planner modality to decide adjustments to the control parameters of the cupola. These parameters included: blast rate, oxygen enrichment, coke to metal ratio (CMR) and steel to cast ratio (SCR). 


\subsection{Results and Analysis of Demo Runs}

One of the runs was aimed at ensuring that the cupola instrumentation were working properly and the interface between the $\mathrm{I}^{3} \mathrm{PSC}$ and the data acquisition system as well as the integrated system at ALRC are working properly. It was also used to test the effect of changing the CMR on the cupola operating conditions. The subsequent runs aimed at demonstrating the ability of $\mathrm{I}^{3} \mathrm{PSC}$ to control the carbon content of the molten iron by adjusting the composition of the iron stream, the melt rate and temperature of the molten iron within an appropriate range.

The first of these runs illustrates the ability to change the carbon content of the molten iron from $2.8 \%$ Carbon to $3 \%$ while maintaining the metal temperature and the melt rate constant. It also illustrates the ability of the $I^{3} P S C$ controller to reject disturbances in the form of unknown metal stream that is being introduced into the cupola. Partial results of the first of these runs are shown in Figure 5-2 through

Figure 5-7. 
\%Carbon

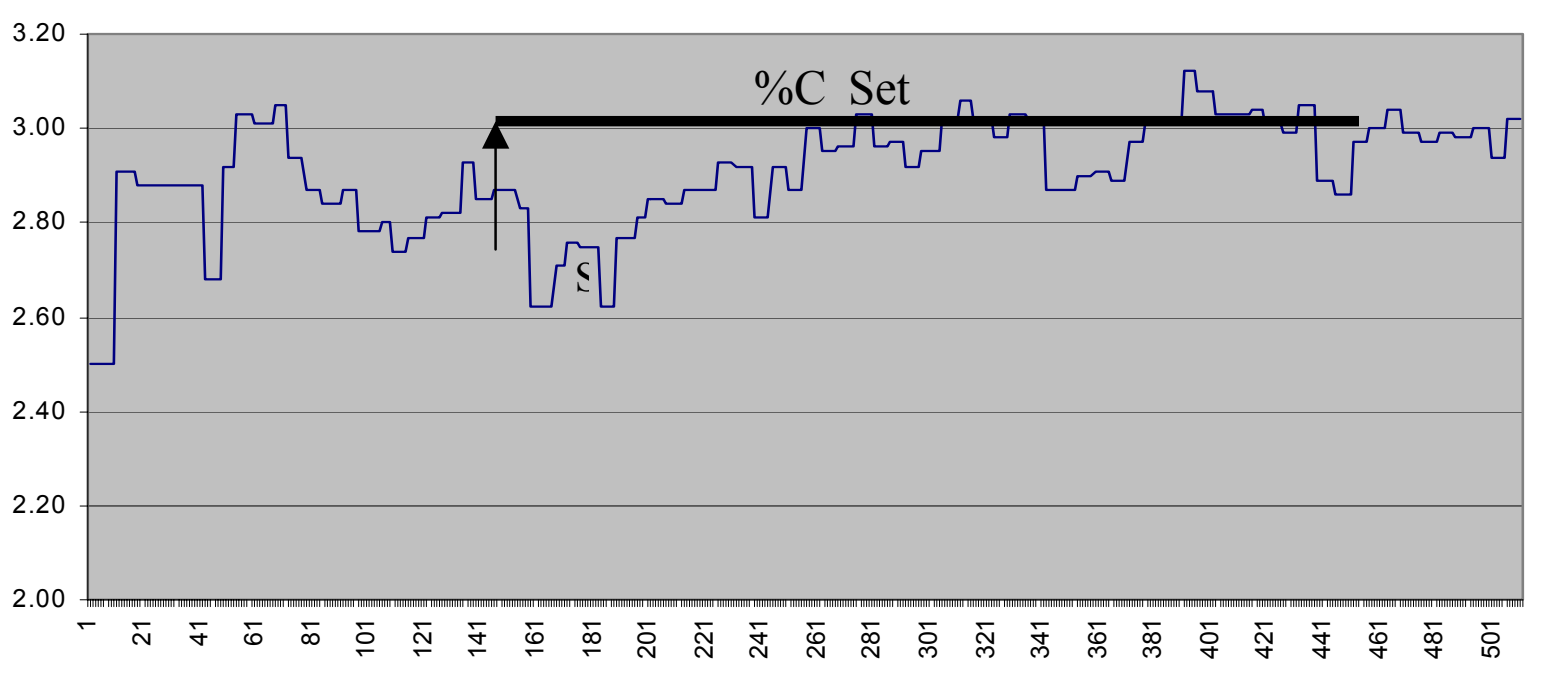

Figure 5-2 Control of Carbon Content, Run \#2

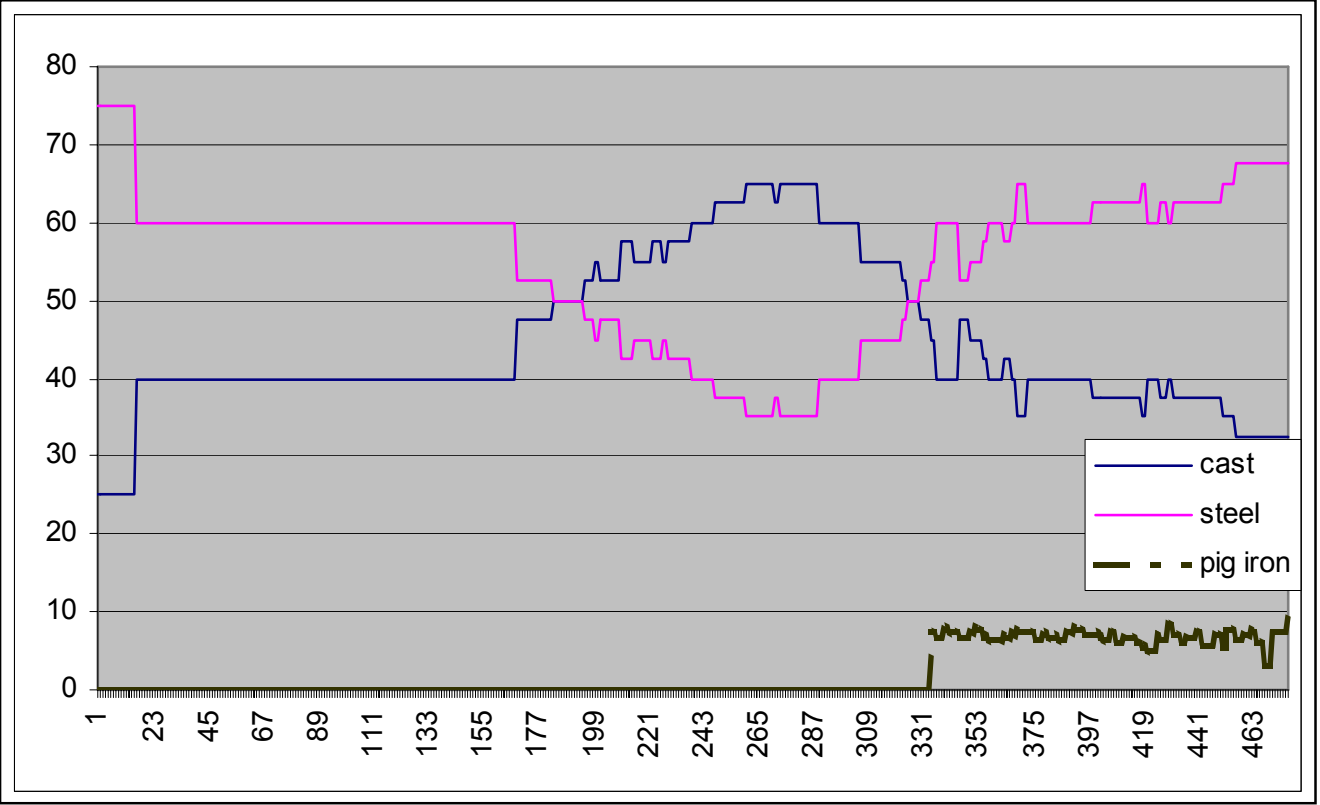

Figure 5-3 Metal Stream Changes suggested by I3PSC for control of $\% \mathrm{C}$ for Run \#1 


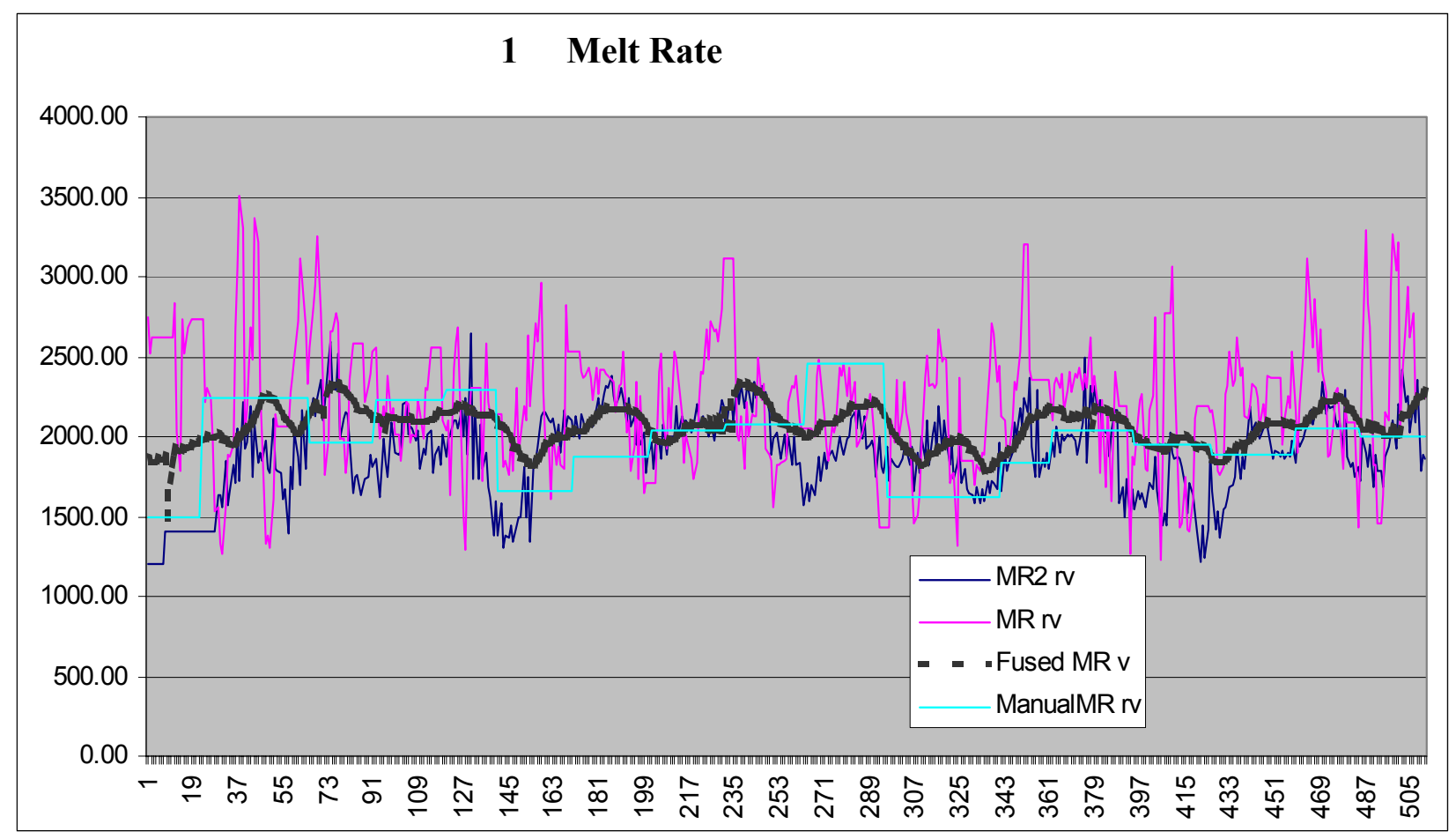

Figure 5-4 Individual Measurements and Fused Melt Rate for Run \#1

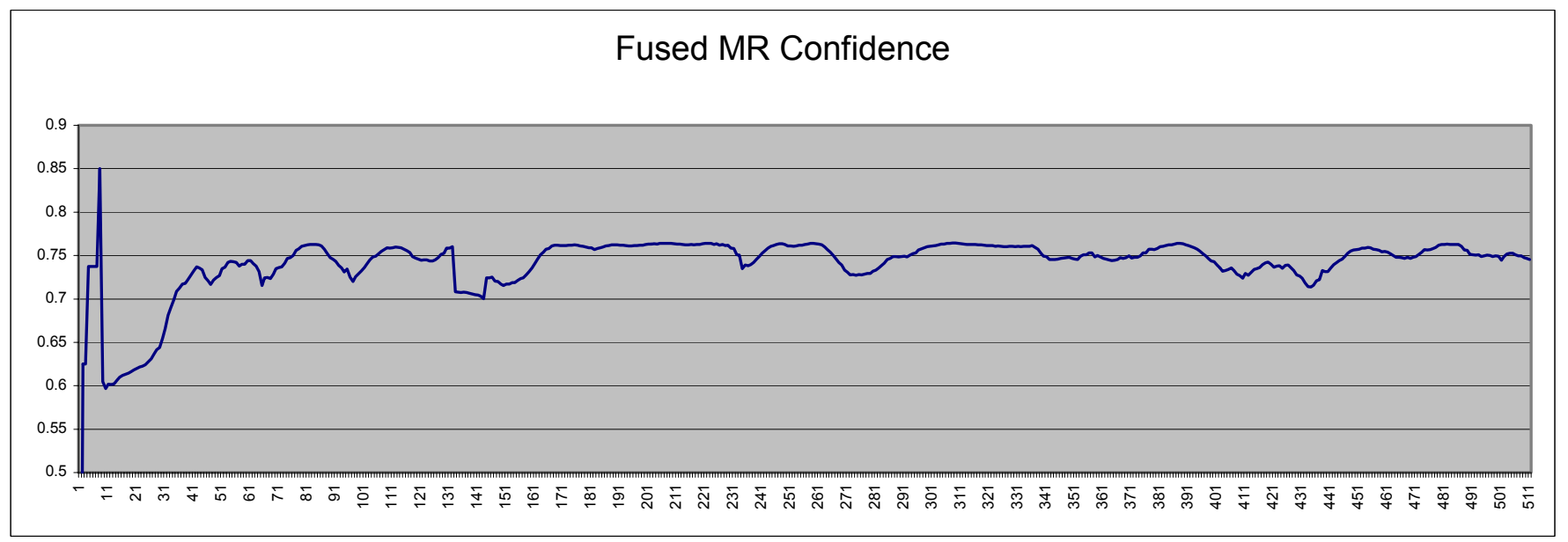

Figure 5-5 Confidence of Fused MR 


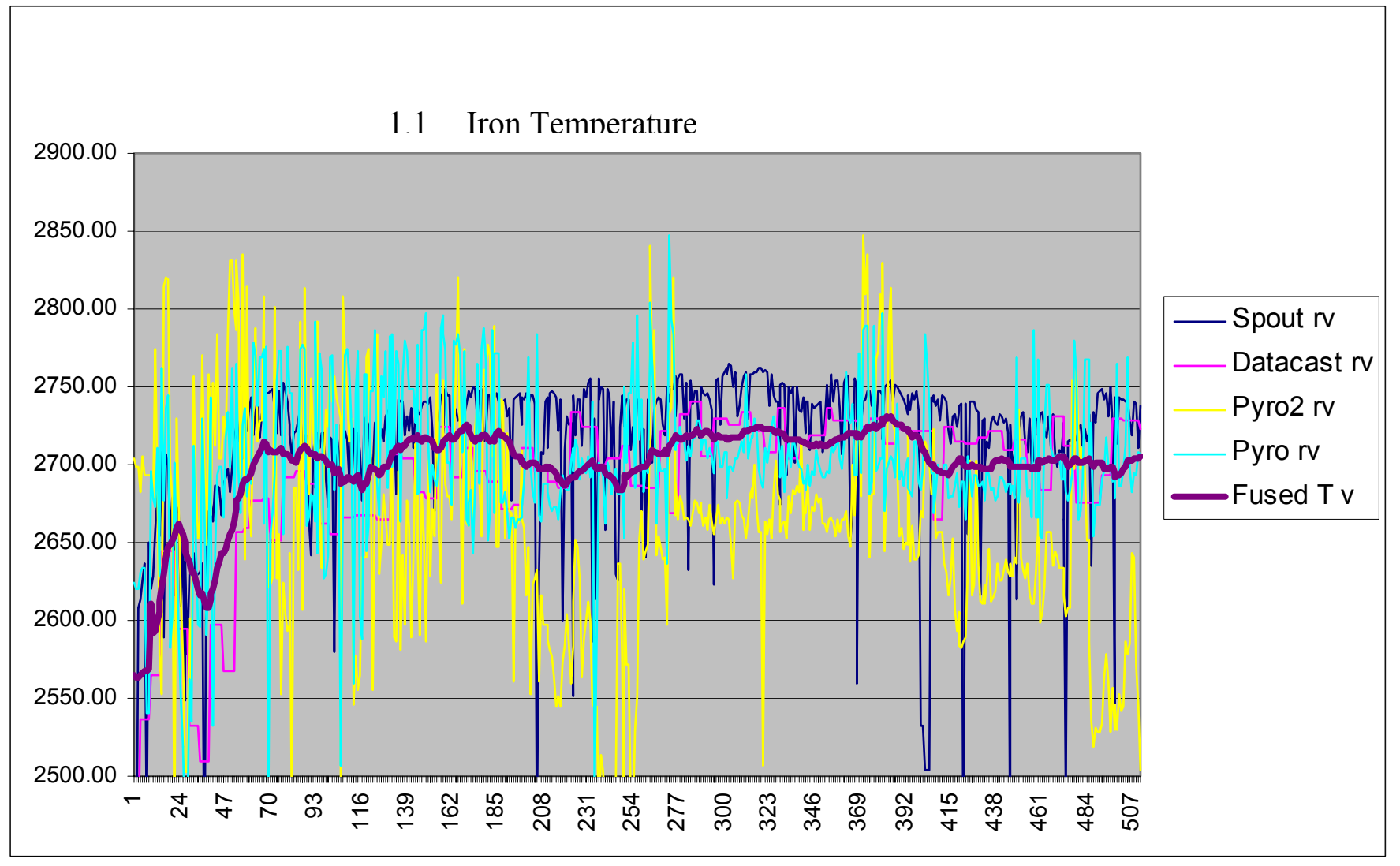

Figure 5-6 Individual Measurements and Fused Temperature

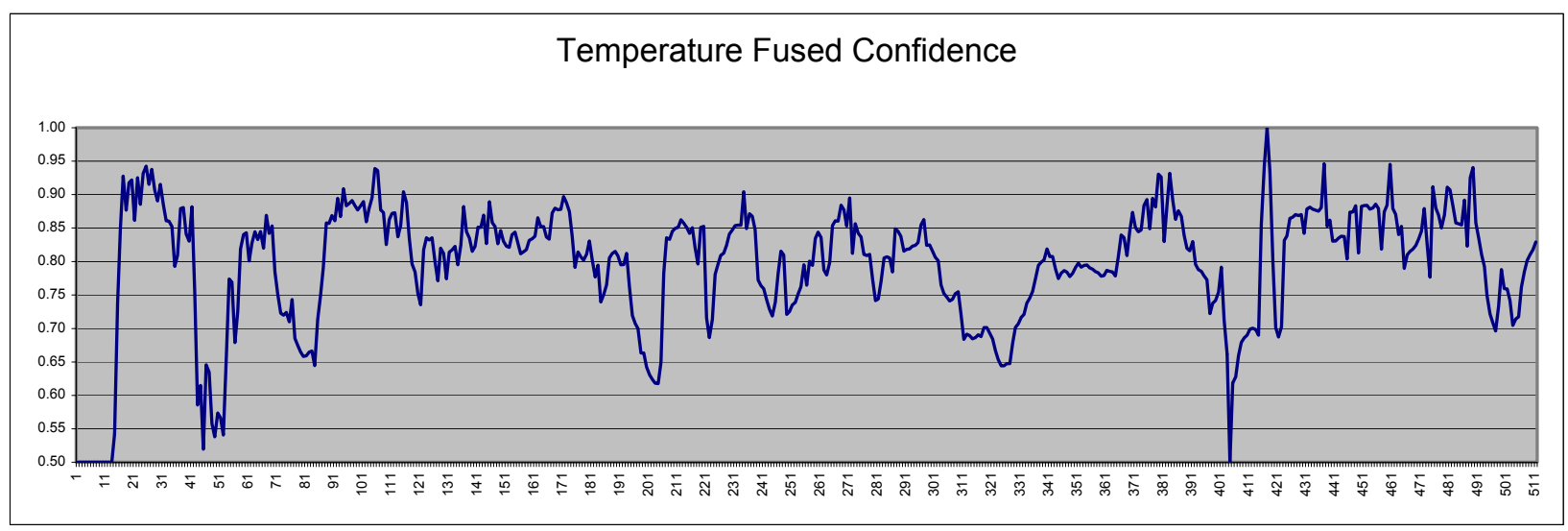

Figure 5-7 Confidence of Fused Temperature 


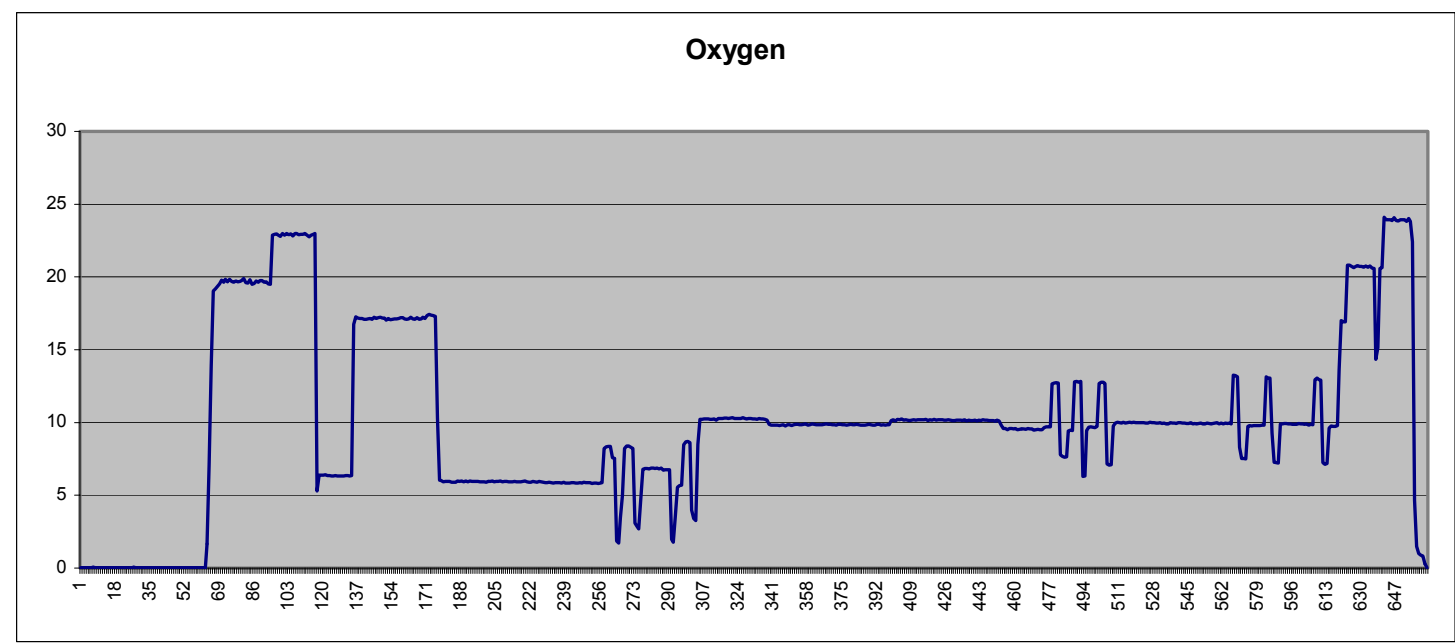

Figure 5-8 Oxygen Enrichment for Temperature Control for Run \#1

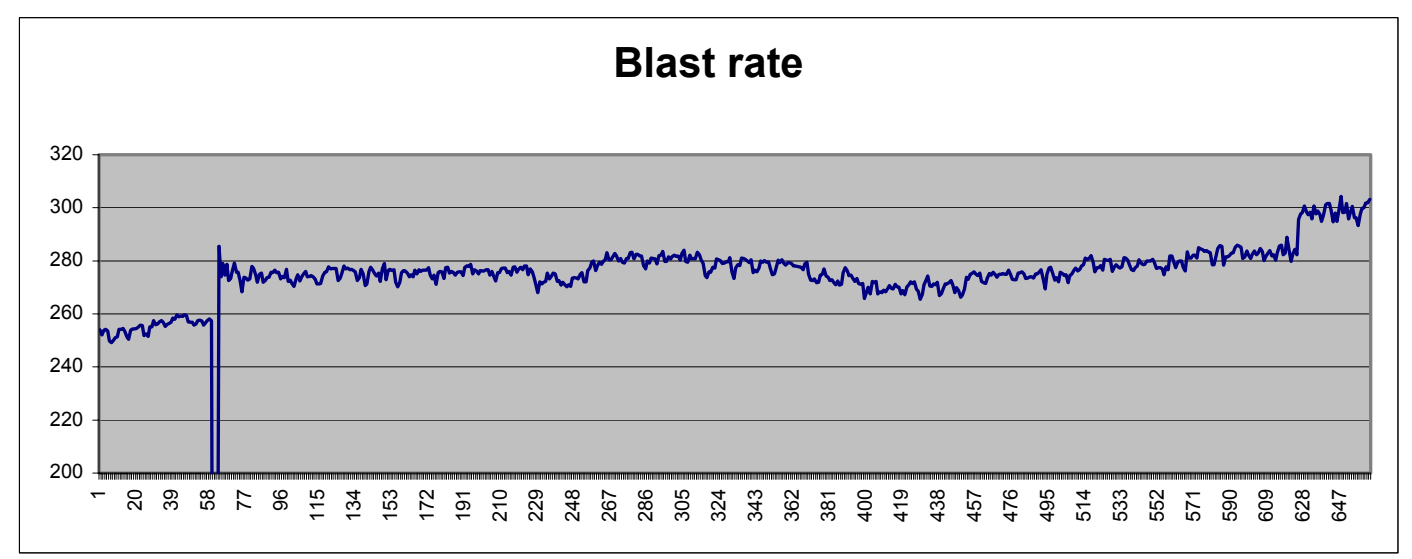

Figure 5-9 Blast Rate for Melt Rate Control for Run \#1

Figure 5-2 illustrates the change in the carbon content between the current carbon content and the desired content of $3 \%$. It should be noted that a stream of pig iron was added to the metal stream, as a disturbance, in place of part of the steel in the charge, as illustrated in 
Figure 5-3. The controller corrects for the disturbance by increasing the amount of steel in the charge. Adjustments in oxygen enrichment and blast rate to maintain the temperature and melt rate are illustrated in Figures 5.8 and 9. The temperature and melt rate obtained from individual measurements are shown in Figure 5-4 and Figure 5-6. Changes in the confidence in the fused values reflecting agreement between the different measurements are shown in Figure 5-5 and

Figure 5-7.

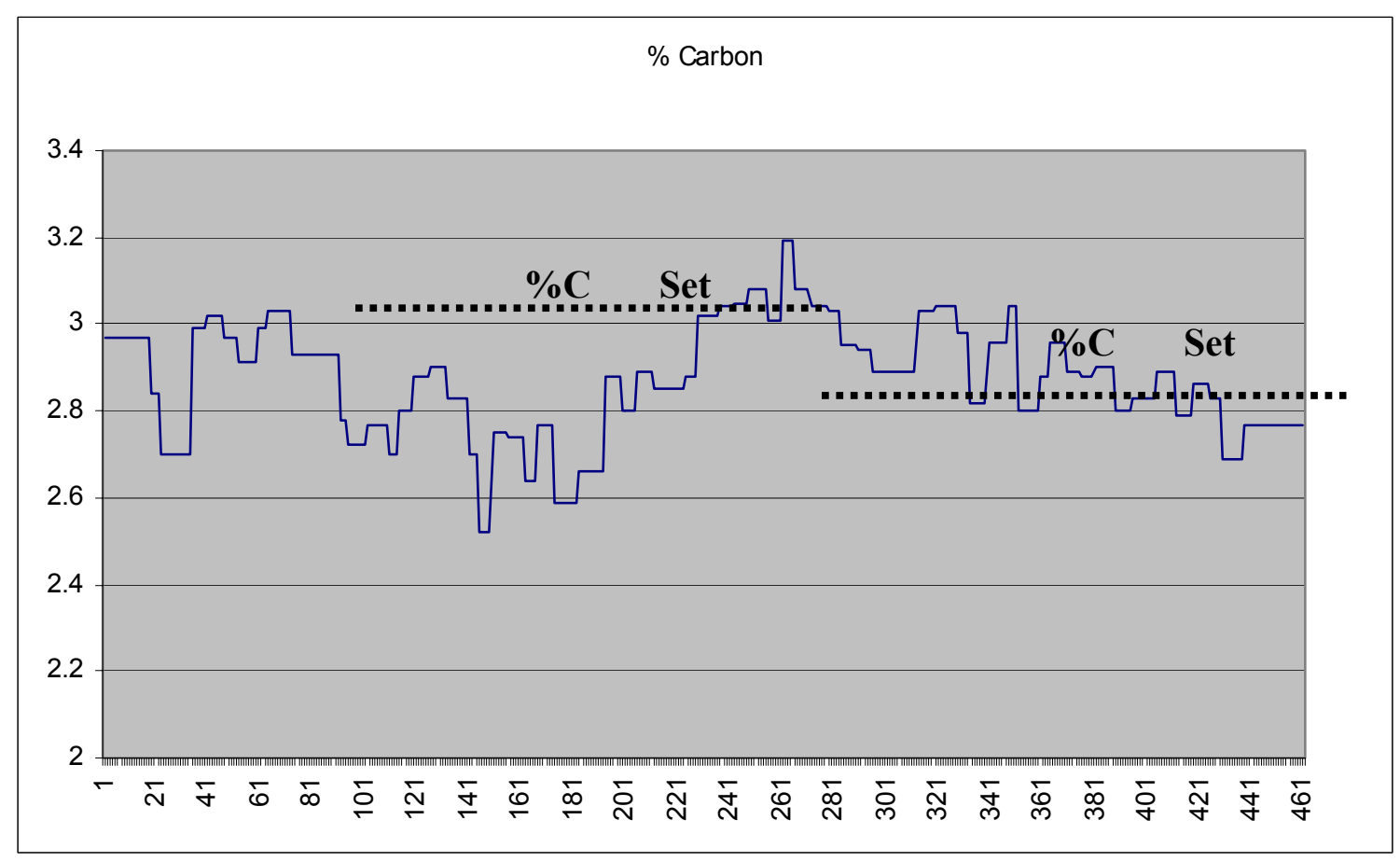

Figure 5-10 Control of \%C during Run \#2 


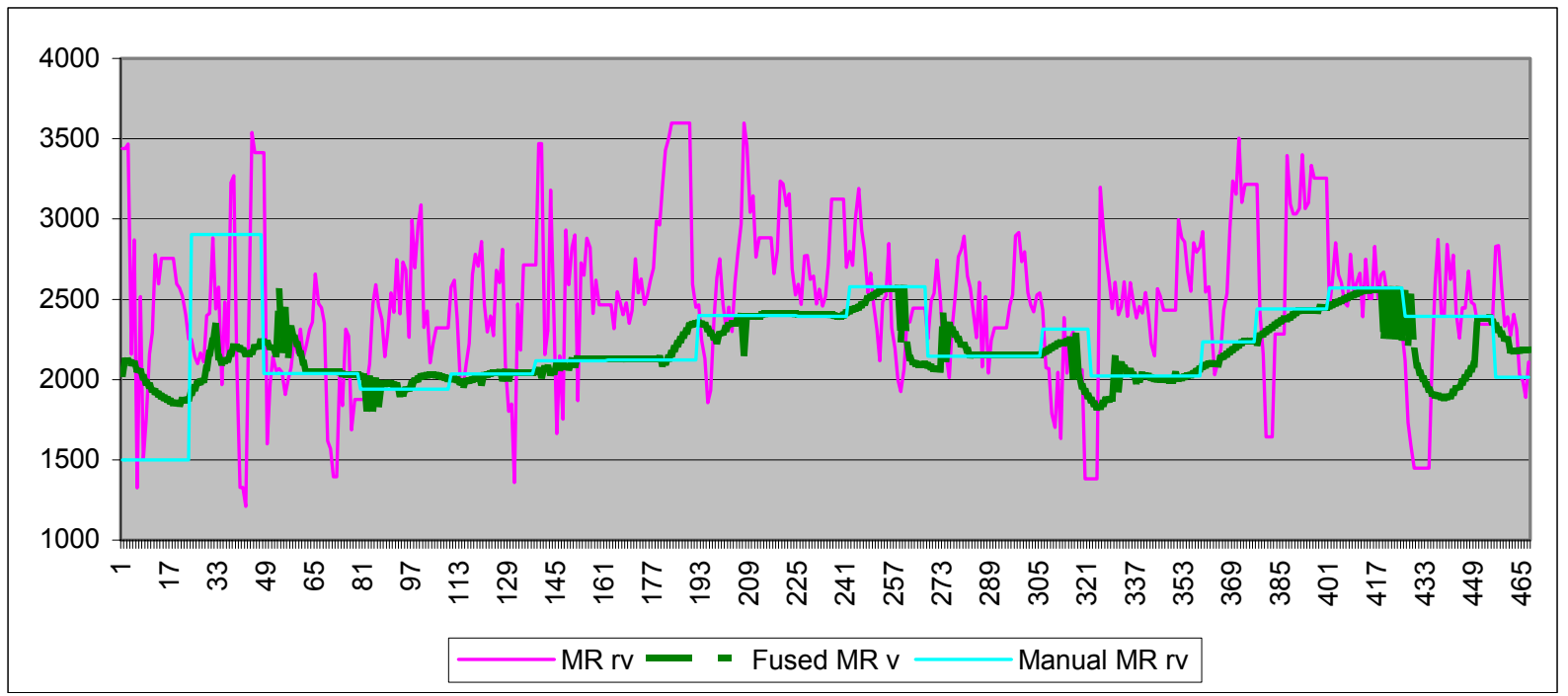

Figure 5-11 Changes in MR during Run \#2

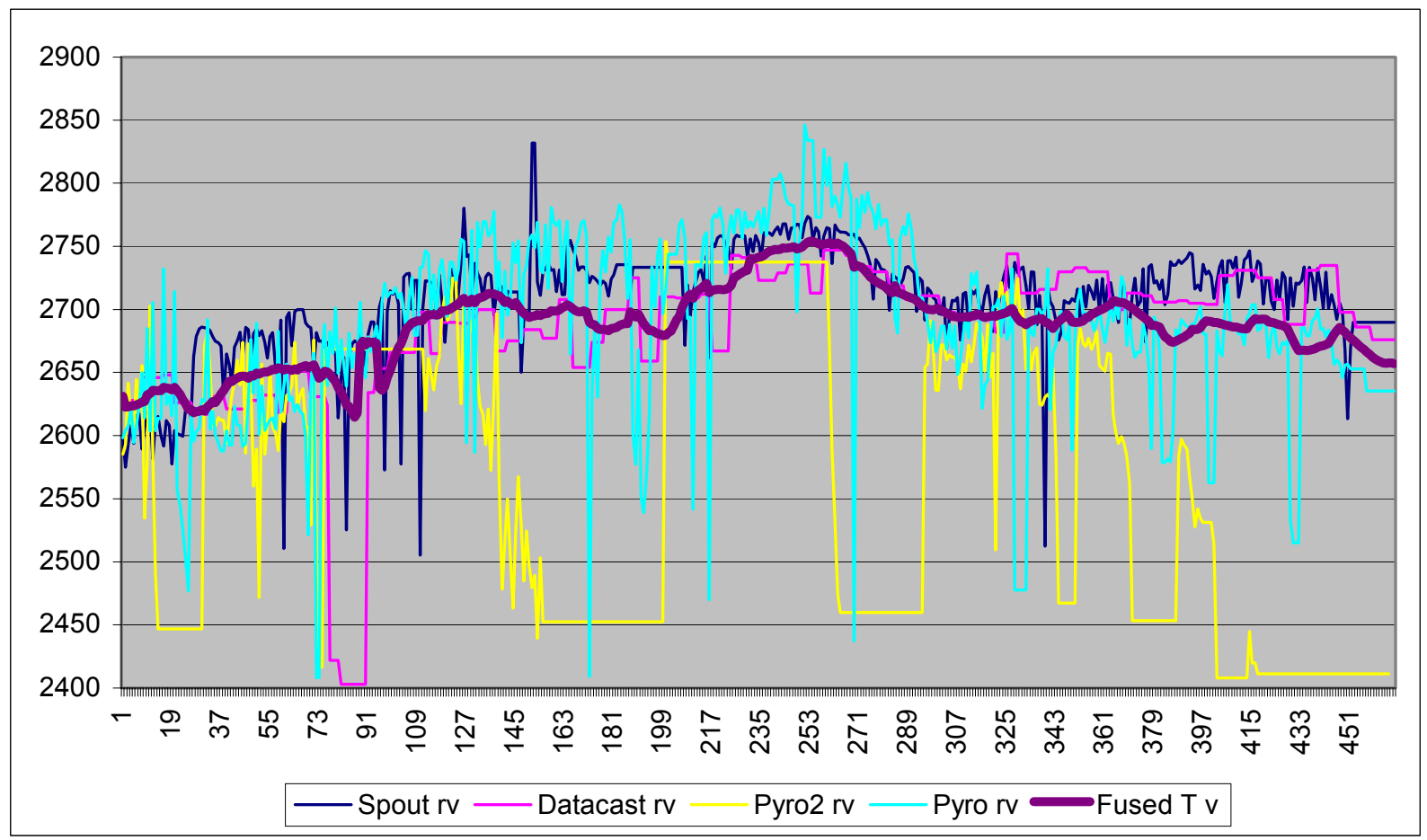

Figure 5-12 Changes in MR during Run \#2 
The second run aimed at showing the ability to quickly change the carbon content of the iron. Two set points for the carbon were desired. The first set point was 3\% and the second set point was $2.8 \%$. It should be noted that the first set point took longer to achieve than the second set point, as the cupola did not reach steady state when the controller was initially turned on. The change in the Carbon content is shown in Figure 5-10. The change in set points was also accompanied by a requested change in the melt rate as shown in

Figure 5-11.

Figure 5-12 shows the change in the temperature of the molten iron during the run. Figure 5-13 shows the change in the metal stream going into the cupola as suggested by $\mathrm{I}^{3} \mathrm{PSC}$ along with a disturbance in the form of pig iron stream replacing part of the cast iron.

The last run demonstrated the ability to drastically reduce the melt rate while maintaining the carbon content and the iron temperature within appropriate ranges. This was achieved by a change in the CMR prior to the reduction in the blast rate. The change in the CMR was accompanied by a change in the metal stream to compensate for the expected effect of the increase of CMR on the carbon content. The main reduction in the melt rate was produced by a drastic cut in the blast rate and oxygen enrichment. It should be noted that the forward change in the CMR reduce the required decrease in the blast rate and keeps the metal temperature within the 
desired range after such drastic cut in the blast rate and oxygen enrichment. These changes are illustrated in Figure 5-14 to Figure 5-20. Figure 5-14 shows the change in $\mathrm{CMR}$ in anticipation of the request for a change in the MR and the corresponding adjustments in the metal streams to compensate for that change. Figure 5-16 and Figure 5-17 show the change in the blast rate and oxygen enrichment to achieve the desired MR.

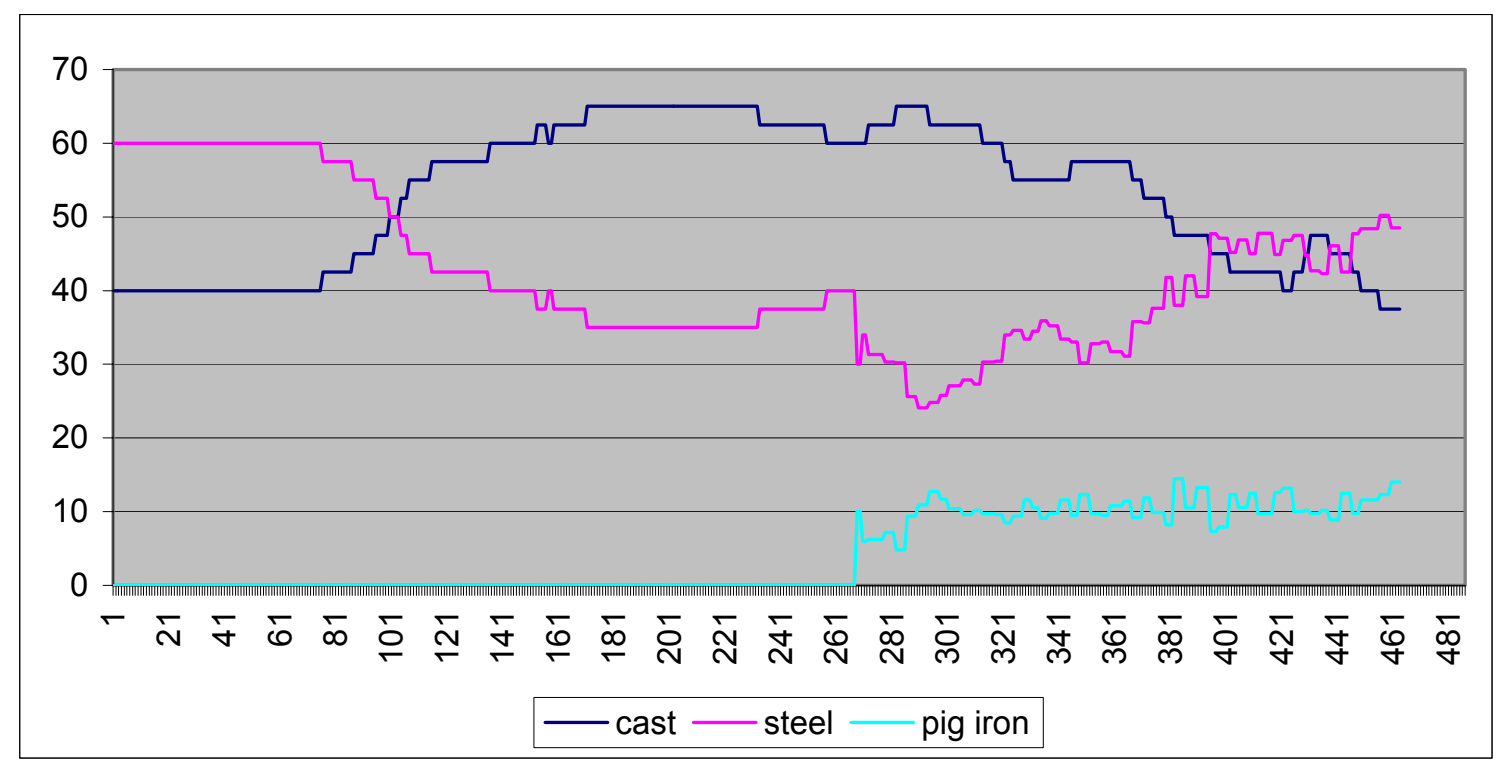

Figure 5-13 Metal Stream Changes control of \%C for Run \#2 


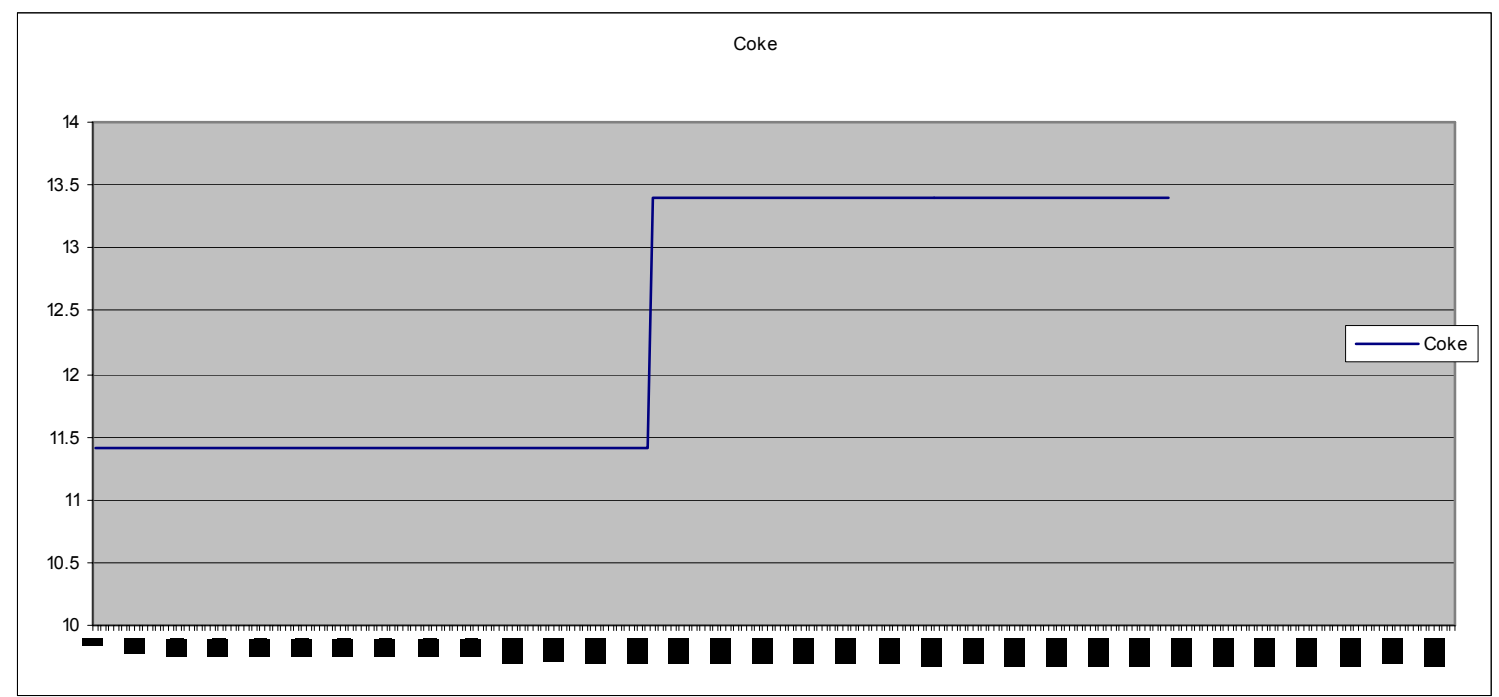

Figure 5-14 Forward Change in CMR to Achieve Large Change in MR (Run \#3)

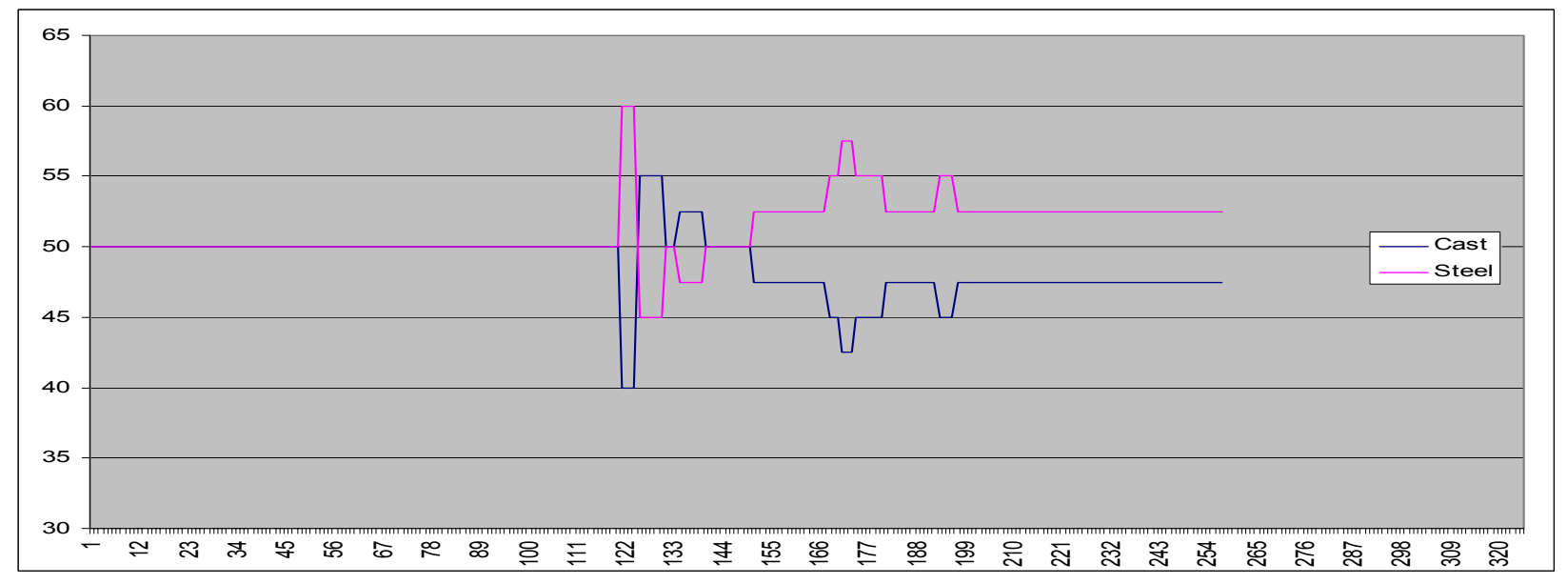

Figure 5-15 Changes in Metal Stream to compensate for Change in CMR 


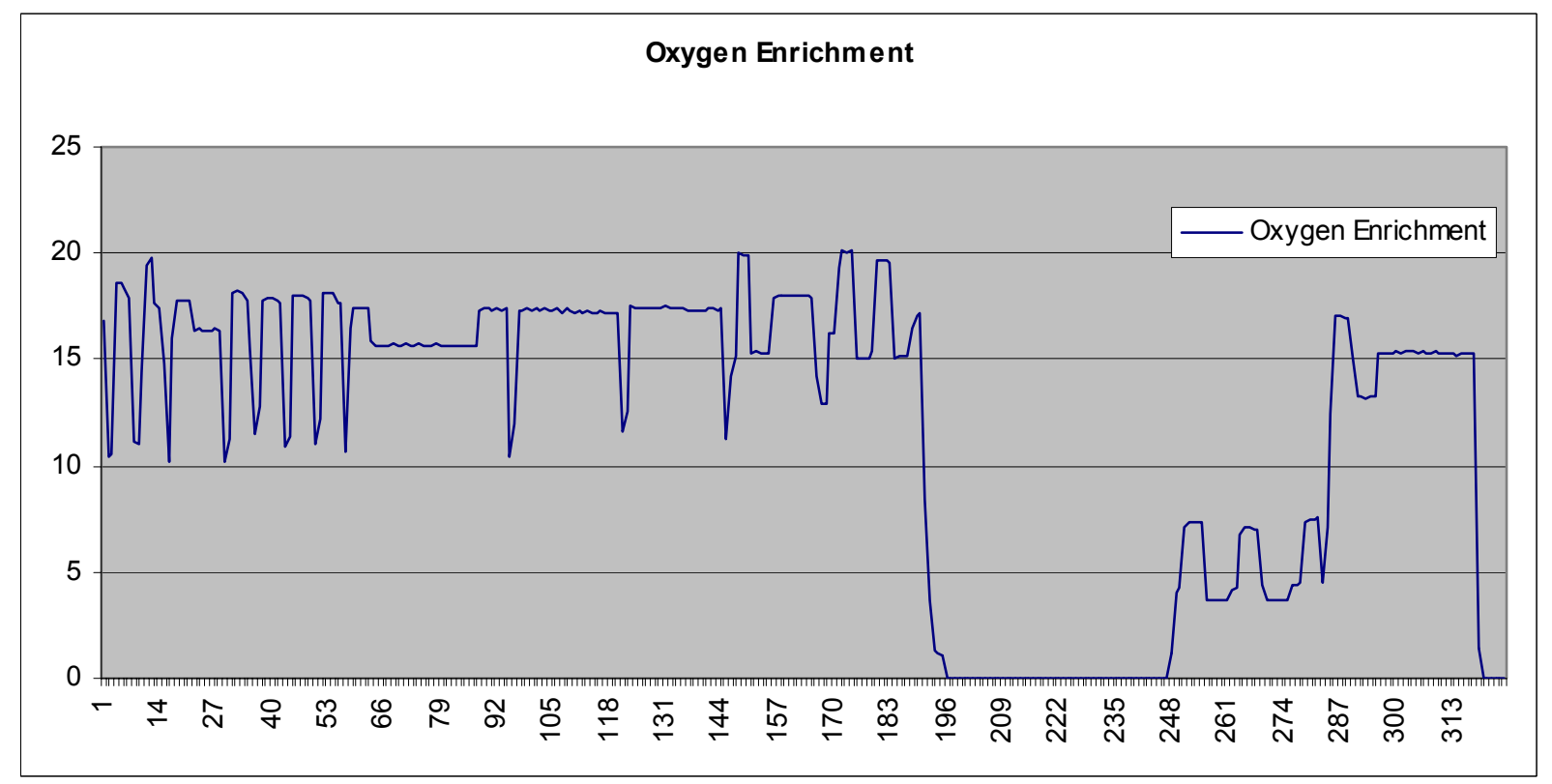

Figure 5-16 Changes in Oxygen Enrichment (SCFM) during Run \#3

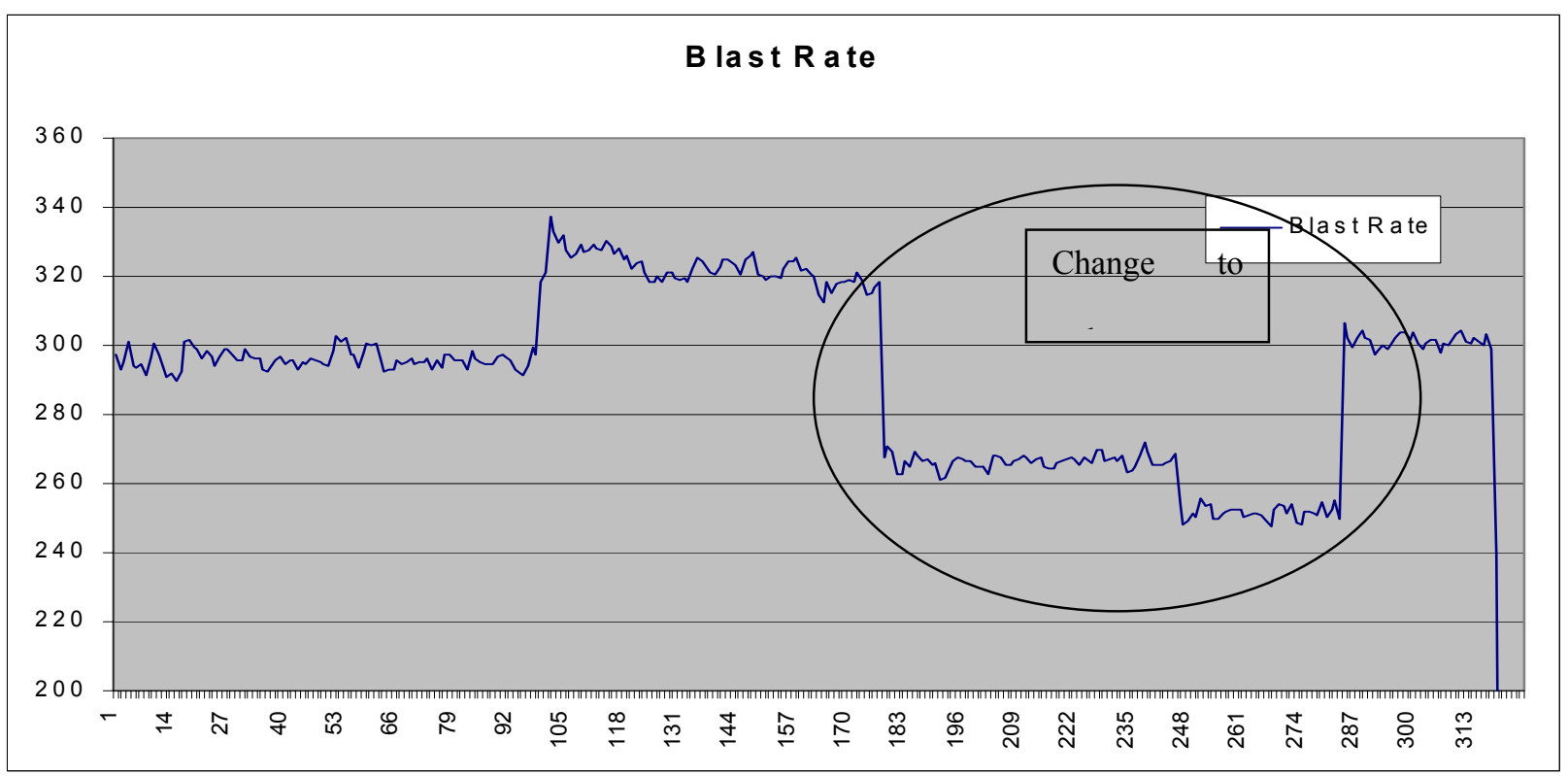

Figure 5-17 Changes in Blast Rate (SCFM) during Run \#3 


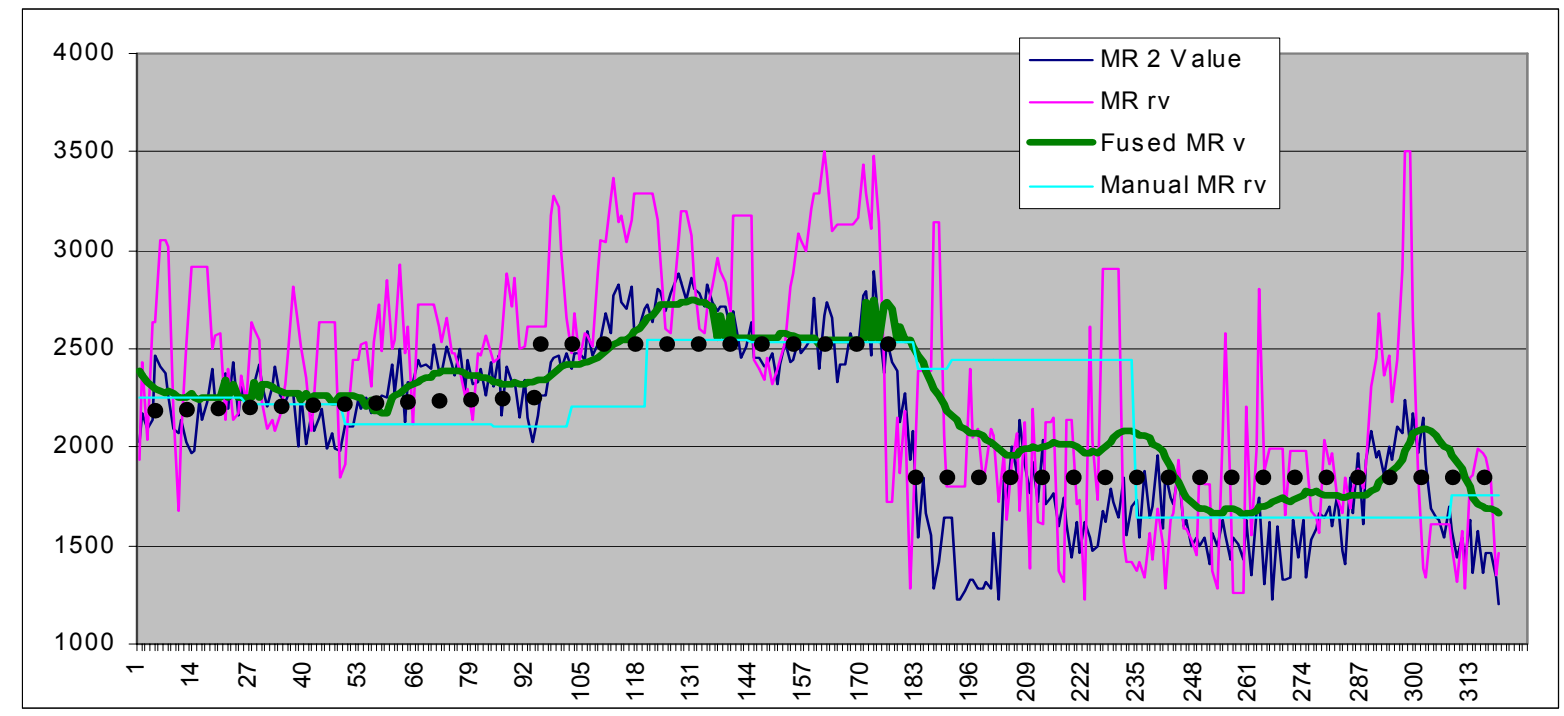

Figure 5-18 Control of Melt Rate during Run \#3

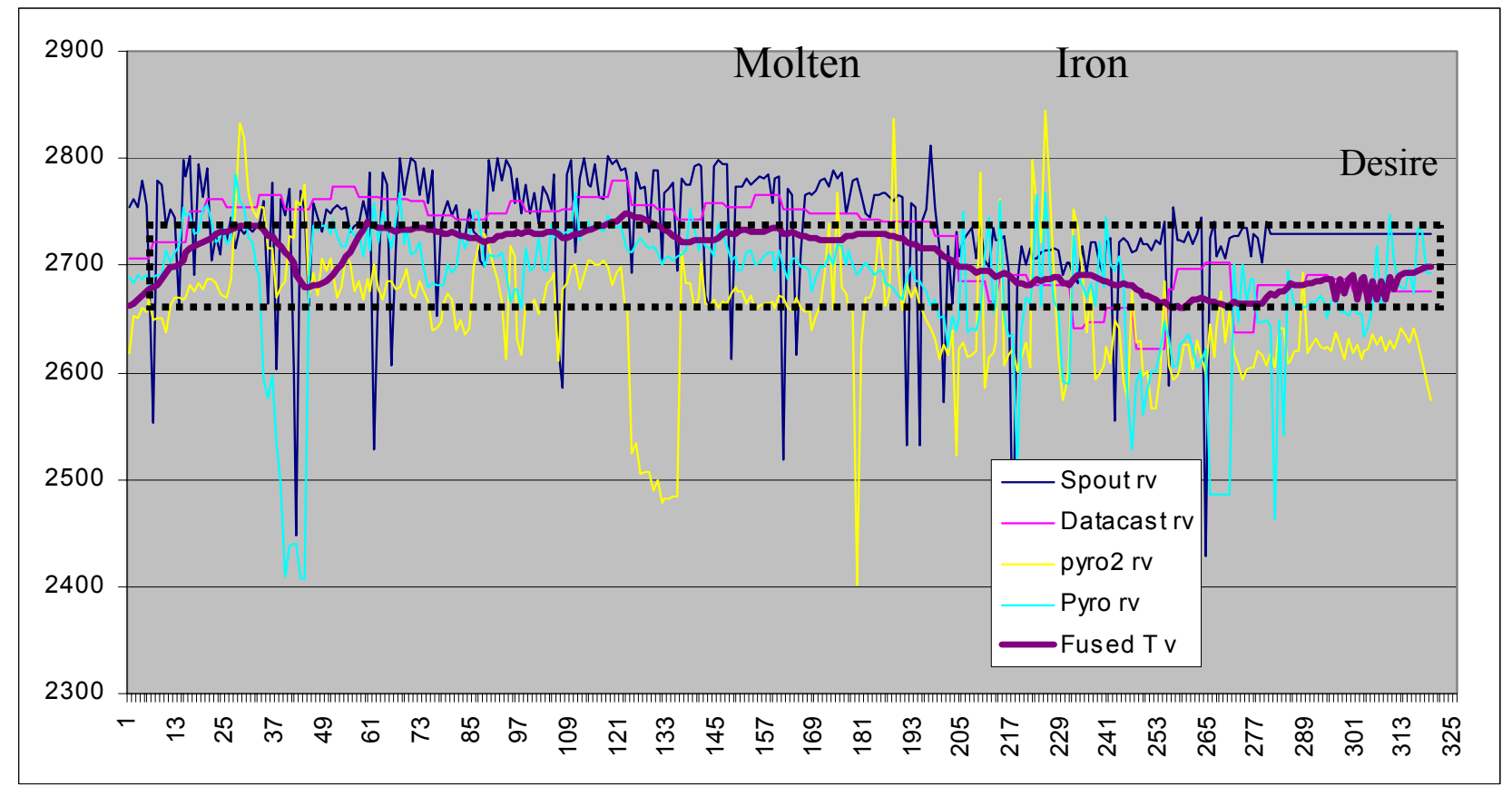

Figure 5-19 Changes in Iron Temperature deg F during Run \#3 


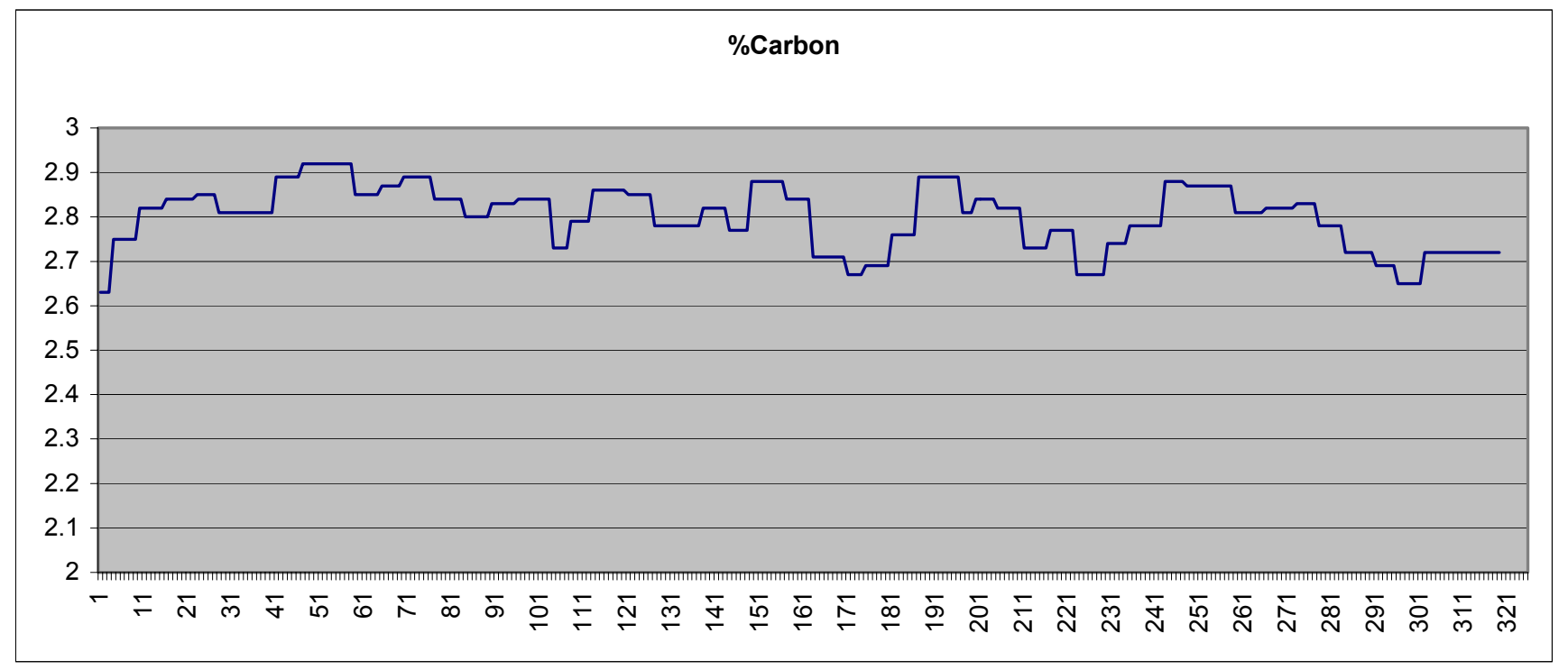

Figure 5-20 Changes in \% Carbon during Run \#3

Figure 5-21 and Figure 5-22 illustrate a different capability of the $I^{3}$ PSC. As we mentioned earlier one of the modalities of $\mathrm{I}^{3} \mathrm{PSC}$ is a monitoring modality that can be directed to monitor the trends of specific variables. This modality can also be directed to monitor for a set conditions on multiple variables including specified trends and absolute values. In the case illustrated here, the monitoring modality detects the occurrence of a bridging condition in the cupola through the monitoring of two parameters, namely the cupola exit temperature and the cupola back pressure. These variables are easily measured and continuously monitored. The two variables, as shown in Figure 5-21 and Figure 5-22, show a simultaneous increase during the marked window. The simultaneous increase of both variables is a good indicator of 
the occurrence of bridging in the cupola. The operator could, thus, be alerted for the bridging and an action to alleviate the problem.

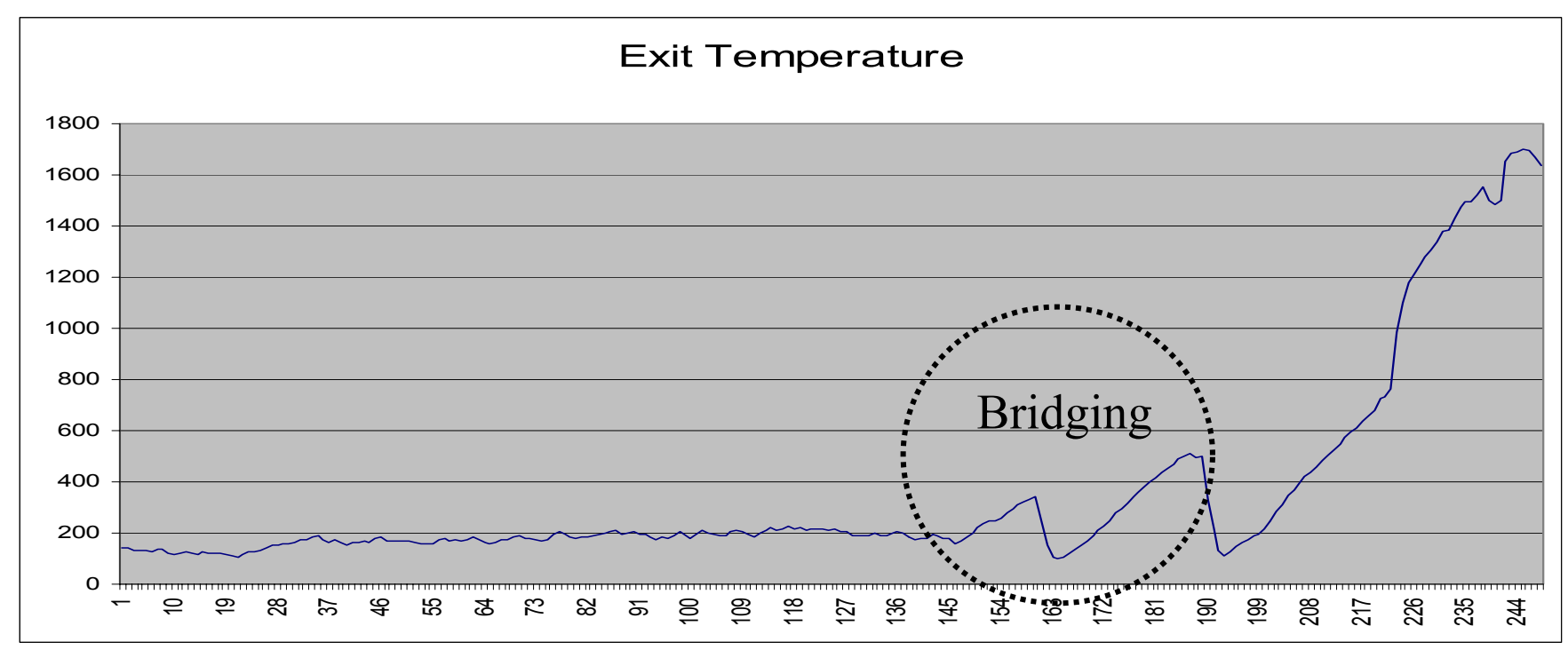

Figure 5-21: Detection of Bridging in the Cupola-Changes in Exit Temperature 


\section{Cupola_Press.}

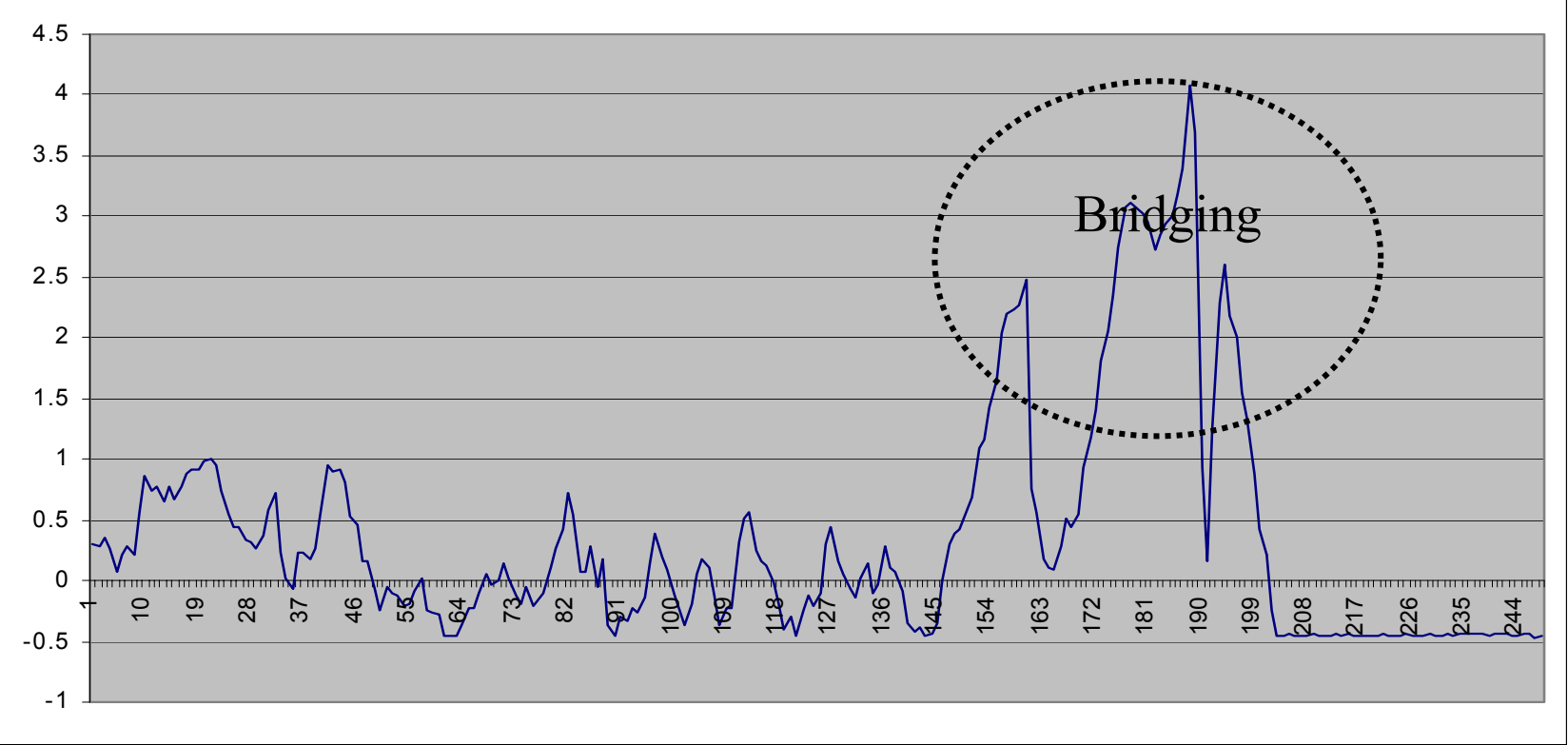

Figure 5-22: Detection of Bridging in the Cupola-Changes in Cupola Pressure 


\section{Appendix 5.A}

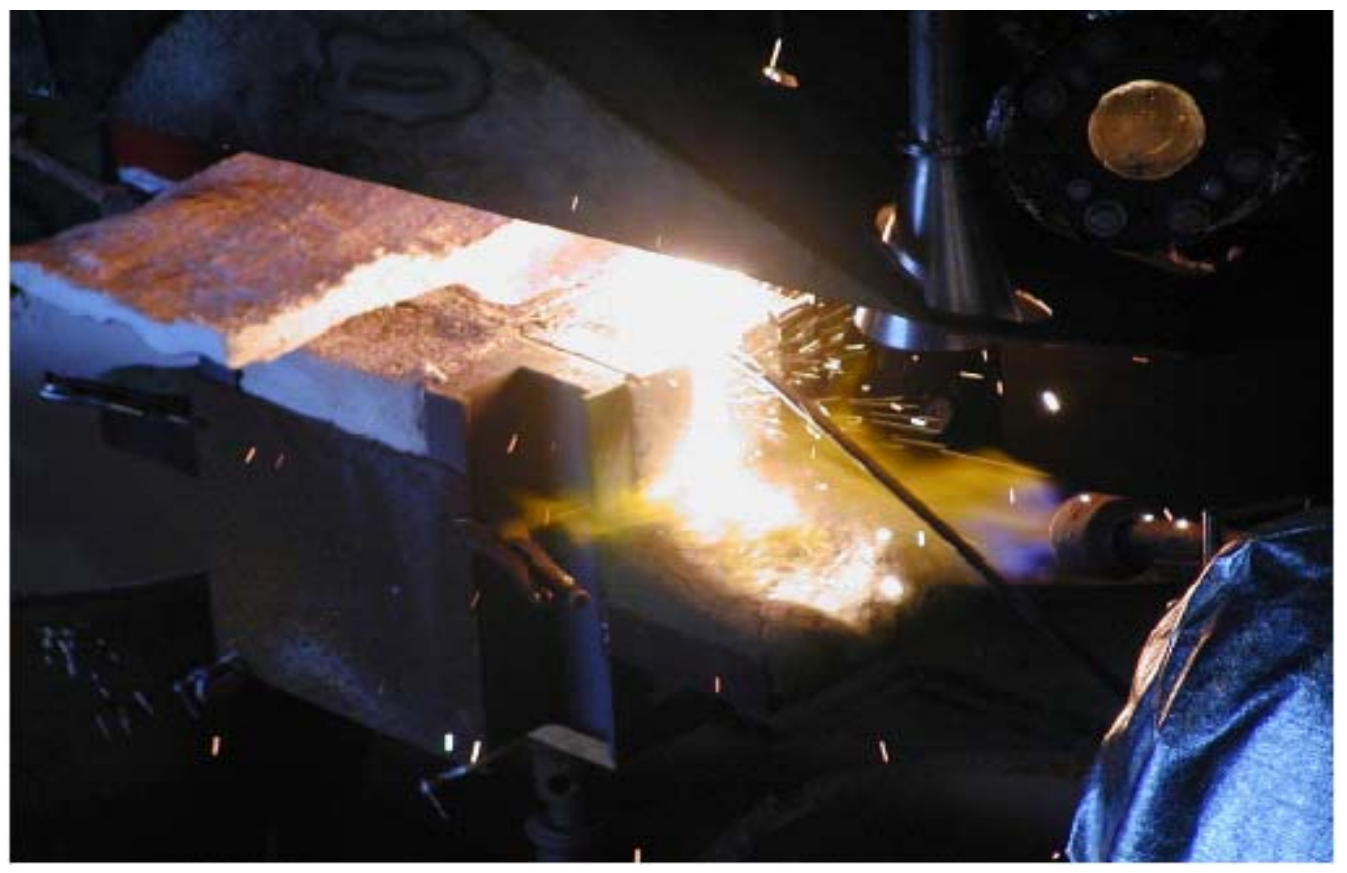

Figure 5-23: Opening the Tap-hole at ALRC Cupola

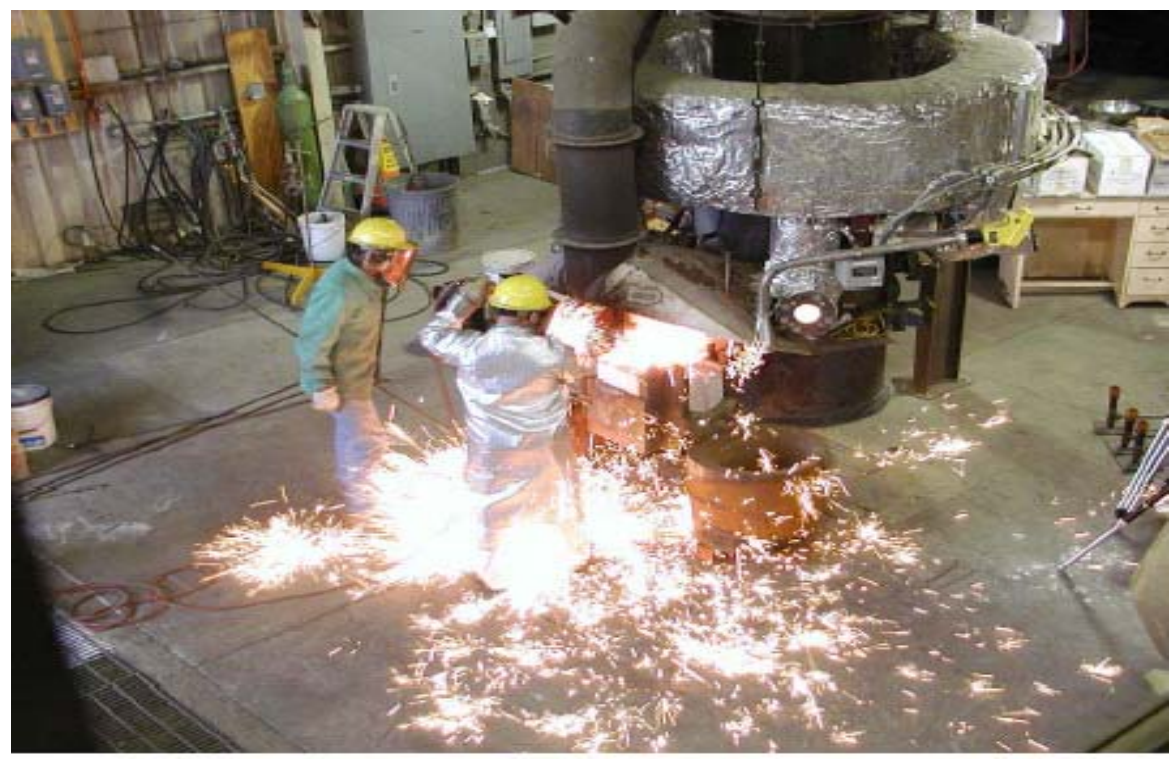

Figure 5-24: Cupola Always Provides Operational Challenges 


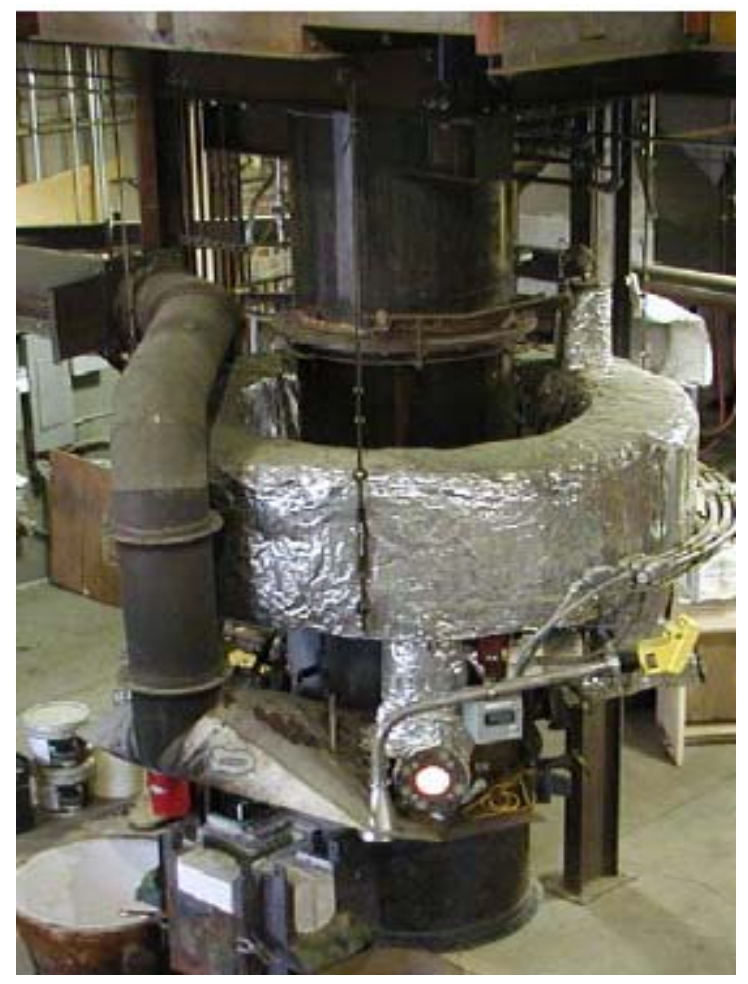

Figure 5-25: An Overview of the ALRC Research Cupola

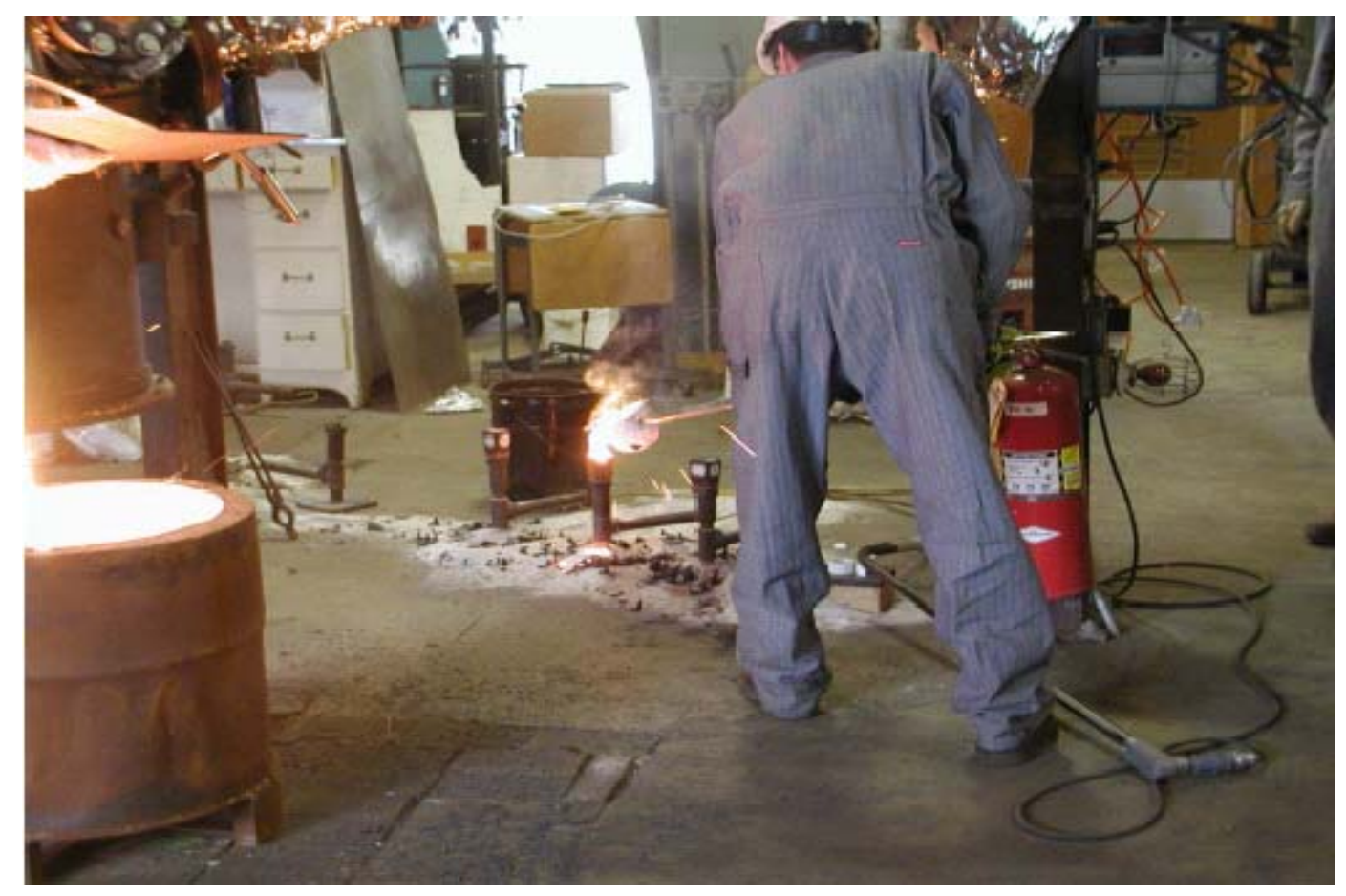


Figure 5-26: Manual Sampling and Quick Analysis of Molten Iron

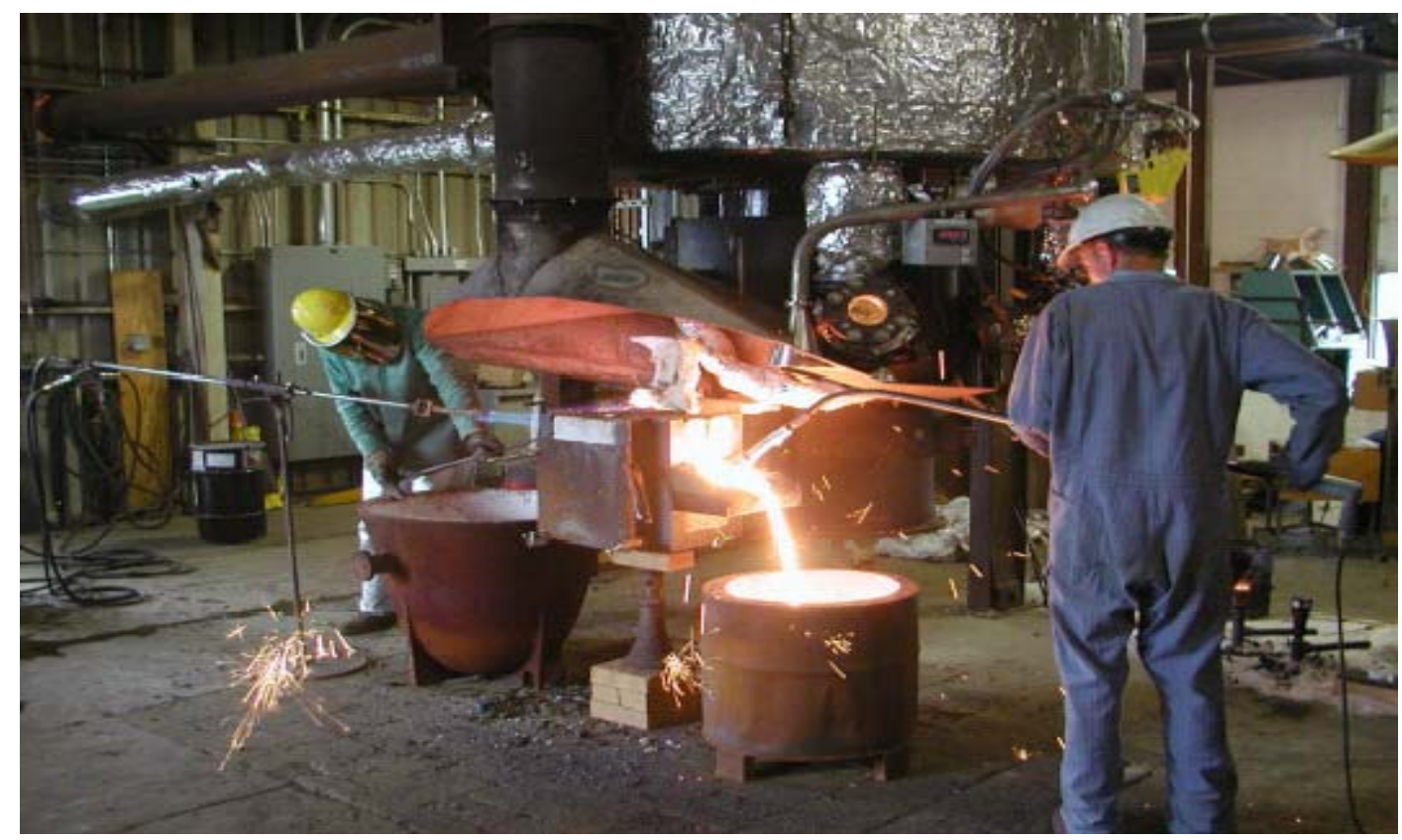

Figure 5-27: Manual Measurement of Temperature of Molten Iron

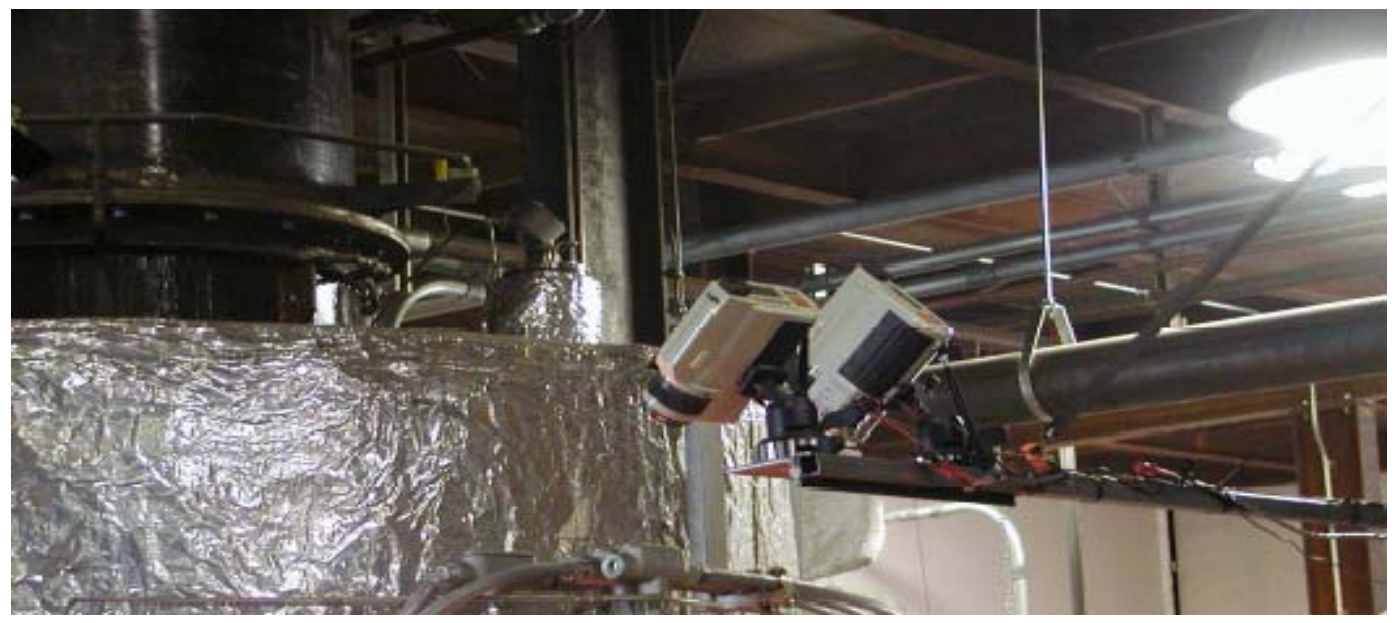

Figure 5-28: Optical Pyrometers for Continuous Measurements of Iron

Temperature 


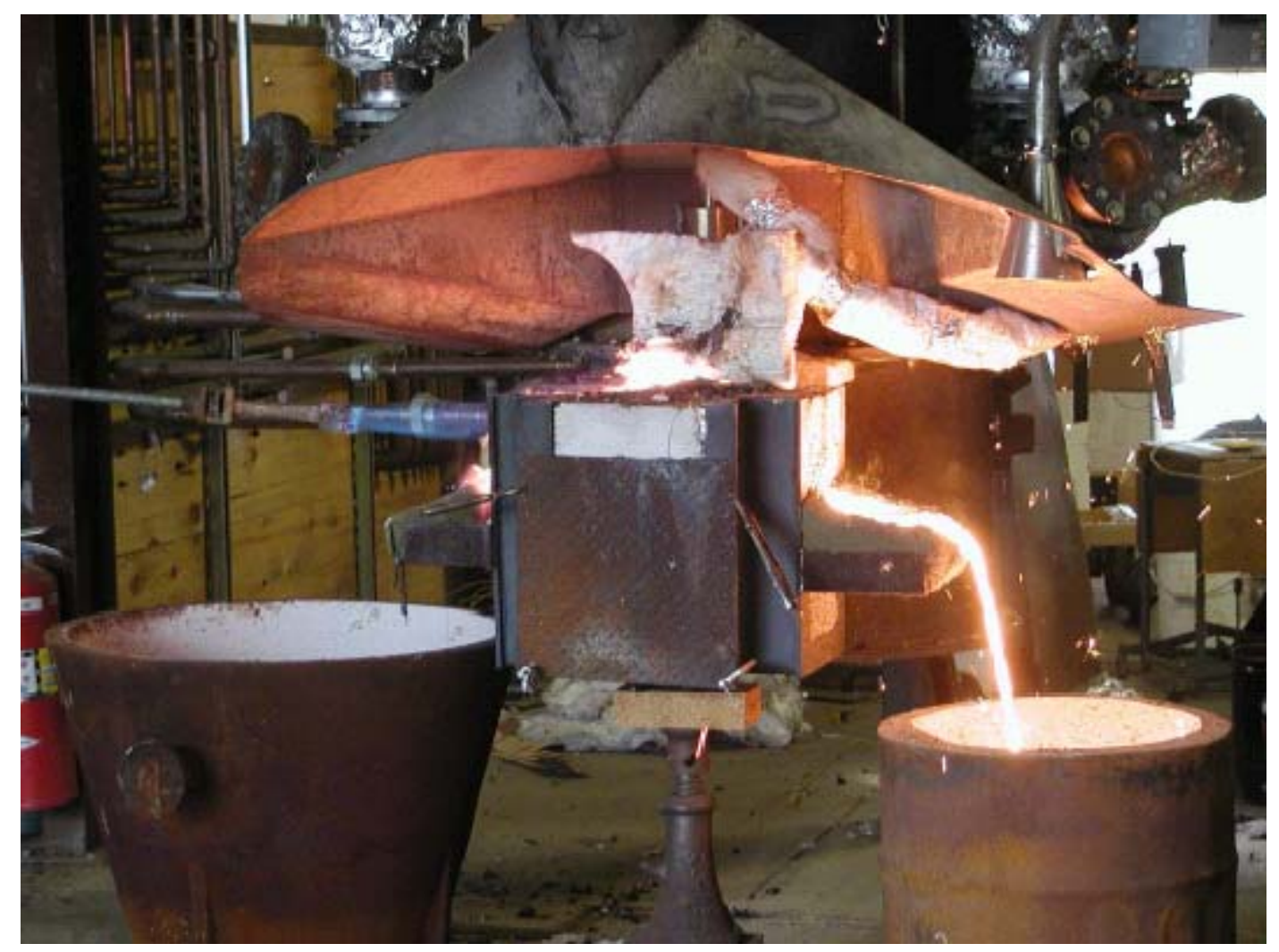

Figure 5-29: A Dip Thermocouple for Continuous Temperature Measurement

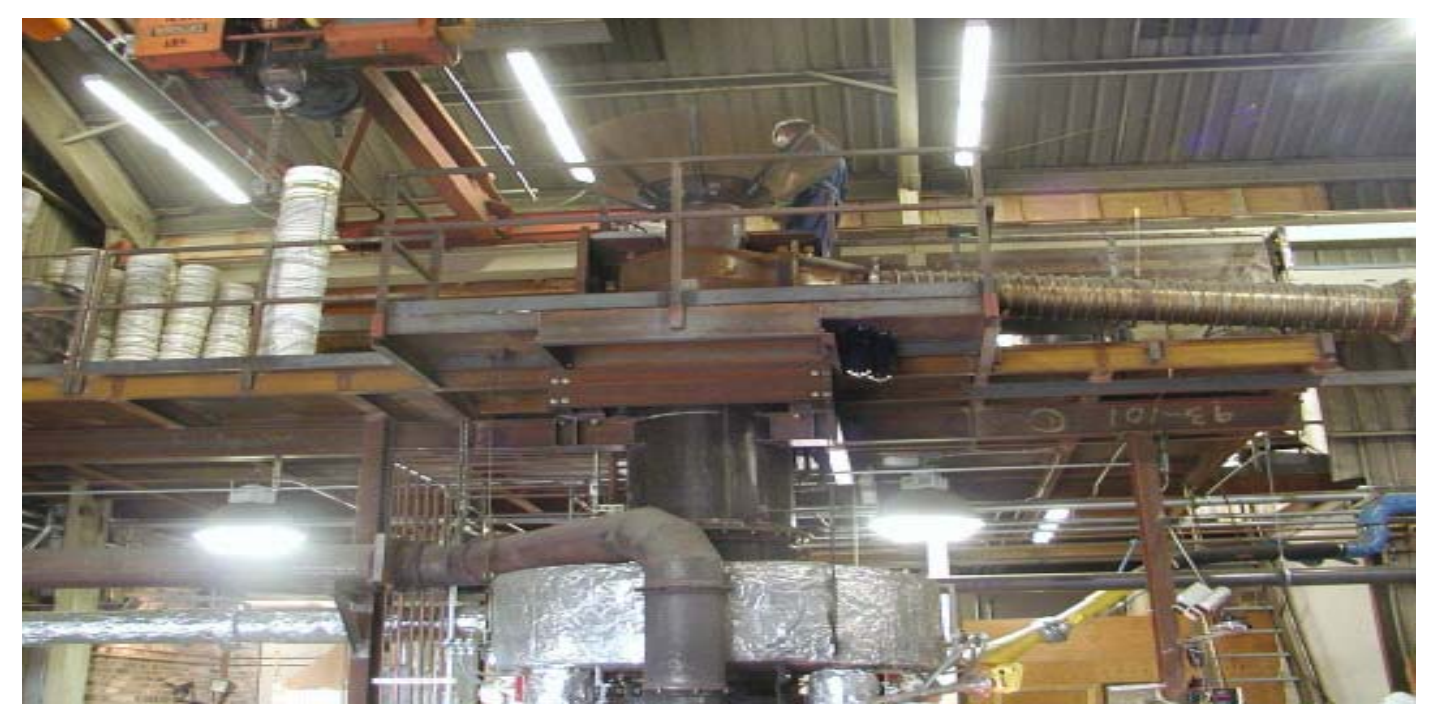

Figure 5-30: Charging Deck of the Cupola at ALRC 


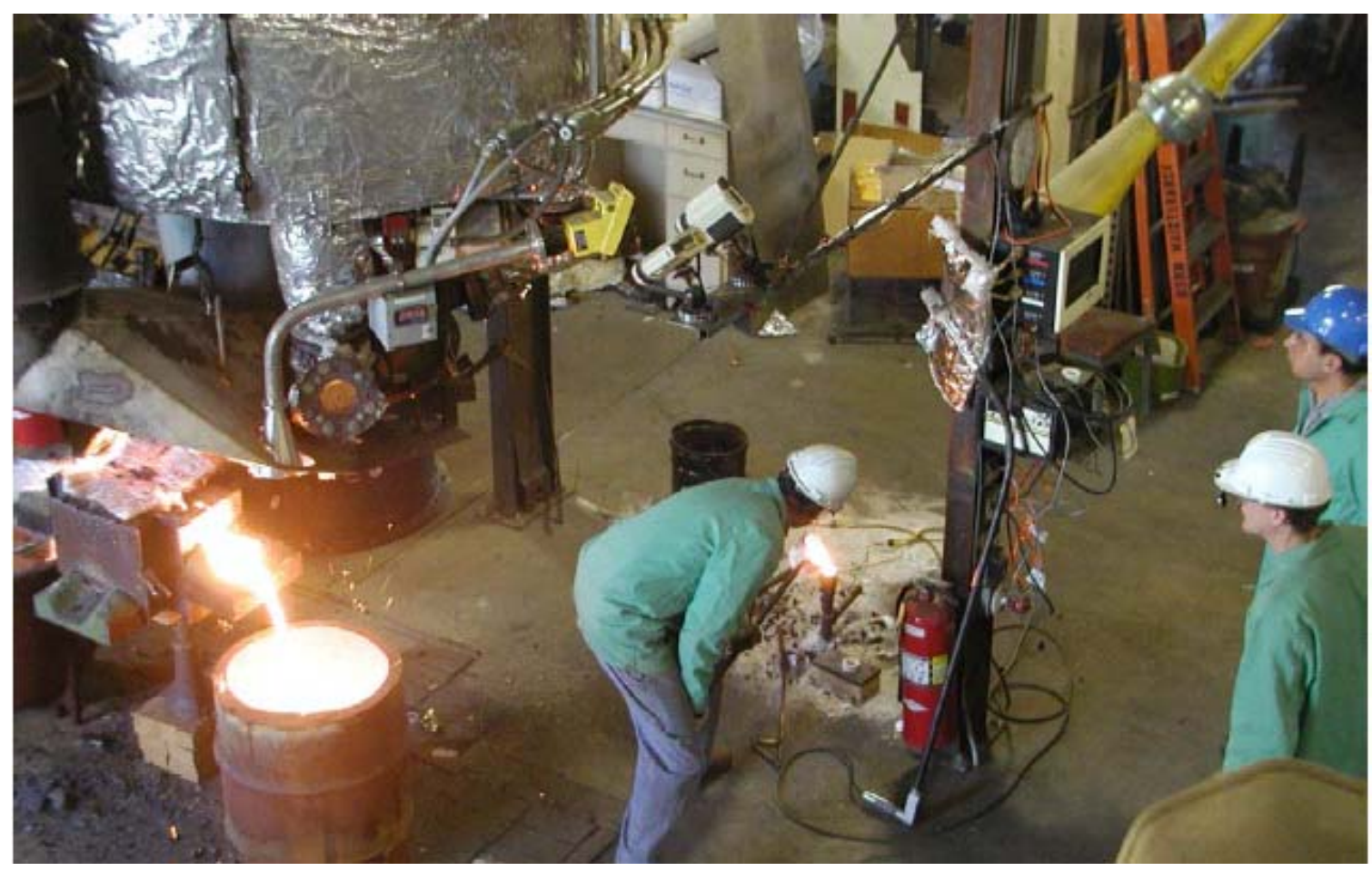

Figure 5-31: Measurement of Melt rate, Chemical Composition, and Temperature

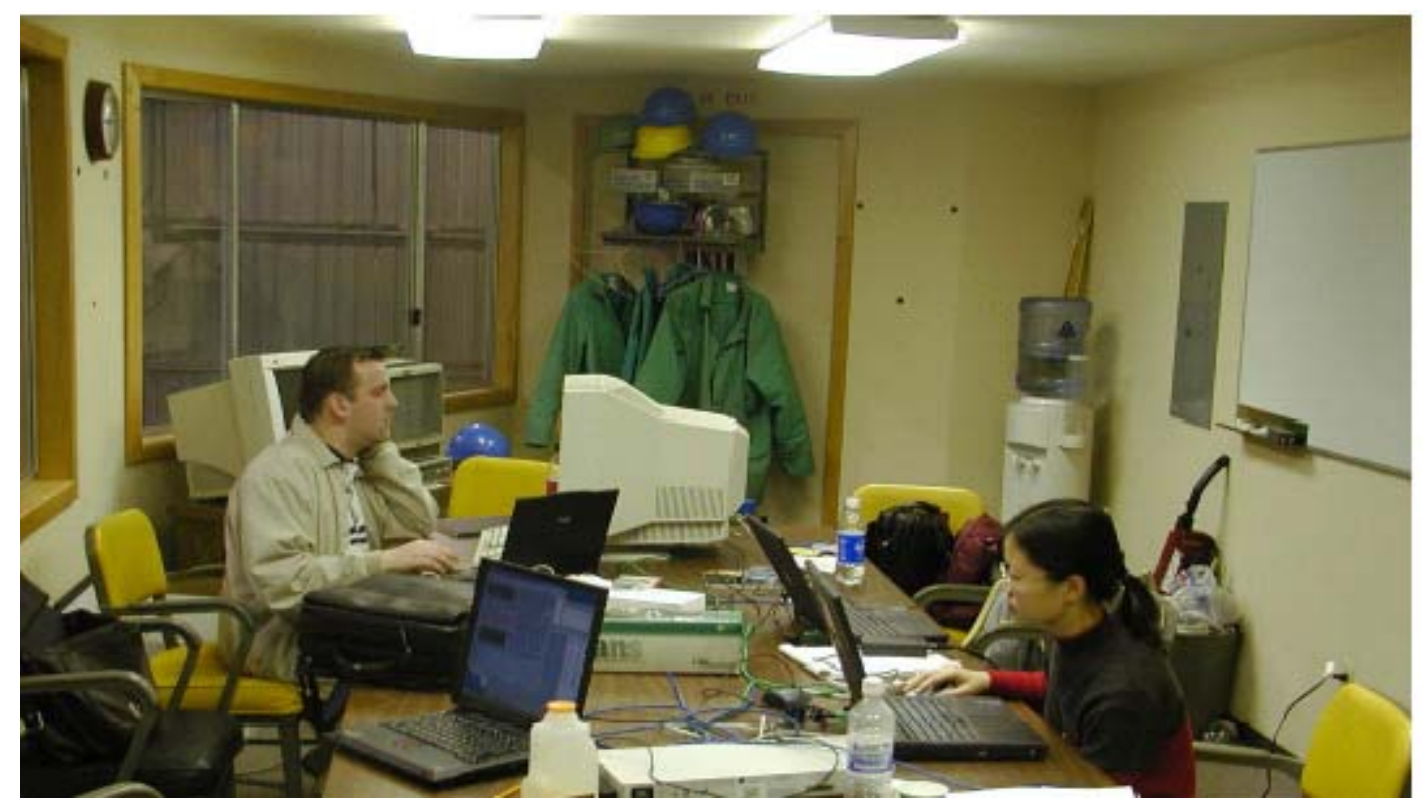

Figure 5-32: Remote Monitoring and Control of the Cupola during Demo Runs 


\section{Chapter 6}

\subsection{Summary and Conclusions}

Section 1 of this report has reviewed major highlights of the project including the management activities, development of algorithms for multiple sensor fusion and integration of sensing and control, and demonstration runs on a cupola iron-melting furnace in Albany research center. The project involved in addition to the algorithms development, the creation of a flexible software package based on object-oriented methodology that integrates the different components of the developed system. The software package includes algorithms for offline analysis as well as online operation. Details regarding the use of software package are provided in Appendix A.

The technical achievements of the project can be highlighted through the refereed journal and conference publications to interested professionals in the field of sensors and control as well as professionals within the metal casting industry. Up to this point fourteen papers and seven theses, that were supported by the project, have been published. The lists of papers and theses are provided in Appendices 1.A and 1.B. Some of the technical details were not included in this report to protect the intellectual property of the participants. 
The developed system (I $\left.\mathrm{I}^{3} \mathrm{PSC}\right)$ was tested in a series of demonstration runs. These runs have demonstrated the ability of the system to:

1- be easily interfaced into an existing cupola foundry with its own data acquisition equipment, sensors and networks,

2- $\quad$ be adapted to incorporate the available sensors and modalities,

3- fuse the available information sources and provide a best estimate as well as a confidence measure on the estimate,

4- monitor trends of individual variables as well as combination of variables and provide early warning on potential problems such as bridging that might be developing in the cupola,

5- $\quad$ integrate sensing and control algorithms to provide a closed loop automatic control system that can aid in maintaining the important operational cupola parameters such as carbon content, melt rate and iron temperature with specified boundaries at various conditions of operation requirements. Specific examples that were illustrated included the ability of the system to change the carbon content quickly during a run while maintaining the temperature and varying the melt rate. Another example showed the ability of the system to plan a large reduction in the melt rate while maintaining the carbon content and the temperature within acceptable ranges, and 
6- $\quad$ reduce the transition period to steady state operation by changing the initial charge setup in the cupola.

The demonstration runs, the publications and the developed software package illustrate that the project have achieved the proposed objectives. Full utilization of the developed algorithms, software and hardware within the scope of the industries of the future depends on other factors that are technical and economical. The next section discusses these issues in more details. 


\subsection{Future Recommendations}

As we have mentioned earlier, the $I^{3} \mathrm{PSC}$ system has achieved the technical objectives set at the start of the project. The system was tested using a state of the art research cupola furnace. It has not yet been adopted and tested by a commercial facility. Cupola foundries are in general conservative in adopting new technology especially under current economic conditions. Certain issues need to be considered towards achieving acceptance of the system in cupola foundries. Although the I PSC system was designed to be generic, certain modalities such as virtual sensors and the automatic controller need to be setup to address the specific needs of a foundry and thus would require the investment of time and resources. Sensors for monitoring of key parameters in a foundry such as temperature and chemical composition have to be installed and operated, if not already available. A training period for personnel in the foundry would be necessary. The investigators have used and continue to use professional meeting and personal contacts to increase awareness of the cupola foundries to the benefits of the $\mathrm{I}^{3} \mathrm{PSC}$ and the possible economic and environmental impact of its utilization. Avenues for support of the first industrial implementation of I $\mathrm{I}^{3} \mathrm{PSC}$ in a cupola foundry using private as well as government funds are currently being explored.

From a different perspective, I I PSC was intended to be generic and applicable to other applications that require the integration of sensing and control. Thus, another 
avenue to pursue is to seek funding for the adaptation of the developed system in other applications within the scope of the industries of the future. 


\section{REFERENCES}

[1] Nagrath, I.J and Gopal, M, Control Systems Engineering, Second Edition, New Age International (P) Ltd., Publishers, 1995.

[2] Maciejowski,J.M, Multivariable Feedback Design, Addison-Wesley Publishers Ltd., 1990.

[3] Richard R.Brooks and S.S.Iyengar, Multi-Sensor Fusion - Fundamentals and Applications with Software, Prentice Hall, Inc., New Jersey, 1998.

[4] Ren C.Luo and Michael G Kay, "Multiple Integration and Fusion in Intelligent Systems," IEEE Transactions on Systems, Man and Cybernetics, vol. 19, no. 5, September 1989.

[5] R.C.Luo, M.Lin, and R.S.Scherp, "Dynamic multi-sensor data fusion system for intelligent robots," IEEE Journal Robotics and Automation, vol. RA-4, no. 4, pp. 385$396,1988$.

[6] Keith E. Holbert, A.Sharif Heger and Nahrul K. Alang-Rashid, "Redundant Sensor Validation by Using Fuzzy Logic," Nuclear Science and Engineering, vol. 118, pp. 54-64, 1994.

[7] Asok Ray and Rogelio Luck, "An Introduction to sensor Signal Validation in Redundant Measurement Systems," IEEE Control Systems Magazine, vol. 11, no. 2, pp. 43, Feb 01, 1991. 
[8] Marcello R Napolitano, Charles Neppach, Van Casdorph , Steve Naylor, Mario Innocenti and Giovanni Silvestri, "Neural Network Based Scheme for Sensor Failure Detection, Identification and Accomodation," Journal of Guidance, Control and Dynamics, vol. 18, no. 6, Dec 1995.

[9] Mohamed Abdelrahman and Senthil Subramaniam, "An Intelligent Signal Validation System for Cupola Furnace - Part 1 and Part 2," American Control Conference, San Diego, 1999.

[10] Janice C, Yang and David Clarke, "A Self-Validating Thermocouple," IEEE Transactions on Control Systems Technology, vol. 5 no. 2 March 1997.

[11] M.P.Henry and D.W.Clarke, "The Self-Validating sensor: Rationale definitions, and examples," Control Eng. Practice, vol. 1, no. 4, pp. 585-610, 1993.

[12] T.M.Tsai and H.P.Chou, "Sensor fault detection with the single sensor parity relation", Nuclear Science and Engineering," vol. 114, pp. 1411993

[13] Mathieu Mercadal, "Sensor Failure detection using Generalized Parity relations for Flexible Structures," Journal of Guidance, Control and Dynamics, vol. 12, no. 1, Feb 1989.

[14] Jeff Frolik, C.V.PhaniShankar and Steve Orth, "Fuzzy Rules for Automated Sensor Self-Validation and Confidence Measure," In Proceedings of American Control Conference, June 2000.

[15] Bernard Friedland, Advanced Control System Design, Prentice Hall, Inc., New Jersey, 1996. 
[16] K.J.Astrom and B.Wittenmark, Adaptive Control, Addison-Wesley Publishing Co., Reading, MA 1989.

[17] Liu Hsu; Aldayr D. de Araujo; Ramon R. Costa, "Analysis and design of I/O based variable structure adaptive control. (input-output variable structure model reference adaptive control systems)," IEEE Transactions on Automatic Control, vol. 39, no.1, pp. 4, Jan 1994.

[18] E. Burdet, A. Codourey, "Evaluation of parametric and nonparametric nonlinear adaptive controllers (Nonlinear controllers)," Robotica, vol. 16, no. 1, 1998.

[19] Judith Hocherman-Frommer; Sanjeev R. Kulkarni; Peter J. Ramadge, "Controller switching based on output prediction errors," IEEE Transactions on Automatic Control, vol. 43, no. 5, pp. 596, May 1998

[20] Michel Barbeau; Froduald Kabanza; Richard St.-Denis, "A method for the synreport of controllers to handle safety, liveness, and real-time constraints," IEEE Transactions on Automatic Control, vol. 43, no. 11, pp. 1543, November 1998.

[21] Specht, D.F., "Probabilistic Neural Networks," Neural Networks, November 1990.

[22] Ronald R. Yager and Dimitar P. Filev, Essentials of Fuzzy Modeling and Control, John Wiley \& Sons, 1994. 
[23] Jeff Frolik and Mohamed Abdelrahman, "Synreport of Quasi-Redundant sensor Data: A Probabilistic Approach," In Proceedings of American Control Conference, 2000.

[24] Hassan K. Khalil, Nonlinear Systems, Second edition, Prentice Hall Inc., 1996

[25] Mohamed Abdelrahman, Kevin Moore, Eric Larsen, Denis Clark and Paul King, "Experimental Control of a Cupola Furnace," In Proceedings of American Control Conference, 1998.

[26] Pascal Gahinet, Arkadi Nemiroviski, Alan Laub, and Mahmoud Chilali, "LMI Control toolbox 1.0," The Math Works Inc.

[27] Jeff Frolik, C.V.Phanishankar and Steve Orth, "Fuzzy Rules for Automated Sensor Self-Validation and Confidence Measure”, Proc. of American Control Conference, 2000, pp. 2912-2916.

[28] Mohamed Abdelrahman, Parameshwaran Kandasamy and Jeff Frolik, " $A$ Methodology for the Fusion of Redundant Sensors", Proc. of American Control Conference, 2000, pp. 2917-2922.

[29] Jeff Frolik and Mohamed Abdelrahman, "Synthesis of Quasi-Redundant sensor Data: Probabilistic Approach", Proc. Of American Control Conference, 2000, pp. 2922-2926.

[30] Vipin Vijayakumar, Mohamed Abdelrahman, Jeff Frolik, "A Convenient Methodology for the hardware implementation of fusion of Quasi-Redundant 
Sensors", Proc. Of $32^{\text {nd }}$ South-Eastern Symposium on System Theory, Florida, Mar 2000, pp. 349-353.

[31] Mohamed Abdelrahman, Min Luo, and Jeff Frolik, "Wavelet-Based Sensor Fusion for Data with Different Sampling Rates," in Proceedings of American Control Conference, Washington D.C., June 2001.

[32] Mohamed Abdelrahman et al. Integrated Intelligent Industrial Process Sensing and Control: Applied to and Demonstrated on Cupola Furnaces. Progress Report, Year 1, DOE contract DE-FC02-99CH10975, March 2000.

[33] Mohamed Abdelrahman et al. Integrated Intelligent Industrial Process Sensing and Control: Applied to and Demonstrated on Cupola Furnaces. Progress Report, Year 2, DOE contract DE-FC02-99CH10975, March 2001.

[34] Mohamed Abdelrahman et al. Integrated Intelligent Industrial Process Sensing and Control: Applied to and Demonstrated on Cupola Furnaces. Progress Report, Year 3, DOE contract DE-FC02-99CH10975, March 2002. 


\title{
APPENDIX A
}

\author{
$I^{3}$ PSC CUPOLA INTERFACE APPLICATION \\ USER MANUAL
}

Version 2.1

January 4, 2003 


\section{Table of Content}

\section{APPENDIX A 166}

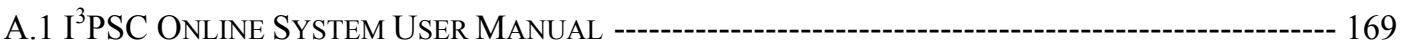

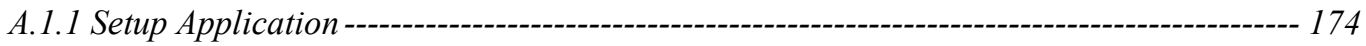

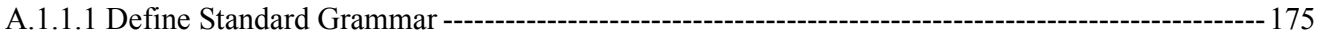

A.1.1.2 Create/Modality Standard Grammar--- 177

A.1.1.3 Select Modalities \& Variable \& Interface ------o-

A.1.1.4 Select Variable Properties---o- 179

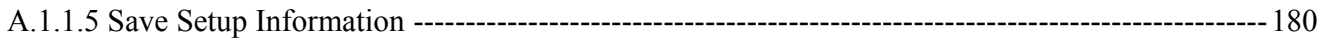

A.1.1.6 Modality Setup ----o- 180

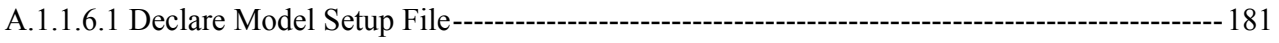

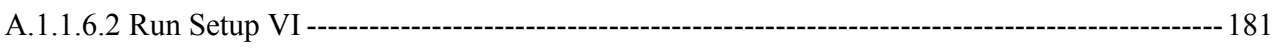

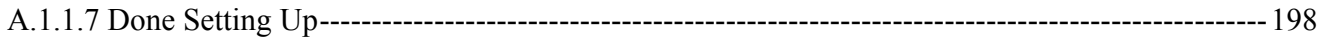

A.1.2 I'PSC Running ----- 199

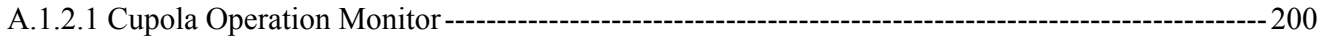

A.1.2.2 Change the Control Option Here--- 203

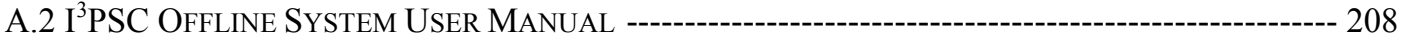

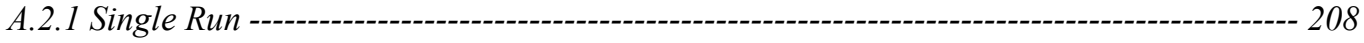

A.2.2 Single Variable Correlation -------------------------------------------------------------------- 209

A.2.3 Multi-Variable Correlation----------- 210

A.2.4 Nominal-Multiple Correlation ------------------------------------------------------------- 211

A.2.5 View Single Variable Graphs ------------------------------------------------------------ 212

A.2.6 View Multi-Variable Graphs ------------------------------------------------------------- 213

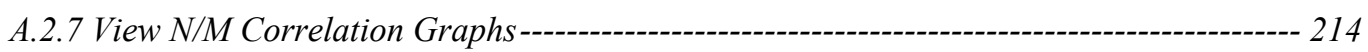

A.3 ONLINE ANALYSIS------------------------------------------------------------------------------------------ 216

A.3.1 Online Setup-------------------------------------------------------------------- 216

A.3.2 Simulate Data Collection -------------------------------------------------------------------- 217

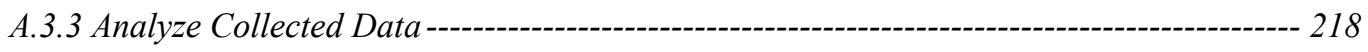

A.3.4 View Results ----_--- 219

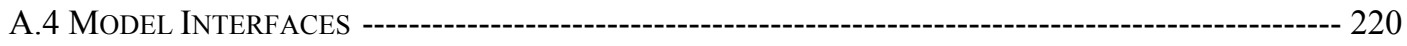

A.4.1 AFS Model Interface --------------------------------------------------------------------- 220

A.4.1.1 AFS Setup---1- 221 


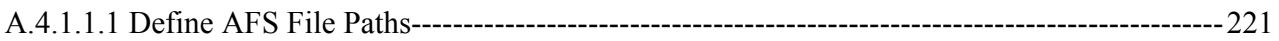

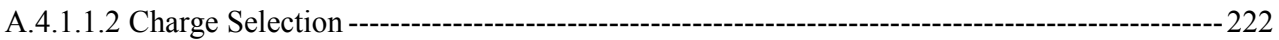

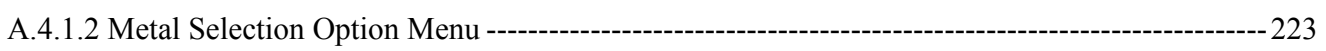

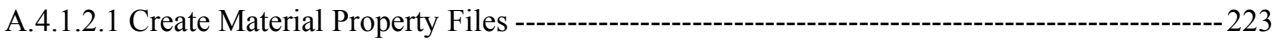

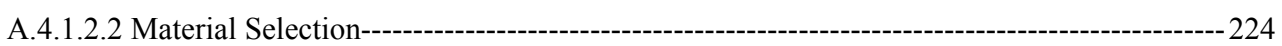

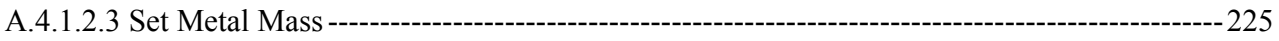

A.4.2 AFS Preprocessor -------------------------------------------------------------------------------- 225

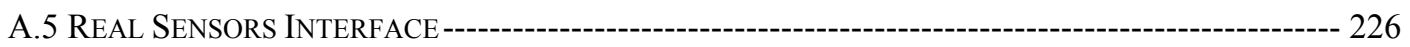




\section{A.1 I ${ }^{3}$ PSC Online System User Manual}

The user can start I I PSC (Intelligent, Integrated, Industrial Process Sensing and Control) online system by running Cupola Interface.vi. The path is $\mathrm{C}: \mathrm{I}^{3}$ psc $\backslash$ Application $\backslash$ Cupola Interface.vi. This VI's front panel is as shown in Figure A-1.

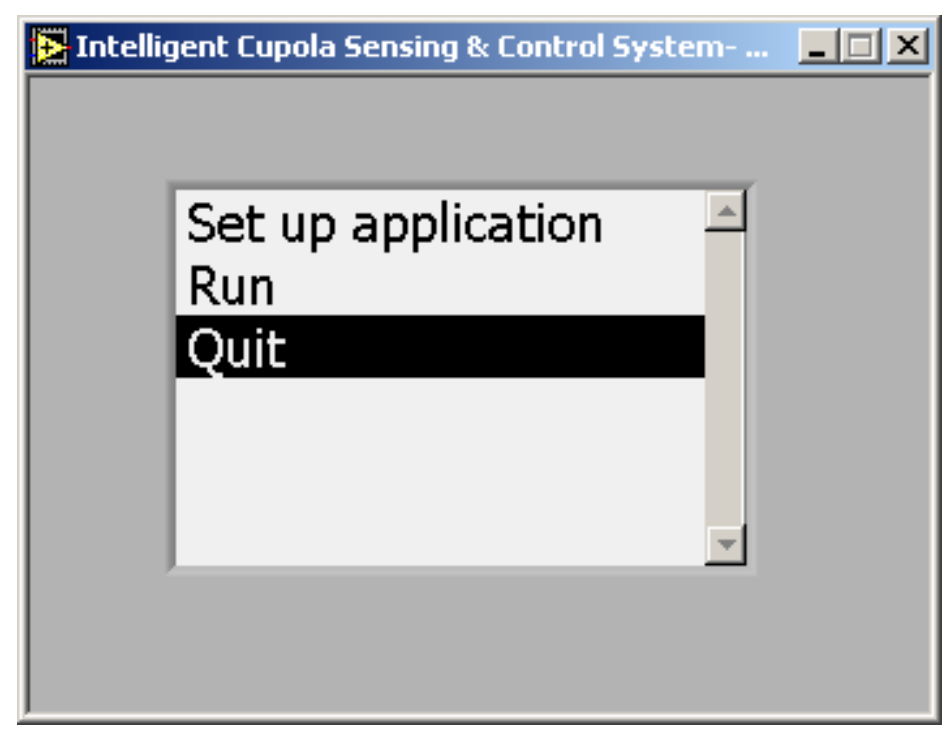

Figure A-1 I ${ }^{3}$ PSC Online System Top-Level Menu

Three options are listed in this menu, namely Set up application, Run and Quit. If you need to run $I^{3}$ PSC for a new application, you should double click on "Set up application". The dialog as shown in Figure A-6 is popped up. The procedure of setting up an application will be introduced in section 2. If the user wants to run an existing application, double click on "Run". The dialog (Figure A-44) is popped up. The running of the system will be introduced in section 3. Double clicking on "Quit" will exit the $I^{3}$ PSC system. The complete flow chart of using I ${ }^{3} \mathrm{PSC}$ is given in Figure A-3. Figure A-4 shows the procedure of setting up a new application and Figure A-5 shows the procedure for setting up a modality. The modalities that represent different system functions such as data acquisition, data fusions, controller, etc. are the components to build the system. As shown in Figure A-2, each modality contains a set of variables and each variable has an associated set of properties that get calculated by the application. This data structure is a 
3-D parallepoid with modalities, variable, and properties representing of the axis. The time represents the $4^{\text {th }}$ axis.

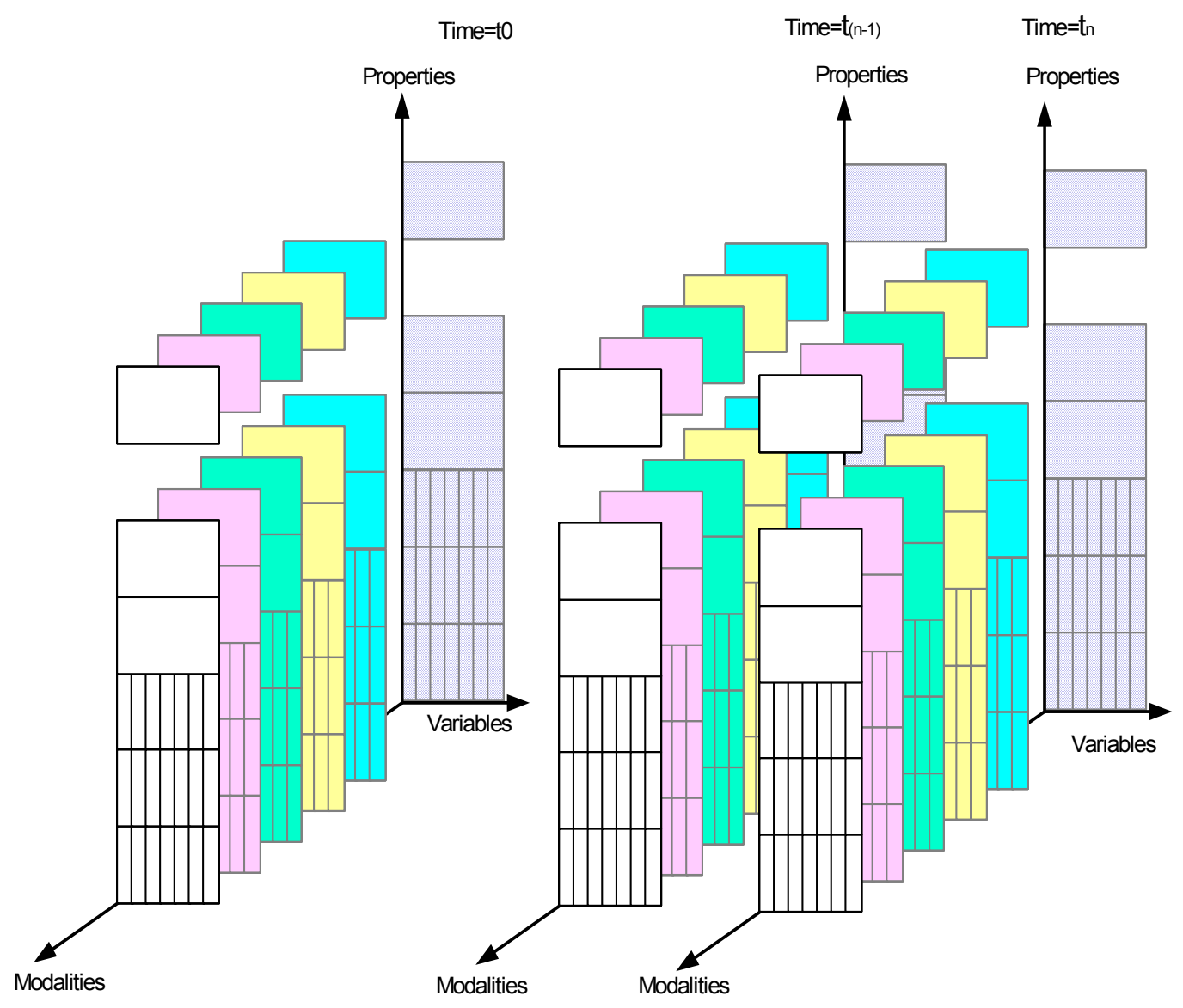

Figure A-2 Data Strucuture Model 


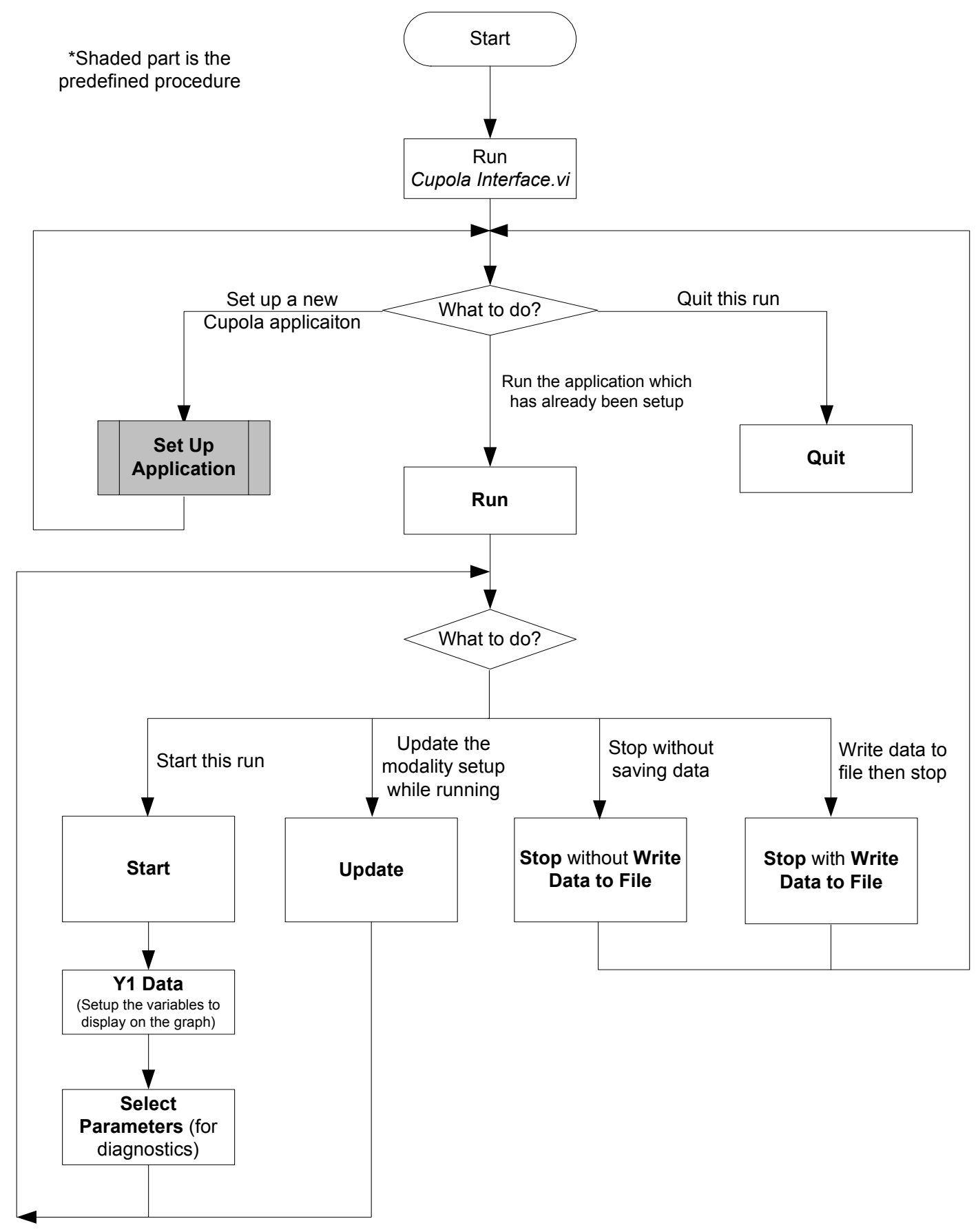

Figure A-3 Top-level Procedure 


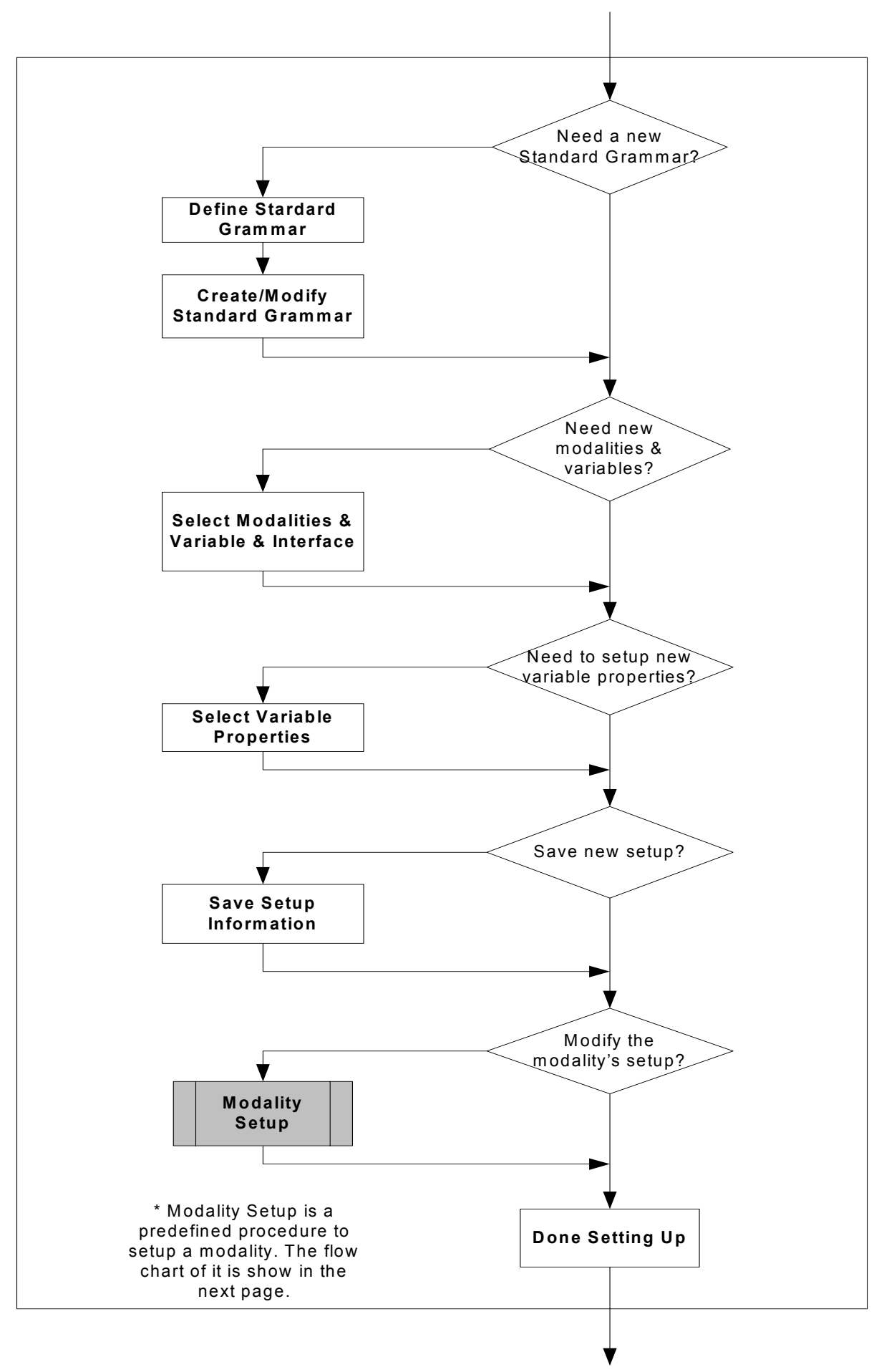

Figure A-4 Procedure of Setting Up Application 


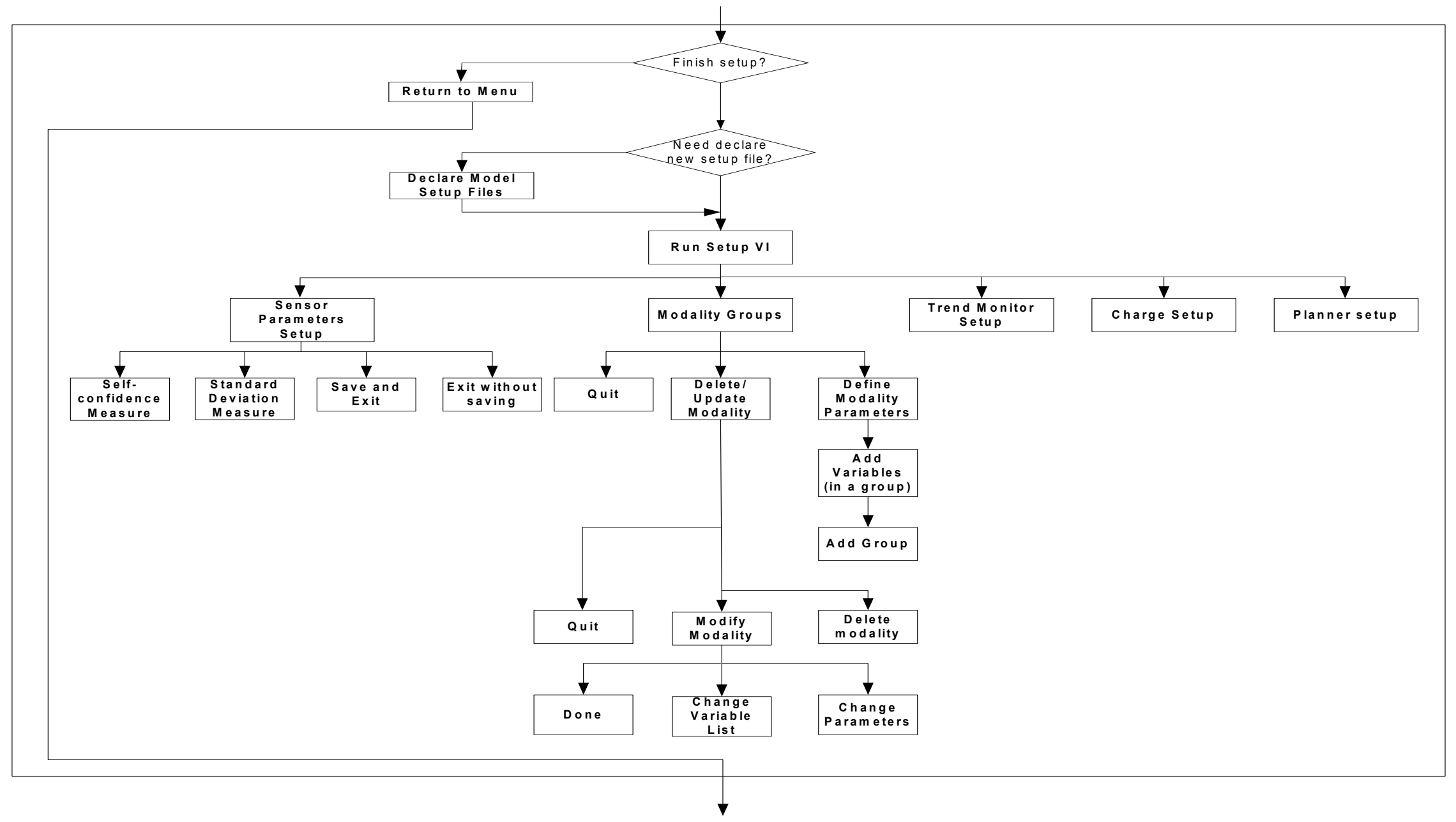

Figure A-5 Procedure of Modality Setup 


\section{A.1.1 Setup Application}

The main purpose of setting up an application is to setup the modalities to build a $I^{3}$ PSC system. I ${ }^{3}$ PSC contains a generic interface in order to include different modalities required by the application. Planner, Controller, Plant, Virtual Sensor, Fusion, Monitor, and Expert are eight modalities that have already been given. A system can be built with these modalities. Every modality has several groups. Each group has input variables, output variables, and an execution engine. The information inside the system is organized as a data structure. Node is the basic component in the data structure. Modality, variable, and property are three elements of a node. All the nodes with the time form the fourdimension data structure. Therefore, to build a system for a new application, the user needs setup the modalities, the variables in the modality, and the property of the variables. The user can setup these in the dialog shown in Figure A-6.

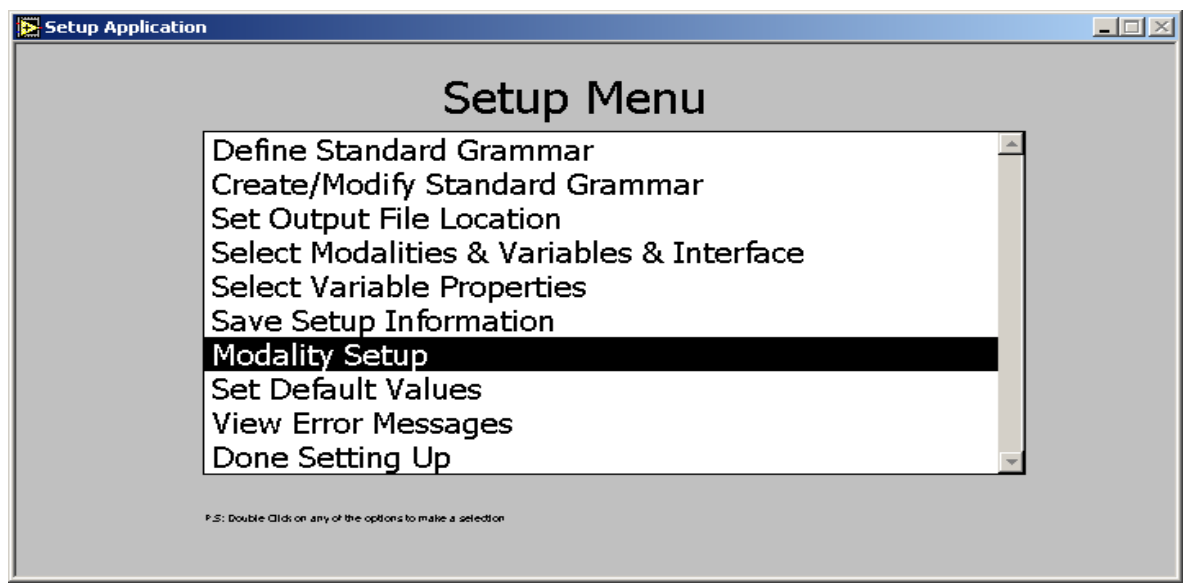

Figure A-6 Application Setup Menu

The main procedure of setting up a new application is followed:

1. Define Standard Grammar (refer to section A.1.1.1)

2. Create/Modify Standard Grammar (refer to section A.1.1.2)

3. Select Modalities \& Variable \& Interface (refer to section A.1.1.3)

4. Select Variable Properties (refer to section A.1.1.4)

5. Save Setup Information (refer to section A.1.1.5)

6. Modality Setup (refer to section A.1.1.6)

7. $\quad$ Done Setting Up (refer to section A.1.1.7) 


\section{A.1.1.1 Define Standard Grammar}

Standard Grammar is the basic reference to describe the structure of the data structure of the system. The structure of a standard grammar file is shown in Figure A-8. The following rules must be followed in order to run the system properly.

1. The first row bears a descriptive name of the column or of the modality. The first six columns are fixed. They contain different information. Starting from column seven, the modalities appear. In the example shown in Figure A-8, the modalities are Planner, Controller, Plant, Virtual Sensor, Fusion, Monitor, and Expert. The order of the modalities in the standard grammar presents the execution order of the modalities in the system. So the modalities should be in a specified order. Up to eight modalities can be included in the system.

2. The second row has "INPUT" which is a flag value to determine the start of the input variable in each modality. At the end of the list of input variables, there is a blank row and a row with "OUTPUT" which is a flag to determine the beginning of the output variable in each modality.

3. The name in the first column is a globe name representing a variable, however, each modality can have a local variable name that corresponds to the variable in the first column of the standard grammar. This local variable name is used in the current implementation to interface the system to the DAQ.

To define an existing standard grammar file, double click on "Define Standard Grammar" in Setup Menu (Figure A-6). The dialog in Figure A-7 will be opened.

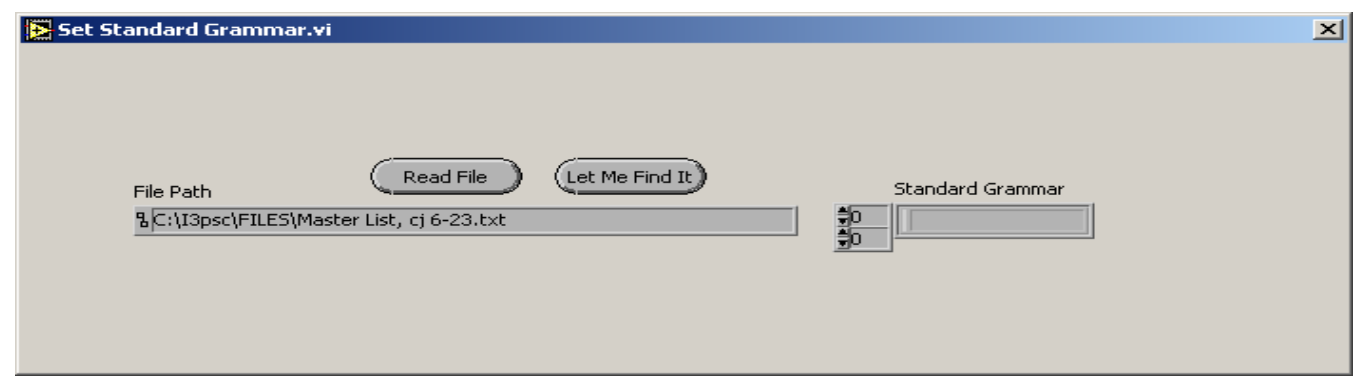

Figure A-7 Set Standard Grammar File

If the standard grammar file is stored in the default path shown in the file path controller, click "Read File" button will load the file into your setup. Otherwise, you need click "Let Me Find It" button to search and load the file. 


\begin{tabular}{|c|c|c|c|c|c|c|c|c|c|c|c|c|}
\hline standard gran & description & default metric & default metric & default British & default British & Planner & Controller & Plant & Virtual Senso & Fusion & Monitor & Expert \\
\hline \multicolumn{13}{|l|}{ INPUTS } \\
\hline coke in charg & weight of cok & 4.672 & $\mathrm{~kg}$ & 10.3 & $\mathrm{Ibm}$ & -- & -- & - & -- & -- & -- & -- \\
\hline coke ratio & weight of cok & 10 & $\%$ & 10 & $\%$ & coke ratio & coke ratio & CMR SP & -- & -- & -- & coke ratio \\
\hline cupola diame & diameter of $\mathrm{th}$ & 0.559 & $m$ & 22 & in & -- & -- & -- & -- & -- & -- & -- \\
\hline cupola well di & diameter of th & 0.457 & $m$ & 18 & in & -- & -- & -- & -- & -- & -- & -- \\
\hline blast rate & volume of air & 0.1339 & $\mathrm{~m}^{\wedge} 3 / \mathrm{s}$ at $0 \mathrm{C}$ & 300 & scfm at $60 \mathrm{~F}$ & blast rate & blast rate & Blast Rate RF & - & -- & -- & blast rate \\
\hline Blower Freq. & volume of air & 0.1339 & $m^{\wedge} 3 / s$ at $0 C$ & 300 & scfm at $60 \mathrm{~F}$ & -- & -- & Blower Freq. & -- & -- & -- & -- \\
\hline pressure drop & pressure drop & 0.1339 & $\mathrm{~m}^{\wedge} 3 / \mathrm{s}$ at $0 \mathrm{C}$ & 300 & scfm at $60 \mathrm{~F}$ & -- & -- & Blast Rate & -- & -- & -- & -- \\
\hline oxygen additi & volume of axy & 0.003125 & $\mathrm{~m}^{\wedge} 3 / \mathrm{s}$ at $0 \mathrm{C}$ & 7 & scfm at $60 \mathrm{~F}$ & O2_Enrich & -- & O2 Enrich & -- & -- & -- & -- \\
\hline O2 Flow Rate & volume of axy & 0.003125 & $m^{\wedge} 3 / s$ at $0 C$ & 7 & scfm at $60 \mathrm{~F}$ & -- & -- & O2 Flow Rate & -- & -- & - & -- \\
\hline blast tempera & temperature & 699.82 & $\mathrm{~K}$ & 800 & $\mathrm{~F}$ & -- & -- & Blast Temp & -- & -- & -- & -- \\
\hline blast fraction & fraction of bla & 90 & $\%$ & 90 & $\%$ & -- & -- & -- & -- & -- & -- & -- \\
\hline actual BR & actual volume & 0.1339 & $m^{\wedge} 3 / s$ at $0 C$ & 300 & scfm at $60 \mathrm{~F}$ & - & -- & actual BR & actual BR & - & actual BR & - \\
\hline total oxygen if & percent oxyge & 22.7 & $\%$ & 22.7 & $\%$ & - & oxygen additi & O2 RP & -- & -- & -- & -- \\
\hline Time & time correspo & 7:00:38 & AM & 7:00:38 & AM & -- & -- & Time & -- & -- & -- & -- \\
\hline & & & & & & & & & & & & \\
\hline \multicolumn{13}{|l|}{ OUTPUTS } \\
\hline Offgas CO & amount of cal & 12.746 & mole $\%$ & 12.746 & mole $\%$ & -- & -- & $\mathrm{CO} \%$ & -- & -- & Offgas C0 & -- \\
\hline combustion e & amount of CQ & 52.8 & $\%$ & 52.8 & $\%$ & - & combustion $\mathrm{e}$ & $\% \mathrm{CE}$ & -- & - & $\%$ CE & -- \\
\hline melt rate & amount of iro & 19.541 & tonne/hr & 21.4951 & ton/hr & melt rate & melt rate & Melt Rate & Melt Rate & Melt Rate & melt rate & melt rate \\
\hline Kalman MR & amount of iro & 19.541 & tonne/hr & 21.4951 & ton $/ \mathrm{hr}$ & -- & -- & -- & Kalman MR & -- & -- & -- \\
\hline Pyrometer Te & metal temper & 2019.907 & $\mathrm{~K}$ & 3177.8326 & $\mathrm{C}$ & Pyro._Temp & tap temp & Pyro._Temp & Pyro._Temp & Pyro._Temp & tap temp & metal temper \\
\hline \begin{tabular}{|l|} 
Kalman PT \\
\end{tabular} & metal temper & 2019.907 & $\mathrm{~K}$ & 3177.8326 & $\mathrm{C}$ & -- & -- & \begin{tabular}{|l|l|}
-- \\
\end{tabular} & Kalman PT & & -- & -- \\
\hline 2nd Pyromete & metal temper & 2019.907 & $\mathrm{~K}$ & 3177.8326 & C & 2nd Pyromet & 2nd Pyromete & 2nd Pyromete & 2nd Pyromete & 2nd Pyromete & 2nd Pyromete & 2nd Pyromete \\
\hline Datacast Tem & metal temper & 2020.907 & $\mathrm{~K}$ & 3179.6326 & $F$ & Bath Temp & -- & Bath Temp & Bath Temp & -- & Bath Temp & -- \\
\hline Spout Tempe & metal temper & 2019.907 & $\mathrm{~K}$ & 3177.8326 & C & Spout Temp. & Spout Temp. & Spout Temp. & -- & Spout Temp. & Spout Temp. & Spout Temp. \\
\hline Final Carbon & amount of can & 3.69 & $\%$ & 3.69 & $\%$ & $\% \mathrm{C}$ & $\% \mathrm{C}$ & $\% \mathrm{C}$ & $\% \mathrm{C}$ & -- & $\% \mathrm{C}$ & Final Carbon \\
\hline Melt Rate 2 & amount of iro & 19.541 & tonne/hr & 21.4951 & ton/hr & -- & Melt Rate 2 & Melt Rate 2 & Melt Rate 2 & Melt Rate 2 & Melt Rate 2 & Melt Rate 2 \\
\hline metal temper & metal temper & 2019.907 & $\mathrm{~K}$ & 3177.8326 & $\mathrm{C}$ & - & tap temp & Pyro._Temp & Pyro._Temp & Pyro._Temp & tap temp & metal temper \\
\hline Final Carbon & amount of can & 3.69 & $\%$ & 3.69 & $\%$ & -- & $\% \mathrm{C}$ & $\% \mathrm{C}$ & $\% \mathrm{C}$ & $\% \mathrm{C}$ & $\% \mathrm{C}$ & Final Carbon \\
\hline radar level & level & 6 & Foot & & radar & -- & radar & radar & radar & radar & radar & radar \\
\hline Cupola Exit & metal temper & 2019.907 & $\mathrm{~K}$ & 3177.8326 & C & -- & -- & Cupola Exit & -- & -- & Cupola Exit & -- \\
\hline Cupola Press & pressure drop & 0.1339 & $\mathrm{~m}^{\wedge} 3 / \mathrm{s}$ at $0 \mathrm{C}$ & 300 & scfm at $60 \mathrm{~F}$ & -- & -- & Cupola Press & -- & - & Cupola Press & -- \\
\hline Manual Melt & amount of iro & 19.541 & tonne/hr & 21.4951 & ton/hr & u24:Melt rate & Manual Melt & Manual Melt H & Manual Melt F & Manual Melt H & Manual Melt F & Manual Melt \\
\hline
\end{tabular}

Figure A-8 Example of Standard Grammar 


\section{A.1.1.2 Create/Modality Standard Grammar}

The Standard Grammar can be created or modified using this function. Double click on "Create/Modality Standard Grammar" in Setup Menu (Figure A-6) will open the dialog shown in Figure A-9 clicking on button "Create New" will open the dialog (Figure A-10) to create a new standard grammar. Clicking on button "Modify Existing" will open the dialog in Figure A-11 to modify the existing standard grammar.

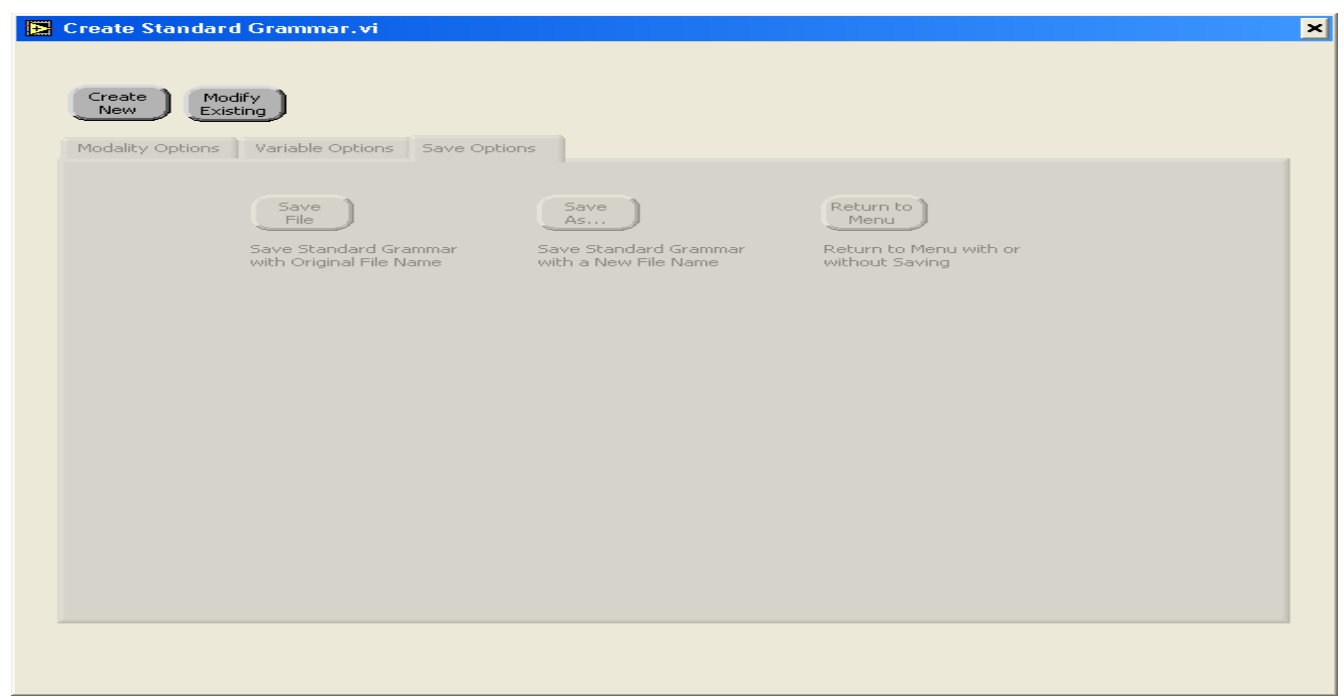

Figure A-9 Create/Modify Standard Grammar

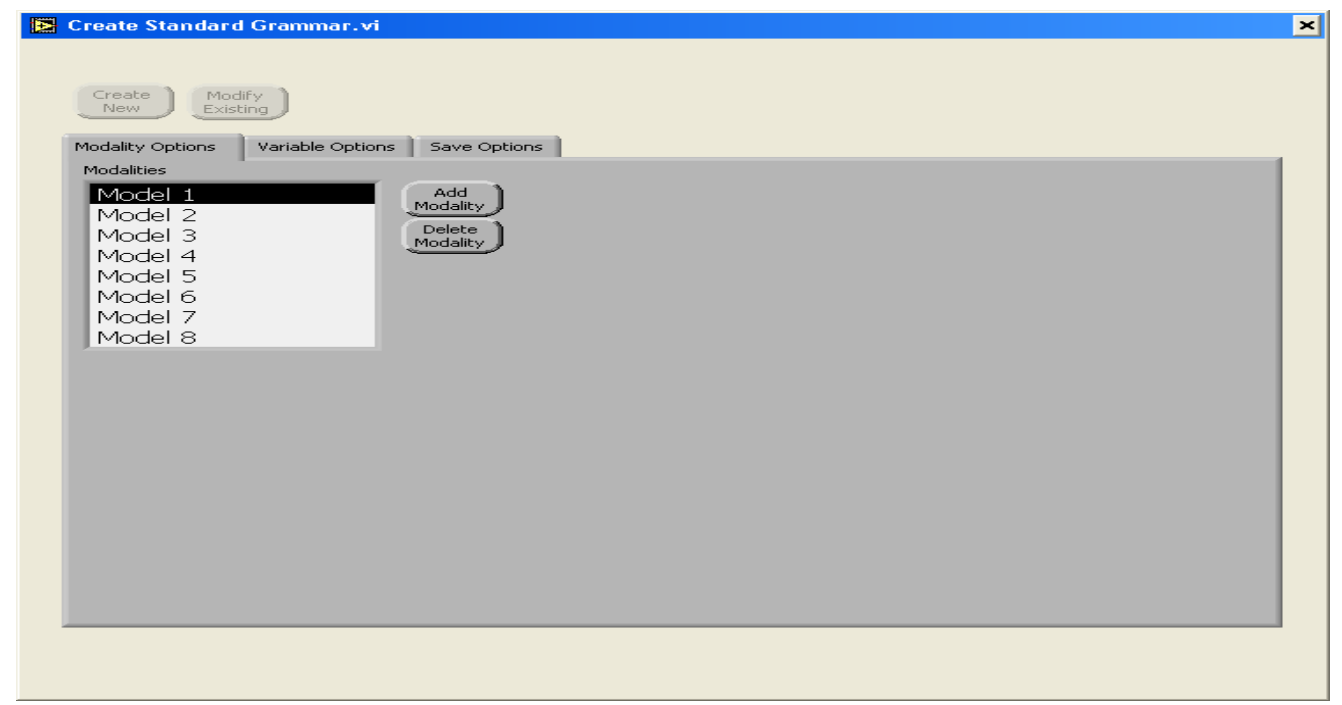

Figure A-10 Create New Standard Grammar 


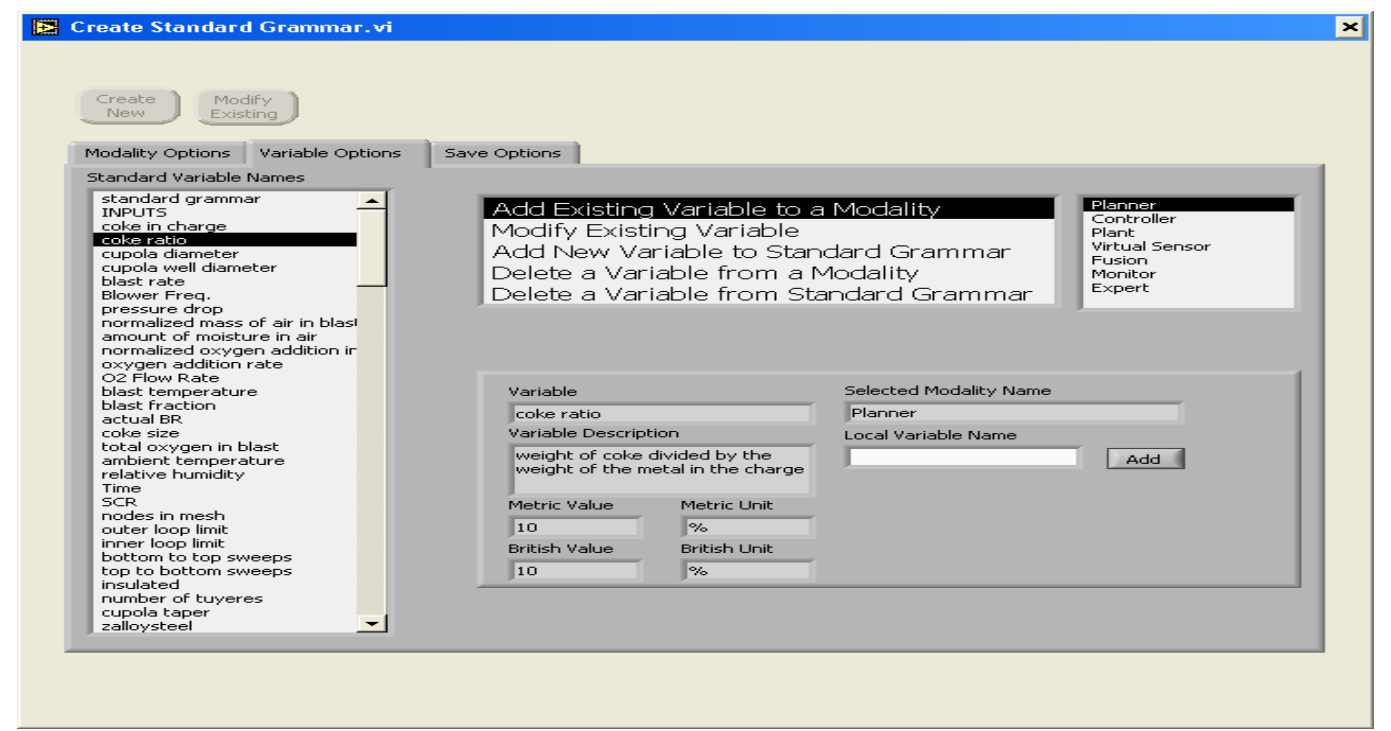

Figure A-11 Modify Existing Standard Grammar

There are three same tabs on these two dialogs. On the "Modality Options" tab, the user can create a new modality or delete an existing modality. On the "Variables Options" tab, the user can create a new variable or delete an existing variable in a modality. On the "Save Options" the user can exit this function with or without saving the new or modified standard grammar.

\section{A.1.1.3 Select Modalities \& Variable \& Interface}

In this option, the user can select the modalities that need to be included in the $I^{3}$ PSC system. Also the variable and the interface related to the modalities can be selected. The interface of Select Modalities \& Variable \& Interface is in Figure A-12. All modalities appearing in the Standard Grammar File appear in the Modalities window. Once the modalities are selected, they become available for the creation of modality groups. The variables in the modality can also be selected. If that variable exists in other modalities, they are also automatically selected. For each modality, the modality interface VI path is also selected. This interface VI associated with that modality will be called when I ${ }^{3}$ PSC starts running. Click "Continue" button to close the window after selecting the modalities, variables and interfaces. 


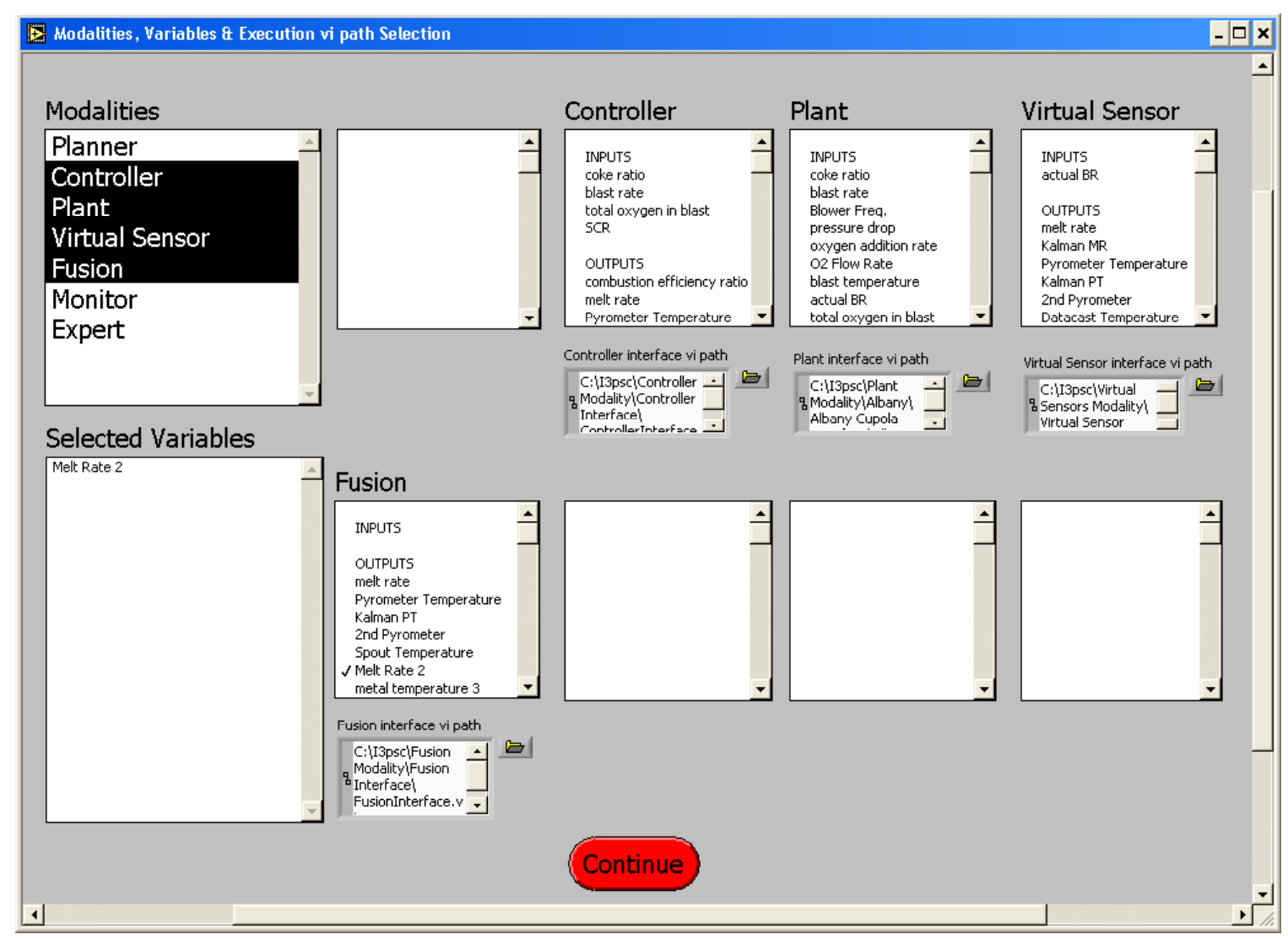

Figure A-12 Modalities, Variables and Interfaces Selection

\section{A.1.1.4 Select Variable Properties}

The user can select variable properties in the dialog shown in Figure 5. Highlight the properties in the parameter list to be added to the data, and then click on "Add to List" button. The "Return to Menu" button will close this dialog.

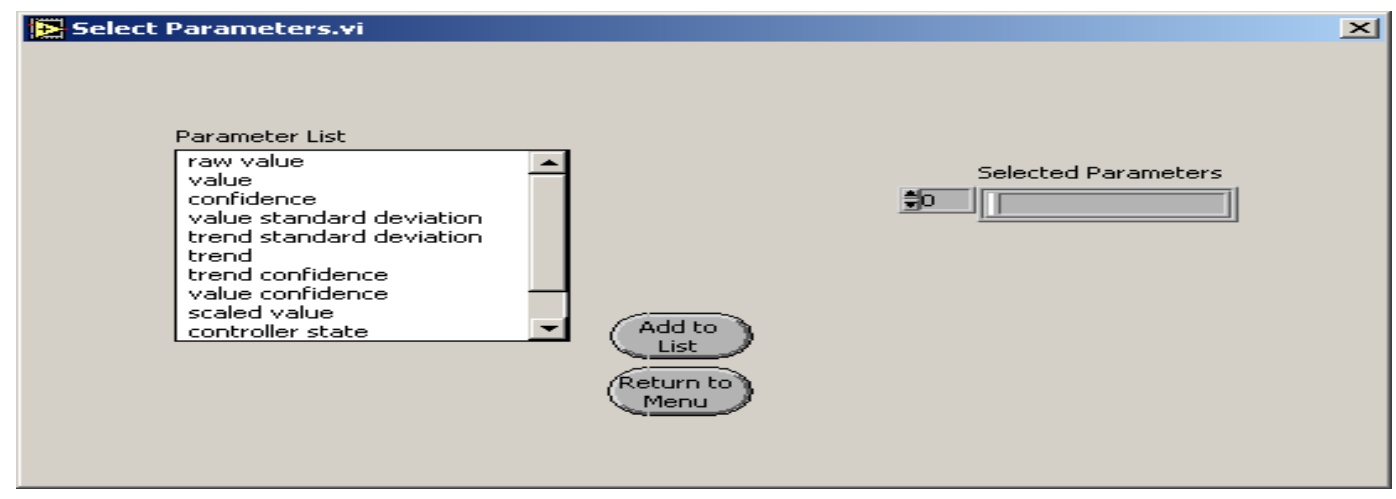

Figure A-13 Select Variable Properties 


\section{A.1.1.5 Save Setup Information}

After you select the modalities, variables and interface for this system in Figure A-12, you should save the setup. These setup information will be used in the following Modality Setup discussed here later. Just double click on the Save Setup Information on Setup Menu (Figure A-6) to save the setup information..

\section{A.1.1.6 Modality Setup}

Once all the modalities needed for system are selected, the Modality Setup option (Figure A-6.) allows you to set up various properties of these modalities like defining groups of the modality (e.g. multiple fusion groups), defining properties of variables in the modality, etc. The Modality Setup window is shown in Figure A-14.

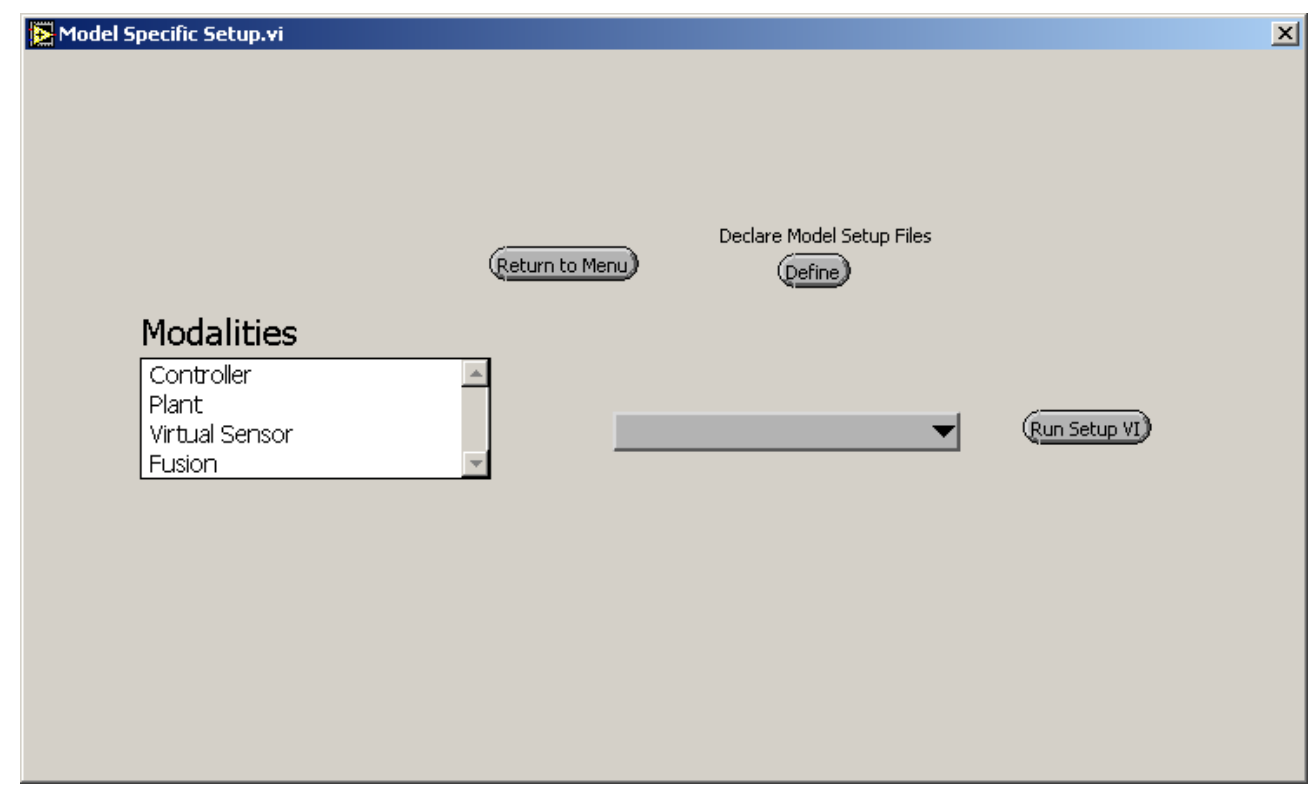

Figure A-14 Modality Specific Setup

In this window, the "Modalities" indicator shows all the modalities which you've already selected previously.

The following steps are taken to setup the modalities:

1. Click on "Define" button to Declare Model Setup File (section A.1.1.6.1) 
2. Select the modality setup VI and then click "Run Setup VI" (section A.1.1.6.2) to run the modality setup VI. For each modality several setup VIs need to be run.

Table 1 shows the Setup VIs that need to be run for each modality.

3. The "Return to Menu" button will exit modality setup and close the dialog in Figure A-14.

Table 1. Setup VI s of Modalities

\begin{tabular}{|l|l|l|l|l|l|l|}
\hline Setup VIs & $\begin{array}{l}\text { Planner } \\
\text { Modality }\end{array}$ & $\begin{array}{l}\text { Controller } \\
\text { Modality }\end{array}$ & $\begin{array}{l}\text { Plant } \\
\text { Modality }\end{array}$ & $\begin{array}{l}\text { Virtual } \\
\text { Sensor }\end{array}$ & $\begin{array}{l}\text { Fusion } \\
\text { Modality }\end{array}$ & $\begin{array}{l}\text { Monitor } \\
\text { Modality }\end{array}$ \\
\hline Modality groups.vi & $\mathbf{X}$ & $\mathbf{X}$ & $\mathbf{X}$ & $\mathbf{X}$ & $\mathbf{X}$ & $\mathbf{X}$ \\
\hline SensorParameters.vi & & & $\mathbf{X}$ & $\mathbf{X}$ & $\mathbf{X}$ & \\
\hline Planner setup.vi & $\mathbf{X}$ & & & & & \\
\hline Monitor setup.vi & & & & & & $\mathbf{X}$ \\
\hline Charge setup.vi & & $\mathbf{X}$ & & & & \\
\hline Controller setup.vi & & $\mathbf{X}$ & & & & \\
\hline
\end{tabular}

\section{A.1.1.6.1 Declare Model Setup File}

Click "Declare Model Setup File" button on the Modality Specific Setup dialog (Figure A-14) will open the dialog shown in Figure A-15. The VIs that perform the modality setup are defined here. The setup VIs required by every modality are list in Table 1. Each VI's are selected by clicking the "Look Up" button. The names of these VI's will be appeared in the pull-down menu in the Modality Specific Setup dialog (Figure A-14).

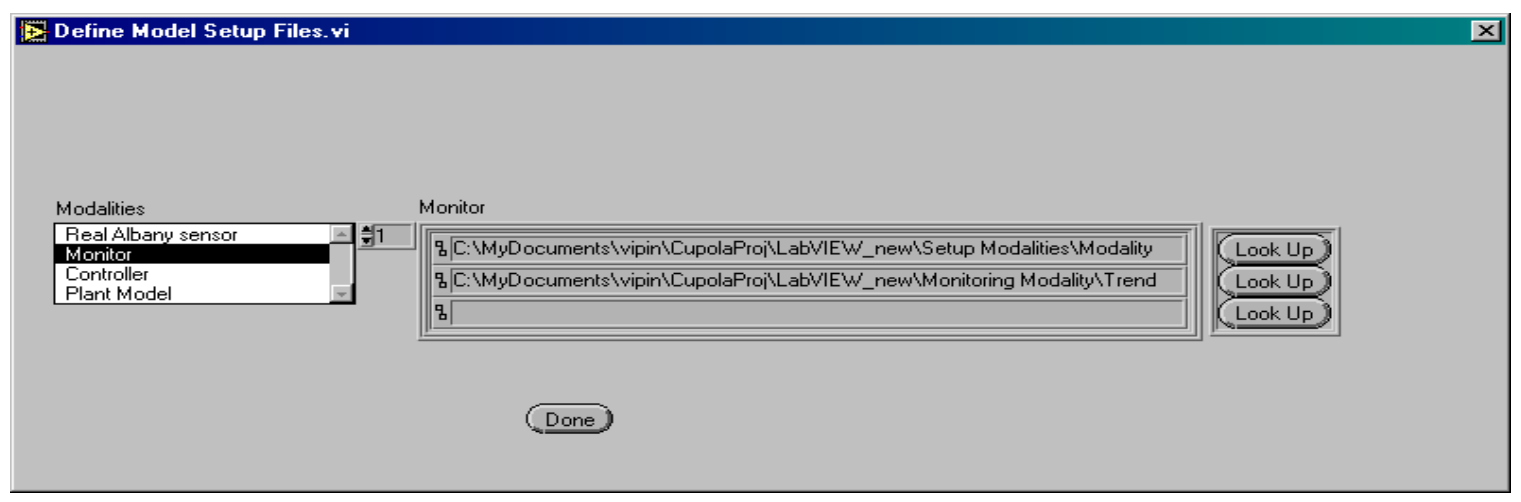

Figure A-15 Declare Model Setup File

\section{A.1.1.6.2 Run Setup VI}

Click "Run Setup VI" button on the Modality Specific Setup dialog (Figure A-14) will run the modality setup VI selected in the list box. 
As shown in Table 1, Modality Groups VI and Sensor Parameters VI are two main modality setup VIs that are required by most of the modalities. Modality Groups.vi is used to define groups in a modality and to delete or update the groups in the modality. Sensor Parameters.vi is used to setup the parameters of the sensors. These two setup procedures will be introduced in the following section A.1.1.6.2.1 and section A.1.1.6.2.2. The rest setup VIs will also be introduced in section A.1.1.6.2.3.

\section{A.1.1.6.2.1. Modality Groups}

The dialog of Modality Groups.vi is shown in Figure A-16.

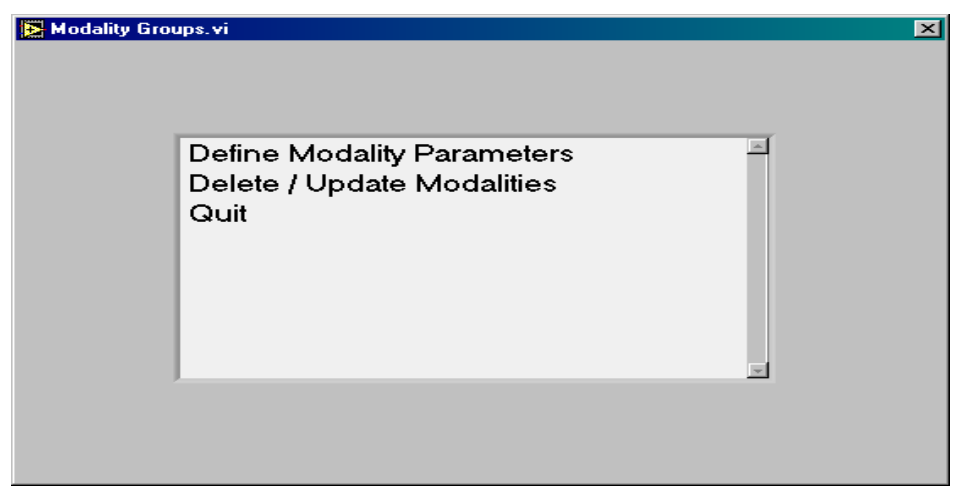

Figure A-16 Modality Groups Main Menu.

Two functions are offered in this dialog, namely Define Modality Parameters and Delete/Update Modalities. Define Modality Parameters can define a new group in the modality and Delete/Update Modalities can modify the existing group in a modality.

\section{A.1.1.6.2.1.1 Define Modality Parameters}

Double clicking the "Define Modality Parameters" option opens an dialog shown in Figure A-17. This dialog allows the user to add variables that form the inputs and outputs of the modality and add them as a group and also to create multiple such groups. The user has to select the variable, its associated modality and property and then click the "Add Variable" button to add that "node" to the modality. Once all the input and output variables are added, this set is classified as a "Modality Group". 


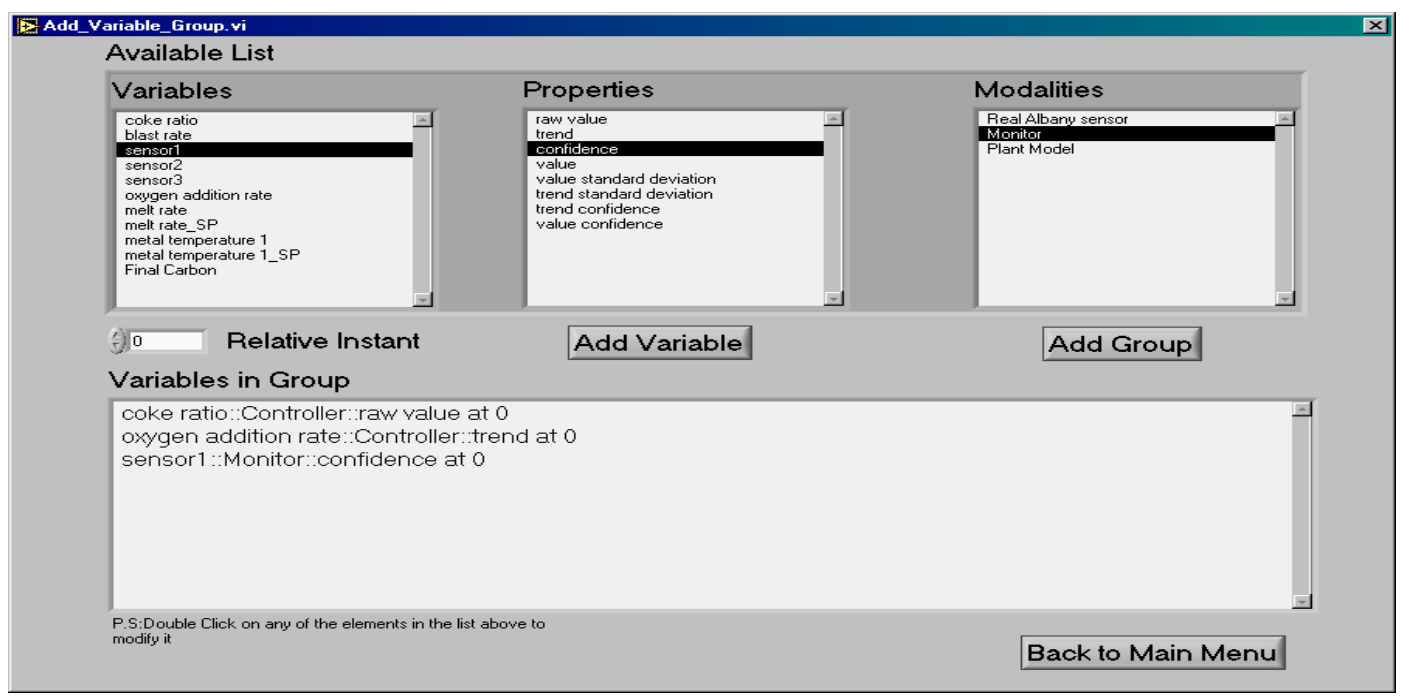

Figure A-17 Select Variables in Group

NOTE:

Whenever you add a node with "value" property, a node which has "trend" property is added automatically. If you only need "value" property node you can delete the "trend" property node. The procedure of deleting a node is double clicking "Delete / Update Modalities" in dialog shown in Figure A-16. Then click the button on each popped up dialogs in the following order: "Modify Modality" (as shown in Figure A-22), "Change Variable List" (as shown in Figure A-23). Double clicking on the variable, you can "Delete" it on the dialog in Figure A-24.

Clicking on the "Add Group" button in Figure A-17 dialog will open another dialog as shown in Figure A-18. This interface allows the user to split all the variables selected in the modality into input and output variables.

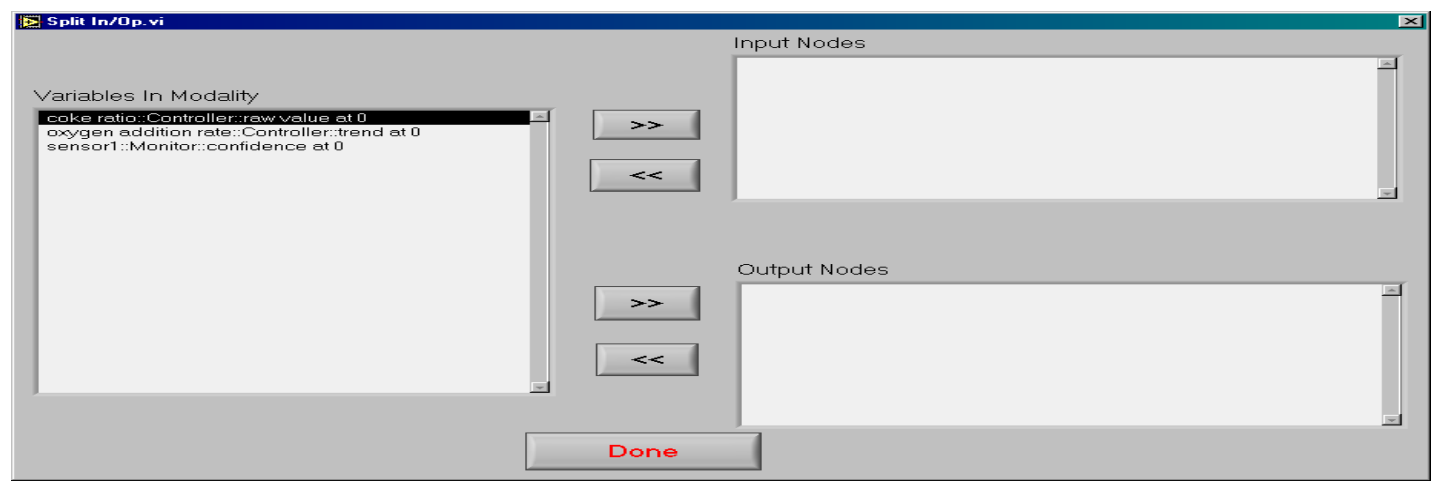

Figure A-18 Split Variables into Input and Output Variables. 
Once "Done", a dialog to name the created group appears. This dialog also allows the parameters of the group to be added and the path of the engine VI to be defined. This dialog is as shown in Figure A-19.

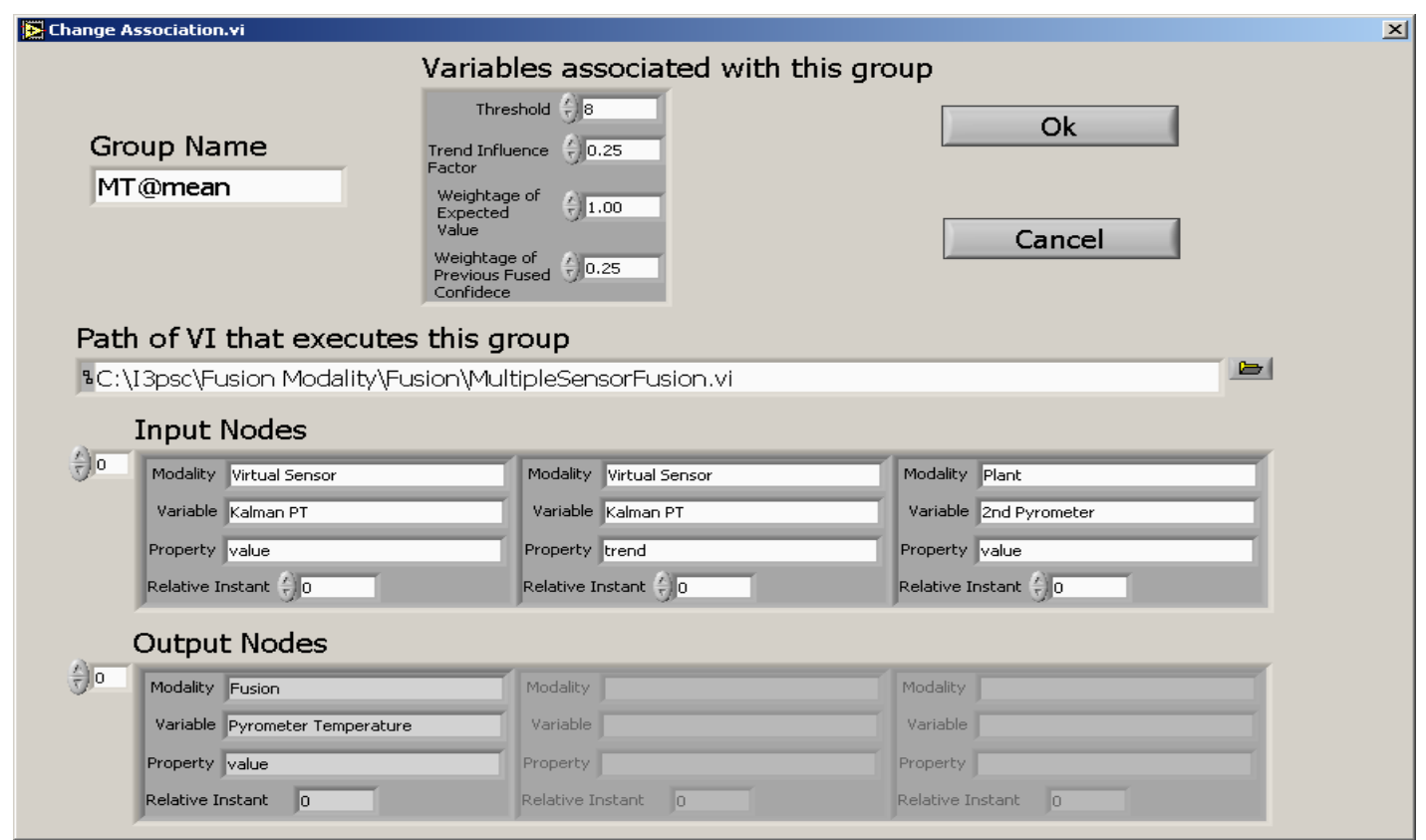

Figure A-19 Add Group Parameters

In this dialog, the user can name a group, define the path of the engine VI, and give the variables associated with that group. The detail explanations are as follows:

1. While defining the group name for a fusion modality group, a suffix is attached to indicate what kind of Standard Deviation (STD) value will be used estimating the fusion value. The format is "GroupName@max". "@max" means using the maxim STD value. “@min” means minimum STD value and “@mean” means the average STD value. The default mode is "mean".

2. Each modality can be executed on different computer. The computer is identified by IP address. In the "Path \& IP of VI that executes this group" control, you can enter the IP address of the computer and the path of that engine VI on that computer. The format is Path::IP. For example, if the engine VI is on machine 149.149.0.1 and its path is $\mathrm{C}: \mathrm{I}^{3} \mathrm{PSC} \backslash$ Fusion $\backslash$ Modality $\backslash$ Fusion $\backslash$ MultipelSensor Fusion.vi, the path will be $\mathrm{C}: \mathrm{I}^{3} \mathrm{PSC} \backslash$ Fusion Modality $\backslash$ Fusion $\backslash$ MultipelSensor Fusion.vi::149.149.0.1. 
3. While defining the group in this modality, the variables associated with this group will be given. These variables are Threshold, Trend Influence Factors, Weight of Expected Value, and Weight of Previous Fused Confidence. Threshold means the number of points to ignore at the beginning of run. Trend Influence Factor is a number of 0-1 that determines how much the trend effects the confidence calculation. Weight of Expected Value is a weight factor that determines the effect of expected value calculated using previous fused value and trend on the fused results. Weight of Previous Fused Confidence is a factor from 0 to 1 that determines the effect of previous fused confidence over the current confidence.

Once all the parameters are added, click on "OK" button to add this group to the modality. The dialog in Figure A-17 will show back for adding more groups.

\section{A.1.1.6.2.1.2 Delete/Update Modalities}

Click "Delete / Update Modalities" in Figure A-16. The dialog as shown in Figure A-20 is popped up to delete or update the details in the existing modality groups.

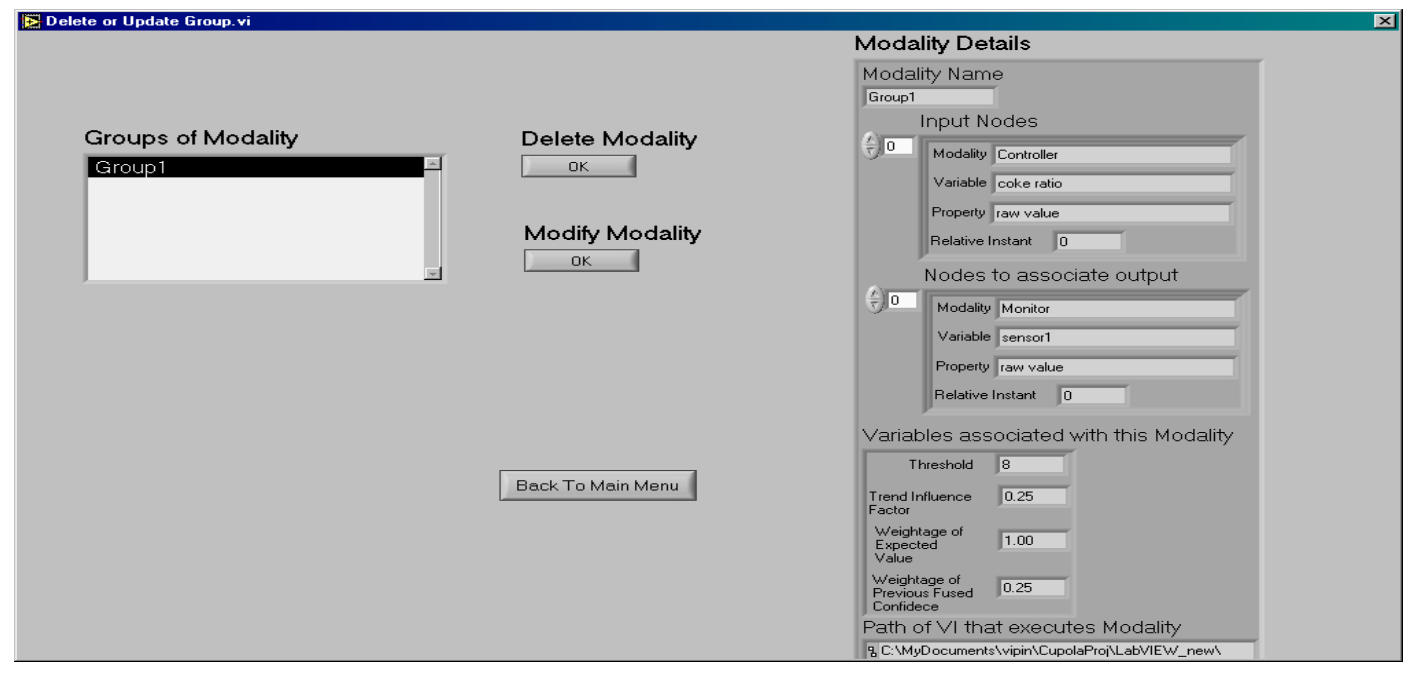

Figure A-20 Delete / Modify Modality

The "Groups of Modality" list box on the left of the screen lists all the groups of the modality created earlier. Selecting one of them, displays the details of that modality group in the "Modality Details" indicator. The user is provided with an option of either deleting the group as such ("Delete Modality") or modifying the contents of the group ("Modify Modality"). If click "Delete Modality" an alert confirmation dialog is popped 
up to request the user to confirm whether he wants to delete the group. The alert dialog is as shown in Figure A-21.

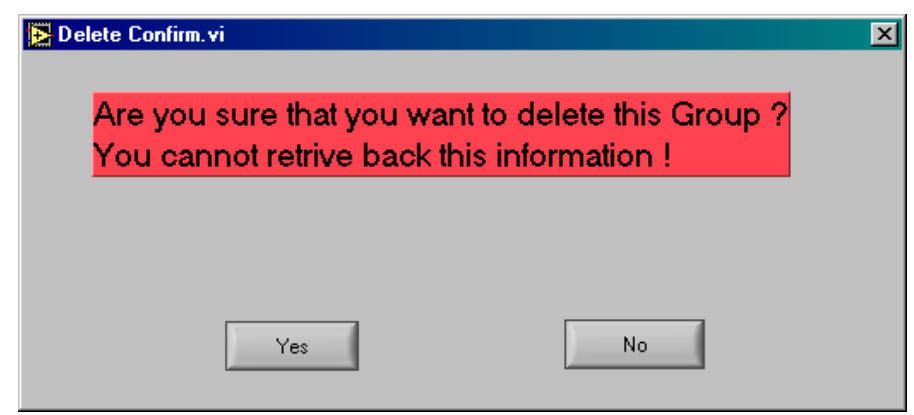

Figure A-21 Delete Confirmation Alert

"Modify Modality" option allows the user to change the details of the modality groups. The dialog of "Update Group" is as shown in Figure A-22.

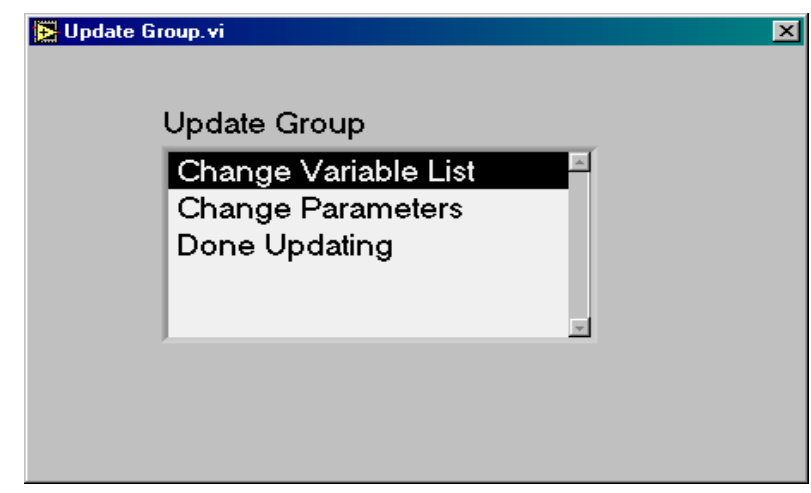

Figure A-22 Update Modality Group Details

The "Change Variable List" option allows the user to change the variable list of the group. The interface is shown in Figure A-23. Double clicking on any of the variables in the list opens an interface as shown in Figure A-24 which allows the user to change the variable.

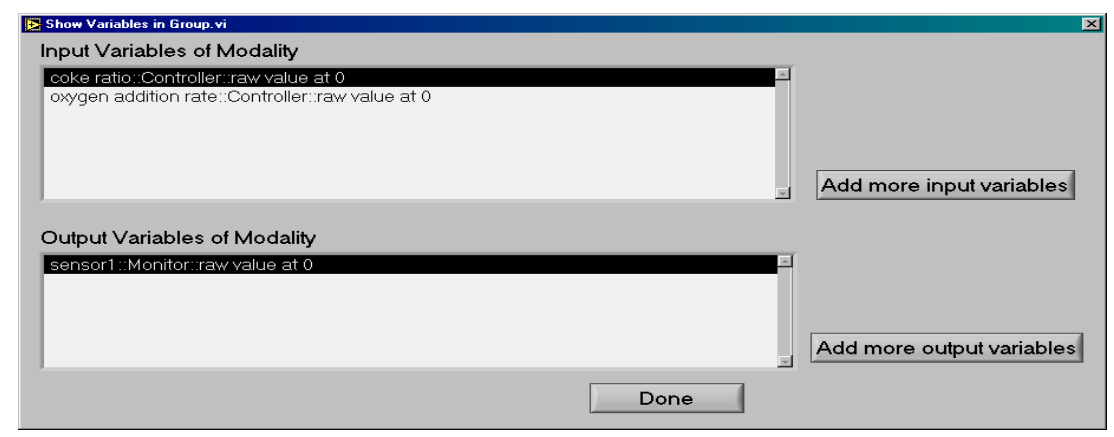

Figure A-23 Change Variable List 


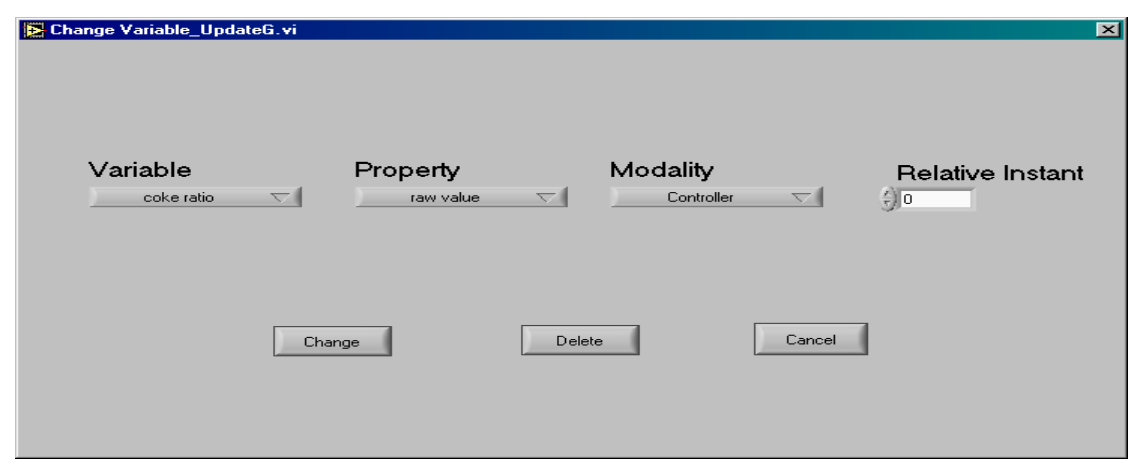

Figure A-24 Change Variable

In case the user wants to add more variables to either the input or the output of the modality, then the user clicks the "Add more input variables" or "Add more output variables" button in Figure A-23. The dialog in Figure A-17 is shown again to add the input/output variables.

When the user selects the "Change Parameters" option in Figure A-22., an interface as shown in Figure A-25 appears. This interface allows the user to change the parameters associated with the Modality Group. The contents in this dialog are same as the contents in Figure A-19.

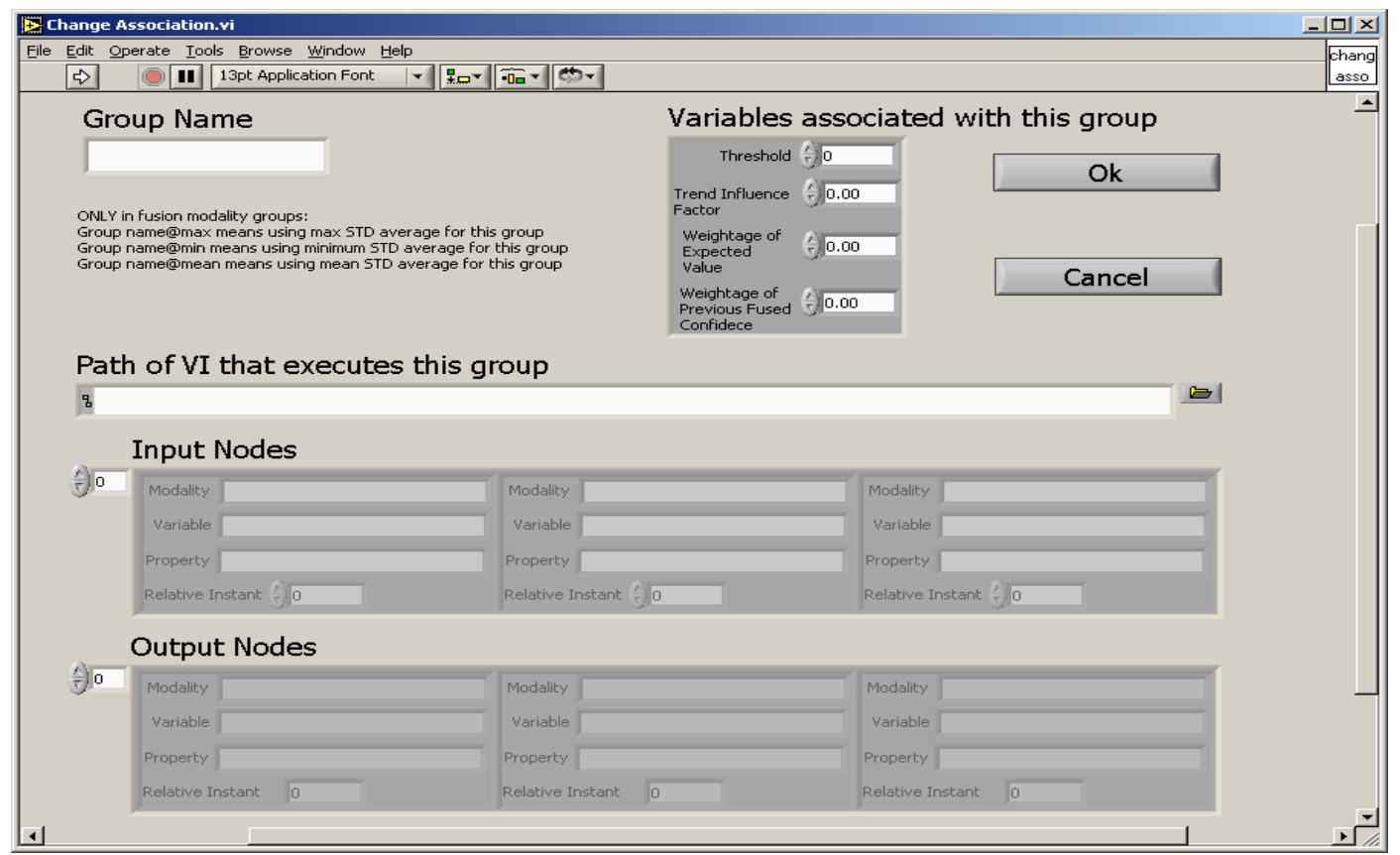

Figure A-25 Change Parameters of Modality Group 


\section{A.1.1.6.2.2. Sensor Parameters Setup}

Sensors monitoring the plant are subjected to self-validation tests to ensure the reliability of the data being read by them. These self-validation tests require the creation of fuzzy FIS files. Also other sensor parameters such as Standard Deviation, etc need to be defined for each sensor. The "Sensor Parameters.vi" is programmed for assigning such sensor parameters. The dialog for setting up sensor parameters is as shown in Figure A-26.

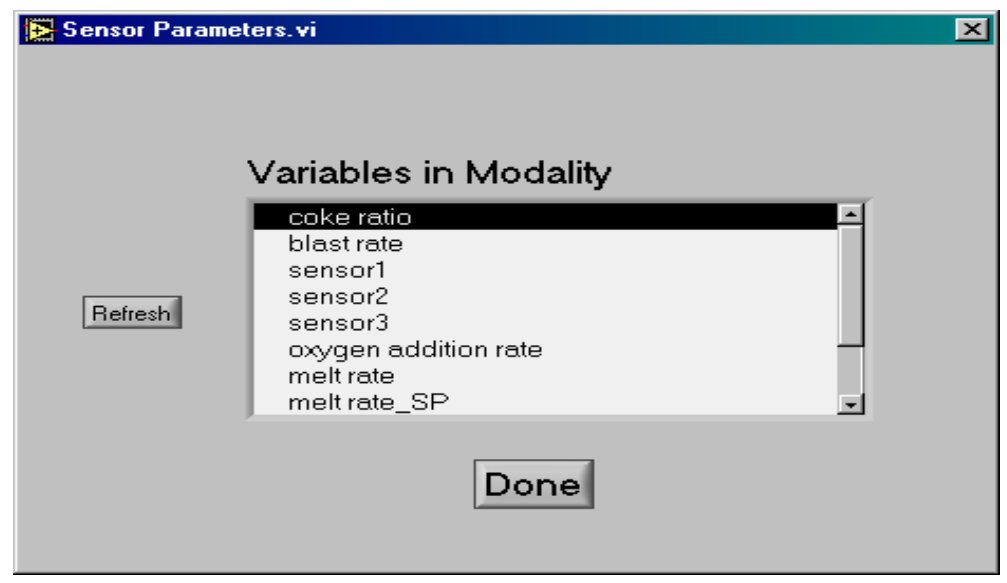

Figure A-26 Sensor Parameters Setup

The "Variables in Modality" list box shows the list of sensors in the modality. To setup the parameters, double clicking on any of the variables. This opens a dialog as shown in Figure A-27.

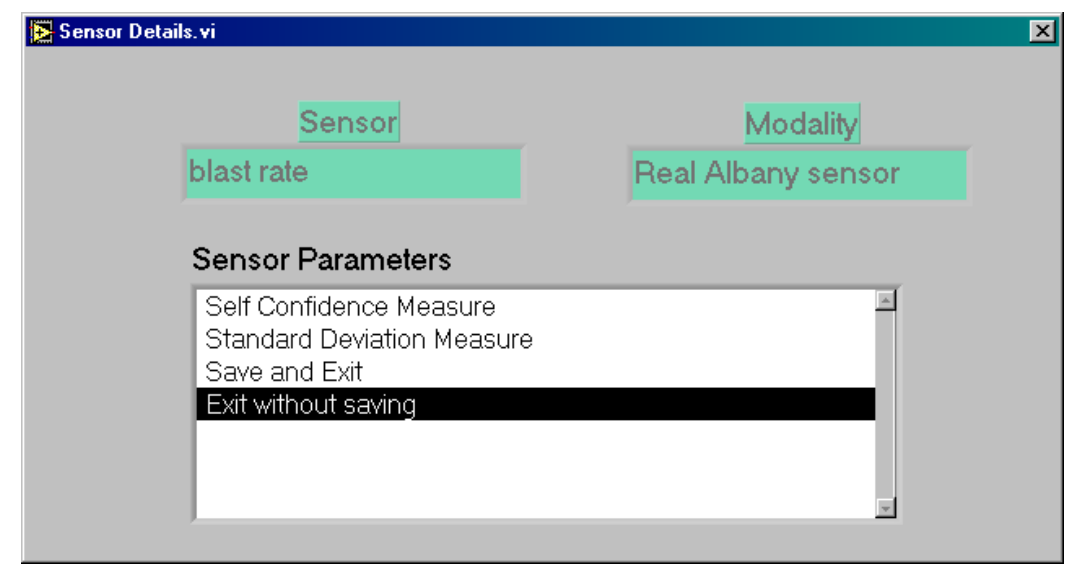

Figure A-27 Sensor Parameters Interface

Clicking on the "Self-Confidence Measure", a dialog shown in Figure A-28 allows the user to set up self-validation by creating FIS file or by assigning self- 
confidence. If the user chooses to create a FIS file a dialog as shown in Figure A-29 appears. The user can create the FIS file.

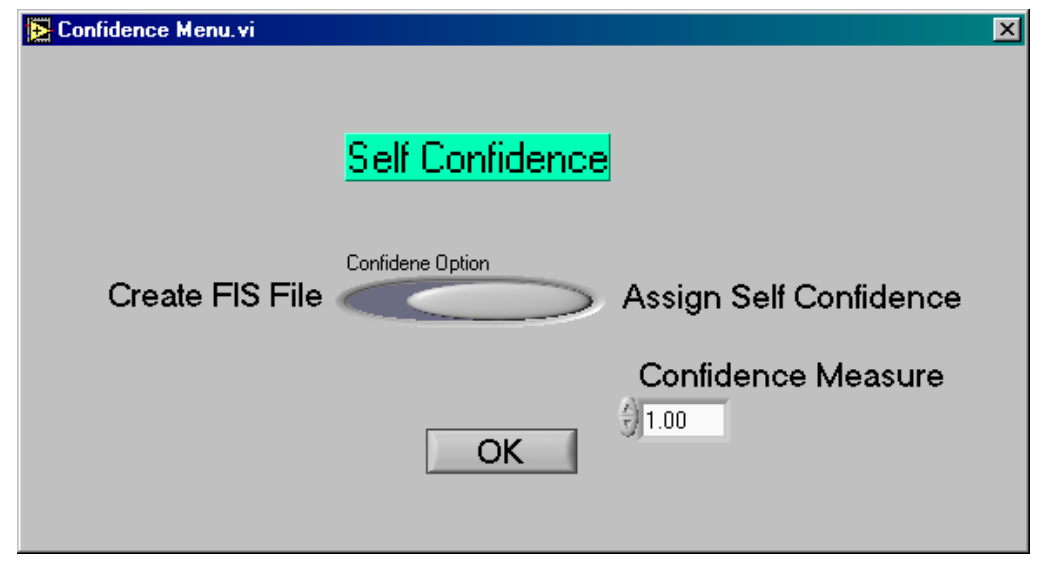

Figure A-28 Sensor self-confidence menu

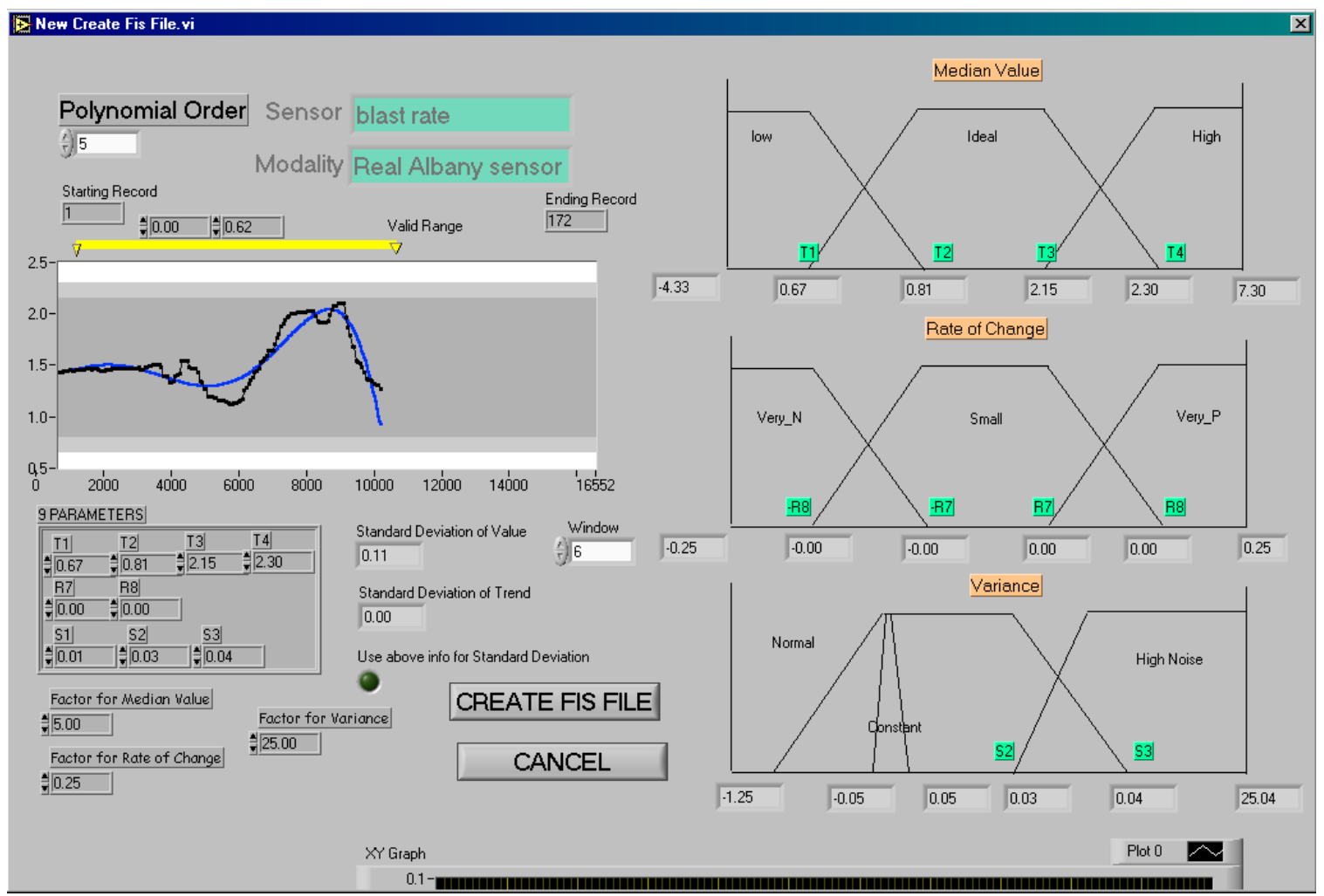

Figure A-29 Create Self-validation Fuzzy FIS File

Double-clicking the "Standard Deviation Measure" in Figure A-27, a dialog, as shown in Figure A-30, is opened to calculate the standard deviation values from history data or to assign the standard deviation value. 


\section{Standard Deviation}

From Historical Data

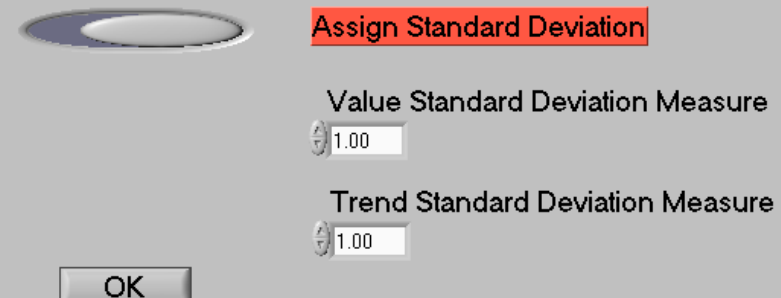

Figure A-30 Standard Deviation Option Menu

If the user selects to calculate the Standard Deviation from historical data, a dialog as shown in Figure A-31 appears for calculating the standard deviation.

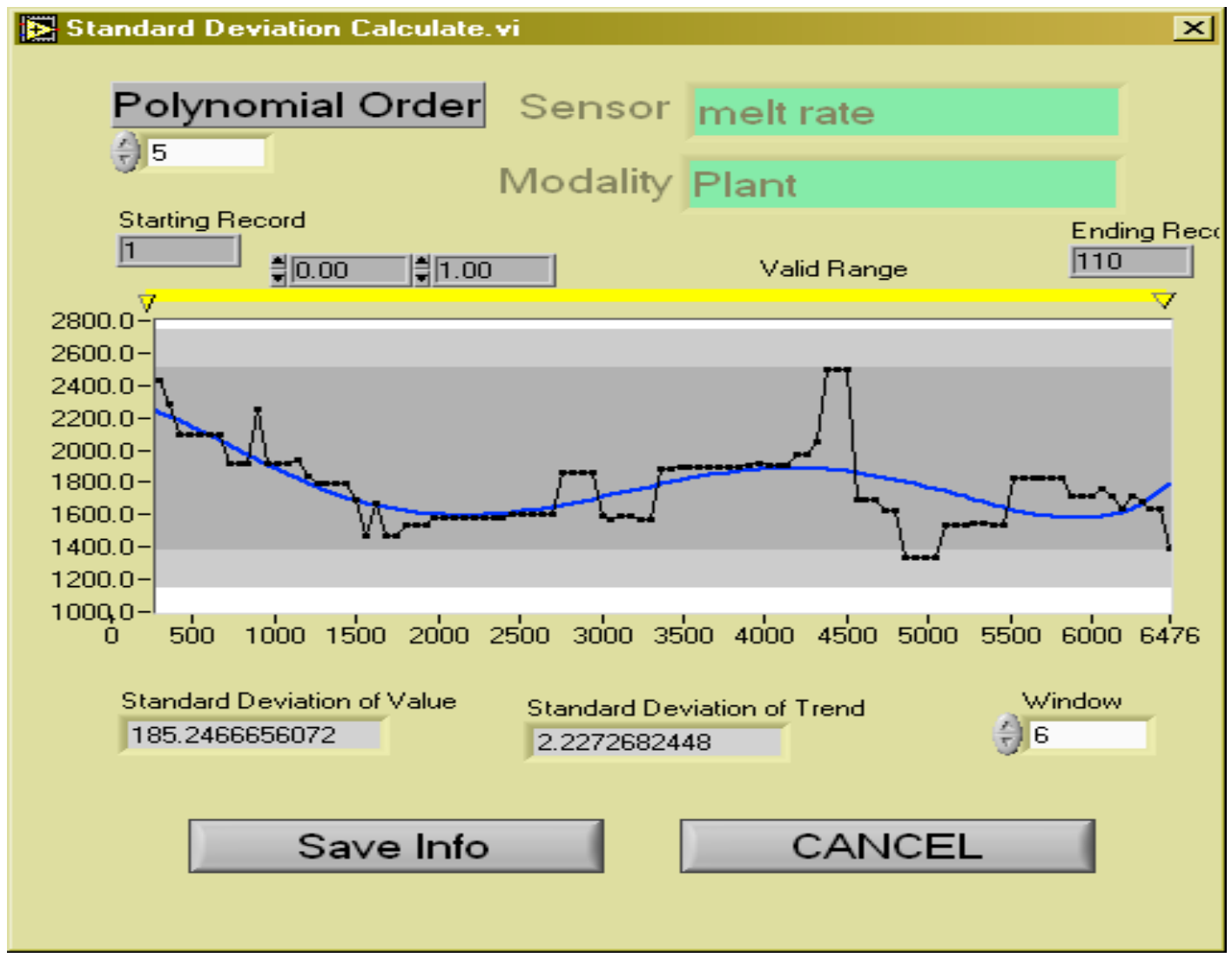

Figure A-31 Standard Deviation Calculation

\section{A.1.1.6.2.3. Other Specific Setup}


There are three more modality setup VIs, namely trend monitor setup, charge setup, and planer setup which are used to setup the specific modalities.

\section{A.1.1.6.2.3.1 Trend Monitor Setup}

The Trend Monitor engine requires some parameters for its operation. The "Trend Monitor setup.vi" is programmed for assigning such parameters needed for calculating the trend. The dialog of Trend Monitor Setup VI is as shown in Figure A-32.

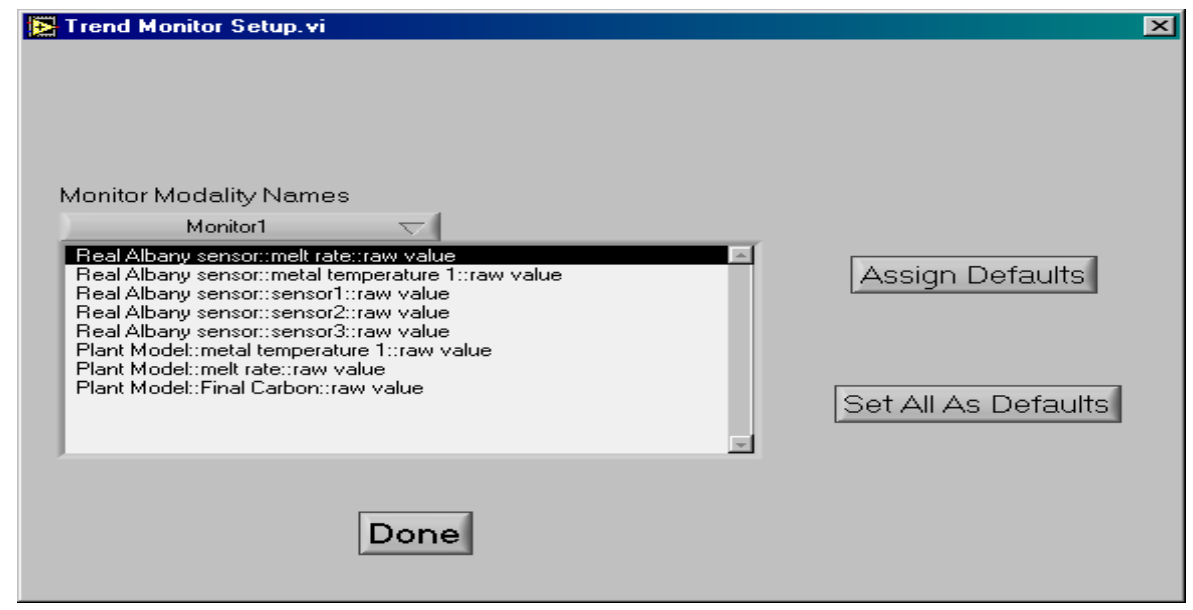

Figure A-32 Interface for setting up trend monitor parameters

The interface shows the list of all groups and the variables in a group of the trend monitor modality. All groups of this modality are listed in the menu ring "Monitor Modality Names". When a particular group is selected all variables in that group get listed in the list box below the menu ring. Double-clicking on any one of the variables, the dialog in Figure A-33 is shown. The user can setup parameters of the trend monitor engine. The window length indicates the number of sample points to be used to calculate the trend. Slow changing variables can be assigned a longer window length. The Trend Monitor also provides with an option wherein it throws an alert when certain trends are encountered. The "Available Cases" list box lists all the possible trend cases and allows the user to add cases to be watched out by using the "Add" and "Remove" button. The added cases appear in the "Cases to be watched out for". The "Preview" shows the trend of the variable for the case selected. 


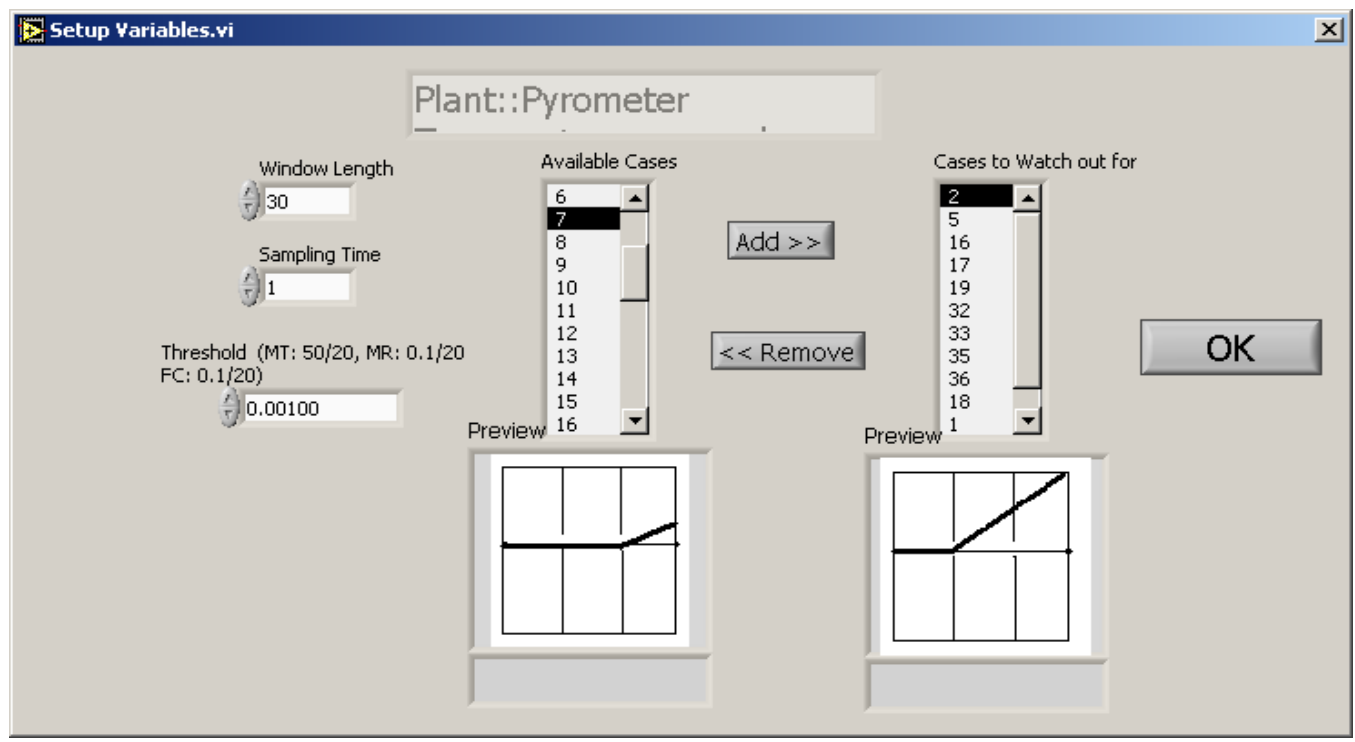

Figure A-33 Assign Parameters for trend monitor

The "Assign Defaults" button in the Figure A-32 dialog allows the user to setup the default parameters. The interface is exactly the same as shown in Figure A-33.

\section{A.1.1.6.2.3.2 Charge setup}

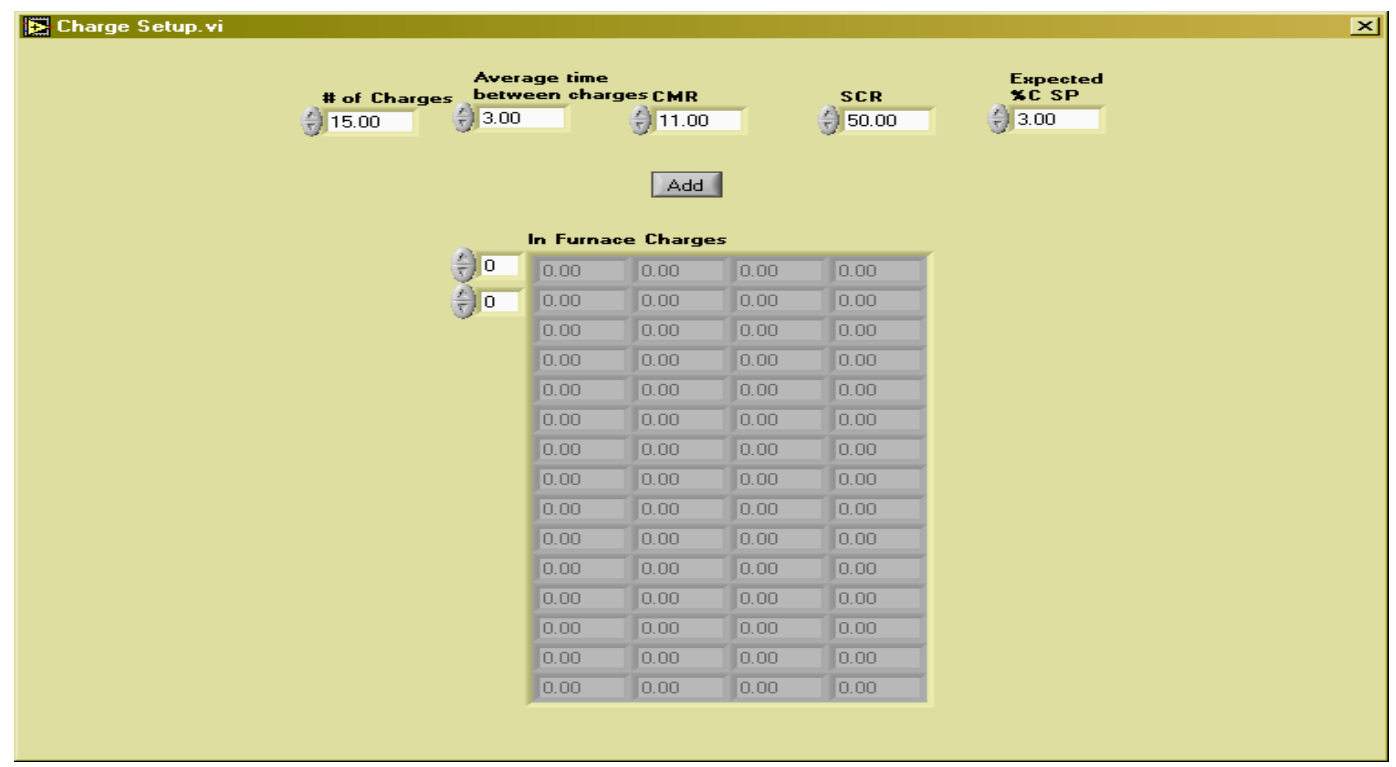

Figure A-34 Setup Charges 
This VI is designed to setup the number of charges that were in the furnace. The dialog is as shown in Figure A-34. The the user can define the number of charges, average time between charges, CMR, SCR, and Expected \%C SP in this dialog.

\section{A.1.1.6.2.3.3 Planner setup}

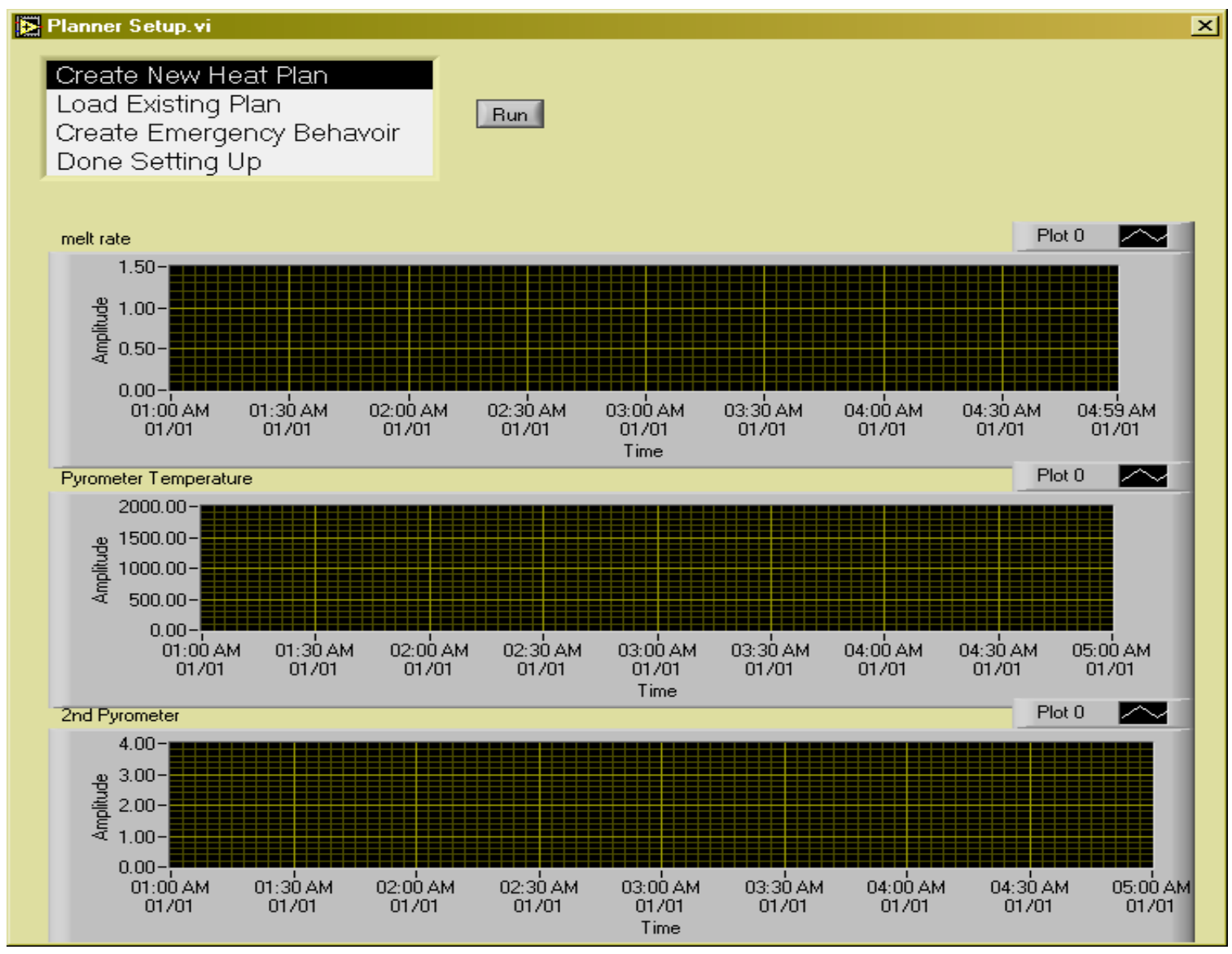

Figure A-35 Planner Setup

The planner setup VI produces the dialog in Figure A-35 to setup the planner. Highlight on Create New Heat Plan then click on Run to create a new plan. The dialog in Figure A-36 is used to add a new plan. User can add the start and stop time, the start up time, the steary burn period, the transition and the shutdowm by highlight the menu and clicking Run. 


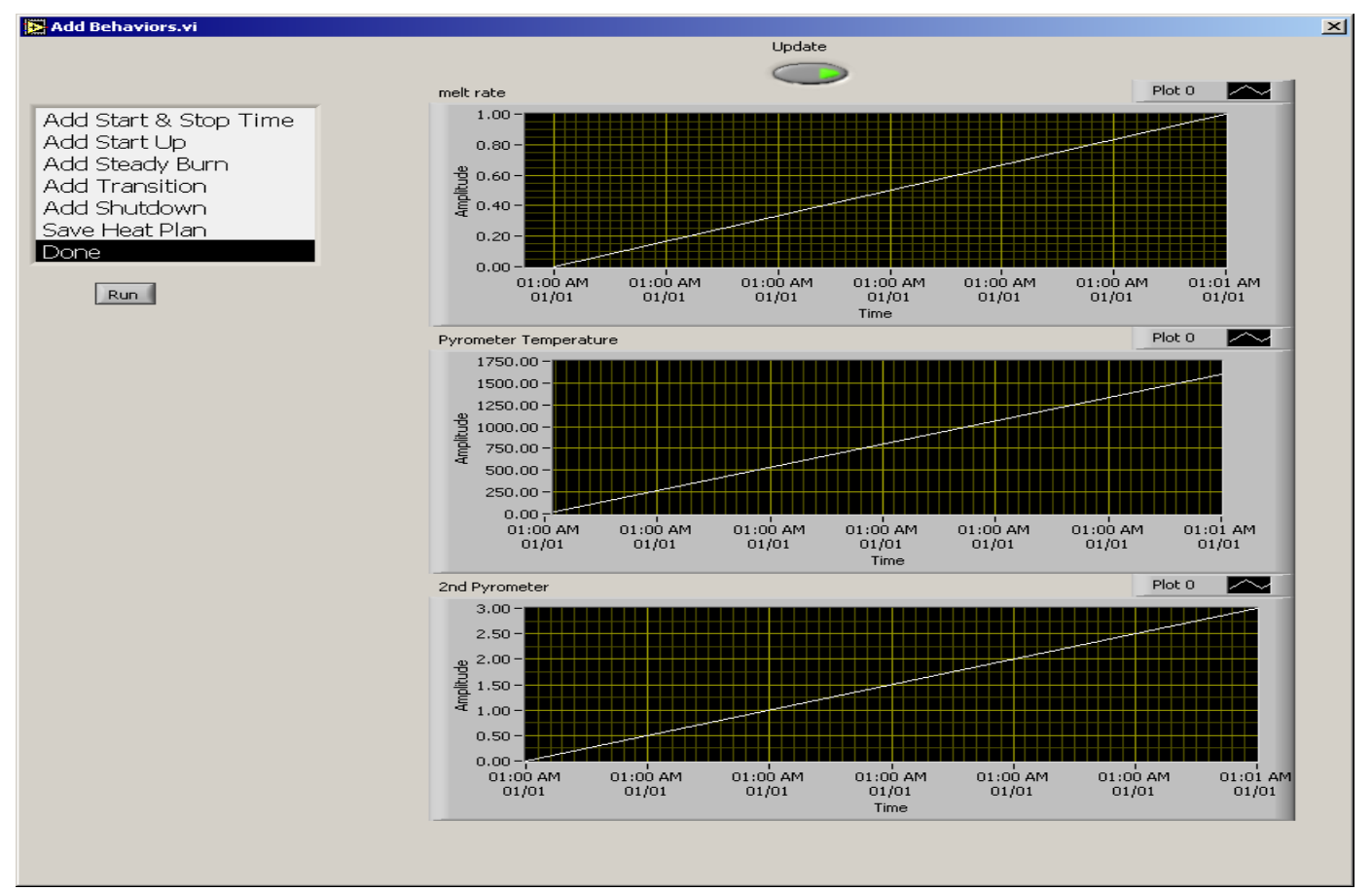

Figure A-36. Add new plan

In the dialog shown in Figure A-37, user setups the start and stop data and time. The startup points can be setup in the dialog in Figure A-38.

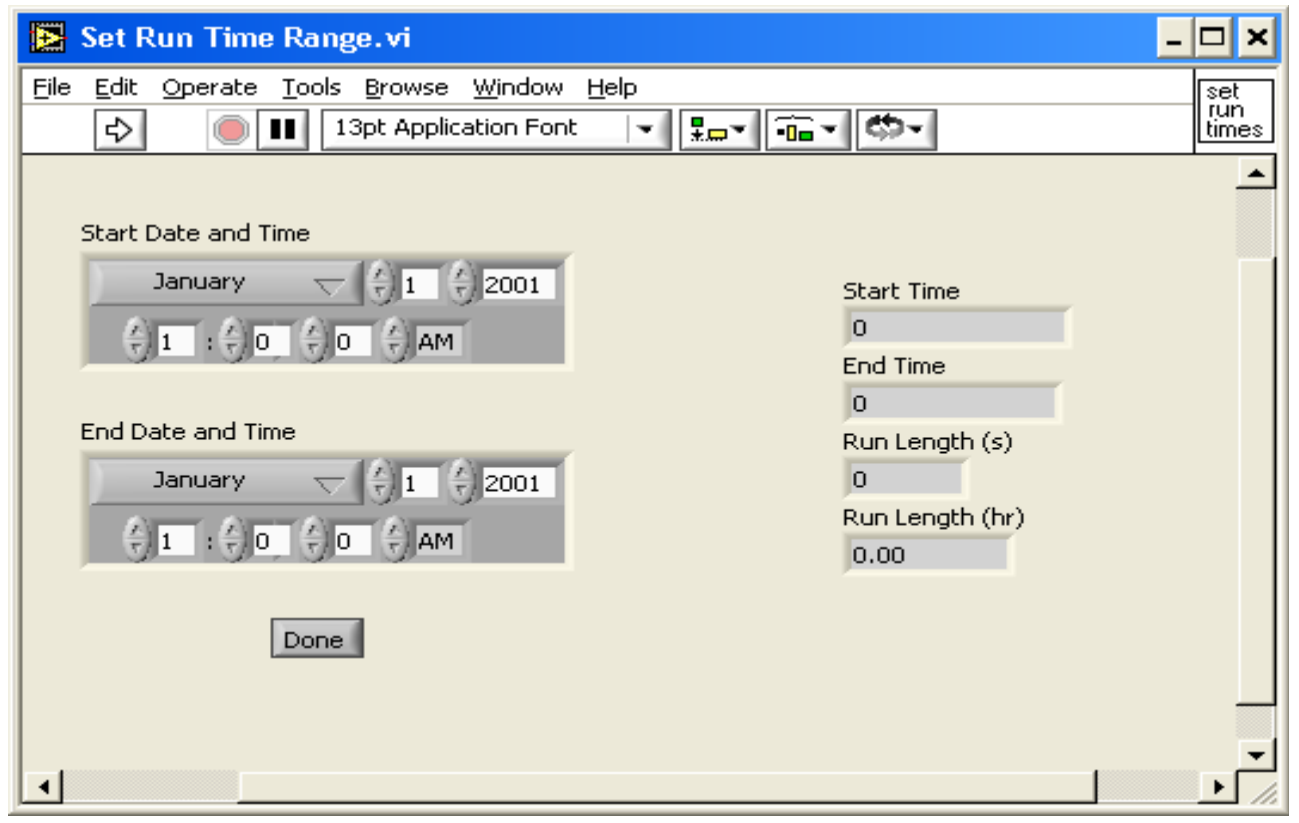

Figure A-37 Setup the start and stop time of a plan 


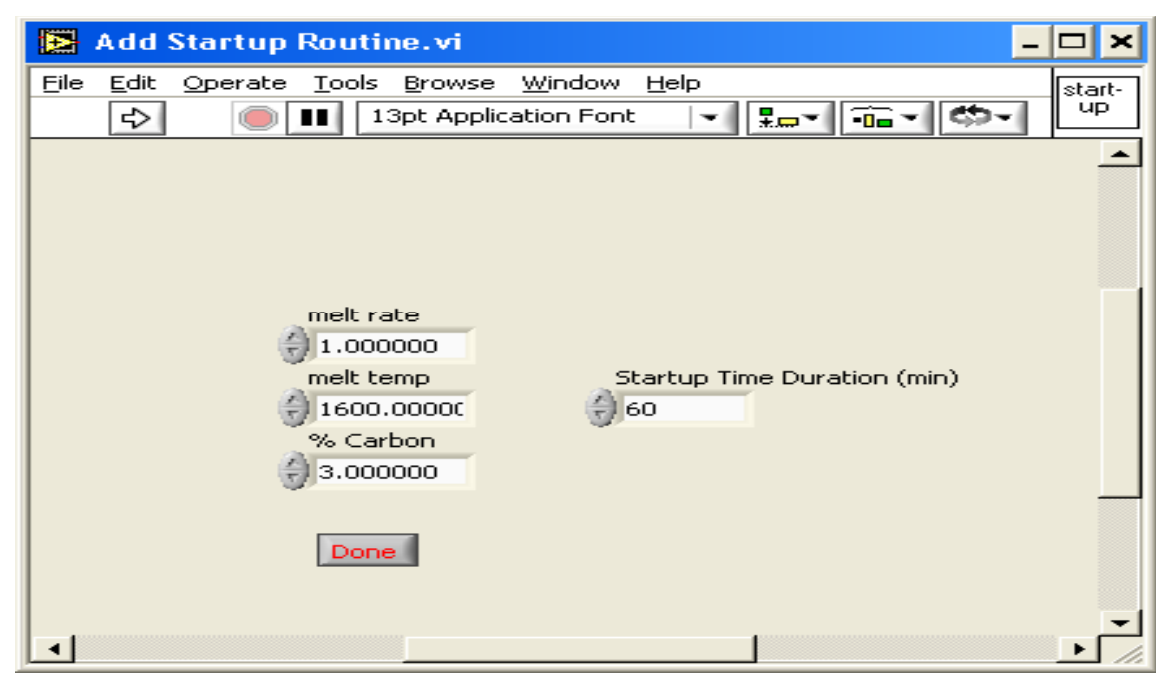

Figure A-38 Add startup routine

The steady burn period is setup in the dialog showm in Figure A-39. Figure A-40 is the dialog to setup the transition. In Figure A-41 user can setup the shut down time.

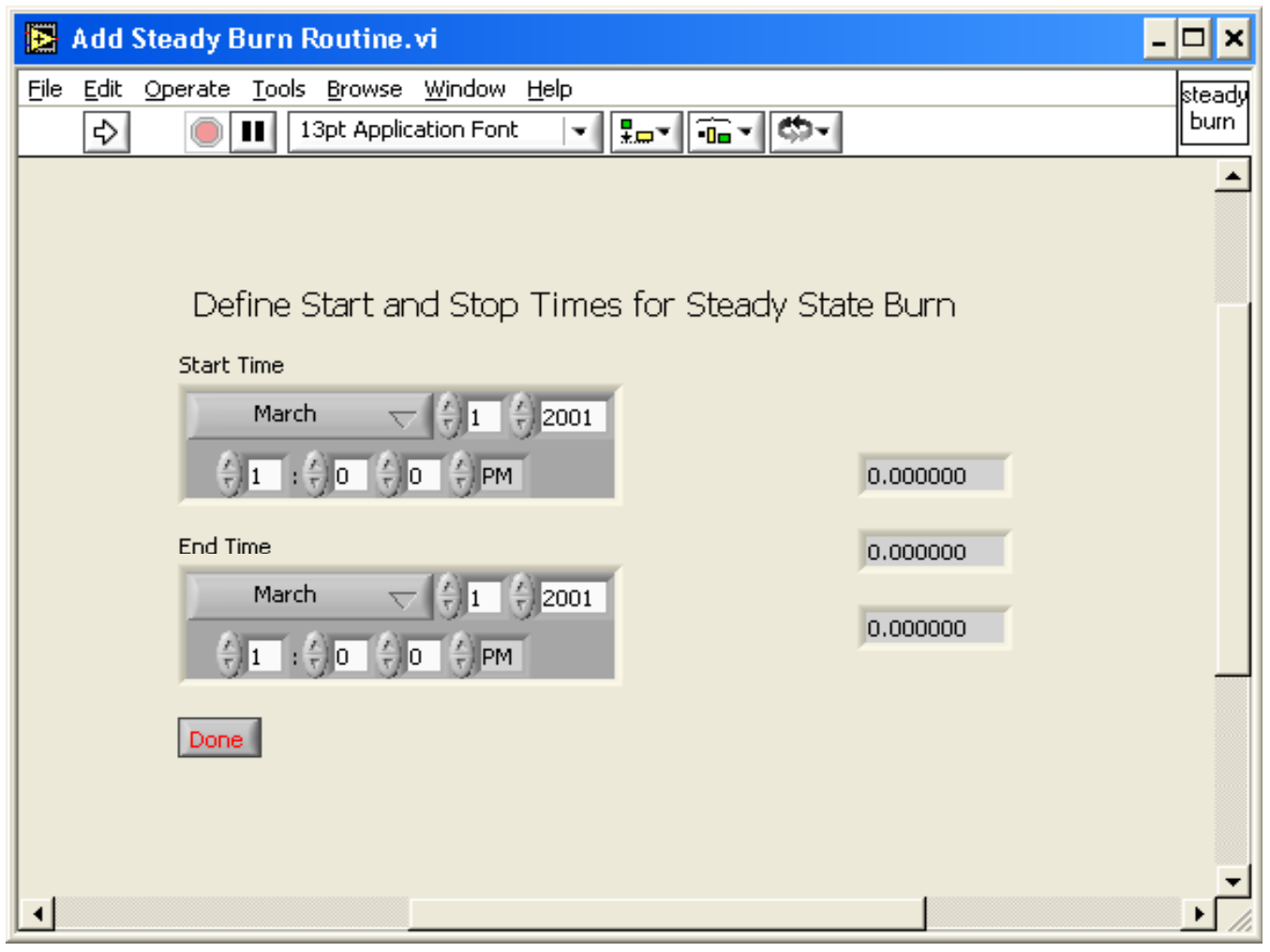

Figure A-39. Set up steady burn routine 


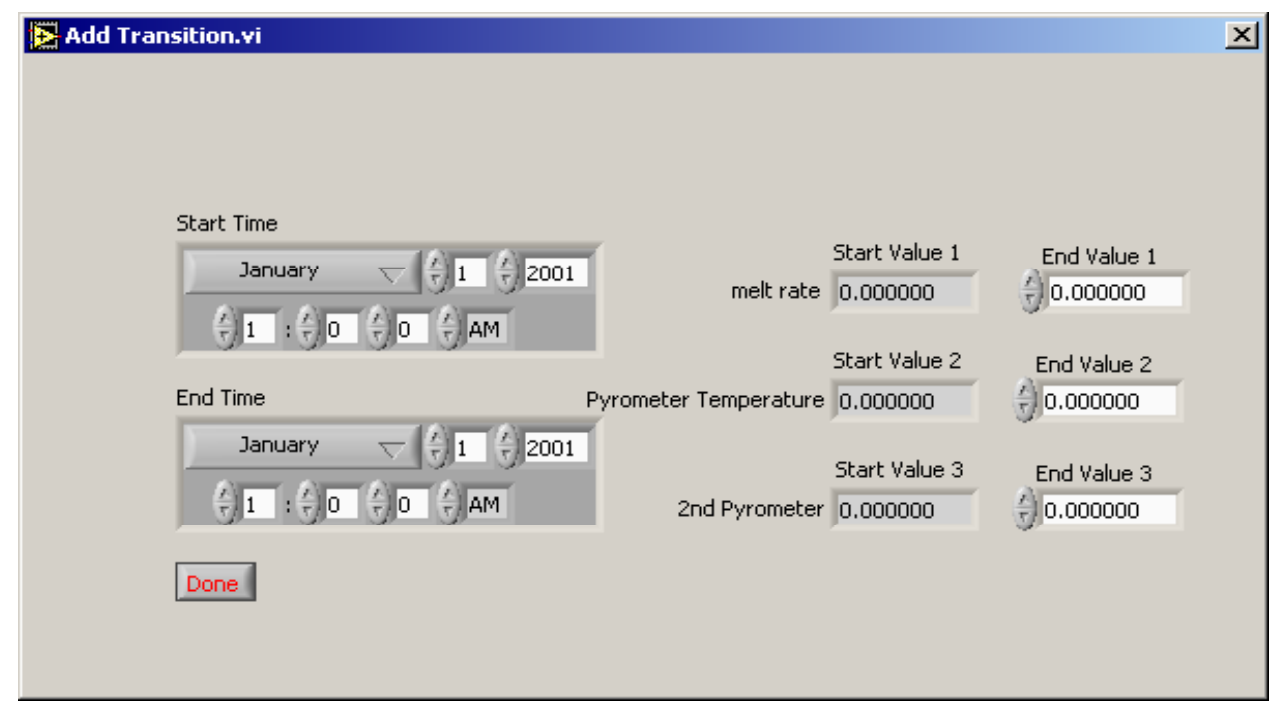

Figure A-40. Add transition

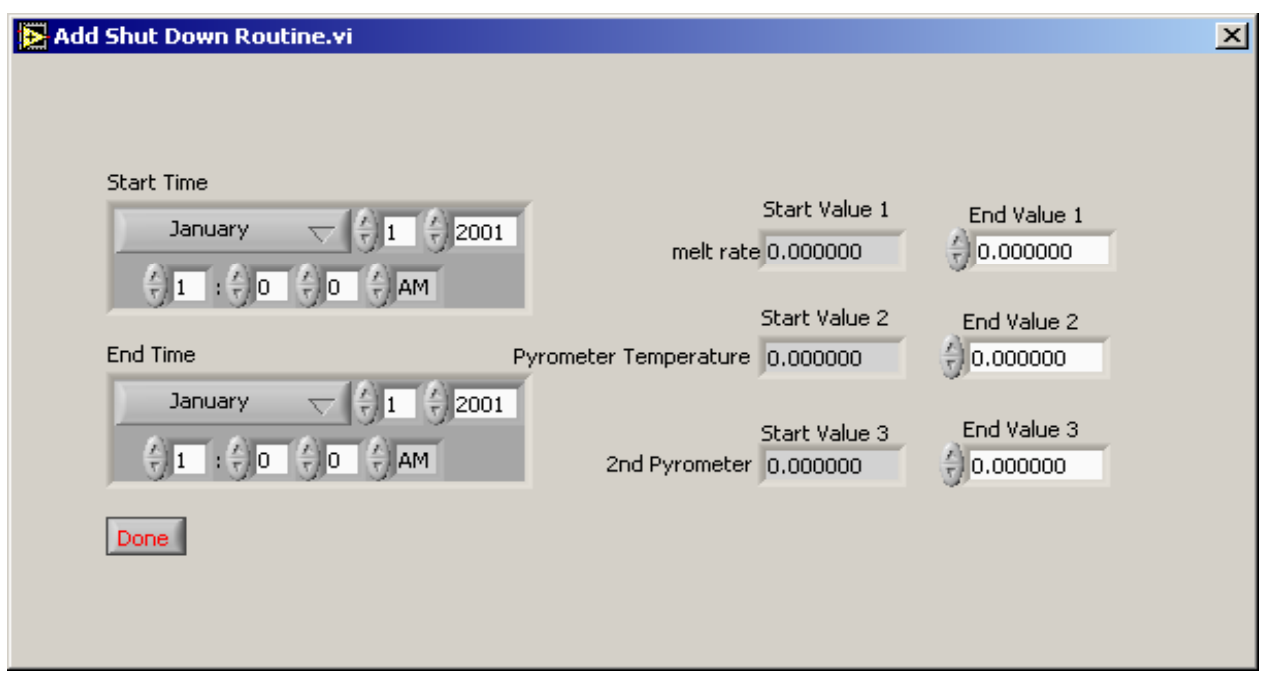

Figure A-41. Add shutdown time

\section{A.1.1.6.2.3.4 Controller setup}

Controller setup is used to setup the parameters of the controller variables which are listed in the menu box in Figure A-42. Highlight the controller variable then click on OK. The parameters setup dialog will popped up as shown in Figure A-43. 


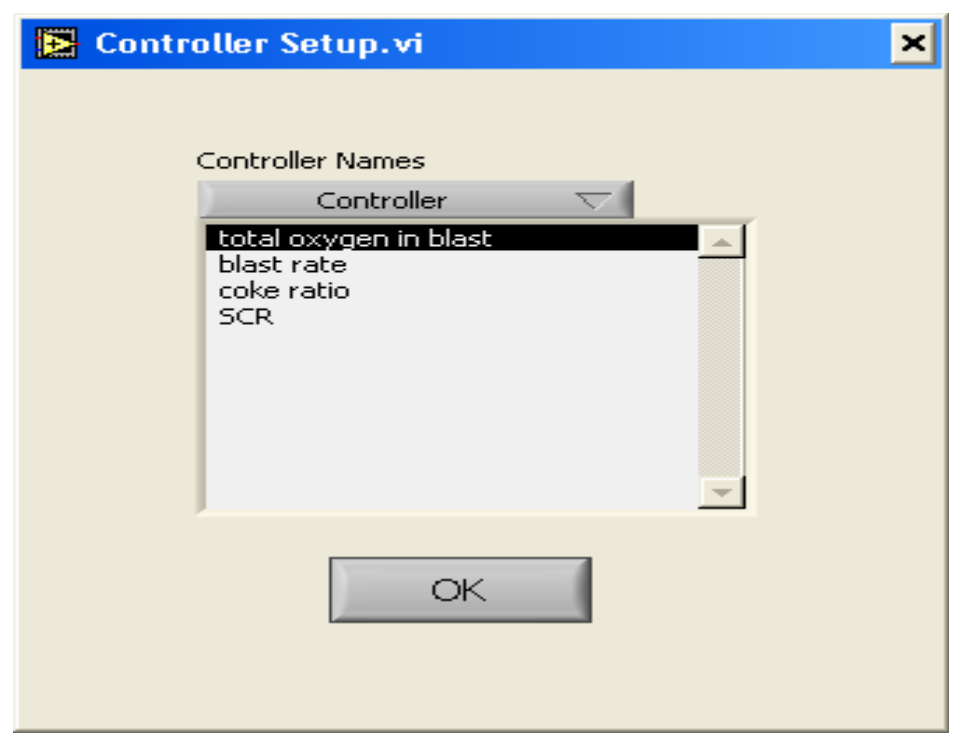

Figure A-42. Controller setup

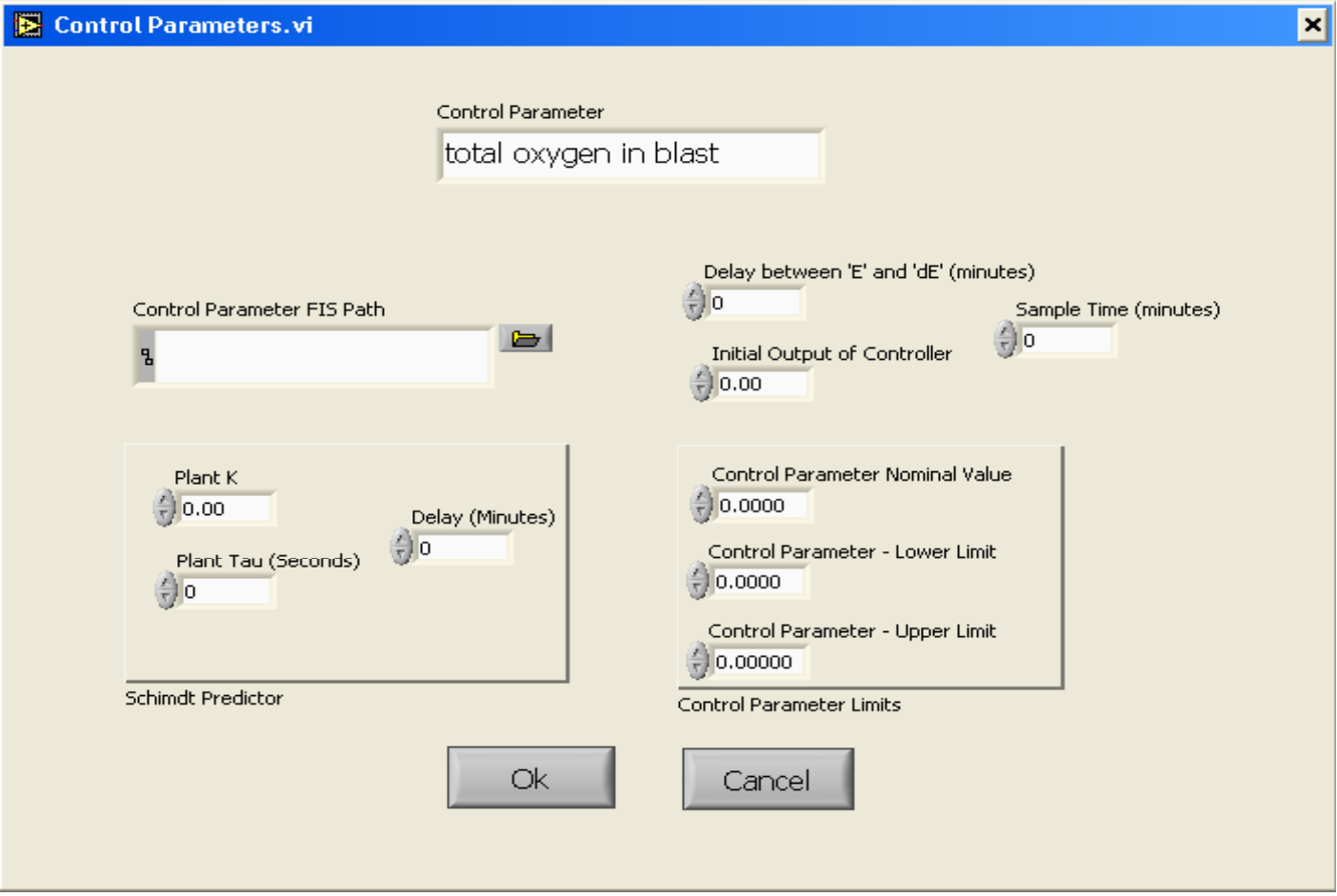

Figure A-43. Setup the cotrollel parameters 


\section{A.1.1.7 Done Setting Up}

After setting up all the modalities for the new application, double click Done Setting Up will save all the modality setup and close the application setup window (Figure A-6). The system returns back to the top-level menu (Figure A-1). 


\section{A.1.2 I ${ }^{3}$ PSC Running}

After setting up the new application, double click "Run" on the panel shown in Figure A-1. The dialog of $I^{3}$ PSC Running (Figure A-44) is popped up.

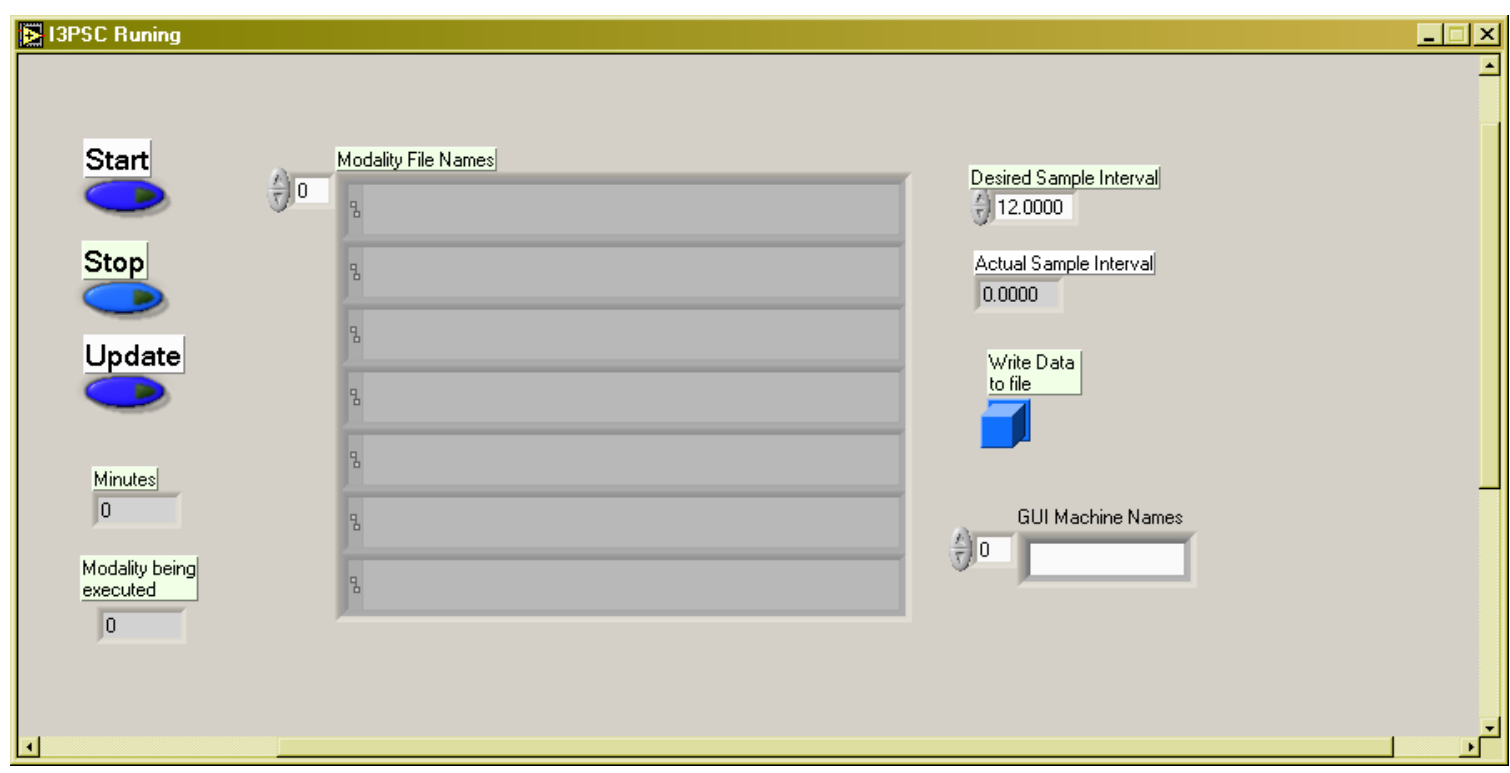

Figure A-44 I ${ }^{3}$ PSC Running

In this dialog,

1. "Desired Sample Interval" is the sample interval of the system. The default value is 60 seconds.

2. "Start" will active the run of all modalities.

3. If you want to monitor the system on another computer, you can type that machine's IP address in the "GUI Machine Name".

4. Press "Write Data to File" will record all the running data into an Excel file and this file will be opened after you "Stop" the running.

5. In the "Modality File Name" box, the user can check all the setup modalities that are executing.

6. While the system is running, the user can setup the modality using the procedure mentioned in section A.1.1.6 and then press "Update" button to update the modality setup. For example, using "Update", the user can add a new variable into a fusion group even the system is still running. 
7. The user can select the variables to be written in the Excel file using the VI whose path is $C: i^{3}$ psc $\backslash$ Datastructurelclasses $\backslash$ Data Structure Export to Excel-New.vi. The variables in the Selected Parameters array will be written.

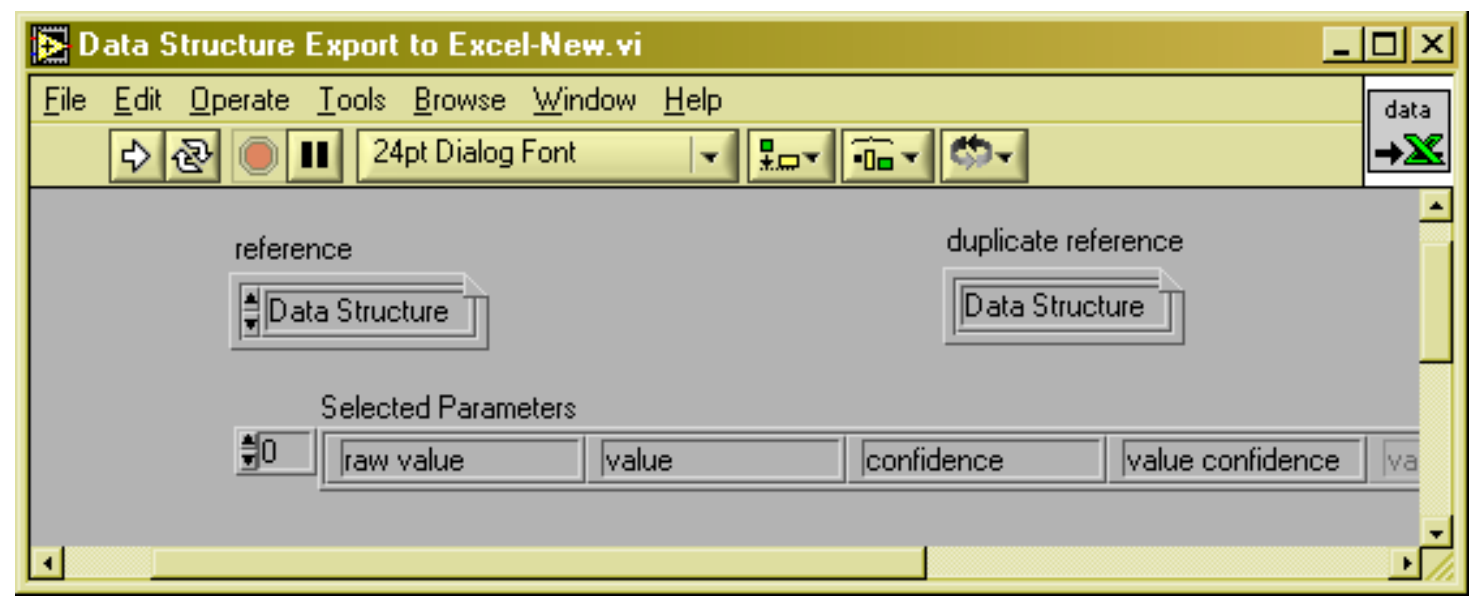

Figure A-45 Excel File Setup

While running I ${ }^{3} \mathrm{PSC}$ 's, the Cupola Operation Monitor window will be popped up. The user can monitor the real running situation of the controlled cupola furnace by this monitor. At the same time, several other dialogs will be popped up depending on the modalities you set. For example, the Controller Options dialog is popped up if you set controller modality in the system.

\section{A.1.2.1 Cupola Operation Monitor}

At the beginning of $I^{3} P S C$ 's running, Cupola Operation Monitor is popped up. You can monitor the real time running situation of the controlled cupola furnace by this interface. The front panel of the monitor is shown in Figure A-46.

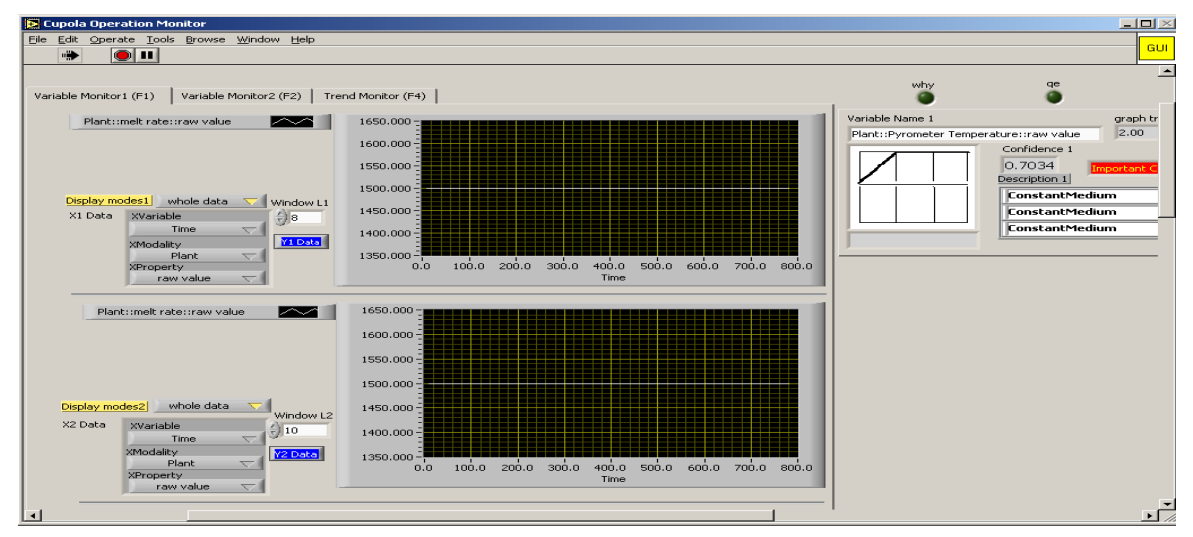

Figure A-46 Cupola Operation Monitor 
There are two tabs for variable monitor and one tab for trend monitor. On each variable monitor tab, four variable waveform windows are arranged. You can select multiple variables in one window to monitor. Select the $\mathrm{X}$ axis node first. All the waveform on the same window must use the same $\mathrm{X}$ axis node. Pull down the menu in $\mathrm{X} 1$ Data list box to select $\mathrm{X}$ axis node. The default $\mathrm{X}$ axis node is Time-Plant-Raw value node. Then, Click on the blue "Y1 Data" button. A YI data setup window will popped up. This window is shown in Figure A-47.

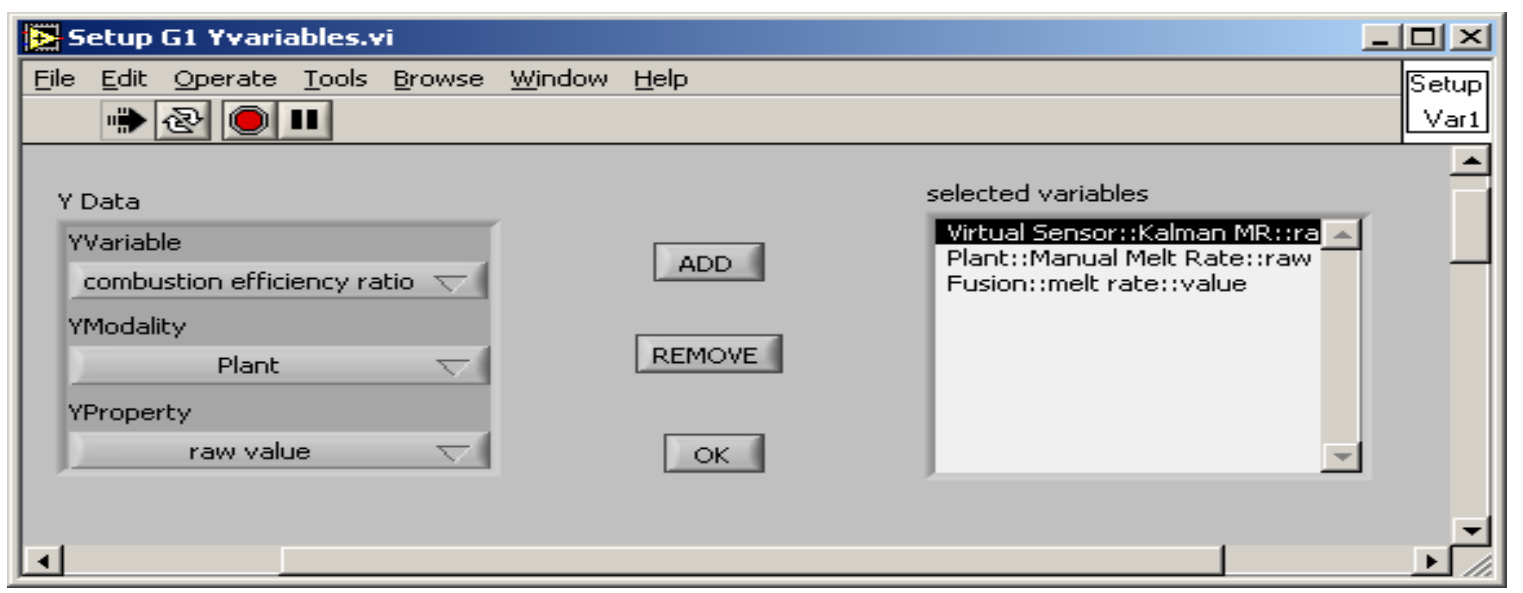

Figure A-47 Setup Variable to Monitor

Select the $\mathrm{Y}$ nodes in the $\mathbf{Y}$ data list box. Then click on "ADD" button to add it into the selected variables list. If you want to delete the selected variables, please select the variable in the selected variables list box then click on "REMOVE" button. After you select variables need to be monitored for this window, click on OK button to return back the Cupola Operation Monitor.

Use the same procedure to select the variables you want to monitor in other windows. There are three display modes, namely whole data, shifting window and fixed segment. Whole data is the default mode that will display the complete data of the running. Shifting window only displays the latest data with the data length defined in Window L1. Fixed segment display the fixed length data statically. The length of the fixed segment is defined by the start point and window length. The Start Point controller only displayed while the fixed segment display mode is selected. 


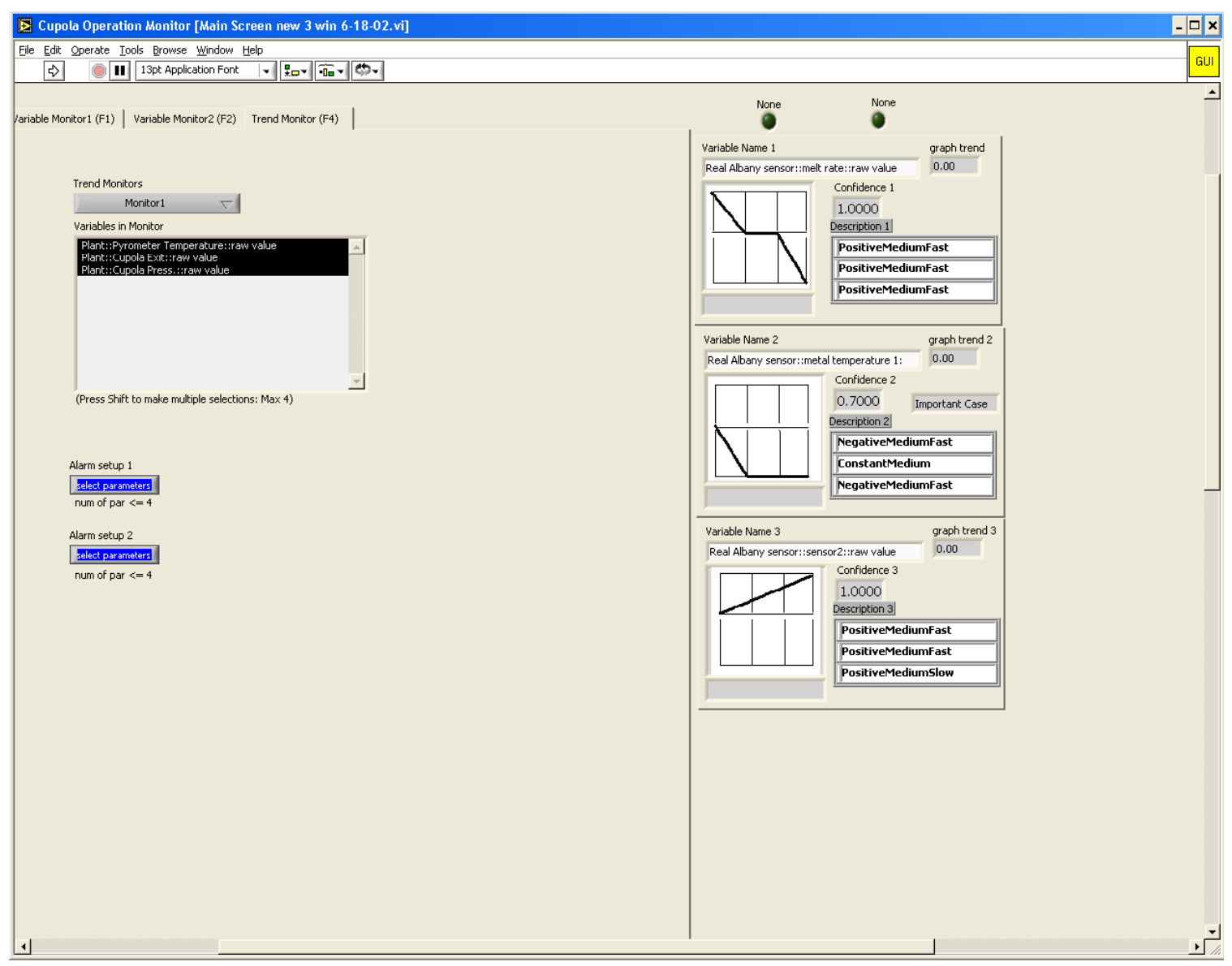

Figure A-48 Trend Monitor

On the trend monitor tab (Figure A-48) you can select the variable, whose trend will be monitored. At most four trend monitor windows are arranged on the right side of the window.

Also, two situation diagnostics can be set up in the same tab. Click on the blue Select Parameters button. The dialog to setup the diagnostics in Figure A-49 is shown. The results of the diagnostics will be displayed by the LED on the Cupola Monitor window. The bright LED indicates the alert situation is happening. On this window you select the diagnostic input nodes using "ADD". "REMOVE" can delete the parameter you've selected. In the Name box you can name your diagnostic. This name will be display on the upper right corner of the Cupola Operation Monitor with the diagnostic alarm led. 


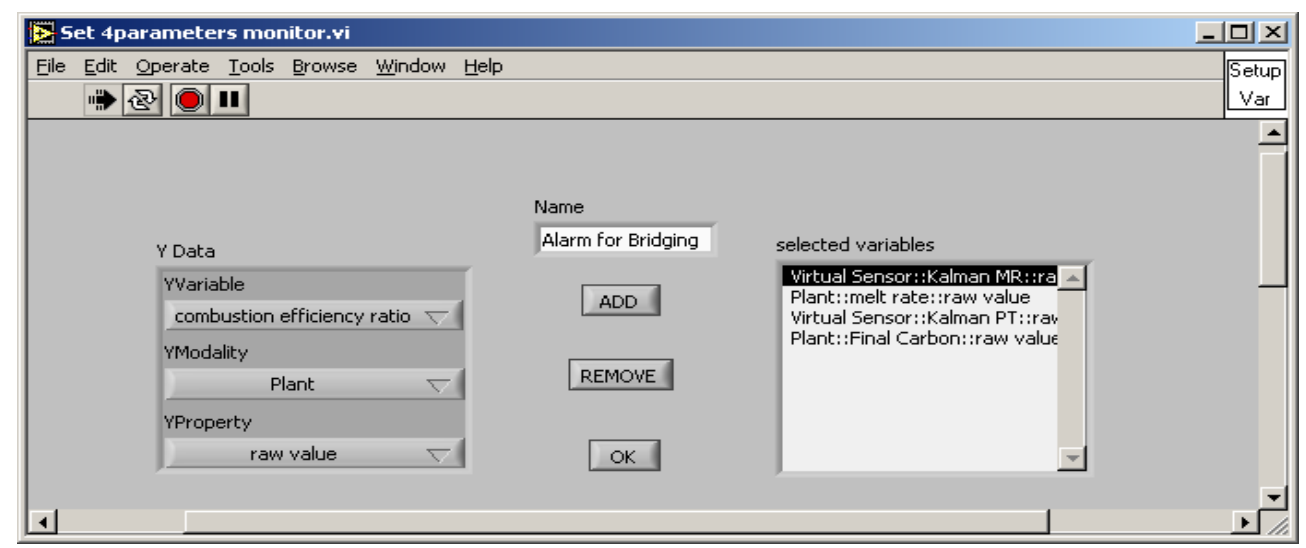

Figure A-49 Setup Diagnostic Parameters

\section{A.1.2.2 Change the Control Option Here}

If a controller is set in the system, the dialog of Change the Control Operation Here is popped up during the running and it will stay on the desktop. So you can change the control operation at any time. There are four tabs on the Change the Control Option Here dialog.

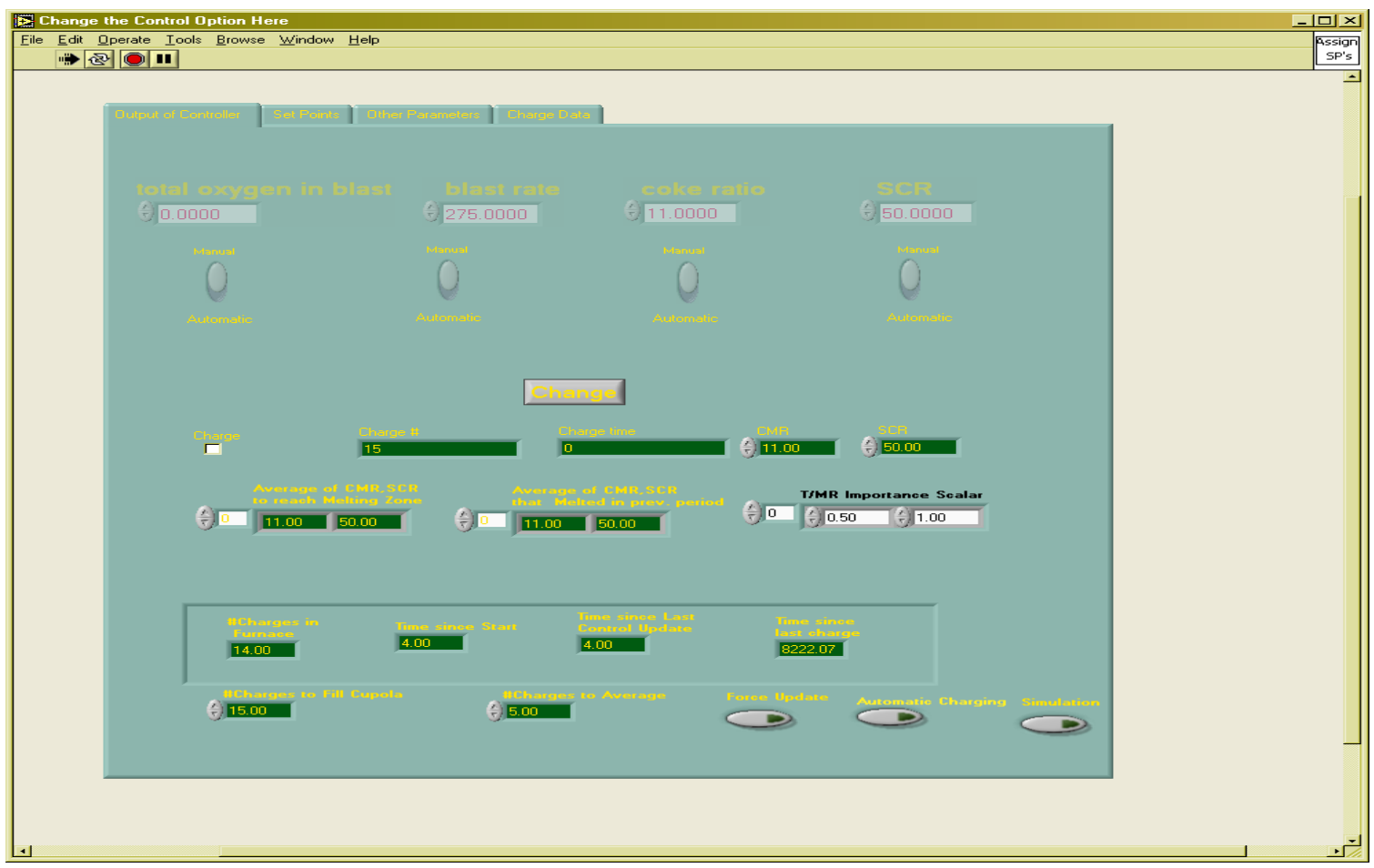

Figure A-50 Change Controller Option - Outputs of Controller 
The first tab is as shown in Figure A-50. It displays information regarding the controller output. It also allows the user to override the controller suggestions by choosing to operate in the manual mode. To change the setting from Manual to Automatic or the reverse. The user presses the Change button, then selects the desired setting, then press the update button. If the value of the controller output is to be changed, the user enters the desired value at the appropriate control. The check box charge is used to indicate that a charge is added to the furnace. The charge number and time is displayed in the two indicators as marked on top of the indicator. The two controls CMR and SCR are used to manually enter the actual coke to metal ratio and steel to cast ration that went in the latest charge added to the furnace. The T/MR Importance Scalar values determines is used by the controller to determine how to adjust the BR and Oxygen since the desired values for both parameters might not be achievable simultaneously. A higher value would favor one parameter over the other. The indicators at the bottom of the screen display important parameters regarding the charges. The control \#Charges to Fill Cupola indicates the average number of charges that can fill the cupola.

The second Tab (Figure A-51) displays a set of parameters used by the controller including the desired set points or temperature, melt rate and the future SP values for the same parameters as well as Carbon. This is important for parameters that require adjustment of charges to avoid the delay resulting from the melting time through the furnace. The delay time is estimated approximately as the time it takes to melt the number of charges inside the furnace. The set of controls marked with Nominal indicate the starting steady state operation values. The controls marked with scalar are adjustable parameters that can be changed if the response of the controller is not satisfactory. The user is given the option of overriding the confidence values calculated by the senor fusion and supplying a constant value. The is done using the switch marker "Assign. Conf./Calc. Conf.". Finally the user can override reading coming from the fusion modality and pass to the controller another set of readings by using the Manual Readings selector. 


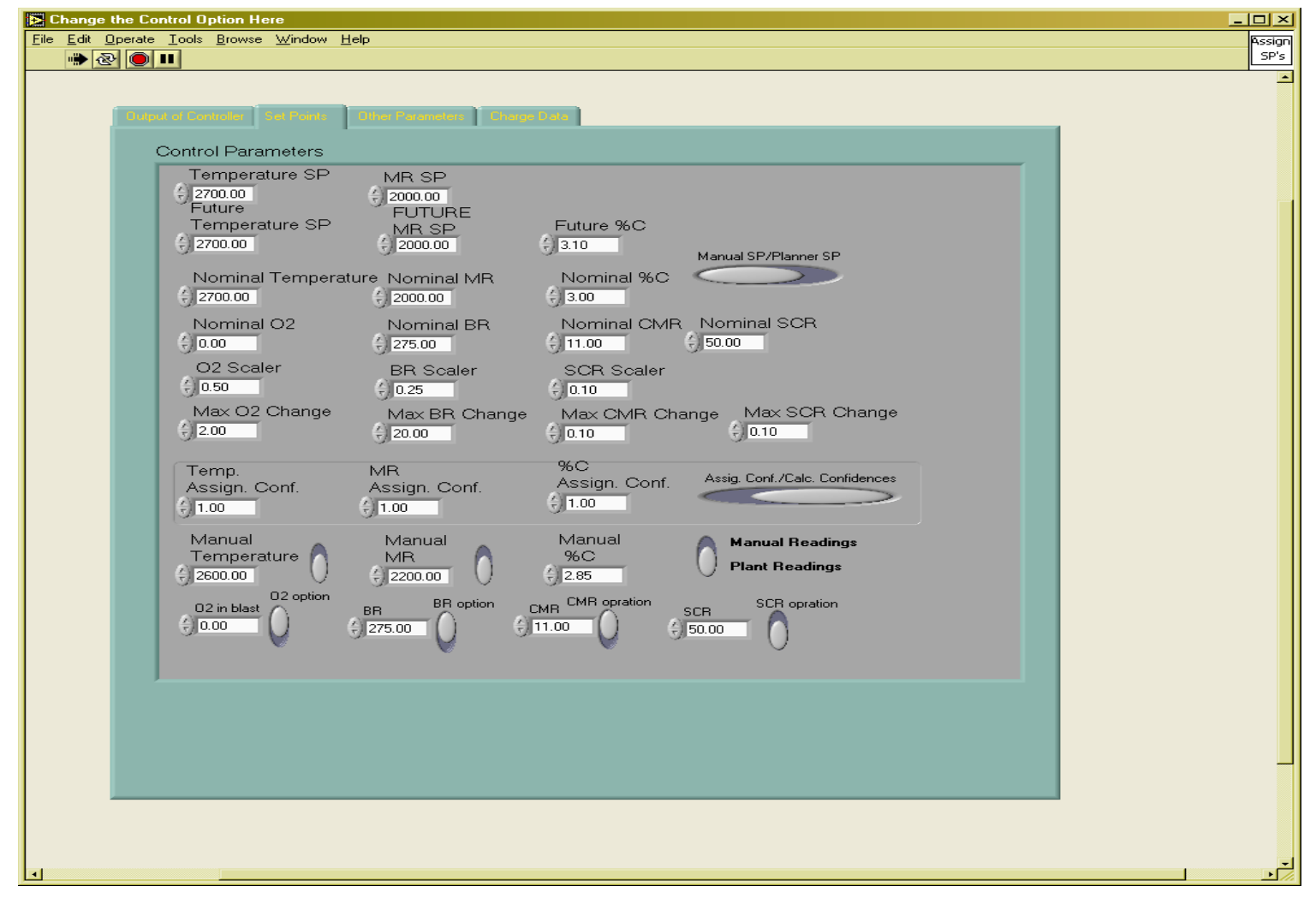

Figure A-51 Change Control Option - Set Points

The third Tab (Figure 3-9), is used by the user to set minimum and maximum values for different parameters in the system. The array marked with $\mathrm{K}$ is used to supply the expected steady state gain matrix of the cupola. This is calculated experimentally or using the AFS model. The vector array marked Tau supplies the time constant that can be used with the matrix $\mathrm{K}$ to form a dynamic linear model for predicting cupola response. The controls marked with update are used to indicated how often the controller updates the corresponding parameter. The number supplied is given in terms of the number of samples. The Confidence Effect Control set of parameters are used to increase sensitivity to the confidence values within a certain range. The power control is a parameter that determines how fast the confidence effect over the controller rolls off as the confidence deviates from the high level. 


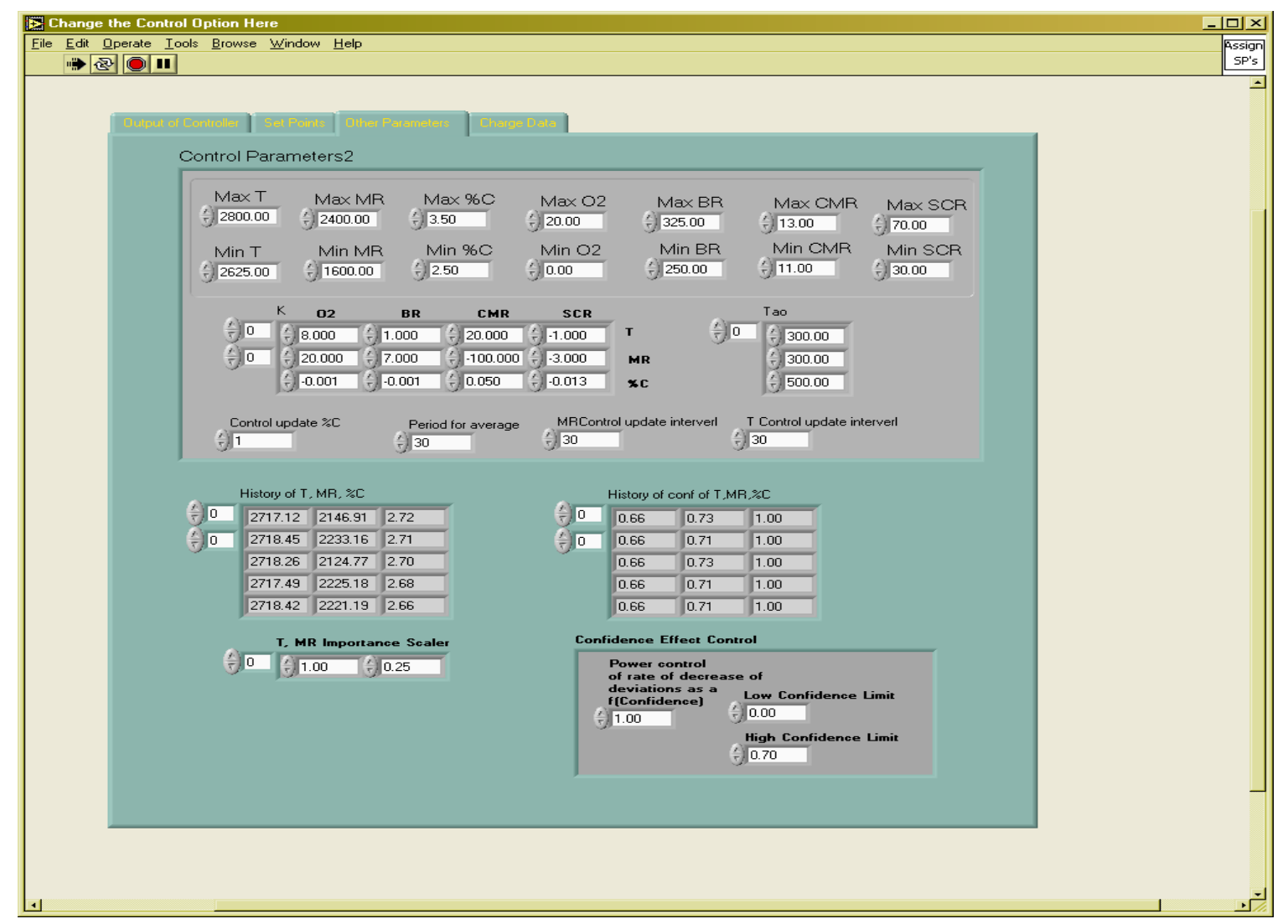

Figure A-52. Change Controller Option - Other Parameters

The fourth tab (Figure 3-10) displays a history of the charge that has been added to the furnace including the charge number, time, the coke to metal ratio, steel to cast ratio as well as the set point for Carbon that was desired at the time the charge was added, the predicted value of carbon when this charge reaches the melting zone and the actual value of Caron measure when this charge reaches the melting zone. 


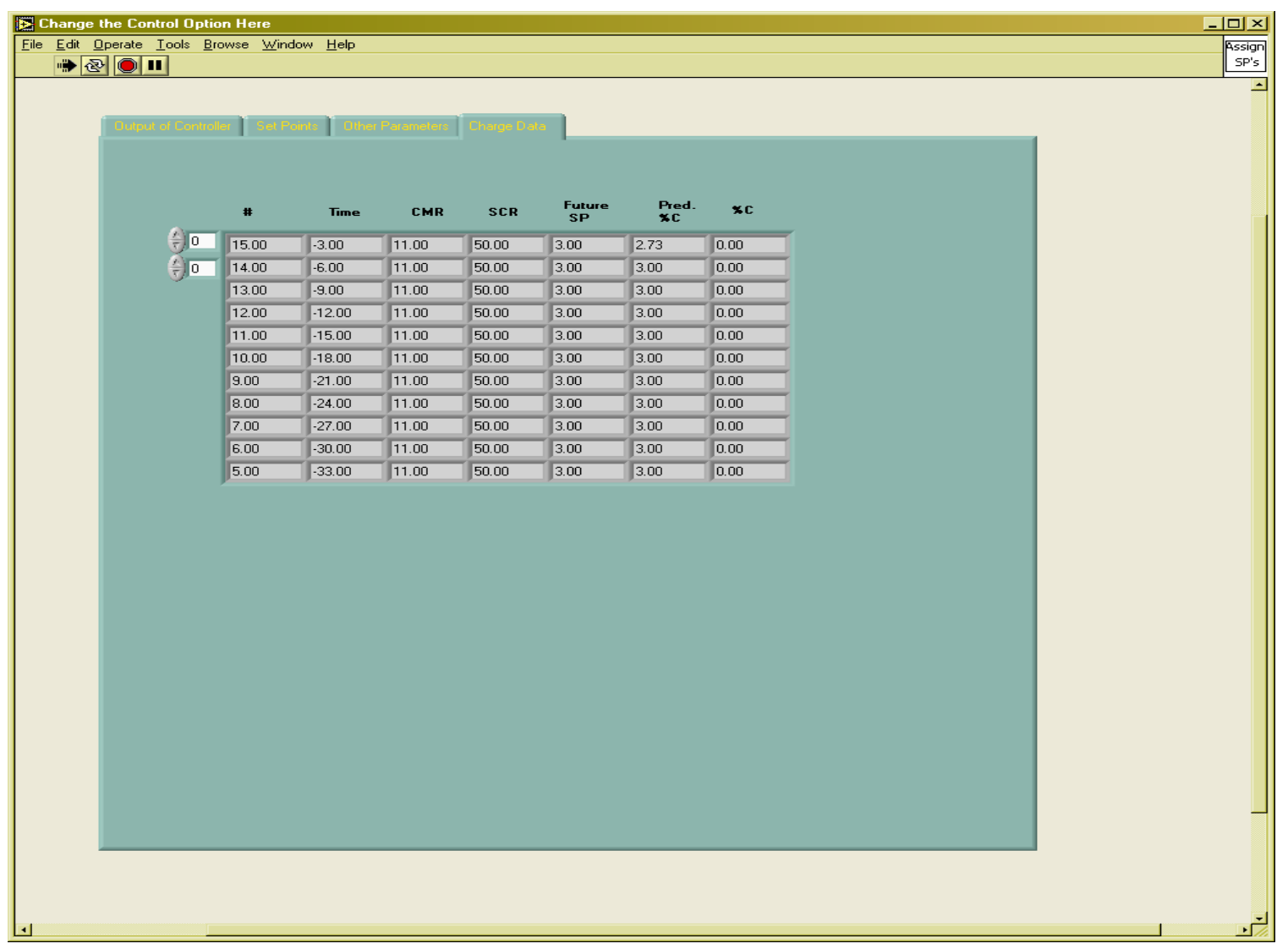

Figure A-53. Change Controller Options - Charge Setup 


\section{A.2 I I PSC Offline System User Manual}

Offline Analysis is concerned with producing Neural Network data sets, doing correlation studies, and interrogating models offline. It consists of tools to setup and process the data sets, and other tools to view the results in graphical format.

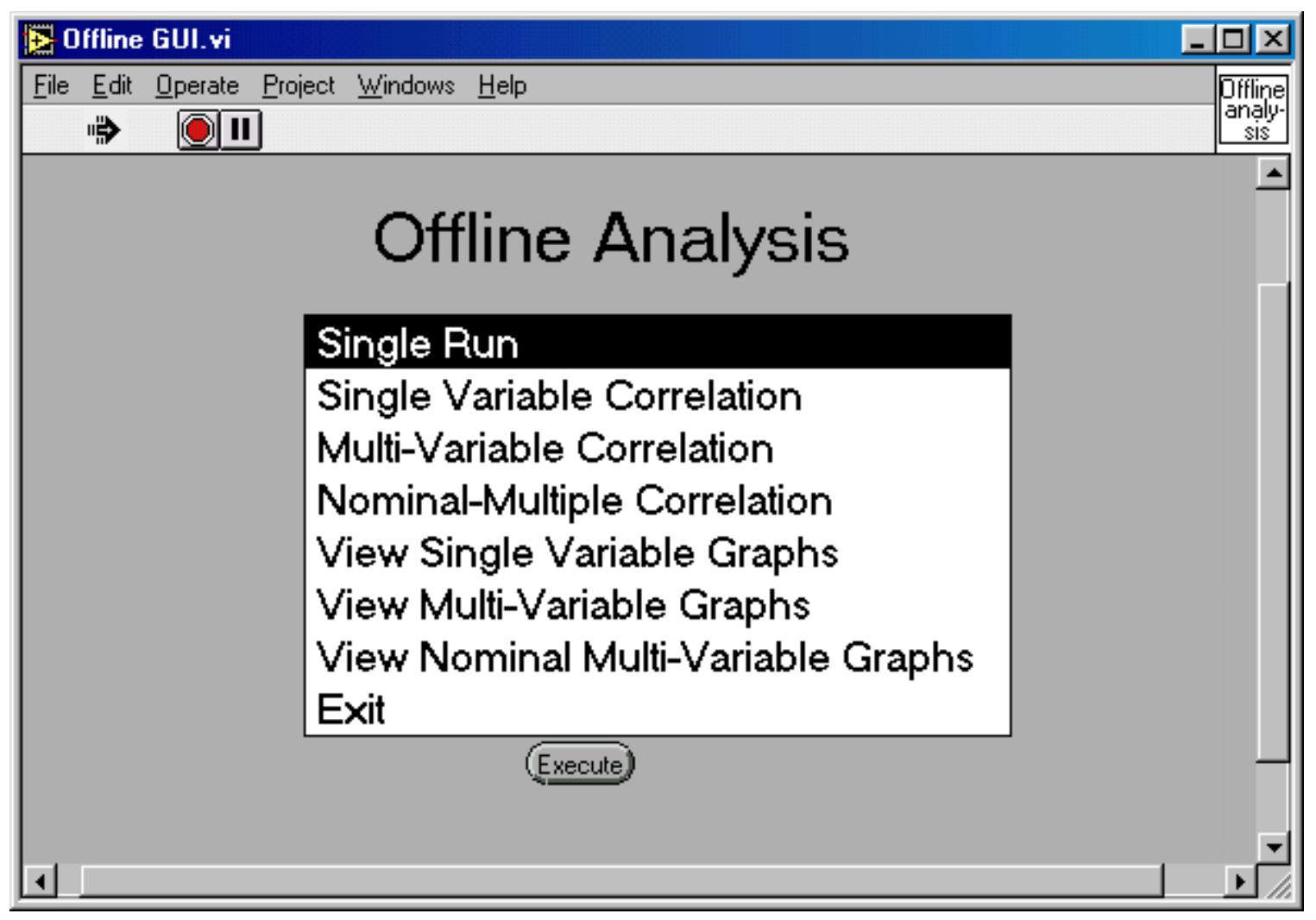

Figure A.54 Offline Analysis Menu Screen - Offline GUI.vi

\section{A.2.1 Single Run}

This application allows the users to set a single set of inputs, pick a model to process those inputs with, and view the outputs. The model is run only once, with one set of inputs. This is useful for seeing quick numerical results, and testing to see that a model is reporting results correctly. The values for the inputs can be changed at this point. Pressing "Return to Main Menu" closes the window and returns control to the offline menu for this and all the other VIs described here. 


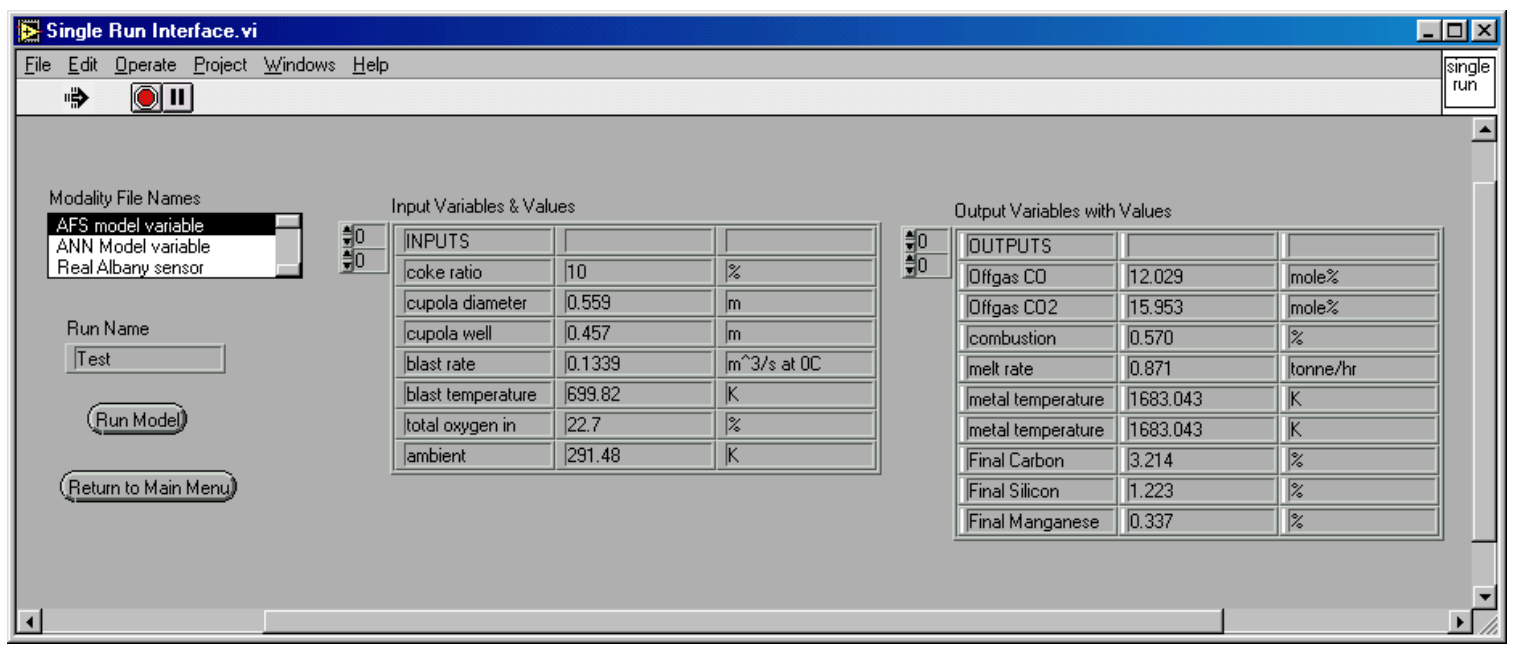

Figure A.55 Single Run - Single Run Interface.vi

\section{A.2.2 Single Variable Correlation}

This application is used to vary a single parameter, while holding all other parameters constant. It is useful for making a large number of iterations on a single variable. The other applications can be configured to accomplish much the same tasks, but it seems to be useful nonetheless. The varied parameter is selected from the list, the default value and unit are displayed for reference, so the user knows an approximate value. The slider in this and the other correlation applications are set to display $1 / 3$ the default value as the lower bound, and 3 times the default as the upper bound. That does not mean that values outside of those bounds can not be set. It also does not guarantee that the bounds make any physical sense. For example, in figure 7, the upper bound for the blast fraction is set at $270 \%$, which does not make sense because the blast fraction cannot surpass $100 \%$. Care should be made to insure appropriate data ranges. 


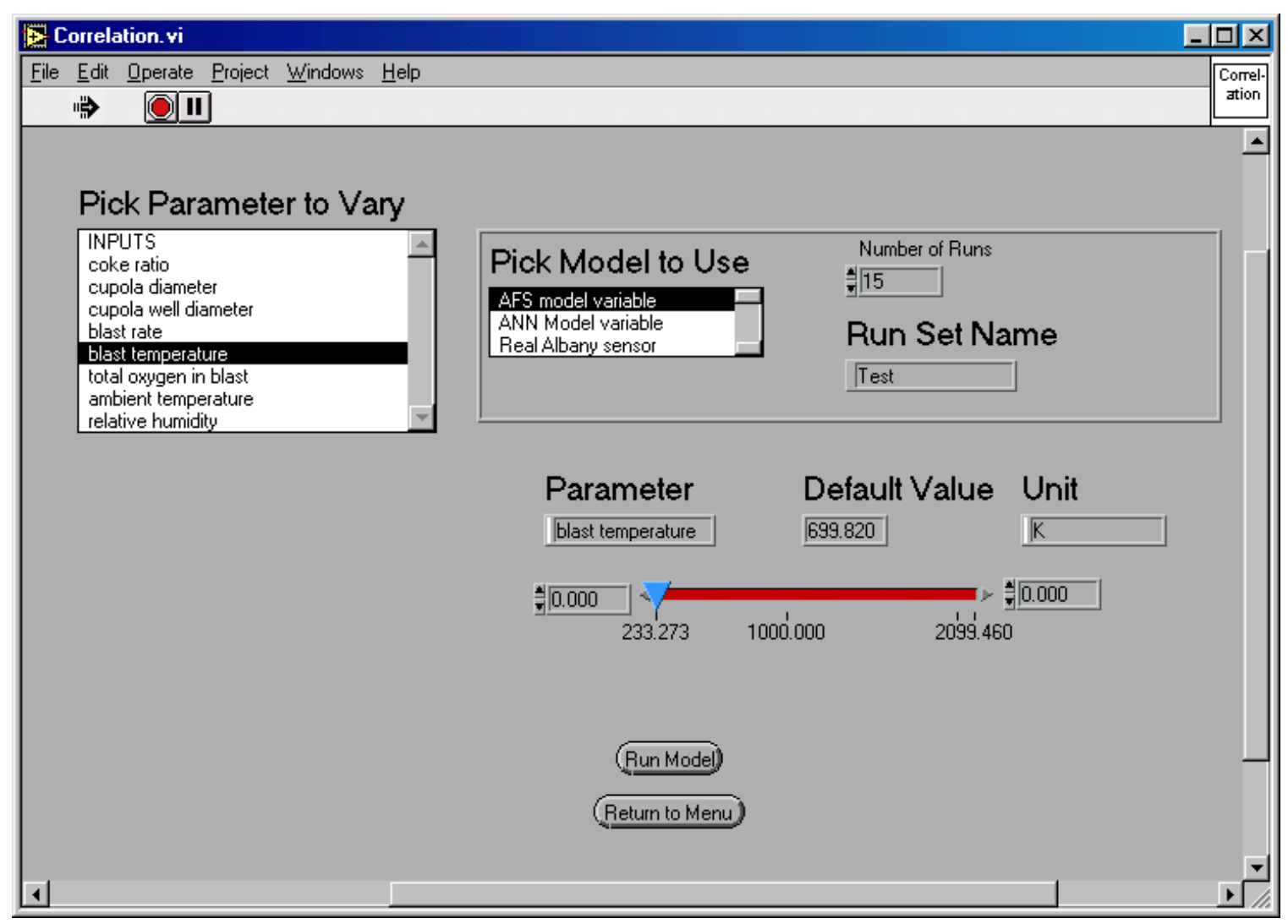

Figure A.56 Single Variable Correlation - Correlation.vi

\section{A.2.3 Multi-Variable Correlation}

The Multi-Variable Correlation application queries the selected numerical model for every possible combination within the input range. As a result, the number of model runs increases exponentially as variables are added and iterations per variable are increased. The number of runs necessary (n) for a complete set is given by the number of variables selected (v), and the number of iterations per variable (i), $n=i^{v}$. In figure 8 , six variables are selected and set to run six times each, resulting in 46,656 model runs. On an average computer this would take about one month. Keeping the total number of iterations below approximately 10,000 allows for completion in about a week. 


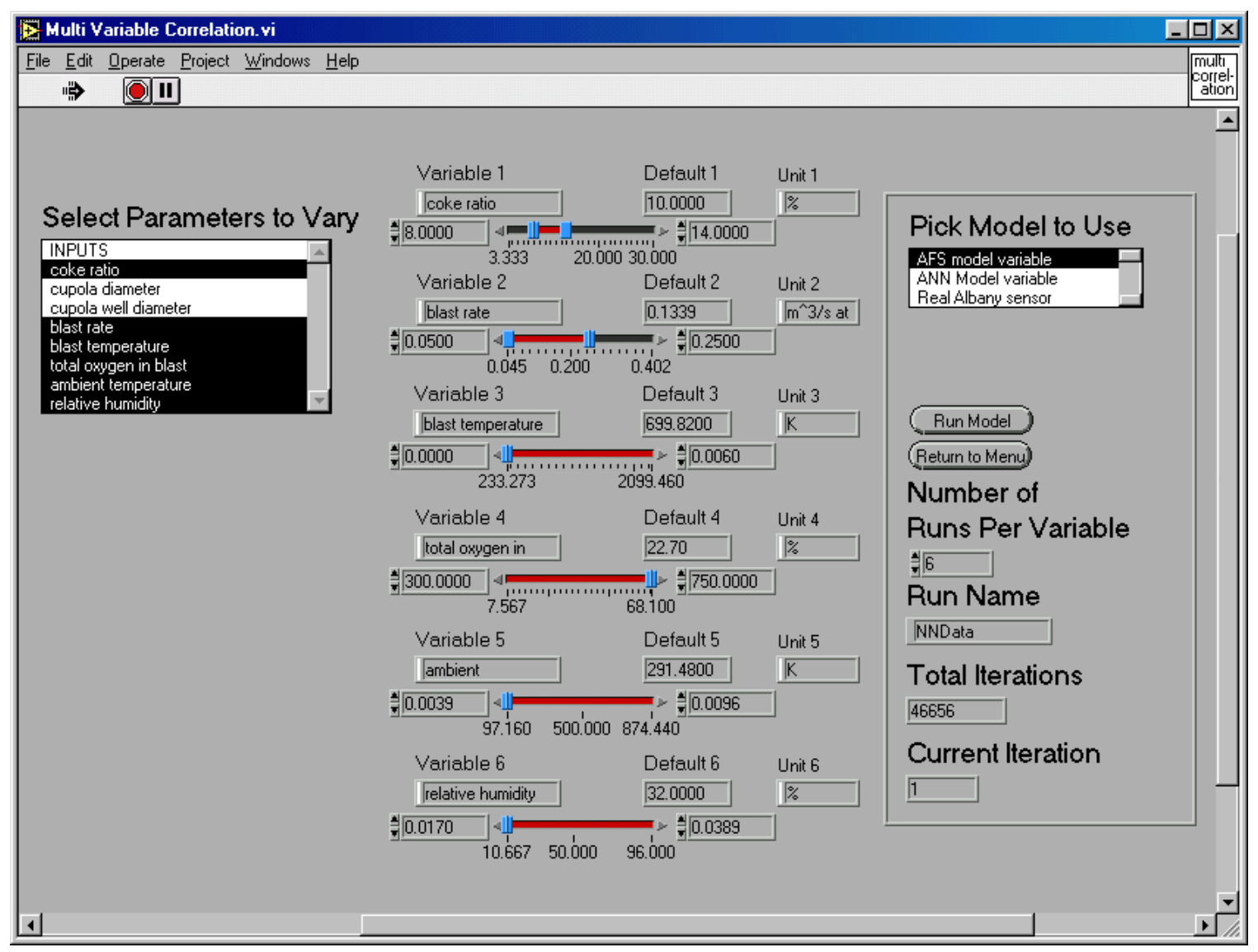

Figure A.57 Multi-Variable Correlation - Multi Variable Correlation.vi

\section{A.2.4 Nominal-Multiple Correlation}

A nominal valued, multiple variable correlation refers to the variation of one parameter while all other parameters are held at their default value. A correlation in this manner allows the user to see the results of a change in one variable as all the others are held constant. There is also a cost advantage to this manner of correlation in that more variables can be selected and the number of iterations per variable can be increased without the exponential relationship. The number of runs is simply the product of the number of variables and the number of iterations. 


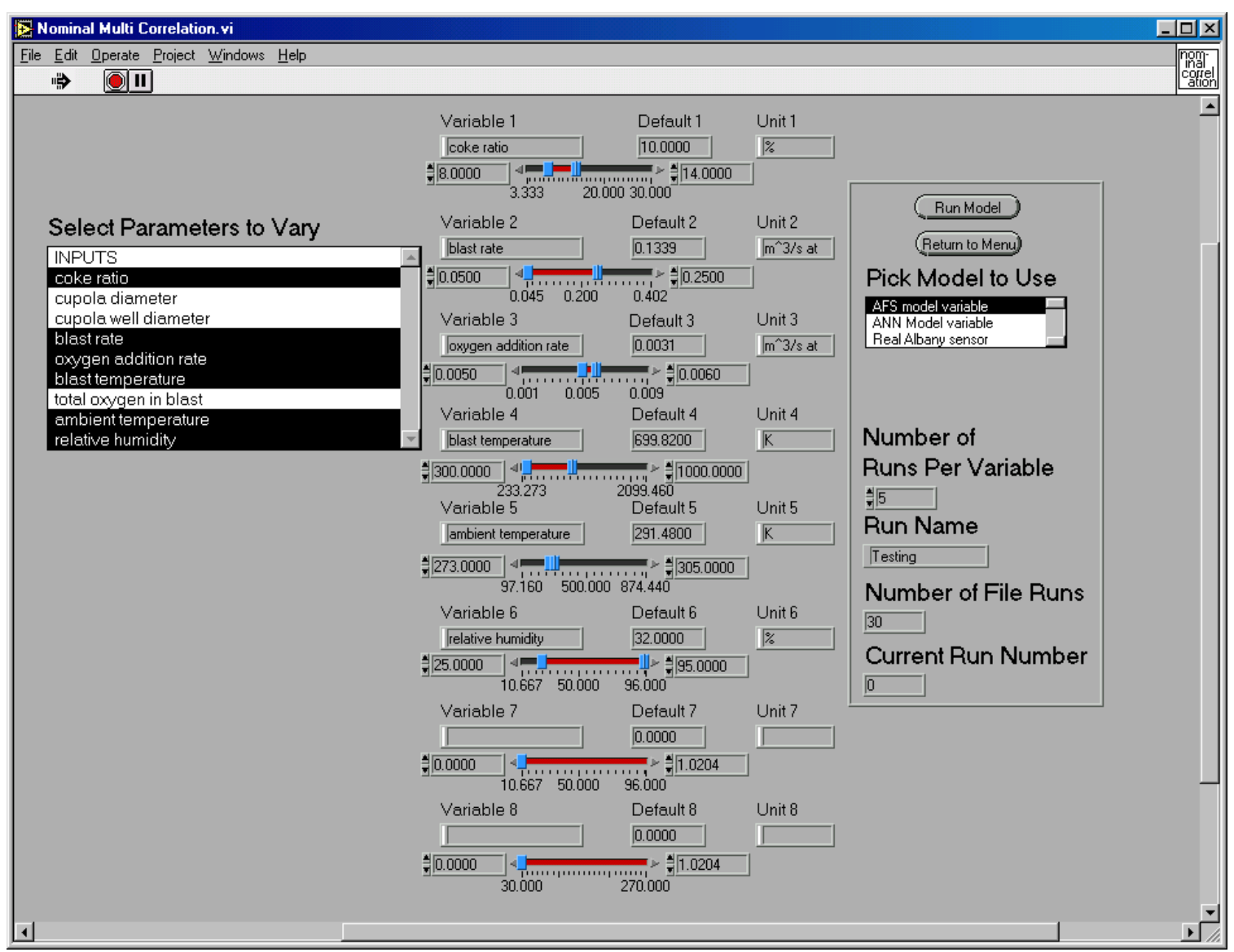

Figure A.58 Nominal Multiple Correlation - Nominal Multi Correlation.vi

\section{A.2.5 View Single Variable Graphs}

All of the correlation applications create tab-delimited spreadsheet files. They are assigned the file extension .xls for easy importation to Microsoft Excel. A list of these files in the output directory path (declared at setup) is displayed in the list box. To view the contents of a file select the file and press "Read New File." To view a single variable correlation, you must use the "View Single Graphs" option, otherwise, the data is not in the correct format. The file will still be read, but it won't make any sense.

For the single variable correlation, the input variable is displayed, and the available outputs are shown in the list box. When a variable is selected, the graph is displayed. When done press "Close Window" and the application will close. 


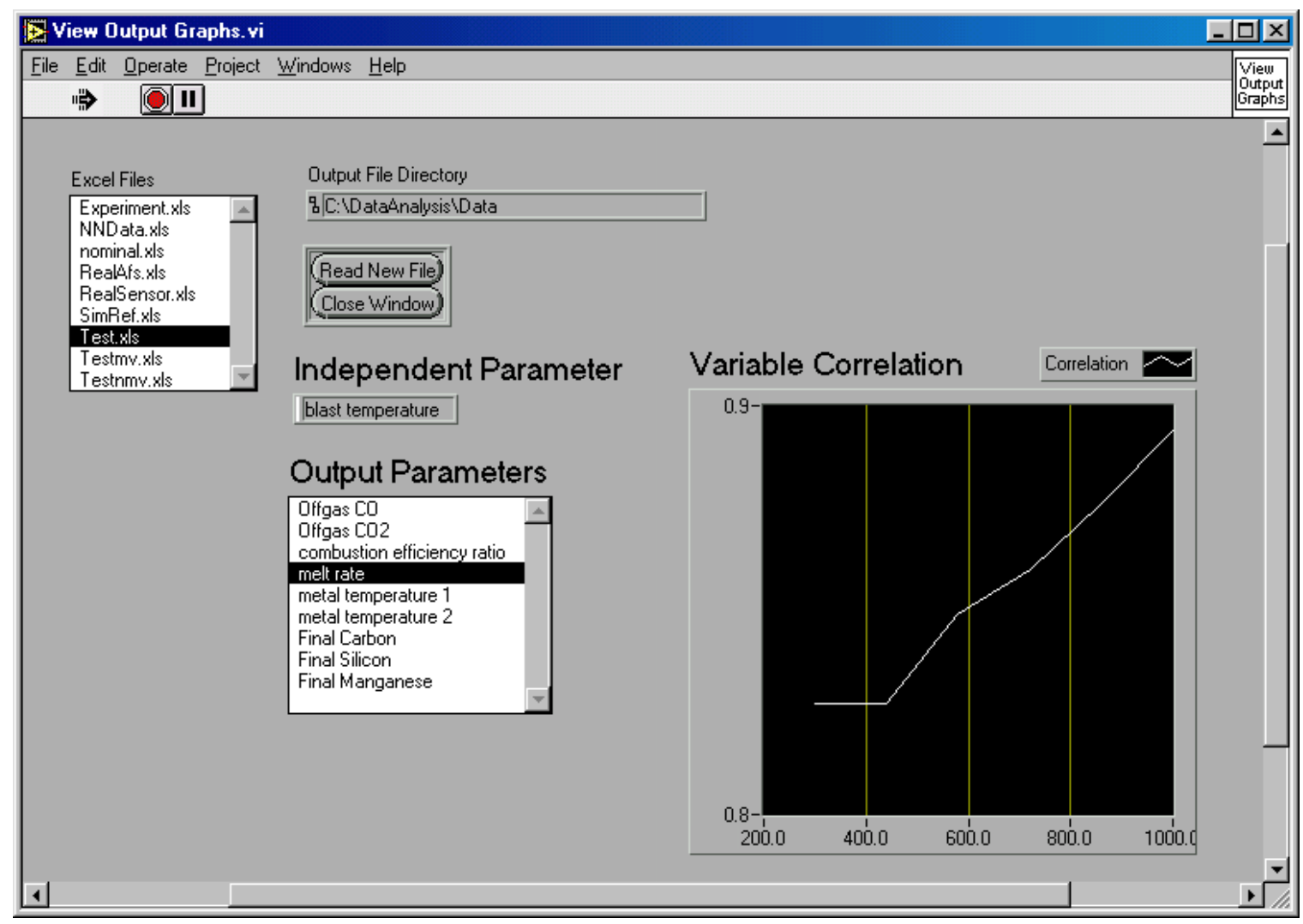

Figure A.59 View Single Variable Correlation Graph - View Output Graphs.vi

\section{A.2.6 View Multi-Variable Graphs}

A multi-variable correlation has a vast amount of information in its database. This graph viewer is designed to cut small slices out of the data and display it on the graph. There are two graphs. The input variable is displayed above the graph and the output variable is displayed to the left. Any combination of inputs and outputs can be selected from the ring boxes. All of the input variables are shown to the right of the graphs, along with the values for each variable that were used in the correlation. This allows the user to "tinker" with the various inputs and view what happens to the variable being graphed. 


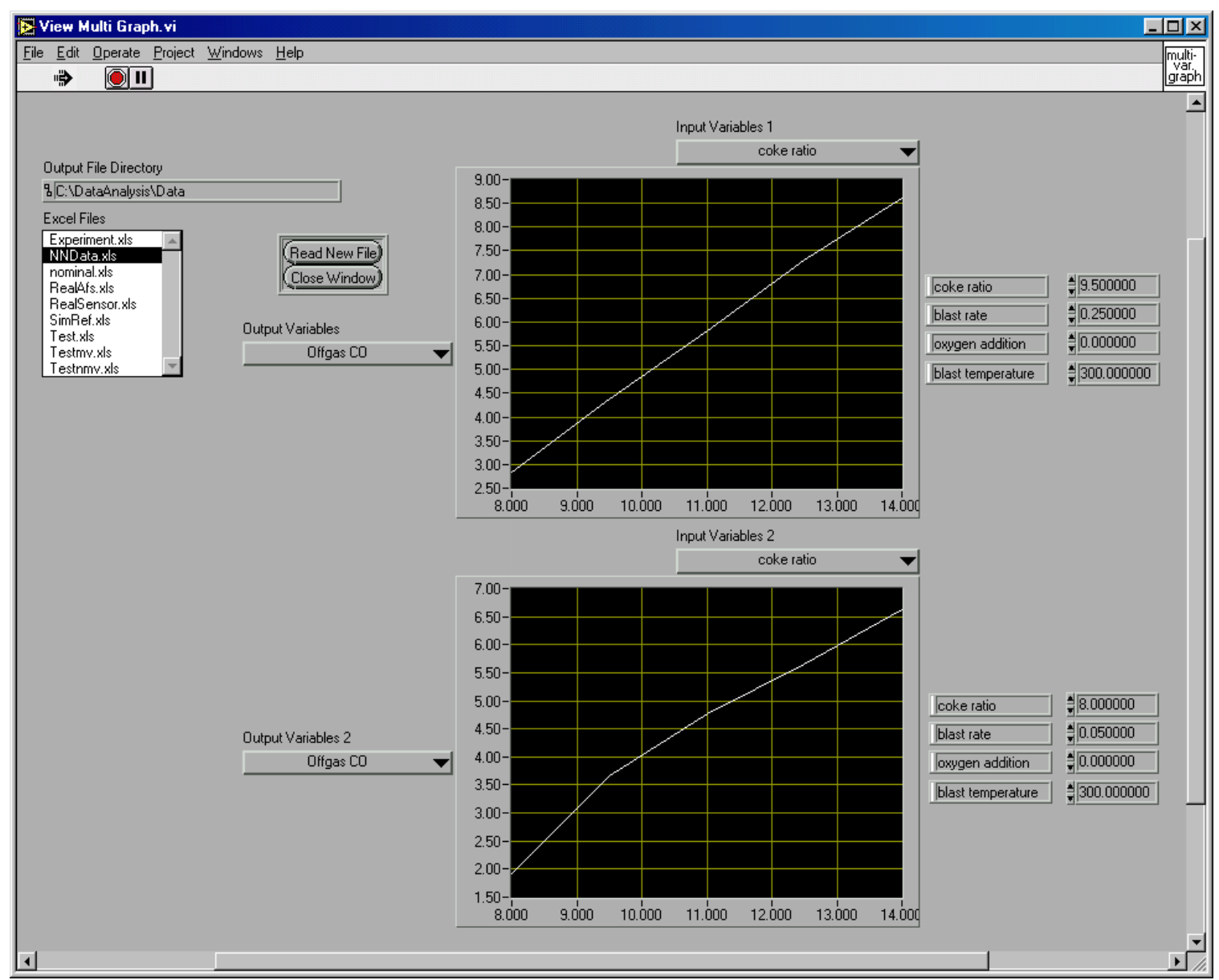

Figure A.60 View Multi-Variable Corr. Graphs - View Multi Graph.vi

\section{A.2.7 View N/M Correlation Graphs}

The nominal-value multi-variable correlation creates a spreadsheet file that shows the relationships between varying a large number of inputs and outputs. The graph application is set up like a matrix. The columns of graphs are all associated with the input parameter shown at the top of the column. The rows are associated with the outputs shown on the left side of the row. This configuration creates a four-by-four matrix of graphs, showing the user the trends of many variables at once. File selection is the same as before. 


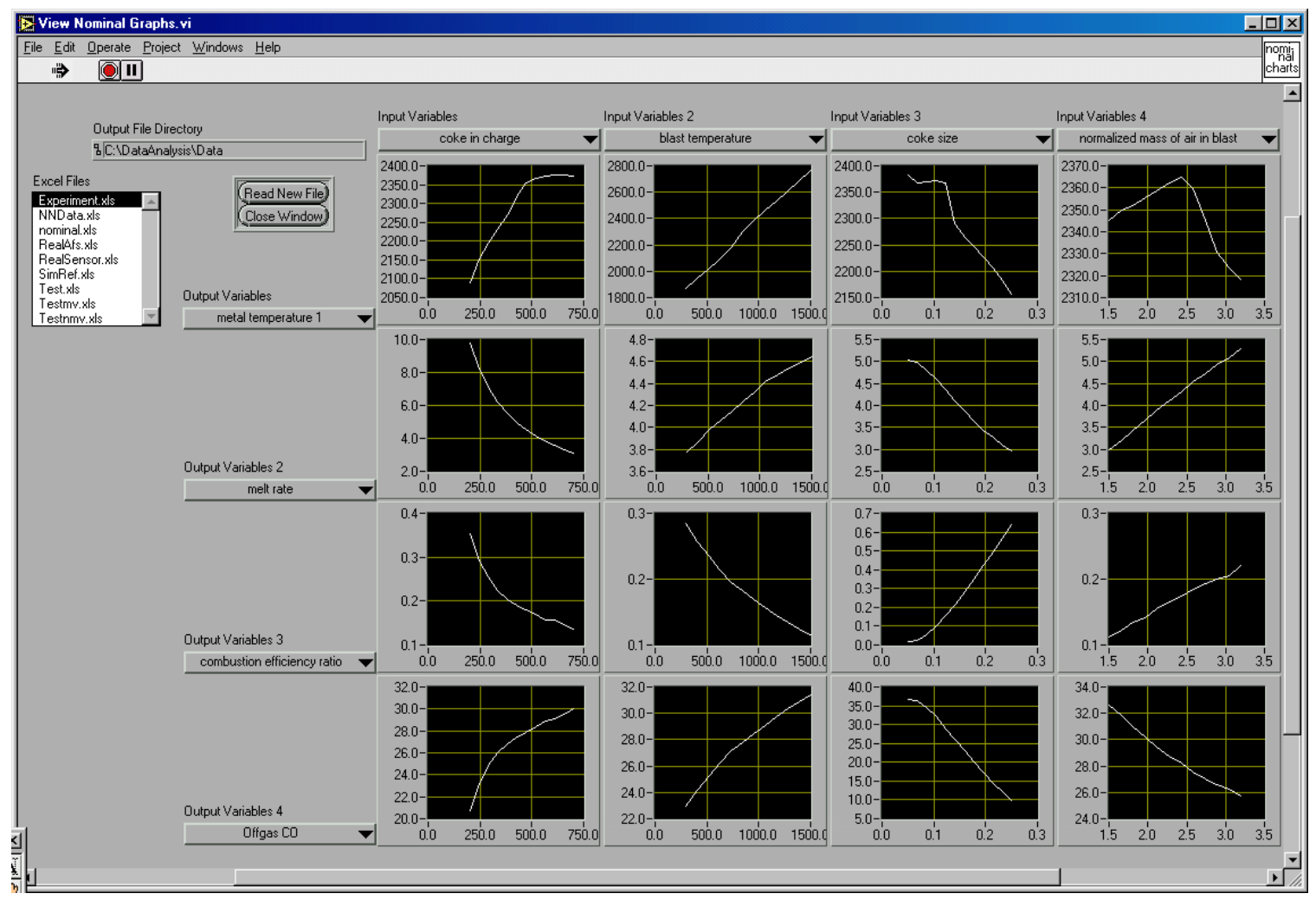

Figure A.61 View N/M Correlation Graphs - View Nominal Graphs.vi 


\section{A.3 Online Analysis}

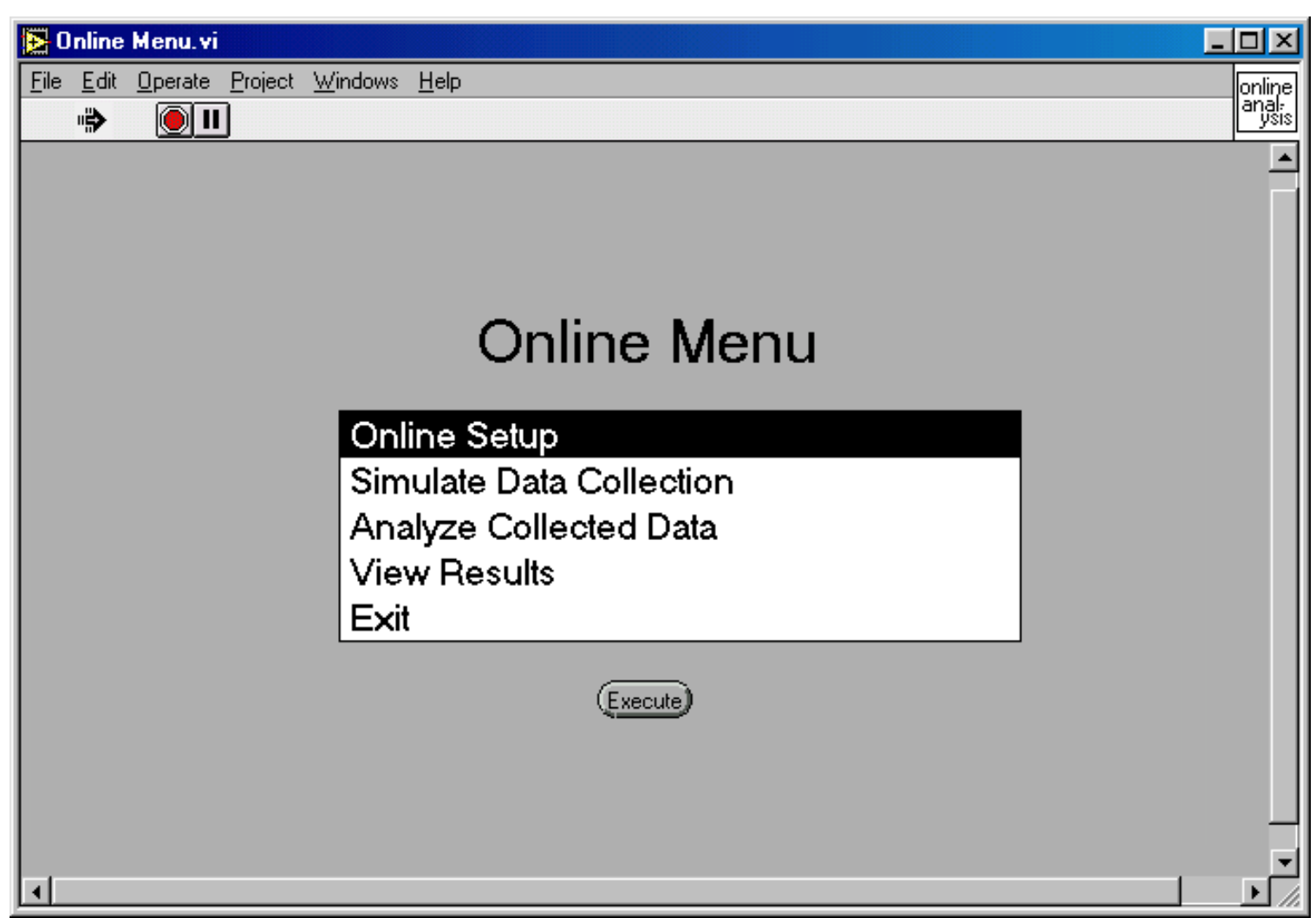

Figure A.62 Online Analysis Menu - Online Menu.vi

The online menu is used to interrogate the models in a real time situation. The 4-D array data structure is setup and populated by the online analysis functions. The 4-D array is stored in a data file that any of the applications (data collection, sensor fusion, controller, interrogator, etc.) on the cupola network will be able to access. A setup file is also created that defines what information is stored, and its location in the data structure. A third file is maintained that keeps count of how many data points have been collected and how many have been processed. This is done so that each separate application can access the information it needs regardless of where the application is running.

\section{A.3.1 Online Setup}

The online setup menu requires the user to input the current run name. Setup then creates the three files discussed in the previous section with the run name as the file 
name. The extensions are .dsc for the data structure file, .dsv for the data structure variable list, and .dsi for the data structure counter file.

The data $<$ run name $>$.dsv file is initialized with the setup data that was chosen during setup, this file should not be modified by any other applications. The <run name $>$.dsc file is initialized with zeroes in the proper dimension sizes according to how many modalities, variables, and variable properties were selected. The time dimension is initialized to five, and expanded dynamically as the data points are collected. The counter file is initialized to zero.

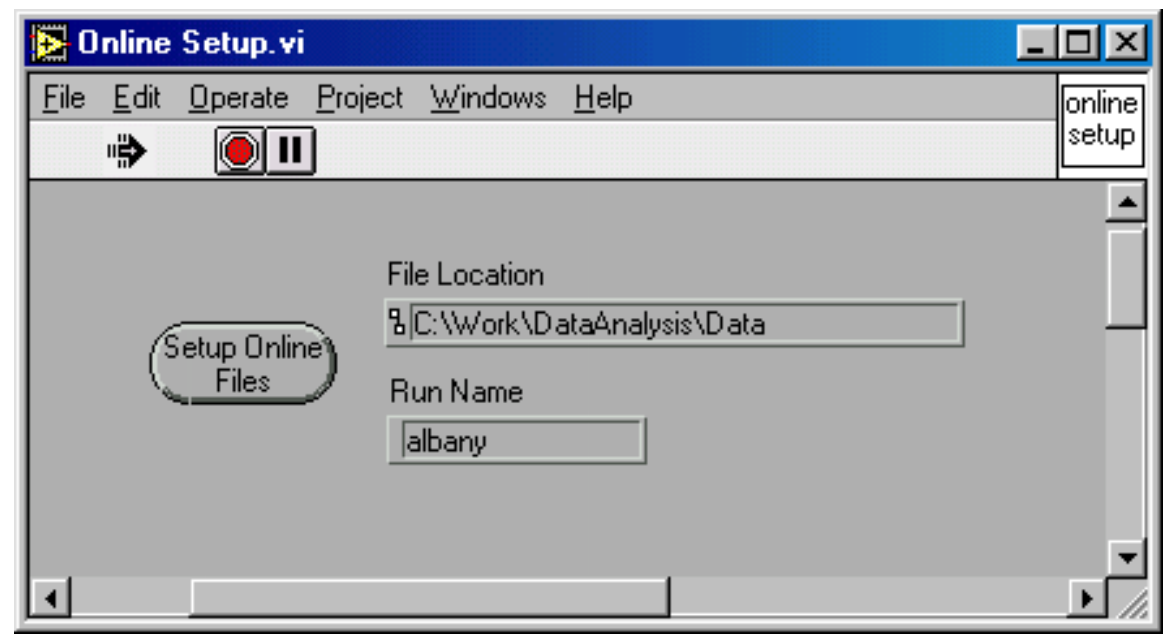

Figure A.63 Online Setup - Online Setup.vi

\section{A.3.2 Simulate Data Collection}

If the data is being collected from an existing data file, this option is selected. The current data file is displayed in the path field. If that needs to be changed, press the "Declare Data File" button and declare the text file with the sensor information and data. The selected modalities are shown in a listbox, select the modality that holds the sensor information that will map the text file data into the standard grammar. Next press the "Declare Run Name" button. Select the run name that was created for the current run. Once this is done the "Collect Data" button becomes enabled, press it and the data is read from the text file into the data structure and written to the data structure file. The counter file is also updated to indicate how many rows of data were collected. While data is being collected, all the buttons are disabled. Once the process is complete, they are 
enabled. You can collect data from another file or return to the previous menu at this point.

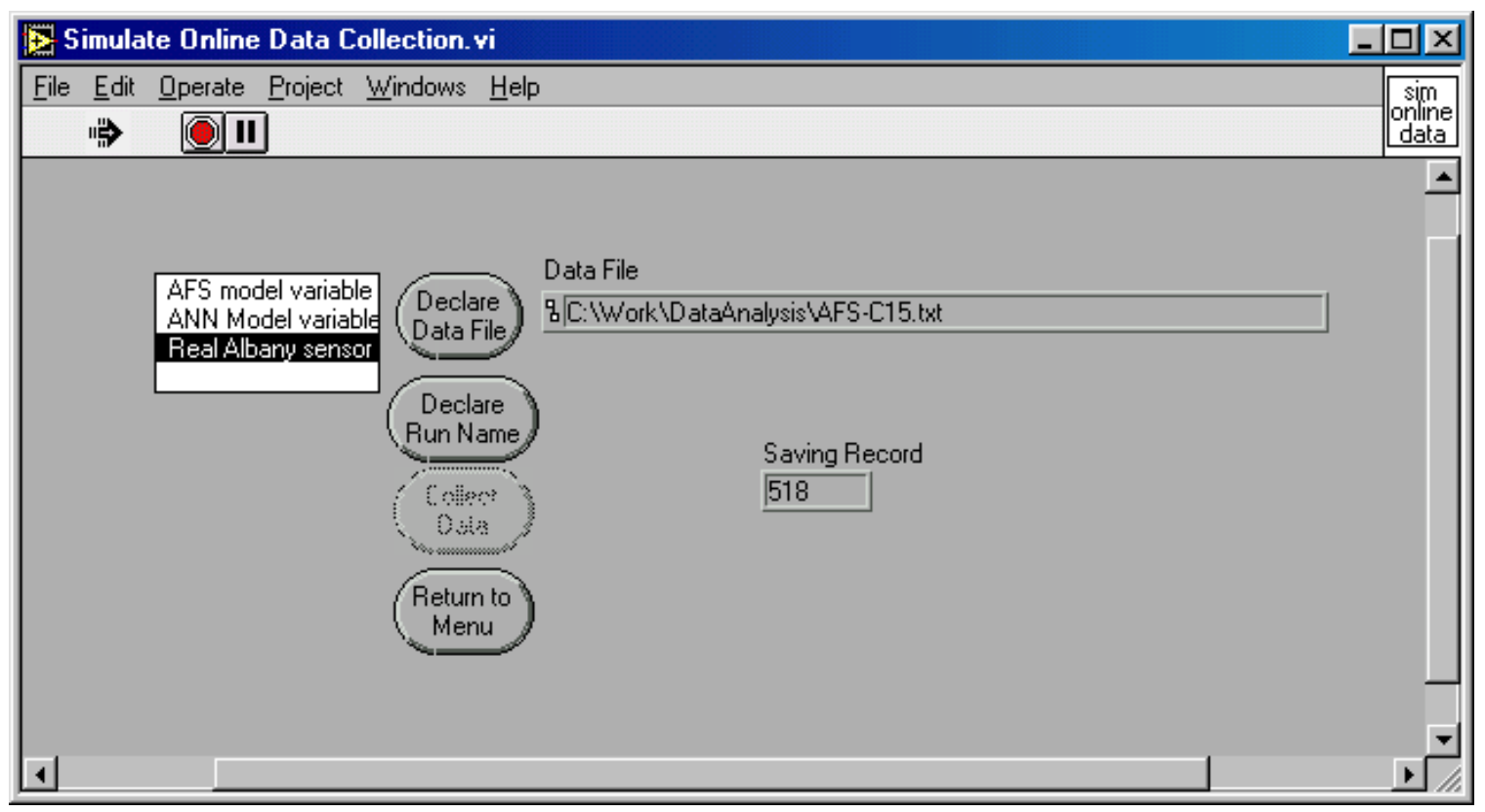

Figure A.64 Simulate Data Collection - Simulate Online Data Collection.vi

\section{A.3.3 Analyze Collected Data}

This function interrogates the models with the data that has been collected. The data comes either from a text file as described in the previous section, or it is being collected from a cupola in real time. Once again, be sure the correct modality is selected and declare the run name first. To start interrogating the models, press the "Run Model Analysis" button. The VI keeps track of how many data sets it has processed, and compares that number to the counter in the counter file. If there are data sets that have not been processed, the VI reads the next data set into the standard grammar and runs it through the models. When a data set has been analyzed, the analysis VI increments a counter. If there is no additional data to analyze, the VI waits and checks again a little later. When there is no more data to collect, either the cupola run is over or there is no more data from the text file, press Return to Menu to end the analysis and close the VI. 


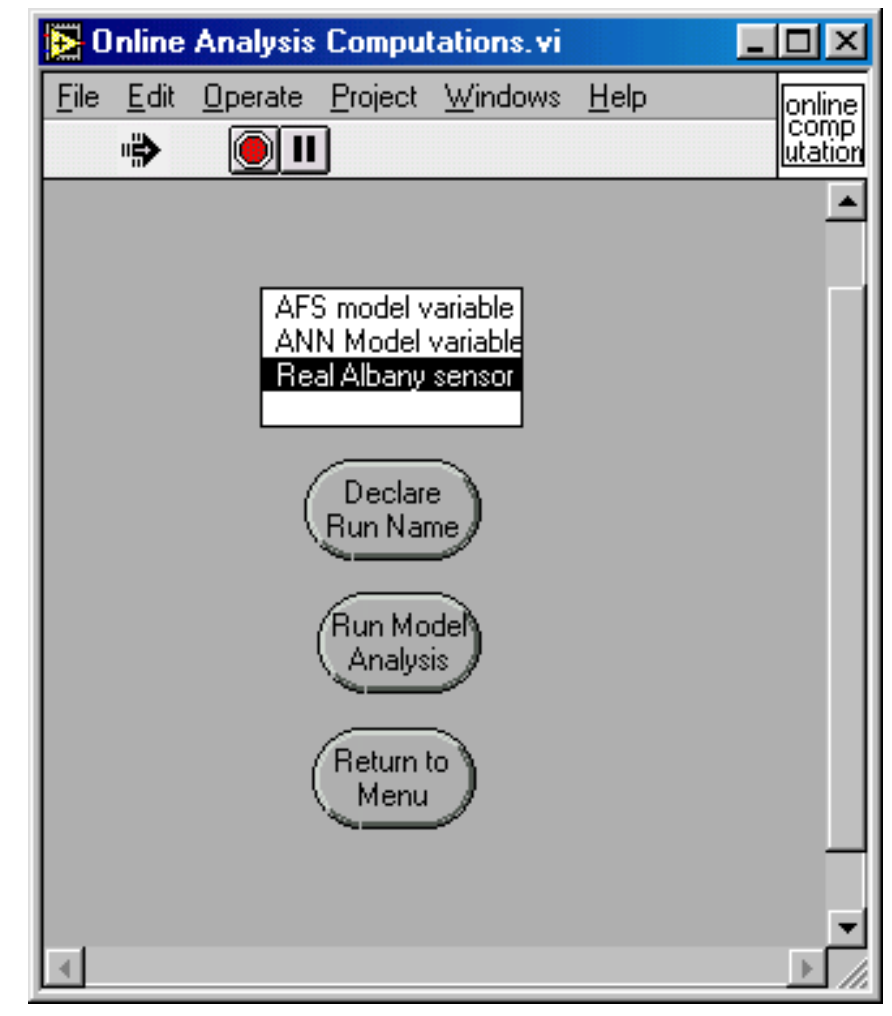

Figure A.65 Figure 24 - Analyze Collected Data - Online Analysis Computations.vi

\section{A.3.4 View Results}

The results of the analysis are viewed with this VI. Declare the run name as before, then press "View Data." The data is read in to the arrays and the variables and modalities are displayed in the ring boxes above the graph. Any combination of inputs, outputs, parameters, and modalities can be selected. Up to three lines of data can be placed on the graph. If you want to select a different run, for instance if you want to see how a previous run looked, press the "Reset Data" button, and declare a new run name and continue as before. 


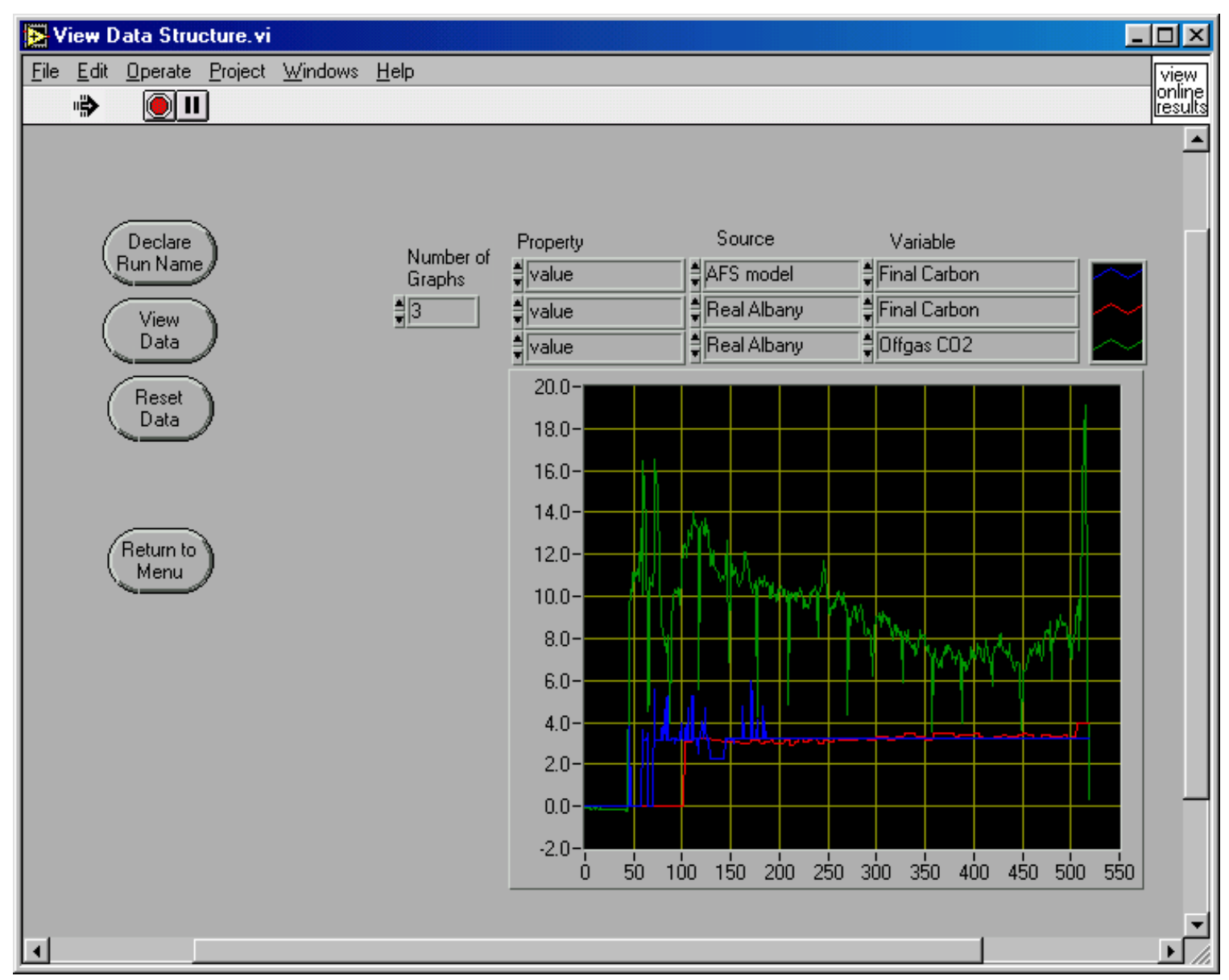

Figure A.66 View Results - View Data Structure.vi

\section{A.4 Model Interfaces}

\section{A.4.1 AFS Model Interface}

The AFS Model is currently the most important model available, much of the previous work was designed with the AFS model in mind, although the interrogator should be easily applied to any model. If used properly, the AFS model interface requires no user intervention in order to run. This is because of the potentially large number of model runs involved in a correlation analysis. 


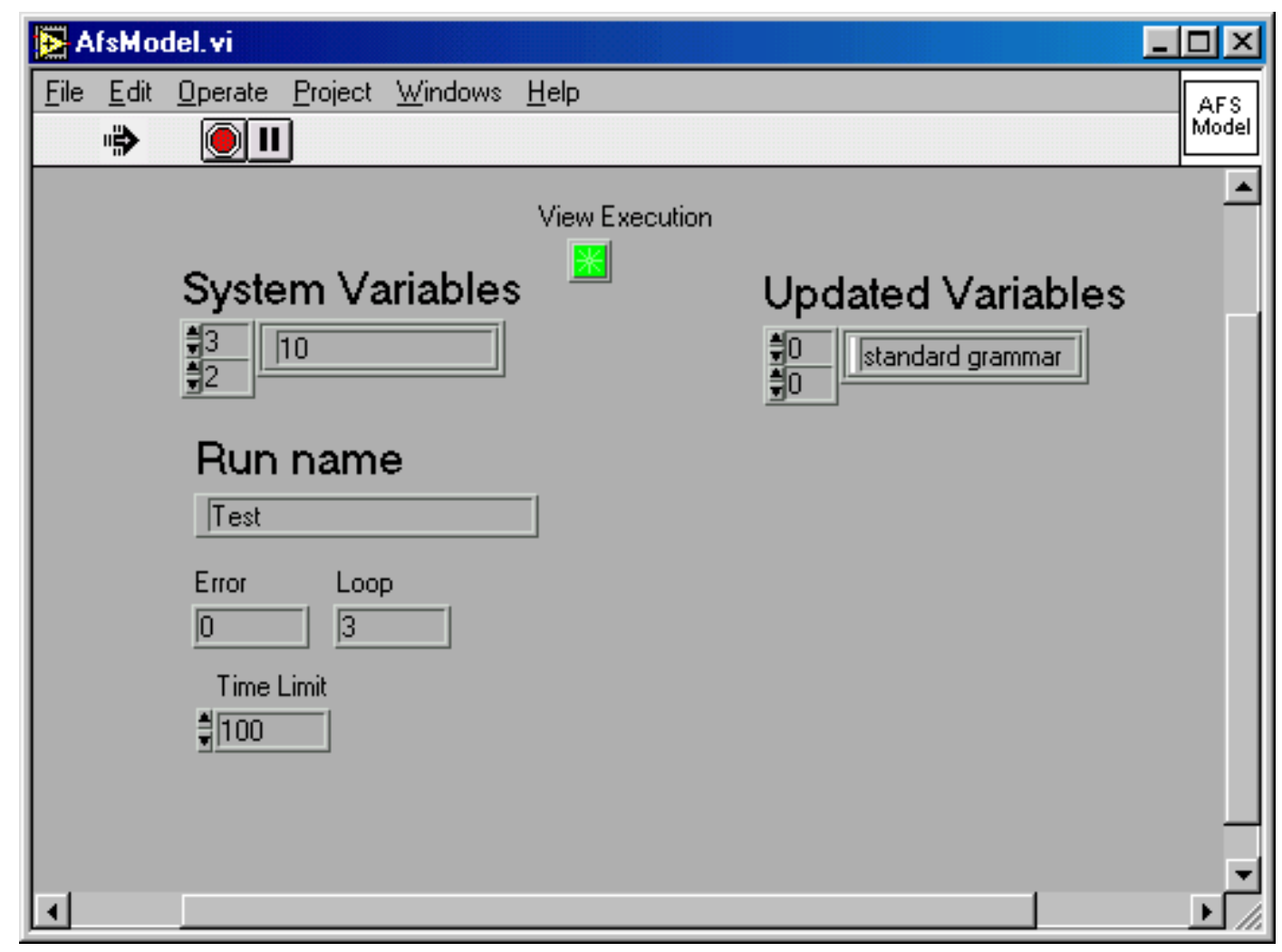

Figure A.67 AFS Model Interface Screen - AfsModel.vi

When the model runs, a DOS window opens and displays some error information about the numerical approximation. The DOS window should be set to close upon execution, this is done by opening a DOS window, selecting the properties button, and checking the box marked "close on exit." This is to avoid having 400 DOS windows open at the end of a correlation.

\section{A.4.1.1 AFS Setup}

There are a few parameters that must be defined at setup in order for the AFS model to work. This is done using the "Additional Model Specific Setup" option on the Setup menu.

\section{A.4.1.1.1 Define AFS File Paths}




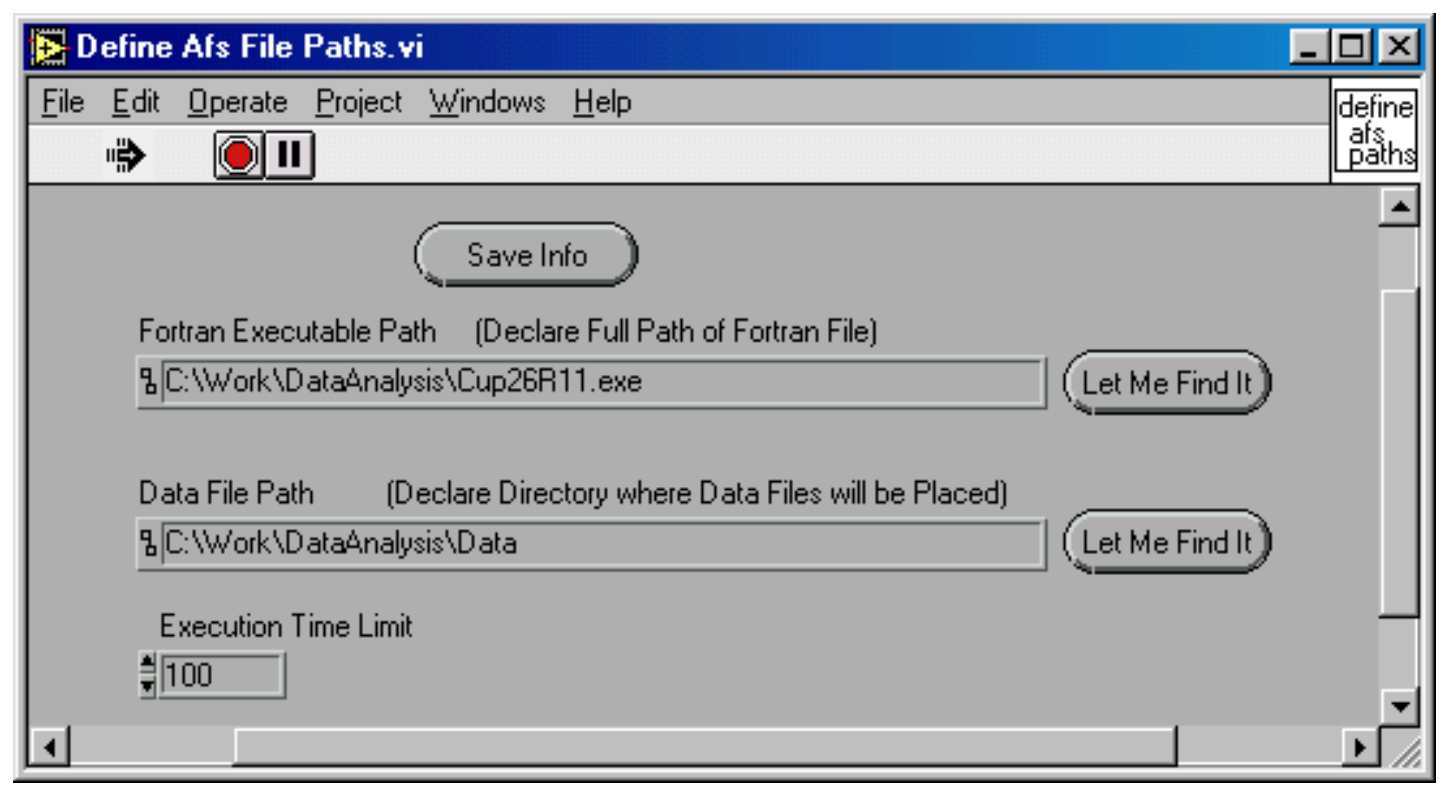

Figure A.68 Define AFS File Paths - Define Afs File Paths.vi

The Fortran executable file needs to be fully declared at this point. The fortran file is called from the DOS prompt, therefore the file path needs to be compatible with DOS rules. The path to the executable should not have any spaces in it, DOS does not handle directory names with spaces in them.

The AFS model creates a large number of data files, the second field defines where they will be written. This may or may not be the same directory where the other data files were placed (the Excel files and data structure files). You may want to choose a different directory to keep the files organized.

There will be combinations of inputs that cause the numerical model to be nonconvergent. The AFS Model interface waits for the numerical model to finish writing the output files before reading the values, if the model hangs so will the interface. To avoid this situation, the interface times out after a set time limit. That time limit is set here, and is dependent on the machine processor speed. A little experimentation should be done in order to determine the best setting for each individual computer. For a $300 \mathrm{MHz} \mathrm{PC}$, 150-200 seconds seemed necessary, for an $800 \mathrm{MHz}$ PC, 100 seconds is sufficient.

\section{A.4.1.1.2 Charge Selection}

The AFS model can accept up to 10 charge materials. There are numerous conditions that these materials must meet in order for the model to run correctly. The following 
procedure is the best that I can come up with for allowing the user to vary the charge material.

The first step is to use the original model interface (the AFS interface) to define the charges to be used (See the documentation with that program for information on how to do this). Run the AFS model using the new charge makeup, then print the cin.264 file that is created. The file that our interface creates must exactly match the other.

\section{A.4.1.2 Metal Selection Option Menu}

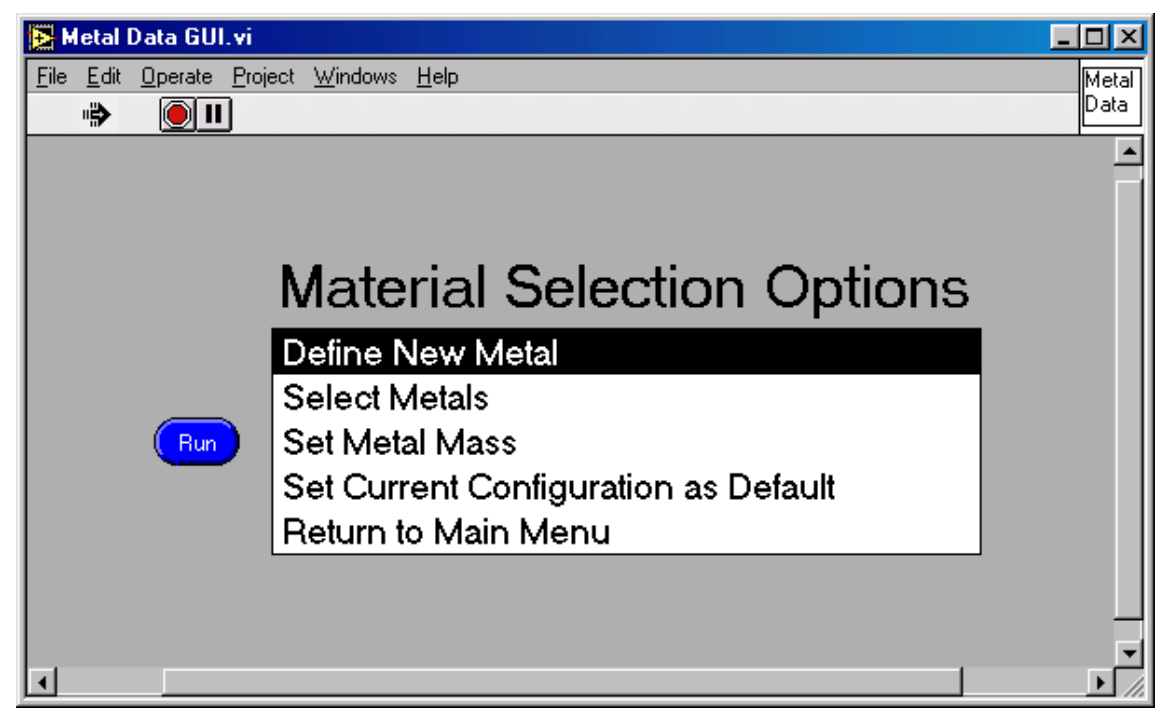

Figure A.69 Material Selection Options - Metal Data GUI.vi

\section{A.4.1.2.1 Create Material Property Files}

Select the first option to open a screen that creates a new metal. This screen has fields for all the variable names that the AFS model needs. Use the cin.264 file to fill in the data for one charge material at a time. It is easiest to copy and paste the data from another application so that the spacing remains correct. Be sure to double check that all the numbers are correct for the metal that is being declared. When all the data is correct for the material, press the green "Save File" button. If there are more metals to declare, fill in the fields as before, otherwise press "Done." 


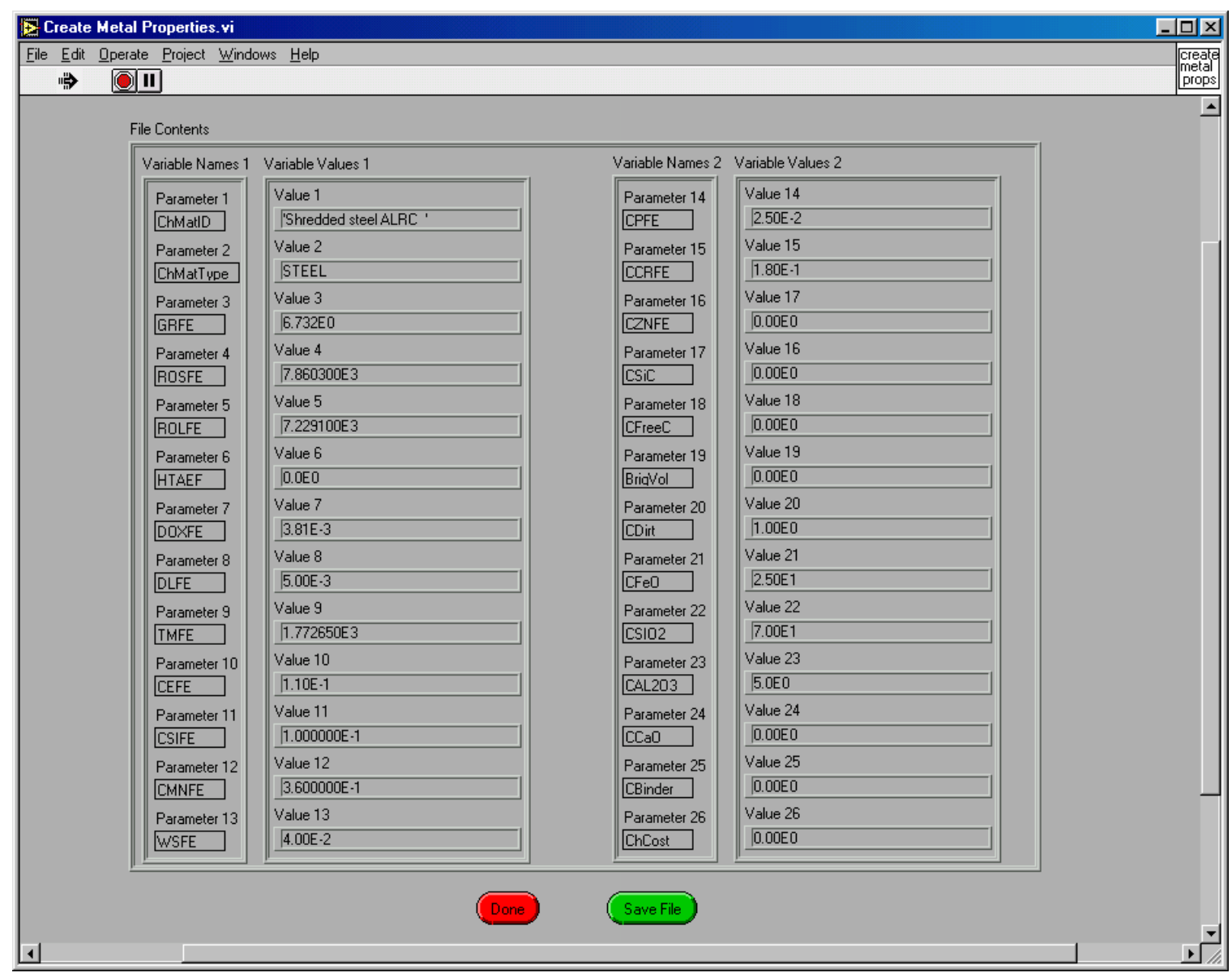

Figure A.70 Material Property File Creation - Create Metal Properties.vi

\section{A.4.1.2.2 Material Selection}

Once all the metals are created, the second option allows the user to select the metals that will be used. At the upper left of the screen there is a field that says "Search Pattern." If you use a common parameter when declaring your metal names (such as ALRC for the Albany Research Cupola), you can use that key to filter out the material files that you won't be using. Select the materials from the lists in the same order that they appear in the cin.264 file or else the AFS model won't execute properly. 


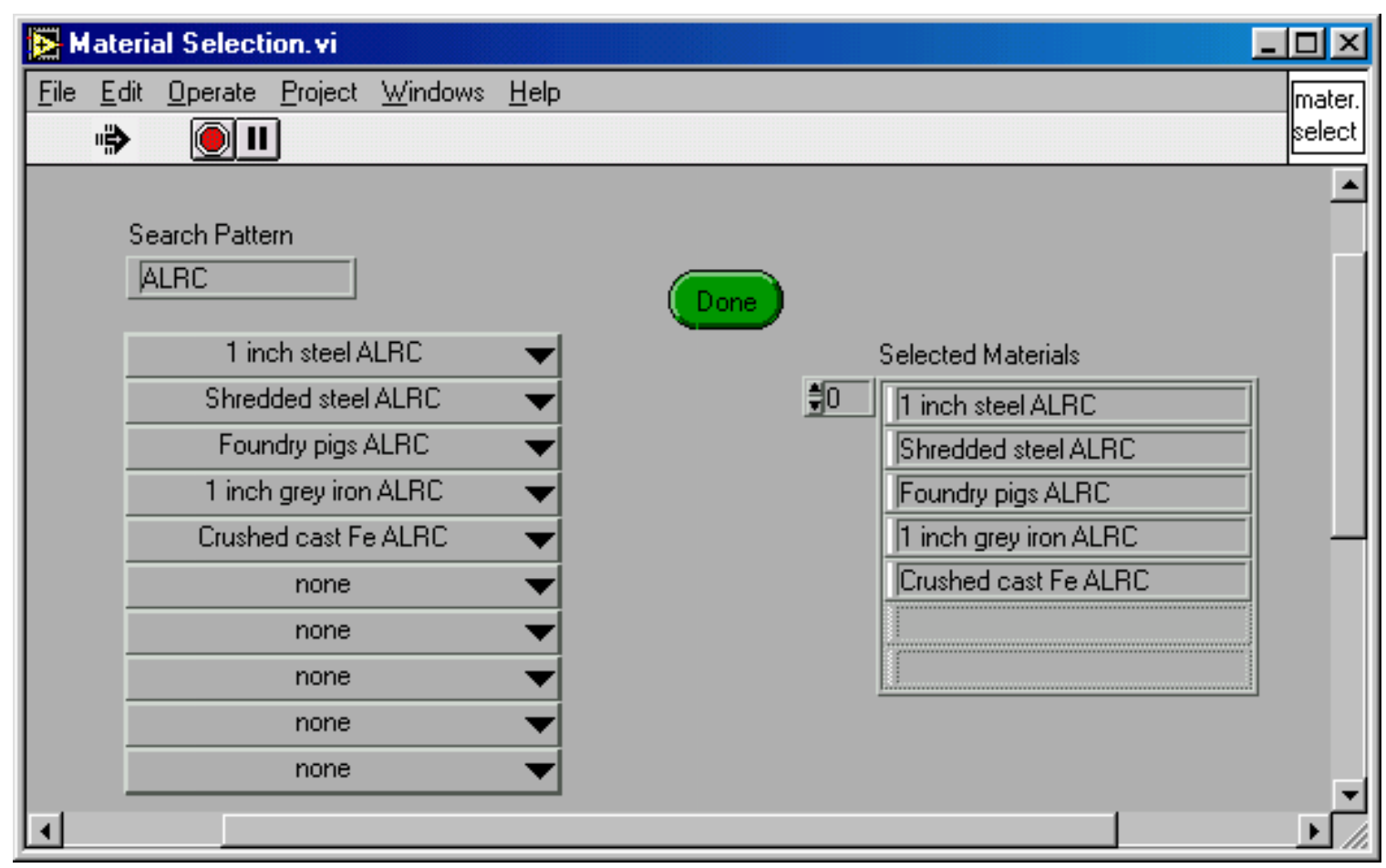

Figure A.71 Material Selection - Metal Selection.vi

\section{A.4.1.2.3 Set Metal Mass}

The one parameter that can be varied from the original cin.264 file is the metal mass. Use this option to change the mass of each material.

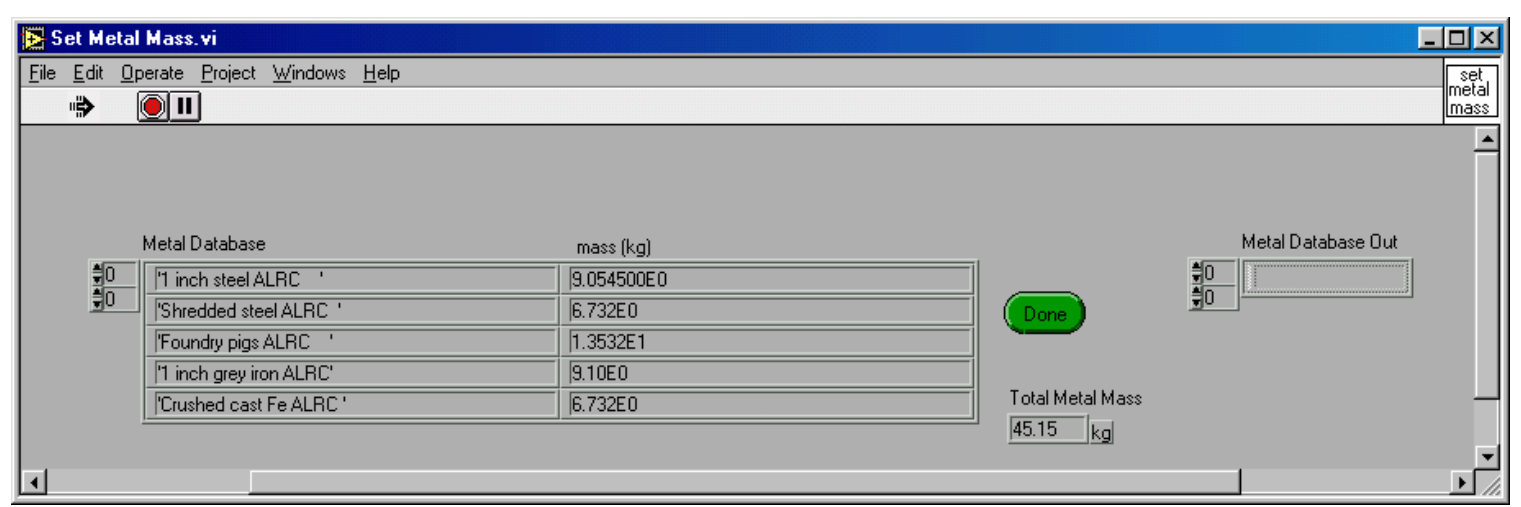

Figure A.72 Set Metal Mass - Set Metal Mass.vi

\section{A.4.2 AFS Preprocessor}

The AFS model has some interesting input parameters that do not correspond directly to commonly used industry terms. A preprocessor was written that converts the common industry terms (a list is included below) to the eccentric terms required by the AFS 
model. If both variables are chosen for inclusion in the database, or in a correlation, the user is asked to choose which variable to use as the input value. If this proves to be a hindrance, perhaps a more elegant solution can be reached. The figure shows the option screen, pressing the button by the desired variable selects that variable and the corresponding value is passed along as an input.

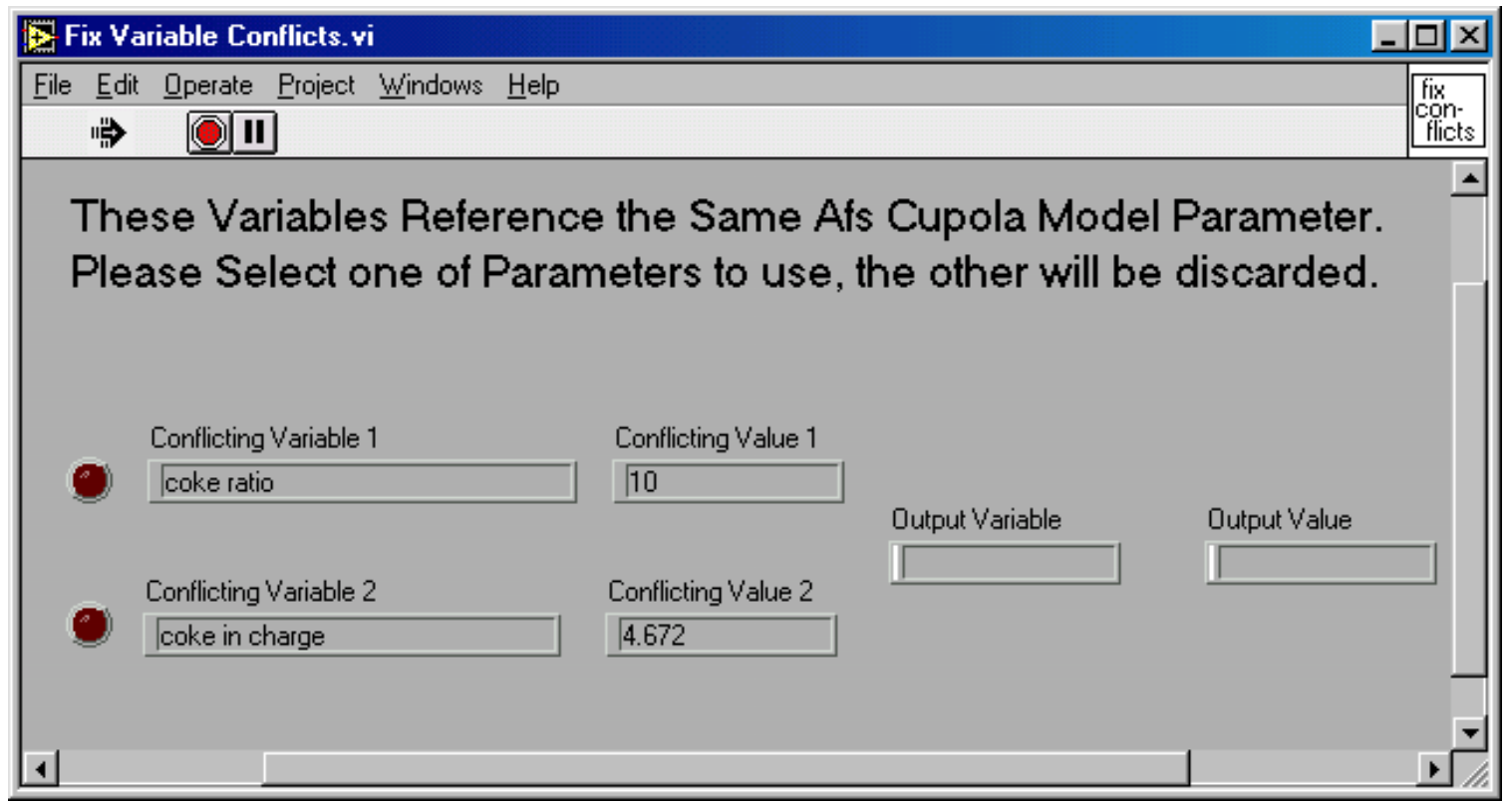

Figure A.73 Conflicting Variable Resolution - Fix Variable Conflicts.vi

The list of conflicting variables is:

AFS Model Variable
normalized mass of air in blast
normalized oxygen addition in blast
coke in charge
amount of moisture in air

AFS Model Variable

normalized mass of air in blast coke in charge

amount of moisture in air 
Unfortunately, most of the values in the sensor data file are expressed in British units; the AFS model requires metric units. The conversions are made by referring to the "Default British Unit" field in the standard grammar. Sub-VIs are called to make the conversion based on the name of the unit. For example if the British unit is F, for degrees Fahrenheit, the function F.vi is called. This function converts from degrees Fahrenheit to Kelvin, the default metric unit. That is why the unit field must always be filled, if it is empty $<$ blank $>$.vi is called, and that file can not exist. The conversion VIs are in fact quite simple to create, so if a new variable (with a new unit) is added to the standard grammar, a sub-VI by that name should be created to handle the conversion. All the unit conversion VIs are stored in DataAnalysis\Online Analysis\Unit Convert directory.

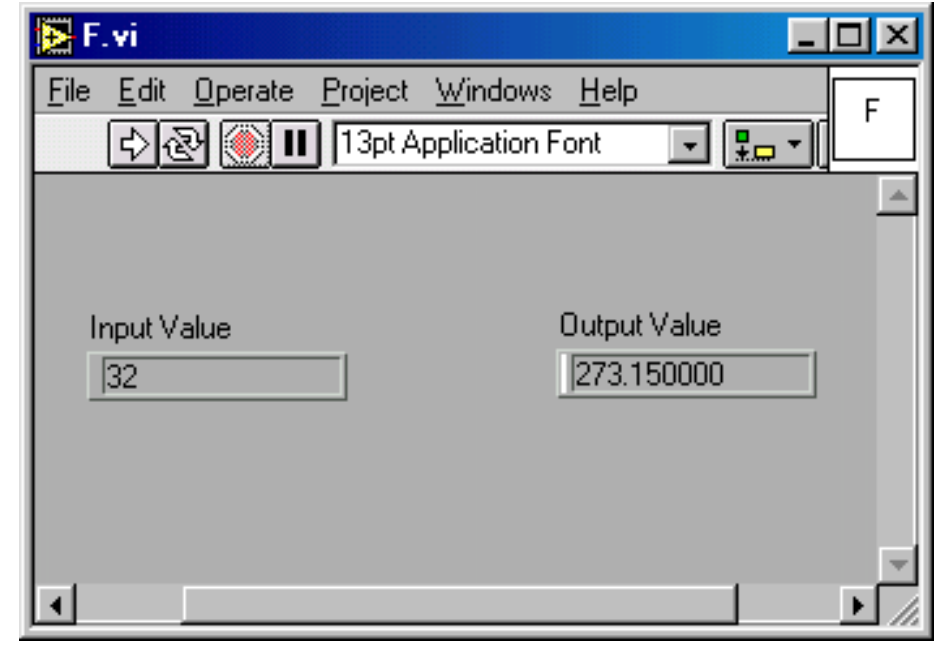

Figure A.74 An example unit conversion - F.vi

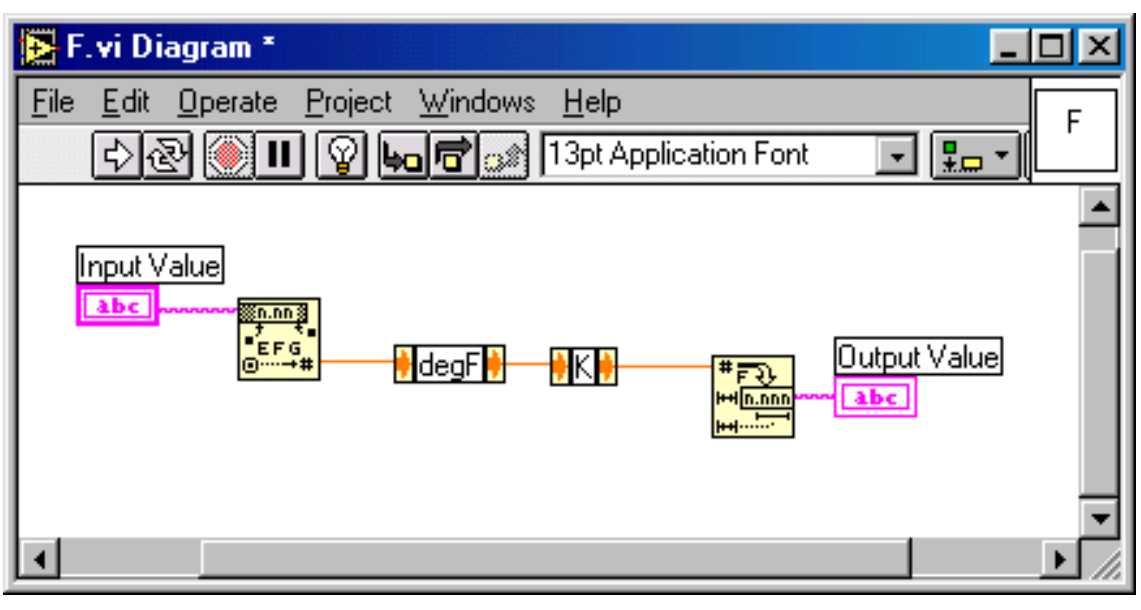

Figure A.75 An example unit conversion diagram - F.vi 
Section 2

\section{Hardware Implementation}


1 YEAR 1 ACCOMPLISHMENTS ....................................................... 5

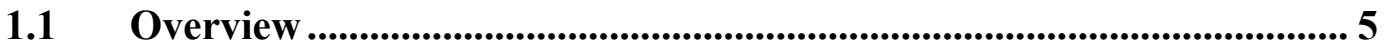

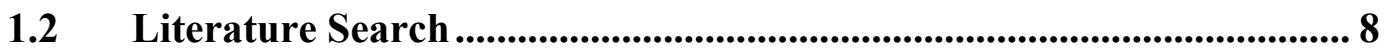

1.3 Hardware Component Acquisitions ................................................ 10

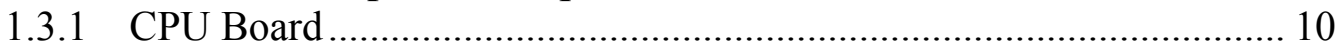

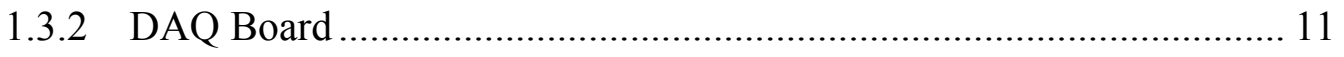

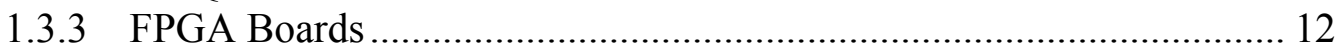

1.4 Analysis and Validation of Algorithms ........................................... 13

1.4.1 Self-Validation Fuzzy Logic ........................................................ 13

1.4.2 Self-Validation Preprocessing ....................................................... 15

1.4.3 Self-Validation Execution Timing.................................................... 16

1.4.3.1 Determination of Timing with Hardware Timer......................... 16

1.4.3.2 Determination of Theoretical Timing ..................................... 17

1.5 Self-Validation Software Implementation .......................................... 18

1.5.1 Self-Validation Fuzzy Logic Code .............................................. 18

1.5.2 Self-Validation Preprocessing Code ............................................... 20

1.6 Communication Software Development - the CPU-to-Host Interface 21

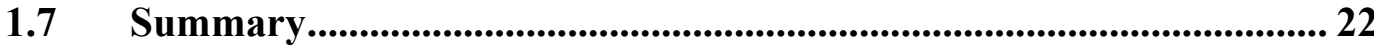

2 YEAR 2 ACCOMPLISHMENTS .................................................. 23

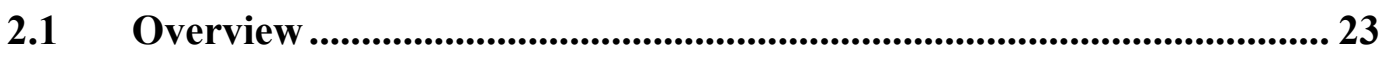

2.2 Communication Protocols ...................................................................... 24

2.2.1 Develop low-level communication protocol..................................... 24

2.2.2 Write and test the low-level communication code for initialization,

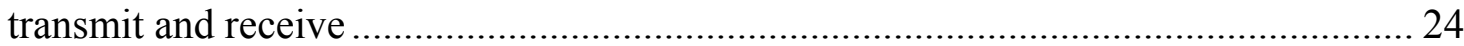

2.2.3 Define specifications for high-level communication protocol details for

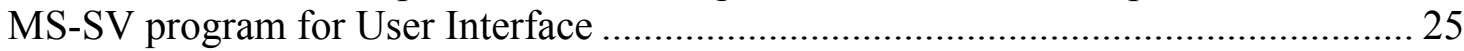

2.2.4 Outline method for modification of SV code .................................. 25

2.3 Develop a Library of Basic Fixed-Point Arithmetic Functions .......... 25

2.4 Implementation of the SV Preprocessing Algorithm.......................... 26

2.5 Develop Architecture of SV Signal Processor Hardware..................... 27 
2.5.1 Select SV procedures for the hardware implementation..................... 27

2.5.2 Separate constants from true Variables............................................ 27

2.5.3 Simplify the fuzzy logic procedures .................................................. 27

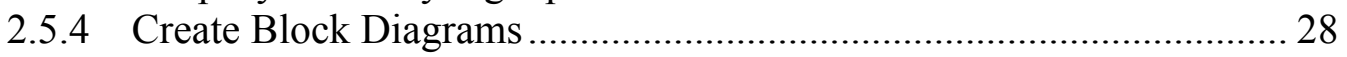

2.5.5 Define Data Structure and Organization.......................................... 28

2.5.6 Define finite state machine controllers .............................................. 28

2.6 Develop Hardware Design of SV Signal processor .............................. 29

2.6.1 Code Hardware Blocks in VHDL ......................................................... 29

2.6.2 Simulate Each Entity Code Separately ................................................ 29

2.6.3 Design of the system interfaces ..................................................... 29

2.6.4 Design of the FSM ............................................................................ 30

2.6.5 Add All Blocks to Top-Level VHDL Entity........................................ 30

2.6.6 Download, Test, and Debug Top-Level SV Signal Processor.............. 30

2.7 Develop Multi-sensor SV Algorithm .................................................. 31

2.8 Develop Multi-Sensor Fusion Algorithm ........................................... 31

2.9 MSF C Code Optimization............................................................ 33

2.10 Develop the MSF fixed-point code.................................................. 34

2.11 Hardware implementation of the MSF code ................................... 35

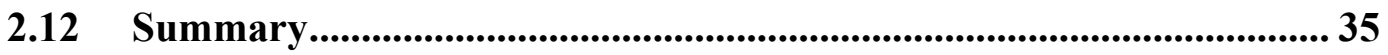

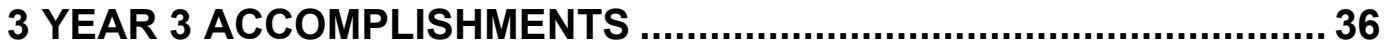

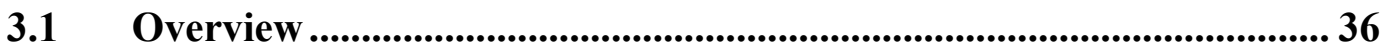

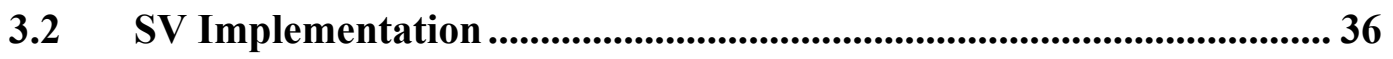

S.3 SV Speedup.................................................................................................... 37

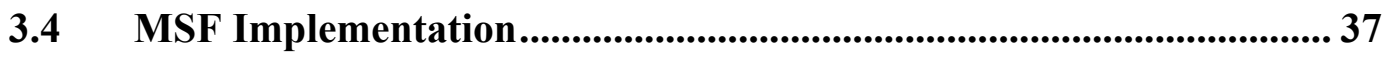

MSF Block diagrams ................................................................................ 44

3.6 Virtex FPGA Board (APS V240) .............................................. 45

3.7 CPU Board ............................................................................................ 46

3.8 Communication ............................................................................. 47

3.9 Host PC Application .................................................................................. 47

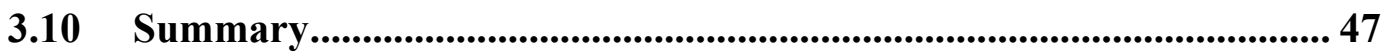




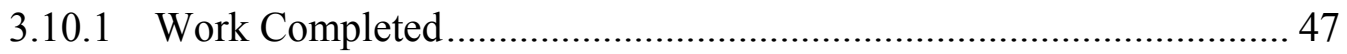

3.10.2 Future Recommendations ......................................................... 49

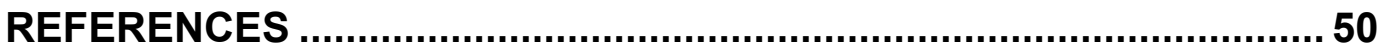

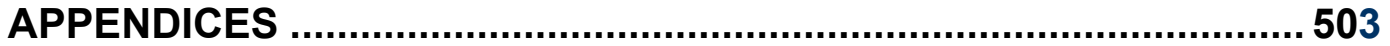




\section{Year 1 Accomplishments}

\subsection{Overview}

During the year 1999, the $I^{3}$ PSC Hardware Team completed much of the background work needed to begin the implementation of the Signal Processing System Hardware portion of the project. We first examined the literature available that was pertinent to our work, which included learning the software tools we needed and the signal processing methods and hardware implementation techniques available. We also

researched the possible system organizations, communication requirements, and commercial boards available for the embedded microcomputer (CPU), data acquisition interface (DAQ), and programmable logic (FPGA - Field Programmable Gate Array) needed for computationally intensive tasks.

After making decisions about our functional and cost requirements, we then selected and purchased the appropriate commercial boards: one CPU, one DAQ, and four FPGA boards. Basic testing and familiarization work was done on the CPU and FPGA boards, while the DAQ board has not yet been tested.

At this time, the overall system consists of two algorithms, Self-Validation and Sensor Fusion. The Self-Validation algorithm, whose hardware implementation is now in progress, inputs the raw time-temperature measurements from sensors, derives some characteristic quantities and filtered outputs, and then applies fuzzy logic to determine a self-confidence value for each sensor. The Sensor Fusion algorithm, whose hardware 
implementation work has not yet begun, combines the filtered inputs and self-confidence values from several sensors into one robust value.

As the first step toward hardware implementation, we analyzed, validated, and refined the Self-Validation algorithms that were supplied by the Intelligent Algorithms Team. The algorithms were divided into two sections for our convenience, specifically referred to as the fuzzy logic and preprocessing portions. Work is proceeding on evaluating the execution timing of the Self-Validation algorithms to assist us in choosing the optimal functions for hardware implementation versus software implementation.

The Self-Validation algorithms were supplied to the Hardware Team in the form of high level Matlab and Excel code. We decided to first implement them in faster, lower-level C-language code, mostly using fixed point arithmetic, and then convert portions to even faster fixed-point hardware implementations in programmable logic on the FPGA boards. After some optimizations and debugging effort, the C-coded versions of the fuzzy logic and preprocessing portions were successfully verified against the original Matlab and Excel results.

The CPU board will eventually have to communicate in three ways:

1. To the host computer for the user's interface to the system,

2. To the DAQ board for data acquisition from the sensors, and

3. To the FPGA boards for signal processing computations.

The CPU-to-Host interface consists of an error detecting/correcting serial communication protocol and its implementation as $\mathrm{C}$ code executing on the CPU board and on the host computer. The protocol has been specified and a limited version of the $\mathrm{C}$ code has been written and verified. Work on the other two communication interfaces has 
not yet begun. The following figure shows the Signal Processing System Hardware and its interfaces.

Signal Processing System Hardware

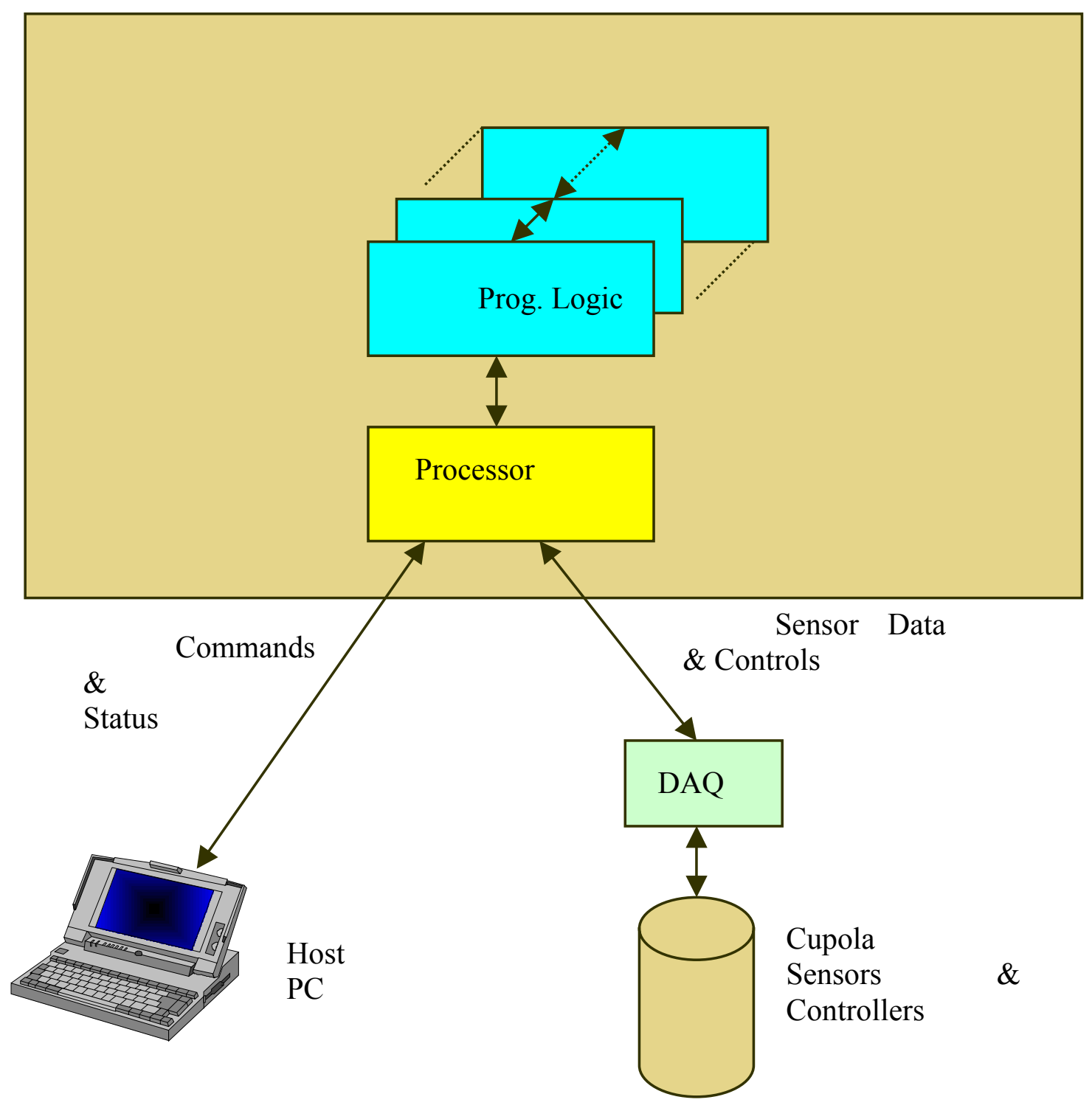

Over the next two years, the Hardware Team will complete the project's hardware implementation. The Self-Validation algorithm will be implemented and tested in programmable logic. The Sensor Fusion algorithm will be analyzed, re-written, and implemented in programmable logic. The communication interfaces to the FPGA board 
and DAQ board will be designed and tested, thus completing the entire hardware/software system.

\subsection{Literature Search}

The Hardware Team conducted a lengthy literature search in different software and hardware areas. The $I^{3} P S C$ project requires knowledge in many software and hardware areas, some of which are outside the Hardware Team areas of expertise.

In order to prepare us for the hardware implementation of the software algorithms, the hardware group members needed to educate themselves about fuzzy logic, neural networks, and data acquisition. This educational process included studying and understanding the basic definitions, terminology, and some of the theories and algorithms in the fuzzy logic, and neural networks areas. The features of the two basic types of fuzzy logic, Mamdani and Sugeno, were studied. The advantages and disadvantages of various neural networks implementation techniques, their learning processes, and their operation were also studied. The group members used all available resources in obtaining information. These resources included published papers, books, and the World Wide Web.

The group members studied and acquired experience in using some of the software commonly used in the fuzzy logic and the neural networks areas such MATLAB, the fuzzy logic toolbox using MATLAB, and the neural network toolbox using MATLAB. The group also studied the use of LabView software, which is often used with Data Acquisition Cards (DAQs).

On the hardware side, the group searched the published literature about hardware implementations of fuzzy logic and neural networks, especially those implementations 
using reconfigurable logic. The methods and techniques used in some of these implementations were studied and summarized for future use. The group also studied the data books of programmable logic devices with concentration on the logic families produced by Xilinx and Altera. The study educated the group, especially the graduate students, about the state of the art families of field programmable logic arrays (FPGAs) in terms of their logic capacities, features, structure, interconnection, and speed. The programmable logic device study was complemented by another search of commercially available FPGA boards. These boards were evaluated based on their logic capacity, speed, external RAM availability, the width of their interface busses, and cost.

The Hardware Team also searched the published literatures about serial and parallel communication protocols. The study helped the group understanding the features, requirements, capabilities, and limitations of various communication protocols. Based on this study, a serial communication protocol was designed to control the traffic between two computers.

We researched the commercially available microprocessor boards. These boards were evaluated based on the type and speed of the microprocessor, the size of RAM available on board, the type and size of their external buses, the software used in downloading programs onto the board, and their cost.

We also researched commercially available DAQ cards. These cards were evaluated based on their sampling rate, number of input channels, number of output channels, programmability, and cost.

The studies about FPGA boards, microprocessor boards, and DAQ boards helped the Hardware Team in preparing the specification list for purchasing these devices. 


\subsection{Hardware Component Acquisitions}

We selected, purchased, and tested an appropriate CPU board, DAQ board, and four FPGA boards for this project. Following are their descriptions.

\subsubsection{CPU Board}

We wanted a compact, inexpensive, and fully PC-compatible (which eases software development) CPU board based on the common PC/104 bus. After considerable research, we decided to purchase a microprocessor board, the SBC2586-166 with options 2586OPT30-8, 2586OPT25, BO/BC3.1 from Micro/Sys, with these primary features:

PC/104 8-bit and 16-bit bus compatible

Pentium 166MHz processor

2 serial ports

$1 \mathrm{EPP} / \mathrm{ECP}$ parallel printer port

512 KB flash ROM

8 MB dynamic RAM

8 MB flash disk

DOS-compatible BIOS

MS-DOS 5.0 software

Borland $\mathrm{C}++3.1$ software and book 
After receiving the board, we studied its documentation and performed a basic operational test on the unit by connecting its monitor port to a PC and downloading some small programs for execution. It passed all the tests and is working well at this time.

\subsubsection{DAQ Board}

We wanted a compact, reliable, 12-bit accuracy DAQ board for interfacing to the analog sensors and controllers of the cupola. After searching through numerous sources, we decided to purchase a data acquisition system, the DaqBook/112 with optional DBK11A from IOtech, Inc., with these principle features:

Link to PC via standard or enhanced (EPP) parallel port

12-bit analog resolution

$100 \mathrm{KHz}$ sample rate

8 differential or 16 single-ended analog inputs

Expandable to 256 inputs

2 analog outputs

Programmable gain of $1,2,4$, or 8 per input channel

4 digital inputs and outputs

Operate on 10 to $20 \mathrm{VDC}$ power source

AC adapter

Packaged in suitable stand-alone enclosure

Screw terminal card with 40 terminal blocks for analog I/O

Drivers for Windows and DOS using $\mathrm{C}$ or $\mathrm{C}++$

Driver for Labview 
DaqView and PostView data acquisition software

We received this board, but it has not yet been tested or interfaced to the rest of the system.

\subsubsection{FPGA Boards}

We purchased four X240 FPGA boards from Associated Professional Systems (APS). Each of these boards is equipped with one Xilinx XLA4085 FPGA chip that has a logic capacity of $180 \mathrm{~K}$ gates. On each of these boards, there are two $128 \mathrm{Kx} 8$ SRAM (Static Random Access Memory) chips. The boards are PC/104 16-bit compatible. The FPGA on each board can be configured from a PC/104 bus, an EPROM, or a parallel port using a Xilinx Xchecker cable. Each board also has a socket for standard clock oscillator. The documentation that came with these boards included an application example program in $\mathrm{C}$ and example FPGA configuration data. The example code allows the user to download the configuration data onto the FPGA chip mounted on the board and to test the on-board SRAM.

Using modified versions of the example code, each of the four boards was tested. An ISA (Industry Standard Architecture) carrier board was used to connect the X240 board to the ISA bus of a PC. Three FPGA boards passed the initial test. The failed FPGA board was sent back to the manufacturer where it was repaired. Upon its return, the board was tested successfully.

The four FPGA boards were then mounted on top of each other, using the PC/104 bus, to create the FPGA system. The provided example code was modified to allow the user to communicate with all the FPGA boards. The new program allows the user to 
specify any of these boards as the target for downloading and also to specify which test to perform. The program was used successfully to test the FPGA system.

\subsection{Analysis and Validation of Algorithms}

The algorithms for self-validation of sensor data were obtained from the Intelligent Algorithm Group in the form of Matlab and Excel files. A sample set of Matlab files are shown in the Appendices. The algorithms were then analyzed and validated for the purpose of successful implementation in hardware. The following sections discuss more in detail about the self-validation fuzzy logic and preprocessing algorithms applied to the sensor data.

\subsubsection{Self-Validation Fuzzy Logic}

Fuzzy logic is a convenient way of mapping an input space to an output space. Fuzzy inference is the process of formulating the mapping from a given input to an output using fuzzy logic. The Matlab Fuzzy Logic Tool Box supports five parts of the fuzzy inference process that includes

- Fuzzification of the input variable

- Application of the fuzzy operator

- Performing the implication operation

- Aggregation of outputs

- Defuzzification 
Fuzzy logic is used for self-validation of the sensor data. A FIS file (text file) specifies the inputs and outputs of the fuzzy logic system, the type of fuzzy logic, the range and shape of membership functions, and every other detail about the fuzzy logic system (see Appendix). Self-validation using fuzzy logic on sensor data is used to determine the confidence level of the input signals.

The following paragraphs discuss changes and enhancements we made to the algorithm: fixed-point considerations, changes made to membership functions, and changes in the choice of the aggregation method.

The raw input signal values (see Appendix) are currently represented as floating point numbers in a data file (later they will be input one-at-a-time from the DAQ as fixed-point numbers). This representation is simplest for the Intelligent Algorithm Team in formulating algorithms and implementing them using Matlab, as Matlab supports floating-point arithmetic for maximum accuracy. However, as far as hardware implementation is concerned, these numbers have to be converted into fixed-point numbers, as ultimately the output from the DAQ Board is in fixed-point. Using fixedpoint numbers also reduces the number of calculations to be carried out in the FPGAs, thereby making it less complicated and less expensive.

Some of the membership functions provided by the Intelligent Algorithm Team exhibited very sharp rising and falling edges. As small variations in floating-point numbers cannot be represented adequately in fixed-point, such sharp transitions in inputs might produce large errors. So the transitions were widened to represent changes in input more accurately using fixed-point numbers. 
The fuzzy logic method adopted for aggregation was the Sugeno method. This method was chosen over the Mamdani method, as it is much less complicated and easier to compute. Specifically, the Sugeno 'constant' method was adopted for processing over the 'linear' method, as it requires fewer parameters to be specified in the FIS file and it is also much easier to compute with no loss in accuracy.

\subsubsection{Self-Validation Preprocessing}

The fuzzy logic code was written to accept preprocessed inputs, consisting of median-filtered temperature, variance of temperature, and rate of change of temperature. However the raw data that is available to us consists of values of time and temperature measured from the sensors. Initially, the Intelligent Algorithms Team did the preprocessing in an Excel spreadsheet. It was then converted to Matlab code (see Appendix for an example Matlab $\mathrm{M}$ file), which we had to convert to $\mathrm{C}$ code. The paragraphs below discuss our changes and enhancements to the preprocessing algorithm: fixed-point considerations and changes made in the algorithm to simplify it.

For reasons previously specified, the code was changed to use only fixed-point numbers. The input signal values, which are floating point numbers at this time, are converted into fixed-point numbers before the preprocessing is carried out.

The preprocessed values derived from the raw input data (time and temperature) are the median-filtered temperature, rate of change of temperature, and the variance of the temperature. These values are determined using the following formulae: 
Median-filtered temperature $\mu_{i}=\operatorname{median}\left(T_{i}, T_{i-1}, T_{i-2}, T_{i-3}, T_{i-4}\right)$ where $\mathrm{T}$ is the measured temperature.

Temperature Rate-of-change $r=\frac{\Delta T}{\Delta t}=\frac{\mu_{i}-\mu_{i-1}}{t_{i}-t_{i-1}}$, where $\mathrm{t}$ is the measured time. Variance $\sigma_{i}^{2}=\frac{1}{5} \sum_{j=0}^{4}\left(T_{i-j}-\mu_{i}\right)^{2}$

Originally, one of the preprocessed results was standard deviation (instead of variance). However, it was changed to variance, since it is relatively complicated and expensive to evaluate the square root of a number (needed for standard deviation) in a hardware implementation.

\subsubsection{Self-Validation Execution Timing}

The processing time of the Self-Validation (SV) code can be reduced if a part of the code is implemented on FPGAs. Parts of the code that have longer processing times are being identified. The following two methods are being used to determine the processing time for the code, namely hardware-based timing measurement and theoretical timing analysis.

\subsubsection{Determination of Timing with Hardware Timer}

First, we tried to calculate the processing time using a standard hardware timer chip called a Programmable Interval Timer (PIT). C code was written to configure the operating modes of the PIT. We achieved partial success in this method. However it had several problems. First, the processing time measurements need a resolution of a few microseconds. The PIT could not measure with such fine resolution. Second, the code 
was running in the Microsoft WINDOWS environment. Background processes and parallel processing are an integral part of this operating system. The relatively random time periods consumed by these processes could not be separated from the actual code timing. The final timing results from multiple runs had a variation of up to $70 \%$ of the average value.

A time consuming solution would have to be developed to eliminate these problems. We decided that the complexity of the solution was not worthwhile. So the Hardware Team decided to drop this hardware-timing method, and try the following theoretical timing method instead.

\subsubsection{Determination of Theoretical Timing}

Theoretical timing, which measures the number of clock cycles required by the system to process the given code, is determined by looking up every instruction's execution time in the microprocessor data book. The main goal of performing this theoretical timing analysis is to compute the average time required (in clock cycles) for processing the SV code.

In order to do this, the code was split into three types of blocks that perform computations. This would help in calculating the average number of clock cycles required for processing the code for a given set of inputs. So the code was divided into straight-line blocks, conditional blocks, or procedure blocks.

A straight-line block consists of statements through which the control flows without any branching to other blocks. For instance, assignment statements, equations, and initializations form part of straight-line blocks. 
A conditional block includes conditional branches and multiple execution paths. Based upon the results of the decisions made in these conditions, program control flows through different paths of execution. As the average execution time is required, a certain weight was associated with each of these blocks. The weight associated depends on the probability that a particular path of execution is followed. Generally, we assumed all branches had equal probability and equal weight.

A procedure block, either a subroutine or a function, is called by other statements elsewhere in the code. The number of clock cycles taken for a call and return from the procedure block was included in the calculations.

An assembly language file of the SV C code was generated. This was the equivalent assembly language code for every line in the $\mathrm{C}$ code. The number of clock cycles required by the instructions was then found from the instruction set description in the Pentium Microprocessor data book.

An MS Excel file was created to document the results (see Appendix). It shows the number of clocks consumed for the execution of every block defined in the code. The total processing time for the code is the sum of the individual block clock cycle counts.

It was found that each input to the Mamdani-style fuzzy logic code required 55,000 clock cycles and each input to the Sugeno-style fuzzy logic code required 15,000 clock cycles.

\subsection{Self-Validation Software Implementation}

\subsubsection{Self-Validation Fuzzy Logic Code}

An introduction to the concept of fuzzy logic was presented earlier in this report. The design and implementation of the fuzzy logic code for Self-Validation began with 
the literature search for fuzzy logic theory and implementations, and with the study of the Matlab Fuzzy Logic Toolbox. Matlab provides a very good, general purpose C-code implementation of fuzzy logic that closely resembles their Fuzzy Logic Toolbox. Our initial version was derived from the Matlab $\mathrm{C}$ code, corrected and simplified for our needs, keeping only a subset of the membership, implication, aggregation, and defuzzification functions. Some of the original code that applied only to its use within Matlab was also eliminated, greatly simplifying the code and increasing its speed and reliability.

Next, the floating-point code was replaced with fixed-point code, which is more suitable for hardware implementation in FPGAs. The fixed-point code uses only the four basic arithmetic functions on fixed-point data words of $8,16,24$, and 32 bits.

Both the Mamdani and Sugeno methods were implemented, even though the project teams have since decided to use only the Sugeno method. We have determined that the less computationally intensive Sugeno method is quite adequate for our application, and thus we intend to only use this method in the future.

At this point, the results from both the floating-point and fixed-point versions of our code were compared to the original Matlab results. We found that the floating-point code produced exactly the same results as Matlab, while the fixed-point code gave results within $1 \%$ of the Matlab results. This accuracy is both reasonable and acceptable for our needs.

Finally, the fuzzy logic source code was reorganized by splitting it into two files, called setup.c and exec.c. The setup file performs the entire house keeping activity. All activities that are performed only once, like reading the input data file and FIS file, and 
printing the results, were grouped into setup.c. The exec file consists of functions that perform the mathematical operations on the input data. This code will be repeatedly executed during normal system operation. Functions in the exec file are most likely to be implemented in FPGAs, while all of the functions in the setup file will continue to be executed as code on the CPU board.

The current version of the code was tested using our preprocessed input data (discussed in the next section). The output of the fuzzy logic code (see Appendix) was compared with that of the Matlab Fuzzy Logic Tool Box (see Appendix). It was observed that most of the results were compatible with $<2 \%$ error, but some input sequences resulted in errors as high as $40 \%$. The cause of the small errors is due to the usage of fixed-point numbers instead of the floating-point numbers used in Matlab. This error is acceptable for our purposes. But the large errors are due to problems in the fuzzy logic membership function definitions and how they relate to the fixed-point arithmetic, which are currently being reconsidered. We fully expect to fix the problems causing these large errors.

\subsubsection{Self-Validation Preprocessing Code}

The algorithm devised by the Intelligent Algorithm team for processing the raw input values to determine the preprocessed input values was coded. The raw input values represented using floating point numbers were converted into fixed-point numbers. The preprocessing was then carried out on these values to generate fixed point preprocessed input values. 
The code was successfully implemented using fixed-point arithmetic using a floating point scaling factor. Scaling factors are constants determined from the range of the floating-point numbers. These factors are used to determine the fixed point equivalent for the floating-point number. A new method has been devised for implementing fixed-point arithmetic using a fixed-point scaling factor. The implementation of the above method is now in progress.

The Matlab floating-point output results (see Appendix) were compared with the ones generated by the fixed-point code (see Appendix). The results were found to be compatible with $<2 \%$ error, which is within expected error bounds.

\subsection{Communication Software Development - the CPU-to-Host Interface}

The CPU-to-host interface consists of a serial communication line connecting the CPU board to the host PC, and the associated communication software. Using standard PC-type RS232 serial ports, it is intended to run at the maximum standard speed of 115200 bits per second. The host PC is the user's interface to this entire signal processing system. The purpose of the CPU-to-host interface is to transfer control commands from the host PC to the CPU board for execution, and to transfer status and computed results back from the CPU board to the host PC. To make this interface robust, a special error detecting/correcting communication protocol was designed, based on a combination of pre-defined message blocks, character counts, checksums, handshaking, and retransmission requests. 
A preliminary version of this communication software has been written. The C code was written initially to implement the complete communication protocol. But then changes were made in the code to enable the receiver to wait for the sender or vice versa for an infinite length of time without timing out. The purpose of including this change was to temporarily avoid error reports due to certain error conditions. The testing was carried out first between 2 PCs, and then between a PC and the CPU board. Only limited success could be achieved regarding the implementation of the protocol. It was observed that the code properly supported serial communication only up to a bit rate of 9600 bits per second. The expected bit rate of 115200 could not be achieved. More work will be done to improve this code to reach full speed using the complete protocol.

\subsection{Summary}

The Hardware Team has successfully begun development of the I ${ }^{3}$ PSC Signal Processing System. We completed the literature search, developed the system architecture, and purchased a CPU board, DAQ board, and multiple FPGA boards to build the system. We analyzed and refined the first of the system algorithms created by the Intelligent Algorithms Team to enhance its suitability for hardware implementation. This Self-Validation algorithm, consisting of two major functions (preprocessing and fuzzy logic) was first implemented entirely in $\mathrm{C}$ code and then tested successfully for compatibility with the original Matlab version. The effort to measure the code's execution time is almost complete at this time. This is needed to determine which functions are most computationally intensive and thus need to be executed on the FPGA 
hardware. Finally, a preliminary version of the CPU-to-Host interface has been designed and tested.

During the first few months of 2000 (contract year 2), the Hardware Team plans to complete the remaining details of the software implementation and timing analysis of the Self-Validation algorithm, and begin its implementation on the FPGA boards. By the end of 2000, we plan to perform similar algorithm analysis, software implementation, timing analysis, and FPGA hardware implementation of the Sensor Fusion algorithm. The remaining interfaces, CPU-to-FPGA and CPU-to-DAQ, will also be developed and tested during this year. The effort in 2001 (contract year 3) will be centered on refining the signal processing system and demonstrating its operation within a working cupola environment.

As discussed earlier, there have been some failures encountered and numerous bugs have been fixed. Most of the development has taken longer than expected, due to unexpected problems and complications that have arisen. But we are confident in our ability to overcome the problems and feel that we are definitely on the road to successful completion of this project.

\section{Year 2 Accomplishments}

\subsection{Overview}

During the year 2000, the I ${ }^{3}$ PSC Hardware Team completed the hardware design and testing of the self-validation algorithm and began working on both the hardware implementation of the multi-sensor fusion algorithm and the communication protocols of 
the ultimate form of the hardware system. In the next few sections we will present the details of all tasks performed by the hardware group in the second year (2000).

\subsection{Communication Protocols}

As stated earlier, the final project will have three different communication protocols. Currently the group is working on the user PC and the FPGA board communication protocol. The work done can be summarized as follows.

\subsubsection{Develop low-level communication protocol}

A low-level communication protocol that controls the communication between the User PC and the CPU Board has been designed. According to this communication protocol all information passes between the User PC and the signal processor in the form of messages. For generality and versatility, a single message consists of 0 or more bytes of information/data preceded and followed by certain message control bytes. Byte \#1 usually represents the function code, which defines the message class and meaning of data. Byte \#2 and \#3 specify the length of the data block, which could be 1-255 or 165535. The information following these bytes in finally followed by the error detection byte, which is the checksum of all other bytes.

\subsubsection{Write and test the low-level communication code for initialization, transmit and receive}

The developed low-level communication code was written in $\mathrm{C}$ and was tested between two CPUs. The system was initialized to operate at a particular baud rate and then it was used to transmit and receive a string of data. The system was tested successfully for various baud rates including the operation baud rate of $115200 \mathrm{bps}$. 


\subsubsection{Define specifications for high-level communication protocol details for MS- SV program for User Interface}

Following the low-level communication protocol, a high-level communication protocol was designed. This protocol specifies the format of the messages that are exchanged between the user PC and the CPU board. The sequence of the messages has also been decided upon. The protocol has been appended with a time-out facility to restart the system or continue from the point just before failure. This task is $100 \%$ complete.

\subsubsection{Outline method for modification of SV code}

The outline for modifying the SV code to include the CPU end of the high-level communication protocol has been completed. The new routines that have to be now included in the $\mathrm{C}$ program are being written. This task will be completed soon.

\subsection{Develop a Library of Basic Fixed-Point Arithmetic Functions}

As previously stated, the code uses the four basic arithmetic functions on fixedpoint data words of $8,16,24$, and 32 bits. To meet these computational requirements of the hardware implementation, a VHDL library of the four basic operations was developed. This library includes adders/subtractors, multipliers, and dividers. These operations were designed as functions so that they can be utilized through function calls. Different designing techniques of these arithmetic units were utilized. The following types were developed for adder/subtractors: 
Ripple carry adders

Carry-Look ahead adders

Look-up table adders

All of these designs were developed for 8,16,32-bit ranges. Pipelined version of each of these types was also implemented. Different designs of the dividers units were also developed. These designs include:

Divider using Shift/subtract algorithm

Divider using single register algorithm

These two designs were developed for 24-bit/16-bit, 16-bit/8-bit. In addition, the following techniques of multipliers designs were implemented.

Array multiplier

Pipelined array multiplier

Dadda-Wallace multiplier

Pipelined Dadda-Wallace multiplier

The multipliers were developed for 8-bit by 8-bit inputs.

All the designs in the VHDL library were coded, simulated, and synthesized. These designed were optimized for the hardware implementation of the SV algorithm on the XC4085XLA FPGA chip. A bit maps for the best designs were then generated, downloaded onto the FPGA chip, and successfully tested.

\subsection{Implementation of the SV Preprocessing Algorithm}

The modified preprocessing algorithm was written with fixed-point code. The code was successfully tested and evaluated with data from the Intelligent Algorithms group. The code was then reorganized and split between the setup and execution file 
codes. A new input files were created for the signal-Validation code. After fixing numerous implementation problems, the code was successfully tested and its results were validated. Finally, the process was completed after inserting appropriate documentation to the code.

\subsection{Develop Architecture of SV Signal Processor Hardware}

\subsubsection{Select SV procedures for the hardware implementation}

We decided to implement in the FPGA hardware all the procedure in the execution code that performs computations on the outputs from the preprocessing stage. These procedures include FisEvaluate(), FisComputeInputMFValue(), FisTrapezoidMF(), FisComputeFiringStrength(), FisArrayOperation(), FisMin(), and FisComputeTSKRuleOutput(). These procedures compute the membership functions of the input parameters; i.e. convert crisp inputs to fuzzy inputs, apply the inputs to the fuzzy rule set, aggregate the outputs of each rule, and compute the outputs. The output of each preprocessed set represents the computed confidence in the corresponding sensor measurement.

\subsubsection{Separate constants from true Variables}

An analysis study of all the code's variables showed that some of the variables are calculated or defined in the setup part and their values do not change during the execution part. Hence they will be implemented once in the setup, not during the execution in hardware.

\subsubsection{Simplify the fuzzy logic procedures}

To simplify the fuzzy logic code for the hardware implementation, variables that do not change during the execution were converted into constants. In addition, all error 
checking and Mamdani code has been removed from the fuzzy logic code. The error checking code was not needed if the Fuzzy Inference Structure (FIS) file, which is the input file to the setup part, is correct. The Mamdani code was not needed since we choose to implement Sugeno type code. After the verification of the code revision, it was tested successfully.

\subsubsection{Create Block Diagrams}

A block diagram for each function was created. They are used to determine the requirements for sequence of events during execution. They are also help to determine how to group functions into hardware blocks, and which sequential functions are reusable. These block diagrams for the hardware-implemented functions are shown in the Appendix.

\subsubsection{Define Data Structure and Organization}

The data structure of the FIS parameters for all sensors that are calculated during the set up phase, and the intermediate results need to stored on the on the FPGA board's SRAM. The data structure and their organization within the SRAM have been defined. A register file was also designed to store the commands

\subsubsection{Define finite state machine controllers}

The different states of a Finite State Machine (FSM) controller were defined. The FSM is used to control all the timing details for the operation of the entire design. The defining ASM chart is shown in the Appendix. 


\subsection{Develop Hardware Design of SV Signal processor}

\subsubsection{Code Hardware Blocks in VHDL}

Each block of code was coded in VHDL using the data flow graphs to help guide the sequencing of operations. All arithmetic operation was done through function calls to the entities of the arithmetic functions library. The coding was done in a way that allows pipelining and parallelism of operations.

\subsubsection{Simulate Each Entity Code Separately}

Each code was simulated separately to ensure its proper functionality. Special debugging codes were inserted to allow monitoring of internal signals during simulation. After fixing all the code problems, the blocks were synthesized to check the amount of logic blocks needed for their implementations. The number of clock cycles needed for each block was recorded. The arithmetic functions were modified to the exact number of bits needed in the implementation.

\subsubsection{Design of the system interfaces}

The system interfaces include a bus interface, a memory interface and a register file. The FPGA-bus interface represents the connection between the PC and the FPGA boards connected to the PC/104 bus. Different signals are used to select one of the FPGA boards and to communicate (read from or write to) with either the processes on the FPGA boards or with the on-board memory. The memory interface connects the on-board memory with the FPGA processes or the host machine. 


\subsubsection{Design of the FSM}

The design of an efficient FSM requires intimate knowledge of the sequence of operations and the number of clock cycles needed for each operation. It coordinates the use of both the address and data buses and memories by the FPGA processes and the host machine and controls all the system's activities. The controller also issues various load, store and Read/Write enable signals.

\subsubsection{Add All Blocks to Top-Level VHDL Entity}

After successfully testing all the signal validation system components, they were integrated with the top-level VHDL entity. The top-level entity acts as the main function in a $\mathrm{C}$ code. The entire hardware code was simulated and synthesized successfully. A bit map file representing the hardware design was then generated.

\subsubsection{Download, Test, and Debug Top-Level SV Signal Processor}

The $\mathrm{C}$ code was modified clean up the preprocessing steps and to include functions that download the hardware design onto the FPGA, to load the system parameters onto memories on the FPGA boards, to read from the on-board memories, to send data to the FPGA processes and read their results. Some of the signal validation code was replaced with FPGA communication tasks. These tasks include send data, send command, and get result Self Confidence. The modifications were done in many stages. In the early stages, A C code was developed to allow testing the on-board memories by writing data onto them and then reading their contents. Currently, the system uses 64 
bytes of on-board memory per sensor and requires about 50 cycles to process each preprocessed set of inputs.

\subsection{Develop Multi-sensor SV Algorithm}

The Self-validation system was originally tested for data acquired from a single sensor. However, in the Cupola system, more than a single sensor's data would be actually fed into the self-validation system. So the single sensor self-validation system was expanded to be a multi-sensor self-validation system. For the testing purpose, the multi-sensor self-validation system accepts the input values of several sensors as a single file where the various sensors' data points are presented as separate columns of temperature and time values. The self-validation system operates on one set of data for one sensor and determines the self-confidence value for that sensor. It then reads the next set of data for the next sensor and determines the self-confidence for that sensor. The output of this system is a set of self-confidence values for the different input data of the various sensors. The system was tested successfully and it can now handle up to 50 sensors. The code will be modified to accept data directly from the sensors instead of a data file.

\subsection{Develop Multi-Sensor Fusion Algorithm}

The Hardware Group Team received the Multi-sensor fusion (MSF) algorithm from the Intelligent Algorithms group in the form of three of MATLAB files and 4 Excel files. The MATLAB files represent MSF code. These files are:

feeddata.m - Serves as the front end file that reads the data from a txt file and feeds it point by point to the sensor fusion function 
ValueConf.m - This is the function that does the multiple sensor fusion. It takes data at every instant from feeddata.m file and returns the Fused Value and its confidence at that instant.

checkindex.m - this function is called by ValueConf.m and it checks whether the maximum value returned by the max function is indeed at the center the trapezoid. Max function in Matlab returns the maximum value and the index of its first occurrence.

The Excel files are data files. These files are::

TestInput1.txt and TestOutput1.txt - One set of Inputs and Expected Outputs TestInput2.txt and TestOutput2.txt - Another set of Inputs and Expected Outputs TestInput3.txt and TestOutput3.txt - Another set of Inputs and Expected Outputs The *.wk1 files can be used to test the $\mathrm{m}$ files as the ${ }^{*}$.m files read the ${ }^{*}$.wk1 files

The Hardware Group studied, analyzed, and validated the MATLAB code for the purpose of understanding its functionality and the enable the development of the hardware implementation code. The algorithm is simple and generic for ' $\mathrm{n}$ ' sensors. It reflects the degree of agreement among sensors, measure the reliability of the measured estimate, and hence minimizes the effect of failed sensors. A sensor's median reading that a deviate sharply from the centroid of the ' $n$ ' membership functions of the sensors readings is assigned a lower confidence. For sampling period, the algorithm reads the median filtered temperature and the corresponding confidence of each of the $\mathrm{n}$ sensors (from the outputs of the self-validation algorithm). Using the sensor reading, the system uses also the historical variance measure of the sensors. For each sensor, the algorithm calculates the four parameters of a trapezoid membership function such that the area of 
each of the trapezoid is the same (one unit). The trapezoids are symmetrical around the sensor reading. The heights of all trapezoids are added together and the position of the maximum height is computed. The value of the normalized area within \pm 3 standard deviations is considered as the overall confidence. On the mean time, the algorithm group converted the MATLAB files into a floating-point C code. All sections of code that apply only to the MATLAB implementation, including redundant error checking, were removed from the floating-point $\mathrm{C}$ code. The $\mathrm{C}$ code was tested successfully and produces comparable results to that of the MATLAB code.

\subsection{MSF C Code Optimization}

Careful examination of the MSF code revealed that the many of the computations performed by the MSF algorithm were not ultimately required. For example, The code calculated the areas before and after the maximum height of the overall graph resulted from adding the trapezoids of all sensors by dividing the total range of the trapezoids into a fixed number of steps and calculating the areas between each step. A large saving in the computational requirements could be achieved by computing the areas as the sum of triangles and rectangles. The areas of trapezoids triangles and rectangles were calculated using basic algebra laws. In addition to be faster and less demanding in terms of computational resources, the new method was more accurate also; it avoided many of the round off errors.

Three versions of The MSF C code were developed to optimize and adapt the floating-point code for the hardware implementation. In the first version, the code was enhanced through the following modifications: 
The calculation of the height sensors' trapezoids has been optimized. This version assumed that the number of fused sensors data is three (constant).

All the static values like number of sensors, number of samples, std_deviation, were made as dynamic values that are read from an input file. The entire code has been optimized by removing redundant calculations. All needed value were calculated once at the beginning of the code execution, and used as many times as needed.

The third version was enhancements included replacing all data structures to arrays (for faster computations). The code for three sensors was also modified to limit the number of points considered to $13(4 * n+1)$ instead of the original 86 points. This has resulted in a significant saving of the computational requirements of the code.

Each of these versions was coded, and tested. The results obtained form the last version have a maximum error of $2 \%$, which is acceptable for our application.

\subsection{Develop the MSF fixed-point code}

As explained before, the floating-point code is not suitable for the hardware implementation. In the final MSF version, the code was implemented as a fixed-point code of $8,16,24$, and 32 bit resolutions. The code was also optimized such that the calculation of any variable is done only once. However, to reduce the accumulation of round off errors, some intermediate variables were calculates every time it is needed. In addition, the number of sensors considered was coded as a dynamic variable whose value is read at the beginning of the execution cycle. The code was also modified to handle a maximum of 10 sensors. The fixed-point code was tested successfully and produced results within the acceptable margin of error. 


\subsection{Hardware implementation of the MSF code}

The reorganized and optimized sensor Multi-sensor fusion was partitioned into modules and a data flow graphs of all these modules were created. Currently, we are working on measuring the execution time for each block and to translate these data flow graphs into VHDL code.

\subsection{Summary}

During the year 2000, the Hardware Team completed the FOGA implementation of the signal validation code. Currently the group is working on the hardware implementation of the multi-sensor fusion algorithm. The group is also working on designing the communication protocols of the final system so that the FPGA system can be connected to the microprocessor board. The group is confident that they will accomplish all the project goals in a timely fashion. 


\section{Year 3 Accomplishments}

\subsection{Overview}

During the final year, extended from 2001 through the first half of 2002, the $I^{3}$ PSC Hardware Team completed most of the project hardware and software as it was initially envisioned.

The multi-sensor Self-Validation (SV) algorithm was designed, simulated, and fully implemented in the FPGA hardware, with significant speedup over a standard microprocessor implementation. The Multi-Sensor Fusion (MSF) algorithm was designed and simulated for the FPGA. A new Virtex FPGA board was purchased and utilized in the system. The CPU board was programmed and tested as the interface between the Host PC and the FPGA board. New serial communication protocols and software were developed and tested. An overall top-level controlling application was created for the system user in Labview on the Host PC. Details of these accomplishments are given in the following subsections.

\subsection{SV Implementation}

The multi-sensor Self-Validation processor was first simulated and validated, using VHDL and Xilinx tools. Next it was successfully implemented on the original FPGA board and then revised to work on the new Virtex FPGA board. The complete system (consisting of the Host PC running Labview code, the CPU board running C code, 
and the FPGA with the SV processor implemented with VHDL code) was debugged and successfully tested.

\subsection{SV Speedup}

The final speedup calculated for the Self-Validation processor on the FPGA was found to be significant. Processing on the FPGA takes only 50 clock cycles versus 15782 clock cycles on a Pentium microprocessor, giving a speedup of 315 . Of course the total processing time also depends on the clock frequency, which is higher on the Pentium, but this is still an important accomplishment for the hardware team.

\subsection{MSF Implementation}

The Multi-Sensor Fusion (MSF) processor was fully developed, simulated, and validated for the original FPGA board. All the VHDL modules were developed. These modules include:

Area_Top_R10.vhd: This module calculates the area to the right and left of the centroid for every sensor (up to 10 sensors). Each sensor calculations requires 6 clock cycles as follows:

Clock \#1: The points of the trapezoid for a sensor are selected. Description of this operation is given in inp_mux module.

Clock \#2a: The local controller compares the 4 points with the centroid and calculates the internal control signals sel, sel_Com. Description of this operation is given in the control module.

Clock \#2b: the 8bit by 8 bit multiplier is used. 
Clock \#3: The $16 * 8$ multiplier multiplies the output of 8 bit by 8 bit multiplier with constant (102).

Clocks \#4, 5: The 24bit /16bit divider divides the product from $16^{* 8}$ multiplier with square of std. of the sensor.

Clock \#6: The quotient of the previous operation is accumulated by the accumulator.

Total number of cycles needed by this multiplier $=6 *$ no. of sensors.

Busifc.vhd: This module contains the PC/104 bus interface for the FPGA signal process. It defines the connections between the PC/104 bus, memory interface, register file, and the SV/MSF processor.

Centroid.vhd: This module is used to compute the centroid of all sensor trapezoids. The module has an accumulator to add all the sensor data values. The centroid is calculates as the result of dividing the resultant sum by the no. of sensors. The divider is implemented in the pdiv16_8 module. The centroid is executed in parallel with val_sen module. All the sensor data, read in the val_sen module, are added by the accumulator in this module.

Conf_control.vhd: This module is used to generate control signals for the selfconfidence process.

Control.vhd: This module is a local controller of the datapath of the area module. It performs the six comparisons needed to determine the location of the centroid with respect to the trapezoid points of all sensors.

Div_8.vhd: This module is an 8 bit / 8 bit divider

Div_16_8.vhd: This module is a 16bit / 8 bit divider. 
Div_16_16.vhd: This module is a 16bit / 16 bit divider.

Div24_16UNS.vhd: The module performs unsigned division of 24 bit / 16 bit. A quotient of 8 bits is generated.

fus_con_top.vhd: This module is used to calculate the fused confidence, which is the area within a span of $3 * \min . \mathrm{STD}$ on either side of the fused value. The operation of this module requires 6 clock cycles as follows:

Clock \#1: The 4 points of the trapezoid for a sensor are selected.

Clock \#2a: The local controller compares the 4 points with the limits of evaluation start conf and end conf. Local controller calculates the internal control signals selfuscon, selmul. The conf_control module describes this operation.

Clock \#2b: The 8bit*8bit multiplier performs multiplication.

Clock 3: The 16bit*8bit multiplier multiplies the output of $8 * 8$ multiplier with the constant 102

Clocks \#4, 5: The 24bit/16bit divider divides the product from $16^{*} 8$ multiplier with square of std. of the sensor.

Clock 6: Appropriate fus_conx is selected based on the condition satisfied. The resulted quotient is accumulated by the accumulator.

Total no. of clock cycles needed for this module $=6 *$ no. of sensors +1 .

The extra clock is used to divide the accumulated result with the number of sensors to calculate the fused confidence.

Inp_mux.vhd: This module calculates the start_base, end_base, start_top, and end-top points of the trapezoid of each sensor. It also calculates the height of each of these trapezoids. A 40 by 18 -bit multiplexer is used to select the element of the trap 
array. The module contains the multiplexers to select the various points of the trapezoid and the points of the array. It takes 2 clock cycles to perform a single selection.

Memifc.vhd: The module contains memory access signals and bus address decoders. This file was modified in order to use the Virtex BlockRAMs as the memory. The MSF process can access 256 words starting at address 1000-10FF.

MSF_Control.vhd: This module generates timing control signals needed for all datapath circuits. It also generates control signals to interface with the system's memory and bus.

MSF_top.vhd: This module is the top-level module of the MSF code. It calls all other MSF modules including the memory interface and the bus interface.

MSF_top_d.vhd: This module is the debugging version of the above MSF toplevel module.

Mux8B_2X1.vhd: This module is an 8-bit 2 to 1 multiplexer.

Mux8B_7X1.vhd: This module is an 8-bit 7 to 1 multiplexer.

Mux8B_10X1.vhd: This module is an 8-bit 10 to 1 multiplexer.

Pdiv16_b.vhd: This module is used to select the inputs of the $16 \mathrm{bit} / 8 \mathrm{bit}$ divider from two possible cases. An 8 bit 2 to 1 multiplexer is used for this purpose. The controller signals for the multiplexer are generated by the master controller.

Pdiv24_16.vhd: This module has a 24-bit/ 16-bit divider. This divider is used called by two modules, the area module and the fus_conf module.

Pmul16_8.vhd: This module has a 16-bit by 8-bit multiplier and a 2 by 1 multiplexer. The MSF code requires 2 instantiation of a 16-bit by 8-bit multipliers. The 2 by 1 multiplexer is used to select one set of these inputs for multiplication. 
Pmul8_8.vhd: This module has an 8-bit by 8-bit multiplier and a 4 by 1 multiplexer. The MSF code requires 4 instantiation of an 8-bit by 8-bit multipliers. To reduce the code size, one multiplier is used to perform the 4 multiplication operations. The multiplexer is used to select the inputs to the multiplier out the possible 4 sets of inputs.

Rearr_un_par.vhd: This module is used to sort all sensors' data points in ascending order using the odd-even sort method. The $\mathrm{C}$ code of this method can be found at http://www.cs.rit.edu/ atk/Java/sorting/sorting.html.

Reg_16.vhd: This is a 16 bit register with enable and reset inputs.

Reg_8.vhd: This is an 8 bit register with enable and reset inputs.

Sen_val.vhd: This module is used to validate the confidence of a sensor. It operate as follows:

Clock \#1a: Read the confidence of sensor $x$ from memory. (Done by the controller)

Clock \#1b: Load the conf into CONF_A register.

Clock \#2a: Read sensor data, and std. deviation from memory for sensor $\mathrm{x}$.

Clock \#2b: Load the sensor data and sensor std deviation into SDATA_A and SSTD_A respectively.

Clock \#3a: Read the confidence of sensor $x+1$ from memory. (Done by the controller)

Clock \#3b: Load the conf into CONF_B

Clock \#3c: Start execution process for values in Set A registers that includes CONF_A, SDATA_A, SSTD_A registers 
Clock \#3d: Send sstd to the $8 * 8$ multiplier.

Clock \#4a: Read sensor data, and std. deviation from memory for sensor $\mathrm{x}+1$.

Clock \#4b: Load the sensor data and sensor std. deviation into SDATA_B and SSTD_B respectively.

Clock \#4c: Read the value on sq_std bus (from multiplier) and continue processing of data.

Clock 5a: Start execution process for values in Set B registers that include CONF_B, SDATA_B, SSTD_B registers.

Clock \#5b: Send sstd to the 8bit*8bit multiplier.

Clock \#5c: Load the output values corresponding to the set A into the output registers.

Clock \#6: Read the value on sq_std bus(from multiplier) and continue processing of data.

Clock \#7a: Read the confidence of sensor $x+1$ from memory. (Done by the controller).

Clock \#7b: Start execution process for values in Set B registers.

Clock \#7c: Send sstd to $8 * 8$ multiplier.

Clock \#8a: Read sensor data, and std. deviation from memory for sensor $\mathrm{x}+1$.

Clock \#8b: Load the output values corresponding to the set B into the output registers.

Clocks 5 through 8 are repeated for each of the remaining sensors. 
Span.Vhd: This module calculates the span of area in the sum trapezoid to determine the fused confidence. The fused confidence is the area on either side of centroid in the sum trapezoid for a span of 3 times the minimum standard deviation.

TopMSF.vhd: This is the top -evel module. It generates all the needed control signals for other modules.

Trap2max.vhd: Prior to starting this module 2 clock cycles are spent for the selection of these points by the inp_mux. The operation of this module is as follow. For all the forthcoming cycles the selection of points is performed in parallel with the execution of trap2max.

Clock \#1: Set inputs to 8bit*8bit multiplier and perform the multiplication.

Clock \#2a: Reset all the registers by asserting Clr_hsum.

Clock \#3_4a: Set inputs to 16bit/8bit divider. Perform division.

Clock 3_4b: The height for the 0th sensor at trap (index) is stored in the accumulator register.

Step \#3_4c: Store the accumulated height in the h_sum array at index location specified by the index input.

Clock \#5-8a: Calculate the height for the 1st sensor at trap (index).

Clock 5_8b: Compare the current $\mathrm{h} \_$sum with the previous $\mathrm{h}$ _sum and store the max. h_sum's index and value.

Repeat clocks 5 through 8 for all the remaining sensors.

Clock \#9-12: Calculate the height for the 2nd sensor at trap (index).

Repeat clocks 1-12 for all the forthcoming index points.

At Clock clkx $=12 *\left(\mathrm{~h} \_\right.$start $-\mathrm{h} \_$end $)+4$ perform the following: 
Step clk $\mathrm{x}+1$ : Store the index of the higher $\mathrm{h} \_$sum in max_ind.

Clock clkx +2 : Access the trap element with index max_ind +1 and store it in trap_mx0.

Clock clkx+4: Access the trap element with index max_ind-1 and store it in trap_mx1.

Clock clkx+6: Access the trap element with index max_ind.

Clock clkx+7a: Calculate the mean of trap(max_ind +1$)$ and trap (max_ind) and trap(max_ind-1) and trap (max_ind).

Clock clkx 7b: Select the mean of the element that is closer to trap (max_ind).

Number of clock cycles used in this module $=12 *\left(\mathrm{~h} \_\right.$start $-\mathrm{h} \_$end $)+11$.

The TopMSF project was created for the implementation of the MSF code with bus interface (Busifc) and memory interface (Memifc).

The MSF code was then enhanced to run on the new Virtex FPGA board, and again simulated successfully. However, it was not implemented and tested on the Virtex FPGA board with rest of the system due to insufficient time available at the end of the project period.

\subsection{MSF Block diagrams}

Block diagrams for all the VHDL modules were created. These diagrams were used to optimize the reuse of primitive components such as the multipliers and the dividers. They were also used to show the sequence of operation for each module. These block diagrams include:

Area Comp: This diagram represents the comparison between the left and right of the sensors' centroid. 
AreaP1- AreaP4: These four diagrams illustrate the computation of trapezoid area.

Centroid: This diagram illustrates the calculation of the centroid.

Dividers: This diagram shows the components needed for the division operation and the sequence of their use.

Fcp1-Fcp5: These five diagrams show the sequence of calculation needed to compute the fused value.

Inpmux: This diagram illustrates the use of multiplexers to choose between different input sets to the dividers or the multipliers

Multipliers: This diagram shows the design of multipliers with different bit-width.

Rearrange: This diagram pictorially shows the sort operation of the $\mathrm{N}^{* 4+1}+1$ points in an ascending order. It calls the sort module to perform the sort operation.

Sort: This diagram shows the details of the sort operation.

Traphtp1-Traphtp5: These five diagrams show the calculation of the height of the trapezoids.

Ue: This module shows the design of a comparator unit.

Validatep1 - Validatep4: these four diagrams are used to show the sequence of Validating sensors' data.

\subsection{Virtex FPGA Board (APS V240)}

The original FPGA board (APS X240) with the Xilinx XC4085 device (about 80,000 gates) was found to be too small to hold the rather large MSF processor and the 
smaller SV processor. So we purchased a replacement FPGA board (APS V240) with a much larger device, the Xilinx Virtex XCV800 with approximately 800,000 gates. As a result, some significant changes had to be made to our system design to accommodate the new device and its improved features. The device download and configuration code on the CPU board was rewritten and tested with the new FPGA, whose configuration details were different from the old FPGA. Since the new FPGA had a significant amount of fast on-chip memory, we decided to eliminate the external memory chips, which caused a significant redesign of the memory system and its interface with the SV and MSF processors. Several other smaller changes were also implemented successfully on the new Virtex board.

\subsection{CPU Board}

We learned all the details necessary to effectively utilize the CPU board on this project. As discussed earlier, it interfaces to the Host PC via one serial line for all command, status, and raw data. We also interfaced with a monitoring laptop PC, using another serial line, to allow us to effectively download and debug the CPU code (written in C). We revised the setup and communication portions of the SV and MSF code to run on the CPU board. We implemented the high-level communications protocol with the Host PC as presented elsewhere. Of course, the CPU board directly controls the Virtex FPGA board, passing low-level commands and raw data to it and receiving back the processed SV data at each sample time. It essentially calls on the FPGA as a high-speed hardware-based subroutine implementing the signal processing algorithms. 


\subsection{Communication}

A sophisticated error-detecting and correcting high-level communication protocol was defined and implemented over the serial communication line between the Host PC and the CPU board, running at the full 115,200 bps speed. The line passes all commands, status, input data and output data between the Host PC and the CPU board. This protocol allows the system to automatically recover from transient communication errors and continue normal operation.

\subsection{Host PC Application}

Control and communication with the CPU board and FPGA were implemented as a Labview application on the Host PC, giving a simple and effective interface to the system operator. This application fully supports all aspects of both the SV and MSF downloading and processing tasks. Each step in the process may be executed independently, simplifying debugging and reducing the total execution time.

\subsection{Summary}

\subsubsection{Work Completed}

During this I3PSC project, the Hardware Team successfully completed most of its planned work, but had insufficient time to finish a few portions.

The team began with a literature search to study the possible signal processing methods, software tools and hardware devices available. We selected and purchased appropriate hardware (CPU Board, FPGA boards, DAQ board) and found effective ways to utilize them. An overall system architecture was created. We developed software 
implementations of the two major algorithms, Self-Validation and Multi-Sensor Fusion to verify their proper operation. We replaced the original floating-point arithmetic with fixed-point versions for efficient hardware implementation. A library of fixed-point arithmetic routines was created in VHDL. We designed and implemented a sophisticated communication structure to tie together all the system elements.

Many enhancements were made to the original algorithms to enhance their performance in hardware. We developed detailed architectures for their implementation into hardware. We partitioned the algorithms into two sections: one section contained computationally-intensive portions destined for hardware implementation, and the other section continued to be implemented in software running on a standard microprocessor.

The hardware portion of the SV and MSF processors was implemented in VHDL and simulated. Two new subsystems were designed specifically for the FPGA, including a PC-104 bus interface and a memory block. Finally, the SV processor was fully implemented and tested on the FPGA, utilizing all of the system components.

In addition to the technical contributions of this project, several graduate students (MS) benefited from this project through the financial support for their work on the project. Sobha Sankaran was a key contributor to the detailed design and implementation of the Self-Validation software and hardware. Srikala Vadlamani was a key designer of the Multi-Sensor Fusion software and hardware under the supervision of Dr. Mahmoud. Jie (Ellen) Chen was responsible for implementing the control and communication software on the Host PC to interface with the CPU board for both SV and MSF. Each of these students contributed significantly to the development of the $\mathrm{I}^{3} \mathrm{PSC}$ hardware system. 


\subsubsection{Future Recommendations}

We recommend that the following tasks, be undertaken to fully utilize the results of the current project.

Complete implementation and testing of the MSF processor on the FPGA board, controlled by the CPU board.

Then combine both processors (SV and MSF) on the same FPGA, running simultaneously.

Complete the hardware/software interface between the CPU Board and the Data Acquisition System (DAQ) to allow direct, autonomous acquisition and processing of the Cupola data without burdening the Host PC.

Test the completely integrated system with the Cupola and live data. 


\section{REFERENCES}

[1] Nagrath, I.J and Gopal, M, Control Systems Engineering, Second Edition, New Age International (P) Ltd., Publishers, 1995.

[2] Maciejowski,J.M, Multivariable Feedback Design, Addison-Wesley Publishers Ltd., 1990.

[3] Richard R.Brooks and S.S.Iyengar, Multi-Sensor Fusion - Fundamentals and Applications with Software, Prentice Hall, Inc., New Jersey, 1998.

[4] Ren C.Luo and Michael G Kay, "Multiple Integration and Fusion in Intelligent Systems," IEEE Transactions on Systems, Man and Cybernetics, vol. 19, no. 5, September 1989.

[5] R.C.Luo, M.Lin, and R.S.Scherp, "Dynamic multi-sensor data fusion system for intelligent robots," IEEE Journal Robotics and Automation, vol. RA-4, no. 4, pp. 385$396,1988$.

[6] Keith E. Holbert, A.Sharif Heger and Nahrul K. Alang-Rashid, "Redundant Sensor Validation by Using Fuzzy Logic," Nuclear Science and Engineering, vol. 118, pp. 54-64, 1994.

[7] Asok Ray and Rogelio Luck, "An Introduction to sensor Signal Validation in Redundant Measurement Systems," IEEE Control Systems Magazine, vol. 11, no. 2, pp. 43, Feb 01, 1991.

[8] Marcello R Napolitano, Charles Neppach, Van Casdorph, Steve Naylor, Mario Innocenti and Giovanni Silvestri, "Neural Network Based Scheme for Sensor Failure Detection, Identification and Accomodation," Journal of Guidance, Control and Dynamics, vol. 18, no. 6, Dec 1995.

[9] Mohamed Abdelrahman and Senthil Subramaniam, "An Intelligent Signal Validation System for Cupola Furnace - Part 1 and Part 2," American Control Conference, San Diego, 1999.

[10] Janice C, Yang and David Clarke, "A Self-Validating Thermocouple," IEEE Transactions on Control Systems Technology, vol. 5 no. 2 March 1997.

[11] M.P.Henry and D.W.Clarke, "The Self-Validating sensor: Rationale definitions, and examples," Control Eng. Practice, vol. 1, no. 4, pp. 585-610, 1993.

[12] T.M.Tsai and H.P.Chou, "Sensor fault detection with the single sensor parity relation", Nuclear Science and Engineering," vol. 114, pp. 1411993 
[13] Mathieu Mercadal, "Sensor Failure detection using Generalized Parity relations for Flexible Structures," Journal of Guidance, Control and Dynamics, vol. 12, no. 1, Feb 1989.

[14] Jeff Frolik, C.V.PhaniShankar and Steve Orth, "Fuzzy Rules for Automated Sensor Self-Validation and Confidence Measure," In Proceedings of American Control Conference, June 2000.

[15] Bernard Friedland, Advanced Control System Design, Prentice Hall, Inc., New Jersey, 1996.

[16] K.J.Astrom and B.Wittenmark, Adaptive Control, Addison-Wesley Publishing Co., Reading, MA 1989.

[17] Liu Hsu; Aldayr D. de Araujo; Ramon R. Costa, "Analysis and design of I/O based variable structure adaptive control. (input-output variable structure model reference adaptive control systems)," IEEE Transactions on Automatic Control, vol. 39, no.1, pp. 4, Jan 1994.

[18] E. Burdet, A. Codourey, "Evaluation of parametric and nonparametric nonlinear adaptive controllers (Nonlinear controllers)," Robotica, vol. 16, no. 1, 1998.

[19] Judith Hocherman-Frommer; Sanjeev R. Kulkarni; Peter J. Ramadge, "Controller switching based on output prediction errors," IEEE Transactions on Automatic Control, vol. 43, no. 5, pp. 596, May 1998

[20] Michel Barbeau; Froduald Kabanza; Richard St.-Denis, "A method for the synreport of controllers to handle safety, liveness, and real-time constraints," IEEE Transactions on Automatic Control, vol. 43, no. 11, pp. 1543, November 1998.

[21] Specht, D.F., "Probabilistic Neural Networks," Neural Networks, November 1990.

[22] Ronald R. Yager and Dimitar P. Filev, Essentials of Fuzzy Modeling and Control, John Wiley \& Sons, 1994.

[23] Jeff Frolik and Mohamed Abdelrahman, "Synreport of Quasi-Redundant sensor Data: A Probabilistic Approach," In Proceedings of American Control Conference, 2000.

[24] Hassan K. Khalil, Nonlinear Systems, Second edition, Prentice Hall Inc., 1996

[25] Mohamed Abdelrahman, Kevin Moore, Eric Larsen, Denis Clark and Paul King, "Experimental Control of a Cupola Furnace," In Proceedings of American Control Conference, 1998. 
[26] Pascal Gahinet, Arkadi Nemiroviski, Alan Laub, and Mahmoud Chilali, "LMI Control toolbox 1.0," The Math Works Inc.

[27] Jeff Frolik, C.V.Phanishankar and Steve Orth, "Fuzzy Rules for Automated Sensor Self-Validation and Confidence Measure”, Proc. of American Control Conference, 2000, pp. 2912-2916.

[28] Mohamed Abdelrahman, Parameshwaran Kandasamy and Jeff Frolik, “ $\boldsymbol{A}$ Methodology for the Fusion of Redundant Sensors", Proc. of American Control Conference, 2000, pp. 2917-2922.

[29] Jeff Frolik and Mohamed Abdelrahman, "Synthesis of Quasi-Redundant sensor Data: Probabilistic Approach”, Proc. Of American Control Conference, 2000, pp. 2922-2926.

[30] Vipin Vijayakumar, Mohamed Abdelrahman, Jeff Frolik, "A Convenient Methodology for the hardware implementation of fusion of Quasi-Redundant Sensors", Proc. Of $32^{\text {nd }}$ South-Eastern Symposium on System Theory, Florida, Mar 2000, pp. 349-353. 
A.1 Self-Validation Example using Matlab ....................................................5 54

A.1.1 Matlab M File (SV4.m).................................................................. 54

A.1.2 Matlab FIS File (SV4.fis)................................................................ 57

A.1.3 Raw Data Input File (RawIn.txt)_................................................... 58

A.1.4 Matlab Input/Output File (sv4_in-out.txt) ............................................ 60

A.2 Self-Validation Code Documents.............................................................. 70

A.2.1 Theoretical Timing Analysis Spreadsheet (Sugeno)............................ 70

A.2.2 Preprocessed Output File (sv4_pp.txt)............................................... 72

A.2.3 Self-confidence output file (sv4_outx.txt) ............................................ 74

A.3 Hardware Block Diagrams........................................................................... 77

A.4 FSM Controller ASM Charts ..................................................................... 95

A.4.1 Self-Validation Controller ASM Charts ……………………………... 95

A.5 Timing Diagrams .................................................................................. 100

A.5.1 Self-Validation Timing .................................................................... 100

A.6 MSF Hardware Block Diagrams ............................................................... 103

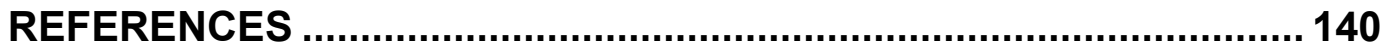




\section{Appendices}

\section{A.1 Self-Validation Example using Matlab}

\section{A.1.1 Matlab M File (SV4.m)}

$\%$ SV4.m

$\%$ Self Validation V4

$\%$ RLH - Modified to print out data for in_out.txt file

$\%$ Reading the three signal data from wk1 file

$\mathrm{a}=$ wk1read('TpDt1');

$\%$ Reading the FIS file to use it to find the self confidence of the

$\%$ sensor readings

fis $=$ readfis('sv4.fis');

$\%$ Read the temperature and the time from the file and seperating

$\%$ them into individual data points

$\%$ and creating the three inputs required, to the fuzzy system.

$\mathrm{sz}=\operatorname{size}(\mathrm{a})$

for $\mathrm{j}=1: \mathrm{sz}(1)$

$\%$ The Preprocessing required on the raw data to form the three input

$\%$ to the fuzzy System.

$\%$ Getting Change in time

if $\mathrm{j}==1$

ch_in_time $=a(j, 1)$;

else

ch_in_time $=a(j, 1)-a(j-1,1)$;

end

$\%$ Finding the current median and previous median temperature - to find

$\%$ the change in temperature value leading to the calculation of one

$\%$ of the input $=$ rate of change in Temperature

if (ch_in_time $==0$ )

temp $(1)=a(j, 2)$;

temp $(2)=a(j, 2)$;

temp $(3)=a(j, 2)$;

temp $(4)=a(j, 2)$;

temp $(5)=\mathrm{a}(\mathrm{j}, 2)$;

else

$\operatorname{temp}(6)=\mathrm{a}(\mathrm{j}, 2)$; 


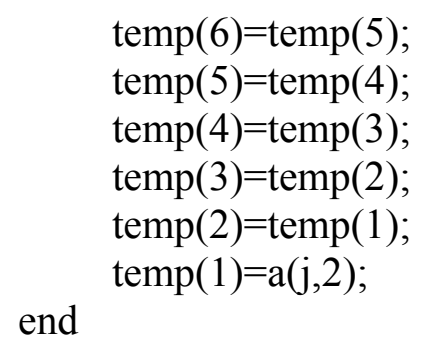

prev_temp $=$ median $(\operatorname{tem} p([2: 6])) ; \%$ Previous Median Temperature. curr_temp $=$ median $(\operatorname{temp}([1: 5])) ; \%$ Current Median Temperature.

$\%$ Setting the current median temperature as one of the input to the $\%$ fuzzy system $=$ Median Temperature med_temp $(\mathrm{j})=$ curr_temp;

$\%$ Finding the second input $=$ rate of change in temperature ch_in_temp $=$ (curr_temp-prev_temp);

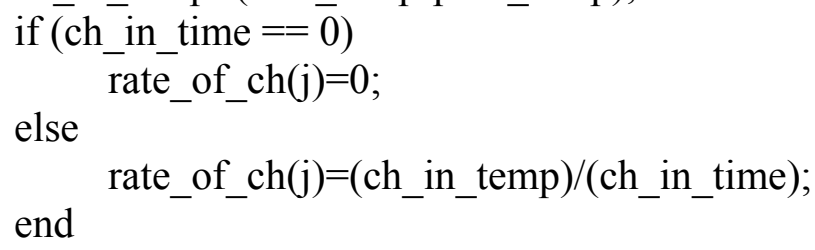

end

figure, $\operatorname{plot}(\mathrm{a}(:, 2))$

title('Raw Temperature');

figure, plot(med_temp)

title('Median-Filtered Temperature');

figure,plot(rate_of_ch)

title('Rate of Change');

figure, plot(vr) 


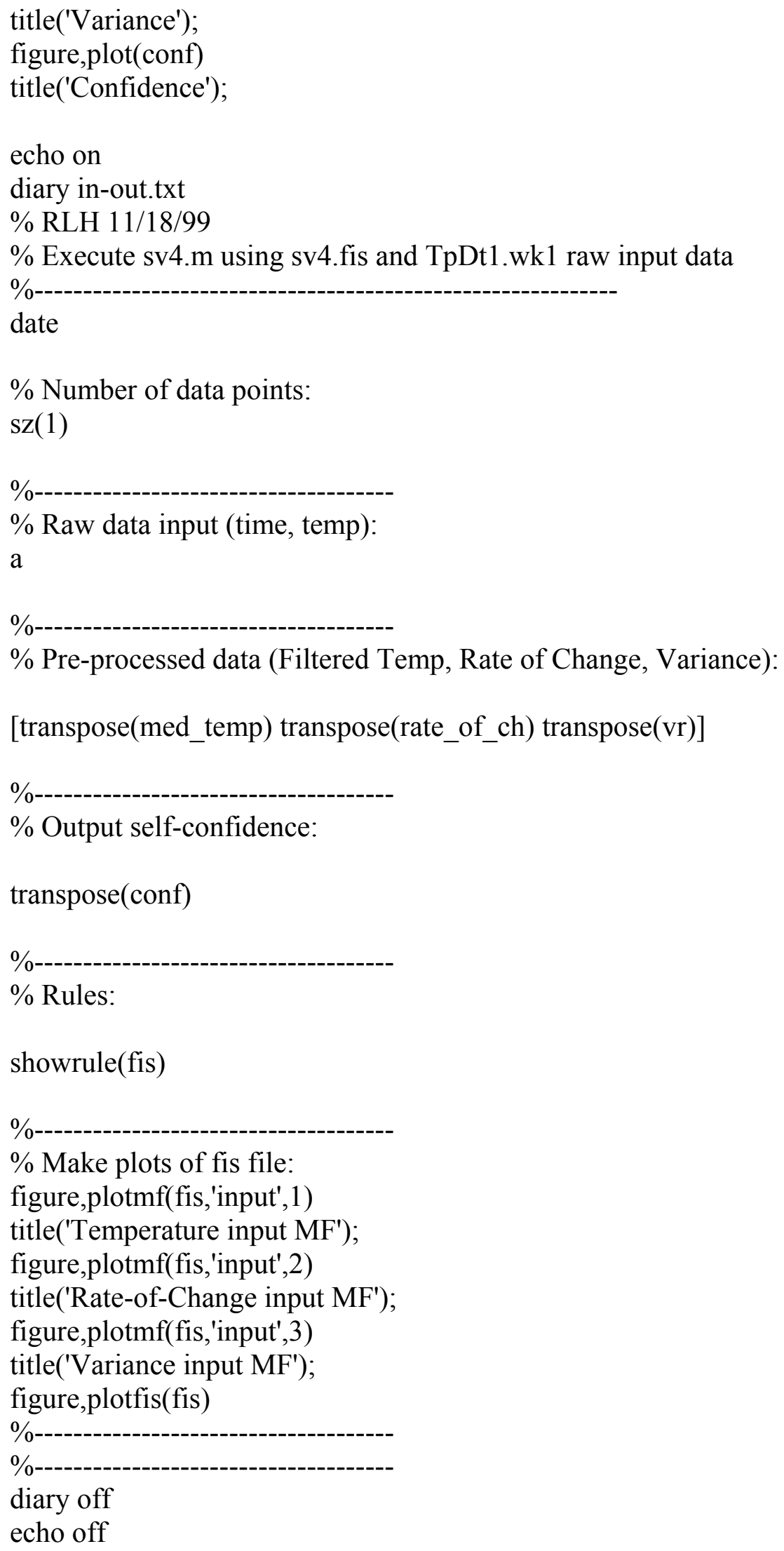




\section{A.1.2 Matlab FIS File (SV4.fis)}

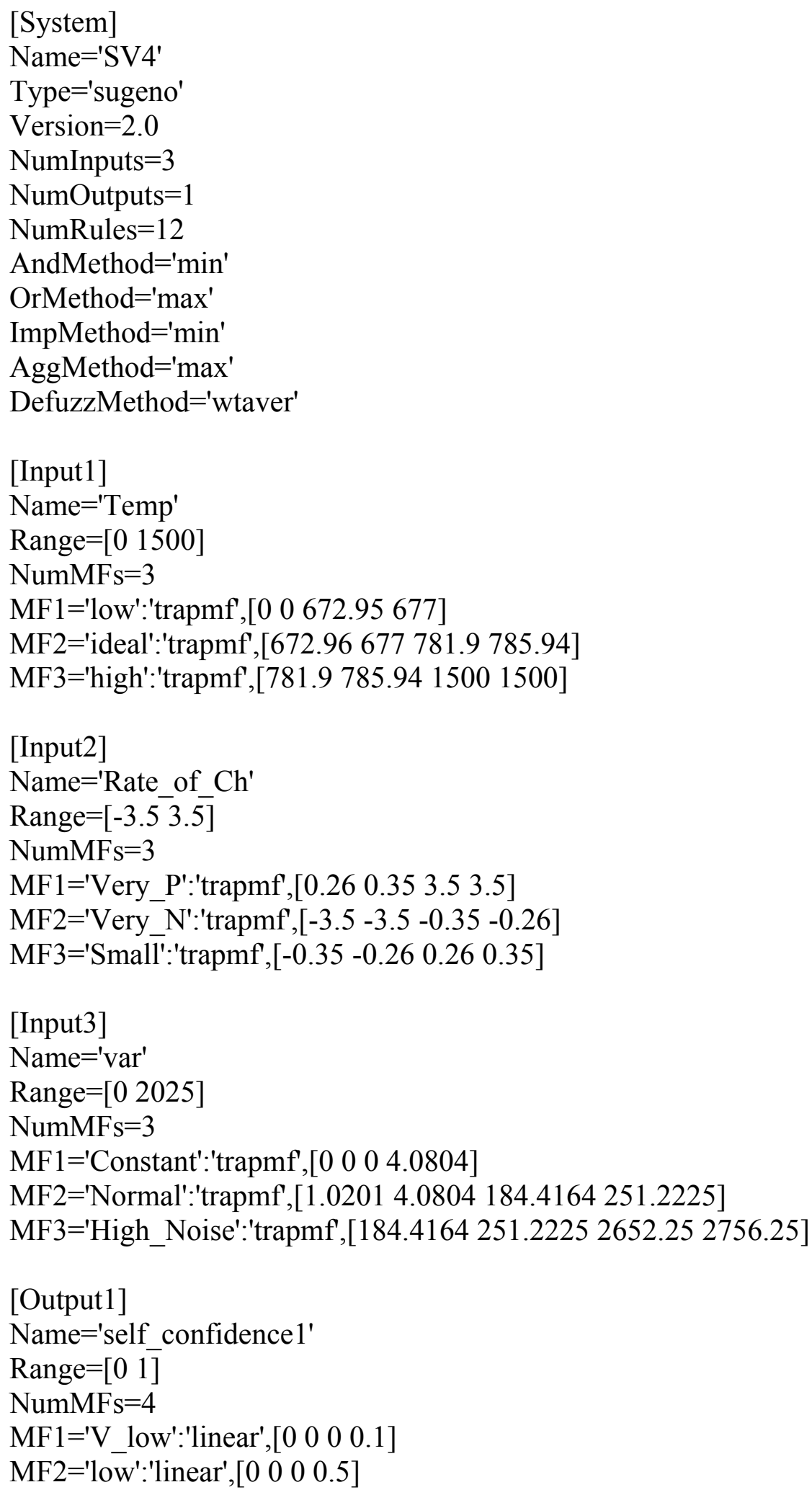




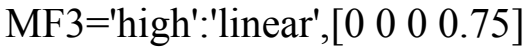

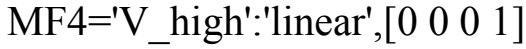

[Rules]

300,2 (1): 1

$100,2(1): 1$

$232,4(1): 1$

$210,2(1): 1$

220,2 (1): 1

00 1, 2 (1): 1

$110,1(1): 1$

$120,1(1): 1$

$320,1(1): 1$

310,1 (1): 1

003,2 (1): 1

$001,2(1): 1$

\section{A.1.3 Raw Data Input File (RawIn.txt)}

$\begin{array}{ll}0 & 779.2346227 \\ 56 & 779.6359885 \\ 118 & 779.1388128 \\ 176 & 776.3767261 \\ 238 & 771.7933885 \\ 295 & 769.1202064 \\ 359 & 765.8402284 \\ 416 & 761.098934 \\ 475 & 759.6134492 \\ 537 & 755.3356674 \\ 599 & 748.8223216 \\ 659 & 746.9354711 \\ 715 & 747.0019339 \\ 779 & 744.2286262 \\ 837 & 740.3850099 \\ 899 & 733.484972 \\ 959 & 729.9123854 \\ 1017 & 731.322776 \\ 1079 & 724.1163191 \\ 1135 & 717.5365106 \\ 1197 & 712.771911 \\ 1255 & 709.5169645 \\ 1319 & 706.4234273 \\ 1376 & 698.6740478 \\ 1439 & 692.4645316 \\ 1496 & 690.1521471 \\ 1559 & 684.3051548\end{array}$




$\begin{array}{ll}1618 & 680.5815166 \\ 1675 & 679.3756931 \\ 1738 & 675.8635272 \\ 1795 & 678.1759117 \\ 1857 & 681.270312 \\ 1914 & 681.9936334 \\ 1977 & 687.0741467 \\ 2035 & 693.6850286 \\ 2098 & 699.1591393 \\ 2154 & 702.9526927 \\ 2216 & 711.3554784 \\ 2279 & 717.8610558 \\ 2335 & 724.1016455 \\ 2398 & 730.0004269 \\ 2454 & 736.1995853 \\ 2516 & 742.0802406 \\ 2579 & 754.6399668 \\ 2636 & 761.786003 \\ 2694 & 765.5182727 \\ 2757 & 769.7865598 \\ 2814 & 775.2563547 \\ 2877 & 778.4569227 \\ 2935 & 776.5441776 \\ 2998 & 776.2334429 \\ 3055 & 780.2635 \\ 3118 & 780.1469745 \\ 3175 & 774.2689087 \\ 3238 & 777.0335849 \\ 3296 & 774.5727382 \\ 3359 & 773.5749344 \\ 3416 & 767.3619656 \\ 3479 & 768.4978738 \\ 3536 & 767.0926621 \\ 3594 & 764.5662159 \\ 3657 & 760.6639053 \\ 3716 & 756.6286692 \\ 3778 & 755.7171806 \\ 3836 & 754.2765798 \\ 3898 & 748.0687899 \\ 3955 & 746.5332423 \\ 4018 & 743.229096 \\ 4075 & 740.0242123 \\ 4138 & 730.3577719 \\ 4195 & 730.4389082 \\ 4258 & 722.5548769 \\ 4315 & 720.7940466\end{array}$




$\begin{array}{ll}4378 & 713.5910424 \\ 4435 & 710.0771502 \\ 4494 & 709.150988 \\ 4557 & 699.3032857 \\ 4615 & 695.9775606 \\ 4678 & 688.4638216 \\ 4735 & 681.8917815 \\ 4798 & 678.0239969 \\ 4856 & 678.0093234 \\ 4944 & 680.8007572 \\ 4975 & 683.0898366 \\ 5035 & 684.0997246 \\ 5094 & 694.8338841 \\ 5154 & 693.0462961 \\ 5217 & 698.3149765 \\ 5274 & 699.6347361 \\ 5336 & 709.5661641 \\ 5394 & 716.8192312 \\ 5456 & 720.3288077 \\ 5514 & 727.5723801 \\ 5576 & 737.6488177 \\ 5633 & 739.3267854 \\ 5696 & 748.6263861 \\ 5753 & 759.2077677 \\ 5816 & 763.4113184 \\ 5873 & 775.0069037 \\ 5936 & 781.3752399\end{array}$

A.1.4 Matlab Input/Output File (sv4_in-out.txt)

\% RLH 11/18/99

$\%$ Execute sv4.m using sv4.fis and TpDt1.wk1 raw input data

\%-------------------------------------------------------------

date

ans $=$

18-Nov-1999

$\%$ Number of data points:

$\mathrm{sz}(1)$

ans $=$

100 


\begin{tabular}{|c|c|}
\hline \multicolumn{2}{|c|}{$\begin{array}{l}\text { \% } \\
\text { \% Raw data input (time, temp): } \\
\text { a }\end{array}$} \\
\hline \multicolumn{2}{|l|}{$a=$} \\
\hline \multicolumn{2}{|c|}{$1.0 \mathrm{e}+003 *$} \\
\hline 0 & .7792 \\
\hline 0.0560 & 0.7796 \\
\hline 0.1180 & 0.7791 \\
\hline 0.1760 & 0.7764 \\
\hline 0.2380 & 0.7718 \\
\hline 0.2950 & 0.7691 \\
\hline 0.3590 & 0.7658 \\
\hline 0.4160 & 0.7611 \\
\hline 0.4750 & 0.7596 \\
\hline 0.5370 & 0.7553 \\
\hline 0.5990 & 0.7488 \\
\hline 0.6590 & 0.7469 \\
\hline 0.7150 & 0.7470 \\
\hline 0.7790 & 0.7442 \\
\hline 0.8370 & 0.7404 \\
\hline 0.8990 & 0.7335 \\
\hline 0.9590 & 0.7299 \\
\hline 1.0170 & 0.7313 \\
\hline 1.0790 & 0.7241 \\
\hline 1.1350 & 0.7175 \\
\hline 1.1970 & 0.7128 \\
\hline 1.2550 & 0.7095 \\
\hline 1.3190 & 0.7064 \\
\hline 1.3760 & 0.6987 \\
\hline 1.4390 & 0.6925 \\
\hline 1.4960 & 0.6902 \\
\hline 1.5590 & 0.6843 \\
\hline 1.6180 & 0.6806 \\
\hline 1.6750 & 0.6794 \\
\hline 1.7380 & 0.6759 \\
\hline 1.7950 & 0.6782 \\
\hline 1.8570 & 0.6813 \\
\hline 1.9140 & 0.6820 \\
\hline 1.9770 & 0.6871 \\
\hline 2.0350 & 0.6937 \\
\hline 2.0980 & 0.6992 \\
\hline 2.1540 & 0.7030 \\
\hline 2.2160 & 0.7114 \\
\hline
\end{tabular}




$\begin{array}{ll}2.2790 & 0.7179 \\ 2.3350 & 0.7241 \\ 2.3980 & 0.7300 \\ 2.4540 & 0.7362 \\ 2.5160 & 0.7421 \\ 2.5790 & 0.7546 \\ 2.6360 & 0.7618 \\ 2.6940 & 0.7655 \\ 2.7570 & 0.7698 \\ 2.8140 & 0.7753 \\ 2.8770 & 0.7785 \\ 2.9350 & 0.7765 \\ 2.9980 & 0.7762 \\ 3.0550 & 0.7803 \\ 3.1180 & 0.7801 \\ 3.1750 & 0.7743 \\ 3.2380 & 0.7770 \\ 3.2960 & 0.7746 \\ 3.3590 & 0.7736 \\ 3.4160 & 0.7674 \\ 3.4790 & 0.7685 \\ 3.5360 & 0.7671 \\ 3.5940 & 0.7646 \\ 3.6570 & 0.7607 \\ 3.7160 & 0.7566 \\ 3.7780 & 0.7557 \\ 3.8360 & 0.7543 \\ 3.8980 & 0.7481 \\ 3.9550 & 0.7465 \\ 4.0180 & 0.7432 \\ 4.0750 & 0.7400 \\ 4.1380 & 0.7304 \\ 4.1950 & 0.7304 \\ 4.2580 & 0.7226 \\ 4.3150 & 0.7208 \\ 4.3780 & 0.7136 \\ 4.4350 & 0.7101 \\ 4.4940 & 0.7092 \\ 4.5570 & 0.6993 \\ 4.6150 & 0.6960 \\ 4.6780 & 0.6885 \\ 4.7350 & 0.6819 \\ 4.7980 & 0.6780 \\ 4.8560 & 0.6780 \\ 4.9440 & 0.6808 \\ 4.9750 & 0.6831\end{array}$




$$
\begin{array}{ll}
5.0350 & 0.6841 \\
5.0940 & 0.6948 \\
5.1540 & 0.6930 \\
5.2170 & 0.6983 \\
5.2740 & 0.6996 \\
5.3360 & 0.7096 \\
5.3940 & 0.7168 \\
5.4560 & 0.7203 \\
5.5140 & 0.7276 \\
5.5760 & 0.7376 \\
5.6330 & 0.7393 \\
5.6960 & 0.7486 \\
5.7530 & 0.7592 \\
5.8160 & 0.7634 \\
5.8730 & 0.7750 \\
5.9360 & 0.7814
\end{array}
$$

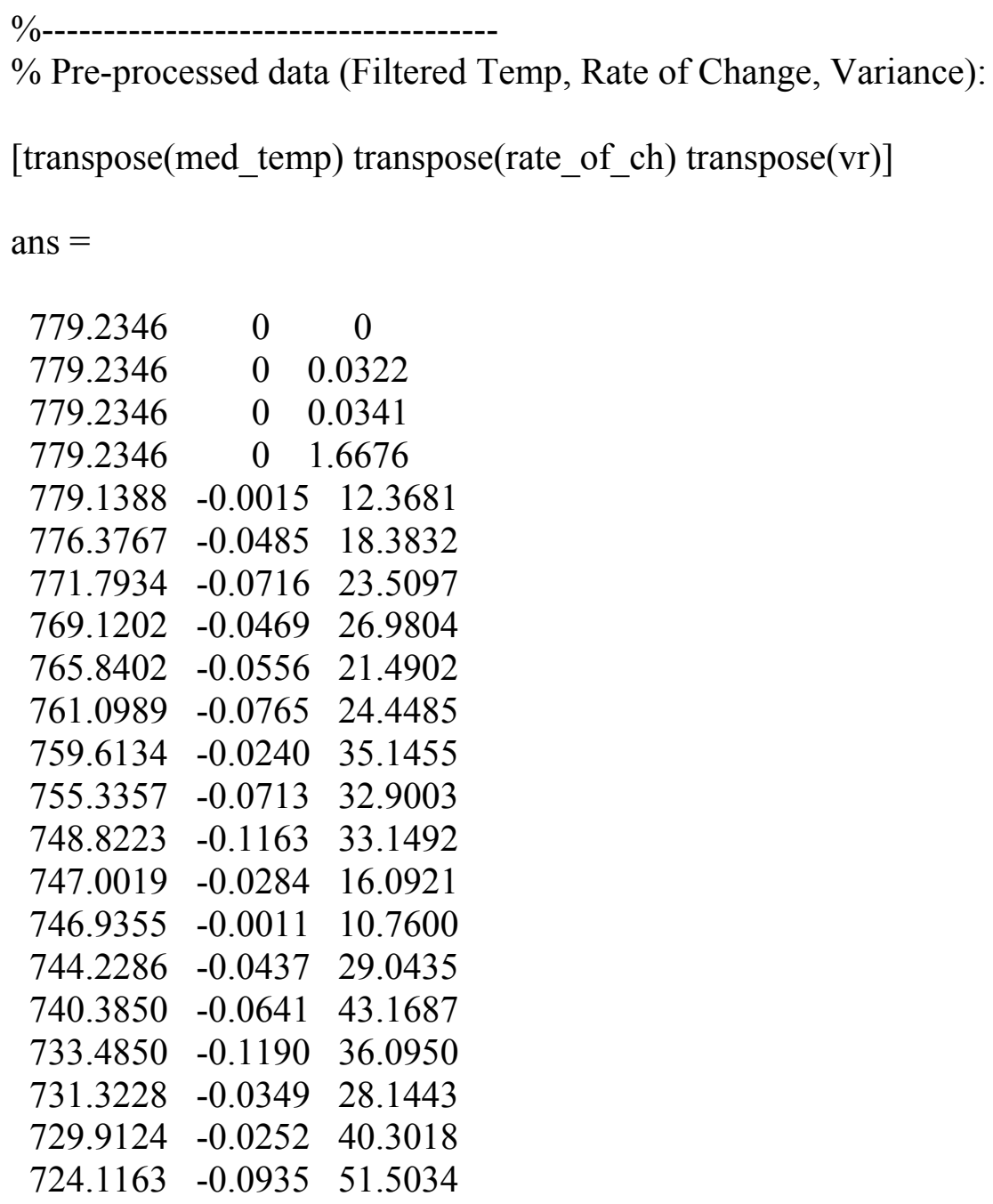




$\begin{array}{lll}717.5365 & -0.1134 & 64.0739 \\ 712.7719 & -0.0744 & 40.4590 \\ 709.5170 & -0.0571 & 40.4093 \\ 706.4234 & -0.0491 & 60.9554 \\ 698.6740 & -0.1360 & 57.7605 \\ 692.4645 & -0.0986 & 61.0663 \\ 690.1521 & -0.0392 & 40.7508 \\ 684.3052 & -0.1026 & 27.7856 \\ 680.5815 & -0.0591 & 25.8352 \\ 679.3757 & -0.0212 & 7.9057 \\ 679.3757 & 0 & 3.7637 \\ 679.3757 & 0 & 4.8436 \\ 681.2703 & 0.0301 & 14.6033 \\ 681.9936 & 0.0125 & 35.5197 \\ 687.0741 & 0.0806 & 49.8494 \\ 693.6850 & 0.1181 & 59.2496 \\ 699.1591 & 0.0883 & 67.8309 \\ 702.9527 & 0.0602 & 78.6293 \\ 711.3555 & 0.1500 & 84.8290 \\ 717.8611 & 0.1033 & 90.1782 \\ 724.1016 & 0.1114 & 76.5131 \\ 730.0004 & 0.0951 & 73.3023 \\ 736.1996 & 0.0984 & 111.8839 \\ 742.0802 & 0.1032 & 145.3136 \\ 754.6400 & 0.2165 & 133.4396 \\ 761.7860 & 0.1134 & 103.4643 \\ 765.5183 & 0.0655 & 49.0632 \\ 769.7866 & 0.0678 & 37.4642 \\ 775.2564 & 0.0943 & 27.3302 \\ 776.2334 & 0.0155 & 9.5115 \\ 776.5442 & 0.0055 & 3.8494 \\ 778.4569 & 0.0304 & 2.9445 \\ 776.5442 & -0.0336 & 6.4174 \\ 777.0336 & 0.0078 & 5.6818 \\ 777.0336 & 0 & 6.7649 \\ 774.5727 & -0.0391 & 7.6432 \\ 774.2689 & -0.0053 & 11.1846 \\ 773.5749 & -0.0110 & 15.4671 \\ 768.4979 & -0.0891 & 13.1891 \\ 767.3620 & -0.0196 & 9.5560 \\ 767.0927 & -0.0043 & 9.9518 \\ 764.5662 & -0.0428 & 20.0147 \\ 760.6639 & -0.0629 & 19.4620 \\ 756.6287 & -0.0696 & 17.1302 \\ 755.7172 & -0.0147 & 17.1748 \\ 754.2766 & -0.0253 & 21.2207\end{array}$




$\begin{array}{lll}748.0688 & -0.0985 & 24.5630 \\ 746.5332 & -0.0269 & 23.1204 \\ 743.2291 & -0.0524 & 42.0565 \\ 740.0242 & -0.0562 & 47.5914 \\ 730.4389 & -0.1521 & 63.5263 \\ 730.3578 & -0.0014 & 49.1593 \\ 722.5549 & -0.1239 & 41.2988 \\ 720.7940 & -0.0309 & 52.5718 \\ 713.5910 & -0.1221 & 32.8590 \\ 710.0772 & -0.0558 & 48.8266 \\ 709.1510 & -0.0160 & 58.2177 \\ 699.3033 & -0.1563 & 68.3216 \\ 695.9776 & -0.0583 & 87.8930 \\ 688.4638 & -0.1193 & 65.2264 \\ 681.8918 & -0.1133 & 54.3268 \\ 680.8008 & -0.0124 & 15.0831 \\ 680.8008 & 0 & 4.3865 \\ 680.8008 & 0 & 6.3251 \\ 683.0898 & 0.0388 & 33.9988 \\ 684.0997 & 0.0168 & 41.4333 \\ 693.0463 & 0.1420 & 42.0253 \\ 694.8339 & 0.0314 & 30.7168 \\ 698.3150 & 0.0561 & 33.6416 \\ 699.6347 & 0.0228 & 87.8179 \\ 709.5662 & 0.1602 & 78.7328 \\ 716.8192 & 0.1251 & 95.1722 \\ 720.3288 & 0.0566 & 96.1207 \\ 727.5724 & 0.1271 & 81.5600 \\ 737.6488 & 0.1599 & 104.9680 \\ 739.3268 & 0.0294 & 124.5435 \\ 748.6264 & 0.1476 & 107.5099 \\ 759.2078 & 0.1856 & 154.9003 \\ 763.4113 & 0.0667 & 138.6848\end{array}$

\%------------------------------------------

$\%$ Output self-confidence:

transpose(conf)

ans $=$

0.5000

0.5000

0.5000

0.5759

1.0000 
1.0000

1.0000

1.0000

1.0000

1.0000

1.0000

1.0000

1.0000

1.0000

1.0000

1.0000

1.0000

1.0000

1.0000

1.0000

1.0000

1.0000

1.0000

1.0000

1.0000

1.0000

1.0000

1.0000

1.0000

1.0000

1.0000

0.9262

1.0000

1.0000

1.0000

1.0000

1.0000

1.0000

1.0000

1.0000

1.0000

1.0000

1.0000

1.0000

1.0000

1.0000

1.0000

1.0000

1.0000

1.0000

1.0000 
0.9454

0.7652

1.0000

1.0000

1.0000

1.0000

1.0000

1.0000

1.0000

1.0000

1.0000

1.0000

1.0000

1.0000

1.0000

1.0000

1.0000

1.0000

1.0000

1.0000

1.0000

1.0000

1.0000

1.0000

1.0000

1.0000

1.0000

1.0000

1.0000

1.0000

1.0000

1.0000

1.0000

1.0000

1.0000

1.0000

1.0000

1.0000

1.0000

1.0000

1.0000

1.0000

1.0000

1.0000

1.0000

1.0000 
1.0000

1.0000

1.0000

$\%$

$\%$ Rules:

showrule(fis)

ans $=$

If (Temp is high) then

(self_confidencel is low) (1)

If (Temp is low) then (self_confidencel is low) (1)

If (Temp is ideal) and (Rate_of_Ch is Small) and (var is Normal) then (self confidencel is $\mathrm{V}$ high) (1)

If (Temp is ideal) and (Rate_of_Ch is Very_P) then (self_confidencel is low) (1)

If (Temp is ideal) and (Rate_of_Ch is Very_N) then (self_confidencel is low) (1)

If (var is Constant) then

(self_confidencel is low) (1)

If (Temp is low) and (Rate_of_Ch is Very_P) then (self_confidencel is V_low) (1)

If (Temp is low) and (Rate_of_Ch is Very_N) then (self_confidencel is V_low) $(\overline{1})$

If (Temp is high) and (Rate_of_Ch is Very_N) then (self_confidencel is V_low) (1)

If (Temp is high) and (Rate_of_Ch is Very_P) then

(self_confidencel is $\mathrm{V}$ _low) (1)

If (var is High_Noise) then

(self_confidencel is low) (1)

If (var is Constant) then

(self_confidencel is low) (1)

$\%$

$\%$ Make plots of fis file:

figure,plotmf(fis,'input',1)

title('Temperature input MF');

figure, plotmf(fis, 'input',2)

title('Rate-of-Change input $\mathrm{MF}^{\prime}$ );

figure,plotmf(fis,'input',3)

title('Variance input $\mathrm{MF}^{\prime}$ );

figure,plotfis(fis) 
\%0----------------------------------------

\%------------------------------------------

diary off 


\section{A.2 Self-Validation Code Documents}

\section{A.2.1 Theoretical Timing Analysis Spreadsheet (Sugeno)}

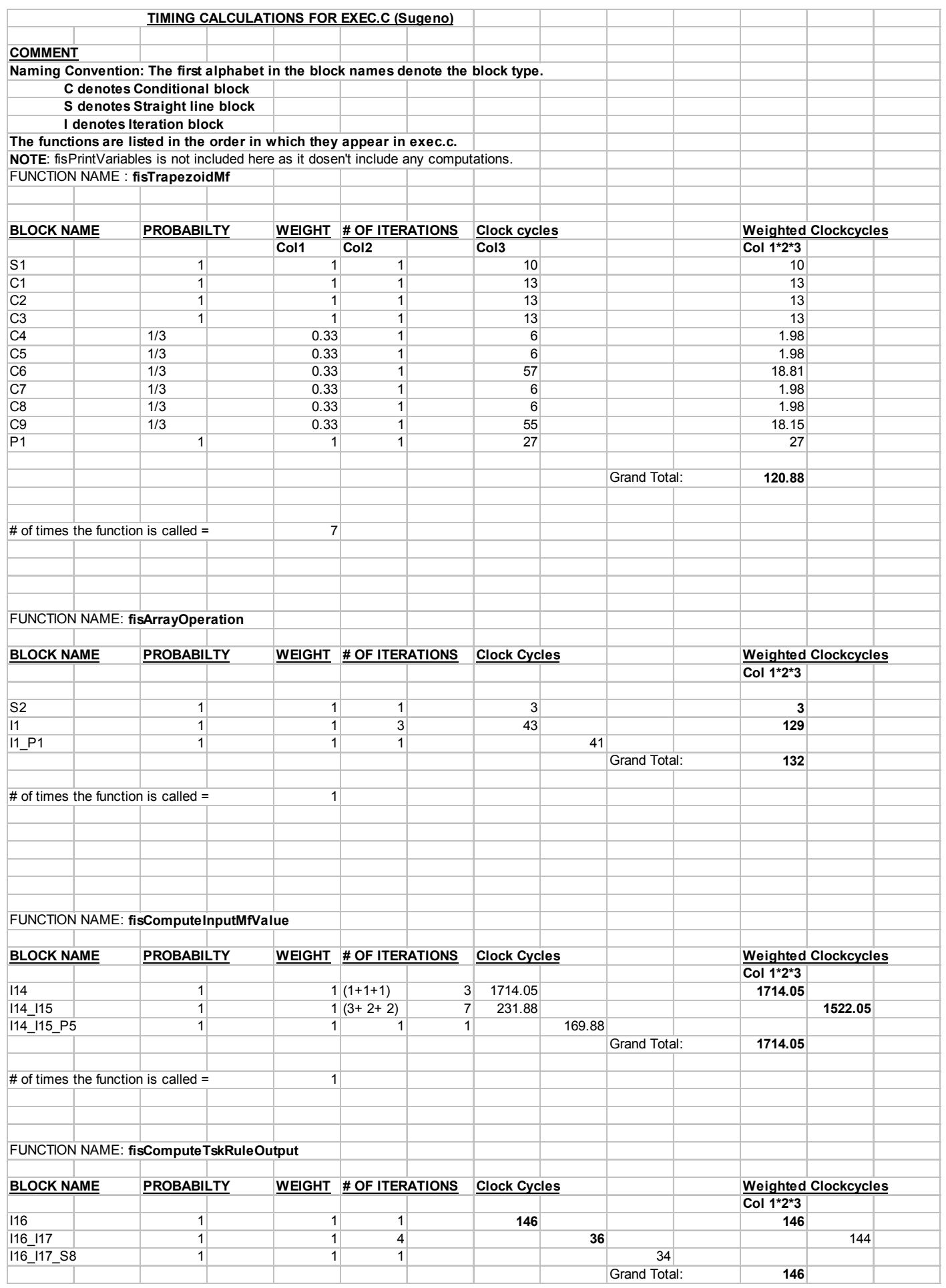


\# of times the function is called $=$

FUNCTION:fisComputeFiring Strength

BLOCK NAME

PROBABILTY

WEIGHT \# OF ITERATIONS

119

119 C18

1

I19_C18_I20

$1 / 2$

119 C18 120 S9

I19_C18_120 C19

I19_C18_I20_C20

$1 / 2 * 1 / 3$

$1 / 2 * 1 / 3$

_C18_120_C21

$1 / 2 * 1 / 3$

119 C18 20 S10

I19_C18_P6

119_C22

119_C22_I20

119 C22 I20 S11

$1 / 2$

$1 / 2$

$1 / 2$

19 C22 $120 \mathrm{C} 23$

$119 \mathrm{C} 22 \mathrm{I} 20 \mathrm{C} 24 \quad 1 / 2 * 1 / 3$

I19_C22_I20_C25 1/2*1/3

119_C22_I20_S12 1/2

$119 \mathrm{C} 22 \mathrm{P} 7$

I19_S13

$1 / 2$

121

1
0.5
0.5
0.5

16
1

Weighted Clockcycles

Col 1*2*3

11820

205
193
10
23
10
17
16
160
205
193
10
23
10
17
16
160
21
70
Grand Total:

201.375

289.5

5
3.795

1.65

$0.165 \quad 1$

$0.5 \quad 1$

$\begin{array}{lll}0.5 & 1 & 160\end{array}$

$\begin{array}{lll}0.5 & 1 & 205 \\ 0.5 & 3 & 193\end{array}$

$0.5 \quad 1$

0.165

0.165

0.5

1
1
1
1
1
16
16

\# of times the function is called $=$

FUNCTION:fisEvaluate

BLOCK NAME

PROBABILTY

$\mathrm{S} 15$

P11

S16

130

130_P13

C30

C30_I32

C32

C32_P14

C32 I33

C32_I33_134

C32_I33_I34_C33

C32 I33 I34_C34

C32_I33_S18

C32_I33_I34

C32_133_C33

C32_133_C34

C33

C33_I35_P15

C33 I35 P16

C34

\# of times the function is called $=$

Setup functions called by exec.c

FUNCTION: fisMin

BLOCK NAME

PROBABILTY
WEIGHT \# OF ITERATIONS

Clock Cycles

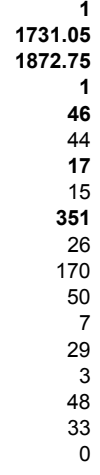

Grand Total:
15782.55

Weighted Clockcycles Col 1*2*3

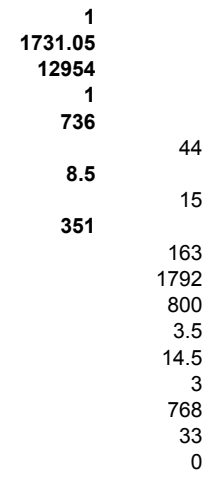

Weighted Clockcycles Col 1*2*3 


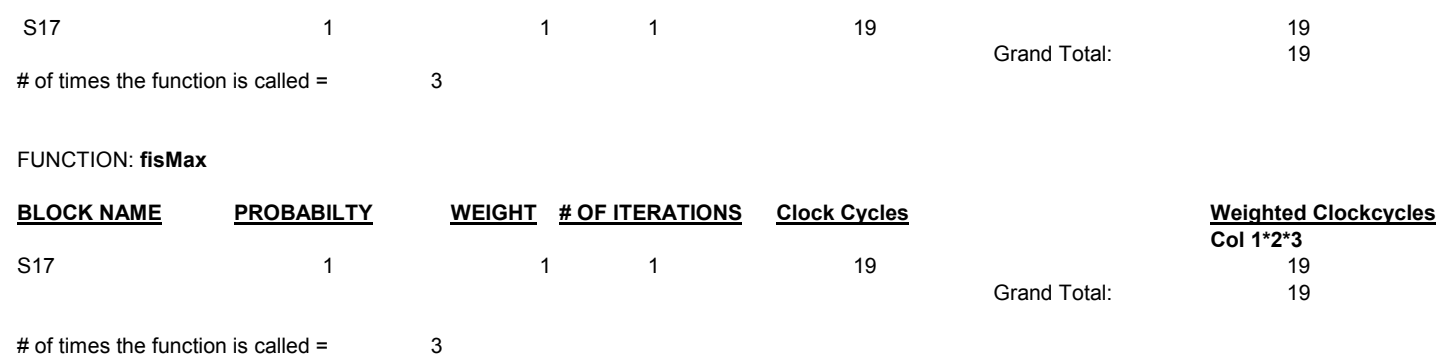

\section{A.2.2 Preprocessed Output File (sv4_pp.txt)}

$\begin{array}{lll}779.412 & 0.000 & 0.000 \\ 779.412 & 0.000 & 0.000 \\ 779.412 & 0.000 & 0.000 \\ 779.412 & 0.000 & 1.730 \\ 779.412 & 0.000 & 13.149 \\ 776.471 & -0.051 & 17.647 \\ 771.765 & -0.071 & 23.875 \\ 769.412 & -0.039 & 26.990 \\ 765.882 & -0.060 & 22.145 \\ 761.176 & -0.076 & 25.260 \\ 759.412 & -0.028 & 34.602 \\ 755.294 & -0.067 & 32.180 \\ 748.823 & -0.115 & 31.834 \\ 747.059 & -0.025 & 15.917 \\ 747.059 & 0.000 & 10.727 \\ 744.118 & -0.046 & 28.374 \\ 740.588 & -0.057 & 43.253 \\ 733.529 & -0.119 & 35.986 \\ 731.176 & -0.037 & 29.066 \\ 730.000 & -0.021 & 40.138 \\ 724.118 & -0.094 & 50.173 \\ 717.647 & -0.110 & 62.976 \\ 712.941 & -0.071 & 40.138 \\ 709.412 & -0.060 & 40.138 \\ 706.471 & -0.046 & 61.592 \\ 698.823 & -0.133 & 57.785 \\ 692.353 & -0.101 & 62.630 \\ 690.000 & -0.039 & 41.176 \\ 684.118 & -0.101 & 27.336 \\ 680.588 & -0.055 & 24.913 \\ 679.412 & -0.018 & 7.266\end{array}$




$\begin{array}{lll}679.412 & 0.000 & 3.460 \\ 679.412 & 0.000 & 4.498 \\ 681.176 & 0.028 & 14.187 \\ 681.765 & 0.009 & 35.640 \\ 687.059 & 0.083 & 51.211 \\ 693.529 & 0.115 & 60.554 \\ 699.412 & 0.094 & 67.474 \\ 702.941 & 0.055 & 76.817 \\ 711.176 & 0.145 & 83.045 \\ 717.647 & 0.101 & 90.311 \\ 724.118 & 0.115 & 79.239 \\ 730.000 & 0.094 & 76.125 \\ 736.471 & 0.101 & 112.111 \\ 742.353 & 0.101 & 143.253 \\ 754.706 & 0.211 & 129.412 \\ 761.765 & 0.110 & 101.384 \\ 765.294 & 0.060 & 49.135 \\ 770.000 & 0.074 & 37.024 \\ 775.294 & 0.090 & 27.336 \\ 776.471 & 0.018 & 8.997 \\ 776.471 & 0.000 & 3.114 \\ 778.235 & 0.028 & 2.422 \\ 776.471 & -0.030 & 5.882 \\ 777.059 & 0.009 & 5.190 \\ 777.059 & 0.000 & 6.228 \\ 774.706 & -0.037 & 6.920 \\ 774.118 & -0.009 & 10.035 \\ 773.529 & -0.009 & 15.225 \\ 768.235 & -0.092 & 14.187 \\ 767.647 & -0.009 & 8.651 \\ 767.059 & -0.009 & 9.689 \\ 764.706 & -0.039 & 20.415 \\ 760.588 & -0.064 & 19.377 \\ 756.471 & -0.069 & 17.993 \\ 755.882 & -0.009 & 16.609 \\ 754.118 & -0.030 & 20.069 \\ 748.235 & -0.092 & 24.567 \\ 746.471 & -0.030 & 23.183 \\ 742.941 & -0.055 & 40.138 \\ 740.000 & -0.051 & 45.329 \\ 730.588 & -0.149 & 61.592 \\ 730.588 & 0.000 & 51.211 \\ 722.353 & -0.129 & 43.253 \\ 720.588 & -0.030 & 52.941 \\ 713.529 & -0.119 & 31.142 \\ 710.000 & -0.055 & 47.405\end{array}$




$\begin{array}{lll}709.412 & -0.009 & 59.862 \\ 699.412 & -0.159 & 69.896 \\ 695.882 & -0.060 & 90.657 \\ 688.235 & -0.119 & 65.052 \\ 681.765 & -0.110 & 52.941 \\ 680.588 & -0.011 & 14.187 \\ 680.588 & 0.000 & 3.460 \\ 680.588 & 0.000 & 5.536 \\ 682.941 & 0.039 & 33.218 \\ 684.118 & 0.018 & 40.484 \\ 692.941 & 0.138 & 41.522 \\ 694.706 & 0.030 & 29.758 \\ 698.235 & 0.055 & 33.218 \\ 699.412 & 0.018 & 90.657 \\ 709.412 & 0.161 & 81.661 \\ 717.059 & 0.131 & 98.616 \\ 720.588 & 0.055 & 95.502 \\ 727.647 & 0.122 & 79.931 \\ 737.647 & 0.159 & 103.806 \\ 739.412 & 0.030 & 125.952 \\ 748.823 & 0.149 & 108.304 \\ 759.412 & 0.184 & 156.055 \\ 763.529 & 0.064 & 136.332\end{array}$

A.2.3 Self-confidence output file (sv4_outx.txt)
0.501960814
0.501960814
0.501960814
0.501960814
1.000000000
1.000000000
1.000000000
1.000000000
1.000000000
1.000000000
1.000000000
1.000000000
1.000000000
1.000000000
1.000000000
1.000000000
1.000000000
1.000000000
1.000000000
1.000000000 
1.000000000

1.000000000

1.000000000

1.000000000

1.000000000

1.000000000

1.000000000

1.000000000

1.000000000

1.000000000

1.000000000

0.501960814

1.000000000

1.000000000

1.000000000

1.000000000

1.000000000

1.000000000

1.000000000

1.000000000

1.000000000

1.000000000

1.000000000

1.000000000

1.000000000

1.000000000

1.000000000

1.000000000

1.000000000

1.000000000

1.000000000

0.501960814

0.501960814

1.000000000

1.000000000

1.000000000

1.000000000

1.000000000

1.000000000

1.000000000

1.000000000

1.000000000

1.000000000

1.000000000

1.000000000

1.000000000 
1.000000000
1.000000000
1.000000000
1.000000000
1.000000000
1.000000000
1.000000000
1.000000000
1.000000000
1.000000000
1.000000000
1.000000000
1.000000000
1.000000000
1.000000000
1.000000000
1.000000000
0.501960814
1.000000000
1.000000000
1.000000000
1.000000000
1.000000000
1.000000000
1.000000000
1.000000000
1.000000000
1.000000000
1.000000000
1.000000000
1.000000000
1.000000000
1.000000000
1.000000000 


\section{A.3 Hardware Block Diagrams}

The following notes help in understanding the block diagrams.

1. All names that start with a 'B', 'P' are signals that connect with BUS Interface and Processor Interface respectively.

2. 4 block diagrams are used to describe the FPGA H/W. They are listed below
a. Top level (Top.vsd)
b. Memory Interface (Memifc.vsd)
c. Bus Interface (BusIfc.vsd)
d. Register file (RegFile.vsd)

3. The Memory Interface depicts the 2 port Interface between RAM and Processor, RAM and CPU.

4. The Bus Interface connects PC/104 Bus to Memory and SP and contains 8 x 16 Register File.

5. The Top Level shows the connections between the three modules.

6. The internal signals that connect the 3 modules, namely PC104 ifc, 2 Port Mem Ifc and Signal Processor application, are marked as 1,2 and 3. The signals that constitute each of these groups are listed here.

a. (BMxxx) -> BMAI, MAI, BMDoutI, BMModeI, BMR_n_I, BMW_n_I, BMCe_n_I, BMDWeI, and MDinI.

b. (PBxxx) -> PBA_I, PBD_I, PBR_n_I, PBW_n_I, PBRF_I.

c. (PMxxx) -> PMA_I, PMDout_I, PMR_n_I, PMW_n_I, PMCe_n_I, and PMDWe_I. 
7. The following equations define the signals used in the Regfile block diagram
a. $\quad \mathrm{RFen}=\left(\mathrm{RF} * \operatorname{RFAdReg}(2)^{\prime}\right)$
b. $\quad$ RFtoBus $=\left(\mathrm{RF} * \mathrm{BR} \_\mathrm{n}^{\prime}\right)$
c. $\quad$ RFtoProc $=(\operatorname{PBRF} *$ PBR_n' $)$
d. $\quad \operatorname{PBen}=\left(\operatorname{PBRF} * \operatorname{PBA}(2) * \operatorname{PBW} \_\right.$' $)$

8. The following equations define the signals used in the BusIfc block diagram
a. BMR_n $=\left(B R \_n ' * B M w r i t e ' * \text { Mem * BMmode }\right)^{\prime}$
b. BMW_n=(BW_n'* BMwrite * Mem * BMmode $)^{\prime}$
c. $\mathrm{BMDWe}=(\mathrm{BMwrite} * \mathrm{Mem} *$ BMmode $)$
d. MDrd=(BR_n' $*$ BMwrite' $*$ Mem * BMmode $)$
e. BMce_n=(Mem * BMmode $)^{\prime}$
f. $\quad$ BDout $=\left(B R \_n ' *\right.$ BrdSel $)$
g. $\quad B D i n=\left(B R \_n *\right.$ BrdSel $)$ 


\section{8k X}

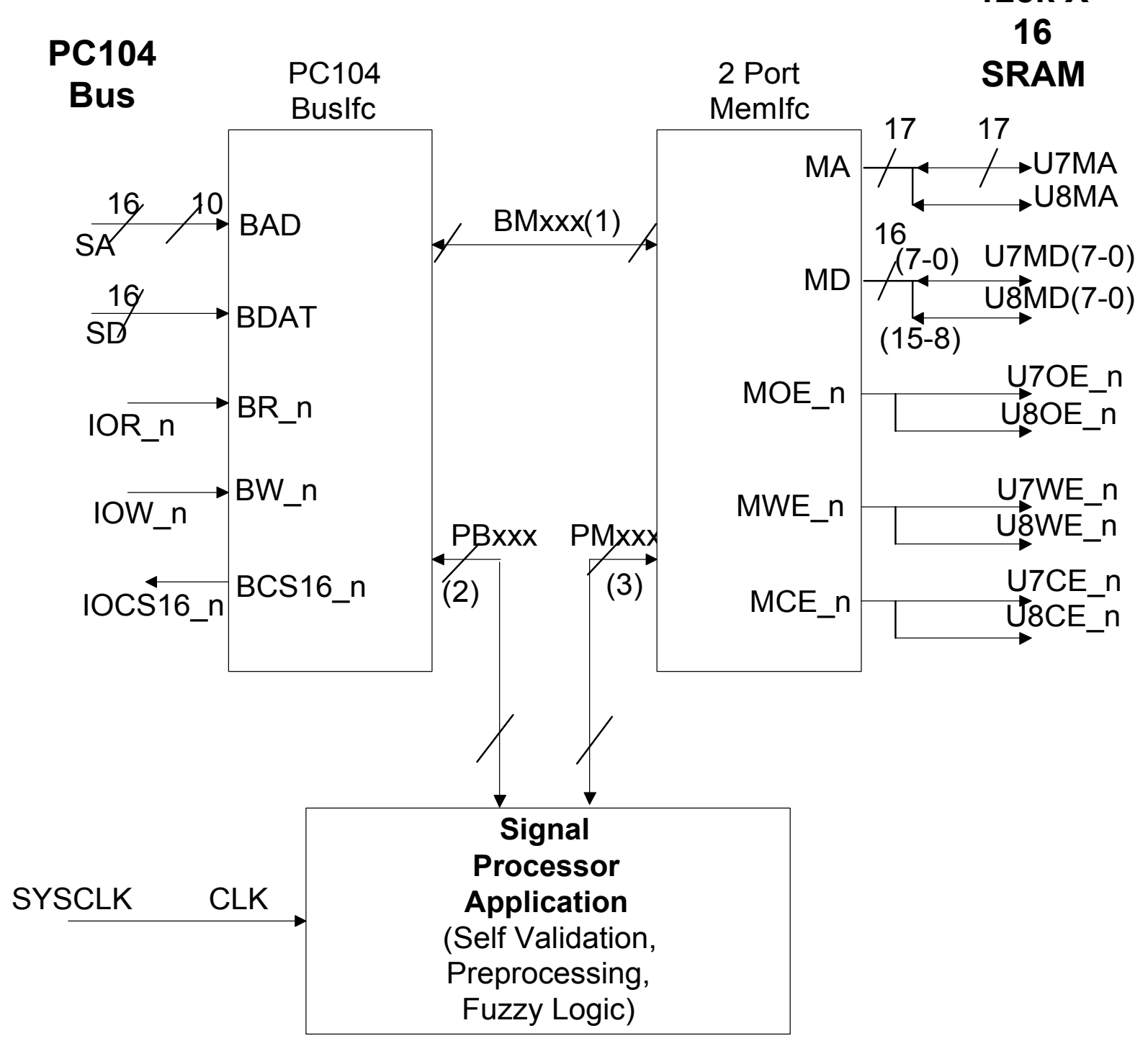

Top Level Hardware Architecture (FPGA) 

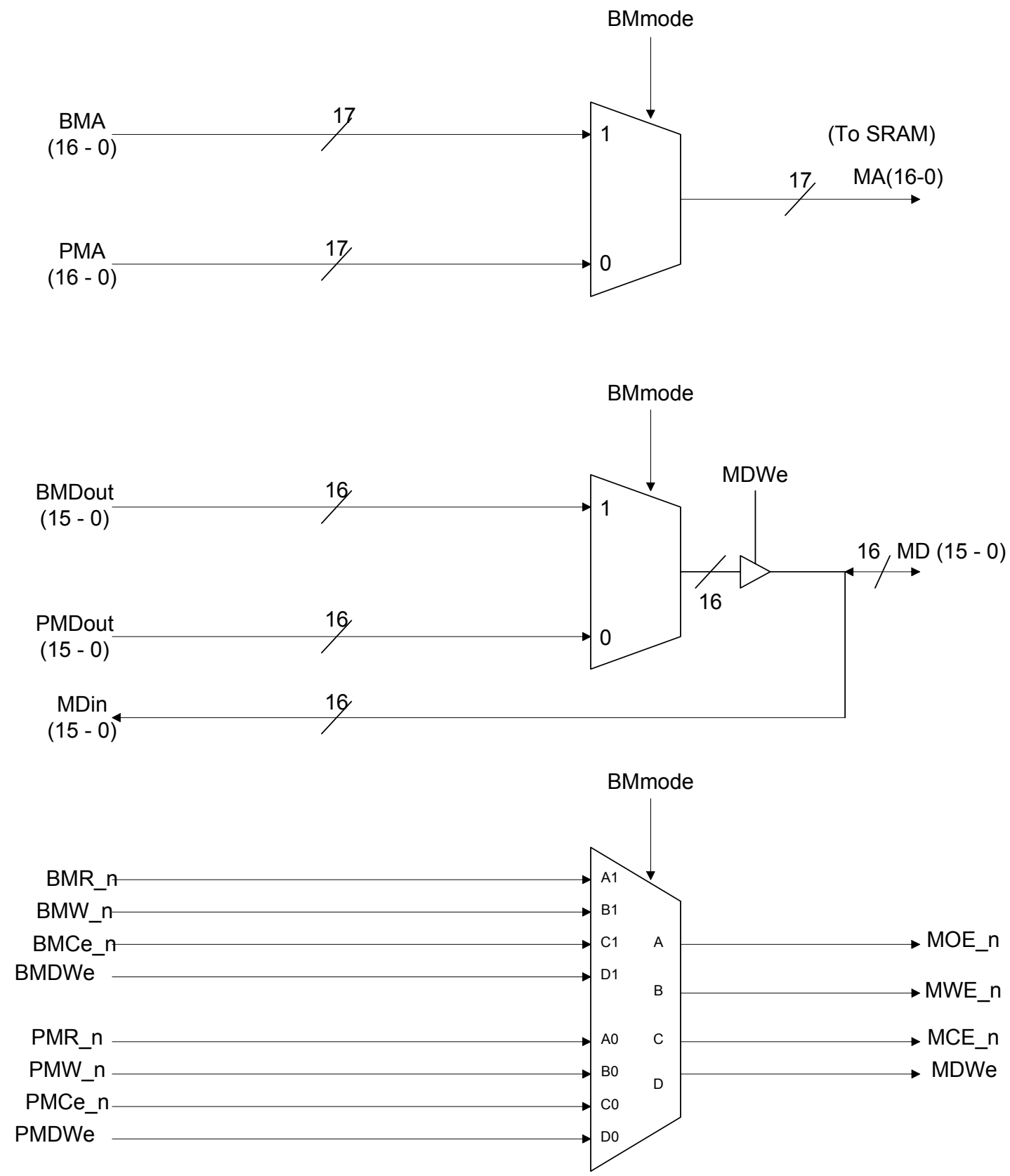

2 - Port Memory Interface (Memlfc) 


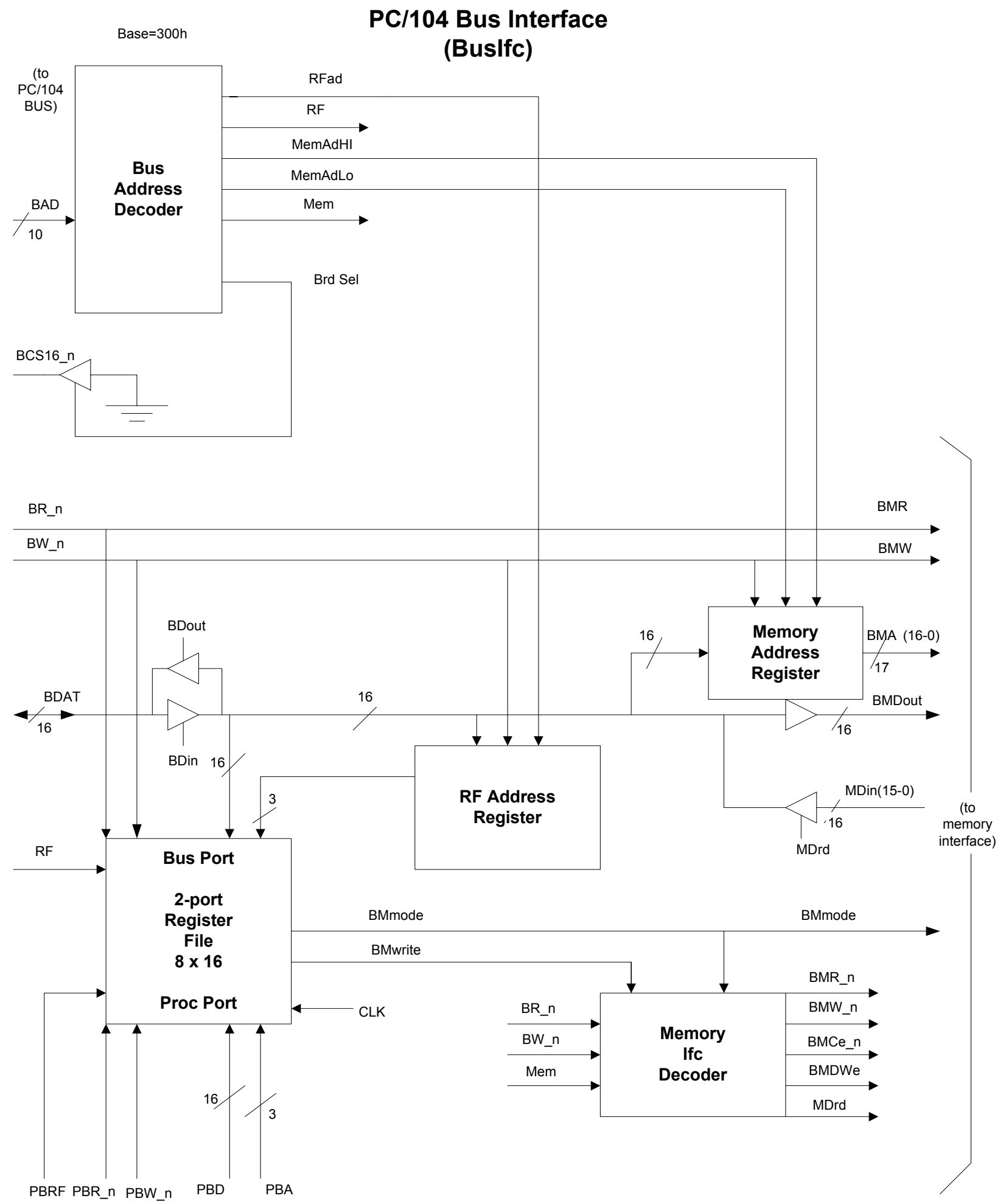


Buslfc-Register File(Reg File)

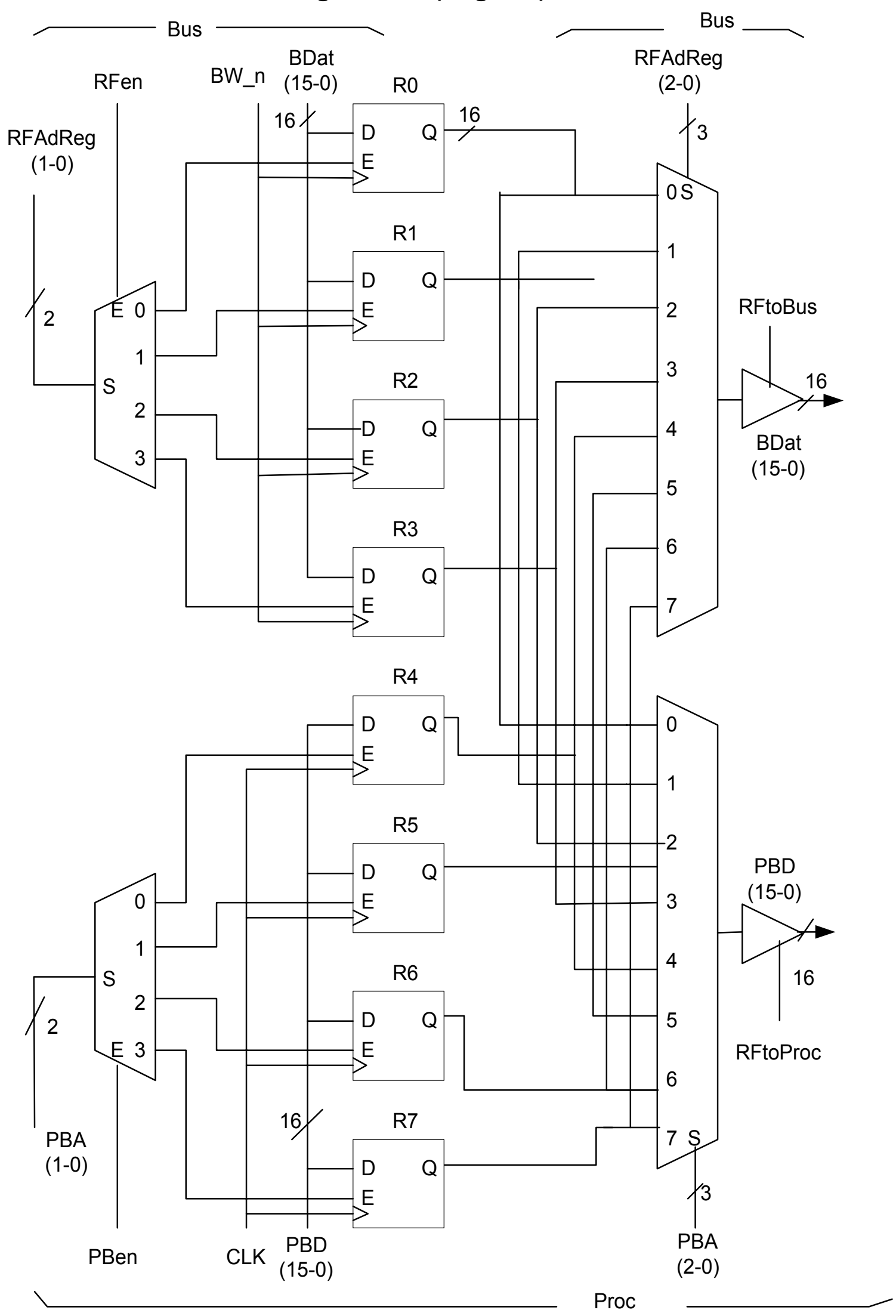




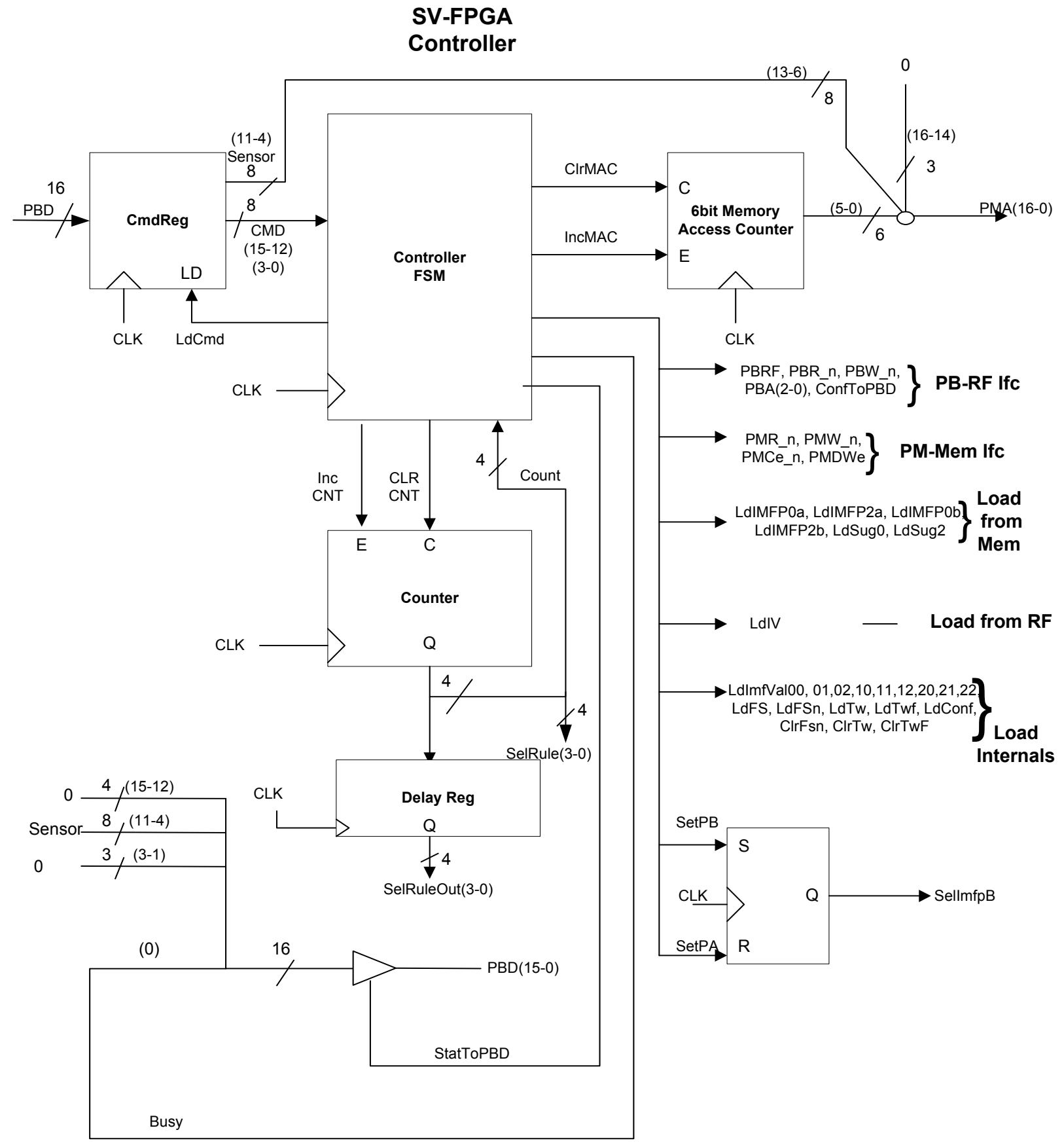


CompFire P.1

HardWired Signals

(fisComputeFiringStrength)

$\stackrel{8}{\longrightarrow}$ mfsRule00 (B00)

$\stackrel{\mathrm{m}}{\longrightarrow} \mathrm{mfsRule01}$ (B01)

$\stackrel{m}{\longrightarrow}$ mfsRule02 (B02)

$\underset{8}{\longrightarrow}$ mfsRule10 (B10)

$\longrightarrow$ mfsRule11 (B11)

mfsRule12 (B12)

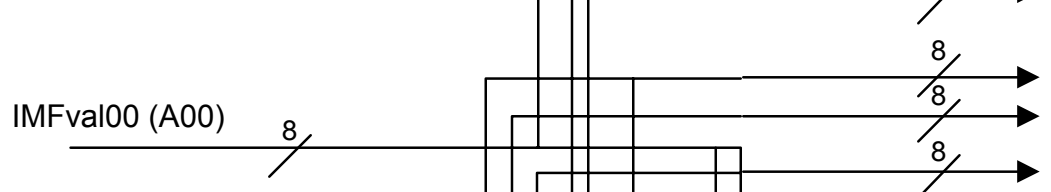

mfsRule20 (B20)

mfsRule21 (B21)

mfsRule22 (B22)

IMFval00 (A01) 8

IMFval00 (A02) 8/

8

mfsRule30 (B30)

mfsRule31 (B31)

mfsRule32 (B32)

IMFval00 (A10)
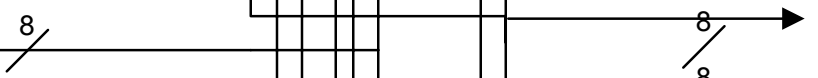

mfsRule40 (B40)

mfsRule41 (B41)

IMFval00 (A11) 8

IMFval00 (A12)

8

IMFval00 (A20) 8

IMFval00 (A21) $8 /$

IMFval00 (A22) 8,

$\stackrel{\rightarrow}{\longrightarrow}$ mfsRule42 (B42)

mfsRule50 (B50)

mfsRule51 (B51)

mfsRule52 (B52)

mfsRule60 (B60)

mfsRule61 (B61)

mfsRule62 (B62)

mfsRule70 (B70)

mfsRule71 (B71)

mfsRule72 (B72)

mfsRule80 (B80)

mfsRule81 (B81)

mfsRule82 (B82)

mfsRule90 (B90)

mfsRule91 (B91)

$\stackrel{\longrightarrow}{\longrightarrow}$ mfsRule92 (B92)

8 mfsRule100 (B100)

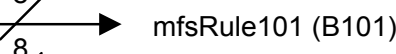

$\longrightarrow$ mfsRule102 (B102)

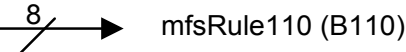

$\stackrel{8}{\longrightarrow}$ mfsRule111 (B111)

$\longrightarrow$ mfsRule112 (B112) 


\section{ComFire}

Multiplexers (fisCompfiringStrength)

P.2

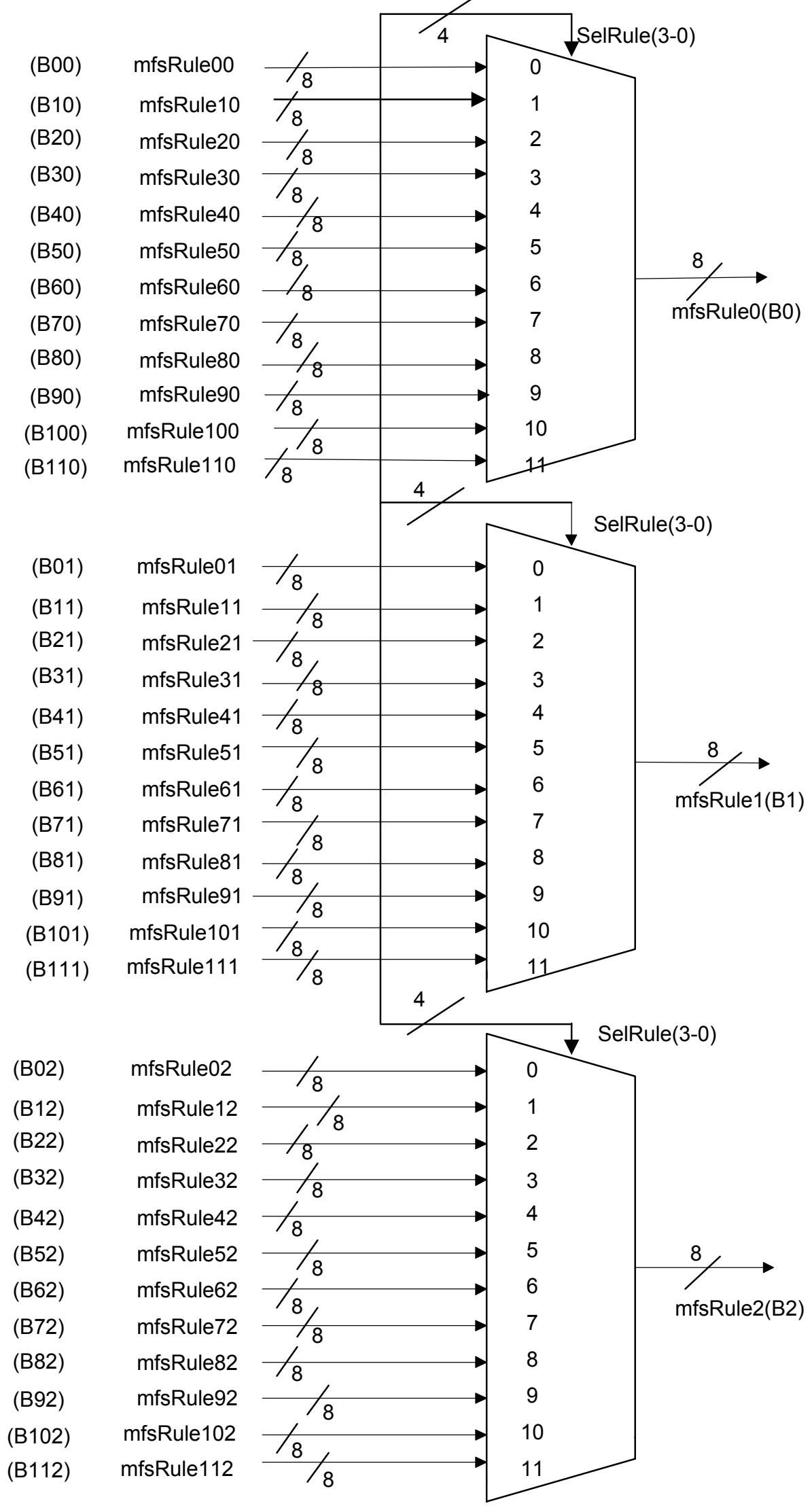


ComFire P.3

(Comparator) fis ComputeFiringStrength

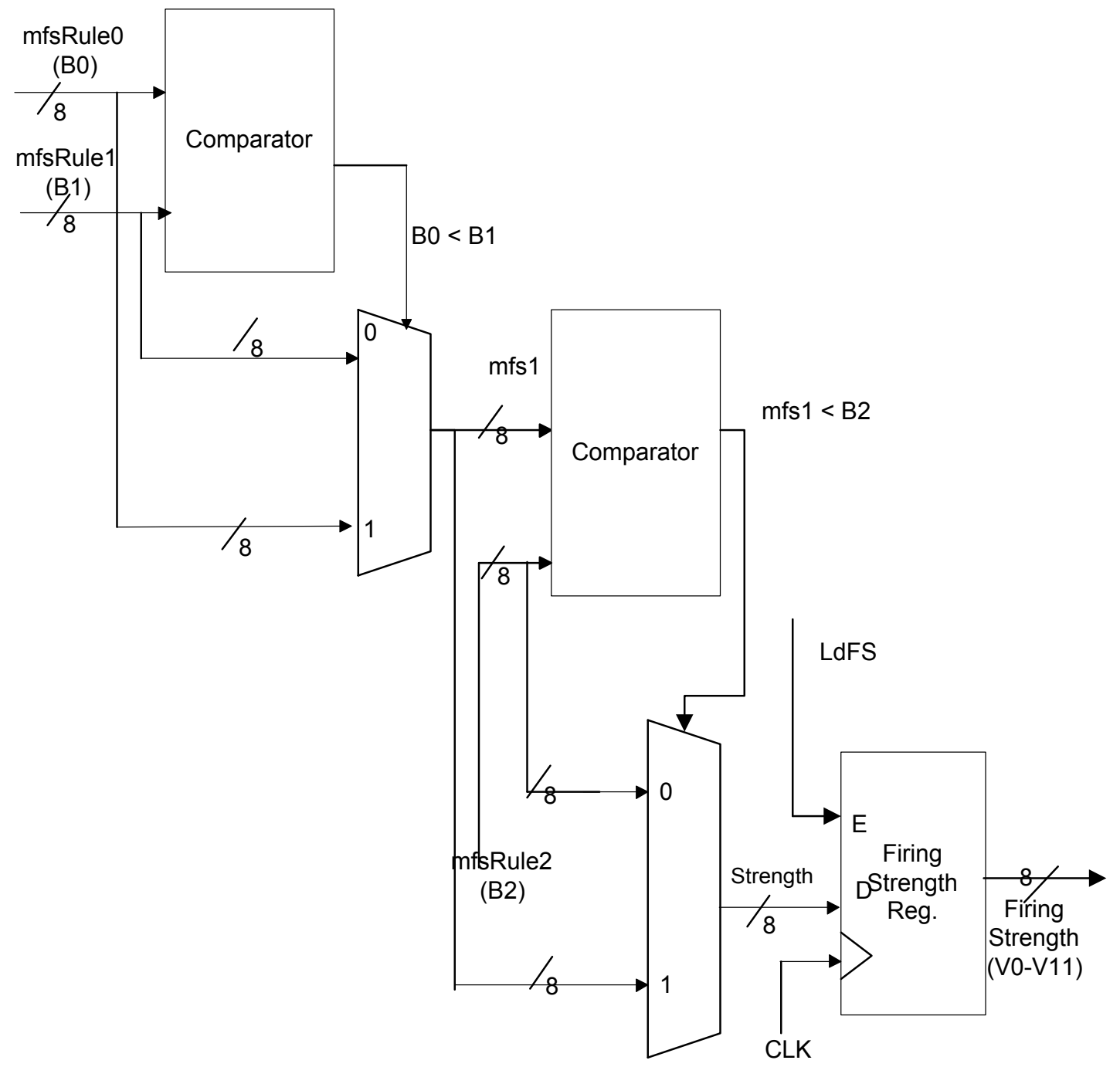


ComplnMF P.4

Output Registers(fisComputelnput, fisTrapezoid)

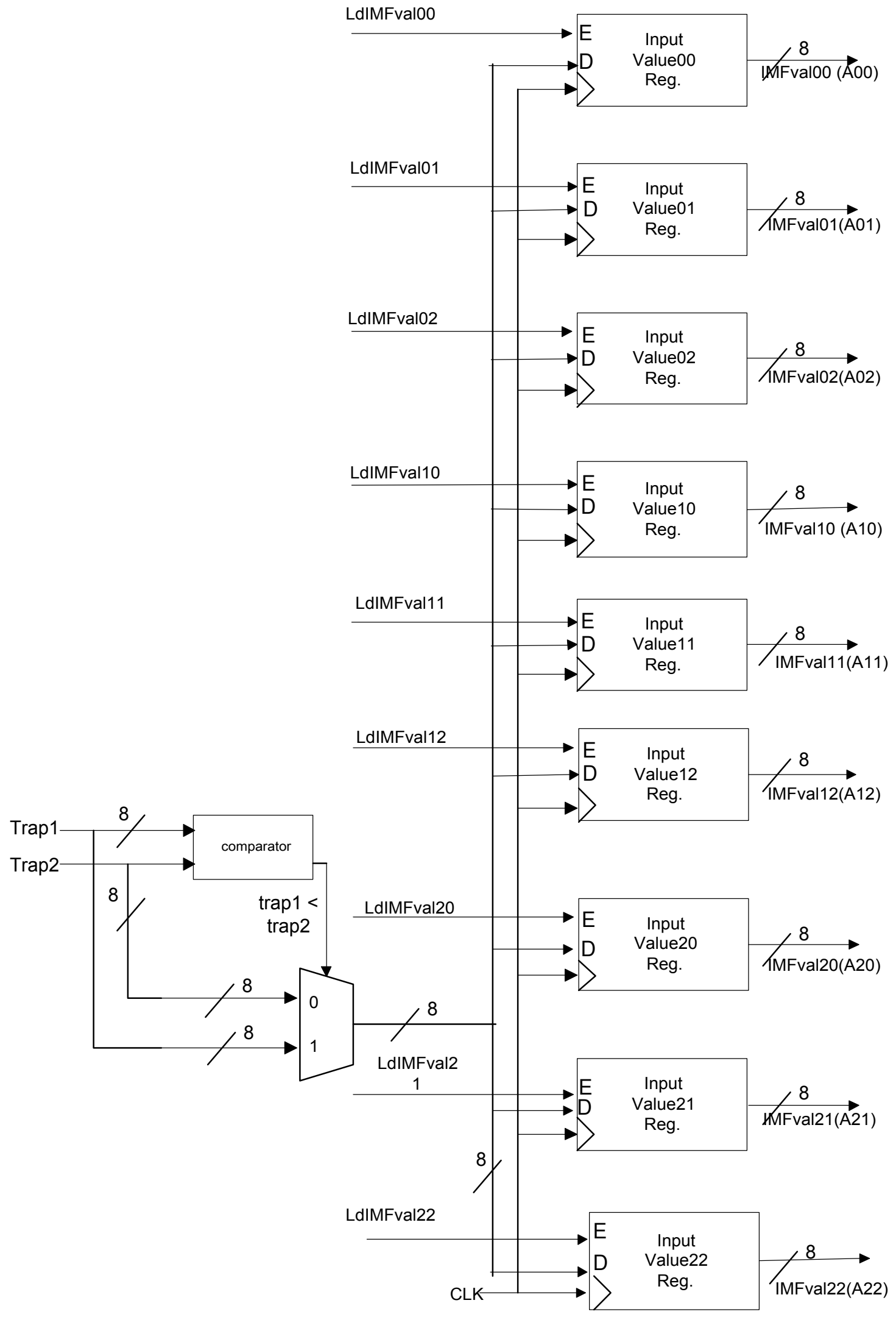




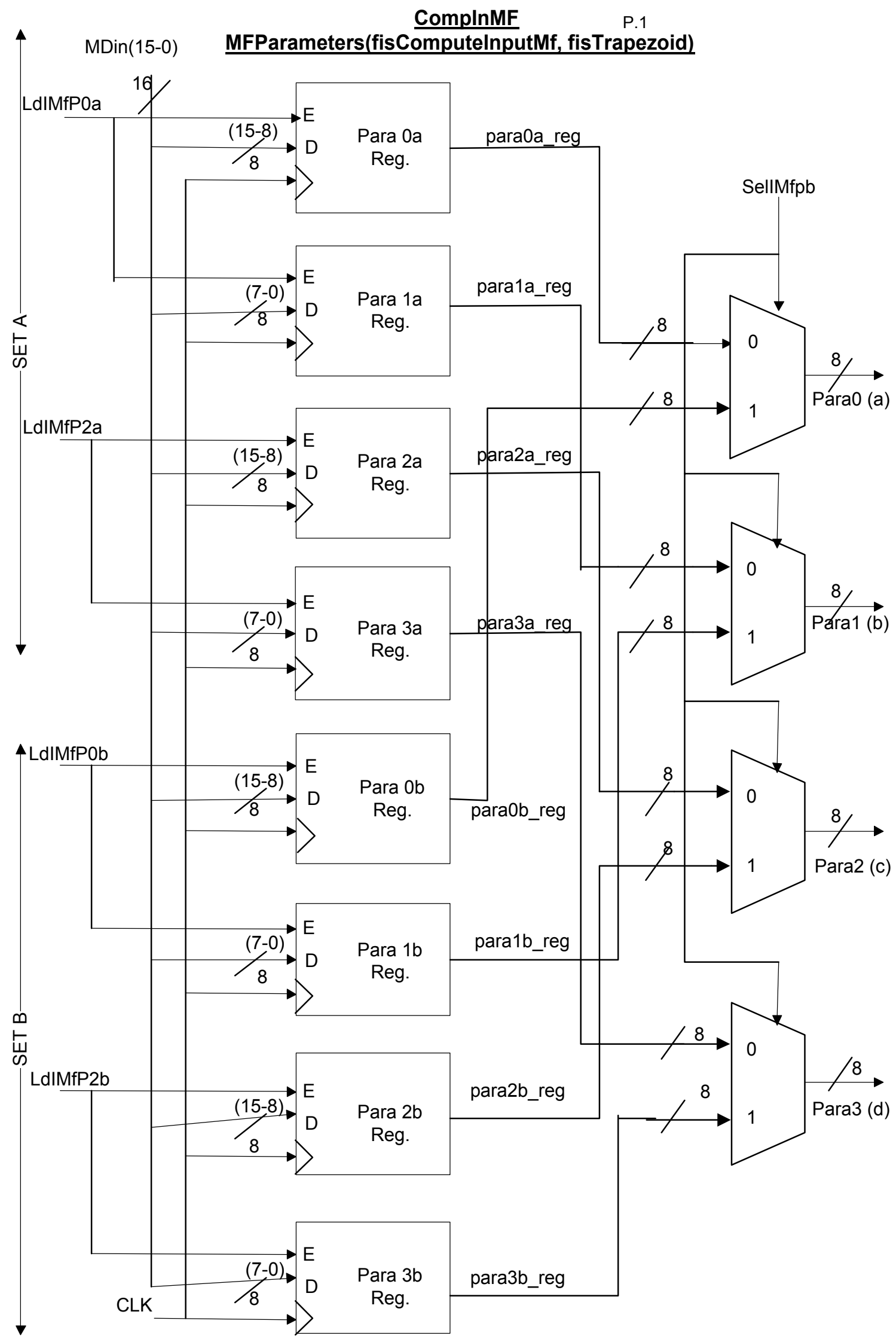




\section{ComplnMF}

TRAP1 (fisComputelnputMf, fisTrapezoid)

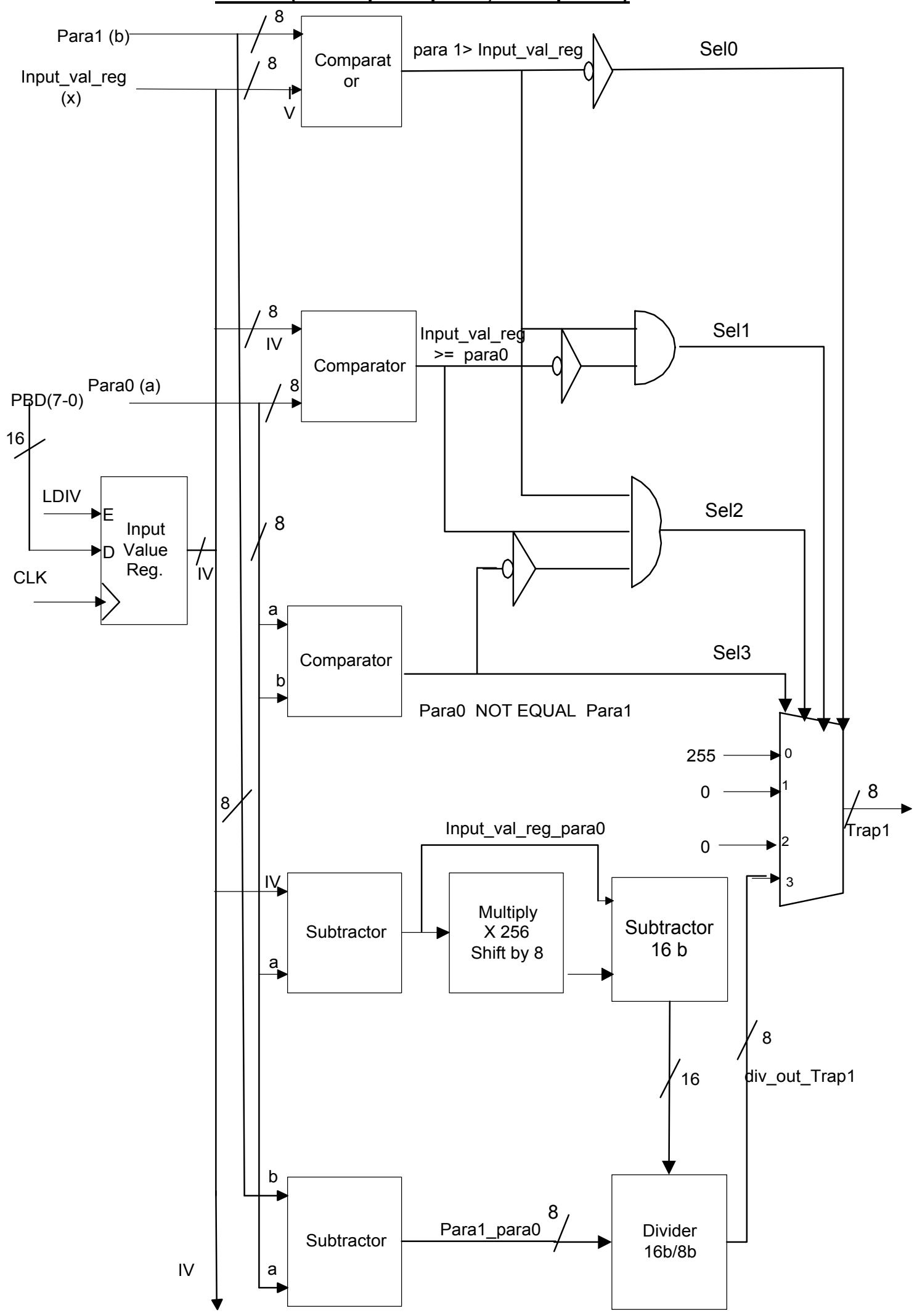




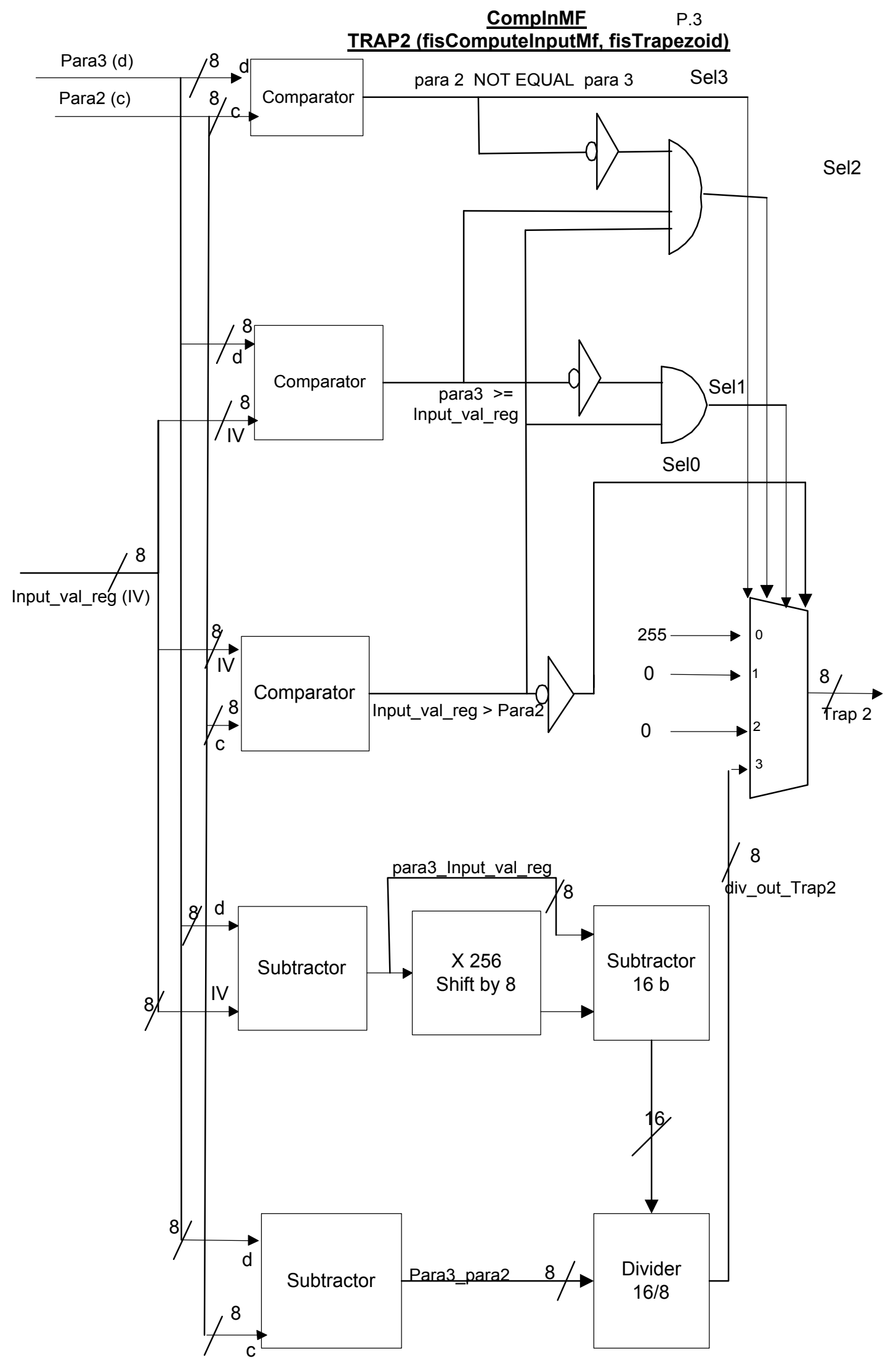




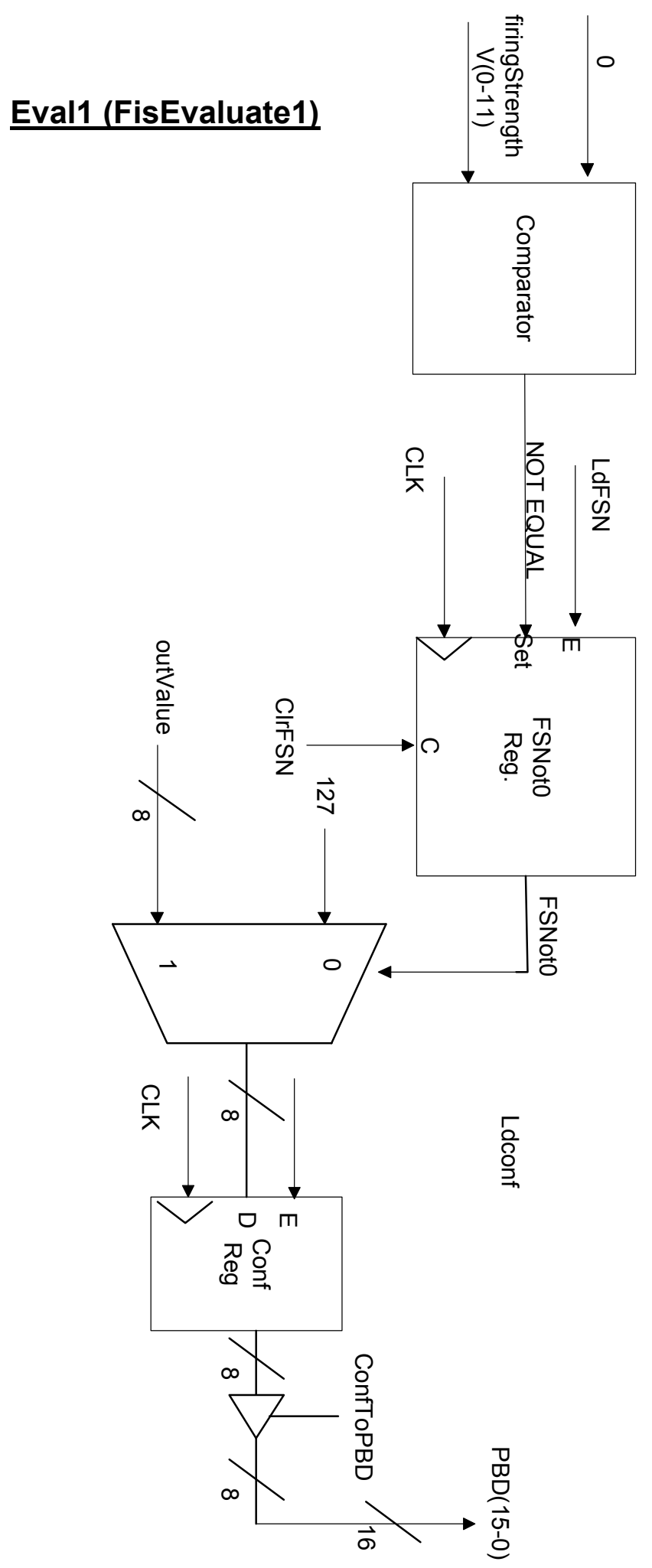


Eval5 P.1

(FisEvaluate3)

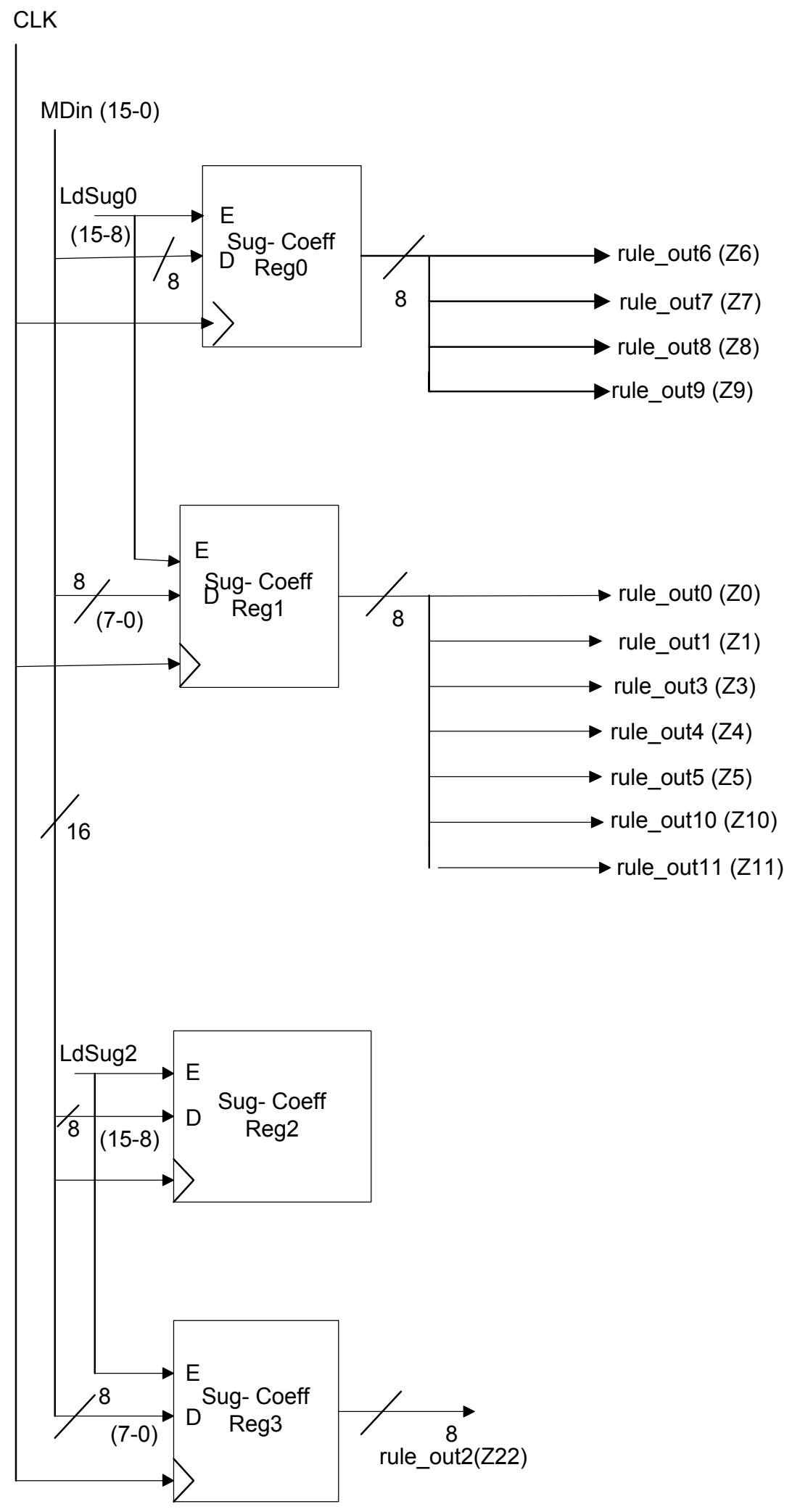




\section{Eval5 $\quad$ P.2}

\section{(FisEval4 and FisEval5)}

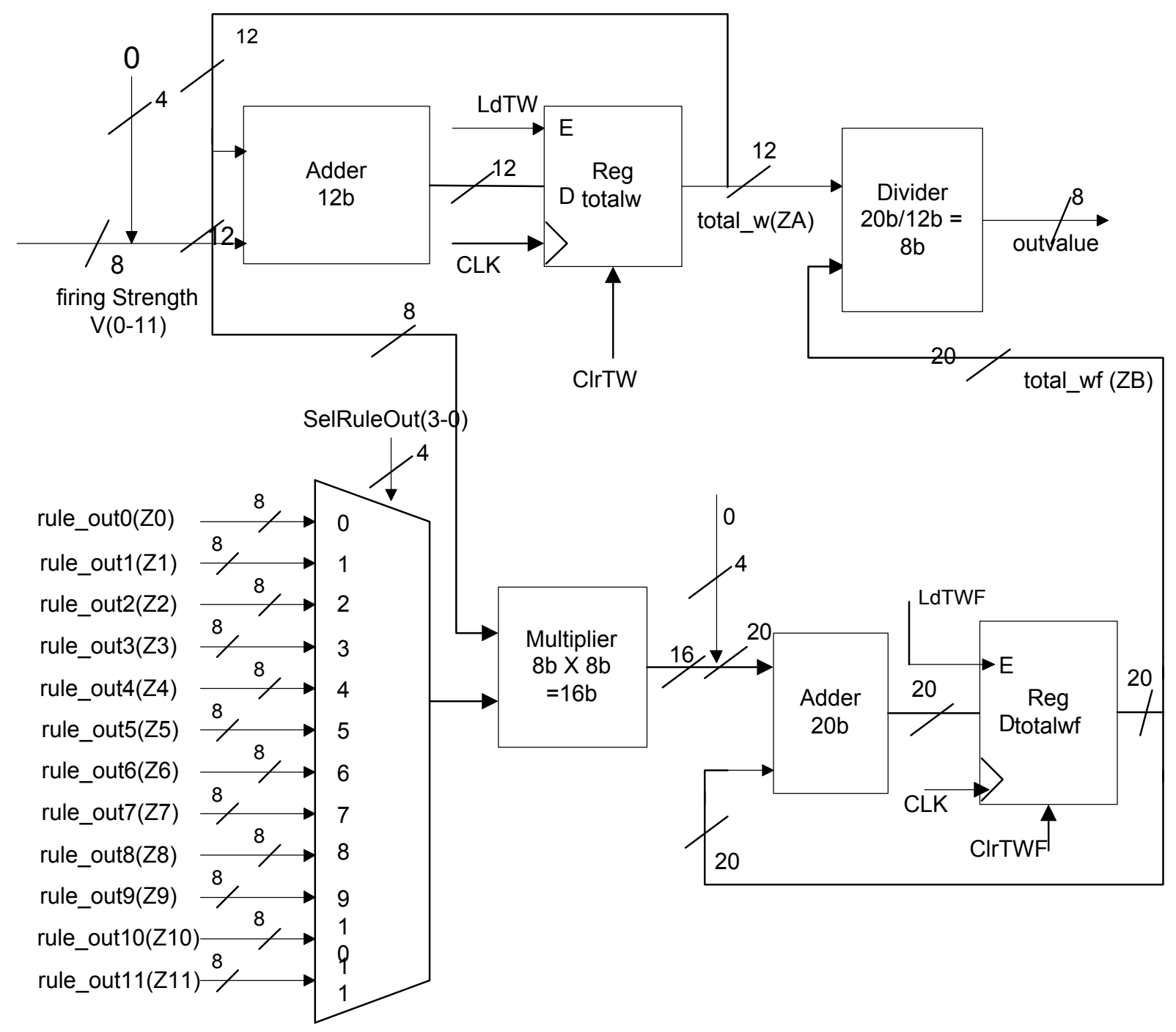




\section{A.4 FSM Controller ASM Charts}

\section{A.4.1 Self-Validation Controller ASM Charts}




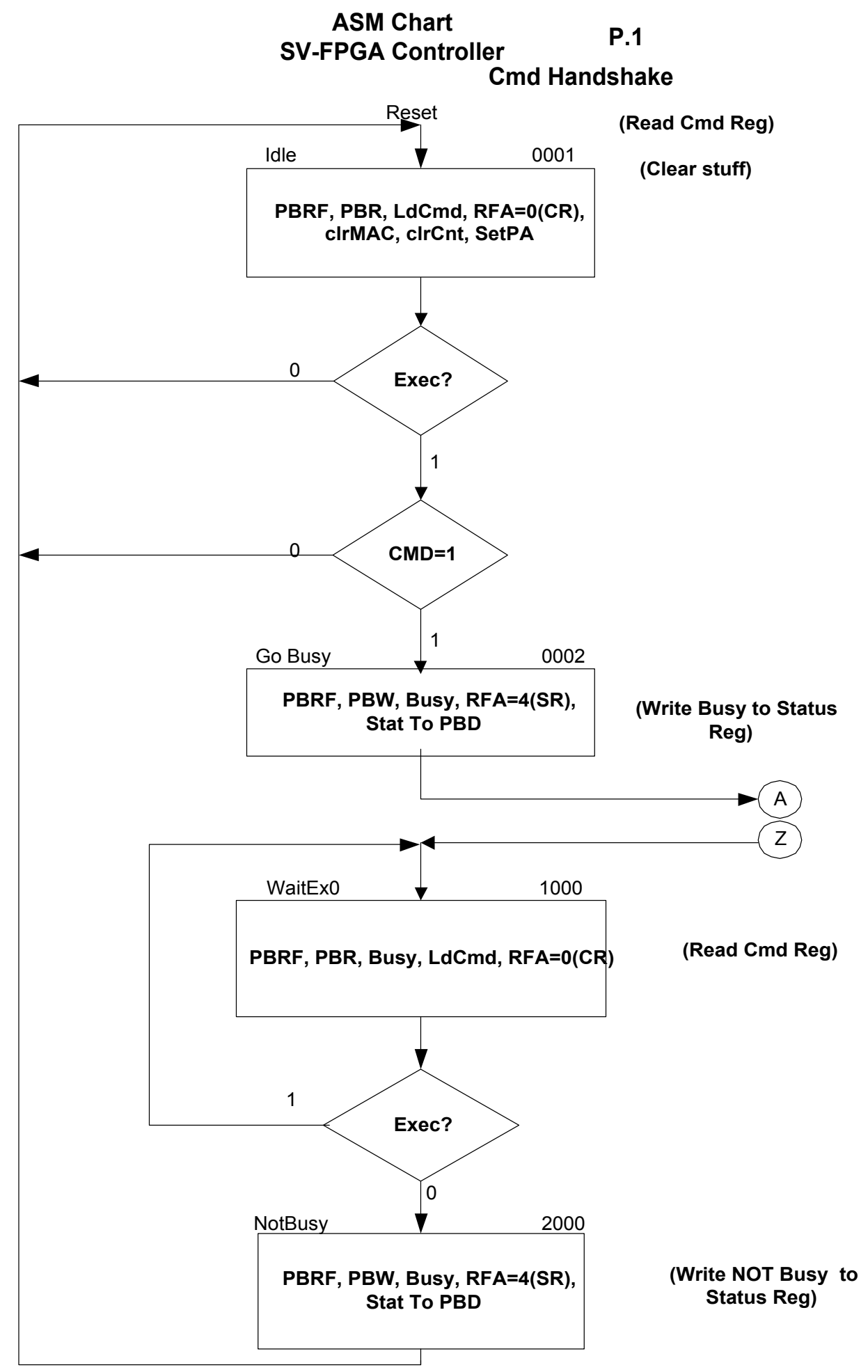


ASM Chart

SV-FPGA Controller

Comp. Input MF Values 1

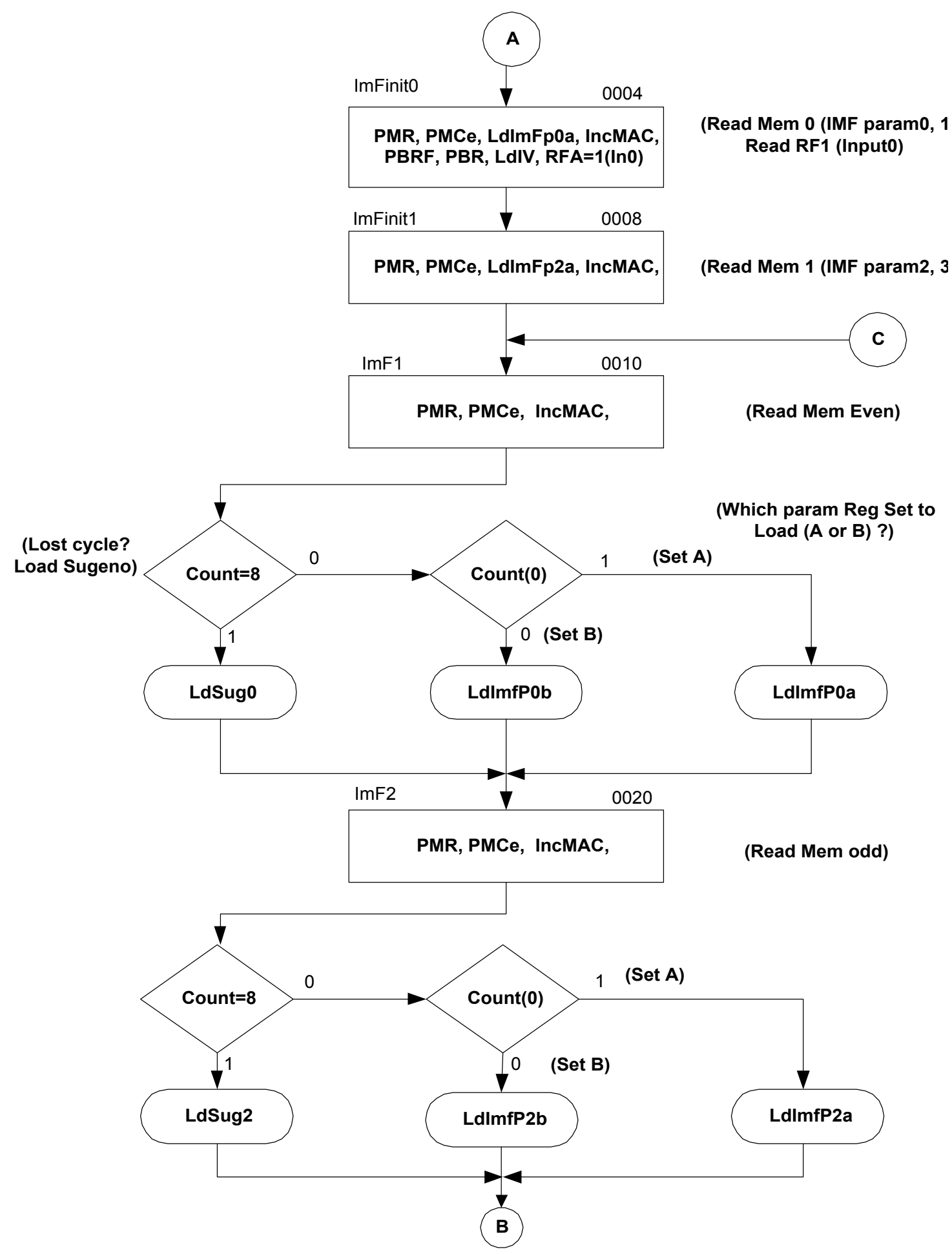




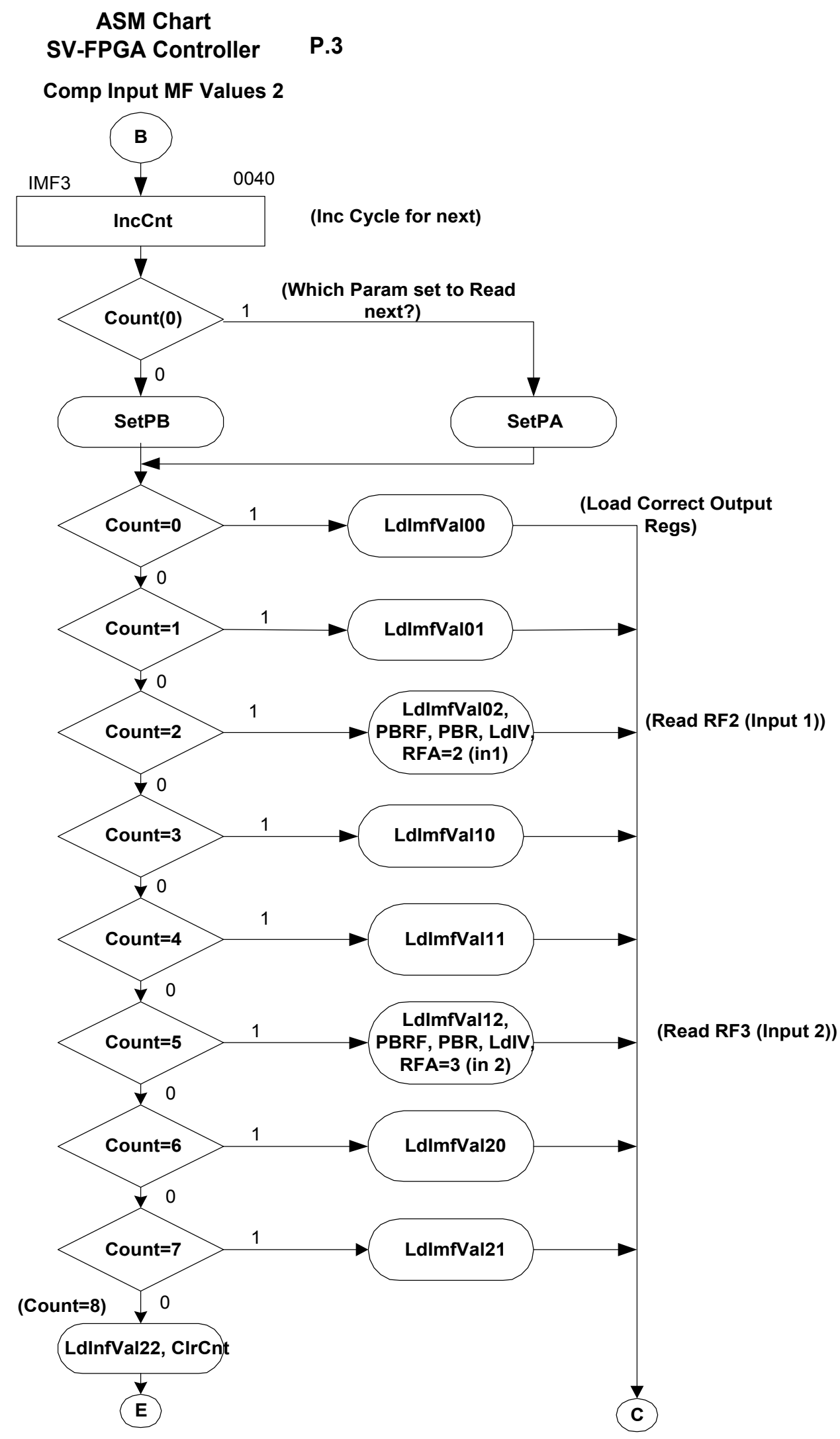




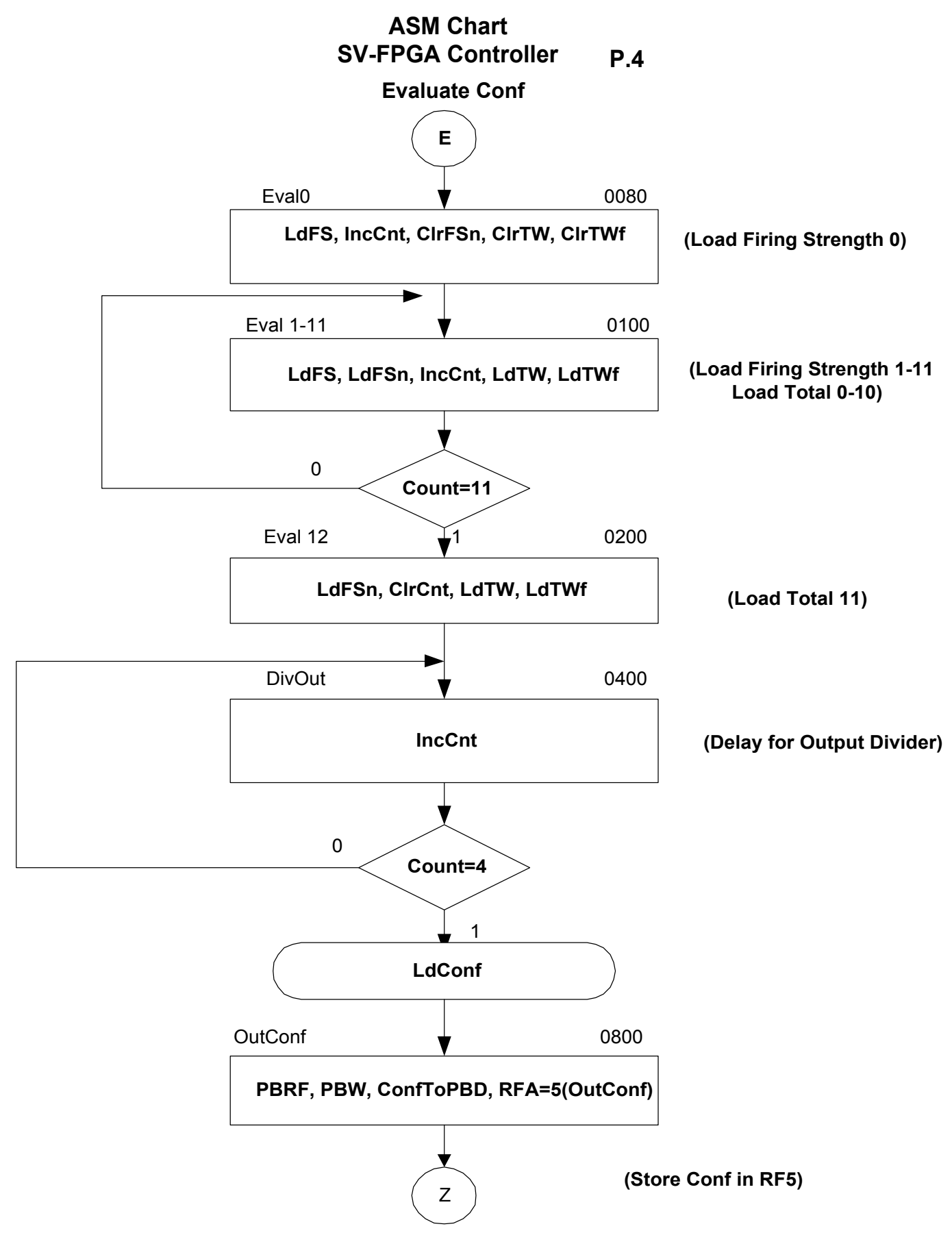




\section{A.5 Timing Diagrams}

\section{A.5.1 Self-Validation Timing}




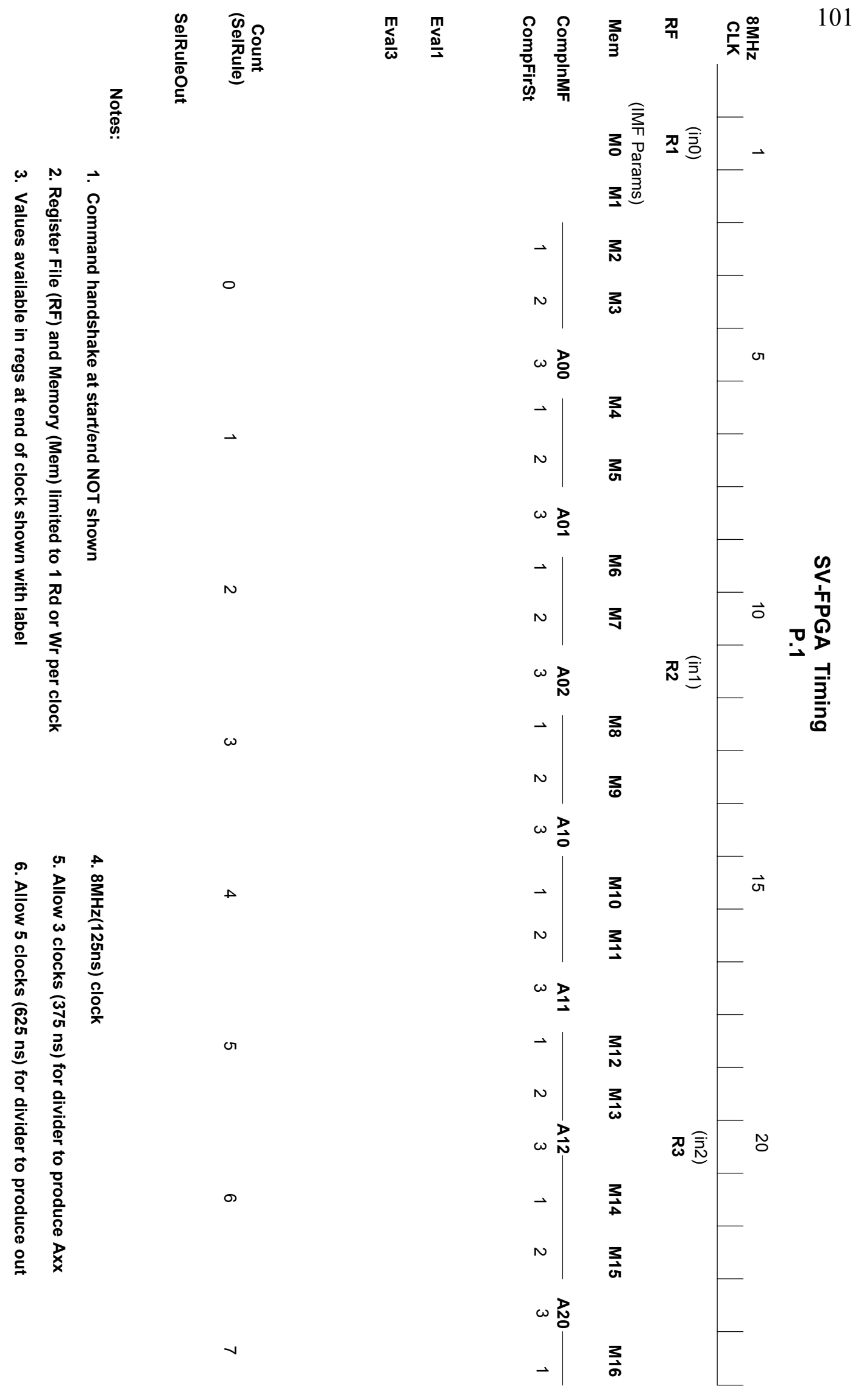




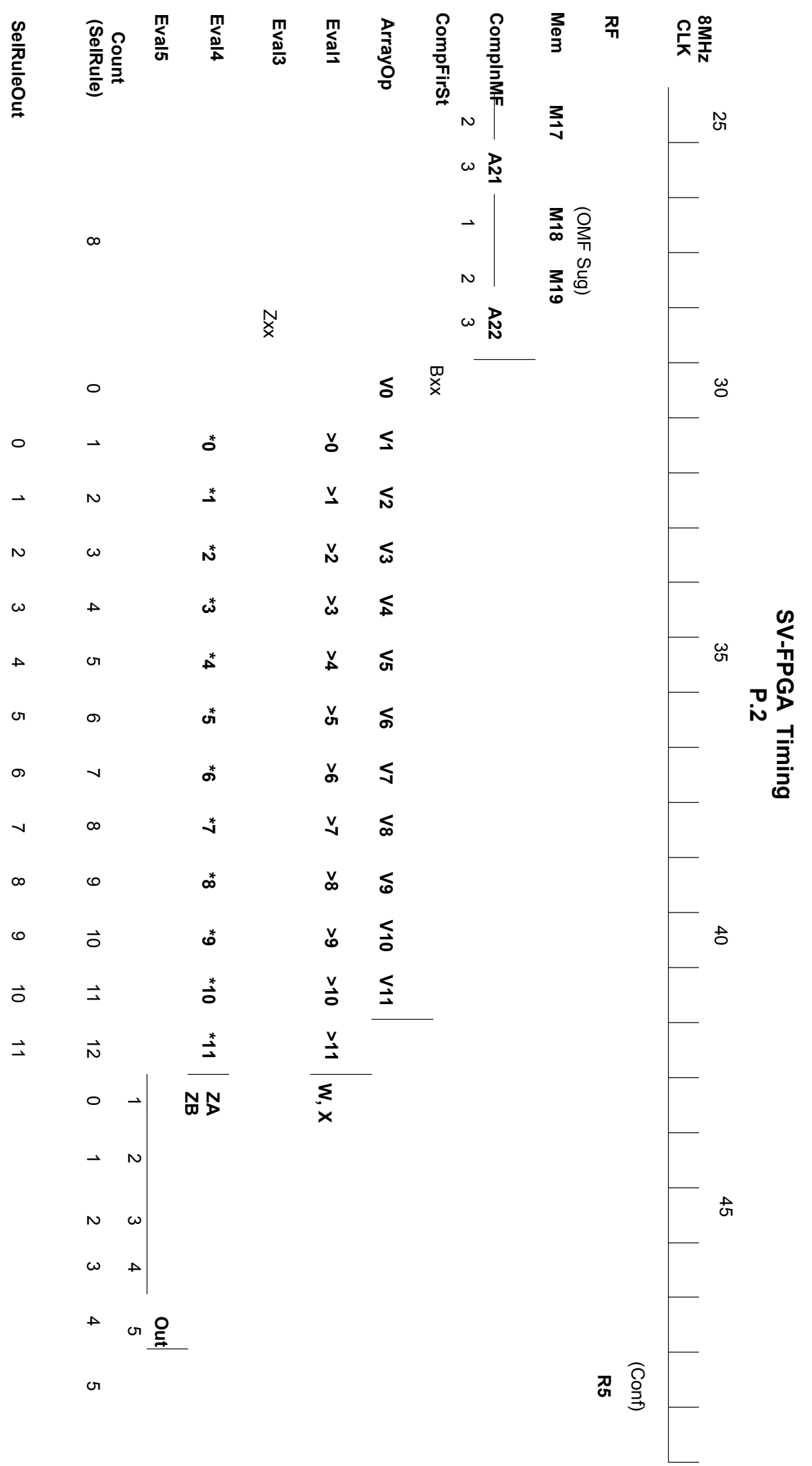




\section{A.6 MSF Hardware Block Diagrams}




\section{Area Comp}

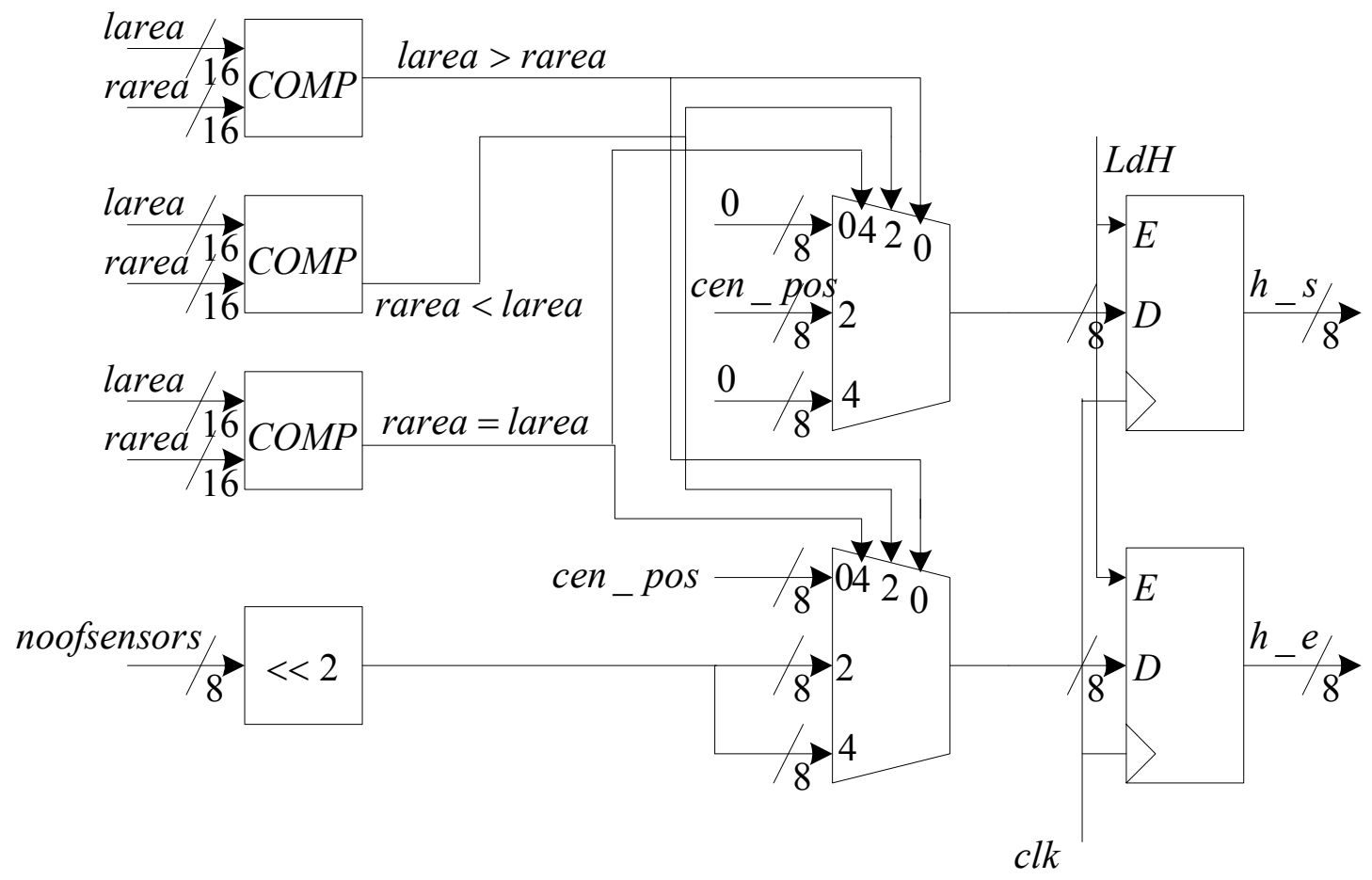




\section{AREA (P1)}

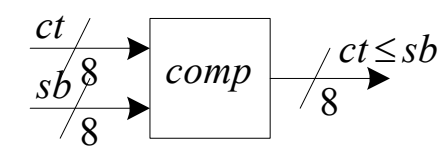

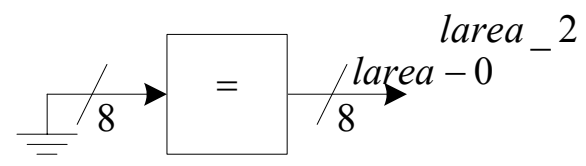

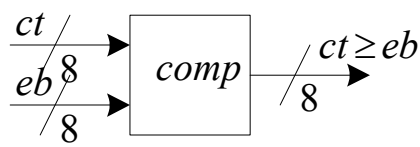

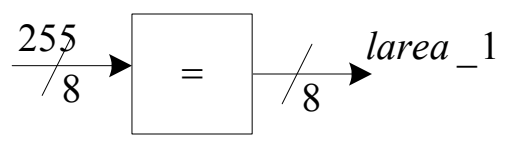

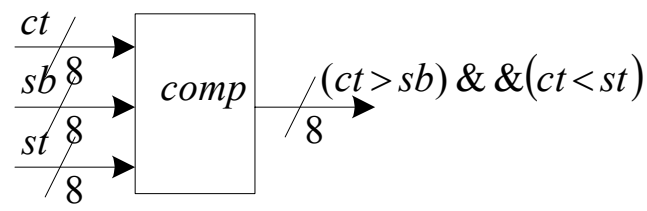

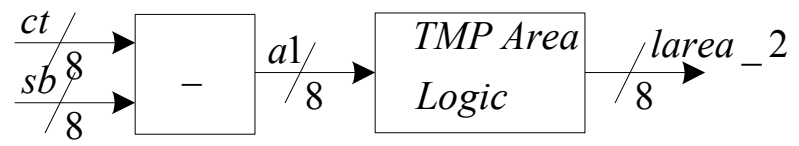

$\underset{s t / 8}{\stackrel{c t}{\longrightarrow}} \operatorname{comp} / \mathrm{ct}=s t$

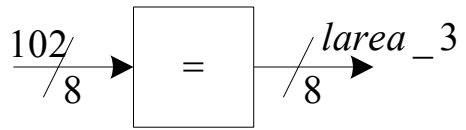
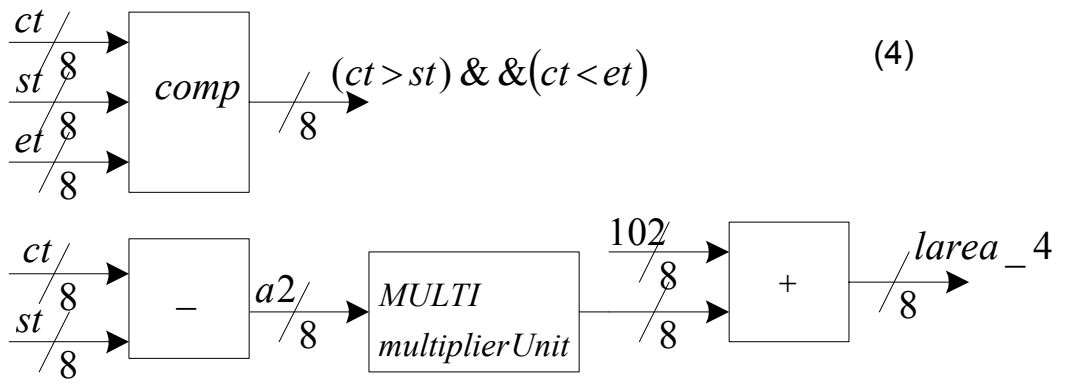


\section{AREA}

(P2)

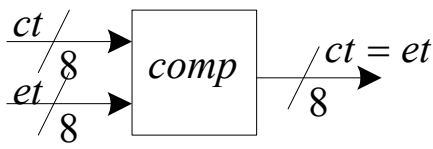

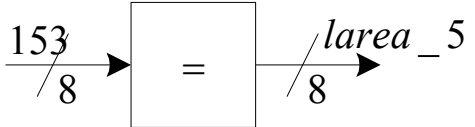

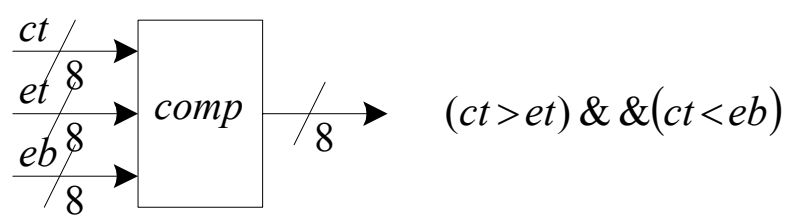

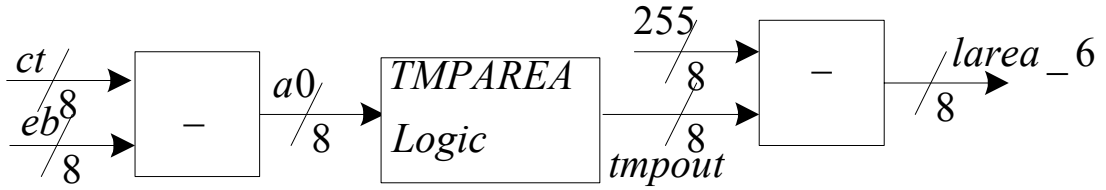




\section{Area (P3)}

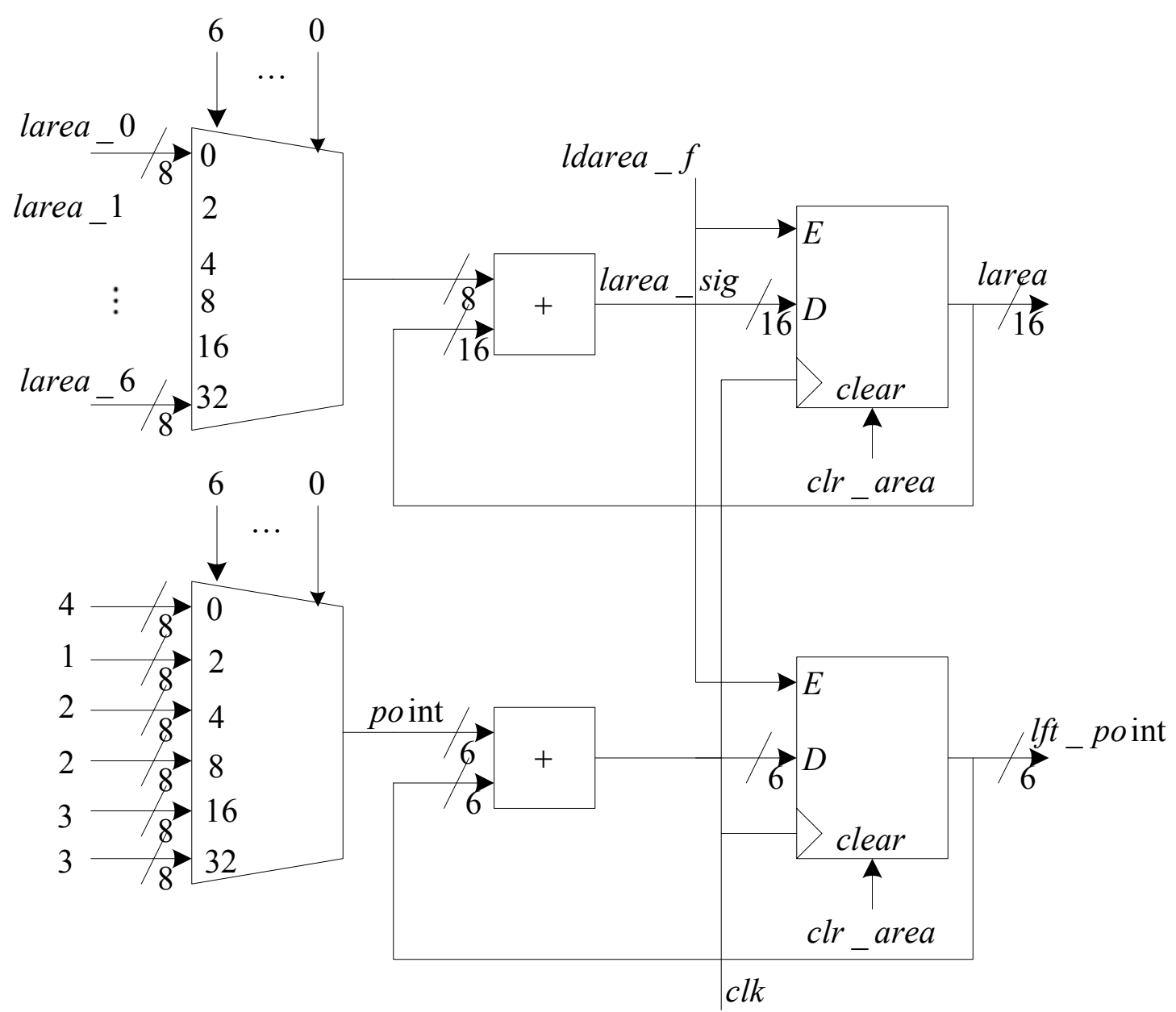




\section{AREA (P4)}

TMP. AREA Logic

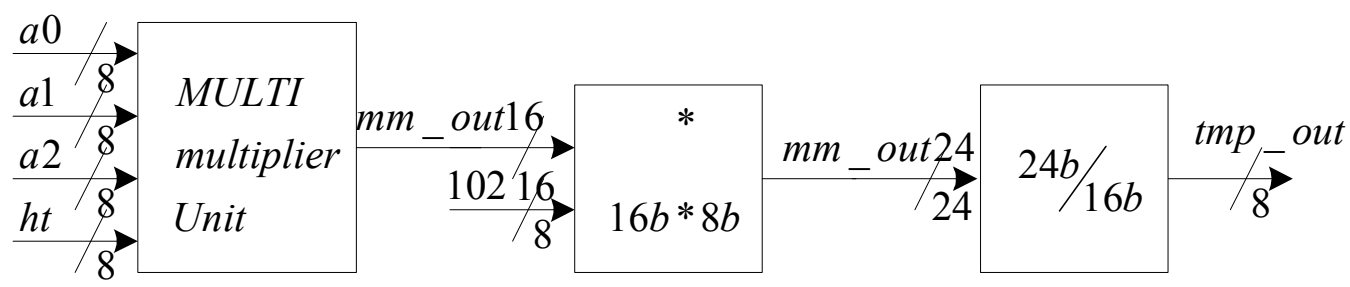

MULTI muliplier Unit

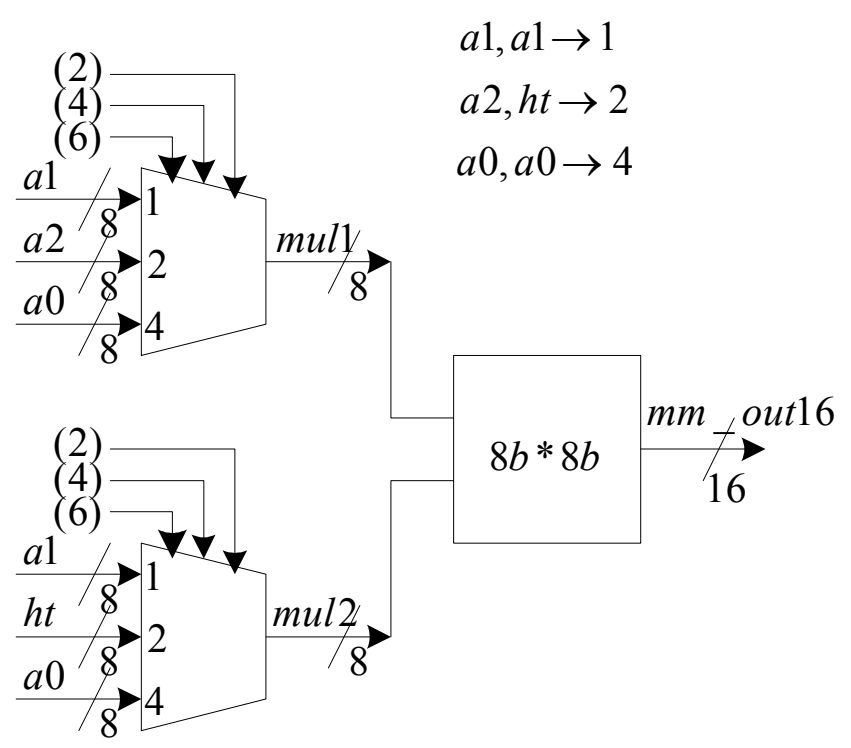

Note: All the multiplier \& divider units are implemented outside the Area module 


\section{CENTROID}

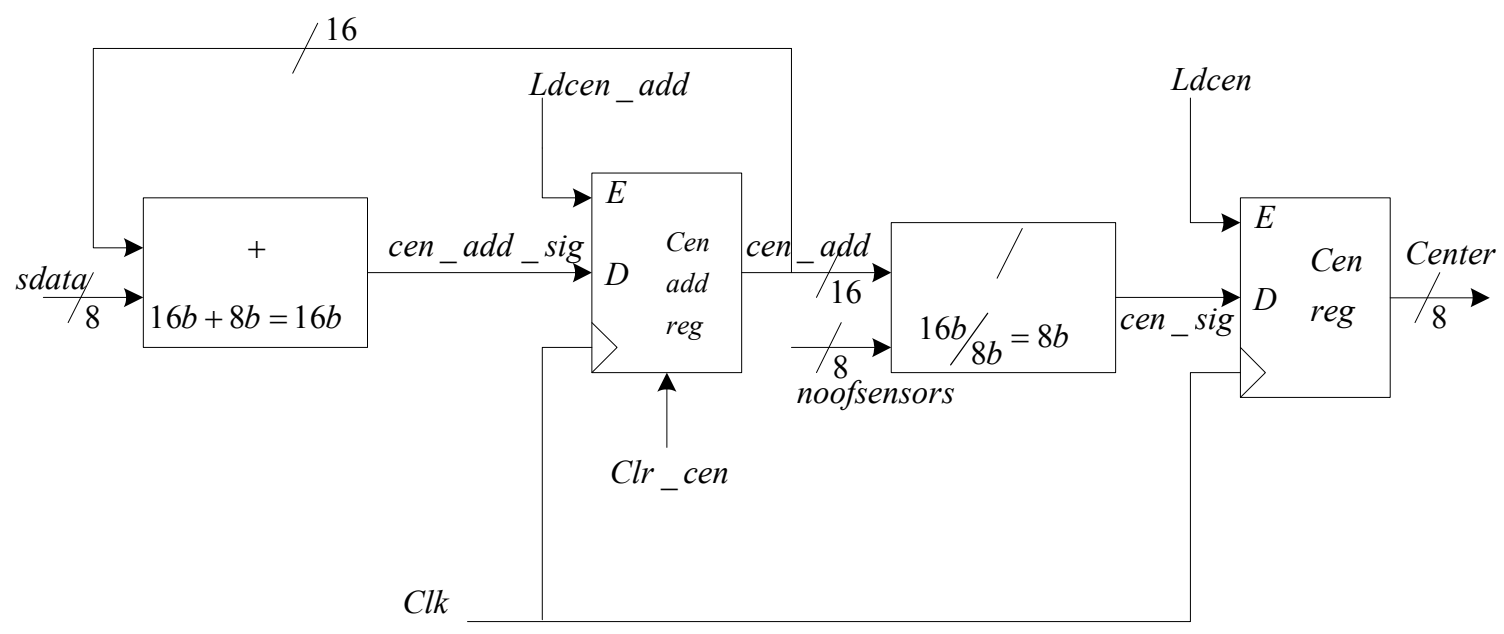


Dividers for MSF
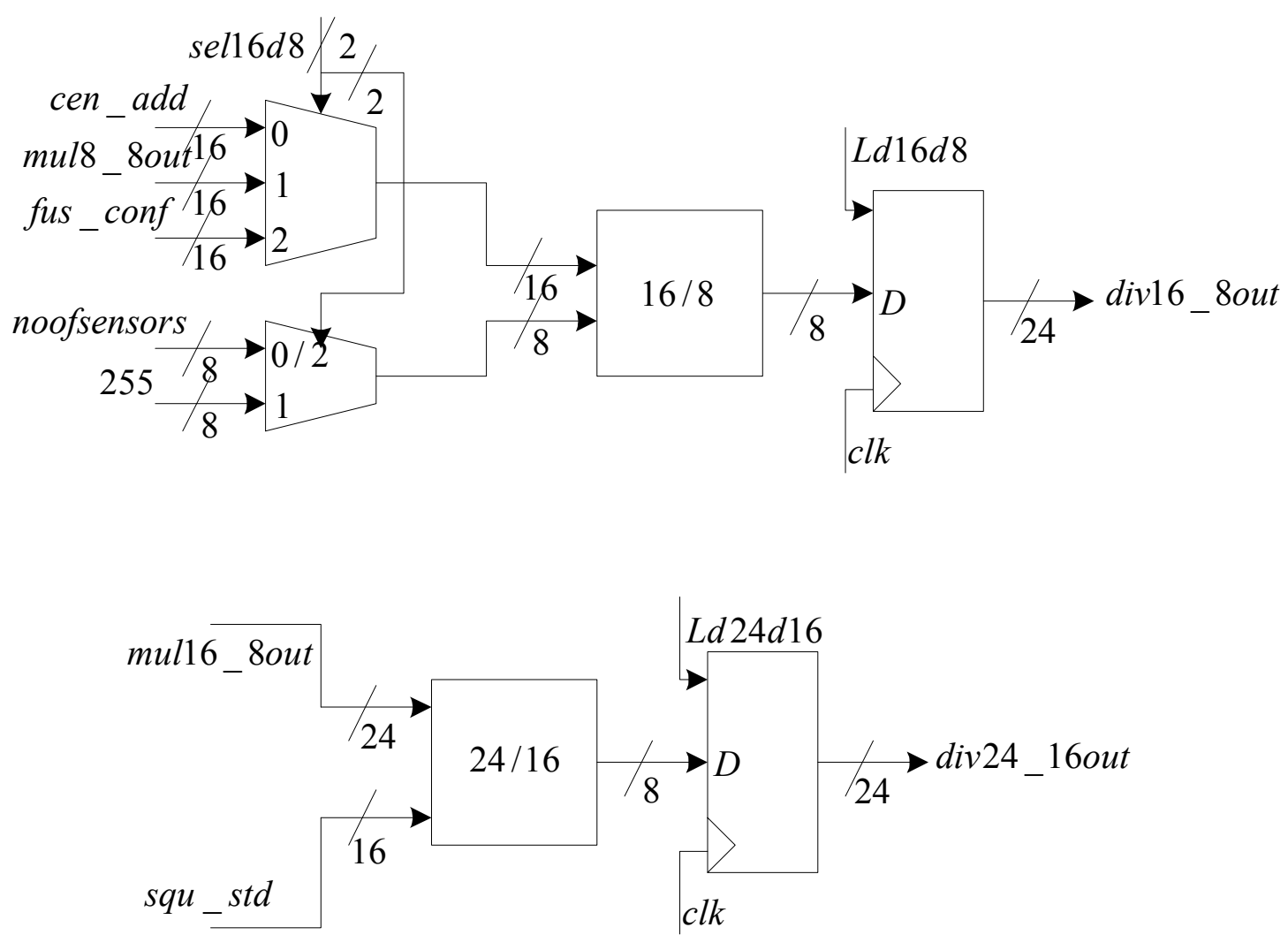

NOTE:

Sel6d_8 $0: \quad \frac{\text { Inputs from }}{\text { centroid }}$

1: $\quad$ Trap Height

2: $\quad$ Fused Conf 
Fused_Conf (P1)

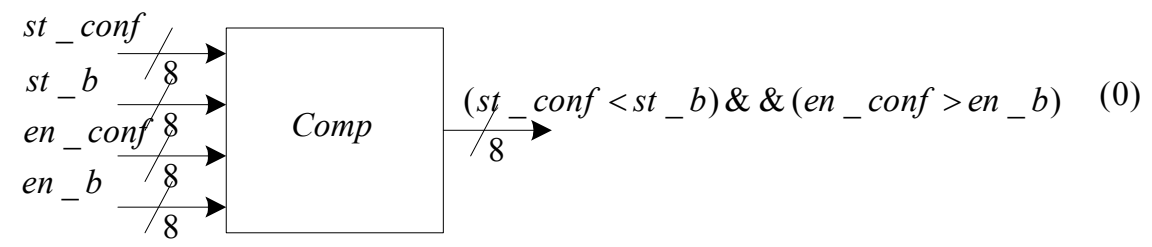

$$
\begin{aligned}
& 255 \longrightarrow / 8 \longrightarrow / 8 \text { fus_conf } 0
\end{aligned}
$$

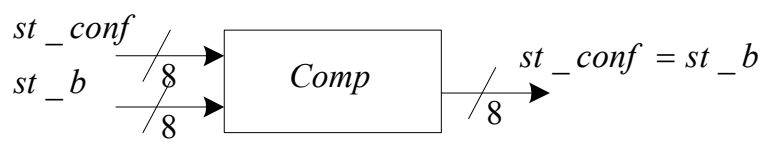

$$
\begin{aligned}
& 255 \longrightarrow / 8 \quad \stackrel{\text { fus }}{\longrightarrow}-\operatorname{conf} 1
\end{aligned}
$$

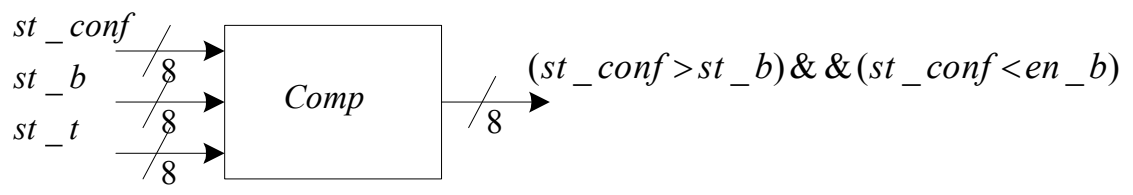

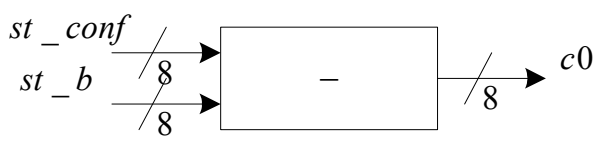

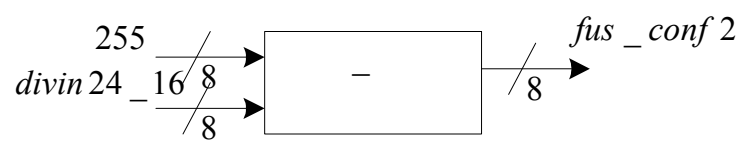

$$
\begin{aligned}
& s t_{-} t \stackrel{\text { conf }}{\stackrel{\text { Comp }}{\longrightarrow} \mathrm{C}} \stackrel{\mathrm{st}}{\longrightarrow} \text { conf }=s t_{-} t
\end{aligned}
$$

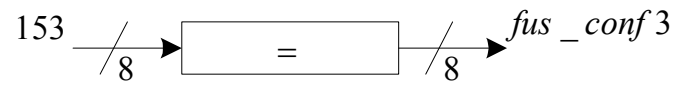

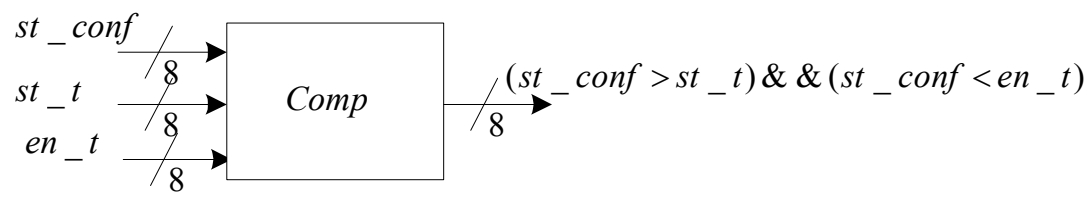

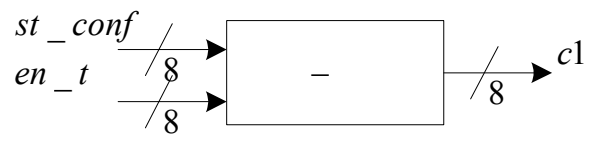

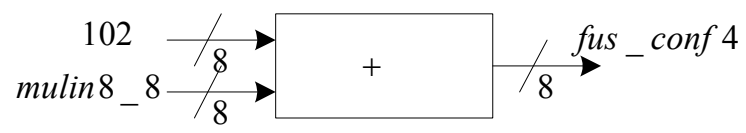

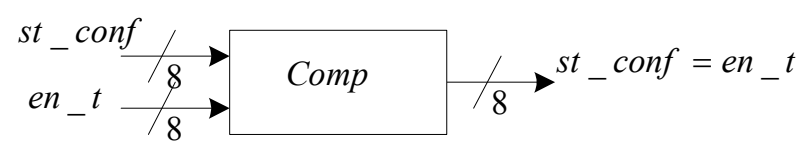

$$
\begin{aligned}
& 102 \underset{8}{\longrightarrow}=/ \text { fus }_{8} \text { conf } 5
\end{aligned}
$$




\section{Fused_Conf (P2)}

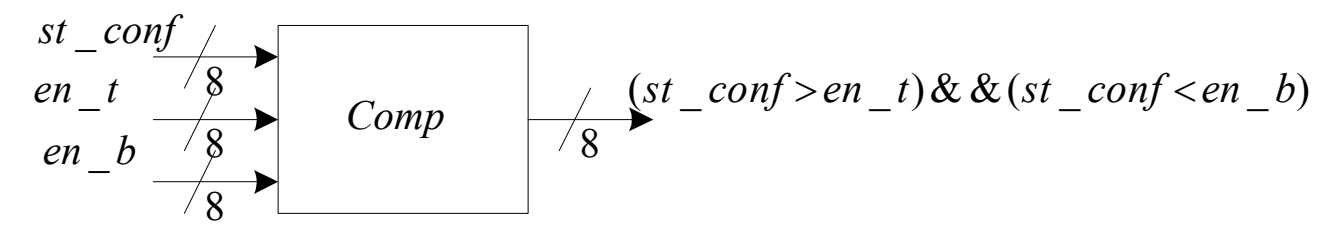
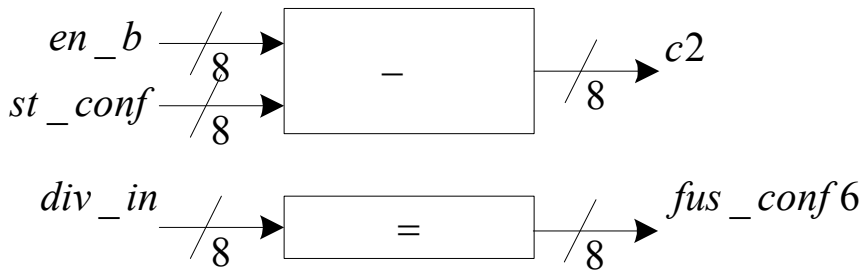

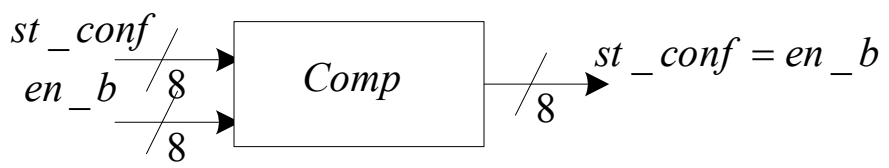

$$
\begin{aligned}
& \stackrel{0}{\longrightarrow} \longrightarrow \quad=\quad f_{8}^{f u s}{ }_{-} \operatorname{conf} 7
\end{aligned}
$$

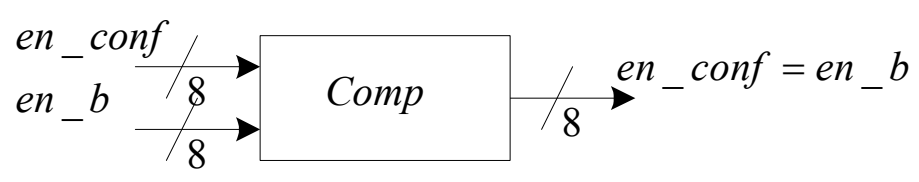

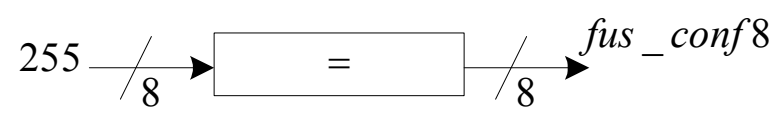

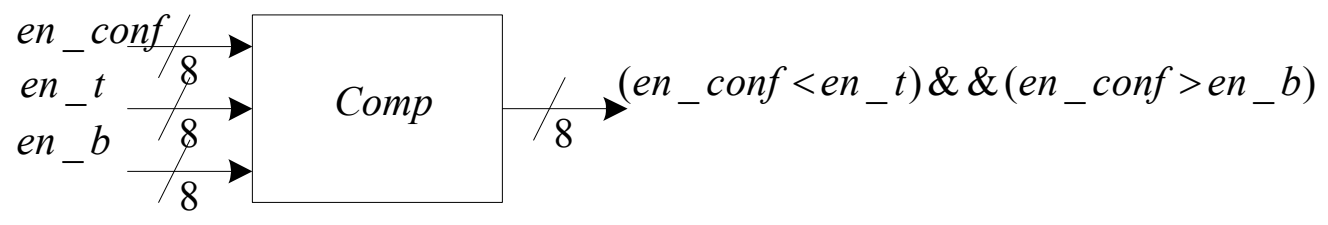

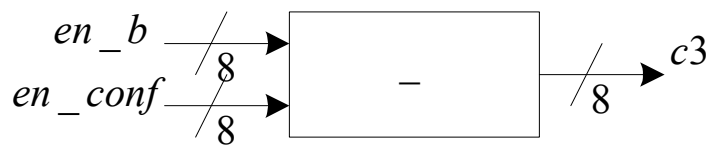

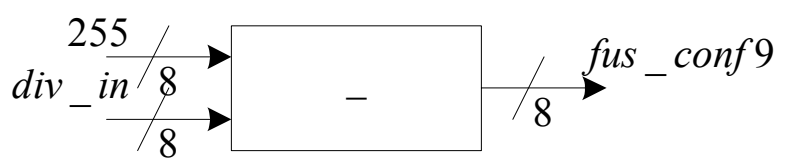




\section{Fused_Conf (P3)}

$$
\begin{aligned}
& \stackrel{\text { en_conf }}{\text { en_t }} \stackrel{\text { Comp }}{\longrightarrow} \underset{8}{\longrightarrow} \text { Con_conf }=e n_{-} t \\
& 153 \underset{8}{\longrightarrow}={ }_{8} \text { fus }_{-} \text {conf } 10
\end{aligned}
$$

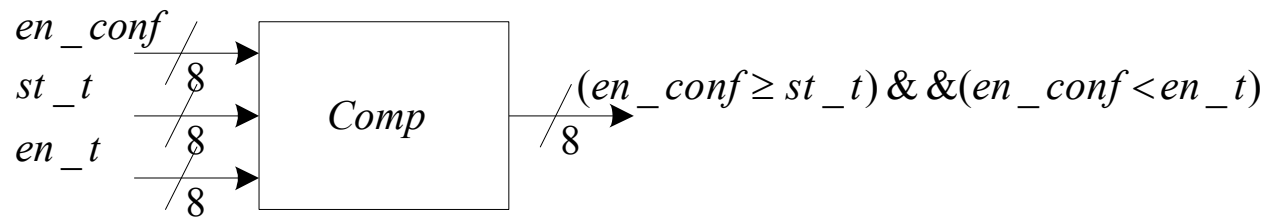
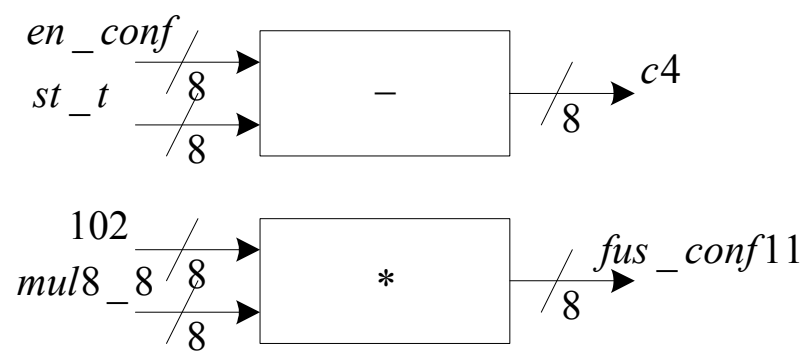

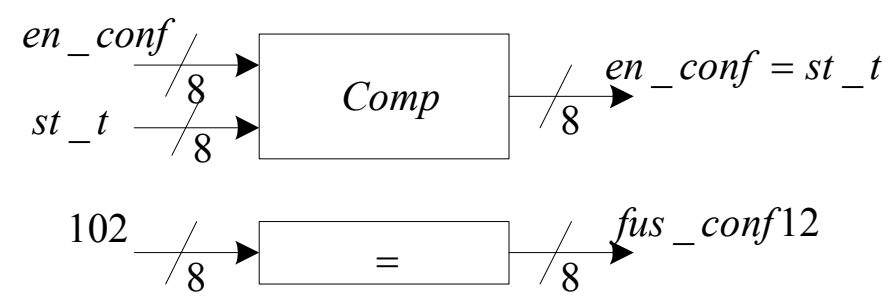

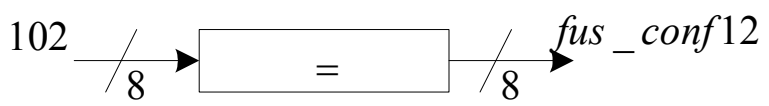

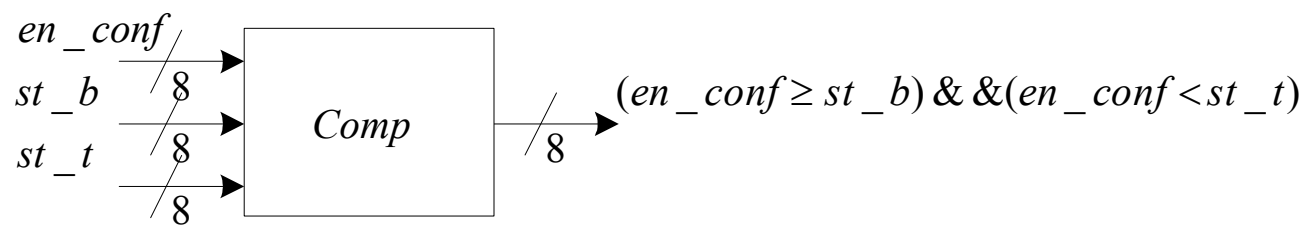

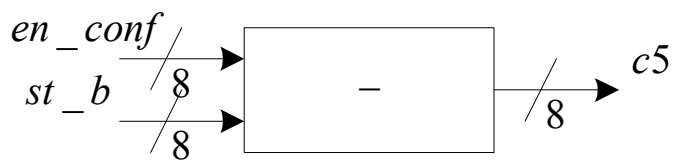

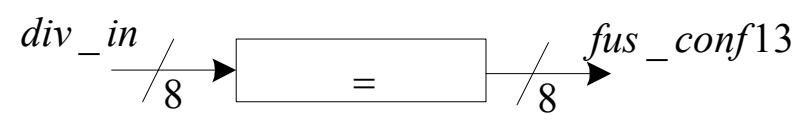




\section{Fused_Conf (P4)}

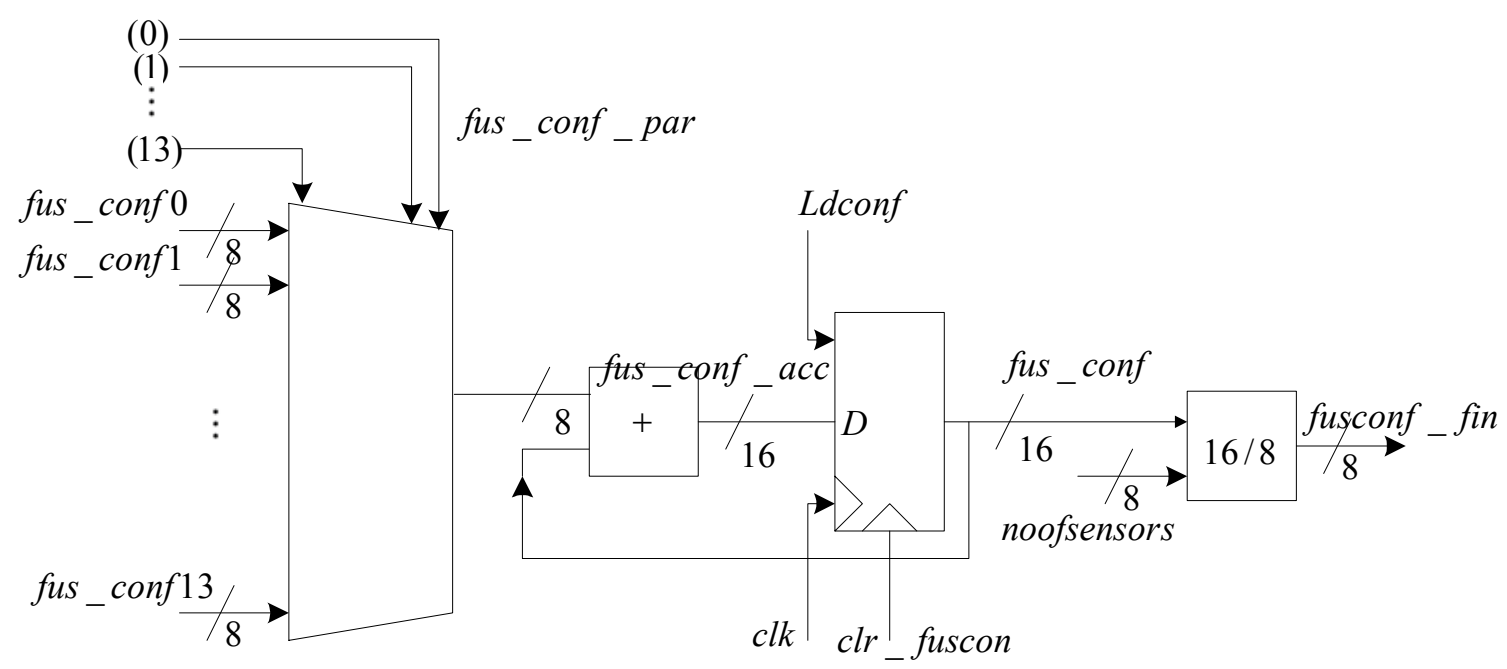

Note: $16 / 8$ divider is implemented outside fus_conf module 
Fused_Conf (P5)
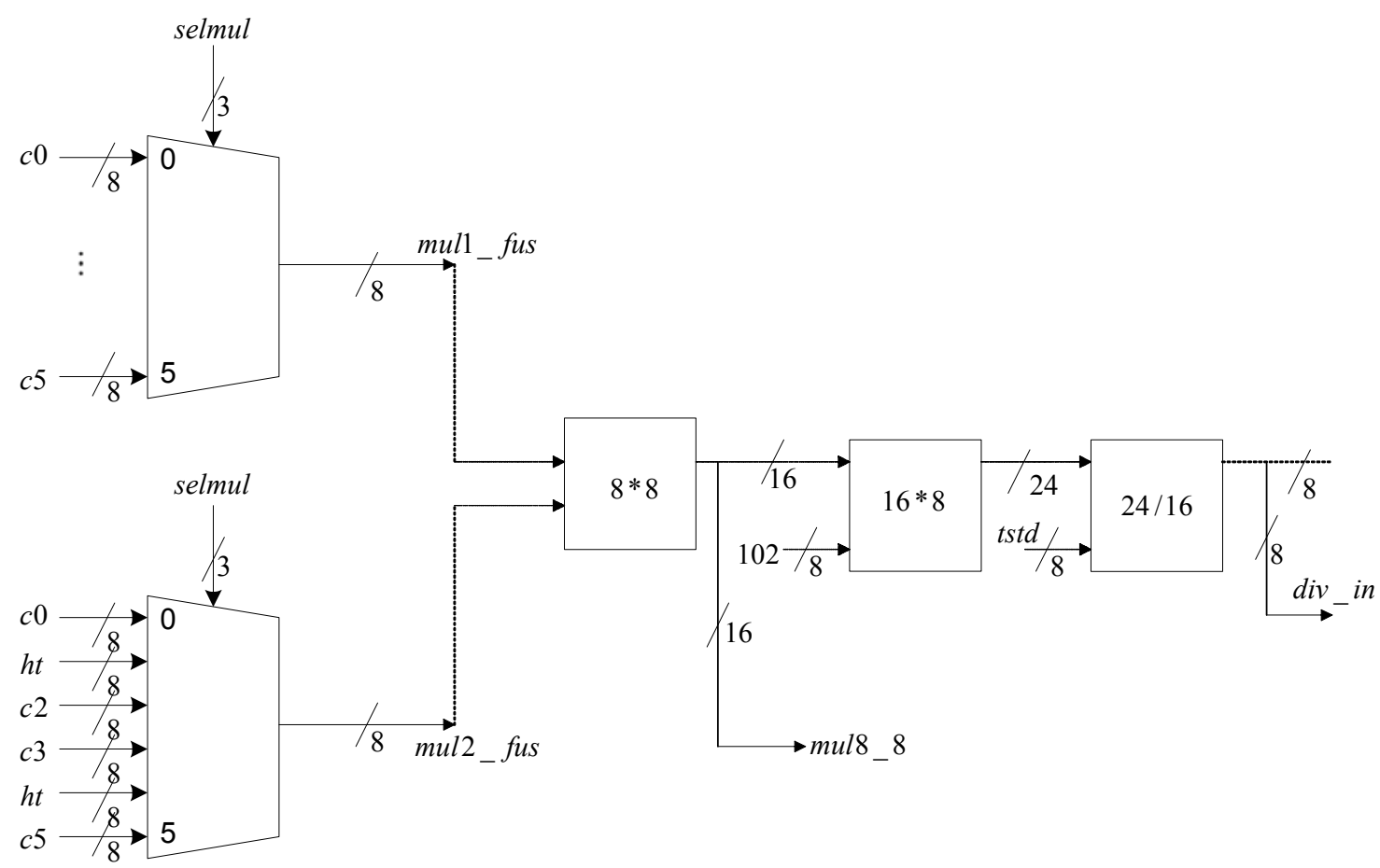

NOTE: All multipliers and dividers are implemented outside the fus_conf module.

The dotted lines show the buses going outside the module 


\section{INPUT MUX}

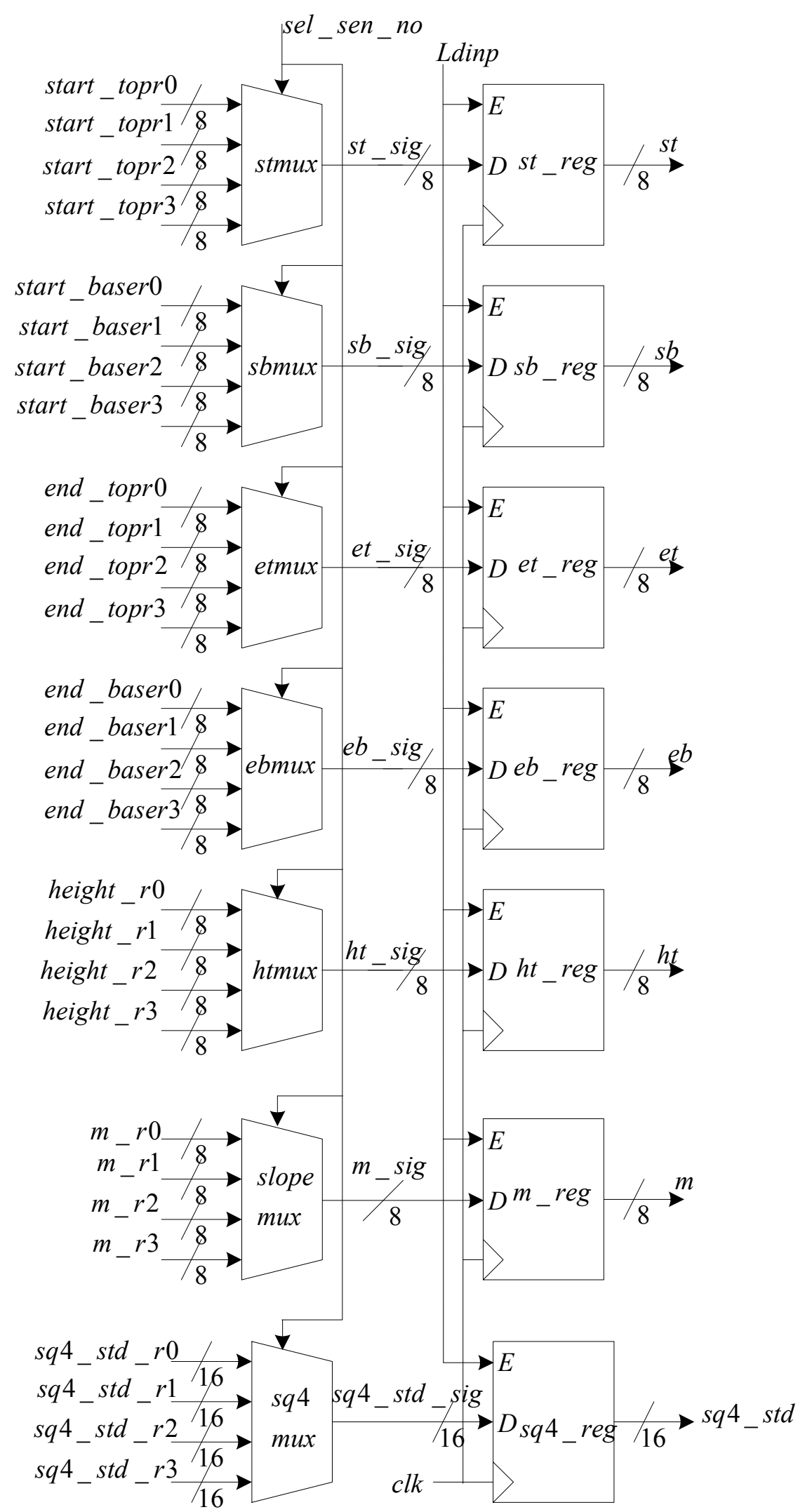


Multipliers for MSF

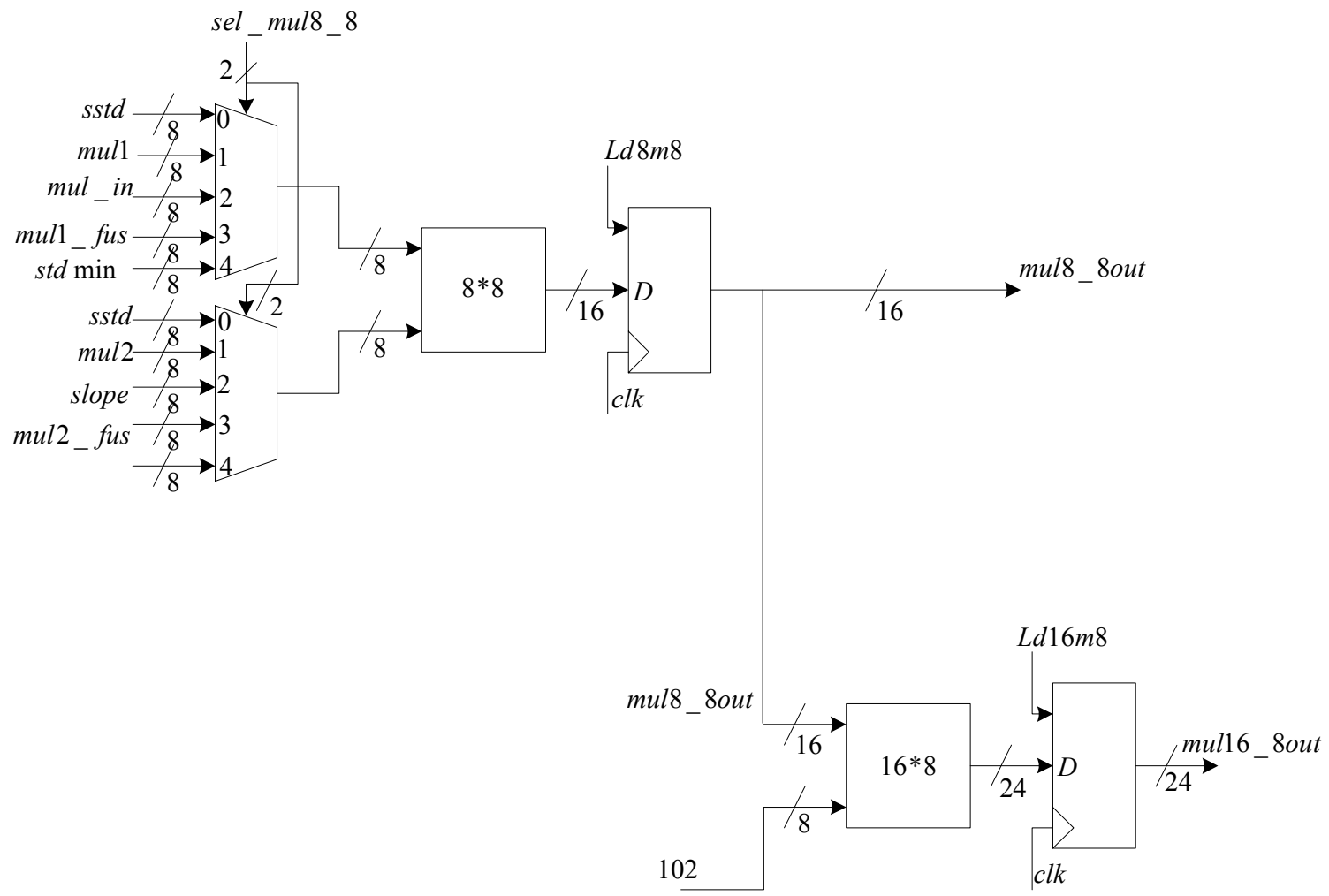

NOTE:

Sel mul8 8 Inputs from

0 : val_sen

1: Area

2: Trap Height

3: $\quad$ Fused Conf

4: Span 
REARRANGE
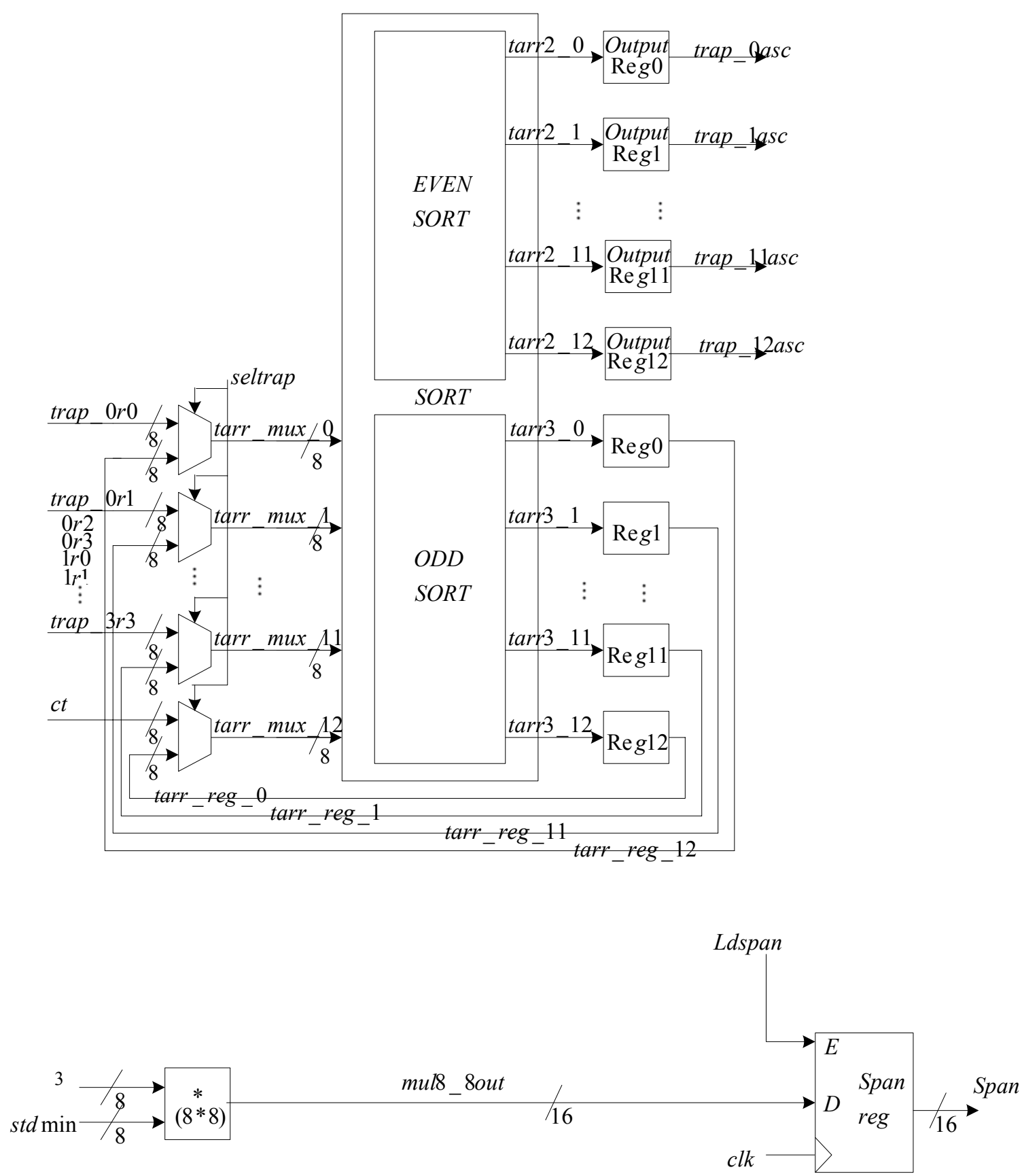

NOTE: $8 * 8$ multiplier is used to calculate $3 *$ stdmin for span module. The multiplier is implemented outside the Rearrange module. 


\section{SORT}

\section{Even Sort}

\section{Odd Sort}

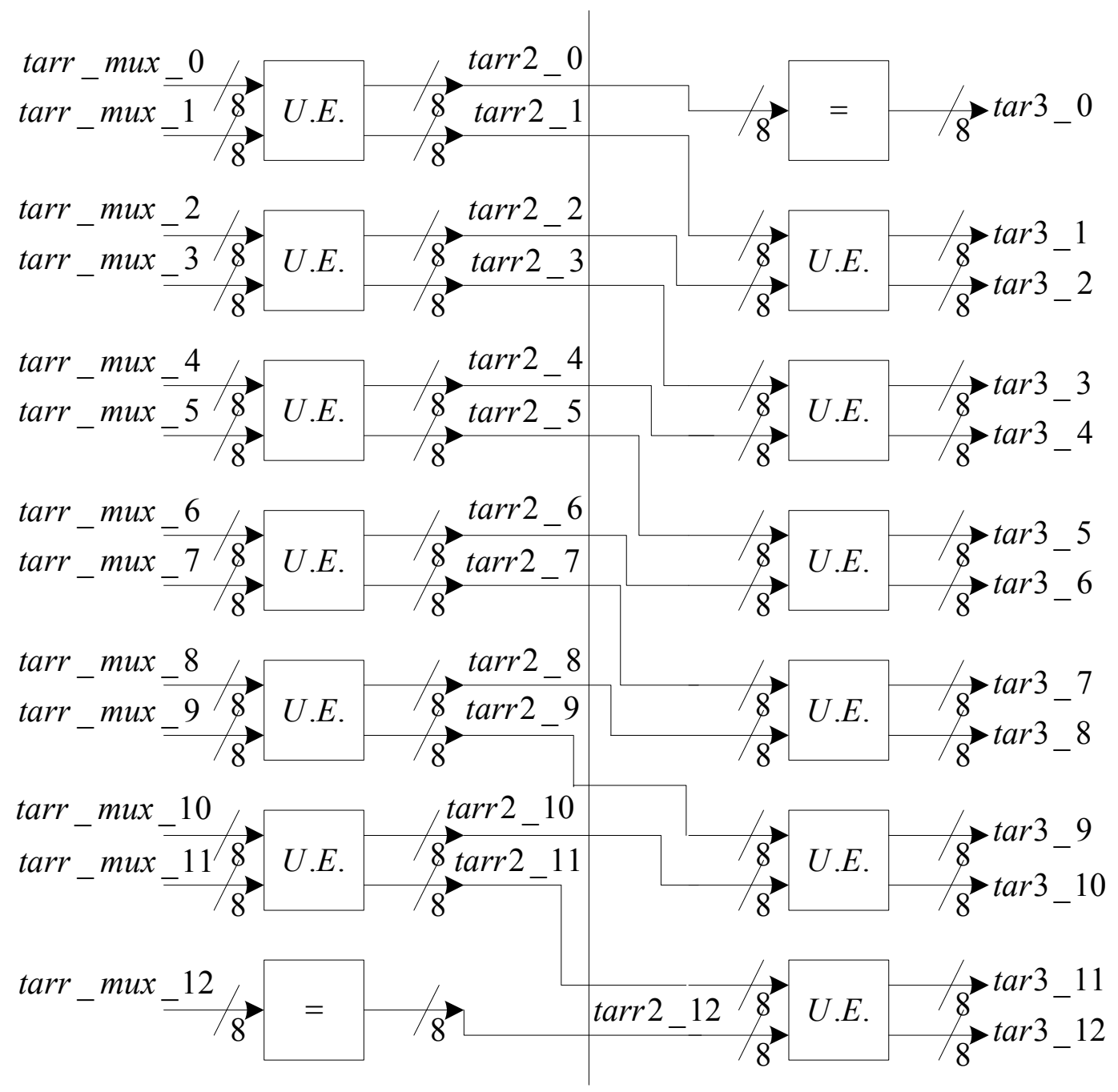




\section{Trap_ht (p1)}
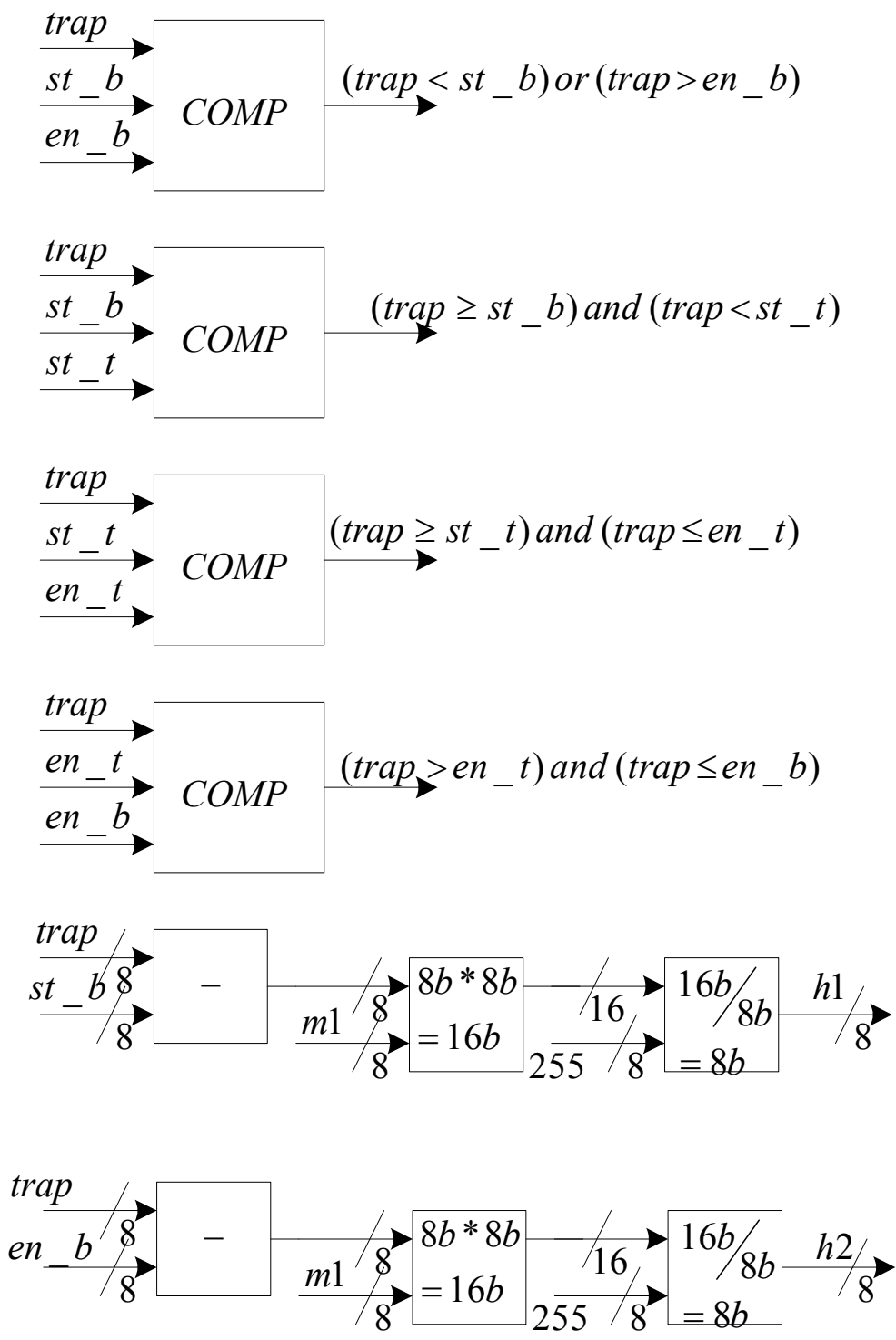
Trap_ht (p2)

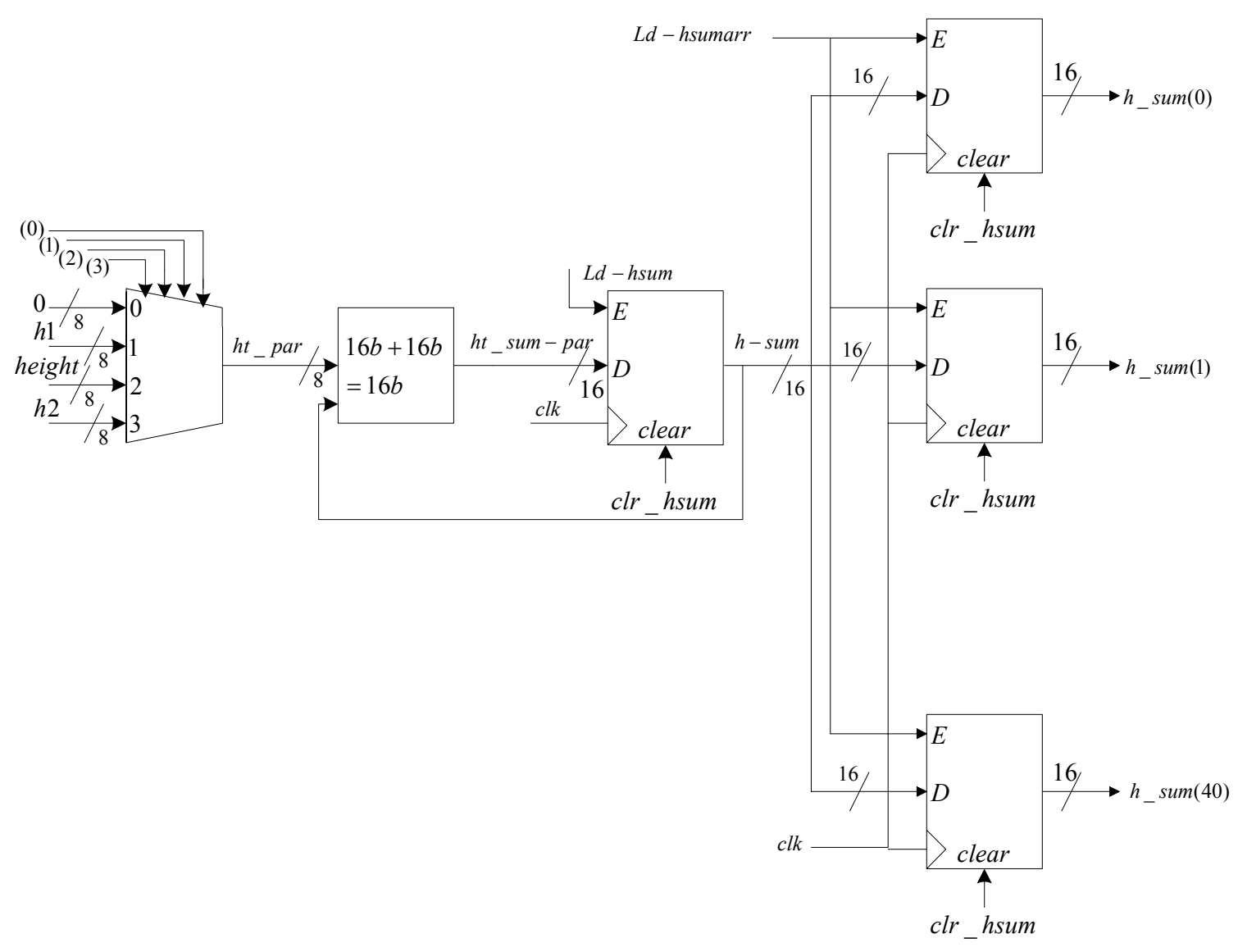


Trap_ht (p3)

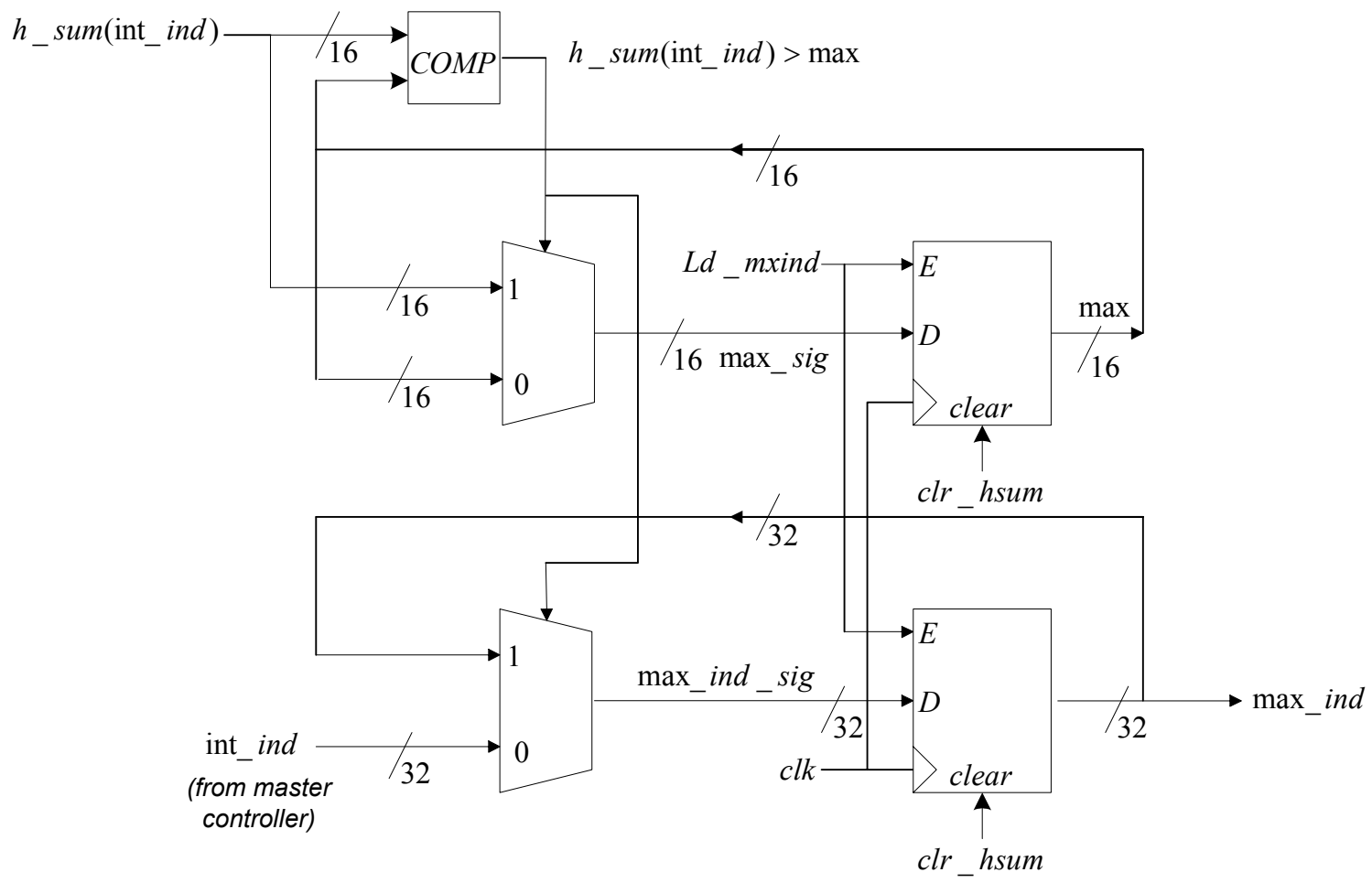




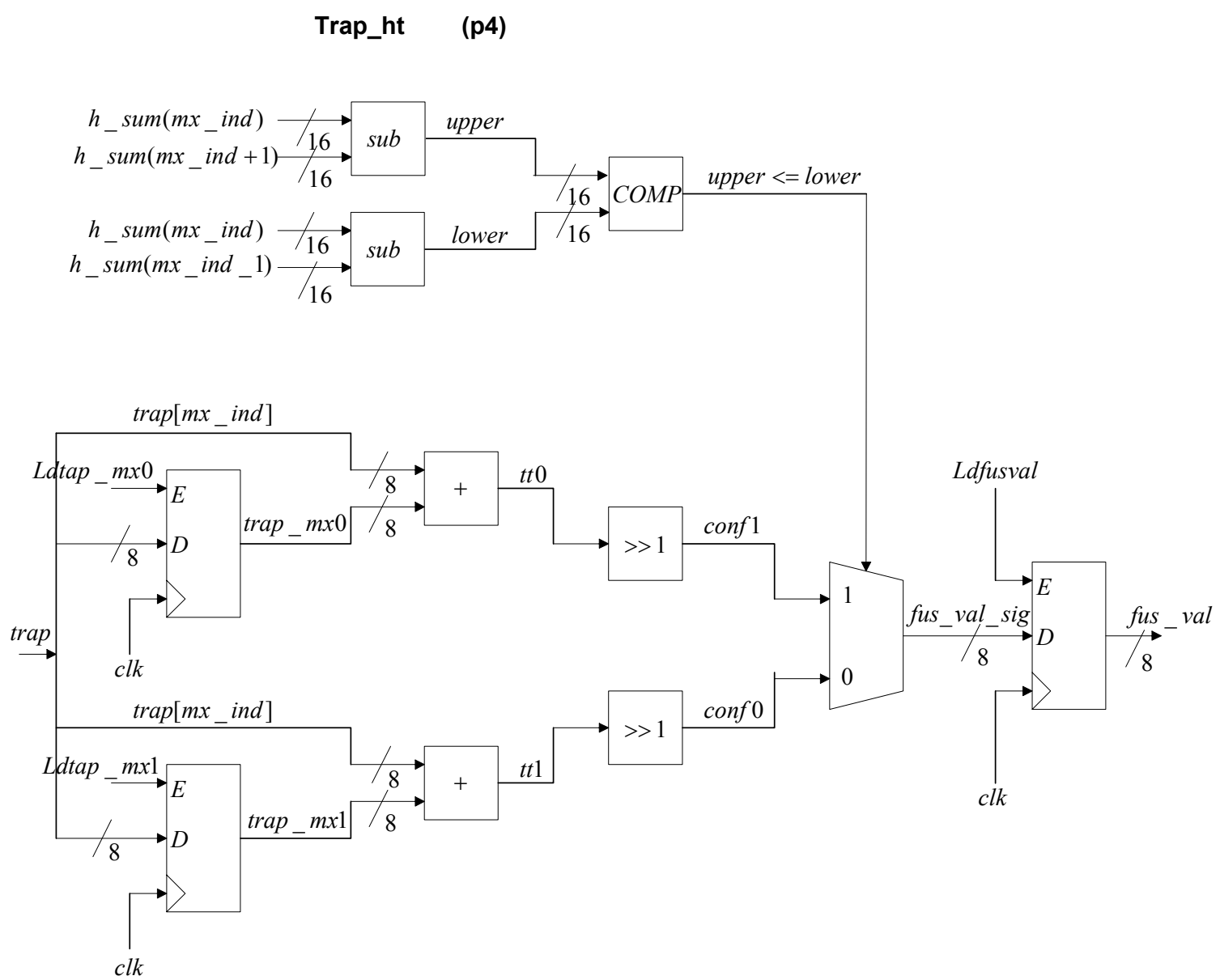

NOTE: Trapht2 and max all run parallel

Trapht2, max, fus_val are combined into a single module trap2max.vhd 
Unit Element (UE)

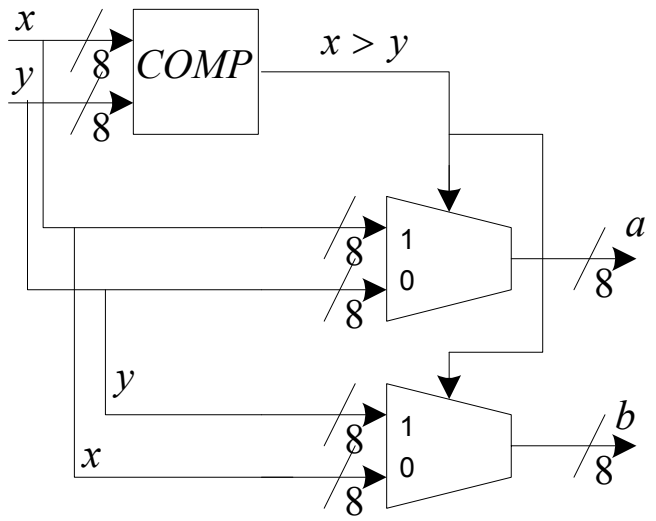




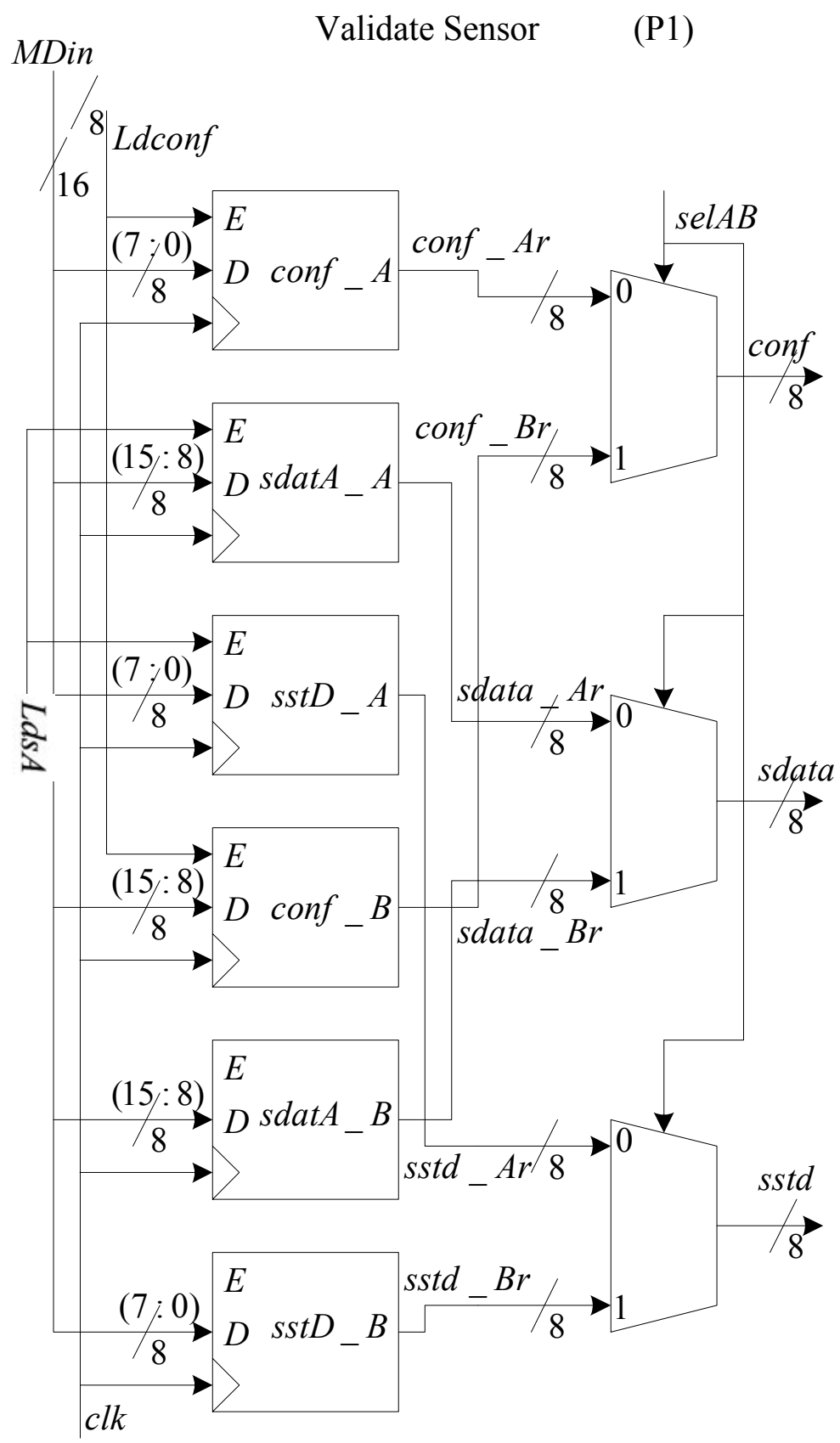


Validate Sensor

(P2)

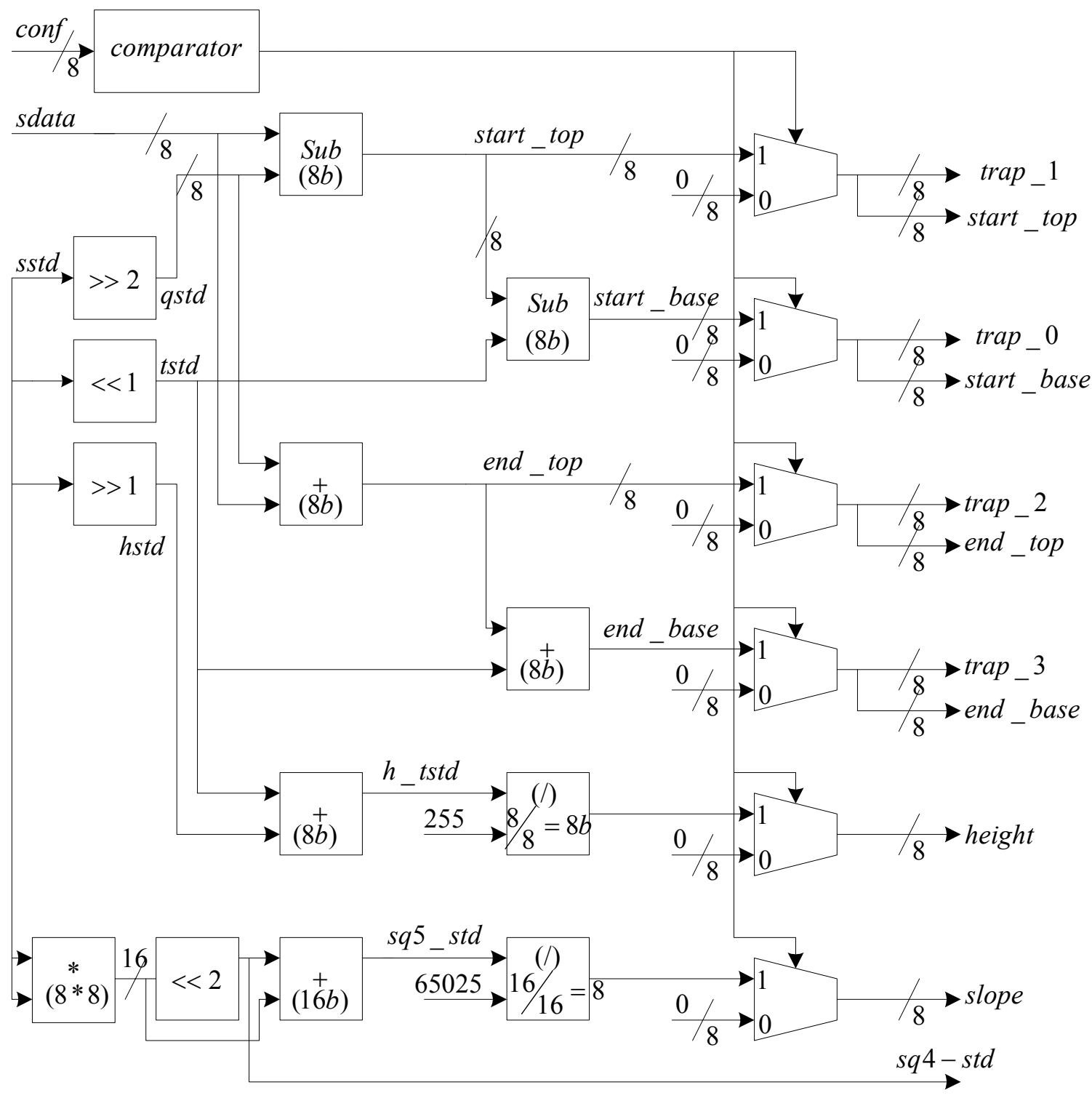




\section{VALIDATE SENSOR (p3)}

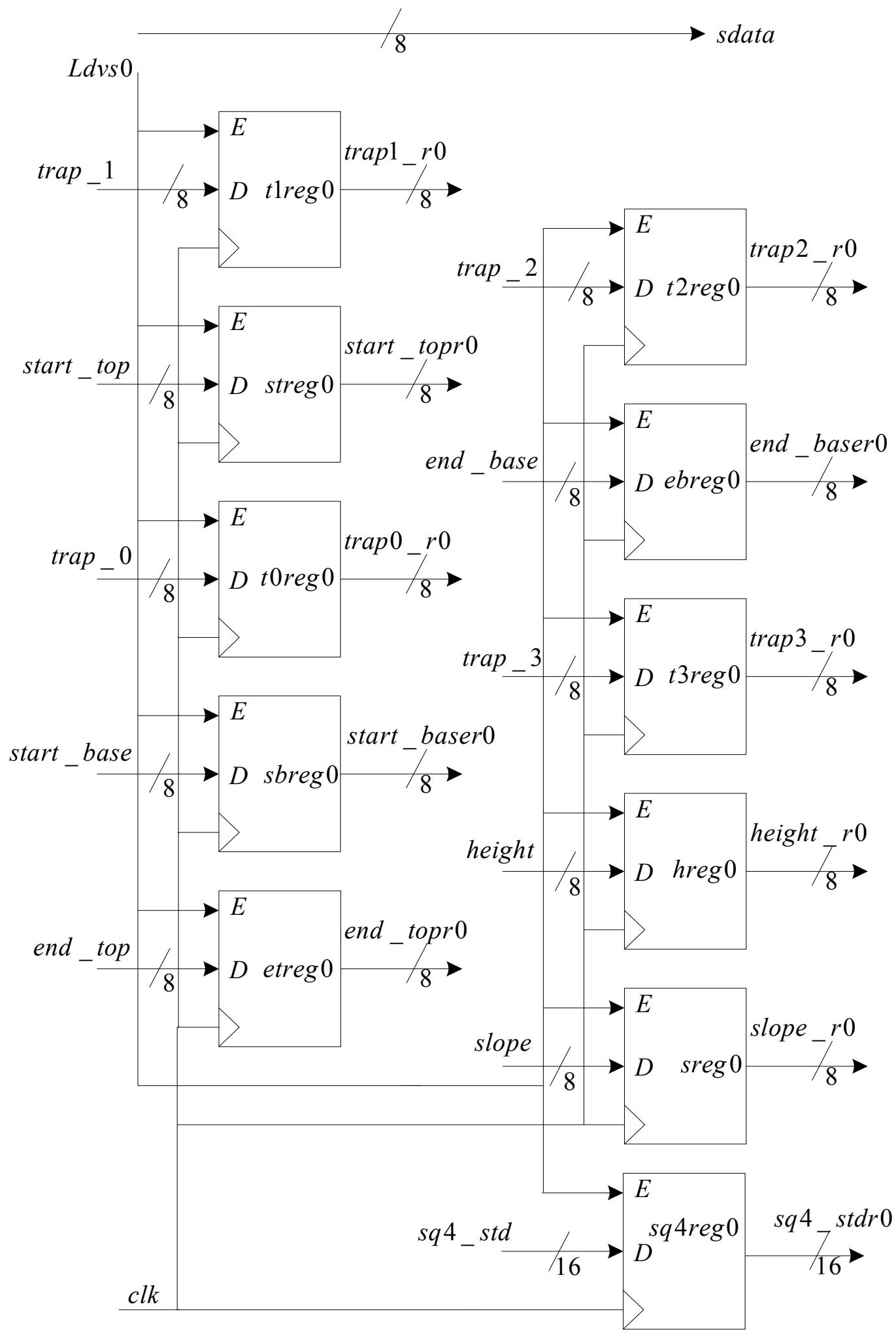




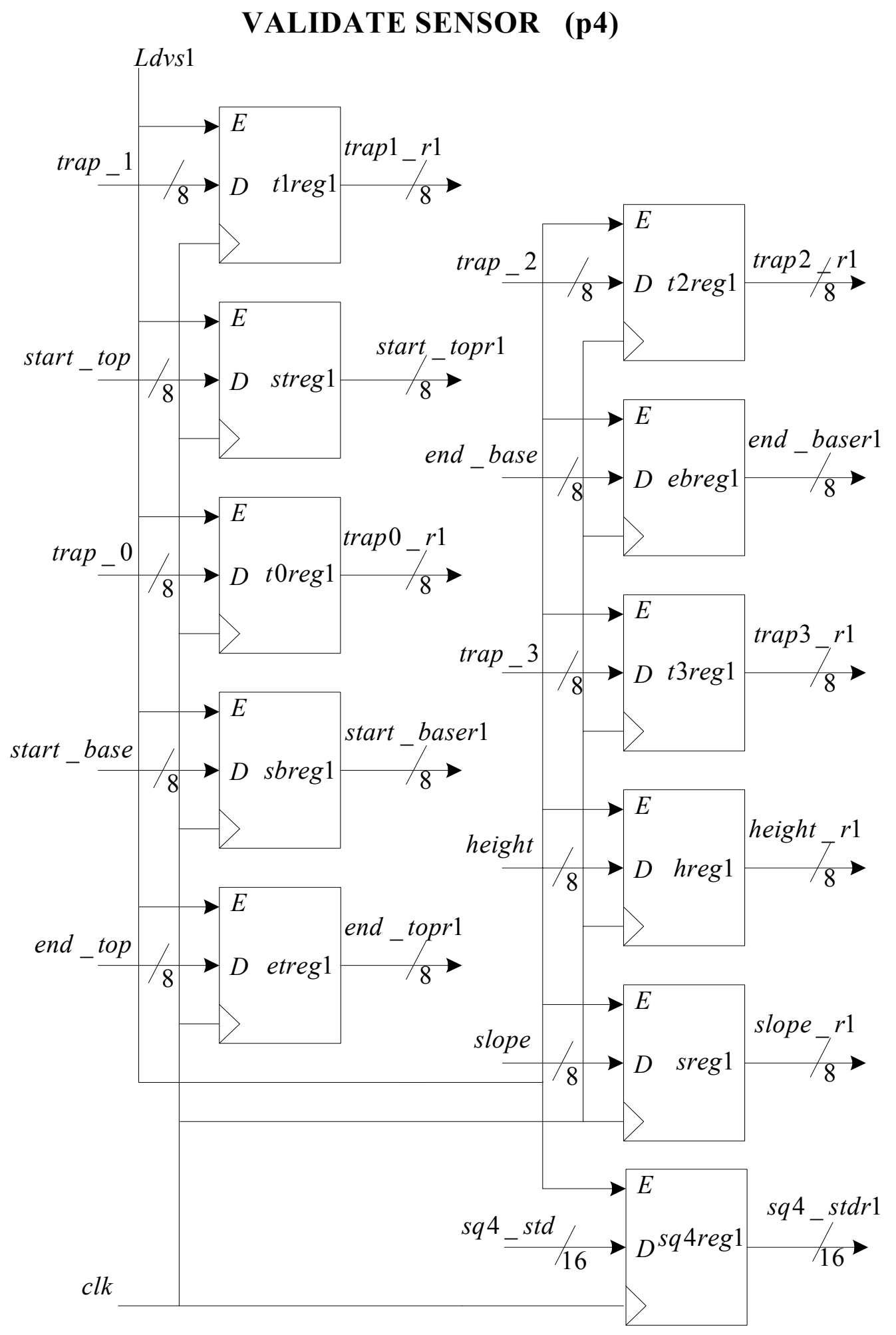

Note: The same diagram can be repeated with appropriate modifications for the rest of the sensors 


\section{REFERENCES}

[1] Nagrath, I.J and Gopal, M, Control Systems Engineering, Second Edition, New Age International (P) Ltd., Publishers, 1995.

[2] Maciejowski,J.M, Multivariable Feedback Design, Addison-Wesley Publishers Ltd., 1990.

[3] Richard R.Brooks and S.S.Iyengar, Multi-Sensor Fusion - Fundamentals and Applications with Software, Prentice Hall, Inc., New Jersey, 1998.

[4] Ren C.Luo and Michael G Kay, "Multiple Integration and Fusion in Intelligent Systems," IEEE Transactions on Systems, Man and Cybernetics, vol. 19, no. 5, September 1989.

[5] R.C.Luo, M.Lin, and R.S.Scherp, "Dynamic multi-sensor data fusion system for intelligent robots," IEEE Journal Robotics and Automation, vol. RA-4, no. 4, pp. 385396, 1988.

[6] Keith E. Holbert, A.Sharif Heger and Nahrul K. Alang-Rashid, "Redundant Sensor Validation by Using Fuzzy Logic," Nuclear Science and Engineering, vol. 118, pp. 54-64, 1994. 
[7] Asok Ray and Rogelio Luck, "An Introduction to sensor Signal Validation in Redundant Measurement Systems," IEEE Control Systems Magazine, vol. 11, no. 2, pp. 43, Feb 01, 1991.

[8] Marcello R Napolitano, Charles Neppach, Van Casdorph , Steve Naylor, Mario Innocenti and Giovanni Silvestri, "Neural Network Based Scheme for Sensor Failure Detection, Identification and Accomodation," Journal of Guidance, Control and Dynamics, vol. 18, no. 6, Dec 1995.

[9] Mohamed Abdelrahman and Senthil Subramaniam, "An Intelligent Signal Validation System for Cupola Furnace - Part 1 and Part 2," American Control Conference, San Diego, 1999.

[10] Janice C, Yang and David Clarke, "A Self-Validating Thermocouple," IEEE Transactions on Control Systems Technology, vol. 5 no. 2 March 1997.

[11] M.P.Henry and D.W.Clarke, "The Self-Validating sensor: Rationale definitions, and examples," Control Eng. Practice, vol. 1, no. 4, pp. 585-610, 1993.

[12] T.M.Tsai and H.P.Chou, "Sensor fault detection with the single sensor parity relation", Nuclear Science and Engineering," vol. 114, pp. 1411993 
[13] Mathieu Mercadal, "Sensor Failure detection using Generalized Parity relations for Flexible Structures," Journal of Guidance, Control and Dynamics, vol. 12, no. 1, Feb 1989.

[14] Jeff Frolik, C.V.PhaniShankar and Steve Orth, "Fuzzy Rules for Automated Sensor Self-Validation and Confidence Measure," In Proceedings of American Control Conference, June 2000.

[15] Bernard Friedland, Advanced Control System Design, Prentice Hall, Inc., New Jersey, 1996.

[16] K.J.Astrom and B.Wittenmark, Adaptive Control, Addison-Wesley Publishing Co., Reading, MA 1989.

[17] Liu Hsu; Aldayr D. de Araujo; Ramon R. Costa, "Analysis and design of I/O based variable structure adaptive control. (input-output variable structure model reference adaptive control systems)," IEEE Transactions on Automatic Control, vol. 39, no.1, pp. 4, Jan 1994.

[18] E. Burdet, A. Codourey, "Evaluation of parametric and nonparametric nonlinear adaptive controllers (Nonlinear controllers)," Robotica, vol. 16, no. 1, 1998. 
[19] Judith Hocherman-Frommer; Sanjeev R. Kulkarni; Peter J. Ramadge, "Controller switching based on output prediction errors," IEEE Transactions on Automatic Control, vol. 43, no. 5, pp. 596, May 1998

[20] Michel Barbeau; Froduald Kabanza; Richard St.-Denis, "A method for the synreport of controllers to handle safety, liveness, and real-time constraints," IEEE Transactions on Automatic Control, vol. 43, no. 11, pp. 1543, November 1998.

[21] Specht, D.F., "Probabilistic Neural Networks," Neural Networks, November 1990.

[22] Ronald R. Yager and Dimitar P. Filev, Essentials of Fuzzy Modeling and Control, John Wiley \& Sons, 1994.

[23] Jeff Frolik and Mohamed Abdelrahman, "Synreport of Quasi-Redundant sensor Data: A Probabilistic Approach," In Proceedings of American Control Conference, 2000.

[24] Hassan K. Khalil, Nonlinear Systems, Second edition, Prentice Hall Inc., 1996

[25] Mohamed Abdelrahman, Kevin Moore, Eric Larsen, Denis Clark and Paul King, "Experimental Control of a Cupola Furnace," In Proceedings of American Control Conference, 1998. 
[26] Pascal Gahinet, Arkadi Nemiroviski, Alan Laub, and Mahmoud Chilali, "LMI Control toolbox 1.0," The Math Works Inc.

[27] Jeff Frolik, C.V.Phanishankar and Steve Orth, “Fuzzy Rules for Automated Sensor Self-Validation and Confidence Measure”, Proc. of American Control Conference, 2000, pp. 2912-2916.

[28] Mohamed Abdelrahman, Parameshwaran Kandasamy and Jeff Frolik, “ $\boldsymbol{A}$ Methodology for the Fusion of Redundant Sensors", Proc. of American Control Conference, 2000, pp. 2917-2922.

[29] Jeff Frolik and Mohamed Abdelrahman, "Synthesis of Quasi-Redundant sensor Data: Probabilistic Approach”, Proc. Of American Control Conference, 2000, pp. 2922-2926.

[30] Vipin Vijayakumar, Mohamed Abdelrahman, Jeff Frolik, "A Convenient Methodology for the hardware implementation of fusion of Quasi-Redundant Sensors", Proc. Of $32^{\text {nd }}$ South-Eastern Symposium on System Theory, Florida, Mar 2000, pp. 349-353. 
